\title{
Mechanistic Studies of Conjugated Fatty Acid Biosynthesis
}

By

\section{Palash Bhar, M.Sc.}

A thesis submitted to the Department of Chemistry

in partial fulfillment of the requirements for the degree of

Doctor of Philosophy

To

The Faculty of Graduate and Postdoctoral Affairs

Department of Chemistry

Carleton University

Ottawa, Canada

December 2011 
Library and Archives

Canada

Published Heritage

Branch

395 Wellington Street

Ottawa ON K1A ON4

Canada
Bibliothèque et

Archives Canada

Direction du

Patrimoine de l'édition

395 , rue Wellington

Ottawa ON K1A ON4

Canada
Your file Votre référence

ISBN: 978-0-494-87740-1

Our file Notre référence

ISBN: $978-0-494-87740-1$

\section{NOTICE:}

The author has granted a nonexclusive license allowing Library and Archives Canada to reproduce, publish, archive, preserve, conserve, communicate to the public by telecommunication or on the Internet, loan, distrbute and sell theses worldwide, for commercial or noncommercial purposes, in microform, paper, electronic and/or any other formats.

The author retains copyright ownership and moral rights in this thesis. Neither the thesis nor substantial extracts from it may be printed or otherwise reproduced without the author's permission.
AVIS:

L'auteur a accordé une licence non exclusive permettant à la Bibliothèque et Archives Canada de reproduire, publier, archiver, sauvegarder, conserver, transmettre au public par télécommunication ou par l'Internet, prêter, distribuer et vendre des thèses partout dans le monde, à des fins commerciales ou autres, sur support microforme, papier, électronique et/ou autres formats.

L'auteur conserve la propriété du droit d'auteur et des droits moraux qui protege cette thèse. $\mathrm{Ni}$ la thèse ni des extraits substantiels de celle-ci ne doivent être imprimés ou autrement reproduits sans son autorisation.
In compliance with the Canadian Privacy Act some supporting forms may have been removed from this thesis.

While these forms may be included in the document page count, their removal does not represent any loss of content from the thesis.
Conformément à la loi canadienne sur la protection de la vie privée, quelques formulaires secondaires ont été enlevés de cette thèse.

Bien que ces formulaires aient inclus dans la pagination, il n'y aura aucun contenu manquant. 


\begin{abstract}
The mechanism of conjugated fatty acid biosynthesis as it occurs in plants has been examined by incubating deuterium-labelled linoleates with a number of yeast strains expressing the requisite FAD2 type enzymes: $\Delta^{8,10}$-desaturase (Calendula officinialis); $\Delta^{11,13}$-desaturase (Aleurites fordii Hemsl.) and $\Delta^{10,90 \mathrm{H}}$-desaturase (Dimorphotheca sinuata).
\end{abstract}

The stereochemistry of calendic acid (octadec-8E,10E,12Z-trienoic acid) formation was probed by monitoring the fate of deuterium from $(R)$ - and $(S)-8-\mathrm{d}_{1}$-octadec-9Z,12Zdienoates and $(R)$ - and $(S)-11-\mathrm{d}_{1}$-octadec-9Z,12Z-dienoates (linoleates). The latter substrates were synthesized by a chemoenzymatic route that involved the use of a yeast $\Delta^{12}$ mutant to introduce the $12 Z$ double bond. Calendate is formed from linoleate by synremoval of the $8 \mathrm{H}_{R}$ and $11 \mathrm{H}_{S}$ hydrogens as expected.

Using similar methodology, it was shown that $\alpha$-eleostearic acid (octadec$9 Z, 11 E, 13 E$-trienoic acid) formation was formed from linoleate by syn-removal of the $8 \mathrm{H}_{S}$ and $11 \mathrm{H}_{R}$ hydrogens. This result required the postulation of a revised substrate conformation. By comparing the deuterium kinetic isotope effects at C-11 (large) and C14 (small), the site of initial hydrogen abstraction was determined to be at C-11.

Dimorphecolate $\quad((R)-9$-hydroxyoctadec-10E,12E-octadecadienoic acid) is biosynthesized from oleate by tandem $12 E$-desaturation /allylic oxidation. It was demonstrated that the latter step is initiated by isotopically sensitive hydrogen abstraction at $\mathrm{C} 11$, followed by hydroxyl rebound to $\mathrm{C}-9$ of the allylic radical intermediate.

All biotransformations could be described by an active site model that is common to all FAD2 type homologues operating on linoleate substrates. 


\section{Acknowledgements}

I would like to take this opportunity to greatly acknowledge my thesis supervisor Dr. Peter H. Buist for introducing me to this ever interesting field of desaturase research. I will always be thankful to him for his sincere guidance, continuous support and enthusiastic encouragement during this tenure of research without that it would have been quite impossible to overcome this passage. I am deeply influenced by his research skills, teaching ability and the unique way of critically analyzing the issues and hope that this lesson will pave my way in future.

Many thanks to those people who assisted in this thesis and were generous enough with their time and advice: Jeff Manthrope (Carleton University) for various consultations during the synthesis of the compounds; John M. Dyer (US Department of Agriculture-Agricultural Research Services Southern Regional Research Center, New Orleans, Louisiana) for supplying the tung FADX plasmid; Matthew Anderson (Dr. Jim Wright's group, Carleton University) for the CLUSTALL comparison; Dr. Clem Kazakoff (University of Ottawa) for GC-MS analyses; Jim Logan (Carleton University) for technical support to kept the computer running and Tony O'Neil (Carleton University) for IR. Nevertheless to mention, the success of this thesis work would not have been possible without the active participation from our collaborator, Dr. Patrick S. Covello and Darwin W. Reed (Plant Biotechnology Institute, National Research Council Canada, Saskatoon). Especially, I am thankful to these two persons for taking care about me and helping me out with the biological experiments during my two visits to Saskatoon. Financial support for this research work from Natural Sciences and Engineering Research Council of Canada (NSERC) and Carleton University are also sincerely acknowledged. 
I would also like to express my gratitude to all of the past and present members of the Delta Force family whose presence in the lab throughout these years was always enjoyable and helpful for friendly discussions going. In particular, I am grateful to Amy E. Tremblay and Christopher Mattice with whom I have had the opportunity to share the museum.

Above all, my sincere gratitude goes to all of my family members especially to my parents, uncle, sisters, nephews and brother-in-law for their unconditional love, support and continuous encouragement throughout all these years and being there whenever I needed them. Finally, I would like to thank my beloved wife Punam for being faithful on me and always supporting myself with her love and affection during various ups and downs in pursuing this $\mathrm{PhD}$ degree. She has been patient enough waiting for me in India while I am studying here and my deepest love goes to her. 


\section{Table of Contents}

Page no.

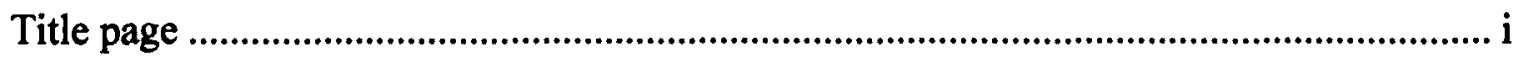

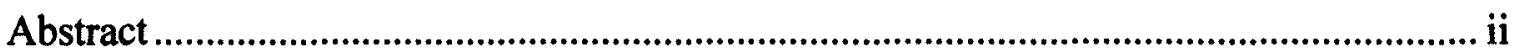

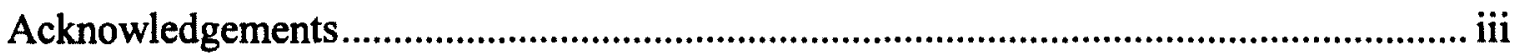

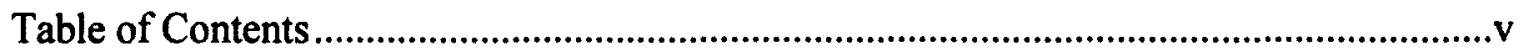

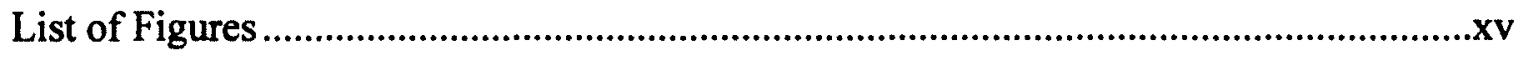

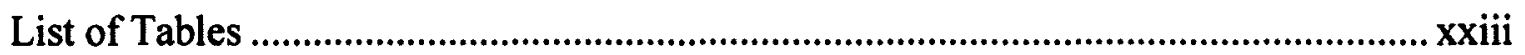

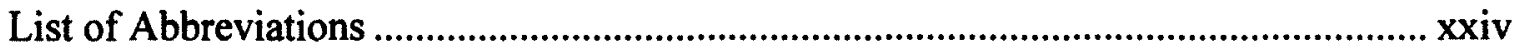

\section{Chapter 1: Biosynthesis of Conjugated Fatty acids}

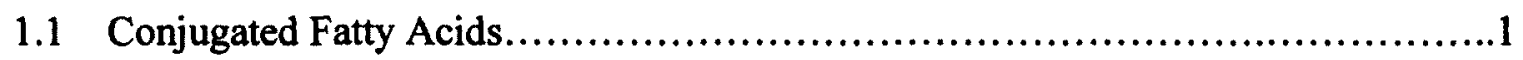

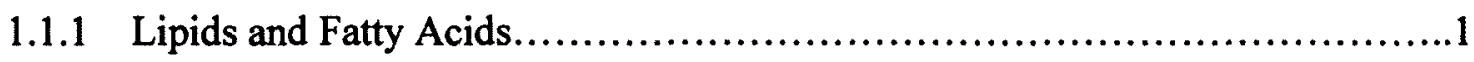

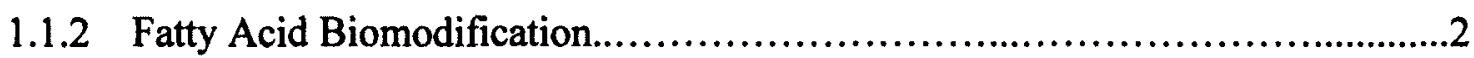

1.1 .3 Conjugated Fatty Acids...................................................

1.1.4 Conjugated Fatty Acids in Animals......................................9

1.1.5 Conjugated Fatty Acids in Insects......................................11

1.1.6 Conjugated Fatty Acids in Plants........................................13 


\section{Table of Contents Continued}

1.2 Fatty Acid Desaturases...................................................... 17

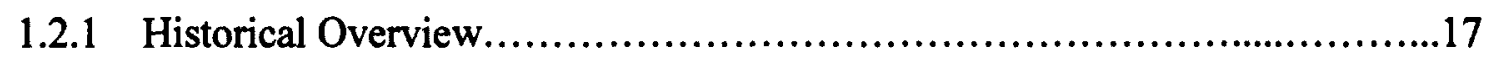

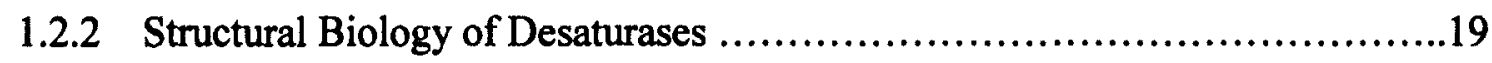

1.2.3 Soluble Plant Desaturases.......................................................19

1.2.4 Membrane-Bound Desaturases............................................22

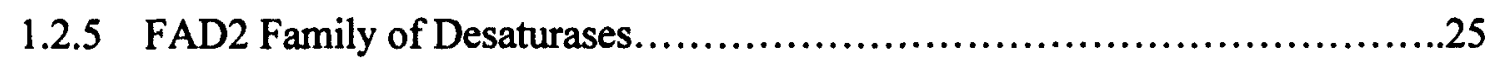

1.3 Mechanistic Investigations of Desaturases...................................26

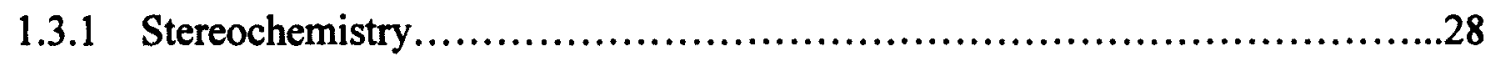

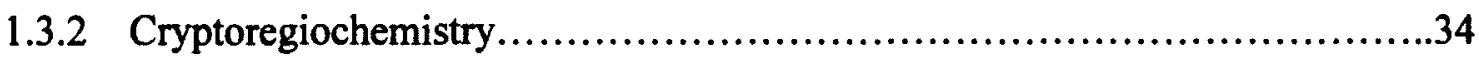

1.3.3 Highly Conserved Active Site Structural Motif ..............................39

1.3.4 Chemoselectivity: Dehydrogenation/hydroxylation Connection...............40

1.4 Mechanistic Investigations of 1,4-Desaturation................................45

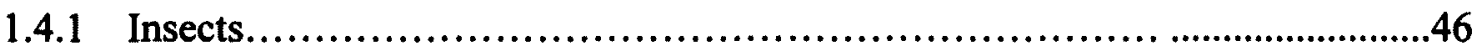

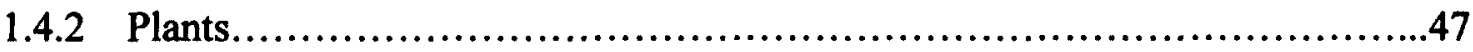

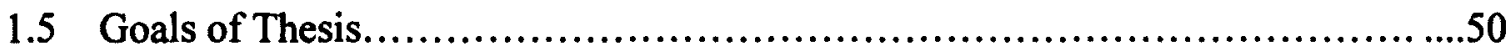

Chapter 2: Stereochemistry of Calendic acid Biosynthesis in Calendula officinalis

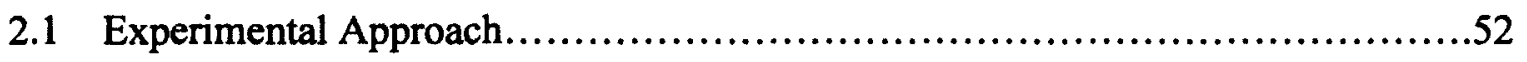

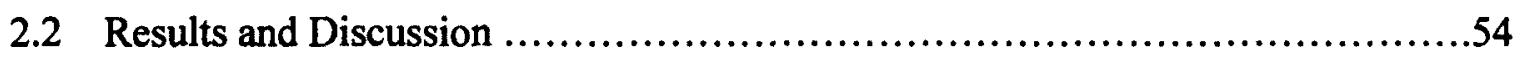

2.2.1 Synthesis of methyl $(8 R)$ - and $(8 S)-\left[8-{ }^{2} \mathrm{H}_{1}\right]$-octadeca- $9 Z, 12 Z$-dienoate,

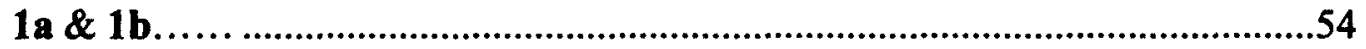

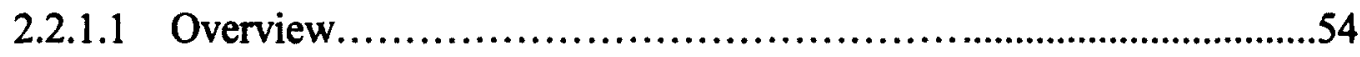

2.2.1.2 Preparation of $(8 S)$ - and $(8 R)$-Ethyl $\left[8-{ }^{2} \mathrm{H}_{1}\right]-8$-Hydroxyoctanoate,

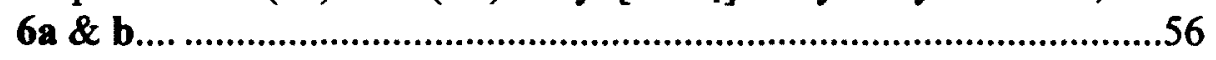




\section{Table of Contents Continued}

2.2.1.3 Stereochemical Analysis of (8R)- and (8S)-Ethyl

$\left[8-{ }^{2} \mathrm{H}_{1}\right]-8$-Hydroxyoctanoate.

2.2.1.4 Conversion of Chiral Ethyl $\left[8-{ }^{2} \mathrm{H}_{1}\right]-8$-Hydroxyoctanoates to Corresponding Chiral $\left[8-{ }^{2} \mathrm{H}_{1}\right]$-Linoleates.

2.2.2 Chemoenzymatic Synthesis of Methyl (11R)- and (11S)-[11- $\left.{ }^{2} \mathrm{H}_{1}\right]-$ Octadeca-9Z,12Z-Dienoate.

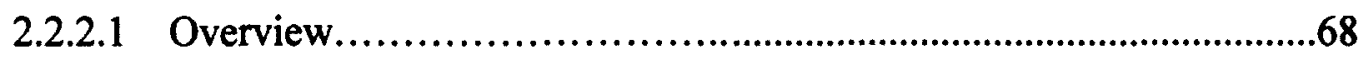

2.2.2.2 Synthesis of Methyl (11S)- and $(11 R)-\left[11-^{2} \mathrm{H}_{1}\right]$-Octadeca-

9Z-enoate, 3a \& 3b

2.2.2.3 Enzymatic Conversion of Methyl (11S)- and $(11 R)-\left[11-{ }^{2} \mathrm{H}_{1}\right]-$ Octadeca-9Z-enoate to Corresponding Linoleates, $\mathbf{2} \mathbf{a}$ and $\mathbf{2 b}$.

2.2.2.4 Attempted Synthesis of Chiral Methyl $\left[11-{ }^{2} \mathrm{H}_{1}\right]$-Octadeca9Z,12Z-Dienoates.

2.2.3 Incubation Experiments Using Chiral Methyl $\left[8{ }^{2} \mathrm{H}_{1}\right]-$ and

$\left[11-{ }^{2} \mathrm{H}_{1}\right]$-Linoleates.

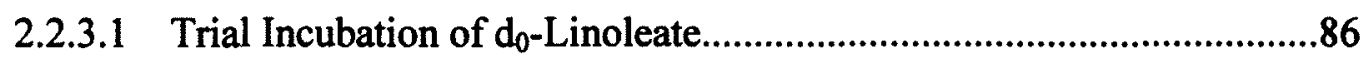

2.2.3.2 Incubation of Chiral $\left[8{ }^{2} \mathrm{H}_{1}\right]-$ and $\left[11-{ }^{2} \mathrm{H}_{1}\right]$-Linoleates...........................

2.2.3.3 Incubation of Racemic $\left[11-{ }^{2} \mathrm{H}_{1}\right]$-Linoleates.....................................94

2.3 Conclusion

Chapter 3: Mechanistic Studies of a-Eleostearate Formation in Aleurites fordii Hemsl.

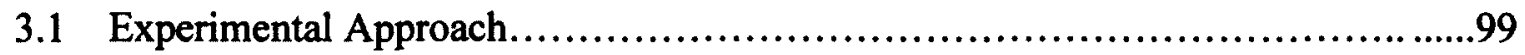

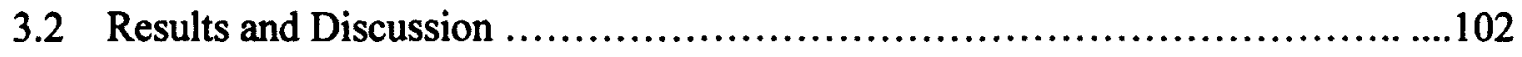

3.2.1 Construction of Yeast Mutant Containing $\Delta^{11,13}$-Desaturase..............................102

3.2.1.1 Amplification of the template DNA, Cloning and

Expression in E. coli. .102 


\section{Table of Contents Continued}

3.2.1.2 Preparation of $S$. cerevisiae mutant expressing $\Delta^{11,13}$-desaturase enzyme from Aleurites fordii Hemsl.

3.2.2 Cryptoregiochemistry of $\Delta^{11,13}$-Desaturation.............................105

3.2.2.1 Synthesis of Methyl $\left[11,11-{ }^{2} \mathrm{H}_{2}\right]$-Linoleate.........................105

3.2.2.2 Synthesis of Methyl $\left[14,14-{ }^{2} \mathrm{H}_{2}\right]$-Linoleate.......................109

3.2.2.3 Incubation of Substrates: Trial Incubation of $d_{0}$-Linoleate.................113

3.2.2.4 Intermolecular Kinetic Isotope Effect (KIE) Studies...........................116

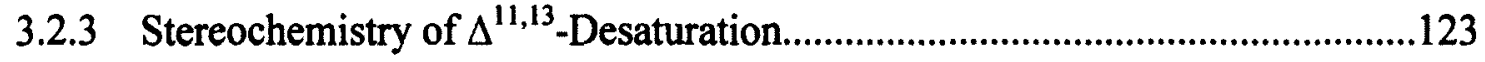

3.2.3.1 Synthesis of Chiral Methyl $\left[14-{ }^{2} \mathrm{H}_{1}\right]$-Octadeca-9Z,12Z-

Dienoates.

3.2.3.2 Stereochemical analysis of Acetylide Anion Attack on Chiral CHD Tosylates.

3.2.3.3 Incubation of Chiral $d_{1}$-Linoleates

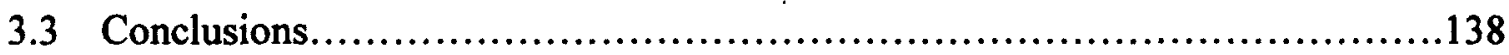

Chapter 4: Stereochemistry of Dimorphecolic acid Biosynthesis in Dimorphotheca sinuata and associated KIE Evaluation

$4.1 \quad$ Experimental Approach..................................................... 140

4.2 Incubation Experiments Using Dideuterated Substrates: KIE Studies.............143

4.2.1 Trial Incubation of $\mathrm{d}_{0}$-Oleate.........................................

4.2.2 Intermolecular Kinetic Isotope Effect (KIE) Studies.......................145

4.3 Incubation of Chiral $d_{1}$-Oleates: Stereochemical Studies.......................150

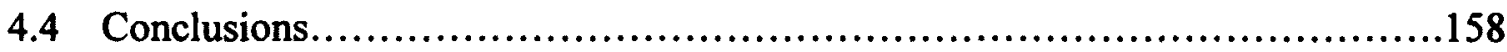




\section{Table of Contents Continued}

\section{Chapter 5: Summary and Future Directions}

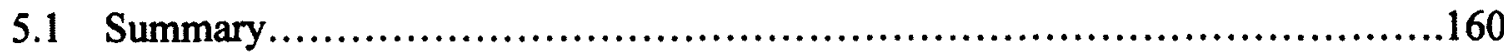

5.2 Future Directions............................................................ 162

\section{Chapter 6: Part I Experimental}

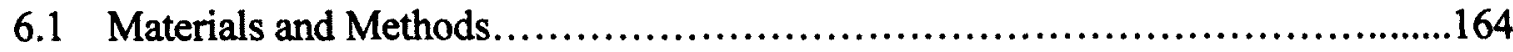

6.2 Synthesis of Deuterium-Labeled Fatty Acids...................................167

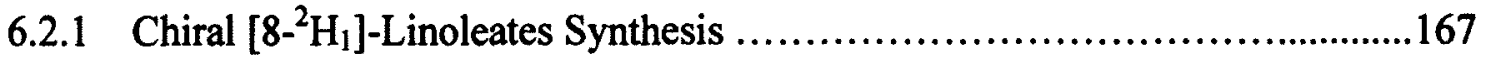

6.2.1.1 Ethyl 8-hydroxyoctanoate .....................................167

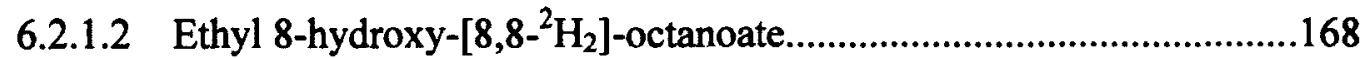

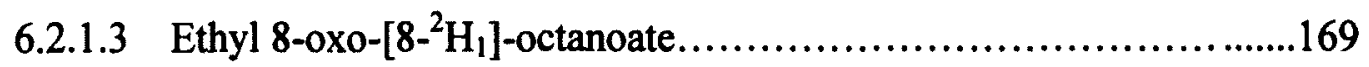

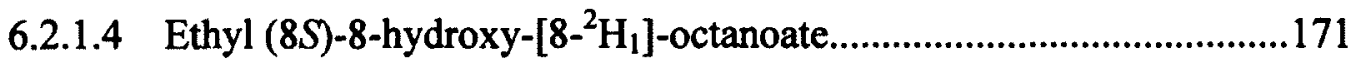

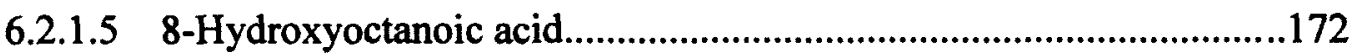

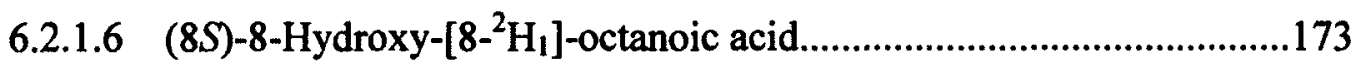

6.2.1.7 8-(p-Tolylsulfonyloxy)-octanoic acid.................................................174

6.2.1.8 (8S)-8-(p-Tolylsulfonyloxy)-[8 $\left.{ }^{2} \mathrm{H}_{1}\right]$-octanoic acid..........................175

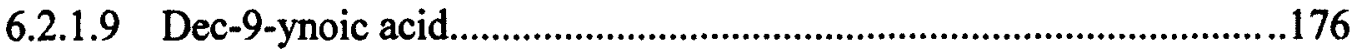

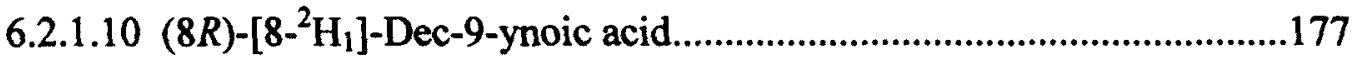

6.2.1.11 Methyl dec-9-ynoate........................................................................178

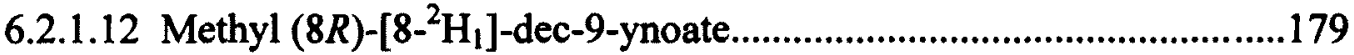

6.2.1.13 1-(p-Tolylsulfonyloxy)-oct-2-yne......................................................180

6.2.1.14 Methyl octadeca-9,12-diynoate...........................................................181 


\section{Table of Contents Continued}

6.2.1.15 Methyl $(8 R)-\left[8-{ }^{2} \mathrm{H}_{1}\right]$-octadeca-9,12-diynoate..................................182

6.2.1.16 Methyl octadeca-9Z,12Z-dienoate...................................................183

6.2.1.17 Methyl (8R)-[8- $\left.{ }^{2} \mathrm{H}_{1}\right]$-octadeca-9Z,12Z-dienoate..............................185

6.2.1.18 Methyl (8S)-[8- $\left.{ }^{2} \mathrm{H}_{1}\right]$-octadeca-9Z,12Z-dienoate.............................186

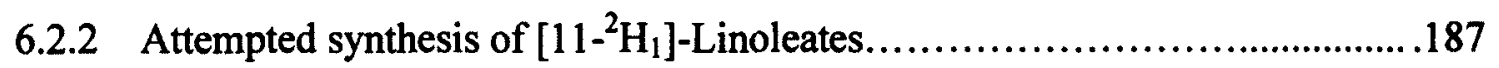

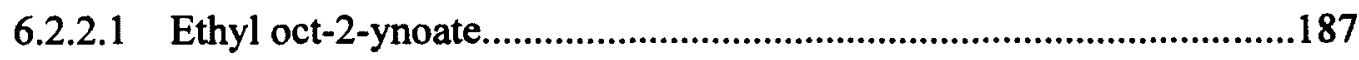

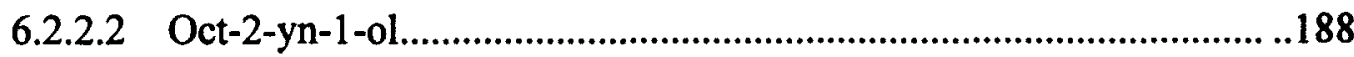

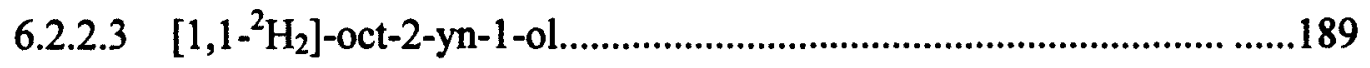

6.2.2.4 2-Octynal.......................................................................................190

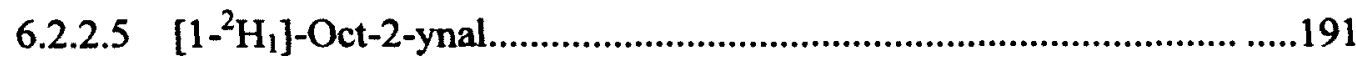

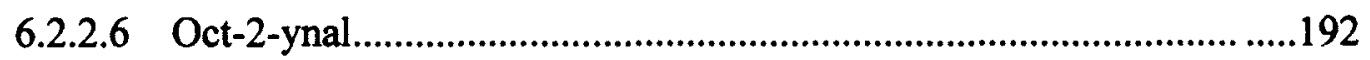

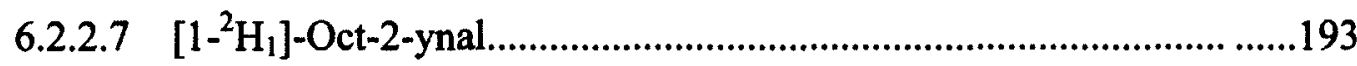

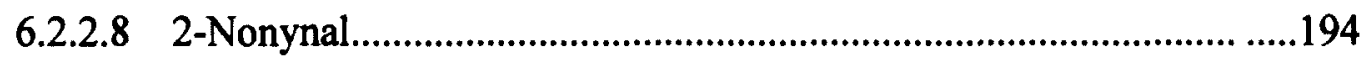

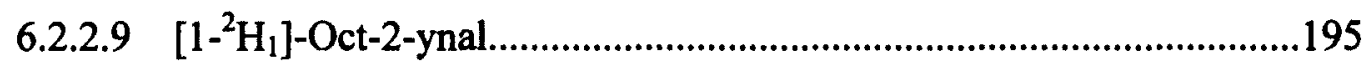

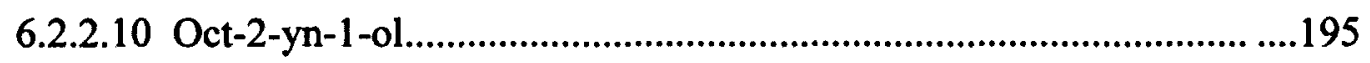

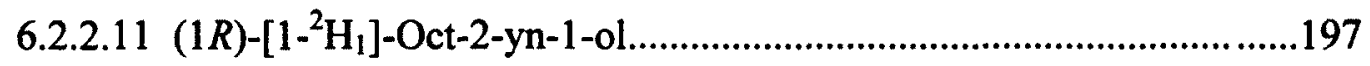

6.2.2.12 (1R)-1-(p-tolylsulfonyloxy)-[1- $\left.{ }^{2} \mathrm{H}_{1}\right]$-oct-2-yne...................................198

6.2.2.13 Octadeca-9,12-diynoic acid................................................................199

6.2.2.14 (11S)-[11- $\left.{ }^{2} \mathrm{H}_{1}\right]$-octadeca-9,12-diynoic acid......................................200

6.2.2.15 Methyl octadeca-9,12-diynoate............................................................201

6.2.2.16 Methyl $(11 S)$-[11- $\left.{ }^{2} \mathrm{H}_{1}\right]$-octadeca-9,12-diynoate..............................202

6.2.2.17 Methyl (11S)-[11- $\left.{ }^{2} \mathrm{H}_{1}\right]$-octadeca-9Z,12Z-dienoate..........................203 


\section{Table of Contents Continued}

6.2.2.18 Methyl $(11 R)-\left[11{ }^{2} \mathrm{H}_{1}\right]$-octadeca-9Z,12Z-dienoate...........................204

6.2.3 Chiral $\left[11-{ }^{2} \mathrm{H}_{1}\right]-$ Linoleates (via $\Delta^{12}$-Desaturation of Chiral $\left[11-^{2} \mathrm{H}_{1}\right]-$ Oleates..205

6.2.3.1 2-(8-Bromooctyl-1-oxy)tetrahydropyran...........................................205

6.2.3.2 2-(8-Iodooctyl-1-oxy)tetrahydropyran.................................................206

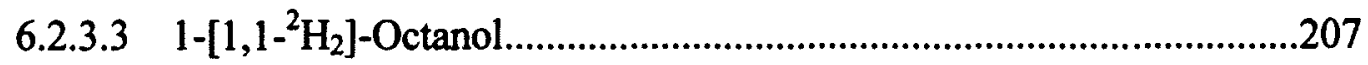

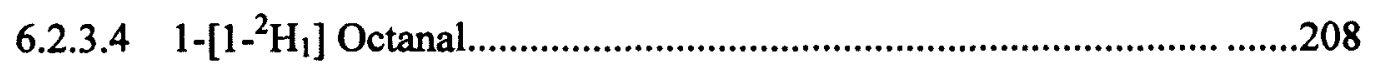

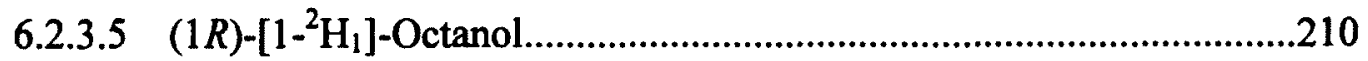

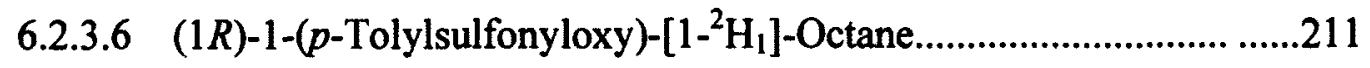

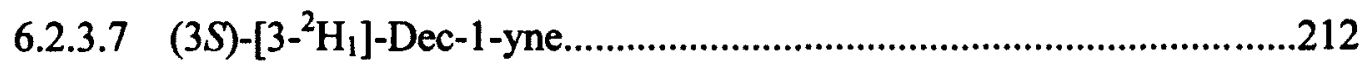

6.2.3.8 2-((11S)-[11. $\left.{ }^{2} \mathrm{H}_{1}\right]$-Octadeca-9-ynyl-1-oxy)tetrahydropyran..............213

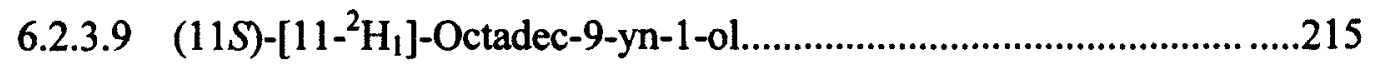

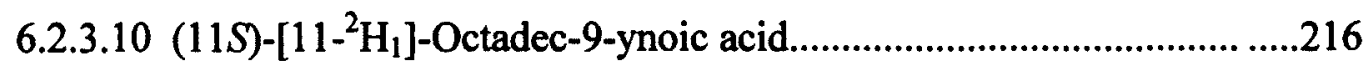

6.2.3.11 Methyl $(11 S)$-[11- $\left.{ }^{2} \mathrm{H}_{1}\right]$-Octadec-9-ynoate...........................218

6.2.3.12 Methyl (11S)-[11- $\left.{ }^{2} \mathrm{H}_{1}\right]-$ Octadeca-9Z-enoate.....................................219

6.2.3.13 Methyl (11R)-[11- $\left.{ }^{2} \mathrm{H}_{1}\right]$-Octadeca-9Z-enoate................................220

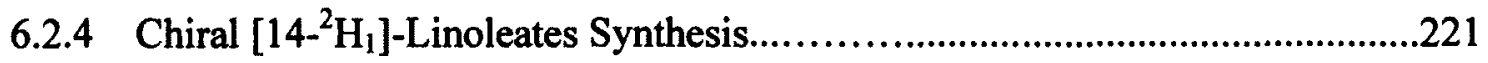

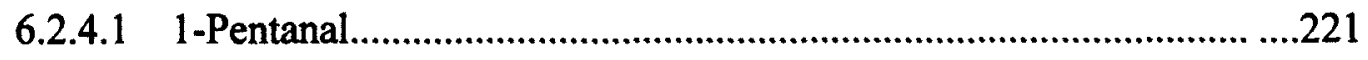

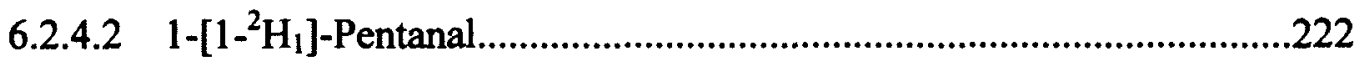

6.2.4.3 1-Pentanol......................................................................................223

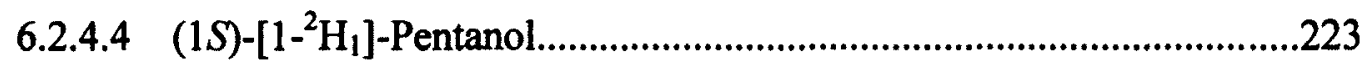

6.2.4.5 1-(p-Tolylsulfonyloxy)-pentane........................................224

6.2.4.6 (1S)-1-(p-Tolylsulfonyloxy)-[1- $\left.{ }^{2} \mathrm{H}_{1}\right]$-pentane.....................225 


\section{Table of Contents Continued}

6.2.4.7 2-((4R)-[4- $\left.{ }^{2} \mathrm{H}_{1}\right]$-octa-2-ynyl-1-oxy)tetrahydropyran...........................226

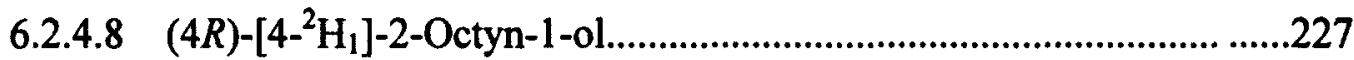

6.2.4.9 (4R)-1-(p-Tolylsulfonyloxy)-[4- $\left.{ }^{2} \mathrm{H}_{1}\right]$-oct-2-yne.............................228

6.2.4.10 Methyl (14R)-[14- $\left.{ }^{2} \mathrm{H}_{1}\right]$-octadeca-9,12-diynoate..............................229

6.2.4.11 Methyl (14R)-[14- $\left.{ }^{2} \mathrm{H}_{1}\right]$-octadeca-9Z,12Z-dienoate.............................231

6.2.4.12 Methyl (14S)-[14- $\left.{ }^{2} \mathrm{H}_{1}\right]$ octadeca-9Z,12Z-dienoate...........................232

6.2.5 Assessment of Enantiomeric Purity of the Chiral Synthetic Intermediates.....233

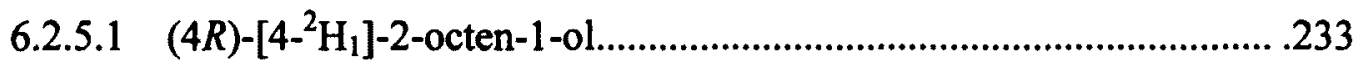

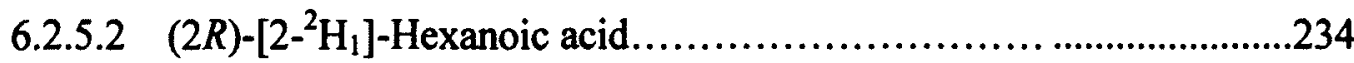

6.2.5.3 (S)-2-Methoxy-2-oxo-1-phenylethyl $(2 R)-\left[2-{ }^{2} \mathrm{H}_{1}\right]$-hexanoate............ 235

6.2.5.4 (S)-2-Methoxy-2-oxo-1-phenylethyl $(2 S)$-[2- $\left.{ }^{2} \mathrm{H}_{1}\right]$-hexanoate.............236

6.2.5.5 (R)-[1(S)- $\left.{ }^{2} \mathrm{H}_{1}\right]$-pentyl 3,3,3-trifluoro-2-methoxy2-phenylpropanoate...........................................................................236

6.2.5.6 (R)-[1(R)- $\left.{ }^{2} \mathrm{H}_{1}\right]$-pentyl 3,3,3-trifluoro-2-methoxy2-phenylpropanoate.

6.2.5.7 (R)-[1(R)- $\left.{ }^{2} \mathrm{H}_{1}\right]-O c t-2-y n e ~ 3,3,3-$ trifluoro-2-methoxy2-phenylpropanoate. .238

6.2.5.8 (R)-[1(S)- $\left.{ }^{2} \mathrm{H}_{1}\right]-\mathrm{Oct}-2$-yne 3,3,3-trifluoro-2-methoxy2-phenylpropanoate. .239

6.2.6 Methyl $\left[11,11-{ }^{2} \mathrm{H}_{2}\right]$-Linoleate Synthesis.................................240

6.2.6.1 $\left[1,1-{ }^{2} \mathrm{H}_{2}\right]-1-(p-T o l y l s u l f o n y l o x y)-o c t-2-y n e \ldots \ldots \ldots \ldots \ldots \ldots \ldots \ldots \ldots \ldots \ldots \ldots \ldots . . . . .240$

6.2.6.2 Methyl $\left[11,11-{ }^{2} \mathrm{H}_{2}\right]$-octadeca-9,12-diynoate....................................241

6.2.6.3 Methyl $\left[11,11-{ }^{2} \mathrm{H}_{2}\right]$-octadeca-9Z,12Z-dienoate...............................242

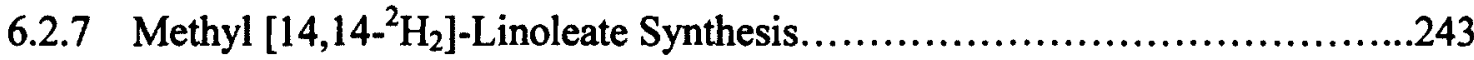




\section{Table of Contents Continued}

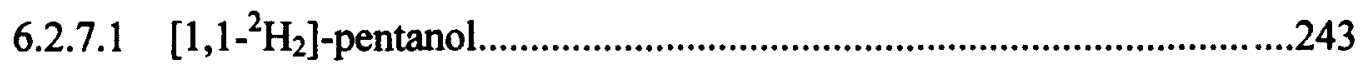

6.2.7.2 1-Bromopentane................................................................................244

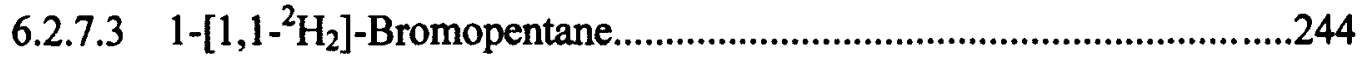

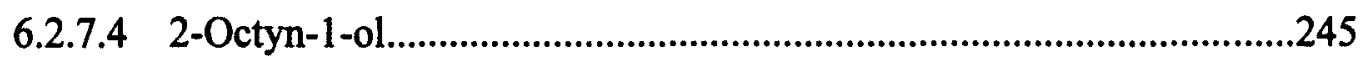

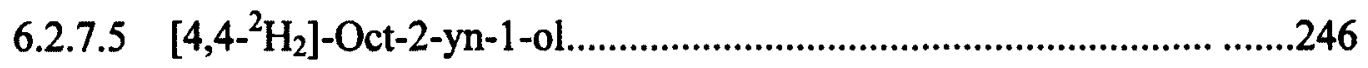

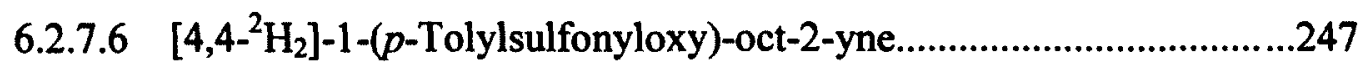

6.2.7.7 Methyl $\left[14,14-{ }^{2} \mathrm{H}_{2}\right]$-octadeca-9,12-diynoate.....................................248

6.2.7.8 Methyl $\left[14,14-{ }^{2} \mathrm{H}_{2}\right]$-octadeca-9Z,12Z-dienoate..............................249

\section{Chapter 6: Part II Biological Experiments}

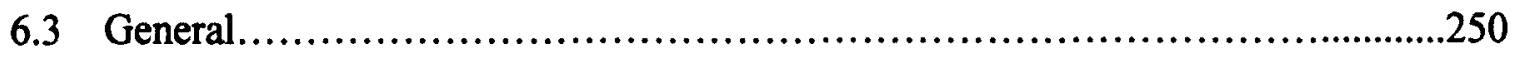

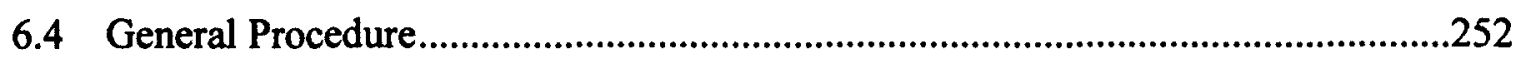

6.4.1 Saponification of Fatty Acid Substrates.........................................................252

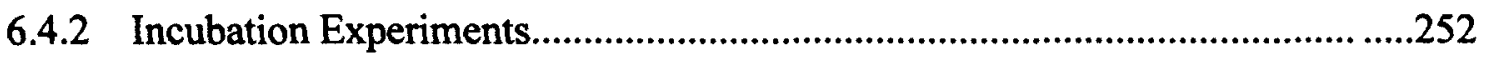

6.4.3 Extraction of the Cellular Fatty Acids............................................................253

6.4.4 Derivatization of Fatty Acids for GC-MS Analysis.........................................254

6.5 Microbial synthesis of methyl $(11 R)$ - and $(11 S)$ - $\left[11-^{2} \mathrm{H}_{1}\right]$-octadeca9Z,12Z-dienoate by $\Delta^{12}$-desaturation of corresponding methyl octadeca-

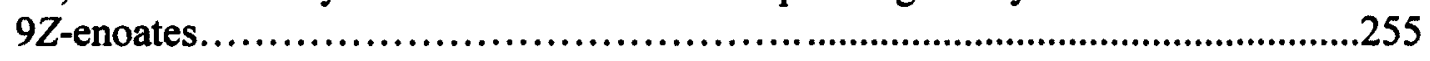

6.6 Construction of the Tung 1,4-Desaturase (FADX)-containing Yeast Mutant......257

6.6.1 Amplification of the Template Plasmid DNA using PCR Reaction............257

6.6.2 TOPO Cloning of PCR Products and Expression in E. coli...........................259

6.6.2.1a LB Medium....................................................................................259

6.6.2.1b LB agar Plates...................................................................................259 


\section{Table of Contents Continued}

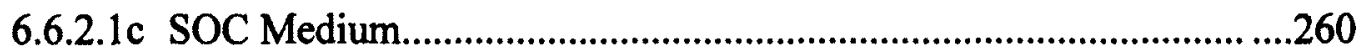

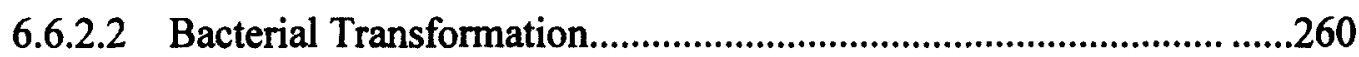

6.6.2.2.1 Preparation of TOPO ${ }^{\oplus}$ Cloning Reaction............................................260

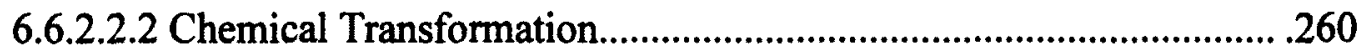

6.6.2.2.3 Long-term storage..................................................................................261

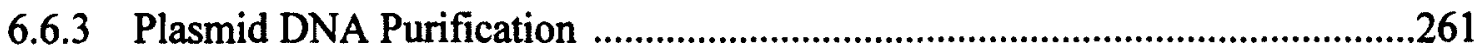

6.6.3.1 Preparation of Bacterial Lysate............................................................261

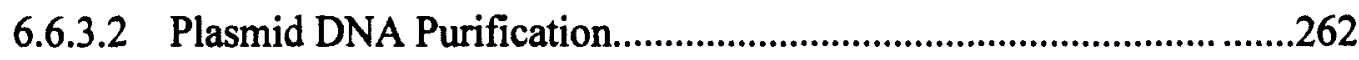

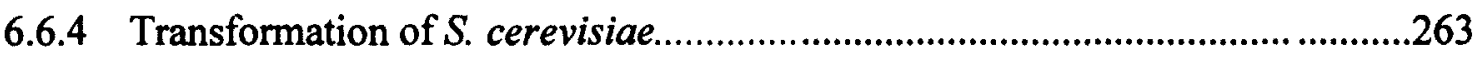

6.6.4.1a Stock Solution...........................................................................................264

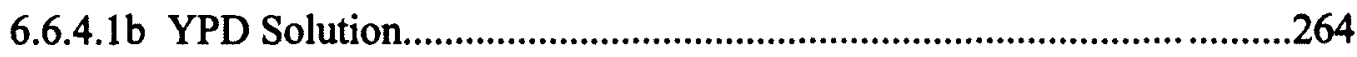

6.6.4.1c YPD Agar Medium...............................................................................264

6.6.4.2 Preparation of Competent Yeast Cells....................................................264

6.6.4.3 Transformation of the Competent Cells................................................265

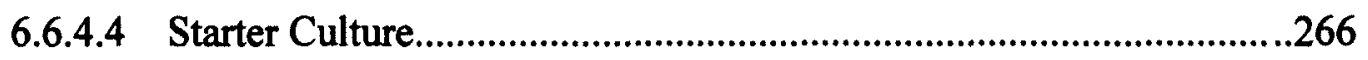

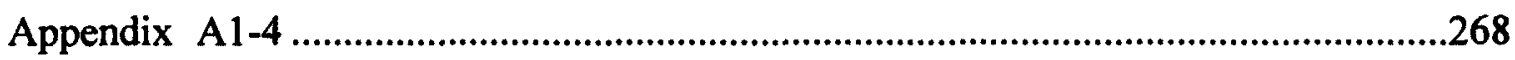

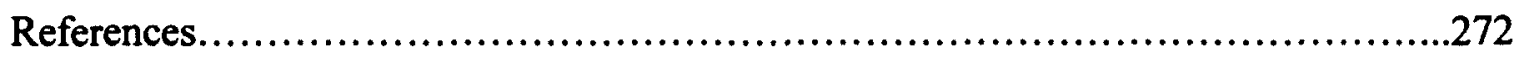




\section{List of Figures}

1.1 Biosynthesis of fatty acids in plants and its subsequent modifications by employing several different enzymes to produce complex lipids.

1.2 Examples of some naturally occurring exotic fatty acids and their derivatives......3

1.3 Representative structures of naturally occurring conjugated fatty acid derivatives from animals, insects, algae and plants. 8

1.4 Biogenesis of CLA in the milk and meat fat of ruminants

1.5 The initial involvement of various membrane-bound desaturases and the bifunctional activity of $\Delta^{11}$-desaturase in generating conjugated fatty acid derivatives in the pheromone blend of female processionary moth, Spodoptera littoralis.

1.6 Catalytic diversity of a multifunctional $\Delta^{11}$-desaturase that is responsible for the biosynthesis of sex pheromone in processionary moth, Thaumetopoea pityocampa.

1.7 Proposed biogenesis of the conjugated fatty acids in plants beginning with $\mathrm{H}$-abstraction at $\mathrm{C}$ - 11 of linoleic acid.

1.8 A prototypical $\mathrm{O}_{2}$-dependent desaturase-mediated biotransformation reaction...17

1.9 Three-dimensional crystal structure of soluble plant stearoyl$\mathrm{ACP} \Delta^{9}$-desaturase.

1.10 Computer model of a soluble plant $\Delta^{9}$-desaturase docked with a stearoylACP substrate in the hydrophobic binding pocket showing the topology of the active site

1.11 Proposed topological model of the membrane-bound $\Delta^{9}$-desaturase (FAD2)......24

1.12 Biosynthesis of several exotic oxidized fatty acid compounds in plants catalyzed by a family of FAD2 variants.

1.13 The currently accepted mechanistic model for fatty acid desaturation.

1.14 Use of chiral monodeuterated substrates as isotopic mechanistic probe to determine the enantioselectivity of $\Delta^{9}$-desaturation

1.15 Determination of the relative stereochemistry of $\mathrm{C}-\mathrm{H}$ bond breaking ….........30

1.16 Application of thia-fatty acid analogue to elucidate the stereochemistry of desaturation. 


\section{List of Figures continued}

1.17 A) Castor stearoyl $\Delta^{9}$-desaturase-mediated chiral sulfoxidation of 18 fluoro-10-thiaoctadecanoylACP; B) Pirkle-binding complexation model for the interaction of $(R)$-AMA with the two enantiomers of 18-fluoro10-thiaoctadecan-1-ol S-oxide; C) its effect on ${ }^{1} \mathrm{H}$-decoupled

${ }^{19} \mathrm{~F}$ NMR spectroscopy

1.18 Use of intermolecular primary deuterium kinetic isotope effect measurement to determine the cryptoregiochemistry of oleate formation by a membranebound $\Delta^{9}$-desaturase

1.19 Schematic diagram preferential 9-sulfoxide formation by membranous $\Delta^{9}$-desaturase

1.20 Determination of cryptoregiochemistry of castor stearoyl soluble-ACP $\Delta^{9}$ desaturase

1.21 Regioselective desaturase-mediated hydroxyl byproduct formation

1.22 Highly conserved active-site structural motif of membrane-bound As well as soluble plant desaturases.

1.23 Comparison of $(R)$-and (S)-9-fluorostearate oxidation by a soluble plant $\Delta^{9}$-desaturase leading to novel hydroxylated and olefinic products.

1.24 Positioning of the iron-hydroxyl species relative to the two carbons of the incipient double bond.

$1.25 \mathrm{C}-4$ Initiated bimodal oxidation of valproic acid.........................................43

1.26 Relationship between 12-hydroxylation / 12-dehydrogenation...................44

1.27 The role of associated ligand environment at the reaction binding site in controlling the switch between the hydroxylation vs. Desaturation channel during the biosynthetic pathway of clavulanic acid.

1.28 CLUSTALL comparison between the amino acid sequences of FAD2 variants responsible for biosynthesis of conjugated fatty acids in plants.

1.29 Topological model for the stereochemical investigation of the biosynthesis of $(10 E, 12 E)$-fatty acetate as a component of sex pheromone blend in moth Spodoptera littoralis using stereospecifically monodeuterated fatty acid analogues.

1.30 Synthesis and use of $\left[8,8,11,11,16,16,17,17-{ }^{2} \mathrm{H}_{8}\right]$-octadec-9-enoic acid as mechanistic probe to test the biosynthesis of calendic acid in marigold seeds. 


\section{List of Figures continued}

1.31 Determination of the cryptoregiochemistry of calendic acid biosynthesis using the KIE measurements.

1.32 Involvement of several variant FAD2 enzymes to generate calendic, $\alpha$-eleostearic and dimorphecolic acid. .50

2.1 Mechanistic model showing the relationship between oxidant position in $\Delta^{12}$-desaturation and the $(1,4)$-desaturase reaction leading to the formation of the conjugated 8,10-diene system of calendate.

2.2 The target chiral monodeuterated linoleates required to investigate the stereochemistry of calendate formation. .54

2.3 Scheme for the synthesis of chiral $(8 R)$-and $(8 S)-\left[8-{ }^{2} \mathrm{H}_{1}\right]$-linoleates..............55

2.4 Scheme for Crombie's method for the synthesis of monodeuterated aldehyde....57

2.5 Preparation of $(+)-(R)$-Alpine-borane and its use in chiral enantioselective reduction of deuterio aldehyde to synthesize the $(S)$ - $\left[1-{ }^{2} \mathrm{H}_{1}\right]$-alcohol

2.6 Explanation of the enantiomeric preference Alpine-Borane ${ }^{\text {reagents in }}$ the reduction of aldehydes.

2.7 Determination of the absolute configuration and enantiomeric purity of the chiral ethyl 8-d $1-8$-hydroxy octanoates using Mosher's esterification method.

2.8 GC-MS chromatogram of the HPLC purified $(8 R)-\left[8{ }^{2} \mathrm{H}_{1}\right]$-linoleate...............62

2.9 GC-MS chromatogram of the HPLC purified $(8 S)-\left[8{ }^{2} \mathrm{H}_{1}\right]$-linoleate.................62

2.10 Mass spectrum of methyl $(8 R)-\left[8{ }^{2} \mathrm{H}_{1}\right]$-linoleate.............................63

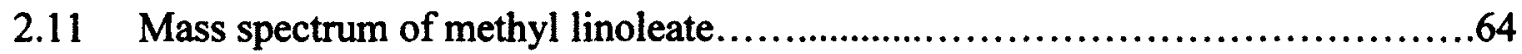

$2.12{ }^{\prime} \mathrm{H}$ NMR spectrum of methyl $(8 R)-\left[8-{ }^{2} \mathrm{H}_{1}\right]$-linoleate.......................65

$2.13{ }^{1}$ H NMR spectrum of methyl linoleate..........................................65

$2.14{ }^{13} \mathrm{C}$ NMR spectrum of methyl $(8 R)-\left[8-{ }^{2} \mathrm{H}_{1}\right]$ linoleate..........................66

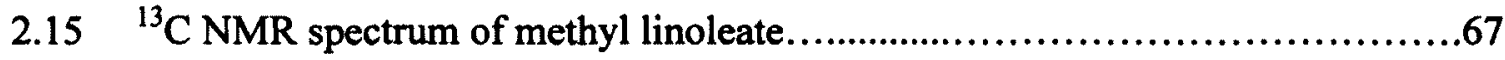

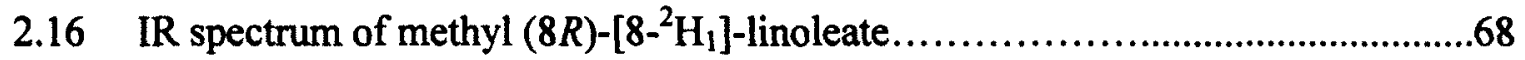




\section{List of Figures continued}

2.17 Scheme for the chemoenzymatic synthesis of chiral $\left[11-^{2} \mathrm{H}_{1}\right]$-linoleates

via $\Delta^{12}$-desaturation of chiral $\left[11-{ }^{2} \mathrm{H}_{1}\right]$-oleate precursor.

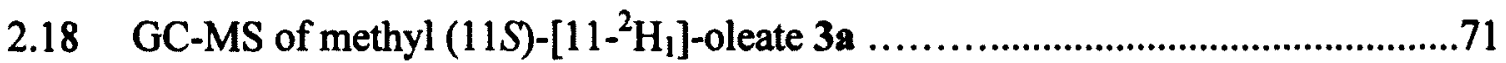

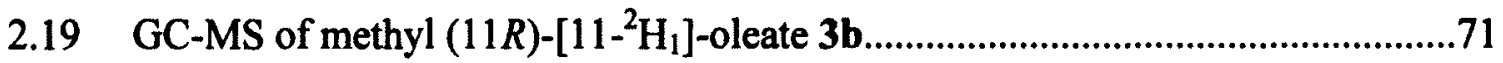

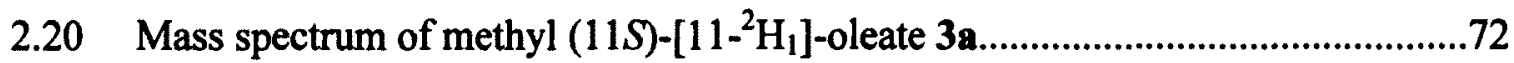

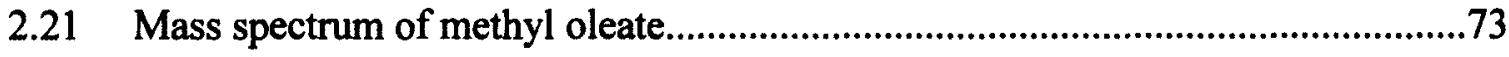

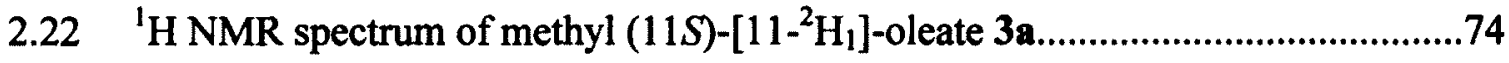

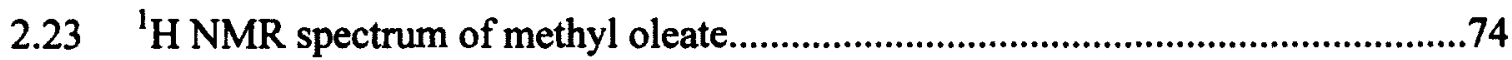

$2.24{ }^{13} \mathrm{C}$ NMR spectrum of methyl $(11 S)-\left[11{ }^{2} \mathrm{H}_{1}\right]$-oleate $3 \ldots \ldots \ldots \ldots \ldots \ldots \ldots \ldots . \ldots . \ldots \ldots$

$2.25{ }^{13} \mathrm{C}$ NMR spectrum of methyl oleate...............................................................76

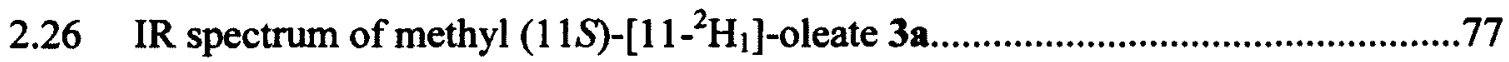

2.27 IR spectrum of methyl oleate..............................................................................77

2.28 GC-MS of biosynthetic methyl $(11 S)-\left[11^{2} \mathrm{H}_{1}\right]$-linoleate 2a..............................79

2.29 GC-MS of biosynthetic methyl $(11 R)-\left[11-{ }^{2} \mathrm{H}_{1}\right]$-linoleate $\mathbf{2 b}$................................79

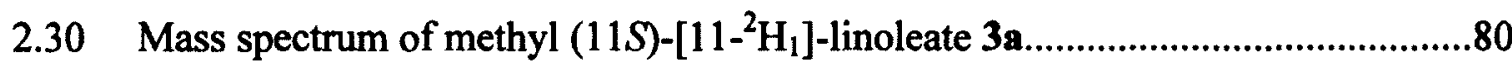

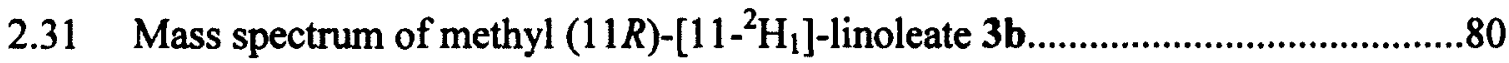

2.32 Scheme for the attempted synthesis of chiral methyl $\left[11-{ }^{2} \mathrm{H}_{1}\right]$-linoleates..........81

2.33 Scheme showing the synthesis of $\left[1-{ }^{2} \mathrm{H}_{1}\right]-2$-octyn-1-al by Crombie et al.........82

2.34 Stereochemical analysis of chiral- $\mathrm{d}_{1}$-alkyn-1-ols using Mosher's esterification method.

2.35 Mass spectrum of $(1 R)-\left[1-^{2} \mathrm{H}_{1}\right]-2$-octyn-1-ol-derived methyl $\left[11-{ }^{2} \mathrm{H}_{1}\right]$-linoleate.

2.36 A: ${ }^{1} \mathrm{H}$ NMR and $B$ : ${ }^{13} \mathrm{C}$ NMR of methyl $\left[11 .{ }^{2} \mathrm{H}_{1}\right]$-linoleate. 85 


\section{List of Figures continued}

2.37 IR spectrum of $(1 R)-\left[1-{ }^{2} \mathrm{H}_{1}\right]$-2-octyn-1-ol-derived methyl

$\left[11-{ }^{2} \mathrm{H}_{1}\right]$-linoleate.

2.38 The cellular fatty acid profile of $S$. cerevisiae grown in a medium supplemented with linoleic acid.

2.39 Mass spectrum of biosynthetic methyl calendate derived from methyl linoleate.

2.40 Representative mass spectrum of biosynthetic methyl calendate derived from methyl $(8 R)-\left[8-{ }^{2} \mathrm{H}_{1}\right]$-linoleate $1 \mathrm{a}$

2.41 Representative mass spectrum of biosynthetic methyl calendate derived from methyl $(8 S)-\left[8-{ }^{2} \mathrm{H}_{1}\right]$-linoleate $1 \mathrm{~b}$.

2.42 Representative mass spectrum of biosynthetic methyl calendate derived from methyl $(11 S)-\left[11-{ }^{2} \mathrm{H}_{1}\right]$-linoleate $2 \mathrm{a}$.

2.43 Representative mass spectrum of biosynthetic methyl calendate derived from methyl $(11 R)-\left[11-{ }^{2} \mathrm{H}_{1}\right]$-linoleate $2 \mathbf{b}$.

2.44 Observed results for calendate formation from stereospecifically deuterated linoleates.

2.45 Comparison of the relative rates of $\mathrm{C}-\mathrm{H}$ vs. $\mathrm{C}-\mathrm{D}$ bond breaking in the two modes of calculating $\mathrm{KIE}$ at $\mathrm{C}-11$

2.46 Mechanistic model for 1,4-dehydrogenation reaction of linoleate to biosynthesize calendate as it occurs in Calendula officinalis.

3.1 A proposed mechanism for the 1,4-desaturase-mediated formation of $\alpha$-eleostearate featuring initial $\mathrm{H}$-abstraction at $\mathrm{C}-11$.

3.2 The dideuterated and chiral monodeuterated linoleates required to investigate the cryptoregiochemistry and stereochemistry $\alpha$-eleostearate formation.

3.3 Agarose gel electrophoresis of the PCR amplified FADX genes.

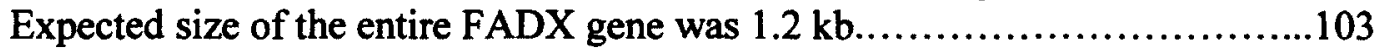

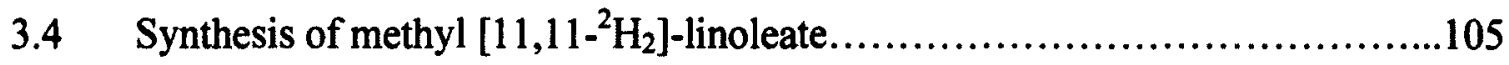

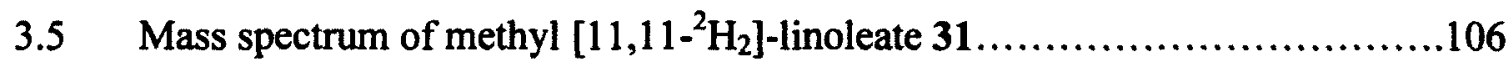




\section{List of Figures continued}

$3.6 \quad{ }^{\prime} \mathrm{H}$ NMR spectrum of methyl $\left[11,11-{ }^{2} \mathrm{H}_{2}\right]$-linoleate. ......................107

3.7 ${ }^{13} \mathrm{C}$ NMR spectrum of methyl $\left[11,11-{ }^{2} \mathrm{H}_{2}\right]$-linoleate........................................108

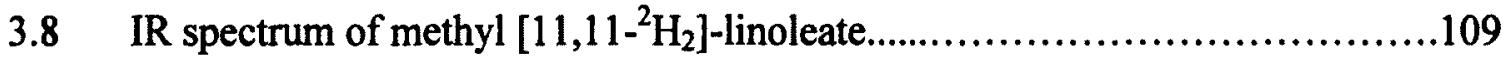

3.9 Synthesis of methyl $\left[14,14{ }^{2} \mathrm{H}_{2}\right]$-linoleate....................................

3.10 Mass spectrum of methyl $\left[14,14-{ }^{2} \mathrm{H}_{2}\right]$-linoleate.............................111

$3.11{ }^{1} \mathrm{H}$ NMR spectrum of methyl $\left[14,14-{ }^{2} \mathrm{H}_{2}\right]$-linoleate. .......................111

$3.12{ }^{13} \mathrm{C}$ NMR spectrum of methyl $\left[14,14-{ }^{2} \mathrm{H}_{2}\right]$-linoleate........................112

3.13 IR spectrum of methyl $\left[14,14-{ }^{2} \mathrm{H}_{2}\right]$-linoleate. .......................................113

3.14 The cellular fatty acid profile of $S$. cerevisiae grown in a medium supplemented with linoleic acid.......................................................115

3.15 Mass spectrum of the biosynthetic methyl $\alpha$-eleostearate derived from methyl linoleate.

3.16 Representative mass spectrum of biosynthetic $\alpha$-eleostearate derived from 1:1 mixture of $\mathrm{d}_{0}$-linoleate and $11-\mathrm{d}_{2}$-linoleate.

3.17 Representative mass spectrum of biosynthetic $\alpha$-eleostearate derived from 1:1 mixture of $\mathrm{d}_{0}$-linoleate and $14-\mathrm{d}_{2}$-linoleate.

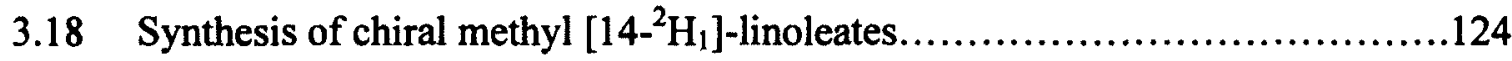

$3.19{ }^{1} \mathrm{H}$ resonances at $\mathrm{C}-1$ of MTPA ester of $(1 S)-,(1 R)-\left[1{ }^{2} \mathrm{H}_{1}\right]$-pentanol (triplet) and $\mathrm{d}_{0}$-pentanol (AB quartet).

3.20 Mass spectrum of methyl $(14 S)-\left[14-{ }^{2} \mathrm{H}_{1}\right]$-linoleate 33a......................127

$3.21{ }^{1} \mathrm{H}$ NMR spectrum of methyl $(14 S)-\left[14-{ }^{2} \mathrm{H}_{1}\right]$-linoleate $33 \mathrm{a} . \ldots \ldots \ldots \ldots \ldots \ldots . \ldots \ldots$

$3.22{ }^{13} \mathrm{C}$ NMR spectrum of methyl $(14 S)-\left[14-{ }^{2} \mathrm{H}_{1}\right]$-linoleate 33a. .....................128

3.23 IR spectrum of methyl $(14 S)-\left[14-{ }^{2} \mathrm{H}_{1}\right]$-linoleate 33a. The C-D stretch due to $\mathrm{C}_{14} \mathrm{HD}$ appears at $2150 \mathrm{~cm}^{-1}$

3.24 Stereochemical analysis of tosylate displacement by alkyne anion 


\section{List of Figures continued}

$3.25{ }^{1} \mathrm{H}$ resonances at $\mathrm{C}-2$ of $(S)-\mathrm{O}$ methyl mandelate (MM) ester of (2S)-, $(2 R)-\left[2{ }^{2} \mathrm{H}_{1}\right]$-hexanoic acid (triplet of triplets) and $\mathrm{d}_{0}$-hexanoic acid (AB quartet).

3.26 Representative mass spectrum of biosynthetic methyl $\alpha$-eleostearate derived from methyl $(11 R)-\left[11-{ }^{2} \mathrm{H}_{1}\right]$-linoleate $\mathbf{2 b}$.

3.27 Representative mass spectrum of biosynthetic methyl $\alpha$-eleostearate derived from methyl (11S)-[11- $\left.{ }^{2} \mathrm{H}_{1}\right]$-linoleate $2 \mathrm{a}$.

3.28 Representative mass spectrum of biosynthetic methyl $\alpha$-eleostearate derived from methyl $(14 R)-\left[14-{ }^{2} \mathrm{H}_{1}\right]$-linoleate 33b.

3.29 Representative mass spectrum of biosynthetic methyl $\alpha$-eleostearate derived from methyl $(14 S)-\left[14-{ }^{2} \mathrm{H}_{1}\right]$-linoleate 33b.

3.30 Predicted outcomes for $\alpha$-eleostearate formation from stereospecifically labeled linoleates.

3.31 The revised topological model and observed results for eleostearate formation from stereospecifically deuterated linoleates

3.32 Schematic diagram showing the possible conformational arrangement of two different substrates at the binding cavity of $\Delta^{13}$-desaturase in T. pityocampa that leads to the formation of various fatty acid products.

3.33 Mechanistic model for 1,4-dehydrogenation reaction of linoleate to biosynthesize $\alpha$-eleostearate as it occurs in Aleurites fordii Hemsl.

4.1 The biosynthetic pathway of dimorphecolic acid formation from oleic acid in the seeds of Dimorphotheca sinuata.

4.2 Proposed mechanistic model for $\Delta^{9}$-hydroxylation leading to the formation of 9-hydroxy- $(E, E)$-10,12-octadecadienoic from trans- $\Delta^{12}$-linoleic acid and showing how iron- $\mathrm{OH}$ is not able to reach the $\mathrm{C} 8 \mathrm{H}$ to form calendate.

4.3 The chiral monodeuterated and dideuterated oleates required to investigate the mechanism of dimorphecolate formation.

4.4 The cellular fatty acid profile of $S$. cerevisiae grown in a medium supplemented with oleic acid....

4.5 Mass spectrum of TMS derivative of the methyl ester of dimorphecolic acid...145 


\section{List of Figures continued}

4.6 Representative mass spectrum of TMS derivative of the methyl ester of dimorphecolic acid derived from 1:1 mixture of 18-d $\mathrm{d}_{3}$-oleate and and $11-\mathrm{d}_{2}$-oleate.

4.7 Representative mass spectrum of TMS derivative of the methyl ester of dimorphecolic acid derived from 1:1 mixture of 18- $\mathrm{d}_{3}$-oleate and $9,10-d_{2}$-oleate

4.8 Representative mass spectrum of TMS derivative of the methyl ester of dimorphecolic acid derived from methyl $(11 S)-\left[11-{ }^{2} \mathrm{H}_{1}\right]$-oleate 3a.

4.9 Representative mass spectrum of TMS derivative of the methyl ester of dimorphecolic acid derived from methyl $(11 R)$ - $\left[1-^{2} \mathrm{H}_{1}\right]$-oleate $3 \mathbf{b}$

4.10 Observed results for dimorphecolate formation from stereospecifically deuterated trans- $\Delta^{12}$-linoleate.

4.11 Comparison of relative stereochemistry of hydroxylation by FAD2 variants in the biosynthesis of ricinoleate and dimorphecolate.....

4.12 Proposed substrate binding modes and reaction outcomes for various substrates with mutant soluble desaturase.

4.13 Mechanistic model for DsFAD2-2 hydroxylase involved biotransformation of trans- $\Delta^{12}$-linoleate to dimorphecolate as it occurs in the seeds of Dimorphotheca sinuata.

5.1 Highly conserved active site model of the membrane-bound family of FAD2 homologues.

6.1 Sequence of the FADX from tung (Aleurites fordii Hemsl.) inserted in the pYES2.1 plasmid.

6.2 Translated gene sequence of tung FADX from Aleurites fordii Hemsl 


\section{List of Tables}

2.1 Isotopic content of stereospecifically labeled monodeuterated linoleates and $\Delta^{8,10}$-desaturated products (calendate)

2.2 Intermolecular isotopic discrimination by the Calendula officinalis $\Delta^{8,10}$-desaturase in the 1,4-dehydrogenation of racemic methyl $\left[11-^{2} \mathrm{H}_{1}\right]$-linoleate.

3.1 Intermolecular isotopic discrimination by the Aleurites fordii Hemsl. $\Delta^{11,13}$-desaturase mediated 1,4-desaturation of methyl $\left[11,11{ }^{2} \mathrm{H}_{2}\right]-$ linoleate 31 and methyl $\left[14,14-{ }^{2} \mathrm{H}_{2}\right]$-linoleate 32

3.2 Intermolecular isotopic discrimination by the Aleurites fordii Hemsl. $\Delta^{11,13}$-desaturase in the 1,4-dehydrogenation of racemic methyl $\left[11-{ }^{2} \mathrm{H}_{1}\right]$-linoleate

3.3 Isotopic content of stereospecifically labeled cellular monodeuterated linoleates and $\Delta^{11,13}$-desaturated products ( $\alpha$-eleostearate)

4.1 Competitive isotopic discrimination in the Dimorphotheca sinuata DsFAD2-1 and DsFAD2-2 desaturase-mediated formation of dimorphecolic acid from $\left[11,11-{ }^{2} \mathrm{H}_{2}\right]$-oleic acid or $\left[9,10-{ }^{2} \mathrm{H}_{2}\right]$-oleic acid vs. $\left[18,18,18-{ }^{2} \mathrm{H}_{3}\right]$-oleic acid.

4.2 Isotopic content of stereospecifically labeled monodeuterated oleates and DsFAD2-1 and DsFAD2-2 involved hydroxylated products (dimorphecolate). 


\section{List of Abbreviations}

(R)-AMA $\quad(R)$-(9-Anthranyl)methoxy acetic acid

$(R)$-MPAA $\quad(R)$-Methoxyphenylacetic acid

$(R)$-TFAE $\quad(R)-(-)-1-(9-$ Anthranyl)-2,2,2-trifluoroethanol

(S)-AMA (S)-(9-Anthranyl)methoxy acetic acid

$(S)-(+)-M P A A \quad(S)-(+)-\alpha-$ Methoxyphenylacetic acid

(S)-TFAE $\quad(S)-(+)-1-(9-$ Anthranyl)-2,2,2-trifluoroethanol

ACP Acyl carrier protein

Amp Ampicillin

9-BBN 9-Borabicyclo [3.3.1]nonane

bp Boiling point

BSTFA $\quad N, O$-Bis(trimethylsilyl)trifluoroacetamide

CIP Cahn-Ingold-Prelog

CLA Conjugated linoleic acid

CLnA Conjugated linolenic acid

CoA Coenzyme A

CS2 Clavaminate synthase 2

CSA Chiral solvating agent

CSM-URA Complete supplement mixture - uracil

DAST Diethylaminosulfur trifluoride

DEPT Distortionless enhancement by polarization transfer

DGPC Deoxyguanidinoproclavaminic acid

DHP 3,4-Dihydro-2 $H$-pyran 


\section{List of Abbreviations continued}

DMAP 4-(Dimethylamino)pyridine

DMF N,N-Dimethylformamide

DMS Dimethyl sulphide

DMSO Dimethyl sulfoxide

DNA Deoxyribonucleic acid

$d r \quad$ Diastereomeric ratio

EDA Ethylenediamine

EDC $\cdot \mathrm{HCl} \quad N$-(3-Dimethylaminopropyl)-N'-ethylcarbodiimide hydrochloride

ee Enantiomeric excess

EI Electronspray ionization

ER Endoplasmic reticulum

EtMgBr Ethylmagnesium bromide

EtOAc Ethyl acetate

EtOH Ethanol

Fac2 Fatty acid conjugase 2

FAD Fatty acid $\Delta^{9}$-Desaturase

FAD2 Fatty acid $\Delta^{12}$-Desaturase

FAD3 Fatty acid $\Delta^{15}$-Desaturase

FAME Fatty acid methyl ester

FAS Fatty acid synthase

GC Gas chromatography

GC-MS Gas chromatography-mass spectrometry 


\section{List of Abbreviations continued}

$\begin{array}{ll}\text { HMPA } & \text { Hexamethylphosphoramide } \\ \text { HPLC } & \text { High-performance liquid chromatography } \\ \text { IR } & \text { Infrared } \\ \alpha \text {-KG } & \alpha \text {-Ketoglutarate } \\ \text { KHMDS } & \text { Postassium bis(trimethylsilyl)amide } \\ \text { KIE } & \text { Kinetic isotope effect } \\ \text { LA } & \text { Linoleic acid } \\ \text { LB } & \text { Lysogeny broth } \\ m \text {-CPBA } & \text { meta-Chloroperbenzoic acid } \\ \text { MeCN } & \text { Acetonitrile } \\ \text { MeLi } & \text { Methyllithium } \\ \text { MeOH } & \text { Methanol } \\ \text { MMA } & \text { Methyl mandelate } \\ \text { MMO } & \text { Methane monooxygenase } \\ \text { mp } & \text { Melting point } \\ \text { MS } & \text { Mass spectrometry } \\ \text { MTPA } & \alpha \text {-Methoxy- } \alpha \text {-(trifluoromethyl)phenylacetic acid } \\ \text { MTPA-Cl } & \alpha \text {-Methoxy- } \alpha \text {-(trifluoromethyl)phenylacetyl chloride } \\ \text { NADH } & \text { Nicotinamide adenine dinucleotide } \\ \text { NADPH } & \text { Nicotinamide adenine dinucleotide phosphate } \\ n \text {-BuLi } & n \text {-Butyllithium } \\ \text { NMR } & \text { Nuclear magnetic resonance } \\ & \end{array}$




\section{List of Abbreviations continued}

\begin{tabular}{|c|c|}
\hline PC & Phosphatidylchloine \\
\hline PDC & Pyridinium dichromate \\
\hline PCR & Polymerase chain reaction \\
\hline PL & Phospholipid \\
\hline PPTS & Pyridinium $p$-toluenesulfonate \\
\hline$p$-TsOH & $p$-Toluenesulfonic acid \\
\hline Py & Pyridine \\
\hline $\mathrm{RuCl}_{3} \cdot n \mathrm{H}_{2} \mathrm{O}$ & Ruthenium(III) chloride hydrate \\
\hline SCD & Stearoyl CoA $\Delta^{9}$-Desaturase \\
\hline SD & Synthetic dextrose \\
\hline SIM & Selected ion monitoring \\
\hline $\mathrm{S}_{\mathrm{N}} 2$ & Bimolecular nucleophilic substitution \\
\hline SOMO & Singly occupied molecular orbital \\
\hline TAG & Triacylglycerol \\
\hline TBS & tert-Butyl dimethylsilyl \\
\hline THF & Tetrahydrofuran \\
\hline THP & Tetrahydropyran \\
\hline TLC & Thin layer chromatography \\
\hline TMS & Trimethylsilyl \\
\hline $\mathrm{TMSCHN}_{2}$ & Trimethylsilyldiazomethane \\
\hline $\mathrm{TsCl}$ & $p$-Toluenesulfonyl chloride \\
\hline YPD & Yeast peptone dextrose \\
\hline
\end{tabular}




\section{Chapter 1: Introduction}

\subsection{Conjugated Fatty Acids}

\subsubsection{Lipids and Fatty acids}

Lipids are essential hydrocarbon-based components of every living organism where they serve as a source of energy and as hydrophobic building blocks of membranes. In the latter role, lipids protect the inner constituents of the cell from the outer environment and selectively allow foreign substances to pass through (cellular transport). Other biological functions performed by lipids include antifeedant activity, regulation of membrane protein activities and chemical signalling. ${ }^{1-4}$ Lipids can be subdivided into two major categories - firstly, fatty acids and their derivatives; and secondly, substances related biosynthetically or functionally to these compounds, such as

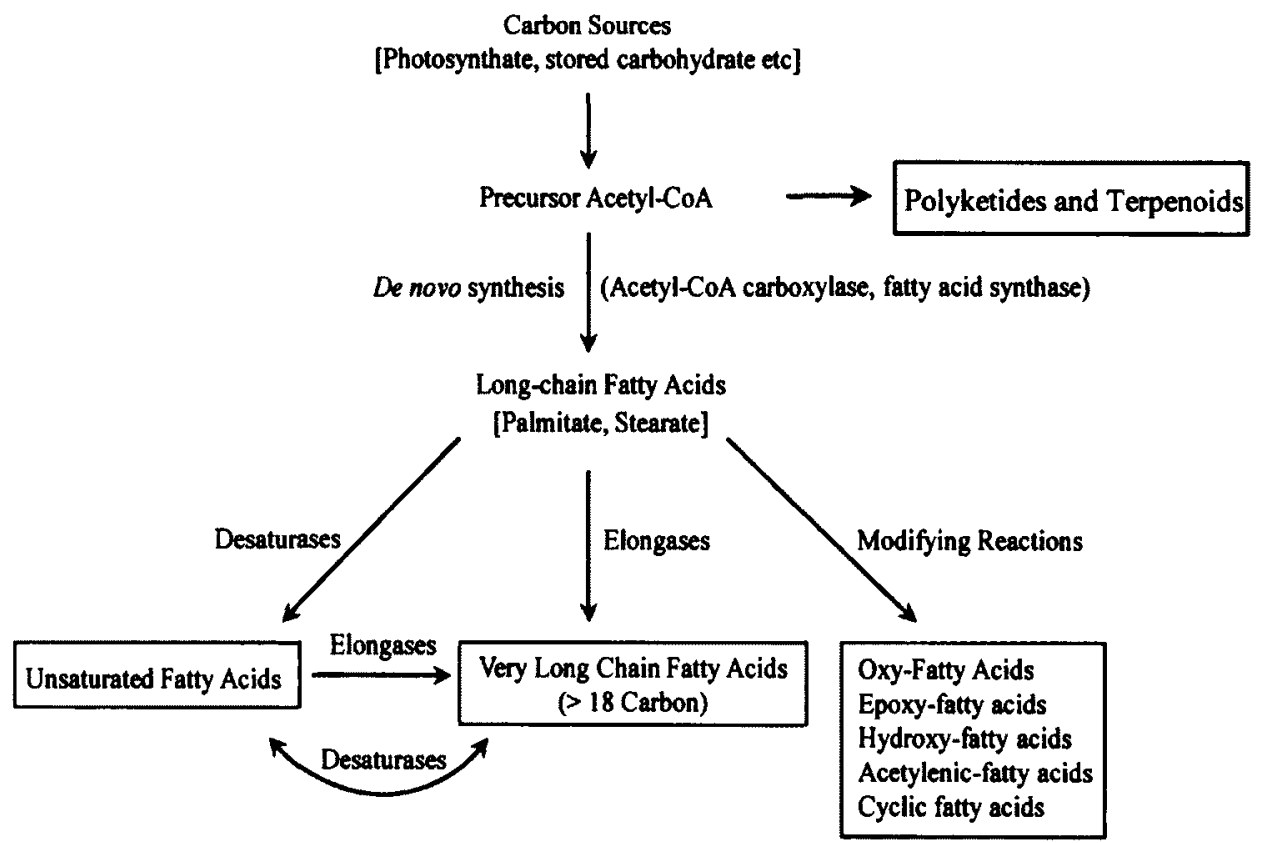

Figure 1.1. Biosynthesis of fatty acids in plants and its subsequent modifications by employing several different enzymes to produce complex lipids. The Figure was adapted from Gunstone et $a l^{5}$ 
polyketides and terpenoids (Figure 1.1). Fatty acids are the long chain carboxylic acid containing compounds that commonly exist in biological systems as esters of triglycerides and phospholipids. ${ }^{6,7}$ In addition to the carboxyl terminus, fatty acids can contain various other functionalities including monoolefinic, polyolefinic (methylene interrupted and/or conjugated), acetylenic, hydroxyl, hydroperoxy, epoxy, keto and cyclopropenyl groups. ${ }^{3,6-8}$ The fatty acyl chain lengths can vary but fatty acids with 16 and 18 carbons are most common. ${ }^{2,7,8}$

\subsubsection{Fatty acid biomodification}

The topic of this thesis is focused on the mechanism of a desaturase-mediated, post assembly fatty acid biomodification known as conjugated fatty acid formation (Panel C, Figure 1.2). To place this biochemical reaction in its proper context, the general subject of biomodified fatty acids will be reviewed. ${ }^{1}$ These compounds arise by transformation of the common saturated (e.g. stearate), monounsaturated (e.g. oleate) and polyunsaturated (e.g. linoleate) fatty acids as shown in Figure 1.2. A common theme that unites many of the biosynthetic pathways is that they are mediated by desaturase or desaturase-like enzymes. ${ }^{1,9}$ The various functional groups generated during these reactions include allene, ${ }^{3}$ cyclopropene and cyclopropane, ${ }^{9}$ epoxide, ${ }^{10}$ alkyne, ${ }^{1,10}$ polyacetylene, ${ }^{9}$ allylic alcohol, ${ }^{11}$ homoallylic alcohol, ${ }^{12}$ conjugated triene and fused polycyclobutane. $^{3}$

Most of these unusual fatty acids are thought to confer antifeedant properties that are used by the corresponding species to defend against various predators in that ecohabitat. ${ }^{1,13}$ Introduction of the allenic moiety in the fatty acyl chain is one of the rarest example of fatty acid modifications that have been observed to present in several 
A
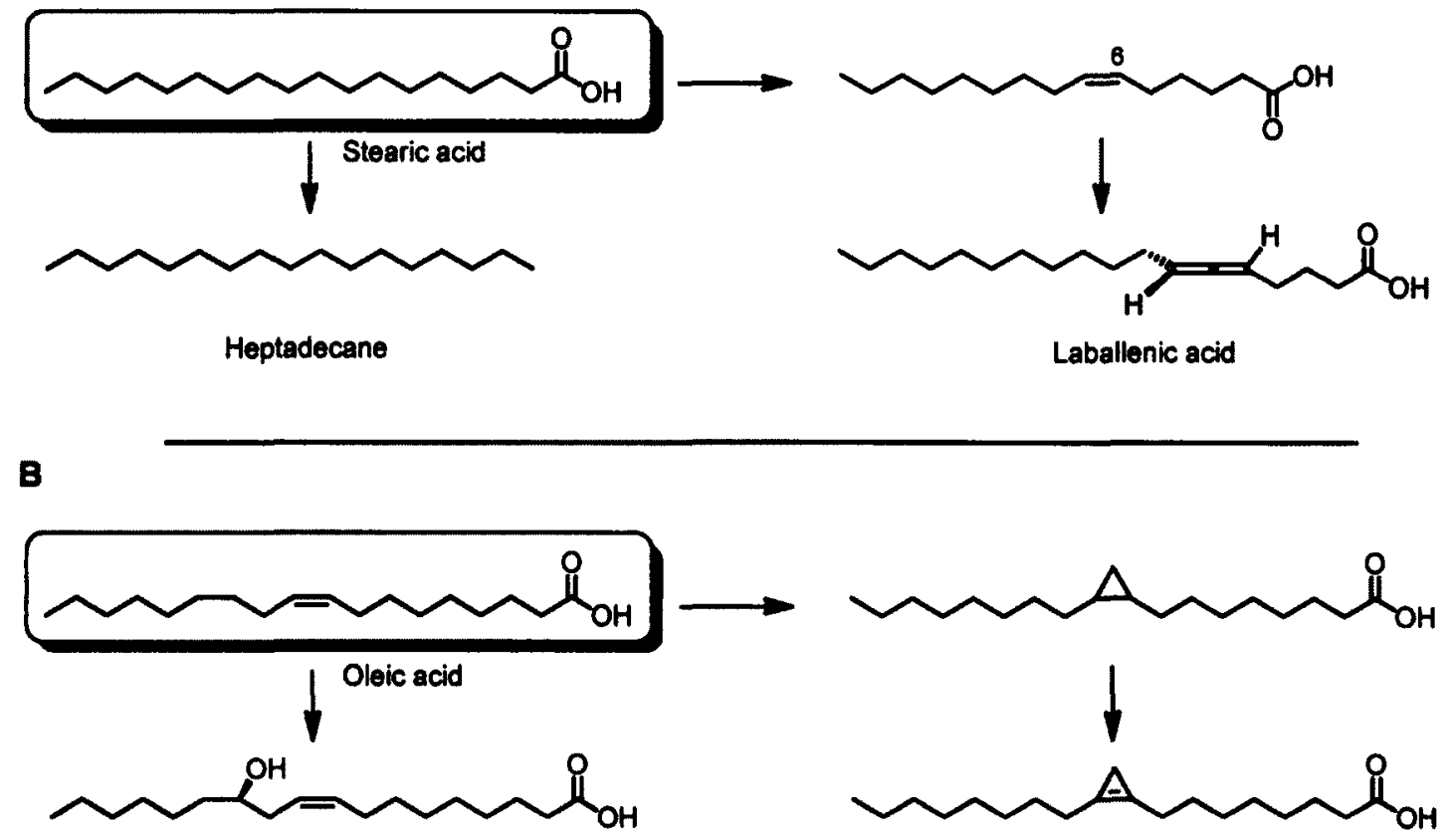

Ricinoleic acid

Sterculic acid

C
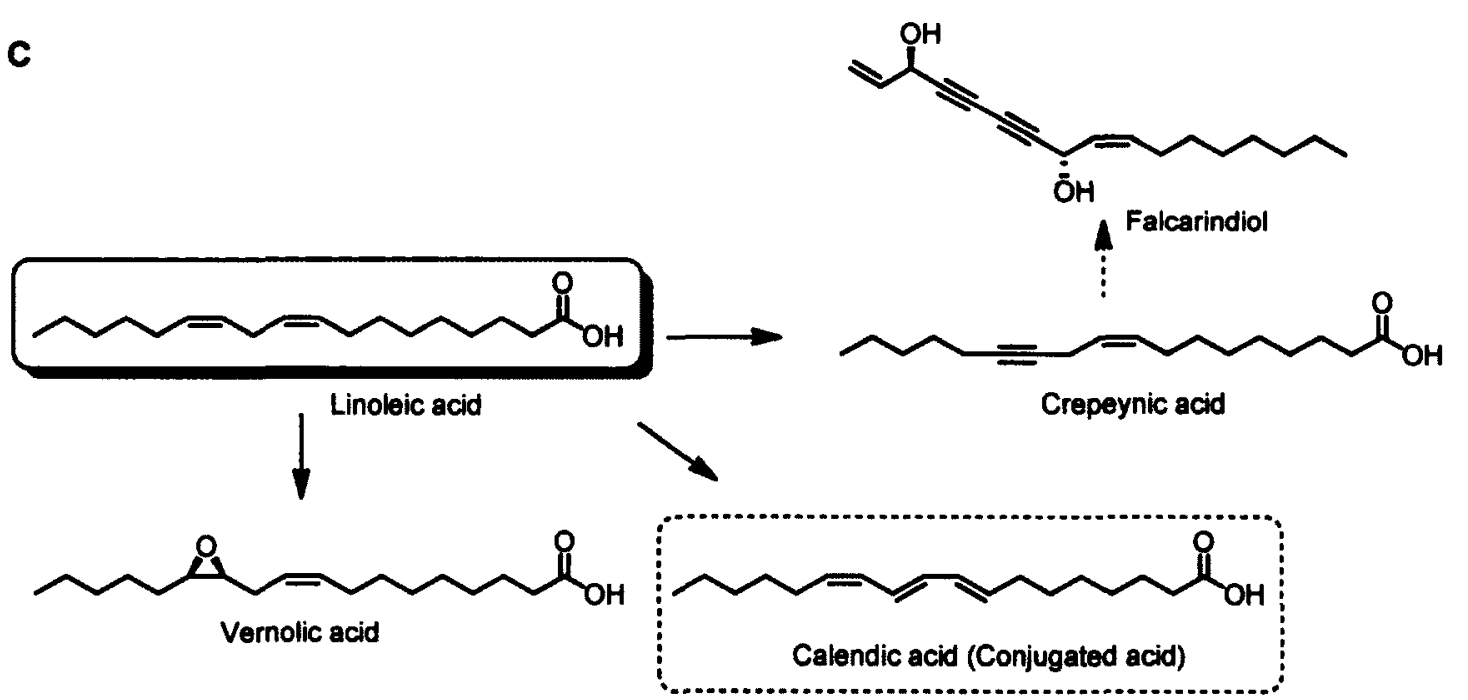

D

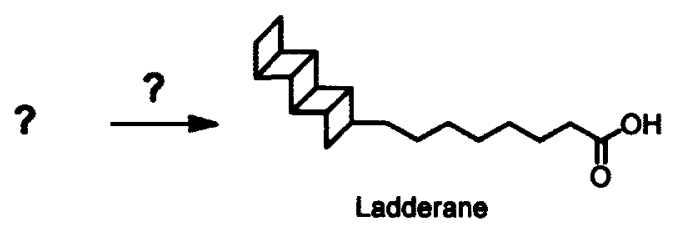

Figure 1.2. Examples of some naturally occurring exotic fatty acids and their derivatives. 
subtropical plants (Panel A, Figure 1.2). ${ }^{14}$ The seed oil of Leonotis neptaefolia (Lion's ear) has been observed to contain $(R)$ 5,6-monoallenic octadecadienoic acid or laballenic acid. ${ }^{15}$ It is thought that the allene is biosynthesized by stepwise dehydrogenation at the 6 , 7 and 5,6 positions.

The production of ricinoleic acid (Panel B, Figure 1.2) in the seeds of castor plant (Ricinus communis L.) has had a high impact in the oleochemical industry as a replacement of petrochemicals in some important processes and as an engine lubricant (Castrol $\left.{ }^{\mathrm{TM}}\right),{ }^{9} 90 \%$ of the total fatty acids in the seed oil of the castor plant is $(R)-12-$ hydroxy-(9Z)-octadecenoic acid or ricinoleic acid and this fact contributes to its bioactivity as a purgative. It has been observed that the plant biosynthesizes this fatty acid from oleic acid using an enzyme that is very closely related to the desaturase responsible for double bond introduction in linoleate biosynthesis. ${ }^{1,9,16}$ The very high toxicity of ricin in the seed coating of this plant complicates its cultivation in large quantity for industrial production. ${ }^{17}$ Cloning of the 12 -hydroxylase gene into more benign plant hosts has been a major biotechnological objective.

Certain tropical plants have also been observed to accumulate a remarkable fatty acid containing highly strained cyclopropene ring in its seeds oil known as sterculic acid (Panel B, Figure 1.2). ${ }^{1}$ Following the first structural elucidation of this fatty acid from the seed oil of Sterculia foetida in 1952 by Nunn, a search for natural products containing cyclopropenyl rings and their biological functions began. ${ }^{18}$ Today it is known that a number of tropical plant species belonging to the families of Sterculiaceae, Bombacaceae, Tiliaceae and Malvaceae possess sterculic acid in their seeds. ${ }^{19}$ Among all the seeds of Sterculia foetida contains the highest amount of this fatty acid as $78 \%$ of the 
total fatty acid content whereas the cotton seed (Gossypium hirsutum) oil contains far less amount ( about $1 \%$ ). Sterculic acid shows strong inhibition of mammalian stearoyl$\operatorname{CoA} \Delta^{9}$-desaturase leading to various disorders, which might explain the reason for the biogenesis of this unusual fat as it part of their antifeedant defence strategy. ${ }^{20-23}$ It is thought that sterculate is generated from a cyclopropyl intermediate via desaturation at C9 and C-10. 9

Vernolic acid is another example of industrially important exotic phyto fatty acid that is derived from a plant source (Panel C, Figure 1.2). Epoxide containing fatty acids are industrially important exotic substances that are commonly used in manufacturing adhesives, varnishes, paints as well as industrial coatings and resins. ${ }^{9}$ The presence of an epoxide ring at the allylic position of a double bond makes it less viscous, easily oxidizable and therefore makes any oil-based paints more durable. That is why it is mostly used as an environment friendly non-volatile solvent for many oil-based paints. ${ }^{9}$ The seeds of Vernonia galamensis, ${ }^{24}$ Euphorbia lagascae, ${ }^{25}$ Crepis palaestina $^{10}$ are the major source of vernolic acid. It is generated by epoxidation of linoleic acid involving a variant desaturase, however the details of the mechanistic pathway are still unknown.

Probably the most remarkable of all fatty acid biomodification features the abstraction of vinylic hydrogens $(=\mathrm{C}-\mathrm{H})$ to generate alkyne (Panel $\mathrm{C}$, Figure 1.2$)$ - the biotransformation process responsible for production of crepeynic acid in Crepis alpina. ${ }^{26-28}$ A similar kind of acetylenase activity has been observed in the moss Ceratodon purpureus with the biofunctional $\Delta^{6}$-desaturase/acetylenase. ${ }^{29}$ These reactions are particularly noteworthy in the sense that it shows the commitment of an enzyme towards a particular mechanistic pathway in preference to the energetically more 
favourable epoxidation or allylic oxidation leading to conjugated fatty acids. ${ }^{1,26}$ Cloning of the cDNA and functional analysis of the acetylenase responsible for the biosynthesis of crepeynic acid from linoleic acid shows high resemblance with parent FAD2 enzyme and another variant FAD2 enzyme (for epoxide formation) found in the genus Crepis itself. $^{9,10,26}$ It has been predicted that the formation of crepenynic acid from linoleic acid is the first step towards the biosynthesis of relatively more widespread, large family of polyacetylenes such as falcarindiol (Panel C, Figure 1.2) $10 .^{27,28,30,31}$ The most common source of polyacetylene compounds are the fungi, algae, marine sponges as well as higher plants and these unusually conjugated natural products have been observed to exhibit a broader range of biological activities including anti-inflammatory, antitumor, antifungal, antimicrobial, insecticidal and many others. ${ }^{30,32}$

Perhaps the most exotic fatty acids are the ladderanes (Panel D, Figure 1.2), first reported by L. A. van Niftrik and M. S. M. Jetten et al. $^{33}$ These lipids are isolated from two anaerobic ammonia-oxidizing bacteria where they create an exceptionally densed, tight protective barrier in the intracytoplasmic compartment to prevent any diffusion of toxic intermediates such as hydrazine during oxidation of ammonia to dintrogen intermediate. ${ }^{33}$ Multiple cyclobutyl rings (as many as five) are fused linearly in cis ring junction fashion such that it looks like a ladder and possibly biosynthesized by anaerobic cyclization of an unsaturated fatty acid. ${ }^{3}$ Such kind of ladderane molecules function as a promising building blocks for optoelectronics. $^{33}$

Isolation, characterization and engineering of the enzymes responsible for the biogenesis of these important lipids in different species is an important task for the biochemist, biotechnologist and bioorganic chemist. ${ }^{3}$ In this way, new drugs against 
various metabolic and terminal diseases may be discovered and the value of green oleochemical feedstocks and renewable energy source (hydrocarbon biofuels (Panel A, Figure 1.2)) may be enhanced.

\subsubsection{Conjugated Fatty Acids}

Conjugated fatty acids are the naturally occurring compounds that have drawn much attention because of their useful applications as nutraceuticals (putative anticancer, antidiabetic, antiobesity properties) as well as feedstocks for industrial uses (paints, inks, varnishes, lubricants, dyes, plastics, resins).${ }^{34}$ Conjugated fatty acids and their derivatives are present in many organisms including algae, plants, insects and mammals. Chain lengths of the conjugated fatty acids can vary from 12 to 22 carbons, but a chain length of 18 carbons is the most common. Variation in position and geometries of the conjugated double bonds as well as the presence of additional functional groups leads to much diversification of the structures of conjugated fatty acids as shown in Figure 1.3.

Three different types of mechanisms have been reported in the literature to date for the biosynthesis of conjugated fatty acids. ${ }^{34}$ Firstly, desaturation $(1,2-$ dehydrogenation) of olefinic fatty acids at the allylic position to give conjugated dienes (Figure 1.3A). ${ }^{35}$ Secondly, an anaerobic isomerisation mechanism that has been used by the marine algae to biosynthesize conjugated trienes and tetraenes (Figure $1.3 \mathrm{C}$ ) ${ }^{36,37}$ It has also been indicated that CLA (conjugated linoleic acid) such as $9 Z, 11 E$-isomer of linoleic acid may be biosynthesized in ruminants via isomerisation of linoleic acid (Figure 1.3A). ${ }^{38}$ Thirdly, a mechanistic variation of 1,2-dehydrogenation (such as 1,4 dehydrogenation) applied to cis-olefinic substrates is featured in the formation of insect pheromones $^{39-41}$ (Figure 1.3B) and conjugated fatty acid accumulation in the seed oils of 
selected higher plants ${ }^{34,42,43}$ (Figure 1.3D). The latter process is catalyzed by desaturase variants.

\section{A Animals}

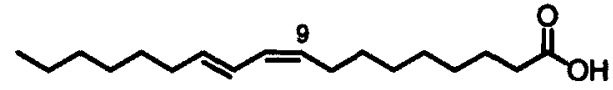

Rumenic acid<smiles>CCCCC/C=C/C=C(\CCCCCCCCC(=O)O)[Ge](Cl)(Cl)Br</smiles><smiles>CCCCCCC=CC=CCCCCCCCC(=O)O</smiles>

CLA Isomer

B Insects<smiles>CC=CC#CCCCCCCCCCOC(C)=O</smiles>

$(10 Z, 12 E)$-Tetradecadienyl acete
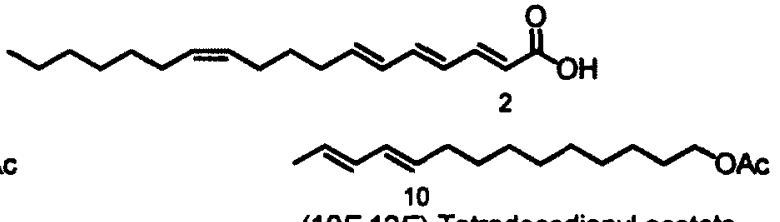

$(10 E, 12 E)$-Tetradecadienyl acetate

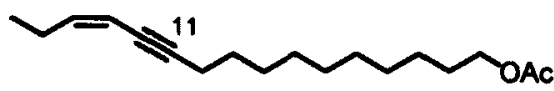

(132)-hexadece-11-ynyl acetate

C Algae<smiles>CCCCC=CC=CC=CC=CCC=CCCCC(=O)O</smiles>

Bosseopentaenoic acid

$(5 Z, 7 E, 9 E, 14 Z, 17 Z)$-Eicosapentaenoic acid

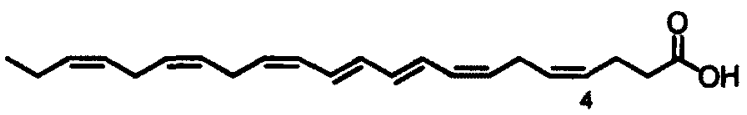

$(4 Z, 7 Z, 9 E, 11 E, 13 Z, 16 Z, 19 Z)-D o c o s a h e p t a e n o i c ~ a c i d ~$

D Plants
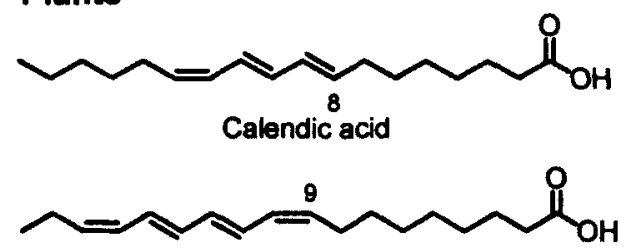

Parinaric acid

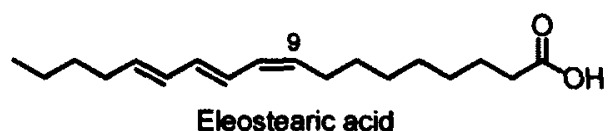

Eleostearic acid

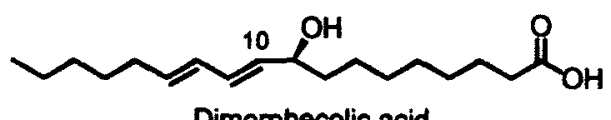

Figure 1.3. Representative structures of naturally occurring conjugated fatty acid derivatives from animals, insects, algae and plants. 


\subsubsection{Conjugated Fatty Acids in Animals}

In animals, conjugated fatty acids predominantly exist as conjugated linoleic acids (CLA). In 1935, Booth et al. reported the presence of conjugated fatty acids in food products derived from ruminant animals. ${ }^{44}$ Subsequently, Parodi et al confirmed the structure of this conjugated linoleic as $18: 2-9 Z, 11 E$ i.e. most commonly now known as rumenic acid. ${ }^{45}$ Milk, meat and other dairy products from ruminants are a rich source of CLA isomers that are thought to have many beneficial properties and are used as healthy food supplements. ${ }^{46-53}$ There are 28 different isomers that have been isolated so far from the rumen CLA but among them the two isomers, $18: 2-9 Z, 11 E$ (about $1 \%$ of the total milk fat and about 80 to $90 \%$ of the total CLA in milk) and 18:2-10E,12Z are present to a major extent. $45,46,50,54,55$

It has been demonstrated that the CLA in ruminants are biosynthesized from dietary forages containing unsaturated fats (linoleic or $\alpha$-linolenic acid) in two pathways where $18: 2-9 Z, 11 E$ is formed as an intermediate or by-product. ${ }^{35,51,55,56}$ In pathway one, CLA is produced as an intermediate during the ruminal biohydrogenation of dietary linoleic or $\alpha$-linolenic acid to stearic acid. Investigations have revealed that biohydrogenation which is a multiple step biochemical process is actually mediated by several different species of rumen bacteria. ${ }^{56,55}$ In pathway two, CLA is biosynthesized in the adipose tissue of mammary glands of the animal from 18:1-11E fatty acid that is formed during biohydrogenation (Figure 1.4).

In 1987 N. K. Grimm and M. W. Pariza et al. first reported the anticarcinogenic properties of conjugated linoleic acid from fried ground beef extract. ${ }^{57,58}$ Following their discovery many other research groups reported numerous physiological activities by 


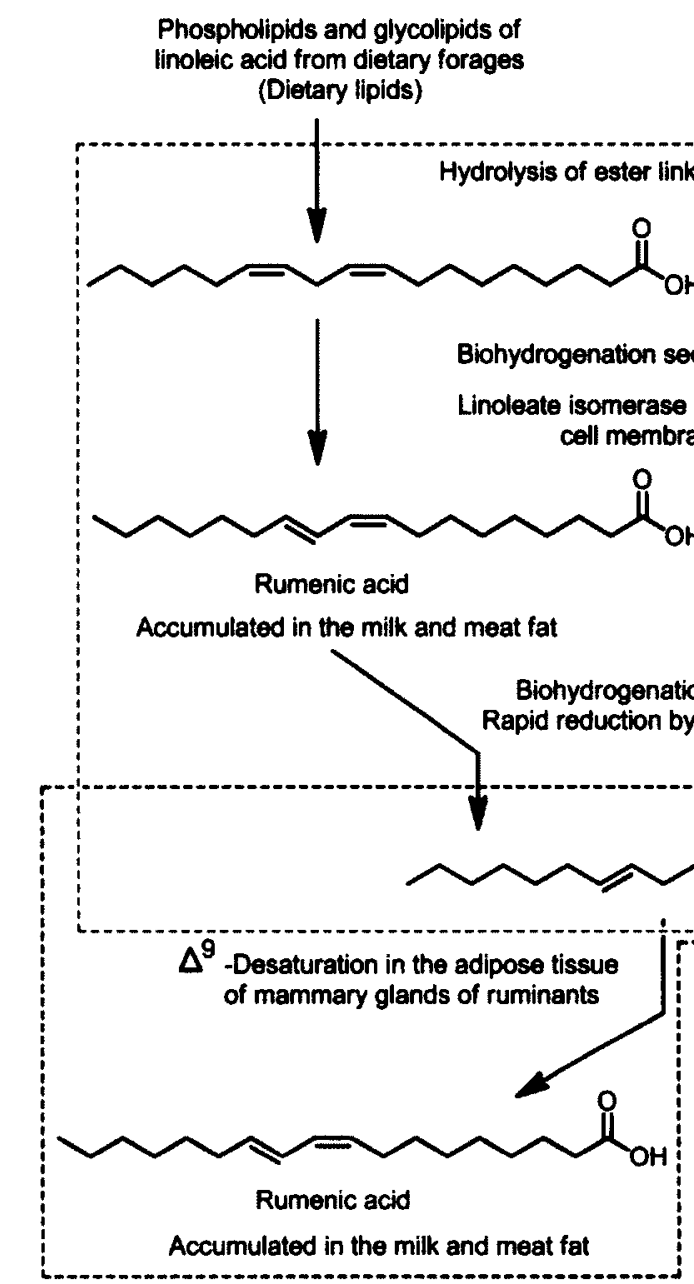

Tissue synthesis of $C L A$
Phospholipids and glycolipids of linolenic acid from dietary forages (Dietary lipids)

=

Figure 1.4. Biogenesis of CLA in the milk and meat fat of ruminants.

this class of compounds. Included in this feature - inhibition of tumorigenesis, modulation of the immune response, antiinflammatory effect, potent antiatheriosclerotic effect as well as play major role in regulating the membrane lipid level in animals body (antiobesity) as evident by reduction in body fat and increase in lean body mass. ${ }^{11,46-}$ 50,53,54,59-66 Conjugated fatty acids have also shown promise for serum lipid lowering effect in mammals, increases the $\mathrm{C}$-reactive protein concentration and lipid peroxidation and to decrease the insulin insensitivity in vivo i.e. has antidiabetic effect. ${ }^{48,52,53}$ In 
particular, $18: 2-9 E, 11 E$ has also been shown to inhibit platelate aggregation ${ }^{67,68}$ and exhibit antiproliferative effect. ${ }^{69}$

Recent studies indicate that average daily requirement of CLA in human body is $<500 \mathrm{mg} /$ day. ${ }^{70}$ However, ruminant milk and meat fat contains maximum up to $1 \%$ (w:w) CLA which is not enough (as food supplement for animals) to consider this as health-promoting food supplement. ${ }^{59}$ Oils that contains high proportions of essential fatty acids such as safflower oil, canola oil, vegetable oil are treated with alkali to generate CLA and supply the health food market.

Research on the physiological effects of conjugated linolenic acid (CLnA) has not progressed as much as work on the benefits of CLA. Only recently, it was reported that this family of conjugated fatty acids also exhibits significant cytotoxic effect on tumor cells, shows anticarcinogenic effect and play critical role in lipid metabolism of animals. ${ }^{34,46,64-66}$ In fact, both in vivo and in vitro studies established that conjugated linolenic acids (CLnA) have much stronger antitumour activity in comparison to CLA. ${ }^{59 .}$ ${ }^{61}$ These findings have increased interest in CLnA.

\subsubsection{Conjugated Fatty Acids in Insects}

Female processionary moths of several lepidopteran species have been observed to use a blend of modified lipids including conjugated $\pi$ systems known as sex pheromones to attract the mate. ${ }^{41,71,72}$ Pheromone blends are usually composed of several long chain lipophilic unsaturated fatty acetates, alcohols or aldehydes where the number as well as the position and stereochemistry of the C-C double bonds can vary along the backbone to constitute a species-specific semiochemical language. ${ }^{72-75}$ Experimental investigations have shown that the desaturation of fatty acyl-CoA esters involving 
membrane-bound acyl-CoA desaturases is the most important reaction that shows high commitment for the production of olefinic pheromone blends. ${ }^{14,74,76}$ Unlike desaturases found in animal and fungi, insect desaturases show enormous enzymatic plasticity and are capable of producing a wide range of unsaturated fatty acyl-CoA esters of varying chain lengths, double bond stereochemistry as well as position. ${ }^{39,41,71,75,77}$

Gemma Fabrias et al. has reported that the female moth pheromone glands contain several $\mathrm{Cl} 4$ acetates including $(9 Z),(11 E)$ and (11Z)-11-tetradecenyl as well as $(10 E, 12 E)$ and $(9 Z, 11 E)$-tetradecadienyl acetates. ${ }^{39,71,76,78}$ Using radiolabeled precursors, (it was) demonstrated that $(9 Z, 11 E)$-tetradecadienoate is biosynthesized from $(11 E)$ tetradecenoate using a $\Delta^{9}$-desaturase, whereas the $(10 E, 12 E)$-tetradecadienoate is derived from (11Z)-tetradecenoate catalyzed by a $\Delta^{\prime \prime}$-desaturase enzyme (Figure 1.5). ${ }^{39,40,71,79}$
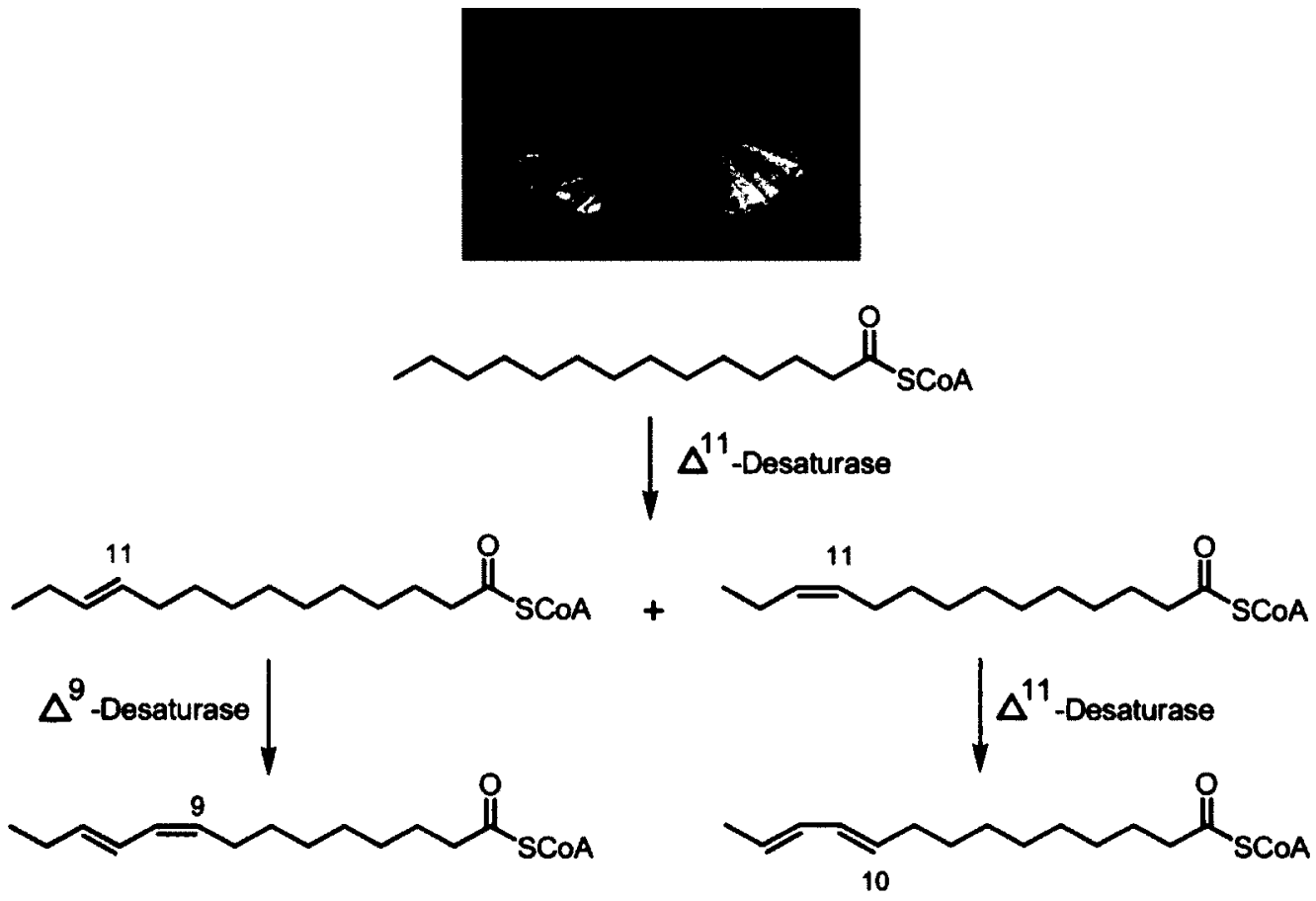

Figure 1.5. The initial involvement of various membrane-bound desaturases and the bifunctional activity of $\Delta^{11}$-desaturase in generating conjugated fatty acid derivatives in the pheromone blend of female processionary moth, Spodoptera littoralis. ${ }^{39,40,41}$ 
The pheromone blend of Bombyx mori was reported to contain a similar conjugated fatty acid derivative, $(10 E, 12 Z)$-hexadecadienoate that was believed to be generated from (11Z)- hexadecenoic acid employing a $\Delta^{11}$-desaturase enzyme that itself catalyzes the formation of (11Z)-hexadecenoic acid from palmitic acid. ${ }^{80}$

Conjugated eneynes are found in another female processionary moth, Thaumetopoea pityocampa. ${ }^{41,75,81}$ It has been observed that there exists a remarkable multifunctional desaturase enzyme which is responsible for exhibiting $\Delta^{11}$-desaturase, $\Delta^{11}$-acetylenase and $\Delta^{13}$-desaturase activities equally (Figure 1.6 ).

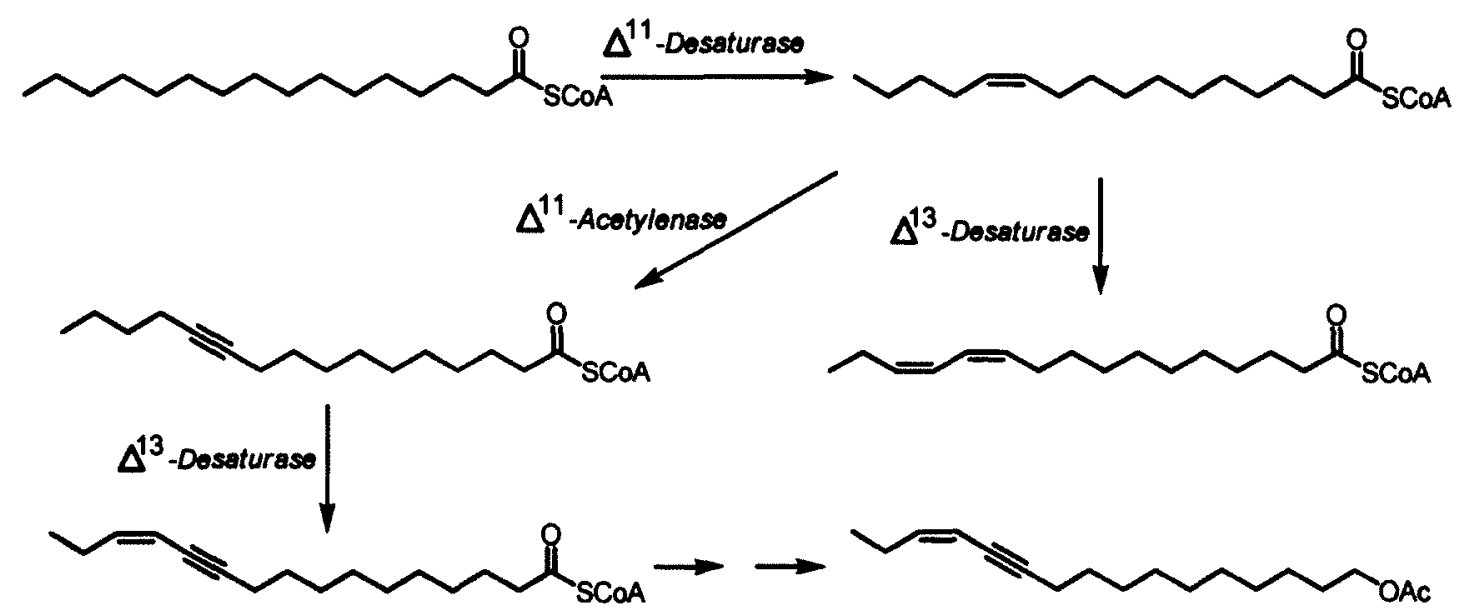

Figure 1.6. Catalytic diversity of a multifunctional $\Delta^{11}$-desaturase that is responsible for the biosynthesis of sex pheromone in processionary moth, Thaumetopoea pityocampa. The Figure was adapted from Gemma Fabrias. ${ }^{75}$

\subsubsection{Conjugated Fatty Acids in Plants}

It is estimated that the seeds of various plants contain thousands of structurally distinct fatty acid compounds in a species-specific distribution pattern. ${ }^{8,82,83}$ Conjugated fatty acids constitute an important subset of the so-called "unusual" fatty acids. Higher 
plants biosynthesize these compounds in developing seed tissues where they are stored primarily in the form of triacylglycerols (TAG) for future use as a source of energy and for structural purposes. ${ }^{83-85}$ An early biogenetic scheme that relates the various conjugated fatty acids to linoleic acid precursor by oxidative pathways is shown in Figure 1.7 .

The existence of $\alpha$-eleostearic acid was first reported by Cloez in $1875^{86}$ and subsequently the chemical structure of this conjugated fatty acid was elucidated by Böeseken and Ravenswaay in $1925{ }^{87}$ It was not until 1957 that L. Crombie and A. G. Jacklin first established the position and configuration of the conjugated trienes in $\alpha$ - and $\beta$-eleosteraric acid as well as punicic acid via total synthesis and analyzing the products based on their physical and spectroscopic properties. ${ }^{88}$ The $\alpha$-eleostearic and punicic acid were synthesized using several stereoselective reactions, whereas $\beta$-eleostearate (all trans isomer) was obtained from $\alpha$-eleostearate following the treatment with $\mathrm{I}_{2}$ in presence of UV-light. Typically the structures of conjugated fatty acids can be determined by a combination of M.P., UV absorbance, molar extinction coefficients, IR data, intensity characteristics of IR bands, quantitative hydrogenation, GC-EI/MS, ${ }^{1} \mathrm{H}$ and ${ }^{13} \mathrm{C}$ NMR, respectively. ${ }^{59}$

18:2-10E,12E CLA is the only non-oxygenated conjugated dienoic fatty acid that has been isolated from a plant source (10\% of the seed oil of Chilopsis linearis). Screening of the seed oil derived from Dimorphotheca species (Dimorphotheca aurantiaca, Dimorphotheca sinuata) revealed that more than $60 \%$ of the total fatty acid content in the oil contains dimorphecolic acid - a unique hydroxylated dienoic acid. Examples of conjugated trienoic fatty acids include $\alpha$-eleostearic acid (Tung oil), 


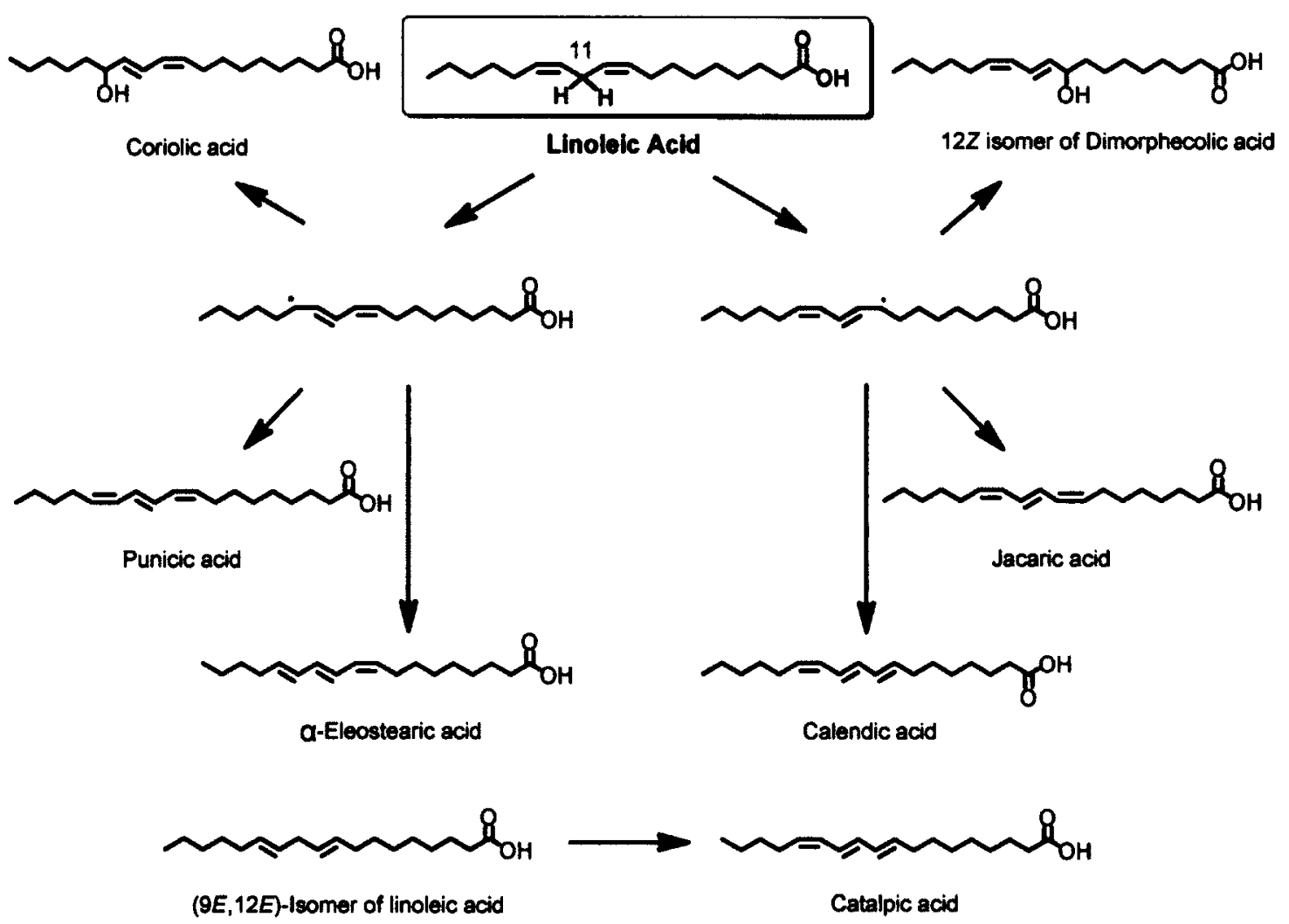

Figure 1.7. Proposed biogenesis of the conjugated fatty acids in plants beginning with $\mathrm{H}-$ abstraction at C-11 of linoleic acid. The Figure was adapted from Crombie et al. . $^{43}$

calendic acid, $\alpha$-parinaric acid, jacaric acid, catalpic acid and punicic acid; these can be found in over nine different plant families. ${ }^{46,62,64,89} \alpha$-Eleostearic acid is the principal fatty acid in the seed oil of tung tree (Aleurites fordii Hemsl.) and contains ca. $80 \%$ of the total fatty acid content. ${ }^{34,90}$ Other sources of $\alpha$-eleostearic acid include bitter gourd (Momordia charantia) where the seeds accounts for $\sim 65 \%$ of this fatty acid, but the flesh contains much less. ${ }^{89,91,92}$ The seed oil of marigold plant (Calendula officinalis) has been reported to contain about $51-53 \%$ of calendic acid. ${ }^{16,63,93} \alpha$-Parinaric acid is the major fatty acid constituent in the seed oil of Parinarium laurinum and Impatiens balsamina plant species where it present as $46 \%$ and $29 \%$ of the total fatty acid content in the oil, respectively. ${ }^{82,94,95}$ 
Oils enriched with conjugated fatty acids have many useful commercial applications such as drying agents in the formulations of inks, paints, plastics, nylon, dyes, resins and varmishes. ${ }^{11,34}$ Conjugated fatty acids are highly unstable and easily prone to oxidation. ${ }^{62,92,96,97}$ As a result, coating materials that contain oil from conjugated fatty acids dry more quickly compared to oil containing methylene interrupted unsaturations. The resultant coating gives more resistance to the extreme weather and adds value to the drying qualities. ${ }^{97}$

Among the medicinal properties of this family of compounds are associated with the anticancer, anti-inflammatory, antiatherosclerotic, antidiabetes, antiobesity (reduce body fat accumulation) properties. ${ }^{46,60-64} \alpha$-Eleostearic acid has shown stronger anticancer activity by inhibiting the growth of tumour cells and inducing the apoptosis of colon cancer cells. ${ }^{60,61,98}$ This fatty acid has also been shown to alter lipid metabolism by lowering the serum lipid level. ${ }^{65}$ Calendic acid has antiinflammatory properties that is used to treat burns, cuts, rashes, any skin wounds ${ }^{99}$ and causes the reduction of body fat content in mice. ${ }^{63,48}$ Dimorphecolic acid is an aromatase and acetycholine esterase inhibitor and is used to treat familial Mediterranean fever, a hereditary inflammatory disorder. ${ }^{100} \alpha$-Parinaric acid is commercially used as fluorescent binding tag that selectively binds to the peroxisome proliferator activated receptors (PPARs), and target proteins for drugs against cancer, diabetes, atherosclerosis. ${ }^{101}$ It is also used as an fluorescent tag to measure lipid peroxidation in cardiomyocytes during ischemia and reperfusion and has also shown promising cytotoxic activity against malignant glioma cell lines. ${ }^{102,103}$ 


\subsection{Fatty acid desaturases}

\subsubsection{Historical Overview}

As illustrated in the previous section, conjugated fatty acids in plants are generated from unsaturated fatty acyl precursors (Figure 1.7) via an $\mathrm{O}_{2}$-dependent dehydrogenation pathway that is catalyzed by a superfamily of enzymes known as fatty acid desaturases (FAD). ${ }^{1,2,104}$ To differentiate this highly exceptional aerobic dehydrogenation of unactivated methylene groups from the anaerobic removal of hydrogens at an activated position, the term "desaturation" was proposed. ${ }^{12,105}$ Fatty acid desaturases are non-heme diiron proteins that introduce double bonds in a manner that cannot be reproduced by synthetic organic catalysts. ${ }^{1,4,106}$ The enzymes are highly regioselective, stereoselective as well as chemoselective and are universally present in every aerobic living organisms examined to-date including bacteria, algae, fungi, plants, insects, protozoa and mammals. Desaturases are unanimously denoted as $\Delta^{\mathrm{n}}$, where " $n$ " signifies the position of the double bond going to be introduced into the fatty acid chain with respect to the carboxyl carbon. For example, a desaturase enzyme that
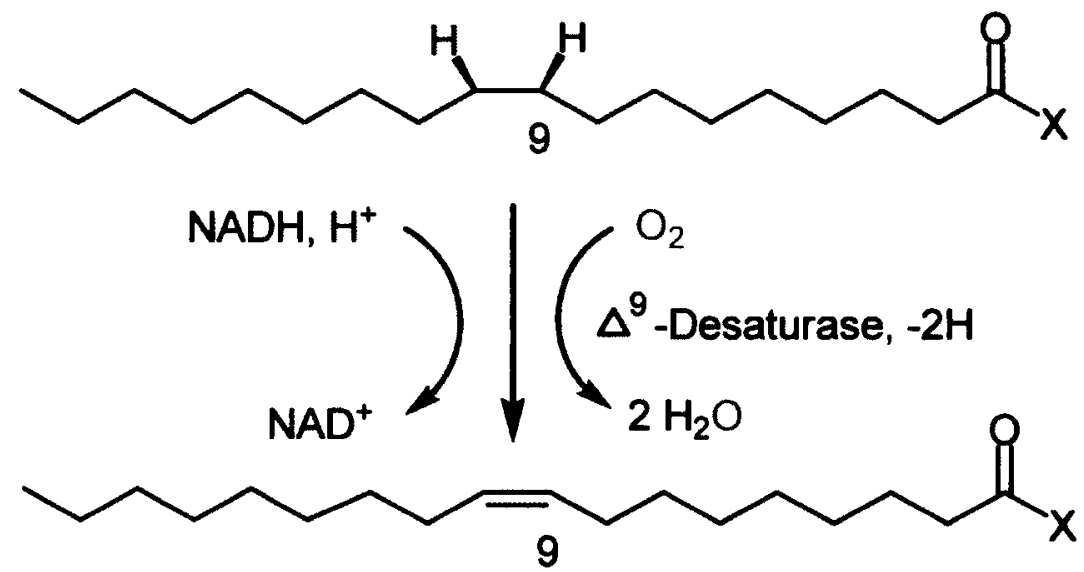

Figure 1.8. A prototypical $\mathrm{O}_{2}$-dependent desaturase-mediated biotransformation reaction (X can be CoA, PL or ACP). 
introduces a double bond at the C-9,10 position of a $18 \mathrm{C}$ fatty acyl chain is referred to as $\Delta^{9}$-desaturase (Figure 1.8).

Some desaturases also use the terminal methyl group as a reference point and introduce the double bond relative to that position. This group of desaturases are designated as " $\omega-n$ ". As an example, a desaturase enzyme that introduces a double bond three carbons away from the methyl terminus, i.e. at C-15 carbon of a 18 carbon fatty acyl chain is designated as a $\omega-3$ desaturase.

The intriguing biosynthetic process was discovered more than 50 years before when Bloch and Bloomfield first revealed that a membranous fraction of yeast, Saccharomyces cerevisiae bioassay can desaturate palmitoyl-CoA (and by implication stearoyl-CoA) to generate oleoyl-CoA in the presence of $\mathrm{O}_{2}$ and NADPH (Figure 1.8). ${ }^{1,107,108}$ The cryptoregiochemistry (site of initial H-abstraction) and stereospecificity of this biotransformation was also subsequently examined by this group by using radiolabelled isotopes; it was demonstrated that the $\Delta^{9}$ desaturase from Corynebacterium diphtheriae is initiating the oxidation by selectively removing the pro $R$ hydrogen at C-9 and then the pro $R$ hydrogen at the C-10 position of substrate. ${ }^{2,109}$ This implies that desaturation is a syn dehydrogenation process. It is now known that the essential biochemistry of this reaction involves four-electron reduction of molecular oxygen to two molecules of water where two electrons come from NAD(P)H via an electron transfer chain involving a $\mathrm{NAD}(\mathrm{P}) \mathrm{H}$ reductase and one or more electron transfer protein such as ferredoxin or cytochrome b5; and other two electrons comes from the fatty acid substrate. $^{1-4,110}$ The electron donors (NADH, NADPH) and the carrier protein (cytochrome b5 or ferredoxin) can vary with the type of desaturase operating. ${ }^{1}$ 


\subsubsection{Structural Biology of Desaturases}

Significant progress have been made in the last few decades in understanding the structural and molecular biology associated with this remarkable class of enzymes. This activity has led to the identification of many more desaturases with unique regioselectivities. ${ }^{110-112}$ Amino acid sequence comparison for a large set of desaturases were examined by Shanklin and Fox and as a result of their effort, it was apparent that desaturases could be divided into two major structurally unrelated categories: a) a smaller group of soluble desaturases that is found only in plants and utilizes specifically the fatty acid substrates as acyl carrier protein (ACP) thioester derivatives ${ }^{113} ;$ b) a relatively large set of widely distributed integral membrane-bound desaturases that act on CoA- or phospholipid (PL)-linked fatty acid substrates. ${ }^{1,112,114,115}$ These two classes of desaturases have also been shown to differ significantly with respect to their reaction kinetics. ${ }^{11}$

\subsubsection{Soluble Plant Desaturases}

An example of a typical soluble plant desaturation reaction is the production of oleoyl-ACP from stearoyl-ACP catalyzed by a soluble plant stearoyl $\Delta^{9}$-desaturase in the castor bean (Ricinus communis L.) (Figure 1.8). ${ }^{2}$ Plant seed oil is the major source of oleic acid where it is stored as triacylglyceride derivative. This observation has led to the investigation of several plant tissues such as safflower seeds, hedera helix (English ivy), soybean cotyledons, spinach and avocado. Considerable attempts have been made in the last two decades to biochemically characterize the soluble plant desaturases which eventually lead to the identification and determination of the crystal structures of several soluble plant desaturases. ${ }^{116-123}$ The most significant breakthrough in desaturase research took place in 1991, when Shanklin and Somerville as well as Thompson et al. 
individually succeeded in isolating and purifying the soluble plant stearoyl-ACP $\Delta^{9}-$ desaturase from castor seed ${ }^{124}$ and safflower embryos ${ }^{125}$ respectively, and overexpressed this protein in E. coli. ${ }^{17,118}$ Lindquist and Shanklin also resolved the three-dimensional crystal structure associated with castor stearoyl-ACP $\Delta^{9}$-desaturase. The crystallographic model revealed the presence of a recombinant homodimeric desaturase (molecular wt $70 \mathrm{kDa}$ ) where each of the monomeric unit contain a diiron cluster in the vicinity of a hydrophobic binding cavity (Figure 1.9a)..$^{118,124,125}$ In agreement with spectroscopic data, ${ }^{118}$ it was demonstrated that the active site of this enzyme features a non-heme diiron core that is

a)

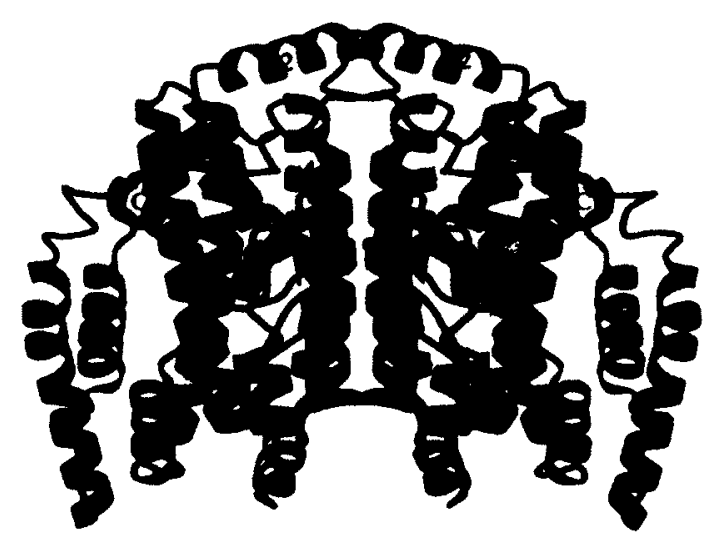

b)

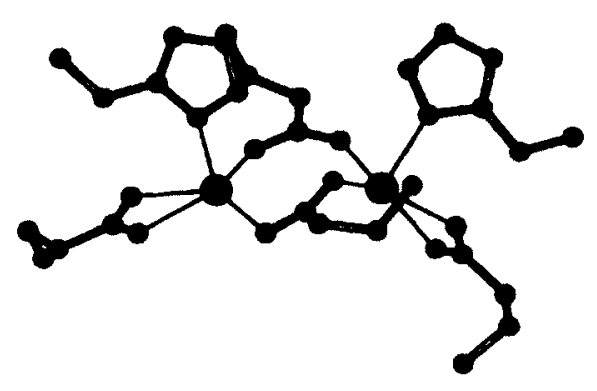

Figure 1.9. Three-dimensional crystal structure of soluble plant stearoyl-ACP $\Delta^{9}$ desaturase. a) View of the homodimer along the $\alpha$-axis. The two subunits are coloured green and blue, respectively and two iron units are indicated as red spheres; b) Stereoview of the diiron centre with ligands. Images taken from Lindqvist et al. ${ }^{118}$ 
coordinated with four carboxylate $\mu$-oxo bridged and non-bridged glutamate ligands and two histidine units of the amino acid side chain (Figure 1.9b). ${ }^{118,126} \mathrm{~A}$ water molecule was also found in the second coordination sphere from the iron atoms. This catalytic core was found to be surprisingly similar to that of well characterized methane monooxygenase (MMO) enzyme that is responsible for the hydroxylation of methane to yield methanol. ${ }^{126}$ Analysis of the structure by Lindqvist et al. suggested a deep hydrophobic binding cavity at the active site that is presumed to accommodate the substrate fatty acid and extends from the surface to the interior of the enzyme. ${ }^{118}$ The topology of this hydrophobic binding channel is boomerang-shaped and forces the substrate fatty acid to adopt a quasi-gauche conformation at the $\mathrm{C} 9-\mathrm{C} 10$ position (Figure 1.10).,2,118 This arrangement allows the C-9 and C-10 pro $R$ hydrogens to come very close to the catalytic

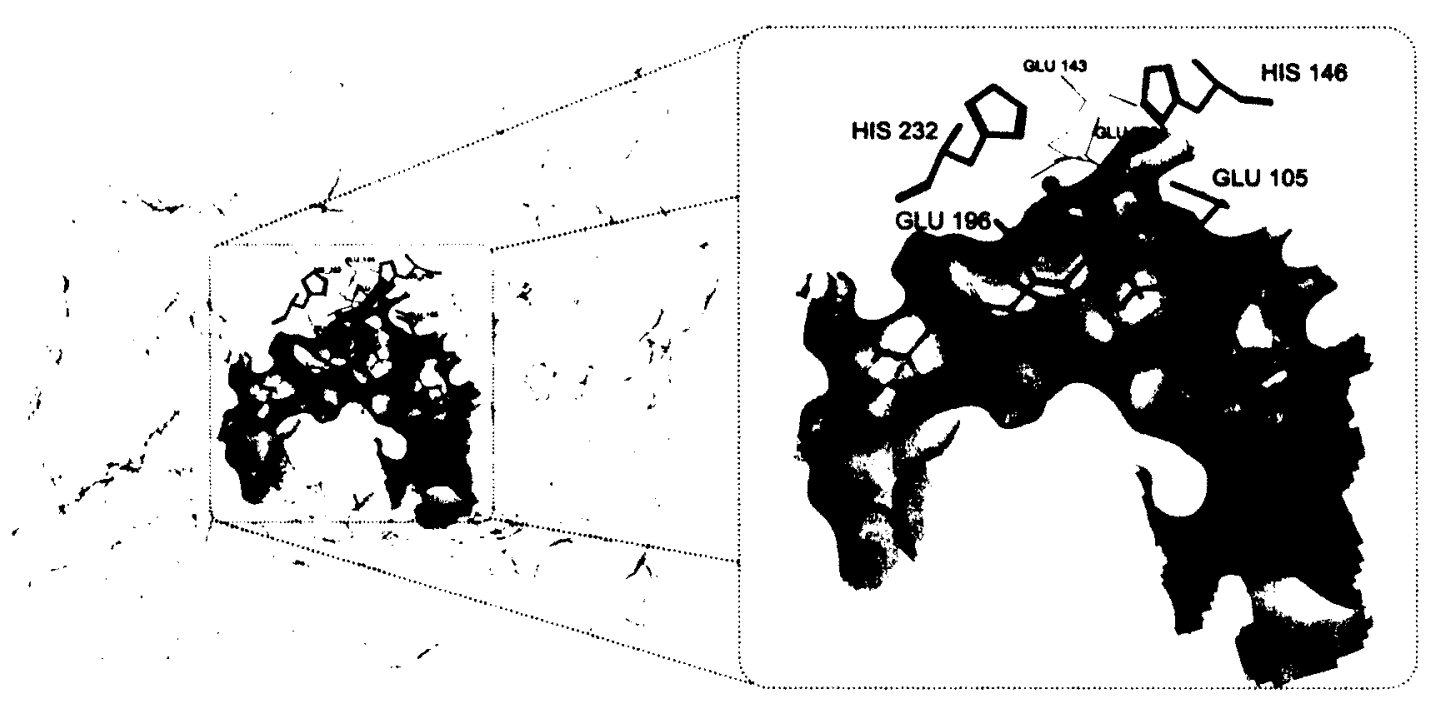

Figure 1.10. Computer model of a soluble plant $\Delta^{9}$-desaturase docked with a stearoylACP substrate in the hydrophobic binding pocket showing the topology of the active site. Image taken from Buist. ${ }^{2}$

centre that would facilitate the regioselective syn dehydrogenation process and generate thermodynamically unfavorable Z-double bond in the product oleoyl-ACP. ${ }^{116,118}$ The 
model was supported by the stereochemical investigation of this enzyme using stereospecifically monodeuterated fatty acid substrates (see Section 1.3.1). ${ }^{127}$ Using thiasubstituted analogues it was demonstrated that the catalytic non-heme diiron core is not actually present at the symmetrical position between C-9 and C-10 carbons, but slightly towards the C-10 carbon (see Section 1.3.2). ${ }^{110}$

It is worthwhile mentioning here that other soluble plant desturase enzymes that show different regioselectivities and chain length specificities exhibit strong similarities to that of the castor stearoyl-ACP $\Delta^{9}$-desaturase in terms of their overall amino acid sequence and diiron binding mode. ${ }^{104}$ Crystal structures of a soluble $\Delta^{4}$-desaturase from Hedera helix was recently documented; sequence comparison showed that this regioselective desaturase enzyme shows $74 \%$ homology in terms of its overall amino acid sequence with castor $\Delta^{9}$-desaturase. ${ }^{117}$ The primary determinant of regioselectivity turned out to be the interaction between the particular amino acid (ASP280 in castor $\Delta^{9}$ desaturase and the corresponding LYS275 in ivy $\Delta^{4}$-desaturase) located at the entrance of the binding cavity and the acyl carrier protein, ACP (phospho-serine 38 in castor $\Delta^{9}$ desaturase and phosphate of the pantetheine in ivy $\Delta^{4}$-desaturase). ${ }^{128}$ Most of the mechanistic, structural and bioinorganic development in this area of desaturase research has been carried out using the castor stearoyl-ACP $\Delta^{9}$-desaturase as a model..$^{3,4}$

\subsubsection{Membrane-Bound Desaturases}

As mentioned previously, dehydrogenation reaction at C-9 and C-10 of a stearoyl substrate has also been found to be catalyzed by a set of membrane-bound $\Delta^{9}$ desaturases. ${ }^{2}$ An example of such biotransformation has already been given in the historical overview section 1.2.1: Bloch first demonstrated that in yeast, $\Delta^{9}$-desaturation 
of stearoyl-CoA generates oleoyl-CoA. In contrast to the situation described above for soluble desaturases, there has not been much progress in isolation, purification and characterization of the membrane-bound desaturases. ${ }^{1,2}$ This is mostly because of the fact that the membrane-bound desaturases are highly unstable outside of the native hydrophobic environment. ${ }^{1}$ Purifying membrane-bound desaturases to generate sufficient quantity for structural and spectroscopic determination has proven to be difficult.' Strittmatter's group first reported the isolation and purification of an integral membranebound $\Delta^{9}$-desatuase along with its electron transport chain consisting of an NADH (nicotinamide adenine dinucleotide) reductase and cytochrome-b5 components by fractionation of rat liver microsomes. ${ }^{128}$ This group has also reported the presence of a non-heme iron functionality instead of a heme group at the active site of rat liver microsomal stearoyl-CoA $\Delta^{9}$-desaturase. ${ }^{129}$ Subsequent comprehensive examination by Shanklin and Fox of the amino acid sequences of a larger set of membrane-bound desaturases from mammals, fungi, insects, higher plants and cyanobacteria revealed that there exists eight essential histidine residues and these are present in three highly conserved $\mathrm{HX}(2-4) \mathrm{H}$ boxes. ${ }^{114}$ This hypothesis was confirmed using site-directed mutagenesis experiments. As described above, the enzymatic properties of desaturation i.e. the requirement of $\mathrm{O}_{2}$ and $\mathrm{NADPH}$ and a catalytic iron centre matches that of hydroxylation by mixed function monooxygenases. ${ }^{130}$ Therefore, it has long been believed that the active site features of both types of enzymes should be similar. Thus, membrane-bound alkane hydroxylases from Pseudomonas oleovorans and related organisms also feature the presence of such three highly conserved histidine boxes. In addition, the existence of a non-heme diiron core at the active site was established for the 
$P$. oleovorans enzyme by Mössbauer spectroscopy. ${ }^{131}$ It is presumed that the essential histidine residues of membrane-bound desaturases/hydroxylases are coordinating with the non-heme diiron center at the catalytic site. ${ }^{1,2}$ A fused cytochrome b5 component i.e. responsible for transporting electrons from NADH reductase to the catalytic diiron center was also documented with a number of membrane-bound desaturases. ${ }^{132}$

At present, only a generalized two-dimensional topological model is available (Figure 1.11) that was developed by hydropathy analyses of the overall amino acid sequence of the rat liver stearoyl-CoA $\Delta^{9}$-desaturase with a homologous yeast $\Delta^{9}$-desaturase. ${ }^{133,134}$ The model demonstrates that the enzyme is actually anchored in the endoplasmic reticulum (ER) membrane where it forms two pairs of closely-spaced membranespanning domains and majority of the enzymatic part is suspended in the cytosolic side of the endoplasmic reticulum (ER) membrane. ${ }^{1}$ However, due to the lack of proper structural information, how the interaction between the substrate fatty acid and amino acid side chains at the active site of these classes of enzymes facilitates such selective dehydrogenation still remains highly illusive.

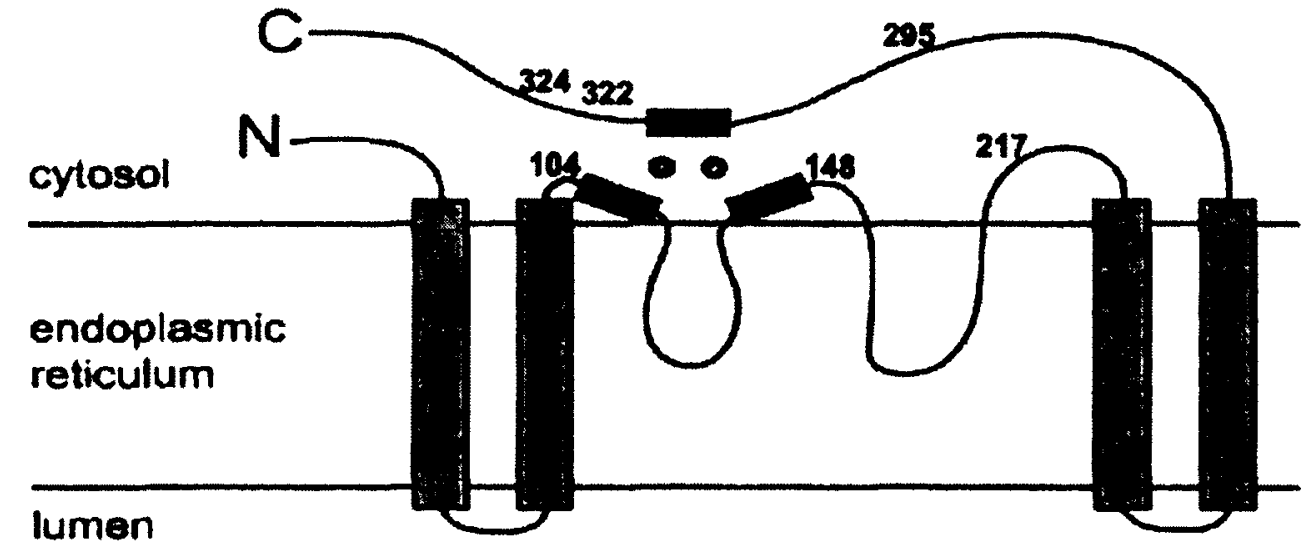

Figure 1.11. Proposed topological model of the membrane-bound $\Delta^{9}$-desaturase (FAD2) showing the presence of two closely spaced membrane-spanning domains in the ER membrane and three essential histidine regions in the cytosolic part are coordinating with the non-heme diiron center at the active site. Image adapted from Meyer et al..$^{135}$ 


\subsubsection{FAD2 Family of Desaturases}

A very important subset of membrane-bound fatty acid desaturases is the family of phytoenzymes that are responsible for the biosynthesis of the essential fatty acid, linoleic acid. ${ }^{136}$ One of the two pathways by which linoleic acid is biosynthesized in plants involves an extraplastidial oleate $\Delta^{12}$-desaturase enzyme (FAD2). ${ }^{2,137}$ In addition, a number of closely related species-specific FAD2 variants generate a number of exotic fatty acids as discussed previously in this thesis (Figure 1.12). In 1996, Pat Covello's group was the first to successfully express FAD2 in Saccharomyces cerevisiae - a convenient yeast strain. ${ }^{138}$ This early triumph greatly facilitated subsequent mechanistic studies of FAD2 and related enzymes using in vivo yeast expression systems instead of plant tissues.

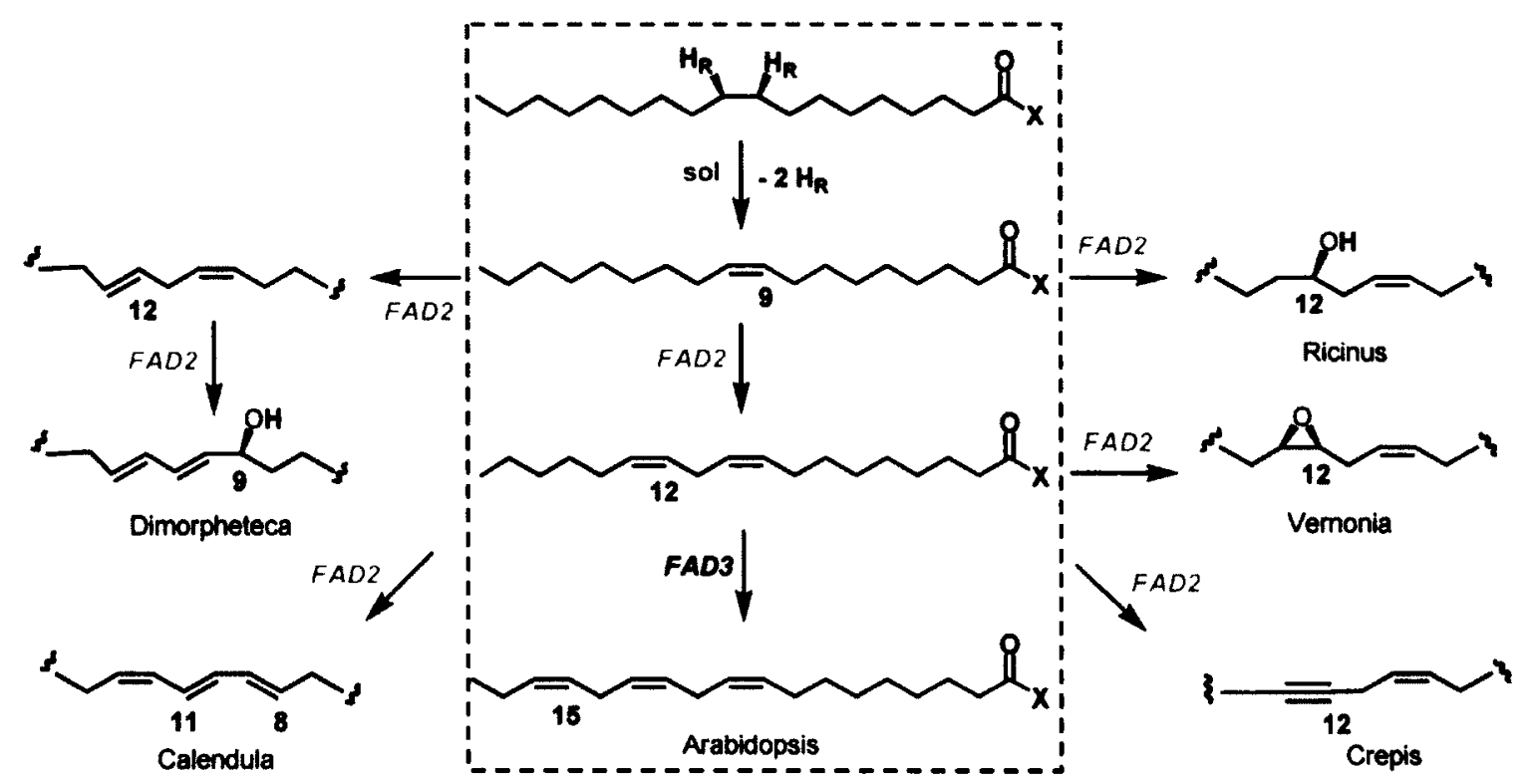

Figure 1.12. Biosynthesis of several exotic oxidized fatty acid compounds in plants catalyzed by a family of FAD2 variants. The Figure was adapted from Buist. ${ }^{1}$ 


\subsection{Mechanistic investigations of desaturases}

The currently accepted mechanistic model for fatty acid desaturation is depicted in Figure 1.13.' This model is equally acceptable for both soluble and membrane-bound desaturases since both of the enzymatic systems utilizes a non-heme diiron catalytic center, molecular $\mathrm{O}_{2}$ and NADH as a source of electrons. ${ }^{1,2}$ Experimental evidence from different sources suggested that the removal of hydrogens from two vicinal carbons takes place in a non-synchronous (step-wise) manner. ${ }^{1,26,127,139}$ It has also been demonstrated that during the course of dehydrogenation a syn removal of hydrogens takes place by abstracting the pro $R$ (or topological equivalent) hydrogens from the vicinal carbons. ${ }^{1,40,109,140}$ A highly constrained active site forces the substrate to bind in a

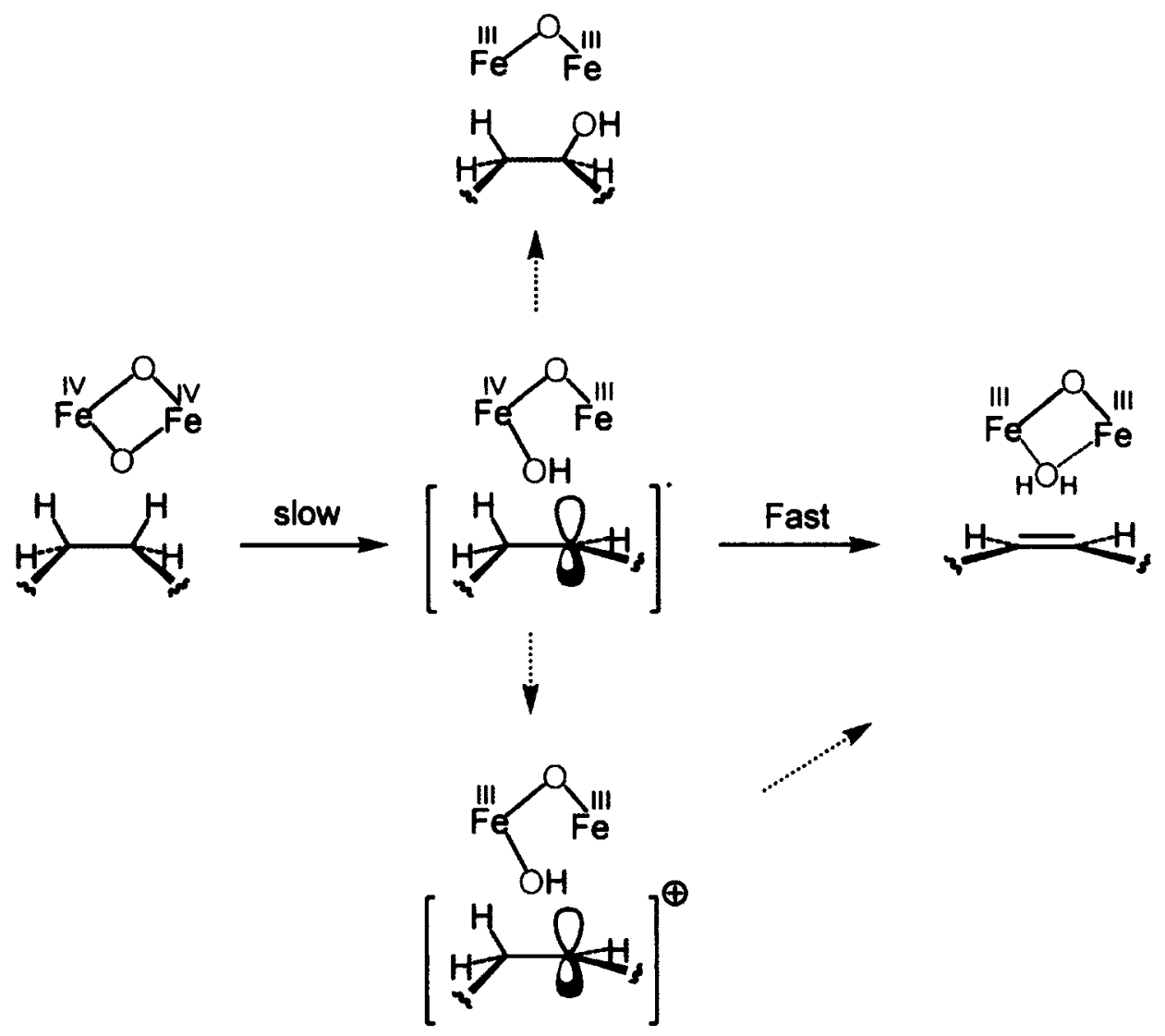

Figure 1.13. The currently accepted mechanistic model for fatty acid desaturation. The Figure was adapted from Buist. ${ }^{2}$ 
quasi-gauche/near-eclipsed conformation. ${ }^{1,2}$ The desaturation is initiated with the abstraction of a hydrogen atom (pro $R$ ) using a compound Q-type high valent iron-oxo species $^{141}$ leading to the formation of a carbon centered radical intermediate. ${ }^{1}$ This step is believed to be kinetically the slowest step of the reaction as it involves the breaking of a very strong, unactivated secondary $\mathrm{C}-\mathrm{H}$ bond. ${ }^{142}$ This radical intermediate is highly unstable and collapses immediately via the elimination of second hydrogen from the adjacent carbon and generating a cis $\mathrm{C}-\mathrm{C}$ double bond along with iron-bound water.,110 According to the current scenario, removal of the second hydrogen could occur either directly or via a one electron oxidation/deprotonation sequence. ${ }^{1,110}$

Support for this model (Figure 1.13) was obtained from many membrane-bound desaturases that produce the corresponding regiospecifically hydroxylated alcohol as low-level error product. ${ }^{16}$ It is thought that generation of hydroxylated product could only be possible if instead of a second hydrogen removal, in fact a competing "hydroxyl rebound" step is followed. ${ }^{2}$ Early evidence by Bloch suggested that the hydroxylated intermediates are not forming as an intermediate during the course of olefin production. ${ }^{143}$ It can be assumed that the relative positioning of the $\mathrm{FeOH}$ species with respect to the radical intermediate as well as the orientation of the second $\mathrm{C}-\mathrm{H}$ bond compared to the half-filled orbital of carbon-centered radical (syn-periplanar for double bond formation) could determine the reaction outcome. ${ }^{1,110}$

In summary, for each desaturase or desaturase variant, three fundamental questions associated with this generic mechanistic model need to be answered: ${ }^{2}$

1. The stereochemical issue - i.e. which hydrogens are removed during the course of desaturation? This is related to the enantioselectivity of desaturation at each prochiral centers. 
2. The cryptoregiochemistry or site of initial oxidation - i.e. assuming there is a preference, then which hydrogen is getting abstracted first by this highly oxidizing iron-oxo species?

3. The chemoselectivity of desaturation - i.e. what factor(s) controls the fate of putative radical intermediate outcome such that the formation of hydroxylated product is prevented?

Answers to the first two questions give critical information on the position of the oxidant with respect to the substrate and help design possible computational experiments to tackle the third. Mechanistic investigations of desaturase-mediated reactions are very much dependent on the use of sterically non-demanding probes that can function as an alternative to the substrate. ${ }^{1}$ For example, the use of methyl substituted fatty acid analogues as alternative substrates by James $e t$ al. where a methyl group was present adjacent to the site of initial oxidation resulted in unproductive substrate binding. ${ }^{144}$ This experimental finding put some limitation over the types of substrate analogues that can be used as mechanistic probes for the active site. In general the substrates bearing isotopic labels such as deuterium $\left({ }^{2} \mathrm{H}_{1}\right)$, tritium $\left({ }^{3} \mathrm{H}_{1}\right),{ }^{18} \mathrm{O}$ and isosteric substitution of hydrogen with fluorine, or methylene unit(s) with sulfur or oxygen are used as possible mechanistic probes. ${ }^{1,2}$ Fatty acid analogues must be enzymatically activated as ACP, CoA or phospholipid ester derivatives which place further restriction on their use. ${ }^{1}$ Critical analysis of all these issues are explained in the following sections.

\subsubsection{Stereochemistry}

There are two stereochemical issues associated with the course of desaturation. These are firstly, the enantioselective way of hydrogen removal from each methylene 
carbons by the reactive diamond-shaped diiron oxo species and secondly, the relative stereochemistry of $\mathrm{C}-\mathrm{H}$ bond breaking at the two adjacent carbons to produce either cis or trans-double bonds.

Traditionally the stereochemistry of hydrogen abstraction is analyzed by using isotopically labelled fatty acid substrates (Figure 1.14). Deuterium or tritium is used as the isotopic label for this purpose. ${ }^{1}$

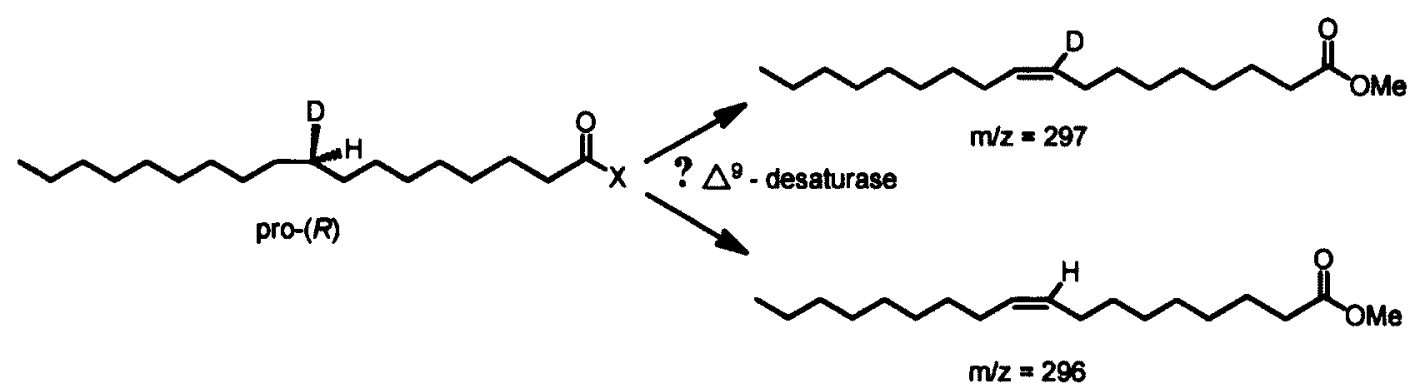

Figure 1.14. Use of chiral monodeuterated substrates as isotopic mechanistic probe to determine the enantioselectivity of $\Delta^{9}$-desaturation.

The approach was first recognized as a powerful tool back in the 1960's when Bloch revealed the stereochemical course of oleate formation in yeast using four stereospecifically monotritiated stearic acids and monitored the loss of isotope labelling with respect to ${ }^{14} \mathrm{C}$-labeled substrate. ${ }^{109}$ These isotopically labelled stearates were synthesized from naturally occurring hydroxystearic acids of known absolute configuration. ${ }^{109,140,145}$ Since then, this general approach continues to be fruitfully applied in investigating fatty acid desaturation. Nowadays, the chiral monodeuterated fatty acids are prepared from alcohols that are produced in high enantiomeric excess using well established synthetic methodology. ${ }^{146}$ In some cases, especially in vivo experiments where chances of isotopic dilution due to endogenous product formation is high, the substrates also contain extra remote deuterium labels to facilitate mass spectrometric 
analysis. ${ }^{40,81}$ The desaturated product of the incubations in vitro or in vivo are analyzed by gas chromatography coupled to mass spectrometry to monitor the loss or retention of deuterium labelling (Figure 1.14). In an alternative way, the chiral monodeuterated substrates can also be generated in situ from $\left[2-{ }^{13} \mathrm{C}, 2-{ }^{2} \mathrm{H}_{3}\right]$-acetate via fatty acid synthase and the loss of isotope labels can be traced at even-numbered carbons using ${ }^{13} \mathrm{C}$ NMR. ${ }^{147,148}$

The second stereochemical issue of desaturation i.e. whether the conversion is operating in syn or anti fashion can be most easily estimated using threo- and erythrodideuterated substrates, which can be synthesized from the corresponding olefinic precursor using stereospecific reduction (see Figure 1.15). ${ }^{1}$ The resultant desaturated product is analyzed by gas chromatography-mass spectrometry to determine whether the biosynthetic process yielded mainly monodeuterated or non-deuterated/dideuterated product.

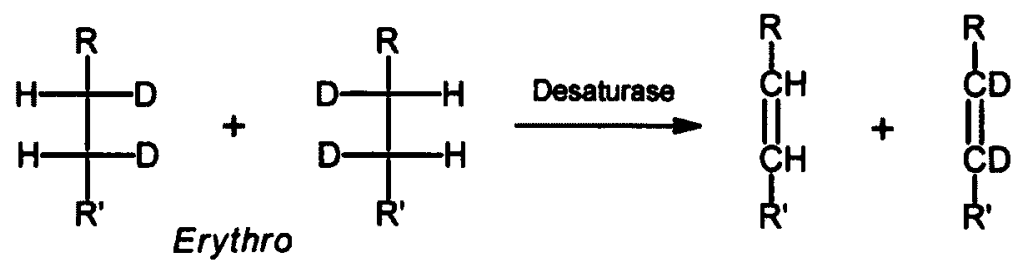

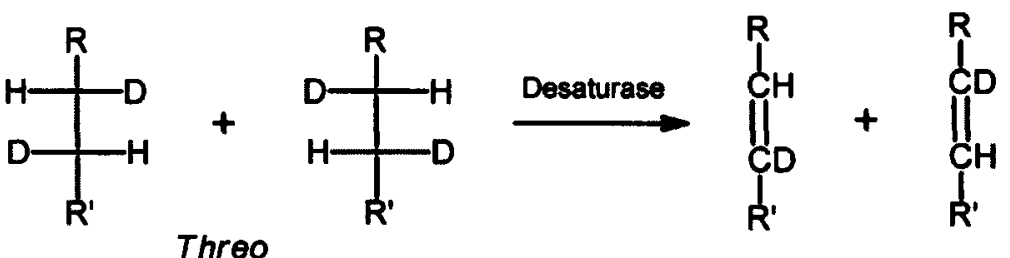

Figure 1.15. Determination of the relative stereochemistry of $\mathrm{C}-\mathrm{H}$ bond breaking via the racemic erythro and threo-dideuterated fatty acids as probes which confirmed the synremoval of hydrogens from the adjacent methylene carbons. 
A novel approach to ascertain the stereochemistry of hydrogen removal has been developed by Buist et al. that exclude the necessity of synthesizing chiral monodeuterated substrates. This approach features the use of fatty acid thia analogues acting as an oxo trap to yield chiral sulfoxides in high enantiomeric excess (Figure $1.16) .^{149,150}$

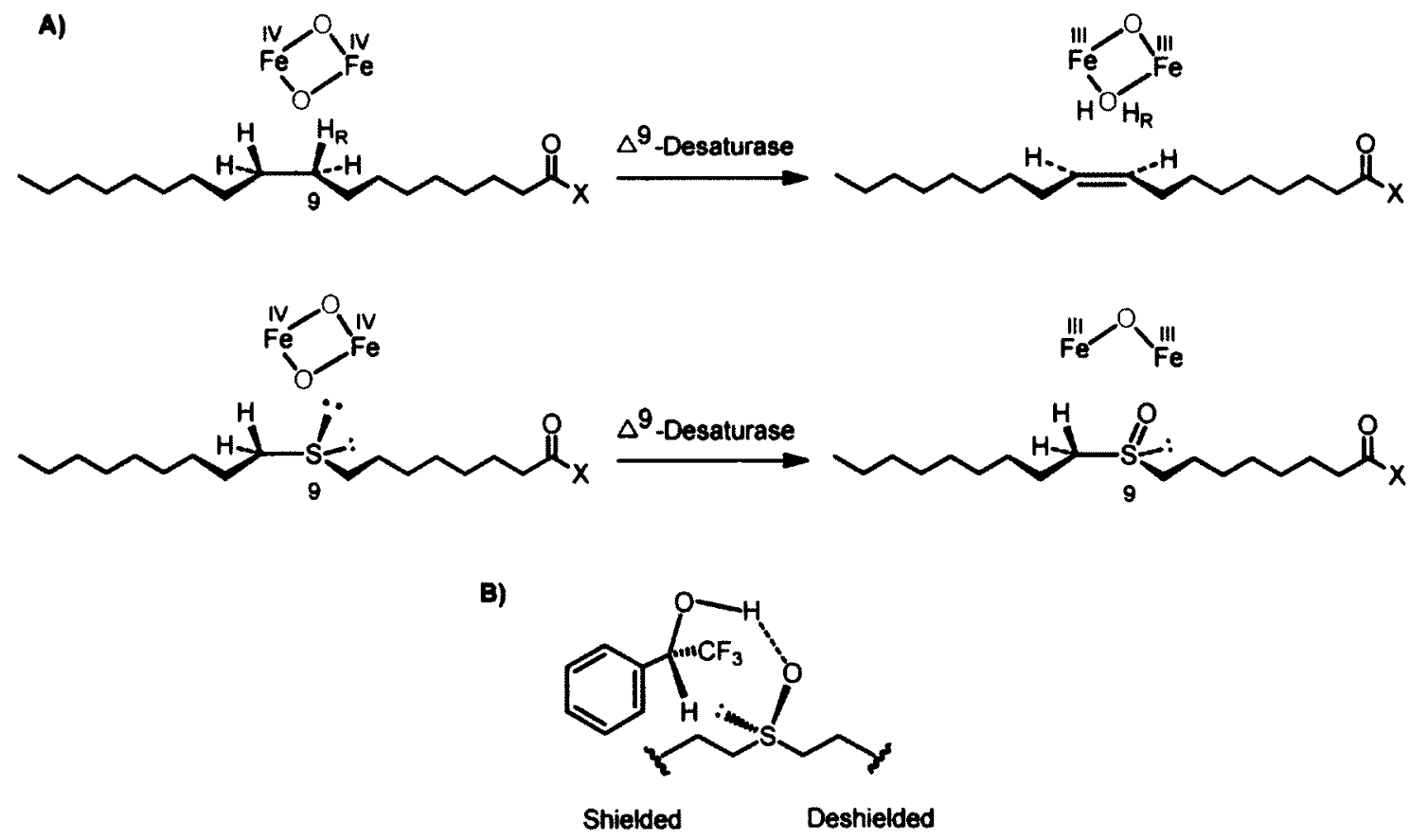

Figure 1.16. Application of thia-fatty acid analogue to elucidate the stereochemistry of desaturation. A) Stereochemical relationship between the fatty acid desaturation and sulfoxidation; B) Determination of the absolute stereochemistry at the sulfoxide center using a chiral solvating agent (CSA) showing the effect of complexation on the chemical shifts of neighbouring atoms in NMR.

The absolute stereochemistry of the sulfoxide center is ascertained through the use of Pirkle-type chiral NMR solvating agents (Figure 1.16B). ${ }^{150,151}$ These form a collision complex by forming a H-bond between the reagent's hydroxyl or carboxyl hydrogen and sulfoxide oxygen. ${ }^{149,152}$ In addition to the hydrogen bond, to account for the specific shielding effects a stabilizing interaction between the lone pair of the sulfoxide 
and the methine of the CSA has also been proposed. Such complexation induces shift non-equivalence in selected NMR signals of the sulfoxide. Use of larger aromatic groups than phenyl, such as in (S)-AMA, $(R)$-TFAE or $(R)$-Naproxen increase the magnitude of non-equivalence on the selected NMR signals which leads to greater separation of the particular peaks in terms of chemical shift $(\delta) .{ }^{153,154}$ The results obtained to date from these oxo transfer reactions have matched exactly with the known enantioselectivity of hydrogen removal as determined via isotopically-labelled fatty acid substrates (Figure $1.16 \mathrm{~A}) .^{151}$

More recently, the sensitivity of this chiral sulfoxide procedure in determining the enantioselectivity of desaturation was further enhanced through the use of $\omega$-fluorotagged thiasubstrates in combination with well-characterized chiral solvating agents (CSA) (Figure 1.17). ${ }^{153,155,156}$ The procedure was first developed by Buist and coworkers who showed that the presence of a fluorine atom at the remote $\omega$-position can be used to differentiate between the two enantiomeric sulfoxides by ${ }^{19} \mathrm{~F}$ NMR /CSA methodology. ${ }^{155}$ Surprisingly, the sense of the non-equivalency generated due to the complexation of the $\omega$-fluoro-tagged chiral sulfoxides with the CSA is opposite to that observed with ${ }^{1} \mathrm{H}$ NMR as was determined using synthetic standards. ${ }^{153,155,156}$ This technique can be applied to resolve the stereochemistry of chiral sulfoxides at the trace level (nanomole scale). The high natural abundance of ${ }^{19} \mathrm{~F}$ isotope, inherently high sensitivity and wide chemical shift range has made the ${ }^{19}$ F-NMR based methodology highly successful.

To date, employing all these mechanistic tools, a syn dehydrogenation pattern has been observed with all desaturases, including soluble and membrane-bound, that again 
support the proposed mechanistic mode (Figure 1.13). ${ }^{1}$ Also for all desaturases, it has been shown that both hydrogens are removed with pro $R$ (or equivalent) selectivity.

A)

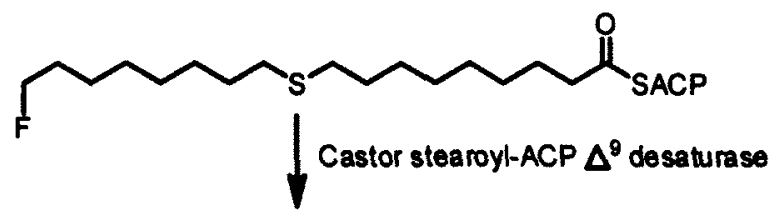

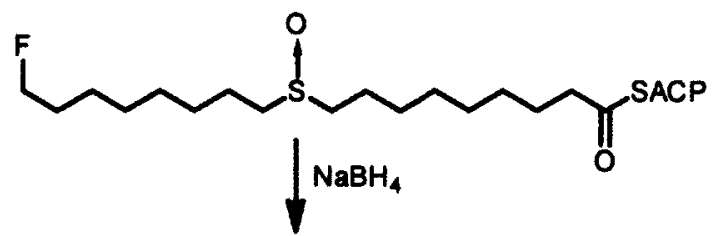<smiles>[O][SH](CCCCCCCCCF)CCCCCCCCCCO</smiles>

B)

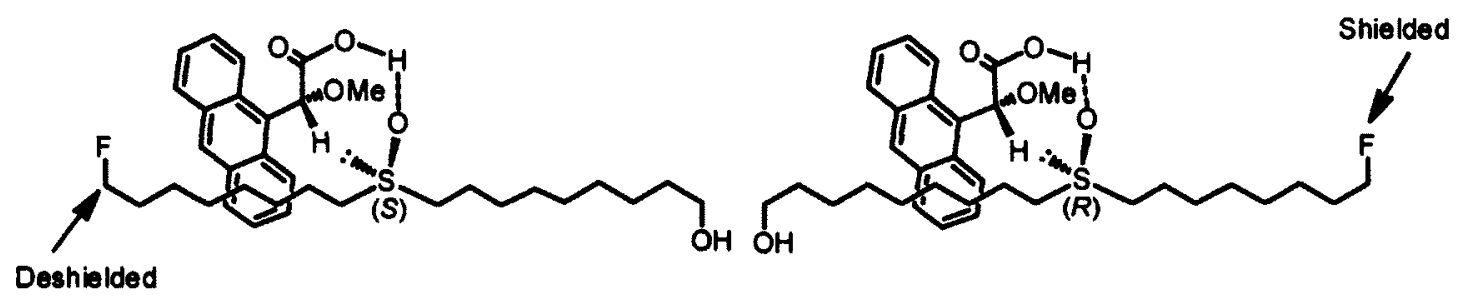

c)

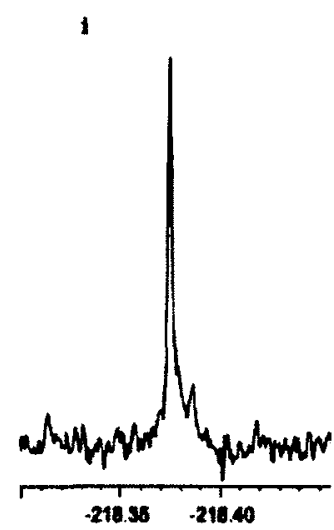

"

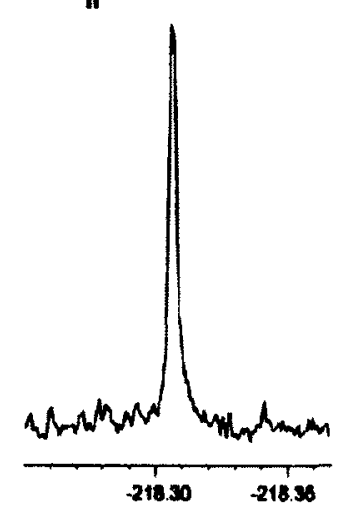

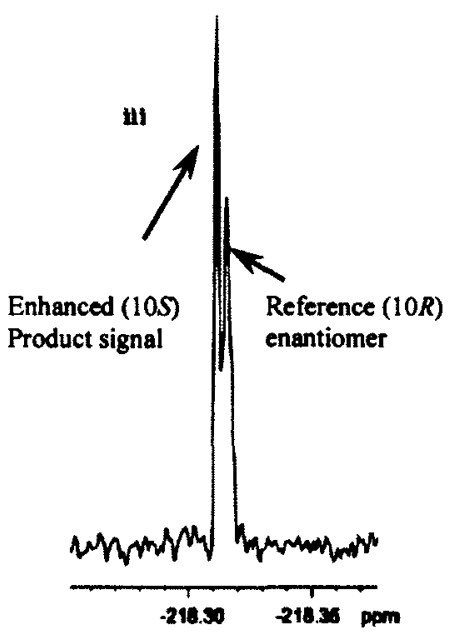

Figure 1.17. A) Castor stearoyl $\Delta^{9}$-desaturase-mediated chiral sulfoxidation of 18-fluoro10-thiaoctadecanoyl ACP; B) Pirkle-binding complexation model for the interaction of $(R)$-AMA with the two enantiomers of 18-fluoro-10-thiaoctadecan-1-ol S-oxide; $C$ ) its effect on ${ }^{1} \mathrm{H}$-decoupled ${ }^{19} \mathrm{~F}$ NMR spectroscopy - i) Enzymatically produced 18-fluoro-10thiaoctadecan-1-ol S-oxide (Biosyn-1), ii) (Biosyn-1) + (R)-AMA, iii) (Biosyn-1) spiked with racemic 18-fluoro-10-sulfoxy-1-octadecanol in presence of $(R)$-AMA. ${ }^{153}$ 


\subsubsection{Cryptoregiochemistry}

Cryptoregiochemistry ${ }^{157}$ is the most general term used to indicate the "site of initial oxidation" during the course of desaturation. As the desaturation reaction involves the abstraction of two hydrogens, the reaction could potentially start from either carbon generating a carbon centered radical that would ultimately yield the same double bond following the second removal of hydrogen. According to the literature, there are three ways to verify the cryptoregiochemistry of hydrogen abstraction. These are - i) the KIE measurement of each $\mathrm{C}-\mathrm{H}$ bond breaking step, ii) use of thia-analogues and evaluating the relative amount of sulfoxide formation and iii) isolation of the hydroxyl product that is formed by a low-level "error" pathway during dehydrogenation.

The most widely used and straightforward methodology of determining the cryptoregiochemistry of double bond formation is via the KIE (kinetic isotope effect) measurement. ${ }^{1,11,157}$ The approach is based on the principle that the abstraction of the first hydrogen by breaking the unactivated, very strong $\mathrm{C}-\mathrm{H}$ bond should be energetically more difficult as compared to the second hydrogen removal. As a result, the initial C-H bond breaking step should be more sensitive towards isotopic substitution than the later one. Therefore, if one can replace that particular $\mathrm{C}-\mathrm{H}$ bond by $\mathrm{C}-\mathrm{D}$ then there should be a kinetic isotope effect associated with its cleavage as breaking the C-D bond is energetically more difficult compare to a $\mathrm{C}-\mathrm{H}$ bond. However, there should be essentially no $\mathrm{KIE}$ associated with the second $\mathrm{C}-\mathrm{H}$ bond cleavage step as it is not kinetically important (fast step). The primary deuterium KIE is only observed at the rate determining

step. ${ }^{157}$ By measuring the primary deuterium KIE on each $\mathrm{C}-\mathrm{H}$ bond breaking step one can easily estimate the site of intial oxidative attack by the active diiron oxo species. To 
measure the primary deuterium KIE a competitive approach has been used, where typically a $\sim 1: 1$ mixture of regiospecifically dideuterated to non-deuterated fatty acids is used as substrates and incubated with a convenient desaturating system. The biosynthetic conversion is quenched at low substrate conversion $(<10 \%)$ and analyzed by using GCMS. ${ }^{1,11,158}$ The primary deuterium KIE is then computed by comparing the $d_{0} / d_{1}$ ratio of the olefinic product with the $d_{0} / d_{2}$ ratio of the substrate. ${ }^{157}$ Racemic monodeuterated substrates can also be used for this purpose and will essentially give the same result. ${ }^{159}$ However, it should be remembered that the anticipated primary deuterium KIE value on each $\mathrm{C}-\mathrm{H}$ bond cleavage step have already some embedded secondary deuterium KIE in it, but this perturbation is expected to be small $(<10 \%) .{ }^{160}$ Differential kinetic isotope

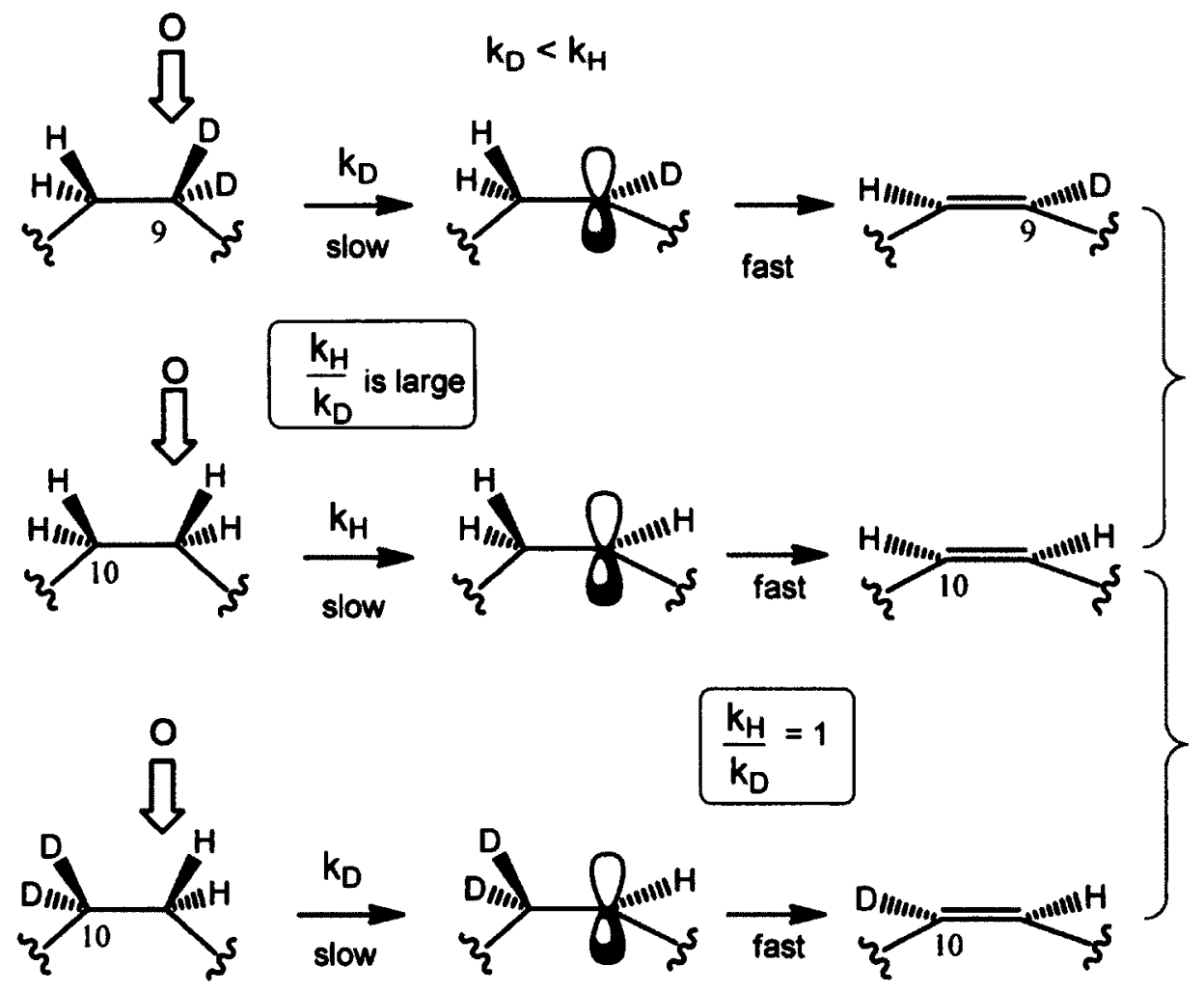

Figure 1.18. Use of intermolecular primary deuterium kinetic isotope effect measurement to determine the cryptoregiochemistry of oleate formation by a membrane-bound $\Delta^{9}$ desaturase. The site of initial oxidation is observed to be at C-9 as demonstrated by large $\mathrm{KIE}$ at $\mathrm{C}-9$ and $\mathrm{KIE}$ of $\sim 1$ at $\mathrm{C}-10$. 
effects on each $\mathrm{C}-\mathrm{H}$ bond cleavage for all membrane-bound desaturases studied to date (one large and one close to unity) clearly support the step-wise nature of the desaturation reaction as originally postulated by Bloch. ${ }^{107,108}$

However, the KIE protocol could not be used to determine the cryptoregiochemistry of dehydrogenation for soluble plant desaturases where it was found that $\mathrm{C}-\mathrm{H}$ bond breaking steps are not kinetically important steps of the reaction. ${ }^{1,161}$ Instead, other enzymatic events such as the substrate binding or product release step were found to be kinetically more important. ${ }^{1}$ Both competitive and non-competitive estimation of the intermolecular primary deuterium KIE on $\mathrm{C}-\mathrm{H}$ bond cleavage at $\mathrm{C}-9$ and $\mathrm{C}-10$ by the soluble plant $\Delta^{9}$-desaturase support this comment (KIE estimated to be 1 in both cases). ${ }^{161,162}$ A more broadly applicable method of determining the site of initial oxidative attack has been developed by Buist and coworkers. ${ }^{1,151,163}$ Systematic replacement of the substrate methylene groups by the isosteric sulphur atom can potentially induce regioselective oxo transfer from the diiron oxo species leading to the formation of sulfoxy-fatty acid (Figure 1.19). ${ }^{1,2}$

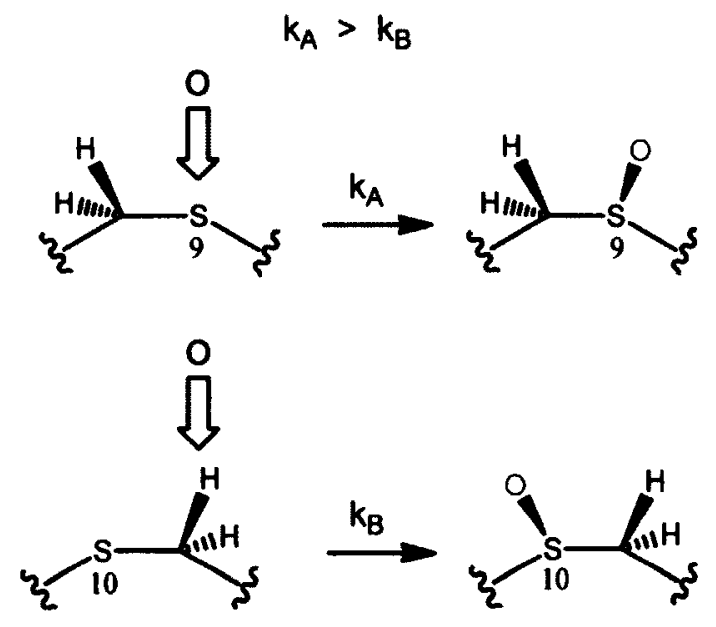

Figure 1.19. Schematic diagram preferential 9-sulfoxide formation by membranous $\Delta^{9}$ desaturase. 
The hypothesis is that the relative amount of sulfoxide production formed from each positional thia-analogue via oxo transfer should indicate the positioning of the putative diiron species relative of the substrate. ${ }^{1,151}$ This approach was initially validated for a membrane-bound yeast $\Delta^{9}$-desaturase as can be seen by comparing the results shown in Figure 1.18 and 1.19. In contrast, cryptoregiochemical investigation of a soluble plant $\Delta^{9}$-desaturase revealed that 10 -thiastearoyl $\mathrm{ACP}$ was efficiently converted into the corresponding sulfoxide, whereas the 9-thia analogue resulted in only a lower amount of (10\%) sulfoxide conversion. ${ }^{2}$

The sensitivity of this thia approach can be further enhanced through the incorporation of a fluorine tag at the remote $\omega$-position (Figure 1.20). ${ }^{164,165}$ Relative changes in the position of sulfoxidation from C-9 to C-10 can greatly affect the chemical shift of fluorine even though this atom is many bonds away from the position of the oxidative event. ${ }^{166}$ The oxidized $\omega$-fluoro-tagged-sulfoxy fatty acid products isolated

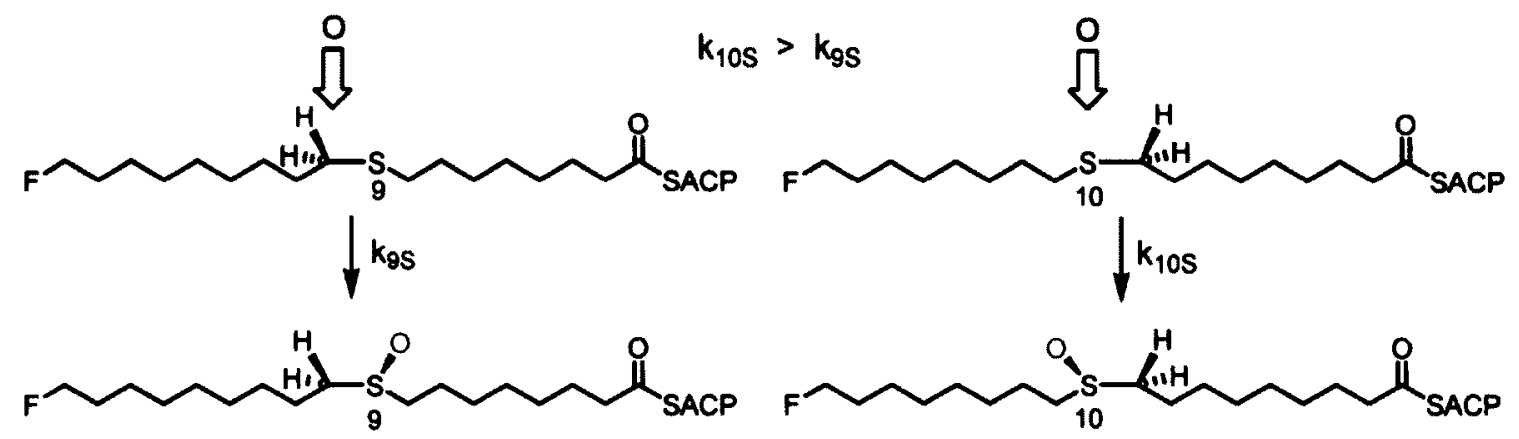

Figure 1.20. Determination of cryptoregiochemistry of castor stearoyl soluble-ACP $\Delta^{9}$ desaturase involved formation oleoyl-ACP by using $\omega$-fluoro-tagged thia-fatty acid analogues as mechanistic probe.

from the cultures and subsequently analyzed by using ${ }^{1} \mathrm{H}$-decoupled ${ }^{19} \mathrm{~F}$ NMR spectroscopy where an efficient measurement can be done even at the nanomole 
scale. ${ }^{165,167}$ The cryptoregiochemical result obtained from this fluoro-tagged thia analysis corroborate well with what have been observed using non-labelled the thia-analogues. ${ }^{166}$

Valuable information about the cryptoregiochemistry can also be obtained through the use of another mechanistic probe, namely by identifying the hydroxyl byproduct that is generated during the course of double bond formation as a low-level error product. ${ }^{16}$ As depicted in Figure 1.13, in a complementary pathway the putative radical intermediate can collapse to give alcohol. ${ }^{1,4}$ In such circumstance, the position and stereochemistry of the hydroxyl byproduct should match with the site of initial oxidation as well as the enantioselectivity of initial hydrogen abstraction. ${ }^{1}$ The yeast membranebound $\Delta^{9}$-desaturase from several sources produces ca. $1 \%$ of 9 -hydroxystearate as byproduct during dehydrogenation which clearly shows the site of initial hydrogen abstraction is at C-9 (Figure 1.21). ${ }^{16}$ Support for this observation has already obtained from the KIE methodology and sulfoxidation strategy ${ }^{168}$ Hydroxylated by-products are usually detected in GC-MS as silylated ether derivatives that dramatically increases the peak intensity. ${ }^{2}$

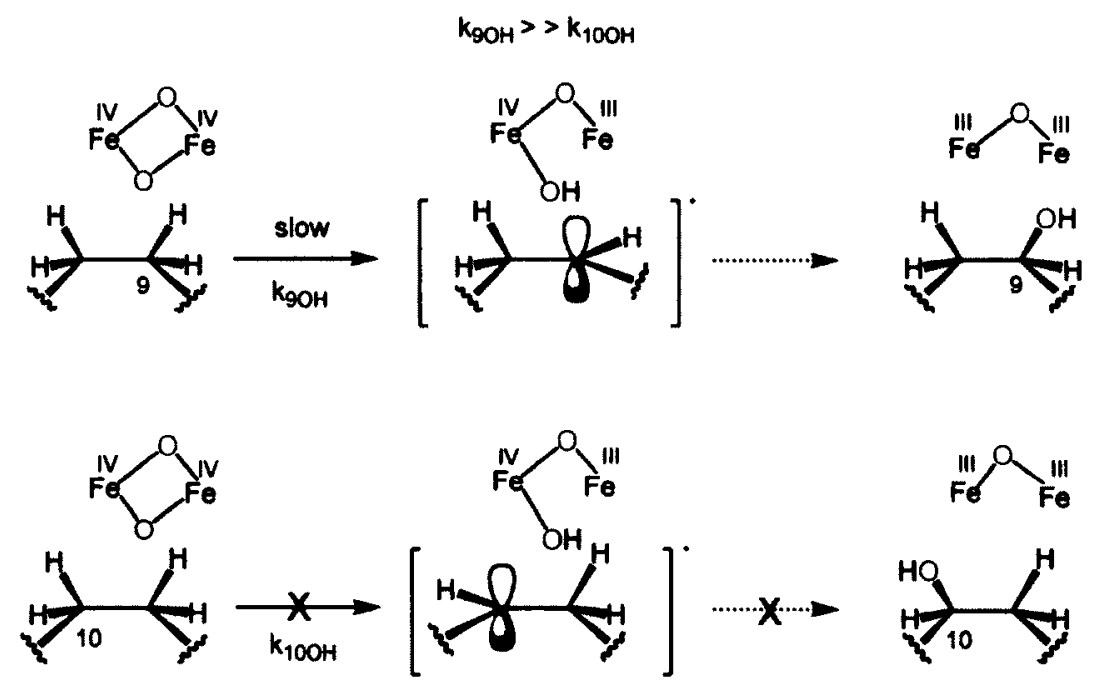

Figure 1.21. Regioselective desaturase-mediated hydroxyl byproduct formation. 


\subsubsection{Highly Conserved Active Site Structural Motif}

By comparing all of the stereo and cryptoregiochemical results presented in the literature for the desaturase-mediated oxidation of fatty acids, it is apparent that for both soluble and membrane-bound desaturases there exists a highly conserved active site structural motif that determines the outcome of those desaturations. ${ }^{1,2,4}$ The active site architecture of soluble plant desaturases is different from that of the membrane-bound enzymes and shows different substrate specificity: for example, the soluble $\Delta^{9}$-desaturase strongly prefers fatty acids with chain length $\mathrm{C} 18$, whereas the membrane-bound $\Delta^{9}$ desaturase can act on the fatty acids with variable chain lengths from $\mathrm{C} 15$ to $\mathrm{C} 19 .{ }^{169} \mathrm{As}$ demonstrated in Figure 1.22, for the soluble plant desaturase, the reaction starts at the carbon that is further from the carboxyl end or closest to the methyl terminus, whereas for the membrane-bound desaturases (1,2-dehydrogenation mode) the site of initial oxidation

Membrane-Bound Desuturies:
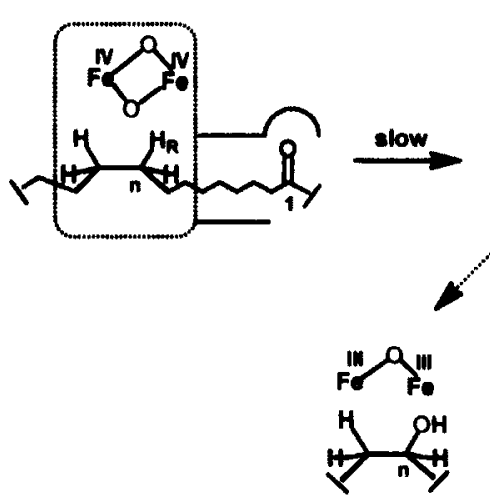
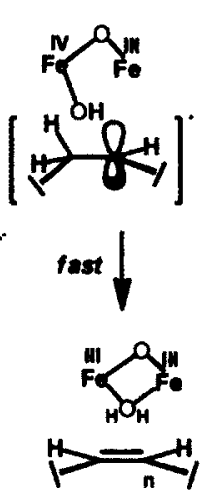

Soluble Dexaturnses:
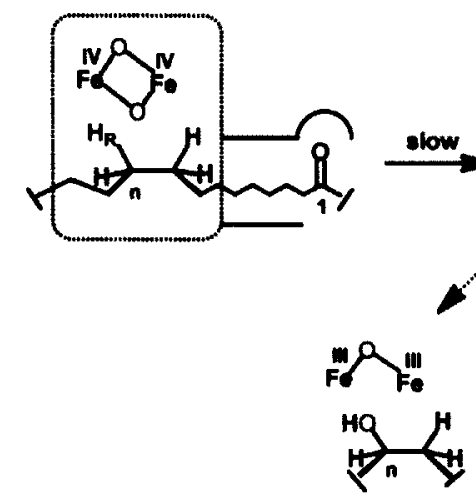
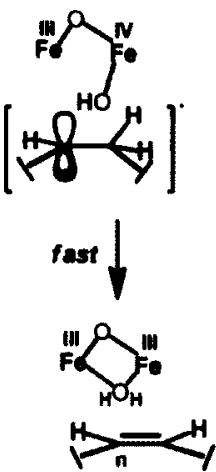

Figure 1.22. Highly conserved active-site structural motif of membrane-bound as well as soluble plant desaturases.

is always at the carbon closer to the carboxyl end. ${ }^{1,161}$ However, the overall enantioselectivity of hydrogen removal is the same for both desaturating systems i.e. the 
pro $R$ hydrogens are abstracted from the prochiral methylene groups during the course of desaturation.

\subsubsection{Chemoselectivity: Dehydrogenation/hydroxylation Connection}

As alluded to before, many membrane-bound desaturases as well as other members of the family of iron-bound proteins, such as non-heme monoiron (MMO) and heme iron (Cytochrome P450) have been documented to produce either dehydrogenated or hydroxylated products. ${ }^{1,16,112,170}$ However, the critical issue that still remains to be understood is the nature of the switch that controls the reaction outcome for those systems. Due to the scarcity of the proper crystallographic information regarding the desaturase-mediated enzyme/substrate complex, proper explanation is difficult. ${ }^{1,2}$ It is thought that there are at least three different factors that may influence the dehydrogenation vs. oxygenation channels. These are the electronic, steric and metal ionbased effects that may be operating either singly or in combination to determine the chemoselectivity of desaturases. ${ }^{1}$

The generic mechanistic model of desaturation (Figure 1.13, 1.22) was developed based on the assumption that both dehydrogenation and hydroxylation reactions proceeds via a common radical intermediate step. As an explanation of the electronic factor, redox potential of this putative radical intermediate plays an important role to decide whether the reaction should direct towards the dehydrogenation or hydroxylation pathway. ${ }^{1}$ While the 'hydroxyl rebound' would lead to the formation of the oxygenated product, the double bond formation can proceed either by direct removal of a second hydrogen or via a rapid one electron oxidation to give a carbocation followed by deprotonation sequence. Thus it can be said that the carbocation formation would facilitate the dehydrogenation 
sequence, whereas the radical intermediate would favor the 'hydroxyl rebound' step. 'As a result, if somehow this radical intermediate is oxidized to a carbocationic intermediate then that will eventually yield the olefinic product. ${ }^{150}$ Support for such a hypothesis has already been obtained from an experimental model where it was observed that the sMMO enzyme, which is typically a hydroxylating protein, can also produce the dehydrogenated product using ethylbenzene as substrate ( $\pi$-cation stabilization at the benzylic position).' On the other hand, castor stearoyl-ACP $\Delta^{9}$-desaturase, which is known to catalyze only the formation of oleoyl product ( $>99.99 \%$ detection limit), produces the 10 -hydroxylated fatty acids upon the introduction of a fluorine atom into the substrate namely as $(R)$ - and (S)-9-fluorostearate (Figure 1.23). ${ }^{127}$ It has been suggested that the strong inductive electron withdrawing effect of highly electronegative fluorine disfavours the formation of a carbocation at the $\beta$-position and allows the radical step to compete, yielding fluorohydrins along with the olefinic products. ${ }^{1,127}$

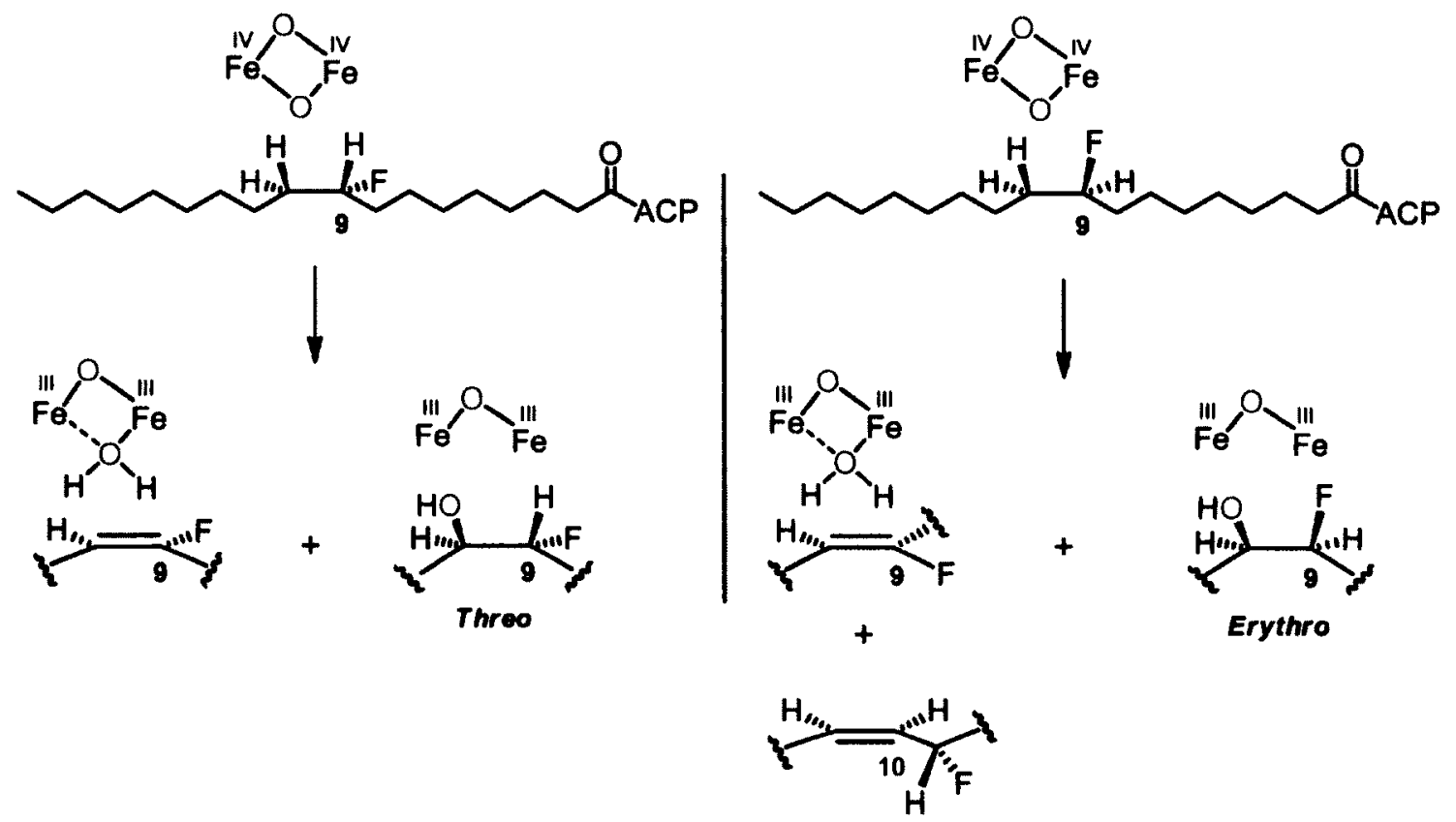

Figure 1.23. Comparison of $(R)$-and $(S)$-9-fluorostearate oxidation by a soluble plant $\Delta^{9}$ desaturase leading to novel hydroxylated and olefinic products. ${ }^{127}$ 
There are two different issues associated with the steric explanation of the chemoselectivity of the desaturase-mediated reaction outcome. These are - firstly, the proper alignment of the $\beta \mathrm{C}-\mathrm{H}$ bond with respect to the singly occupied molecular orbital (SOMO) of the radical intermediate and secondly, the relative positioning of the ironhydroxyl species compared to the two reactive carbon centers (Figure 1.24). ${ }^{1.110}$ Slight changes in the active site architecture of the enzyme could affect these issues that would lead to the alteration of the hydroxylation/dehydrogenation ratio. Briefly, the $\beta \mathrm{C}-\mathrm{H}$ bond must be placed precisely in syn-periplanar arrangement with respect to the half-filled orbital to promote the double bond formation. ${ }^{1}$ In addition, the iron-hydroxyl species

A)
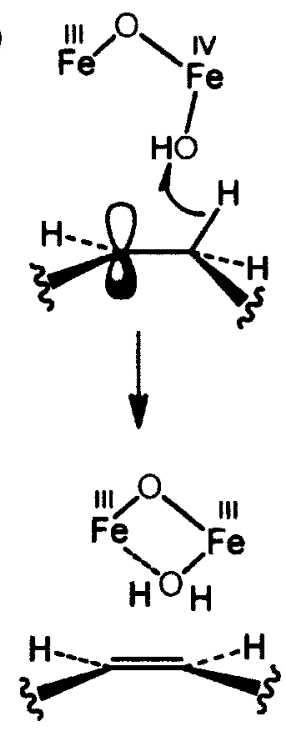

B)
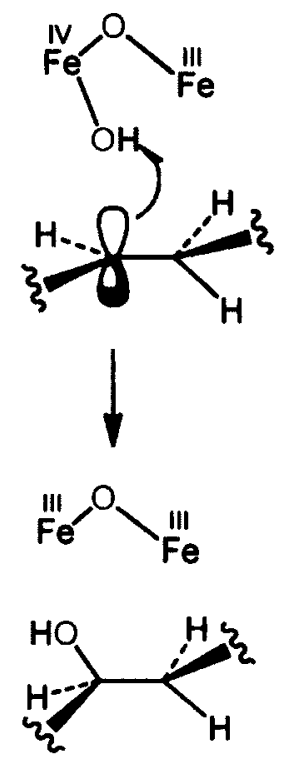

Figure 1.24. Positioning of the iron-hydroxyl species relative to the two carbons of the incipient double bond. Active site scenarios leading to A desaturation and B hydroxylation.'

should also reside close to the second hydrogen so that a favorable removal takes place (Figure 1.24). Violation of any one of these two conditions that favor dehydrogenation would lead to the hydroxylation. 
Hepatic cytochrome P450 family of enzymes are known to catalyze primarily hydroxylation reactions, as in the example found by Baillie et al. in the hydroxylation/dehydrogenation of the epileptic drug, valproic acid (Figure 1.25). ${ }^{1,171}$
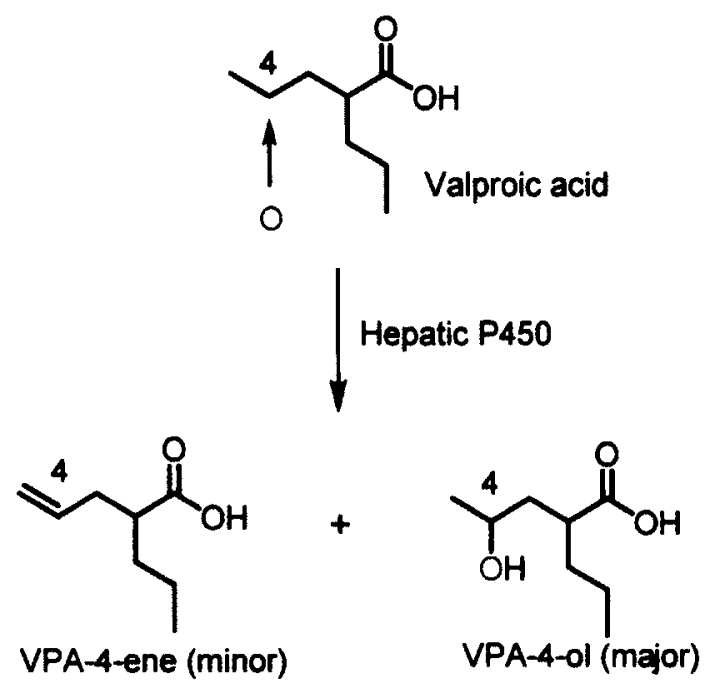

Figure 1.25. C-4 initiated bimodal oxidation of valproic acid.'

This can be rationalized since cytochrome $\mathrm{P} 450$ families of enzymes are known to act on a wide variety of substrates, which means that the active site morphology for these class of enzymes are highly flexible to accommodate various substrates. In contrast, the binding cavities of desaturases are highly conserved for a particular fatty acid chain length and its conformation. Therefore, the alignment required to get dehydrogenation can't be sufficiently controlled by P450 enzymes and that's why they rarely exhibit desaturase activity.' Moreover, this concept of steric factors is further supported through the result obtained from a recent site-directed mutagenesis experiment on a bifunctional membrane-bound 12-hydroxylase/12-desaturase enzyme. ${ }^{172}$ The result demonstrated that a relatively small change of the hydrophobic residues in the substrate-binding cavity 
significantly influenced the relative ratio of hydroxylated vs. dehydrogenated product formation. Both enzymes proceed via a C-12 carbon-centered free radical.

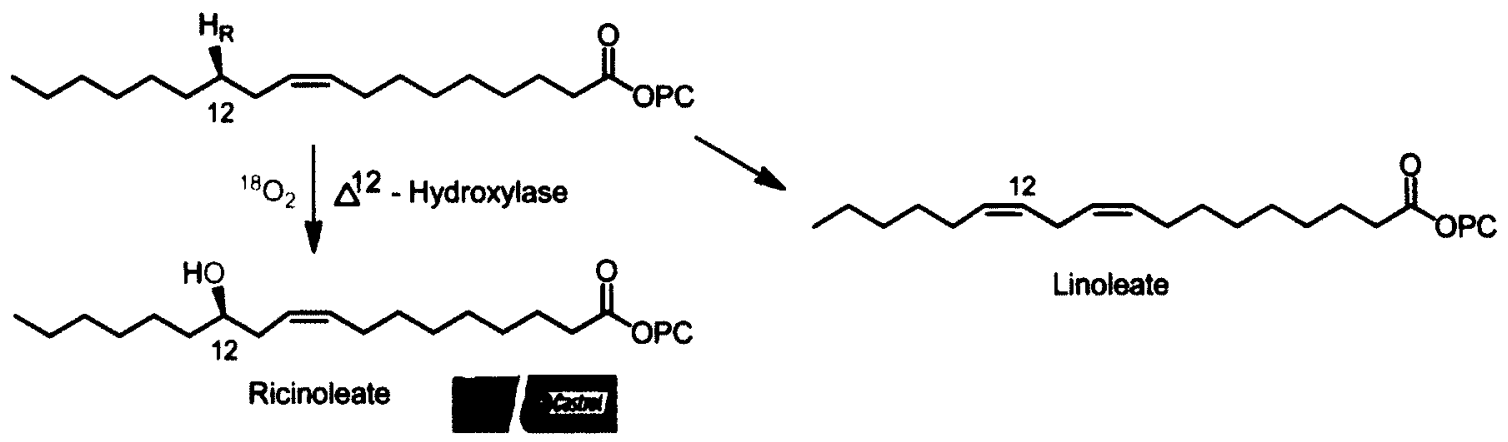

Figure 1.26. Relationship between 12-hydroxylation / 12-dehydrogenation.

In consideration of the metal ion-based effect, the ligand environment of the iron center and the relative energies associated with the coordination of the iron center with the water product (desaturation) vs. no ligand (hydroxylation) may act to drive the selection of reaction pathway. ${ }^{1,170}$ A case in point is a non-heme monoiron-based enzyme, clavaminate synthase 2 that selectively catalyzes the hydroxylation of deoxyguanidinoproclavaminic acid (DGPC) during the course of clavulanic acid biosynthesis, but also generates desaturated product (with DPC) depending upon the nature of a pendant amino substituent present at the $\gamma$-position relative to the site of initial oxidation (Figure 1.27). ${ }^{170}$ It has been postulated that the switch between the hydroxylation and desaturation reaction is determined by the relative stability of the coordinated iron-water complex, which is in turn is affected by how the different substrates bind in the active site pocket. The greater the stability of the coordinated ironwater complex, the more the reaction favours the dehydrogenation sequence over hydroxylation (Figure 1.27). ${ }^{170}$ 
<smiles>CC(C(=O)O)[C@@H](C(=O)O)[C@@H](O)CCCNC(=N)N</smiles><smiles>NCCCC(C(=O)O)N1CCC1=O</smiles>

(DPC)<smiles>NCC=C[C@H](C(=O)O)N1CCC1=O</smiles>

Major product

Figure 1.27. The role of associated ligand environment at the reaction binding site in controlling the switch between the hydroxylation vs. desaturation channel during the biosynthetic pathway of clavulanic acid. ${ }^{170}$

\subsection{Mechanistic Investigations of 1,4-Desaturation}

FADs responsible for the introduction of the conjugated double bonds by 1,4dehydrogenation reactions have been investigated in several higher plants and insects. ${ }^{34,39,40,43,63,71}$ Examples of such fatty acid modifications are already illustrated in the previous sections $1.1 .5 \&$ 1.1.6. In the early literature, the enzymes responsible for 1,4-dehydrogenation process were termed as "conjugases". This misleading name is better replaced with "1,4-desaturase" or " $\Delta$,m desaturase" where " $n$ " and " $m$ " represent the carbon number of the incipient diene system. Sequence analysis of the enzymes responsible for 1,2- and 1,4-dehydrogenation reveals a close relationship between them (Figure 1.28). ${ }^{1,34,63}$ 


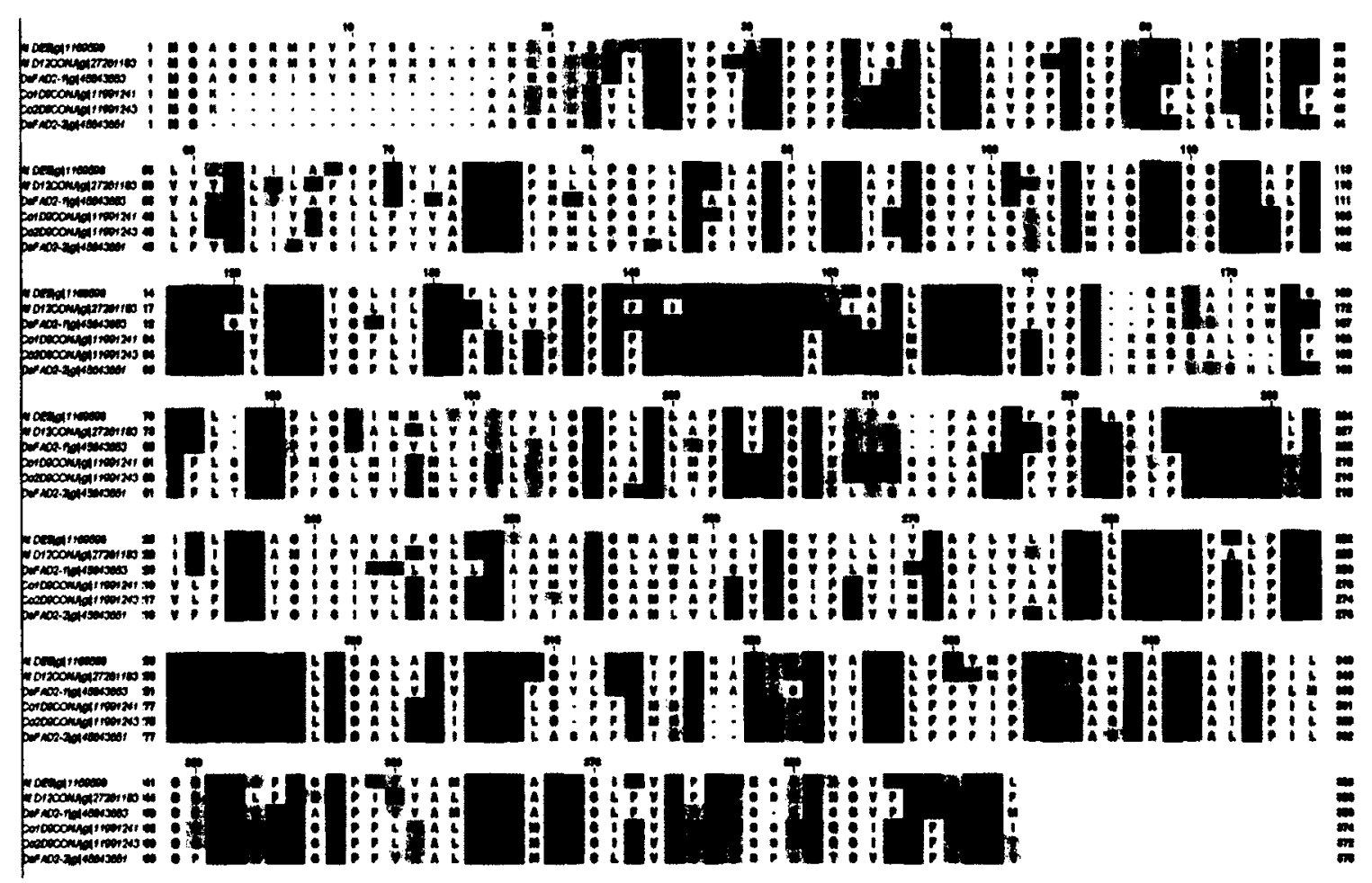

Figure 1.28. CLUSTALL comparison between the amino acid sequences of FAD2 variants responsible for biosynthesis of conjugated fatty acids in plants.

The detailed descriptions of their mechanism of action both in insects and plants are given below.

\subsubsection{Insects}

A description of the conjugated fatty acids found in insect pheromone blends and their species-specific uses as chemical signals in different moth species has already been described in section 1.1.5. In vivo mechanistic work by Gemma Fabrias and coworkers showed that $(10 E, 12 E)$-dodecadienoic acid is produced from $(Z)$-11-tetradecenoic acid by the action of a bifunctional $\Delta^{11}$-desaturase (Figure 1.5 ). ${ }^{39,40}$ The compound generated by $\Delta^{11}$-desaturation of tetradecanoic acid is further used as a substrate by the same $\Delta^{\prime \prime}$ desaturase to introduce the conjugation at $\mathrm{C}-10, \mathrm{C}-12$ position (1,4-dehydrogenation) via the modification of the existing double bond at C-11. Stereochemical analysis of this 
bifunctional enzyme by employing stereospecifically monodeuterated fatty acids revealed that the 1,4-dehydrogenation is taking place via the syn removal of two hydrogens from the non-adjacent positions $\mathrm{C}-10$ and $\mathrm{C}-13$ (Figure 1.29$)^{40,71}$; the enantioselectivity of this process is identical to that of $\Delta^{11}$-desaturation of tetradecanoic acid. ${ }^{1,74}$

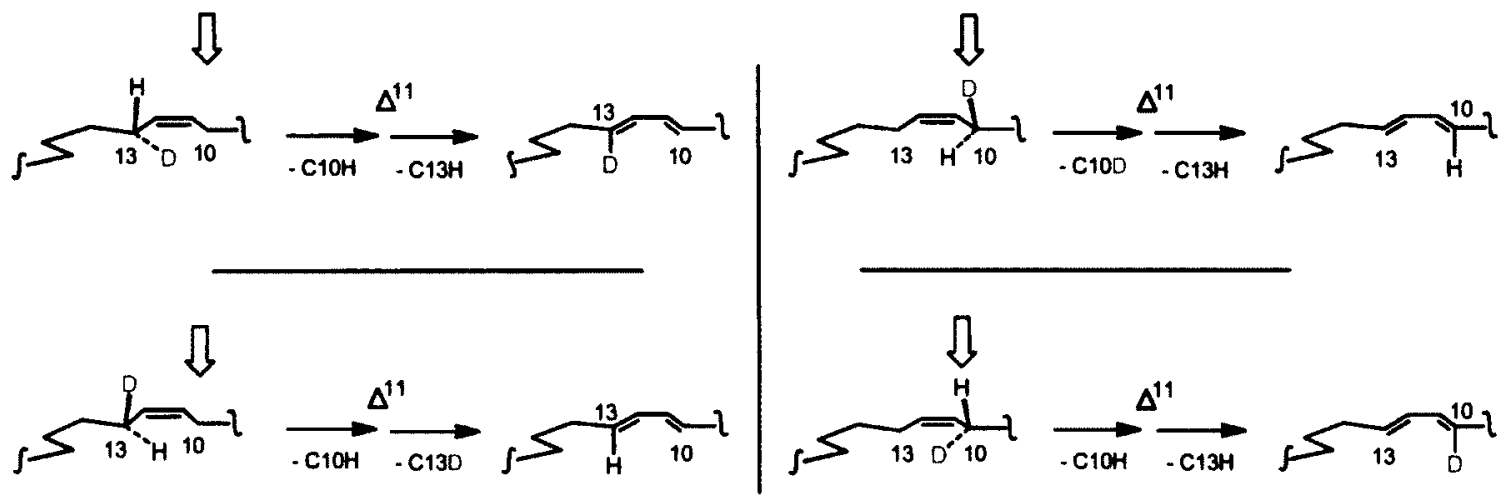

Figure 1.29. Topological model for the stereochemical investigation of the biosynthesis of $(10 E, 12 E)$-fatty acetate as a component of sex pheromone blend in moth Spodoptera littoralis using stereospecifically monodeuterated fatty acid analogues.

Incubating $\sim 1: 1$ mixtures of non-deuterated to regiospecifically dideuterated FA precursors (C-10 and $\mathrm{C}-13)$ and analysis of the resultant primary deuterium kinetic isotope effects showed that the 1,4-dehydrogenation is initiated by rate determining removal of hydrogen from $\mathrm{C}-10 .^{39}$ Thus a large KIE was observed with the C-10 dideuterated species $(10.2 \pm 1.6)$ whereas the $\mathrm{C} 13-\mathrm{H}$ cleavage was found to be insensitive to isotope substitution $(1.2 \pm 0.2)$.

\subsubsection{Plants}

It has been speculated that fatty acids bound to phosphatidylcholine (PC) serve as precursors for the biosynthesis of these conjugated fatty acids from a linoleoyl precursor in the corresponding developing plant seeds. ${ }^{42,62,63,92}$ Isolation and sequence comparison of the genes responsible for the biosynthesis of unusual fatty acids revealed that a family 
of novel closely related divergent FAD2 or FAD2-like desaturase enzymes are actually involved..$^{34,62,63,92}$

1,4-Desaturation in plants was first examined by Crombie and Holloway in 1985; using developing marigold seed homogenates and isotopically labelled fatty acid precursors, it was demonstrated that the calendic acid is biosynthesized in the seeds of Calendula officinalis by an apparent 1,4-dehydrogenation process (Figure 1.30). ${ }^{43}$ Mass spectral analysis of the calendate obtained from a labelled oleic acid precursor

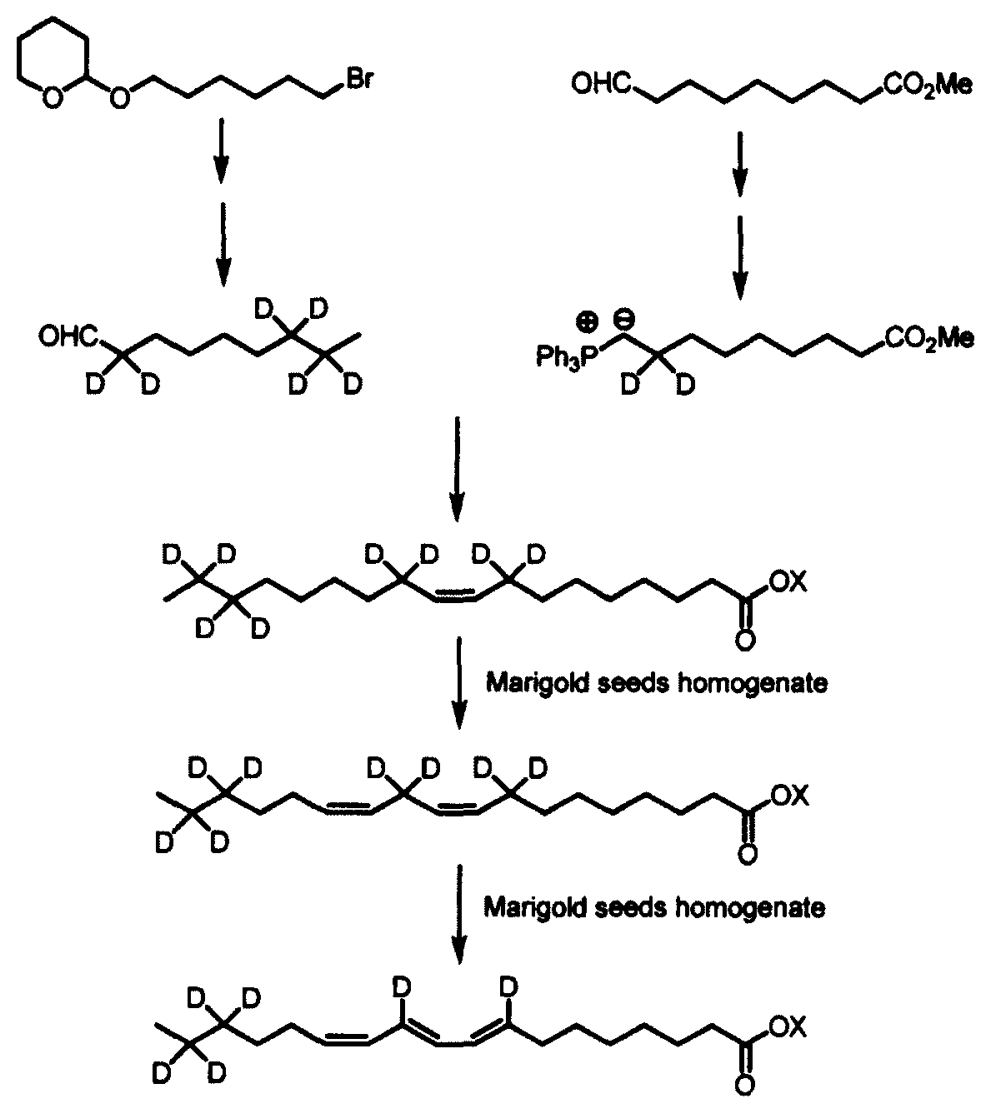

Figure 1.30. Synthesis and use of $\left[8,8,11,11,16,16,17,17-{ }^{2} \mathrm{H}_{8}\right]$-octadec-9-enoic acid as mechanistic probe to test the biosynthesis of calendic acid in marigold seeds.

demonstrated the removal of two non-adjacent hydrogens from C-8 and C-11 positions of the fatty acid precursor. No oxygenated intermediate could be detected. The required 
stereospecifically deuterated linoleates were synthesized by Crombie and Holloway in order to investigate the stereochemistry of this process but insufficient incorporation of labelled precursors in the incubation experiments utilizing plant tissues prevented completion of these experiments.

Cryptoregiochemical analysis of this $\Delta^{8,10}$-desaturase enzyme by Buist et al. using KIE methodology established that formation of calendate was initiated at C-11 (Figure 1.31). ${ }^{158}$ Incubating the appropriately labelled linoleates with a mutant yeast strain expressing the Fac2 - Calendula officinalis gene, it was observed that there is a large $\mathrm{KIE}$ associated with $\mathrm{C} 11-\mathrm{H}$ abstraction $(5.7 \pm 1.0)$ whereas the removal of hydrogen from C-8 position was found to be insensitive to deuterium substitution $(1.0 \pm 0.1){ }^{158}$ This result clearly demonstrates the step-wise nature of the 1,4-dehydrogenation reaction and supports the hypothesis that FAD2 variants initiate the reaction from a position that is closest to C-12 - the site of initial oxidation for the parent FAD2 enzyme that introduces a double bond at 12,13 (Figure 1.26).,
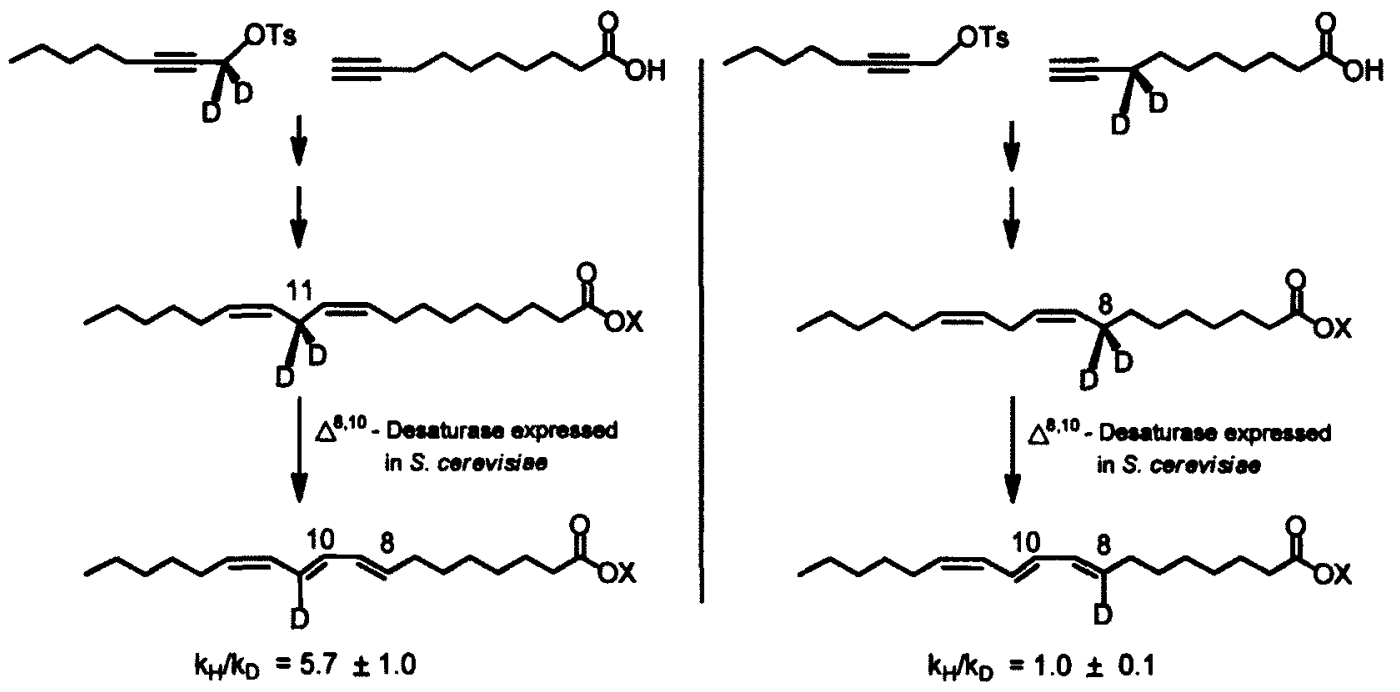

Figure 1.31. Determination of the cryptoregiochemistry of calendic acid biosynthesis using the KIE measurements. 


\subsection{Goals of the Thesis}

Examples of several FAD2-like desaturating systems in various higher plants have been described in the previous sections that are capable of biosynthesizing a diverse array of conjugated fatty acids such as calendic, a-eleostearic and dimorphecolic acid. ${ }^{11,34,62,63,158}$

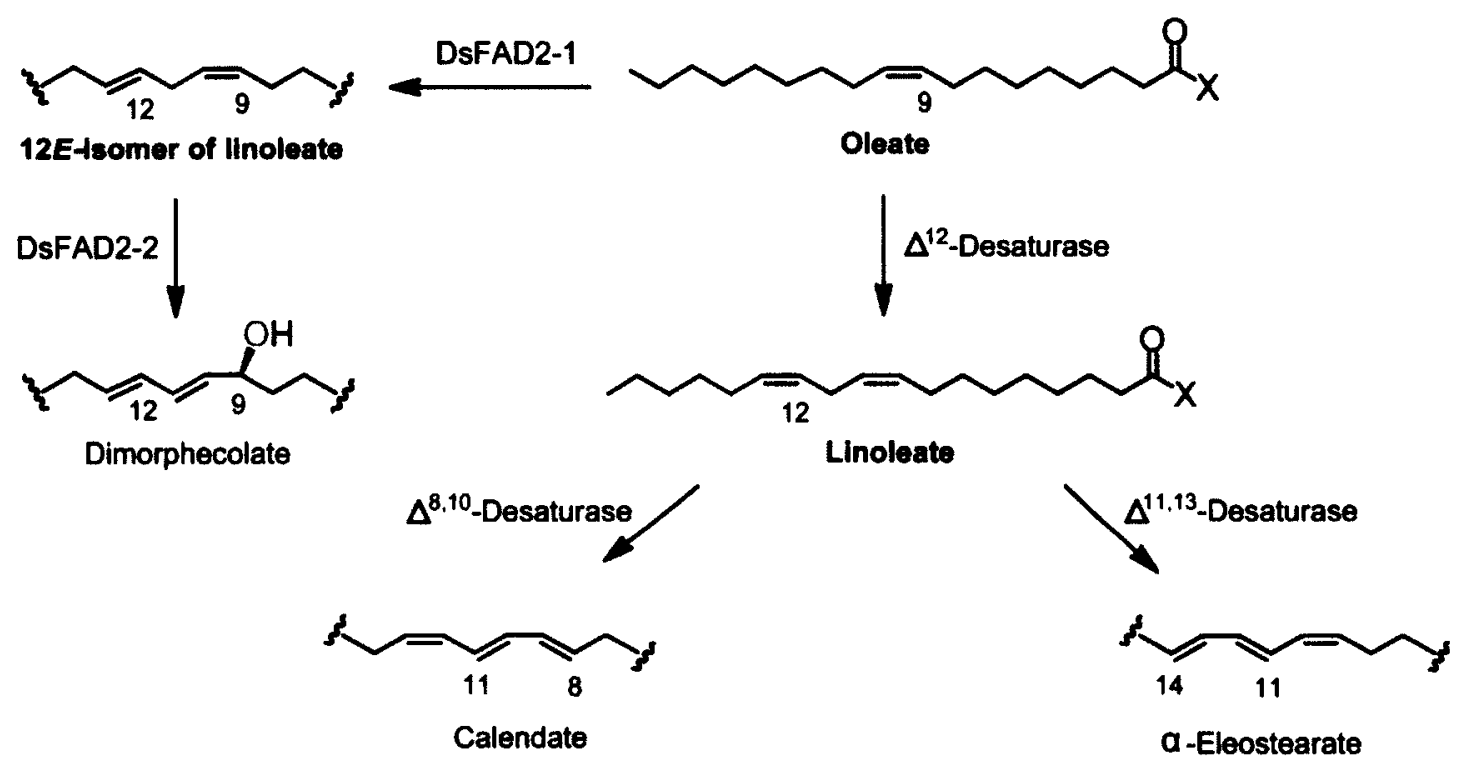

Figure 1.32. Involvement of several variant FAD2 enzymes to generate calendic, $\alpha$ eleostearic and dimorphecolic acid.

However, only one detailed mechanistic study has been carried out on this class of desaturases, namely the determination by our laboratory of the site of initial oxidation for calendate formation. ${ }^{158}$ The enantioselectivity of this 1,4-desaturation has not been reported yet. In addition, the stereochemistry as well as the cryptoregiochemistry of $\alpha$ eleostearic acid formation has not been studied. ${ }^{1,34}$ Detailed functional analysis of the enzymes from Dimorphotheca sinuata showed that there exist two FAD2-like enzymes that work in a tandem fashion to generate the $(R)$-9-hydroxy-(10E,12E)-octadecadienoic acid from oleic acid (Figure 1.32). ${ }^{11}$ However, the detailed mechanism of hydroxylation 
is still not well understood. As part of ongoing research into the structure-function relationships for this class of FAD2-like enzymes, a closer examination of the conjugated fatty acids biosynthesis is clearly necessary. The availability of a convenient yeast ${ }^{11,34,158}$ expression system for these enzymes offers us a unique opportunity to carry out the mechanistic investigations in vivo.

In summary, the objective of the research described in this thesis was to:

1) Elucidate the stereochemistry of calendic acid biosynthesis using the FAD2 variant found in the seeds of Calendula officinalis.

2) Investigate the stereo- and cryptoregiochemistry of $\alpha$-eleostearic acid biosynthesis using the FAD2 variant of developing tung tree, Aleurites fordii Hemsl. seeds.

3) Determine the stereochemistry and KIEs associated with the site of initial oxidation in dimorphecolic acid formation using the FAD2 variants operating in the seeds of Dimorphotheca sinuata. 


\section{Chapter 2: Stereochemistry of Calendic acid Biosynthesis in Calendula officinalis}

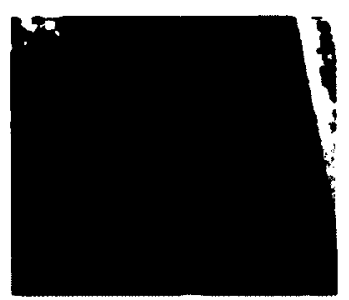

Elie Buist

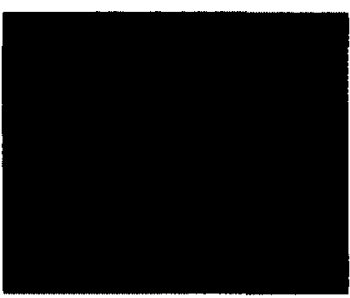

Darwin W. Reed

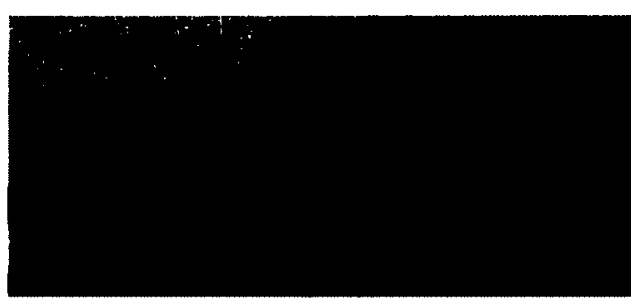

Montreal Botanical Gardens

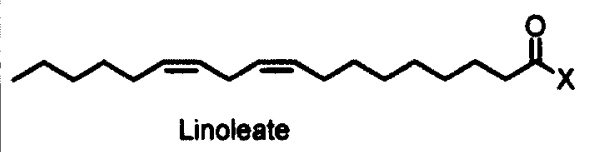

Calendula officinalis

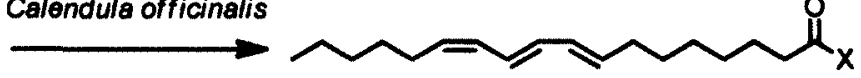

Calendate

\subsection{Experimental Approach}

Previous investigations in our laboratory have shown that calendic acid biosynthesis is initiated via initial abstraction of hydrogen from the $\mathrm{C}-11$ position of the fatty acid chain. ${ }^{158}$ It was proposed that only a slight shift in the position of the highly reactive diiron oxidant (Figure 2.1) with respect to the substrate is enough to switch the mechanism from the parent desaturation (FAD2) to 1,4-dehydrogenation (FAD2 variant).

As shown below, the $\mathrm{C}-8$ hydrogens and $\mathrm{C}-11$ hydrogens of the fatty acid chain are positioned to allow syn dehydrogenation with the same facial selectivity as the syn removal of hydrogens from $\mathrm{C}-12$ and $\mathrm{C}-13$ positions during linoleic acid formation (FAD2). The correctness of this mechanistic model could be tested through the determination of the enantioselectivity of polyunsaturated fatty acid formation using stereospecifically deuterated fatty acids as mechanistic probes. 

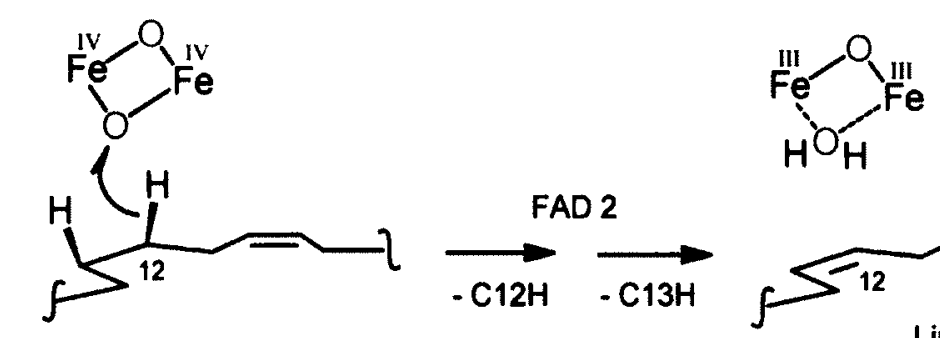

FAD 2
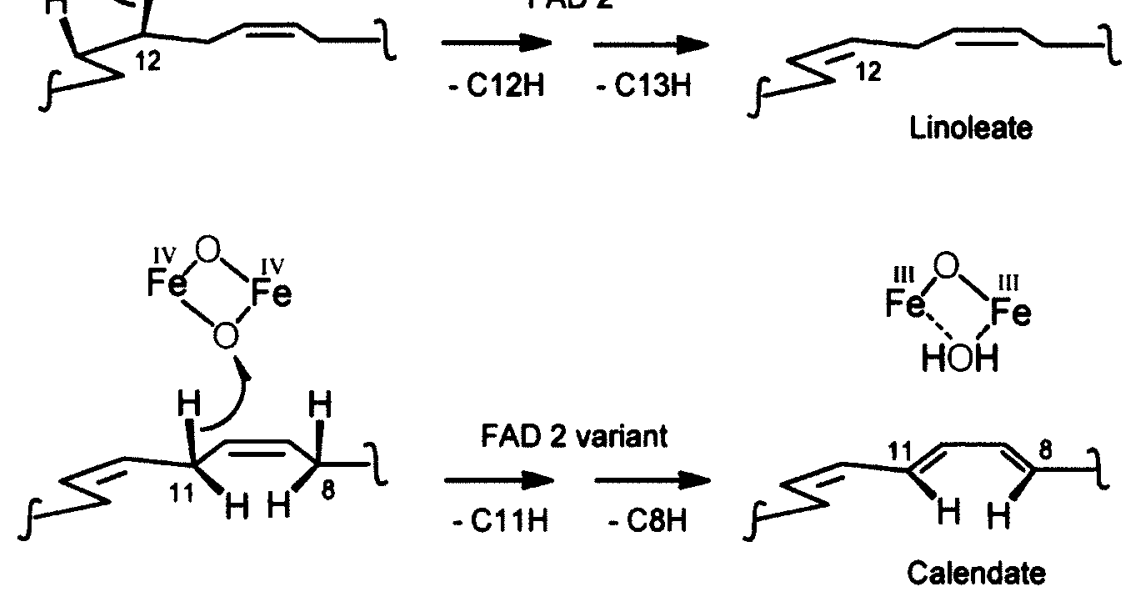

Figure 2.1. Mechanistic model showing the relationship between oxidant position in $\Delta^{12}$ desaturation and the $(1,4)$-desaturase reaction leading to the formation of the conjugated 8,10 -diene system of calendate. ${ }^{158}$ The double bonds are quasi-coplanar.

A convenient Sacharomyces cerevisiae expression system for gene Fac2 from Calendula officinalis that encoded the $(1,4)$-desaturase enzyme was available. ${ }^{93.158}$ The host yeast culture system does not endogenously produce the substrate linoleic acid which made the use of other mechanistic probes such as thia-substituted analogues or unnaturally chain-shortened substrates unnecessary. The use of thia-substituted fatty acid analogues as oxo traps to determine the position of the oxidant (Section 1.3.1) was not a viable option as the required vinyl thioether substrates are difficult to synthesize and are unstable.

Our approach required the synthesis of four stereospecifically monodeuterated methyl linoleates with chirotopic center in one pair at C-8 and in other at C-11 (Figure 2.2) i.e. methyl $(8 R)$ - $\left[8-{ }^{2} \mathrm{H}_{1}\right]$-octadeca- $9 Z, 12 Z$-dienoate $1 \mathrm{a}$, methyl $(8 S)-\left[8-{ }^{2} \mathrm{H}_{1}\right]$-octadeca9Z,12Z-dienoate 1b, methyl (11S)-[11- $\left.{ }^{2} \mathrm{H}_{1}\right]$-octadeca-9Z,12Z-dienoate $2 \mathrm{a}$ and methyl 
(11R)-[11- $\left.{ }^{2} \mathrm{H}_{1}\right]$-octadeca-9Z,12Z-dienoate 2b. It was anticipated that incubation of each isomer with the mutant yeast cultures containing the $\Delta^{8,10}$-desaturase enzyme (FAD2 variant) would generate enough calendate product to allow tracking the fate of deuterium label by gas chromatography - mass spectrometry (GC-MS).<smiles>[2H][C@@H](/C=C\C/C=C\CCCCC)/C=C/CCCCCC(=O)OC</smiles><smiles>[2H][C@H](/C=C/C=C/[C@@H]([2H])[C@H]([2H])/C=C/CCCCCCCC(=O)OC)/C=C/CCCCCC</smiles>

Figure 2.2. The target chiral monodeuterated linoleates required to investigate the stereochemistry of calendate formation.

\subsection{Results and Discussion}

\subsubsection{Synthesis of methyl (8R)- and $(8 S)-\left[8-{ }^{2} \mathrm{H}_{1}\right]$-octadeca-9Z,12Z-dienoate, $1 \mathrm{a} \&$ $1 \mathrm{~b}$}

\subsubsection{Overview}

There are principally two strategies involved in the synthesis of stereospecifically deuterium labeled linoleates - firstly, by preparing a suitably functionalized, long chain, chiral secondary alcohol followed by subsequent $S_{\mathrm{N}}$ 2-type displacement of the corresponding tosylate or mesylate using deuteride $\left(D^{-}\right)^{43,127}$; secondly, via the synthesis of a shorter chain chiral monodeuterated alcohol followed by subsequent $\mathrm{S}_{\mathrm{N}} 2$-type displacement of the corresponding tosylate or mesylate using a terminal acetylide of the correct chain length. ${ }^{40,75,116,127,173-179}$ Alkyne functionality of the synthetic intermediates can be stereoselectively reduced to give the requisite cis-olefins. $^{158,173,180}$ 


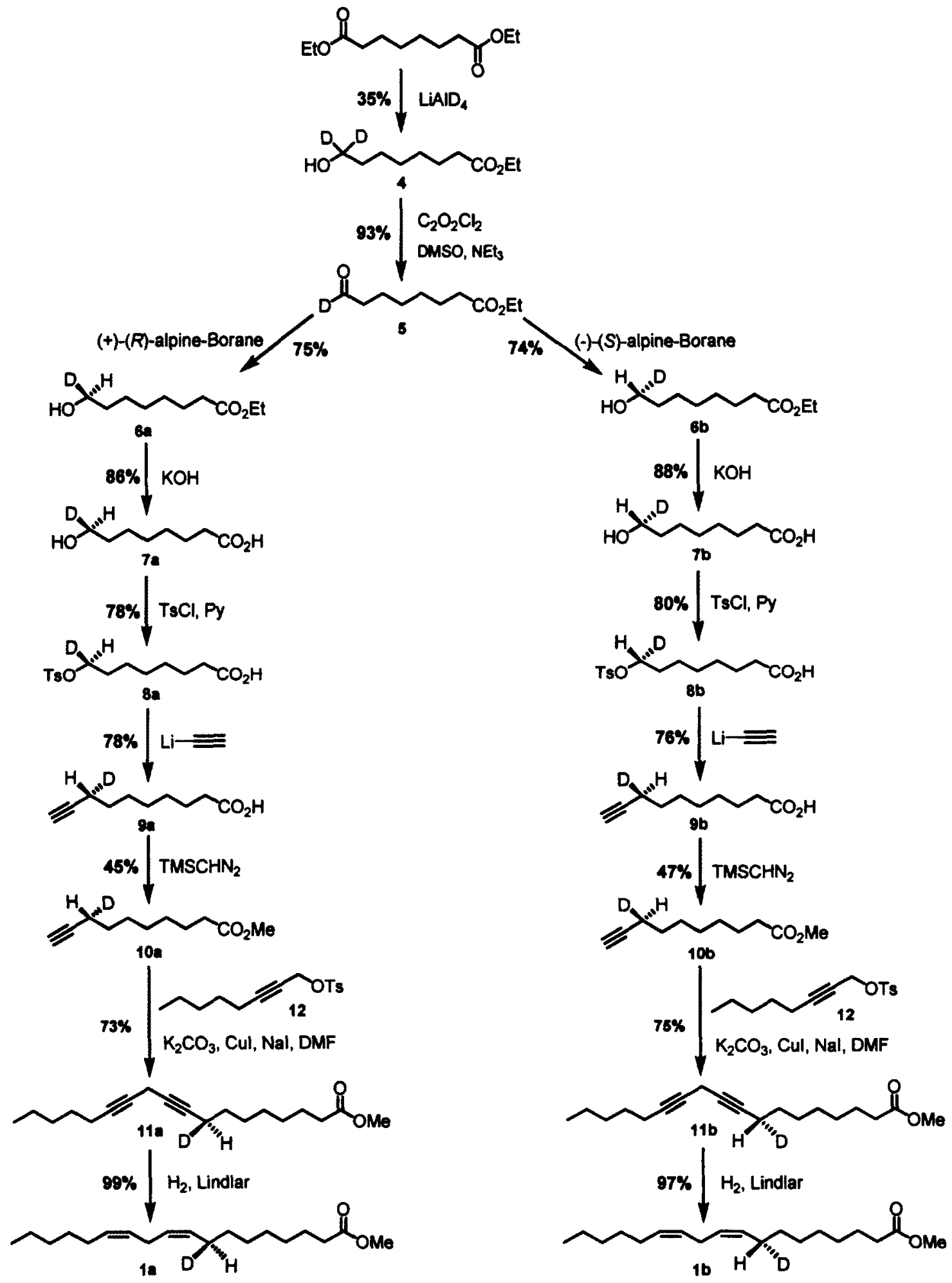

Figure 2.3. Scheme for the synthesis of chiral $(8 R)$-and $(8 S)-\left[8-{ }^{2} \mathrm{H}_{1}\right]$-linoleates. 
In this thesis, the -CHD chiral centre was introduced into the $\mathrm{C} 18$ fatty acid framework by using the approach described in the second method (Figure 2.3). The synthesis of the title compounds $(\sim 4 \%$ overall yield) was adapted from that previously reported by Crombie and Heavers ${ }^{173}$ and features the preparation of both enantiomers of ethyl 8-d $\mathrm{d}_{1}-8$ hydroxyoctanaote 6a, b followed by stepwise elongation using acetylide alkylation reactions and subsequent Lindlar reduction. Trial reactions were carried out using nondeuterated reagents.

\subsubsection{Preparation of (8S)- and (8R)-Ethyl $\left[8-{ }^{2} \mathrm{H}_{1}\right]-8$-Hydroxyoctanoate, $6 \mathrm{a} \&$ b}

Chiral monodeuterated primary alcohols can be synthesized from the corresponding monodeuterated aldehyde which in turn is readily accessible from the respective dideuterated alcohol via various oxidation mechanisms. ${ }^{173}$ As shown in Figure 2.3, diethyl suberate was converted into ethyl 8-hydroxy-[8,8- $\left.{ }^{2} \mathrm{H}_{2}\right]$ octanoate 4 in $35 \%$ yield using half-equivalent of lithium aluminium deuteride in THF. ${ }^{174,179,181,182}$ Flash chromatography was required to remove the diol by-product and starting material. The dideuterated monoethyl ester was then oxidized to the corresponding monodeuterated aldehyde, ethyl 8-oxo- $\left[8-{ }^{2} \mathrm{H}_{1}\right]$ octanoate 5 through the use of the Swern oxidation method (93\% yield). ${ }^{183}$ This approach represented a substantial improvement (33\% vs. $6 \%$ ) over an initial attempt to follow the method of Crombie ${ }^{201}$ as illustrated in Figure 2.4: 


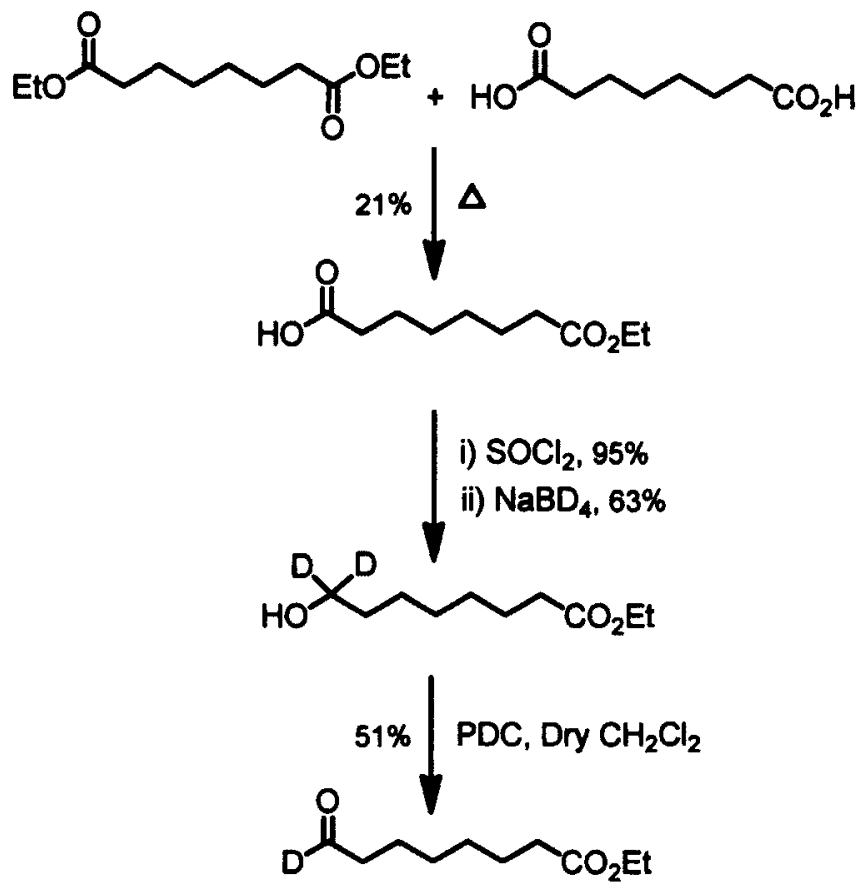

Figure 2.4. Scheme for Crombie's method for the synthesis of monodeuterated aldehyde.

Enantioselective reduction of monodeuterated aldehyde 5 was effected using AlpineBoranes ${ }^{\oplus}$ as the chiral reducing agent ${ }^{184-186}$ (Figure 2.3 and 2.5) rather than the Baker's yeast methodology employed by Crombie (the latter involves somewhat cumbersome product isolation procedures). Analyses of the mass spectra (MS) of the TMS derivatives ${ }^{187}$ of each chiral asymmetric reduced alcohols indicated that the deuterated intermediates consisted entirely of monodeuterated species (within experimental error). The use of chiral boranes was first pioneered by Midland and coworkers and is still widely used for the asymmetric reduction of aliphatic, aromatic as well as $\alpha, \beta-$ unsaturated aldehydes to the corresponding chiral primary 1-deuterio alcohols in a simple, efficient manner with high enantiomeric excess. ${ }^{174,184,185,188}$ It has been observed that the use of $(+)-(R)$-Alpine-Borane ${ }^{\Phi}$ results in the synthesis of $(S)-\left[1-{ }^{2} \mathrm{H}_{1}\right]-$ alcohol from 
deuterio aldehyde (Figure 2.5 ), whereas the $(R)-\left[1-^{2} \mathrm{H}_{1}\right]$-alcohol could be obtained by using (-)-(S)-Alpine-Borane ${ }^{\circledast}$ from the same aldehyde.

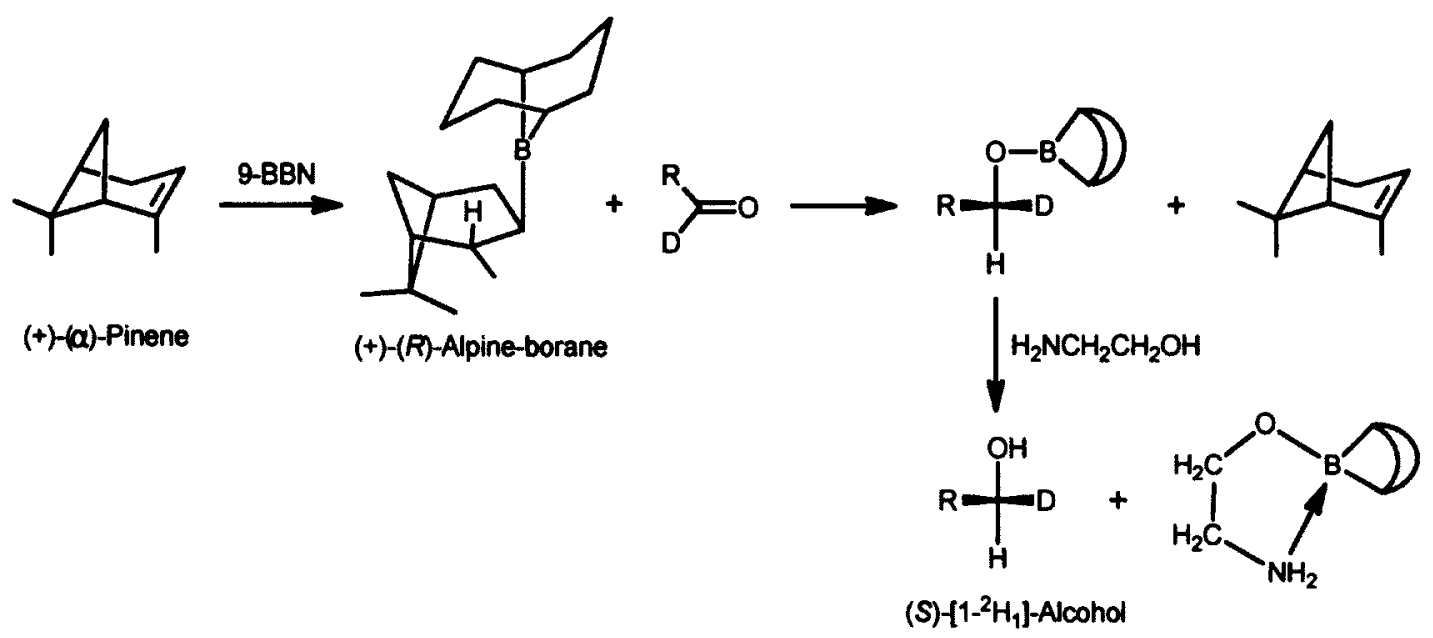

Figure 2.5. Preparation of $(+)-(R)$-Alpine-Borane ${ }^{\circledast}$ and its use in chiral enantioselective reduction of deuterio aldehyde to synthesize the $(S)-\left[1-{ }^{2} \mathrm{H}_{1}\right]-$ alcohol.

The reactions are usually carried out in an inert atmosphere as B(III) is highly sensitive to oxidation in presence of $\mathrm{O}_{2}$. The reaction can be successfully performed under exceptionally mild conditions even at room temperature and has been observed that the use of slight excess of Alpine-Borane ${ }^{~}$ reagents (1.2 equivalent) instead of one equivalent leads to an increase in enantiomeric excess. ${ }^{185,188}$ The chiral $(+)-(R)$-Alpine-Borane ${ }^{\otimes}$ reagent is derived from $(+)-(\alpha)$-pinene and $9-\mathrm{BBN}$, while the chiral $(-)-(S)$-AlpineBorane $^{\otimes}$ reagent is made from the reaction between $(-)-(\alpha)$-pinene and 9-BBN and both of them are commercially available as a solution in THF. The origin of the enantioselective reduction is explained by using the model proposed by Midland et al. as described in Figure 2.6. ${ }^{185,188}$ From the model, it is apparent that during reduction, the aldehyde must approach the chiral borane in such a manner so that the steric interaction between the aldehyde $\mathbf{R}$ group and the pinane methyl group is minimized. 
A

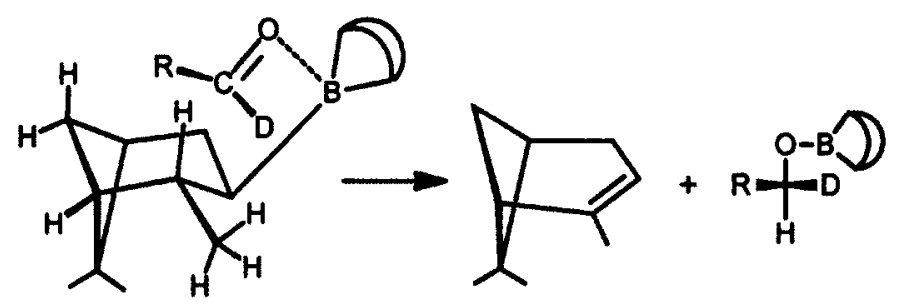

Steric repulsion relaxed in this orientation of the aldehyde
B

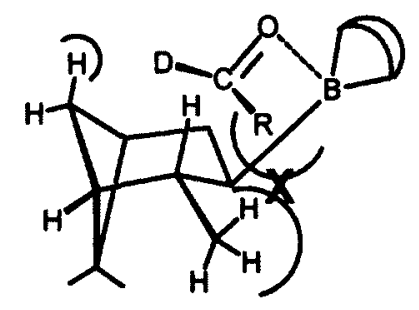

Sterong steric repulsion between $R$ and Me destabilizes the complex intermediate

Figure 2.6. Explanation of the enantiomeric preference Alpine-Borane ${ }^{\circledR}$ reagents in the reduction of aldehydes.

\subsubsection{Stereochemical Analysis of (8R)- and (8S)-Ethyl $\left[8-{ }^{2} \mathrm{H}_{1}\right]-8$-Hydroxyoctanoate}

The absolute configuration and enantiomeric purity of the derived chiral deuterio alcohols were determined using the Mosher's $\alpha$-methoxy- $\alpha$-(trifluoromethyl)phenylacetic esterification (MTPA) derivatization method and comparing the results with previously reported observations. ${ }^{173,189}$ Upon derivatization of primary alcohols with chiral reagents (MTPA-chloride) the enantiotopic hydrogens of the $\mathrm{CH}_{2} \mathrm{OH}$ group become diastereotopic and ${ }^{1} \mathrm{H}$ NMR-nonequivalent due to the presence of another chiral centre nearby. Thus the amount of minor enantiomer in the samples of chiral ethyl 8-d $1-8$-hydroxyoctanoate could be estimated by integration of the peak areas for the ${ }^{1} \mathrm{H}$ signals appearing at diastereotopic positions. 

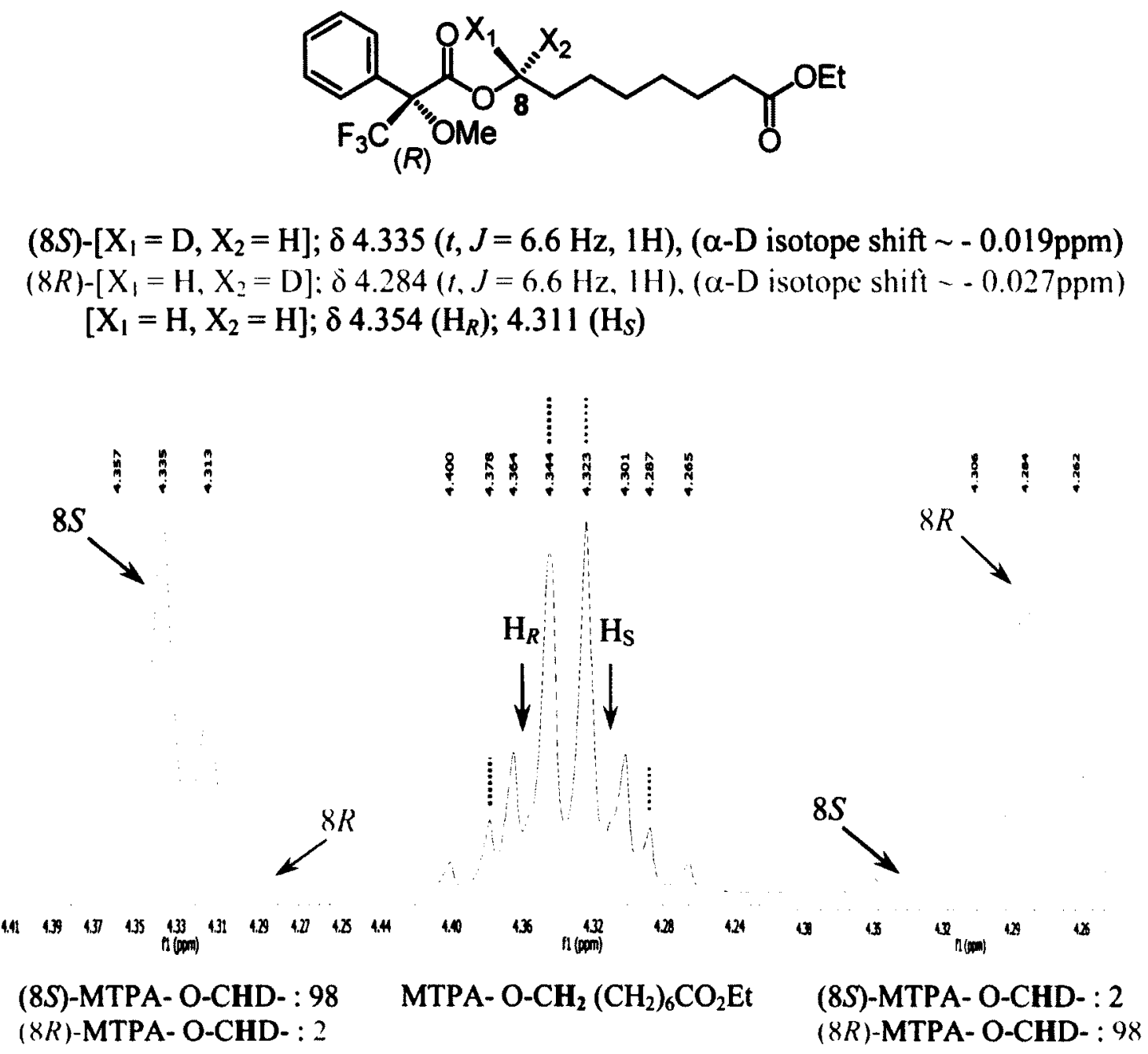

Figure 2.7. Determination of the absolute configuration and enantiomeric purity of the chiral ethyl 8-d $d_{1}-8$-hydroxy octanoates using Mosher's esterification method.

It was observed that the ${ }^{1} \mathrm{H}$ signal of the $\mathrm{CHD}$ group for each Mosher ester was a broadened triplet due to a combination of small ${ }^{2} J$ HD coupling and large ${ }^{3} J$ HH vicinal coupling (Figure 2.7). In contrast, the Mosher ester of the corresponding non-labeled alcohol features an $\mathrm{AB}$ quartet of triplets due to magnetic non-equivalence of the diastereotopic geminal protons. As is well documented in the literature, ${ }^{173}$ the ${ }^{1} \mathrm{H}$ signals at $\mathrm{C}-8$ of the (8S)-alcohol are deshielded relative to the corresponding peaks of the $(8 R)$ alcohol. The enantiomeric excess of the chiral alcohols was estimated to be $\sim 96 \%$. 


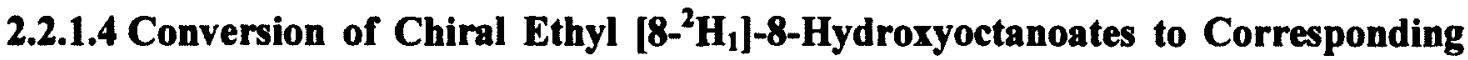 Chiral $\left[8{ }^{2}{ }^{2} \mathbf{H}_{1}\right]-$ Linoleates}

As shown in Figure 2.3, the chiral alcoholic esters 6a and $6 \mathrm{~b}$ were hydrolyzed to the corresponding acids in order to avoid the possibility of ester attack by acetylide in the subsequent steps. The tosylates of the hydroxy acids $7 \mathbf{a}$ and $\mathbf{7} \mathbf{b}^{173,179,182,184}$ were displaced by acetylide ion to give the compounds $9 a$ and $9 b^{39,173}$. The nucleophilic displacement of the tosylate by the acetylide ion is believed to proceed through configurational inversion as demonstrated in an unambiguous manner by Crombie. ${ }^{173}$ Initially the terminal alkynes $9 a$ and $9 b$ were coupled directly with $1-(p$-tolylsulfonyloxy)-oct-2-yne 12 in presence of ethyl magnesium bromide and copper(I) bromide-dimethyl sulfide complex as catalyst as suggested by Crombie ${ }^{201}$, but yields were very low. As an alternative ${ }^{175-178,190}$ the coupling reaction was performed with the terminal alkyne as methyl ester 10a and $10 \mathbf{b}^{191}$ instead of the free acids in the presence of $\mathrm{K}_{2} \mathrm{CO}_{3}$ as a base, copper (I) iodide as a catalyst and sodium iodide ( $\mathrm{Nal}$ ) as an additive in DMF at room temperature. The $\mathrm{Cl} 8$ skipped 1,4-diynes 11a and 11b were obtained in good yields; semi-hydrogenation of these compounds using Lindlar catalyst in hexane afforded the crude target compounds, methyl $(8 R)$ - and $(8 S)-\left[8-{ }^{2} \mathrm{H}_{1}\right]$ octadeca-9Z,12Z-dienoates $(1 \mathrm{a}$ and $1 \mathrm{~b})$ as light yellowish clear oils that were purified first by flash chromatography $\left(1: 19 \mathrm{Et}_{2} \mathrm{O}\right.$-hexane) for spectral characterization and followed by HPLC when incubated with the in vivo yeast culture. ${ }^{158,173}$ The analytical data of the title compounds were in accord with their assigned structures and similar to that previously reported in the literature. ${ }^{173}$ The title compounds were obtained in high purity as determined by the appearance of a large major peak in GC for both of them (Figure 2.8 and 2.9) whose retention time also 
matches with that of the non-labeled linoleate. Minor peaks could be attributed to the unreacted short-chain methyl 8-d $d_{1}-9-$ decenoate, its saturated ester, some of the semipartially reduced C18 eneyne and cis, trans-isomer of the expected product.

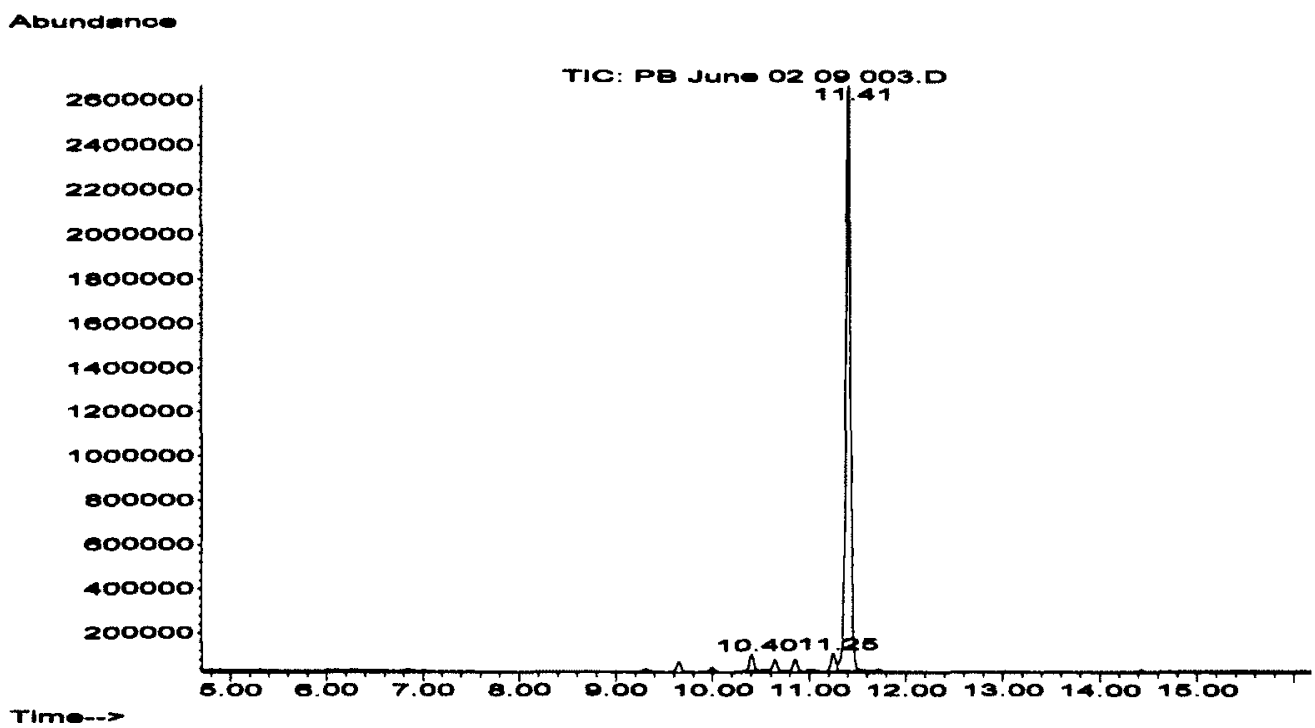

Figure 2.8. GC-MS chromatogram of the HPLC purified $(8 R)-\left[8-{ }^{2} \mathrm{H}_{1}\right]$-linoleate.

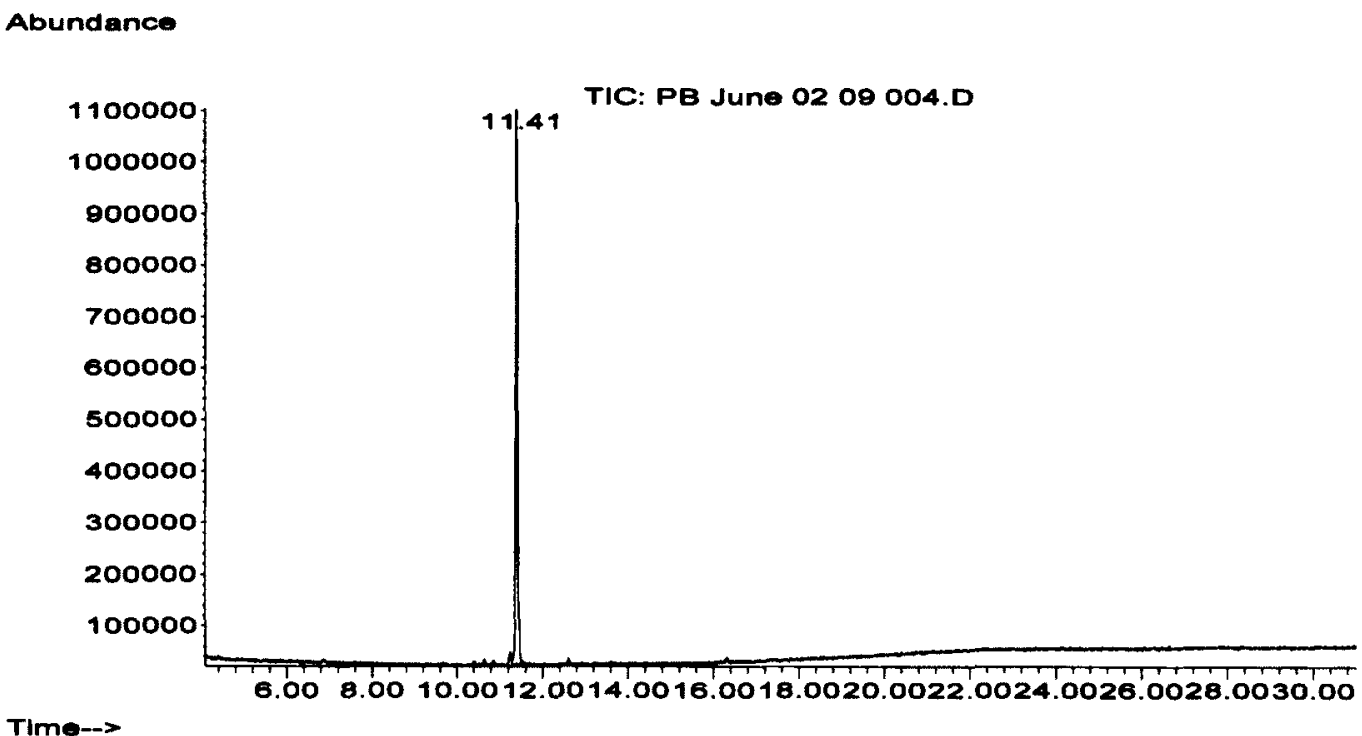

Figure 2.9. GC-MS chromatogram of the HPLC purified $(8 S)-\left[8-{ }^{2} \mathrm{H}_{1}\right]$-linoleate.

The mass spectra (MS) of each enantiomer (e.g. Figure 2.10, methyl $(8 R)-\left[8-^{2} \mathrm{H}_{1}\right]-$ linoleate) are virtually indistinguishable from each other and featured the following 
characteristic fragment ions: $\mathrm{m} / \mathrm{z} 295$ (molecular ion, $\left.[\mathrm{M}]^{+}\right), 280\left(\left[\mathrm{M}-\mathrm{CH}_{3}\right]^{+}\right), 264([\mathrm{M}-$ $\left.\left.\mathrm{OCH}_{3}\right]^{+}\right)$and $\left([\mathrm{M}-74]^{+}\right)$. These are consistent with their assigned structures and corresponds well with the MS of the non-deuterated linoleate ${ }^{192-194}$ (Figure 2.11) as well as with that of the previously reported results. ${ }^{173}$ From the analyses of the MS of each enantiomers it is apparent that the title compounds are obtained mainly as monodeuterated species which is consistent with the isotopic purity of the intermediates and their spectroscopic data.

Abundance

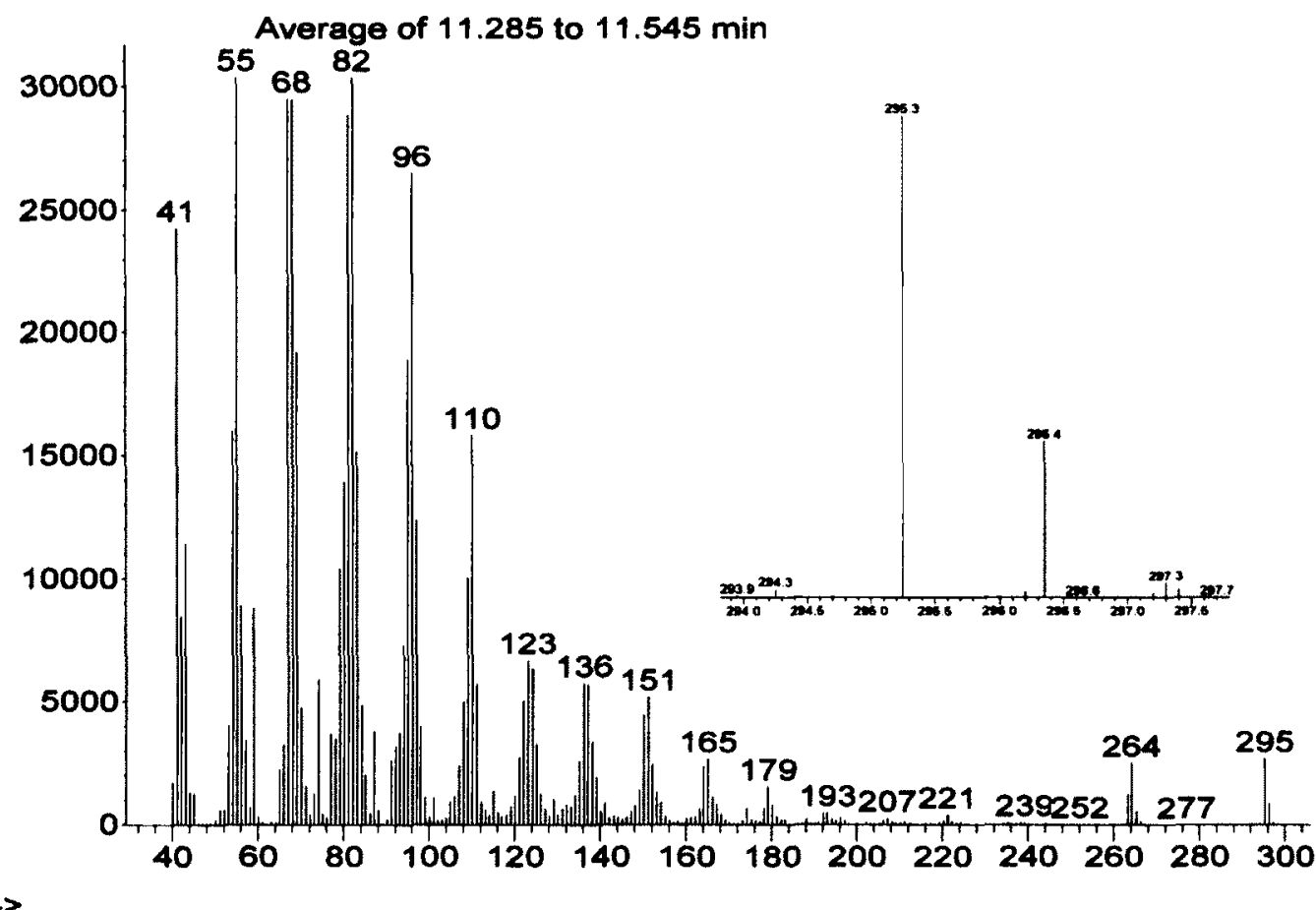
$\mathrm{m} / \mathrm{z}-\mathbf{>}$

Figure 2.10. Mass spectrum of methyl $(8 R)-\left[8-{ }^{2} \mathrm{H}_{1}\right]$-linoleate. 


\section{Abundance}

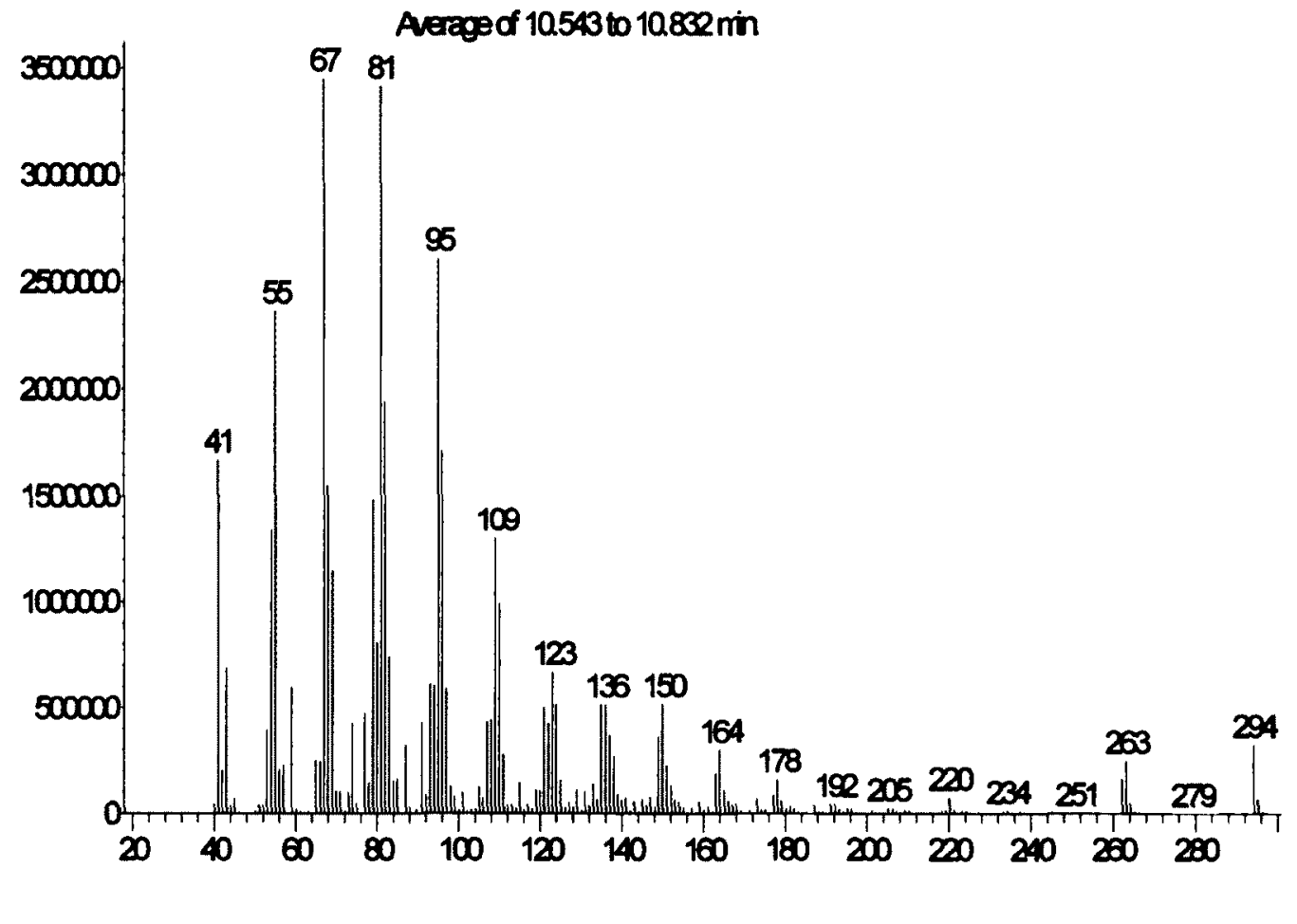

Figure 2.11. Mass spectrum of methyl linoleate.

Since scrambling of allylic deuterium label can occur during catalytic hydrogenation, the location of the deuterium label was confirmed by comparing the ${ }^{1} \mathrm{H}$ and ${ }^{13} \mathrm{C}$ NMR spectra of both enantiomers (1a and 1b) with that of non-labelled methyl linoleate. ${ }^{173}$ Thus, while the ${ }^{1} \mathrm{H}$ multiplet due to the bisallylic hydrogens at $\mathrm{C}-11$ was similar in appearance and integration for the $d_{1}$ - and non-labelled compound (Figure 2.12 and 2.13), the overlapping multiplets at $2.05 \mathrm{ppm}$ due to protons at $\mathrm{C} 8$ and $\mathrm{C} 14$ integrated for 3 and 4 protons, respectively. 


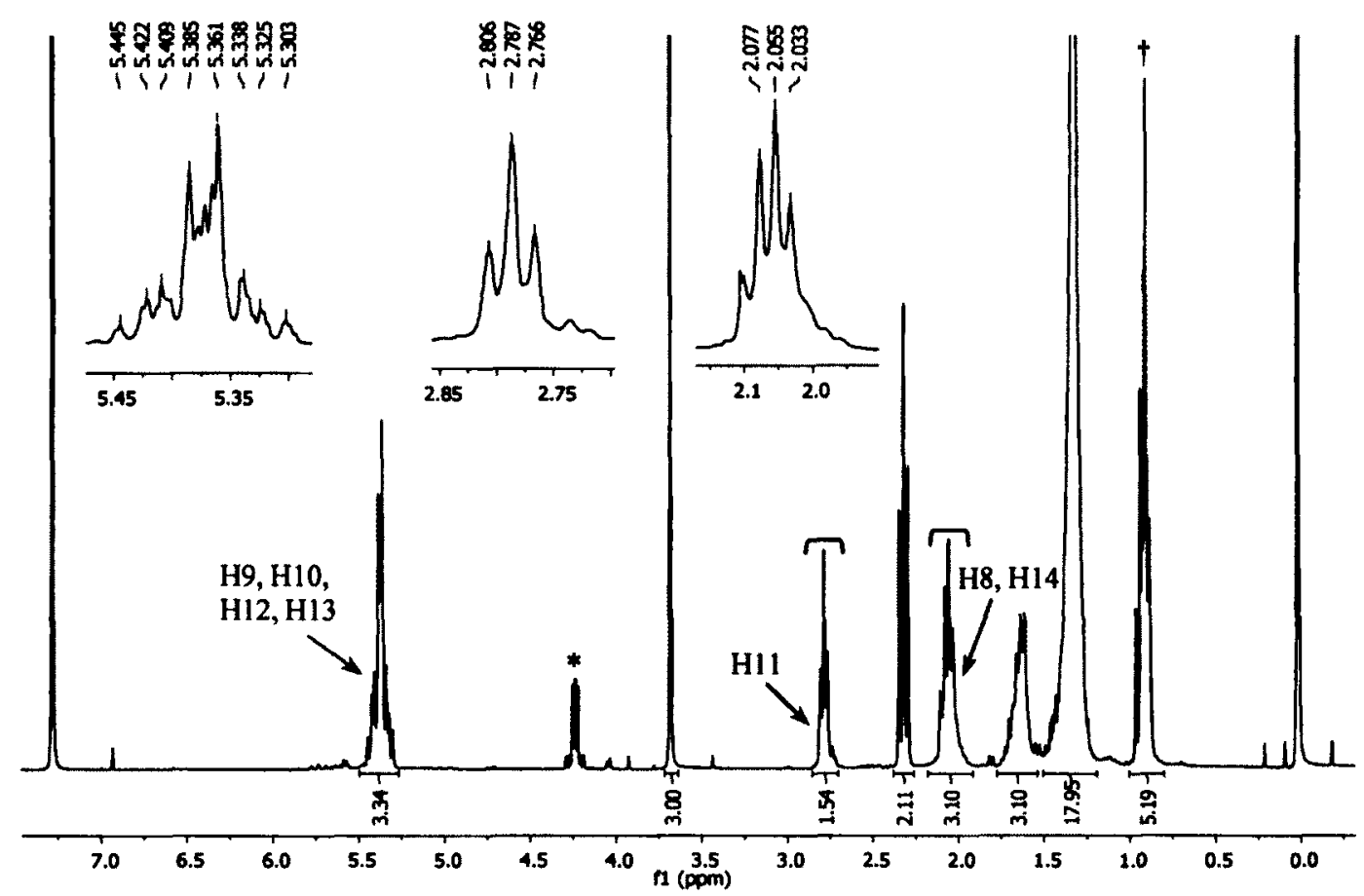

Figure 2.12. ${ }^{1} \mathrm{H}$ NMR spectrum of methyl $(8 R)-\left[8-{ }^{2} \mathrm{H}_{1}\right]$-linoleate. ' due to unknown impuritiy. ${ }^{\dagger}$ Along with the product methyl group, small amount of solvent peak also present.

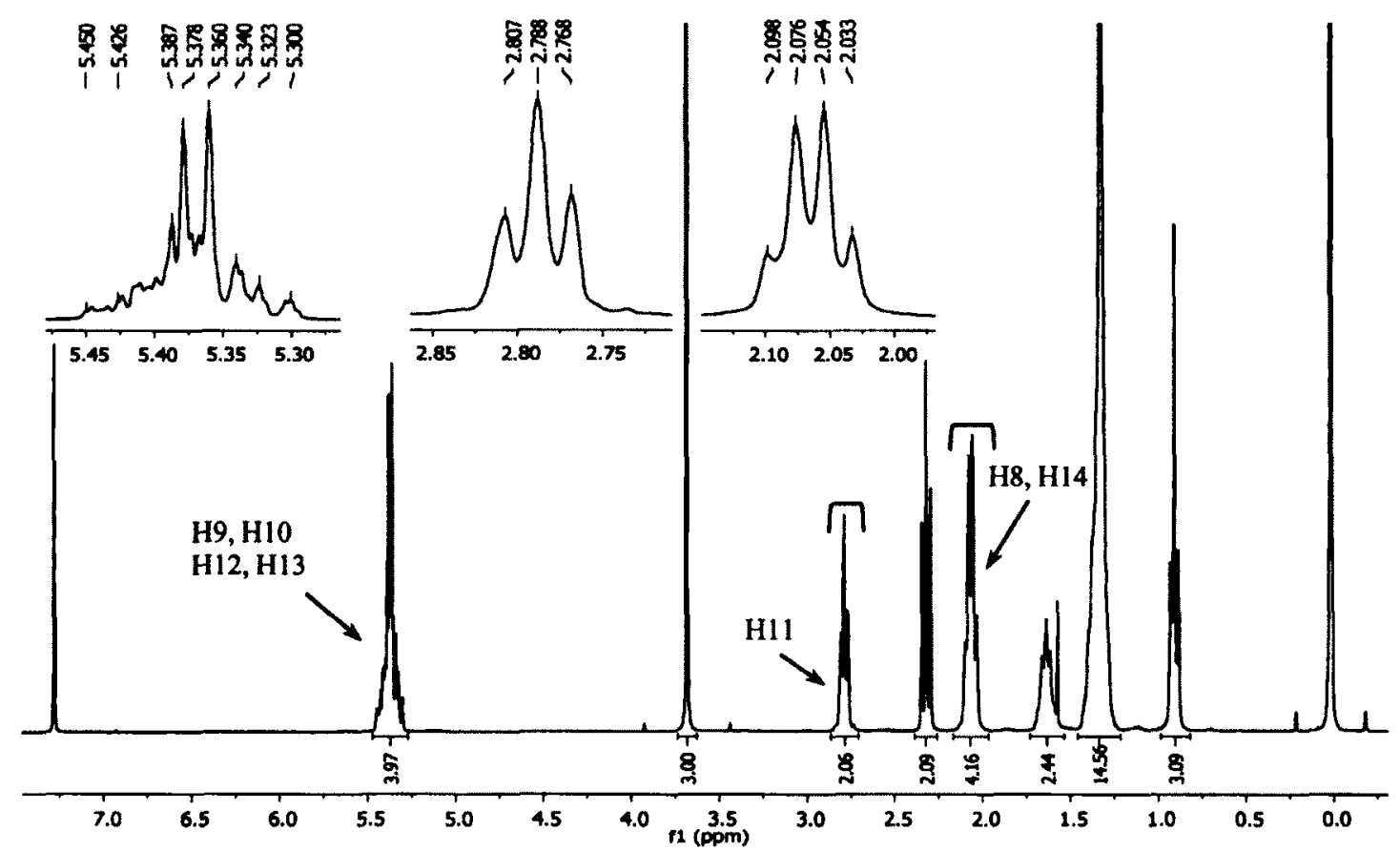

Figure 2.13. 'H NMR spectrum of methyl linoleate. 
The location of deuteration was easily pinpointed in the ${ }^{13} \mathrm{C}$ NMR spectrum of 1a and $1 \mathrm{~b}$. The signal assigned ${ }^{173}$ to $\mathrm{C}-8$ at $\delta 27.20 \mathrm{ppm}$ in the ${ }^{1} \mathrm{H}$-decoupled ${ }^{13} \mathrm{C}$ NMR spectrum of the non-labeled compound was replaced by a triplet $\left({ }^{2} J_{\mathrm{CD}}=19.2 \mathrm{~Hz}\right)$ at $\delta$ $26.84 \mathrm{ppm}(\alpha$-deuterium isotope shift of $-0.362 \mathrm{ppm})$ for $(8 R)-\left[8-{ }^{2} \mathrm{H}_{1}\right]$-linoleate 1a (Figure 2.14). As further detailed in Appendix A, $\beta$-isotope shifts of -0.093 and -0.051 ppm, respectively were also observed for signals assigned to $C-7, C-9$. The same scenario was observed with the (8S)-isomer $1 \mathrm{~b}$ (data not shown).

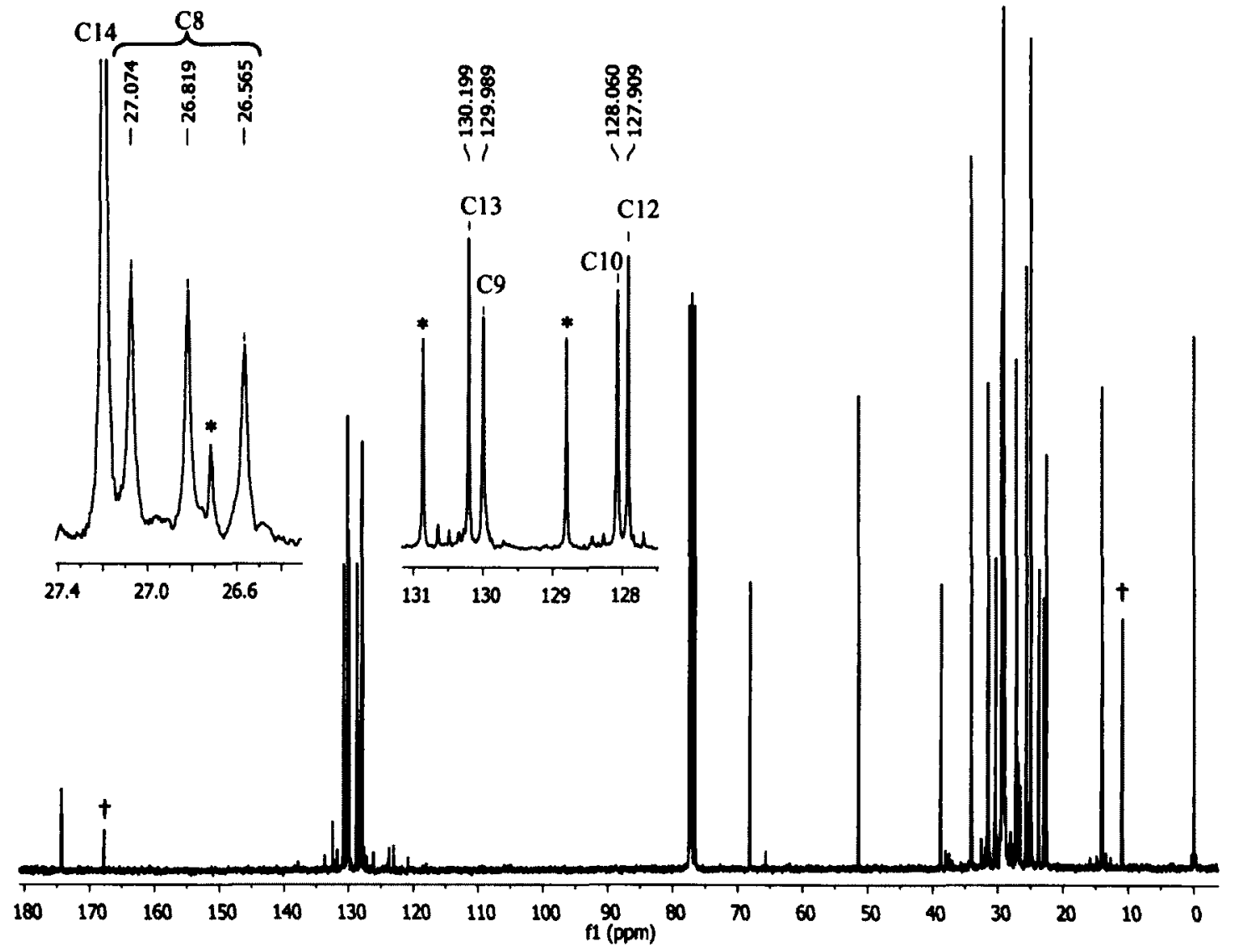

Figure 2.14. ${ }^{13} \mathrm{C}$ NMR spectrum of methyl $(8 R)-\left[8-{ }^{2} \mathrm{H}_{1}\right]$-linoleate. The ${ }^{13} \mathrm{C}$ signals due to C9 (129.99 ppm) and C10 (128.06 ppm) are reduced in intensity due to the presence of deuterium at $\mathrm{C} 8$. ${ }^{*}$ Peaks are due to the presence of unreacted methyl $(8 R)-\left[8-{ }^{2} \mathrm{H}_{1}\right]-$ dec-9enoate. ${ }^{\dagger}$ Unknown impurities. 


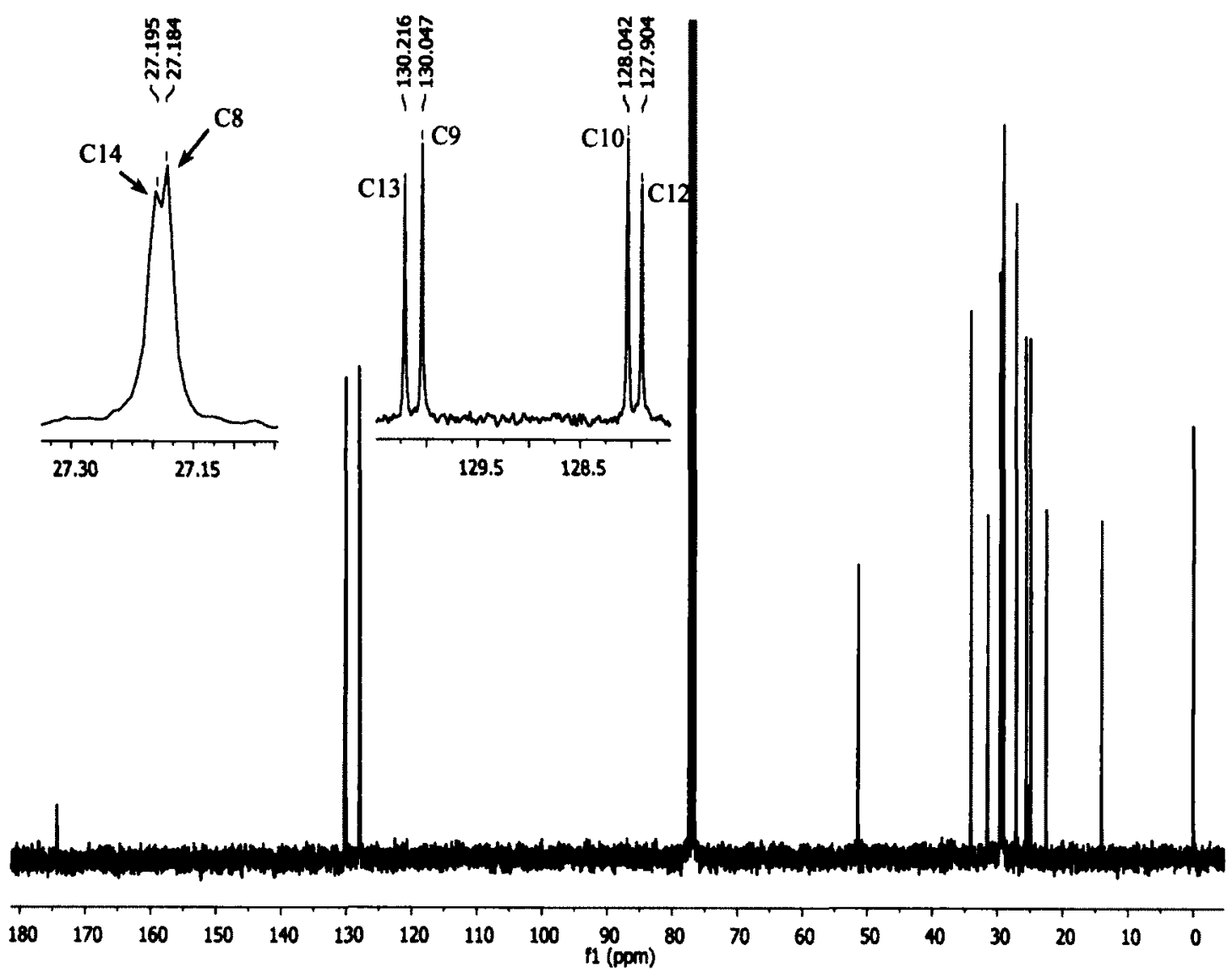

Figure 2.15. ${ }^{13} \mathrm{C}$ NMR spectrum of methyl linoleate.

The presence of deuterium label in $1 \mathrm{a}$ and $\mathbf{1 b}$ was also detected by IR spectroscopy with the appearance of an intense absorption peak at $2145 \mathrm{~cm}^{-1}$ corresponding to a C-D bond stretch ${ }^{173}$ (Figure 2.16). 


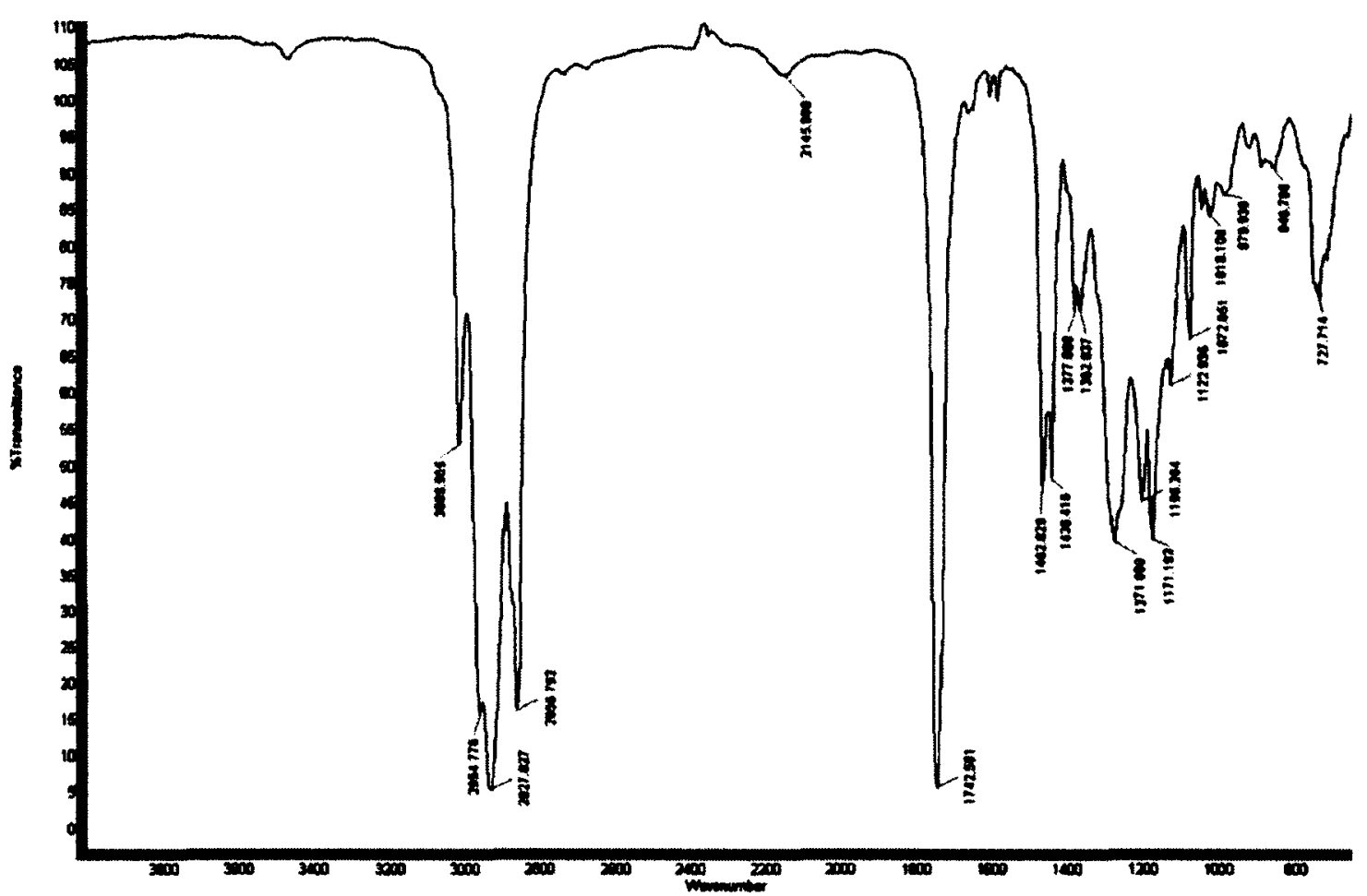

Figure 2.16. IR spectrum of methyl $(8 R)-\left[8-{ }^{2} \mathrm{H}_{1}\right]$-linoleate.

\subsubsection{Chemoenzymatic Synthesis of Methyl (11R)- and (11S)-[11- $\left.{ }^{2} \mathbf{H}_{1}\right]$-Octadeca- 9Z,12Z-Dienoate}

\subsubsection{Overview}

Skipped dienes that are stereospecifically deuterated at the bisallylic position have traditionally been synthesized by enzymatic desaturation of the appropriate chiral precursors. $^{40,195}$ A literature procedure ${ }^{173}$ for a purely synthetic route to the title compounds was initially attempted, as described in the next section, but the products were subsequently discovered to be racemic. The successful route (Figure 2.17), is described here: 


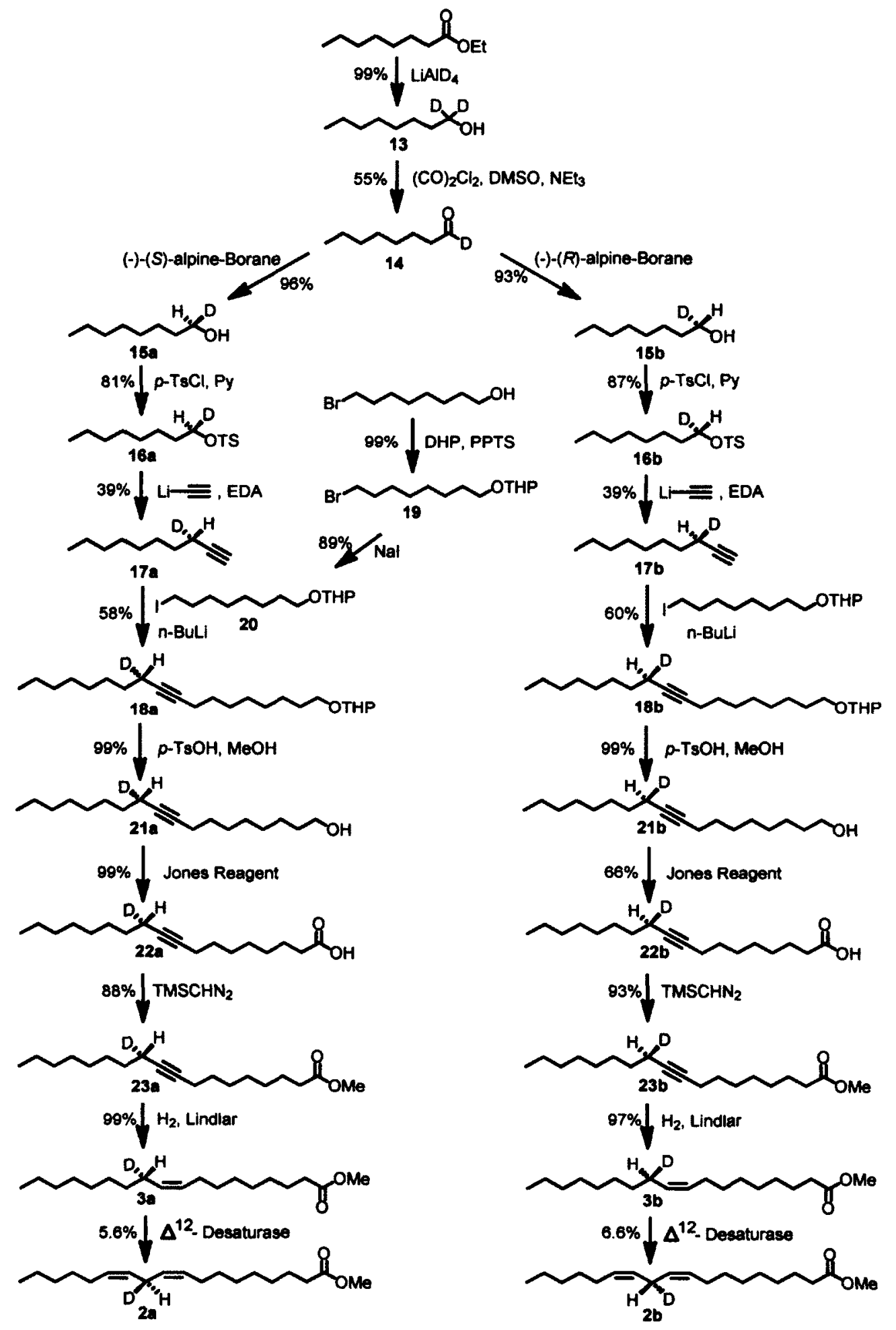

Figure 2.17. Scheme for the chemoenzymatic synthesis of chiral $\left[11-{ }^{2} \mathrm{H}_{1}\right]$-linoleates via $\Delta^{12}$-desaturation of chiral $\left[11-{ }^{2} \mathrm{H}_{1}\right]$-oleate precursors, methyl $(11 S)-\left[11-{ }^{2} \mathrm{H}_{1}\right]$-oleate $3 \mathrm{a}$ and methyl $(11 R)-\left[11{ }^{2} \mathrm{H}_{1}\right]$-oleate $3 \mathrm{~b}$. 


\subsubsection{Synthesis of Methyl (11S)- and (11R)-[11- $\left.{ }^{2} \mathrm{H}_{1}\right]-$ Octadeca-9Z-enoate, 3a \& 3b}

Reduction of ethyl octanoate to the corresponding dideuterated alcohol $13^{174,179}$ using $\mathrm{LiAlD}_{4}$, followed by Swern oxidation furnished $\left[1-^{2} \mathrm{H}_{1}\right]$-octanal $14^{183}$. Enantioselective reduction of the 1-deuteriooctanal employing chiral Alpine-Borane reagents afforded the required chiral monodeuterio octanols $\mathbf{1 5 a}$ and $\mathbf{1 5 b}$ as colorless clear oils. ${ }^{174,185}$ As the expected absolute configuration and high enantiomeric purity (ee $\sim 90 \%)$ of closely related homologous alcohols $\left((1 R)-\right.$ and $(1 S)-\left[1-{ }^{2} \mathrm{H}_{1}\right]$-pentanol) produced in a similar manner had already been confirmed (Section 3.2.3.1), it was

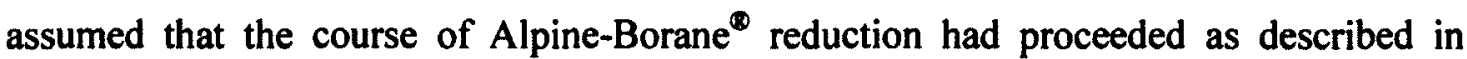
Figure 2.18. The chiral deuterio alcohols were then tosylated ${ }^{174,179,184}$ for the purpose of nucleophilic substitution by lithium acetylide to generate $(3 S)$ - and $(3 R)-\left[3-{ }^{2} \mathrm{H}_{1}\right]-1-$ decyne. $^{39,173}$ The nucleophilic substitution of the tosylate by acetylide is believed to proceed through inversion of configuration as noted above. The acetylide of each chiral deuterio 1-decyne was coupled ${ }^{196}$ with the THP ether derivative of 8-iodo-1-octanol to yield the C18 intermediate $18 \mathrm{a}$ and $18 \mathrm{~b}$. A standard deprotection ${ }^{174,196} /$ oxidation $^{180,196}$ /methylation ${ }^{191} /$ Lindlar hydrogenation ${ }^{158,173}$ sequence generated the required chiral methyl $\left[11-{ }^{2} \mathrm{H}_{1}\right]$-oleates after purification by flash chromatography (1:19 $\mathrm{Et}_{2} \mathrm{O}$-hexane) The extent to which Lindlar semi-hydrogenation produced cis-olefin without positional scrambling and the isotopic content of the $d_{1}$-oleates was checked through the use of GCMS, IR, ${ }^{1} \mathrm{H}$ and ${ }^{13} \mathrm{C}$ NMR spectroscopy. The appearance of a major peak in the gas chromatograms of both enantiomers (Figure 2.18 and 2.19) showed that compounds of high chemical purity were obtained through the use of our synthetic strategy. 


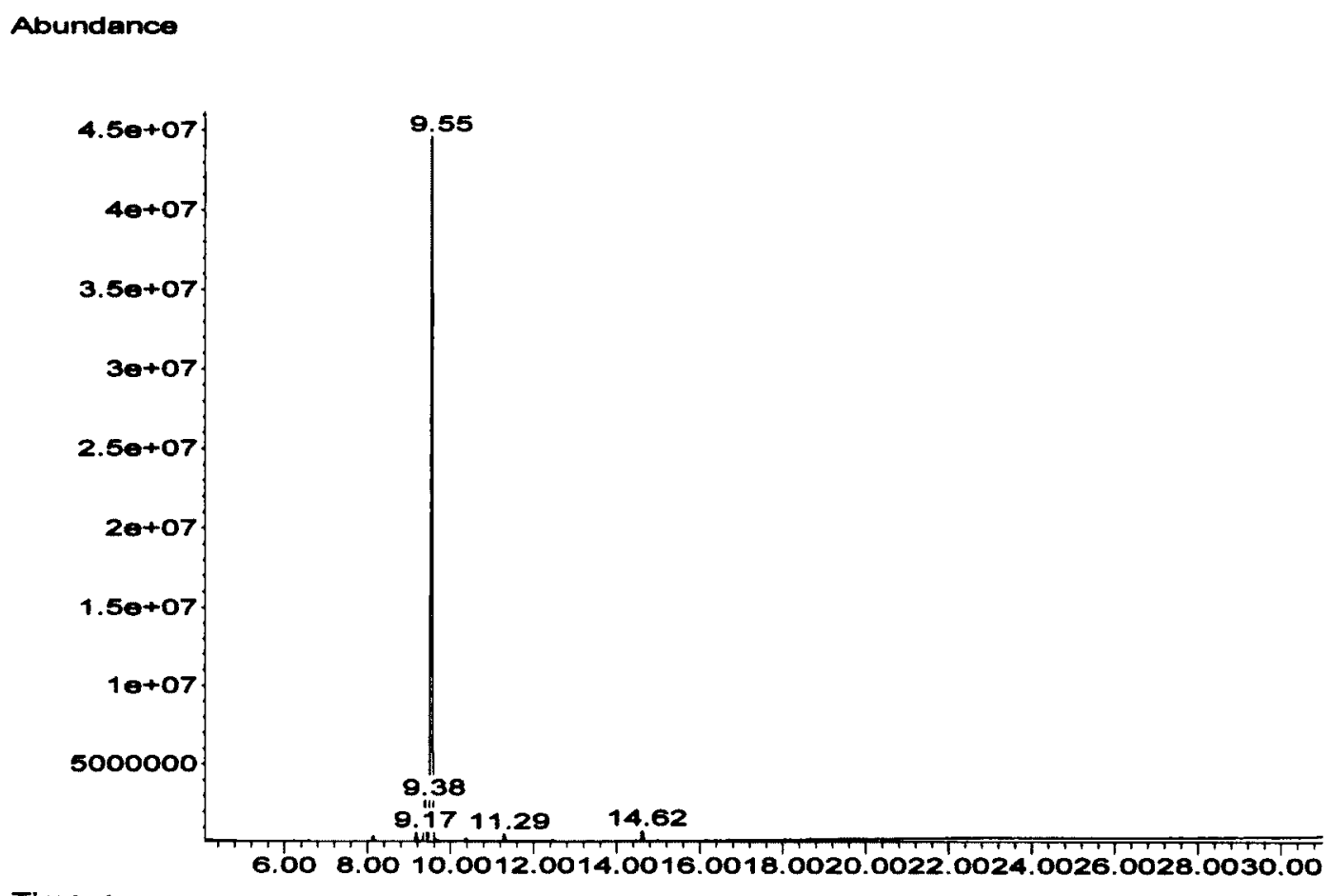

Figure 2.18. GC-MS of methyl $(11 S)-\left[11-{ }^{2} \mathrm{H}_{1}\right]$-oleate $3 \mathbf{a}$.

Abundance

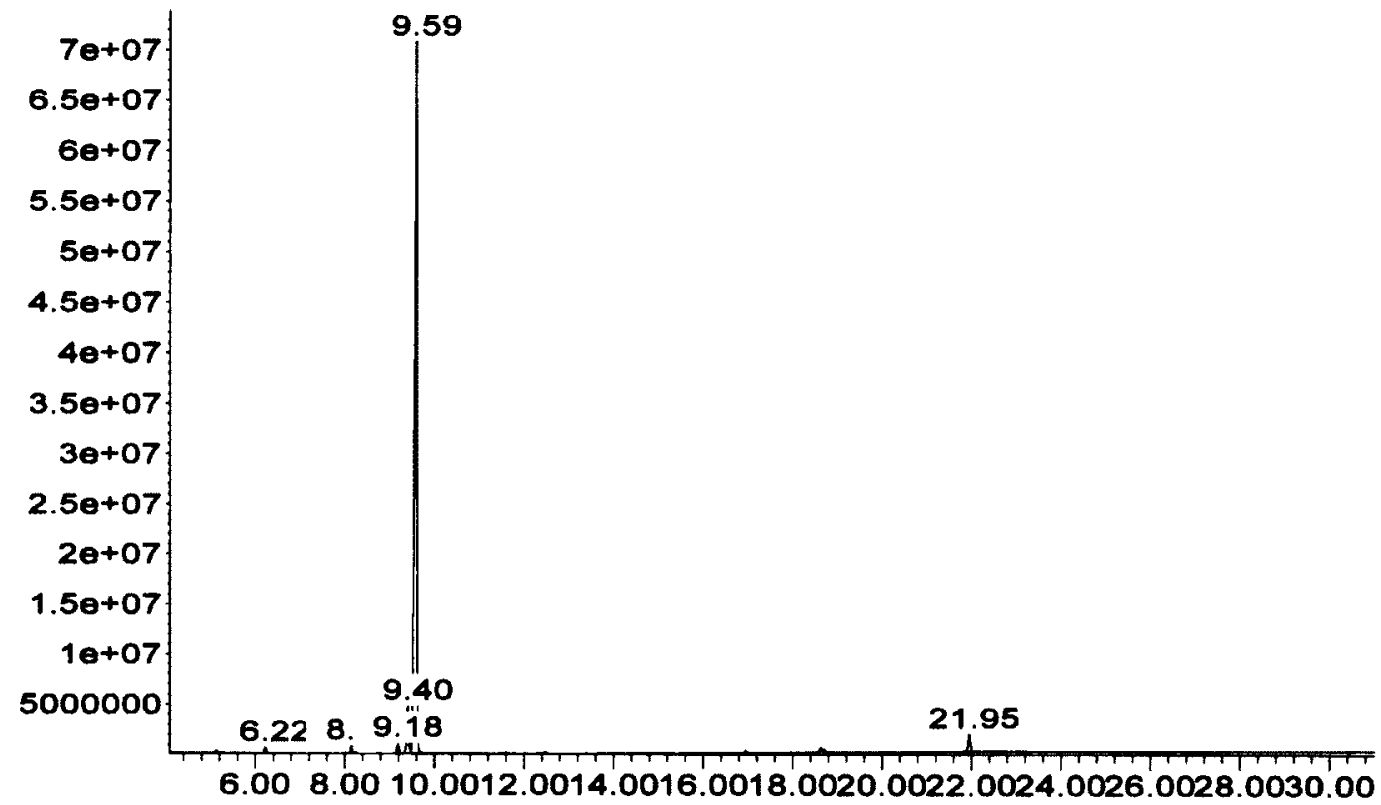

Time-->

Figure 2.19. GC-MS of methyl $(11 R)-\left[11-{ }^{2} \mathrm{H}_{1}\right]$-oleate $3 \mathrm{~b}$. 
The preponderance of monodeuterated species in the target compounds were further confirmed through the mass spectral analysis (e.g. Figure 2.20) of the corresponding GC peak and showed molecular ion 297 that is consistent with the molecular formula $\mathrm{C}_{19} \mathrm{H}_{35} \mathrm{DO}_{2}$. The mass spectral fragmentation pattern was similar (1 mass unit increase) to that observed with the non-labeled analogue $e^{192-194,197}$ (Figure 2.21). The following fragment ions were observed: $297\left([\mathrm{M}]^{+}\right), 266\left(\left[\mathrm{M}-\mathrm{OCH}_{3}\right]^{+}\right), 265\left(\left[\mathrm{M}-\mathrm{CH}_{3} \mathrm{OH}\right]^{+}\right)$and a characteristic peak at $223\left([\mathrm{M}-74]^{+}\right)$and are in accord with our expectations.

\section{Abundance}

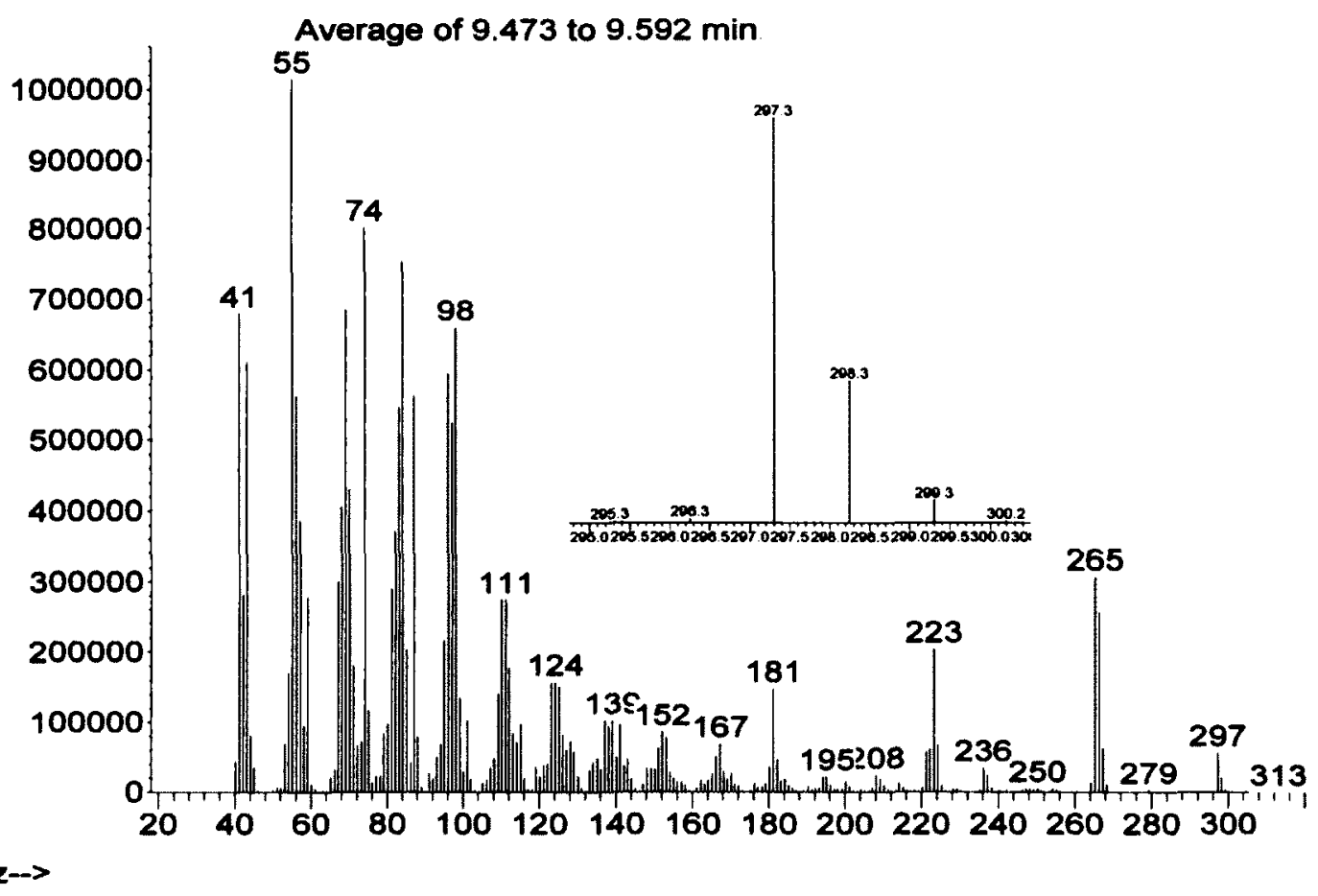

Figure 2.20. Mass spectrum of methyl (11S)-[11- $\left.{ }^{2} \mathrm{H}_{1}\right]$-oleate 3a. 


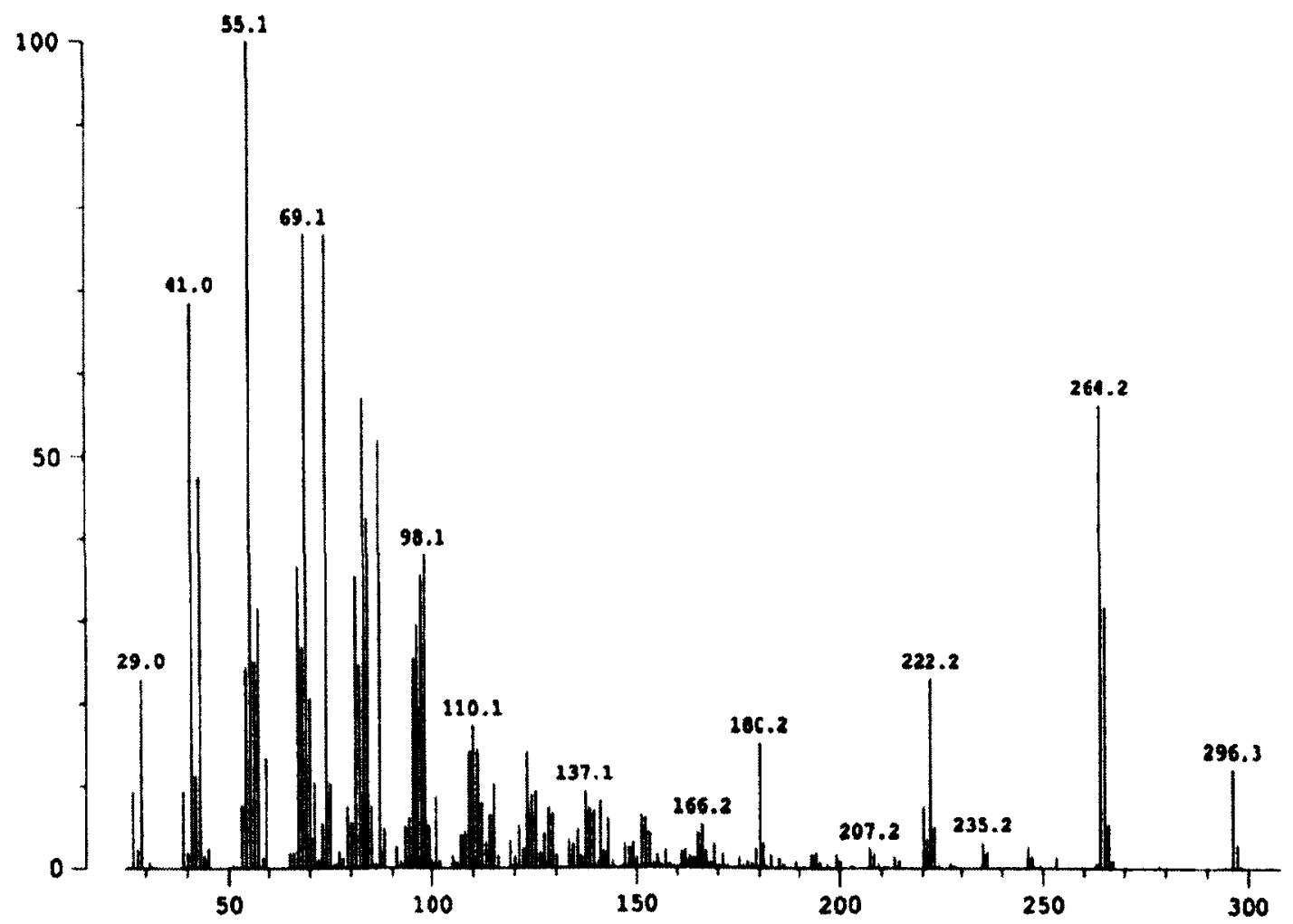

Figure 2.21. Mass spectrum of methyl oleate.

The chemical shifts $(\delta)$ and splitting patterns $(J)$ of the signals in the ${ }^{1} \mathrm{H}$ NMR spectra of methyl (11S)-[11- $\left.{ }^{2} \mathrm{H}_{1}\right]$-oleate 3a (e.g. Figure 2.22) and its enantiomer (data not shown) were in accord with their assigned structures and similar to that observed for the non-deuterio oleate (Figure 2.23). The presence of one deuterium at the allylic position of C-11 was indicated by the reduction of the integral of the multiplet at $\delta 2.01 \mathrm{ppm}$ from $4 \mathrm{H}$ to $3 \mathrm{H}$ relative to that of the unlabelled compound. However, the effect of deuteration on the neighboring and germinal proton's splitting pattern as well as the associated isotopic shifts couldn't be calculated as in most of the cases multiplets were observed due to the overlap of more than one proton signal. 


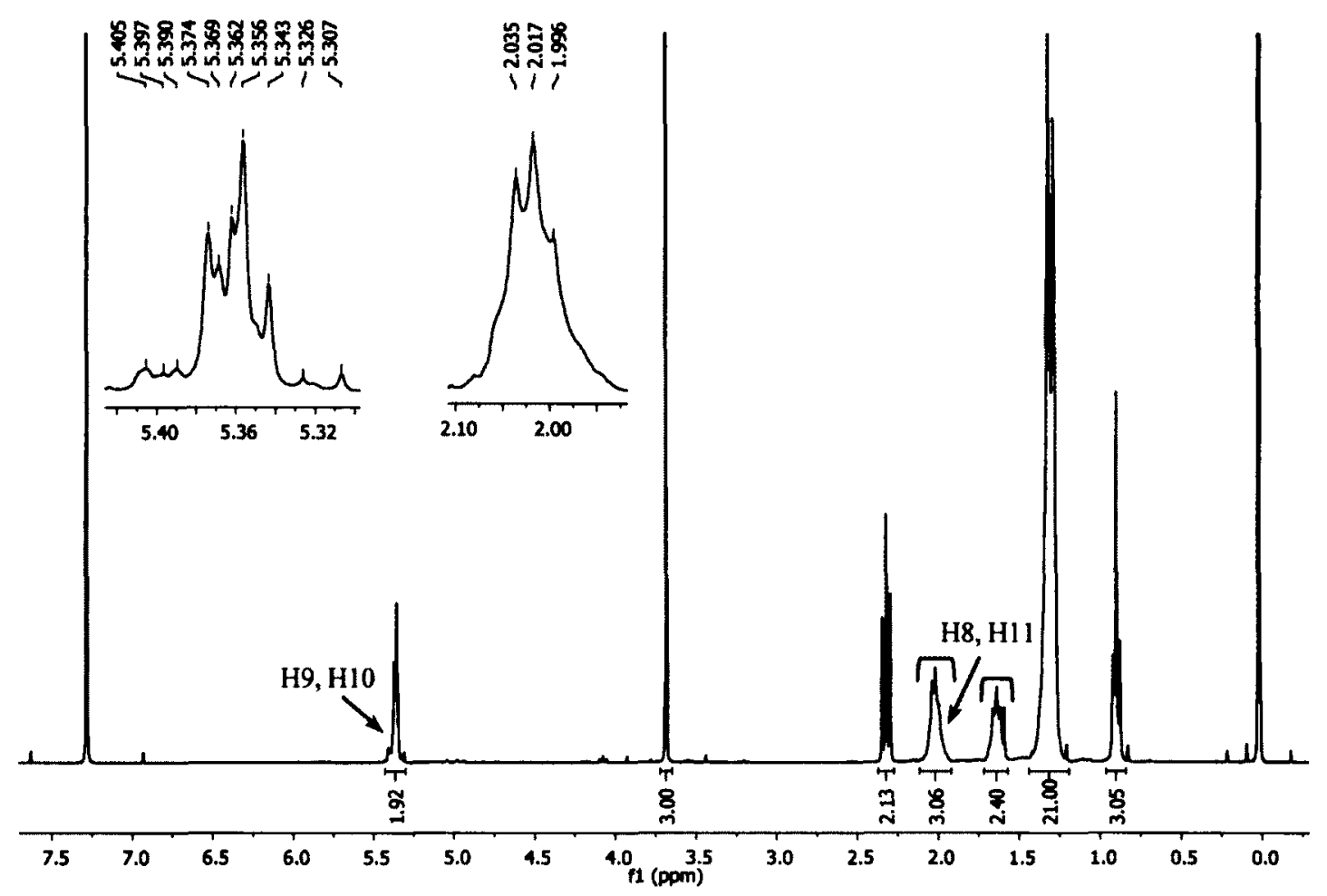

Figure 2.22. ${ }^{1} \mathrm{H}$ NMR spectrum of methyl $(11 S)-\left[11-{ }^{2} \mathrm{H}_{1}\right]$-oleate $3 \mathrm{a}$.
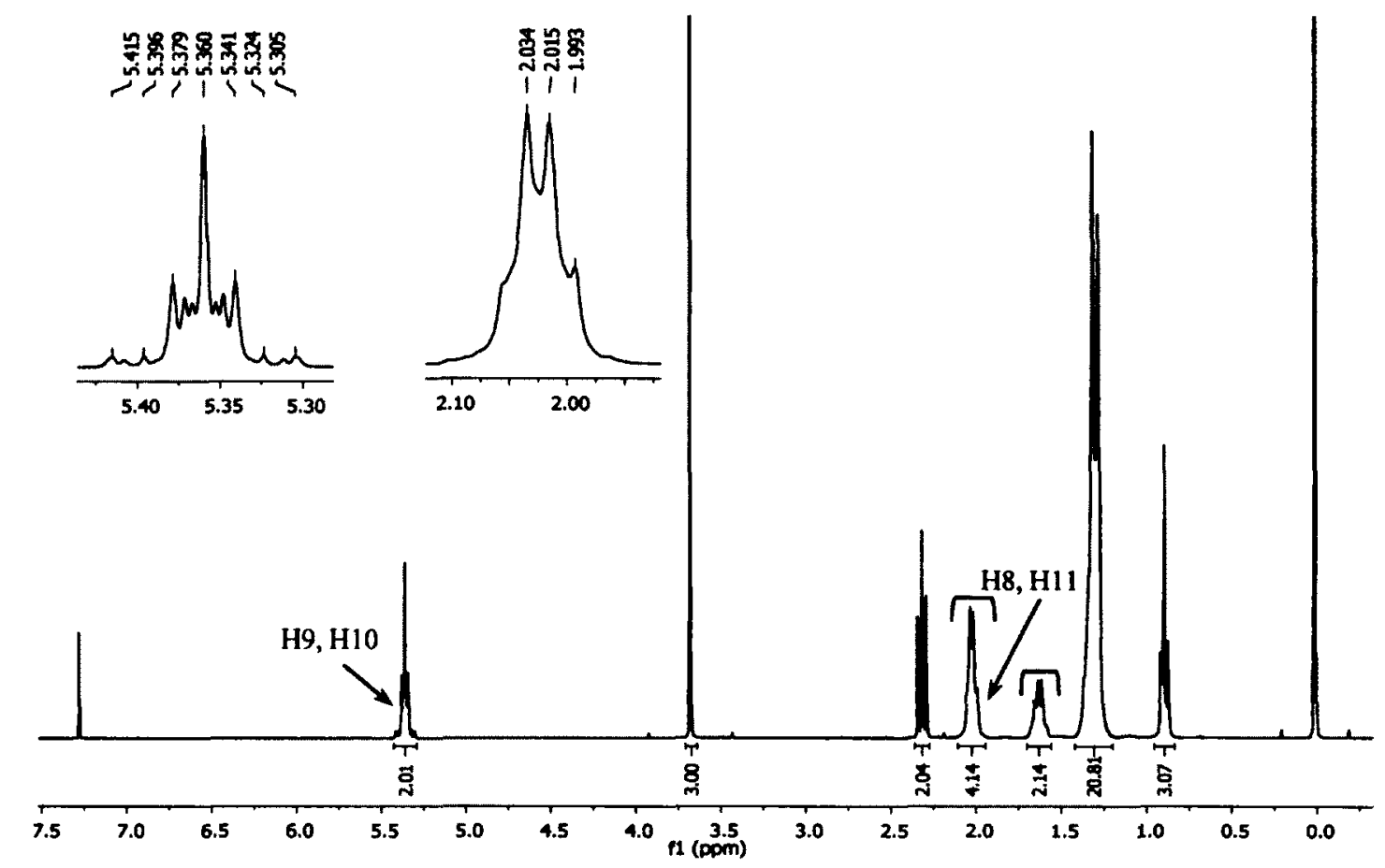

Figure 2.23. ${ }^{1} \mathrm{H}$ NMR spectrum of methyl oleate. 
The ${ }^{13} \mathrm{C}$ NMR spectrum of methyl $(11 S)-\left[11-{ }^{2} \mathrm{H}_{1}\right]$-oleate (Figure 2.24$)$ and its enantiomer (data not shown) featured a triplet $\left({ }^{2} J_{\mathrm{CD}}=19.3 \mathrm{~Hz}\right.$ ) at $26.87 \mathrm{ppm}$ that is upfield shifted ( $\alpha$-deuterium isotope shift) by $0.352 \mathrm{ppm}$ relative to the singlet that appears at $\delta 27.22$ ppm assigned to the C-11 carbon in the ${ }^{13} \mathrm{C}$ NMR of non-deuterated oleate. ${ }^{192-194,197}$ In addition, typical $\beta$-deuterium isotope shifts at $\mathrm{C}-10(-0.04 \mathrm{ppm})$ and $\mathrm{C} 12(-0.08 \mathrm{ppm})$ and $\gamma$-deuterium isotope shifts at $\mathrm{C} 9(0.03 \mathrm{ppm})$ and $\mathrm{C} 11(-0.02)$ were noted (Appendix A3).

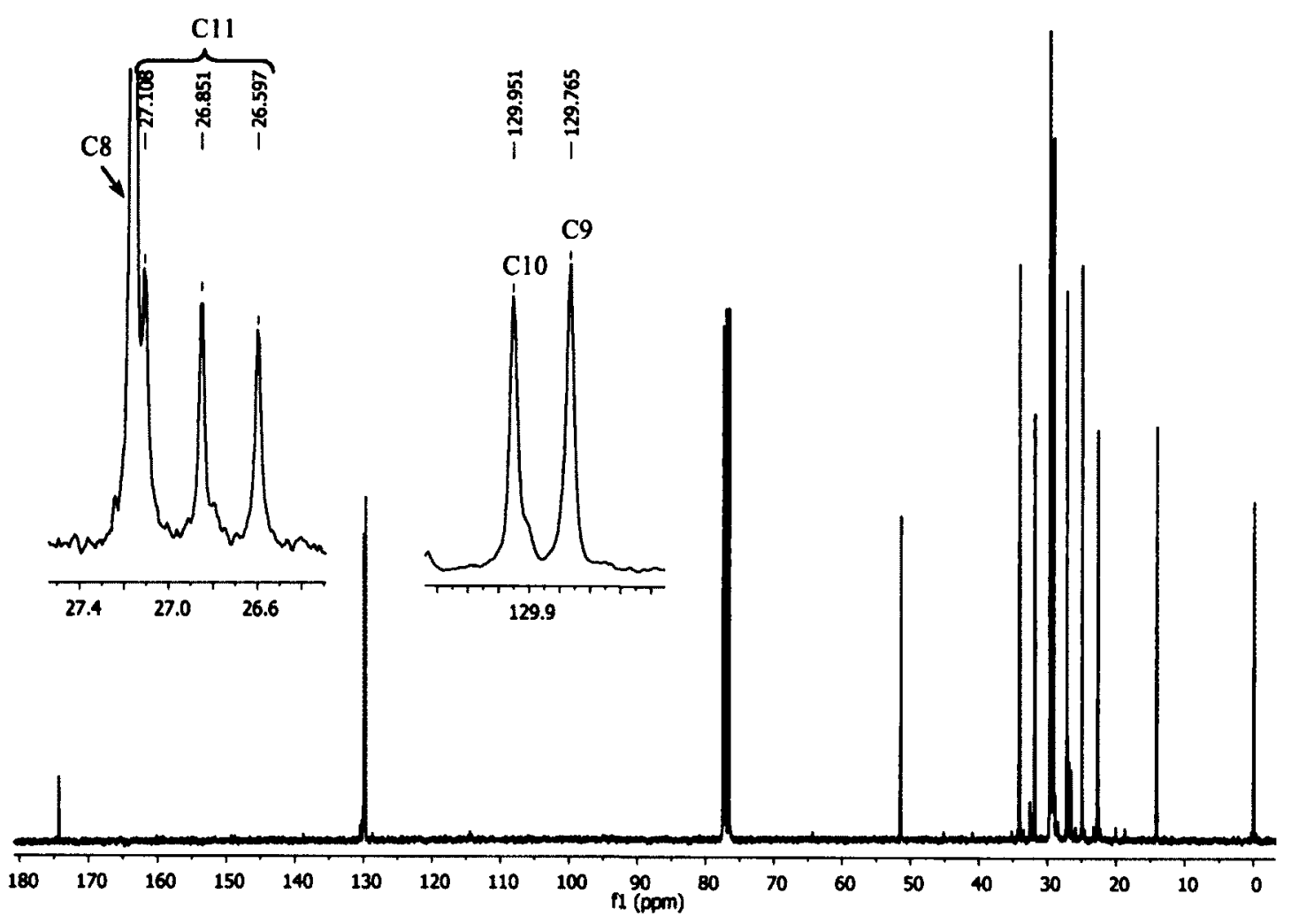

Figure 2.24. ${ }^{13} \mathrm{C}$ NMR spectrum of methyl $(11 S)-\left[11-{ }^{2} \mathrm{H}_{1}\right]$-oleate 3a. The ${ }^{13} \mathrm{C}$ signal due to $\mathrm{C} 11(26.85 \mathrm{ppm})$ splits into a triplet and is significantly reduced in intensity because of coupling with the deuterium atom. Also, the ${ }^{13} \mathrm{C}$ signal due to $\mathrm{C} 9(129.77 \mathrm{ppm})$ and $\mathrm{C} 10$ $(129.95 \mathrm{ppm})$ is reduced in intensity due to the presence of deuterium at $\mathrm{Cl1}$.

The observed chemical shifts for the allylic carbons, C-8 and C-11 at $27.18 \mathrm{ppm}$ and $26.87 \mathrm{ppm}$ respectively are in accord with the assigned Z-configuration of the double 
bond in $3 \mathbf{a}$ and $\mathbf{3 b}$. The corresponding chemical shifts of $E$-configured olefins occur further downfield at $32.64 \mathrm{ppm}$ and $32.66 \mathrm{ppm}$ as documented by Gunstone et al. for the corresponding free acid. ${ }^{192-194,197}$ Absence of such peak in the ${ }^{13} \mathrm{C}$ NMR of methyl (11S)$\left[11-{ }^{2} \mathrm{H}_{1}\right]$-oleate confirmed that the $\mathrm{Z}$-configured isomer was obtained upon reduction.

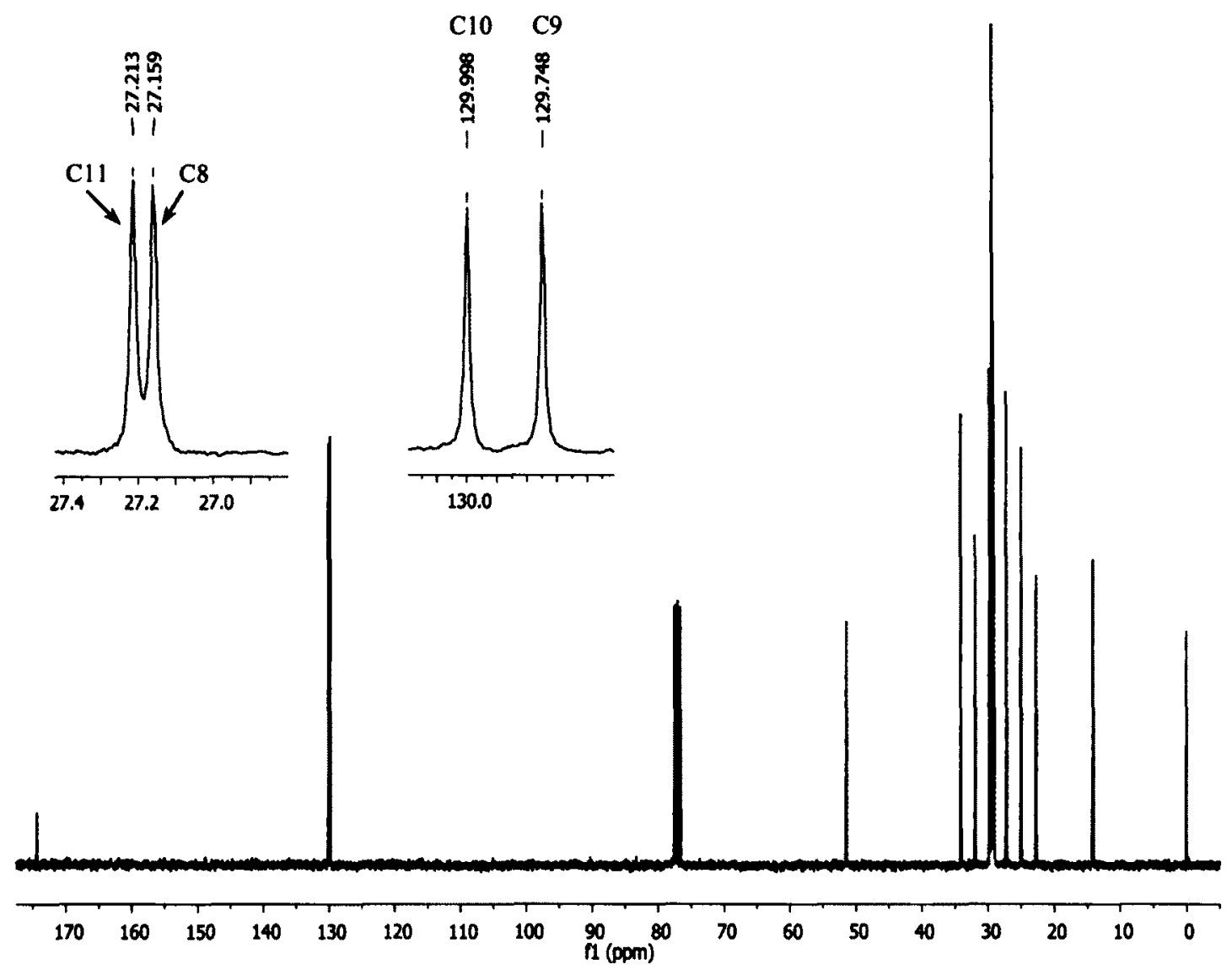

Figure 2.25. ${ }^{13} \mathrm{C}$ NMR spectrum of methyl oleate.

Finally, the presence of a diagnostic C-D stretch was observed in the IR spectra of deuterated samples (e.g. Figure 2.26) at $2149 \mathrm{~cm}^{-1}$. 


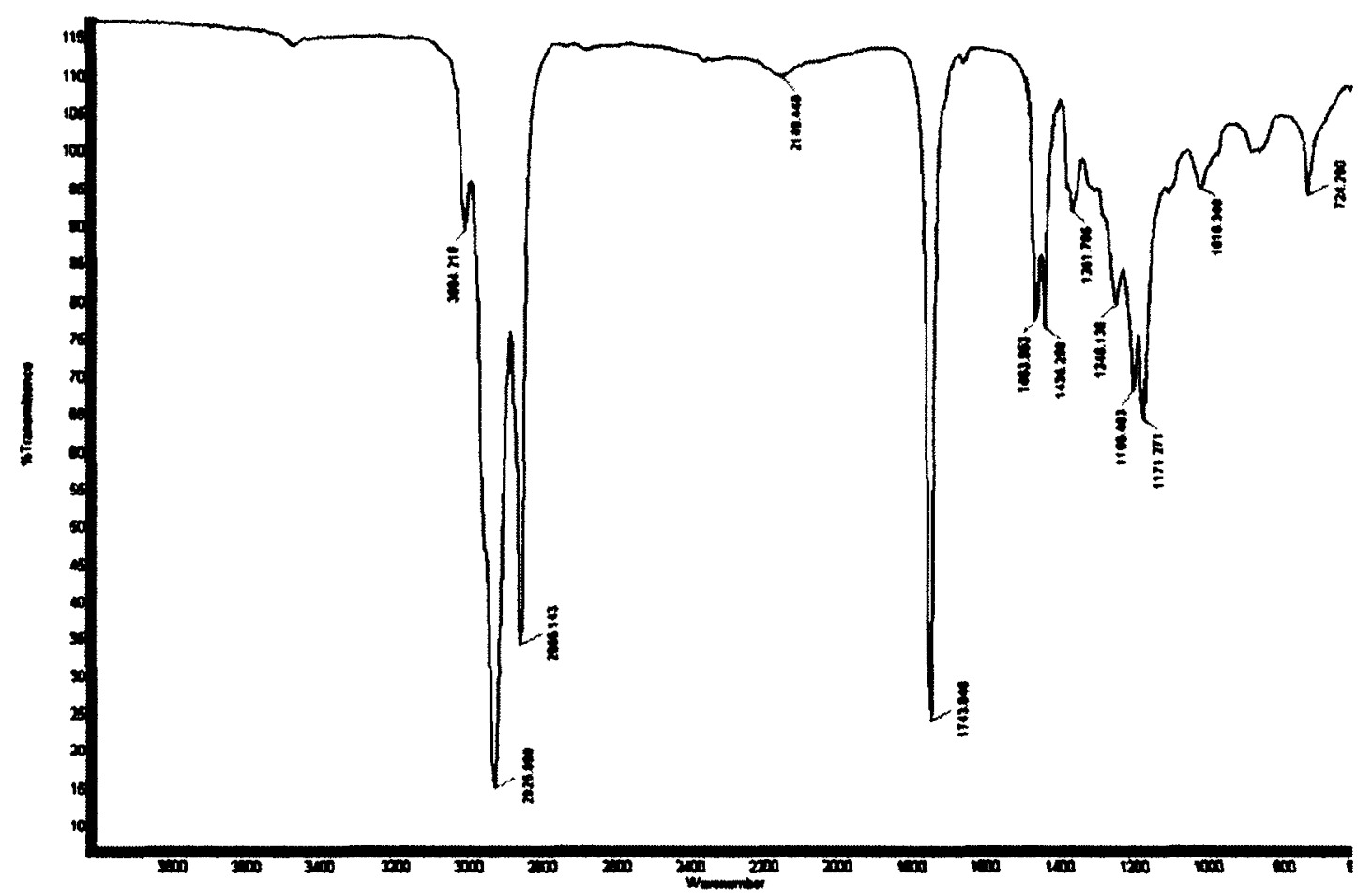

Figure 2.26. IR spectrum of methyl $(11 S)-\left[11-{ }^{2} \mathrm{H}_{1}\right]$-oleate $3 \mathbf{a}$.

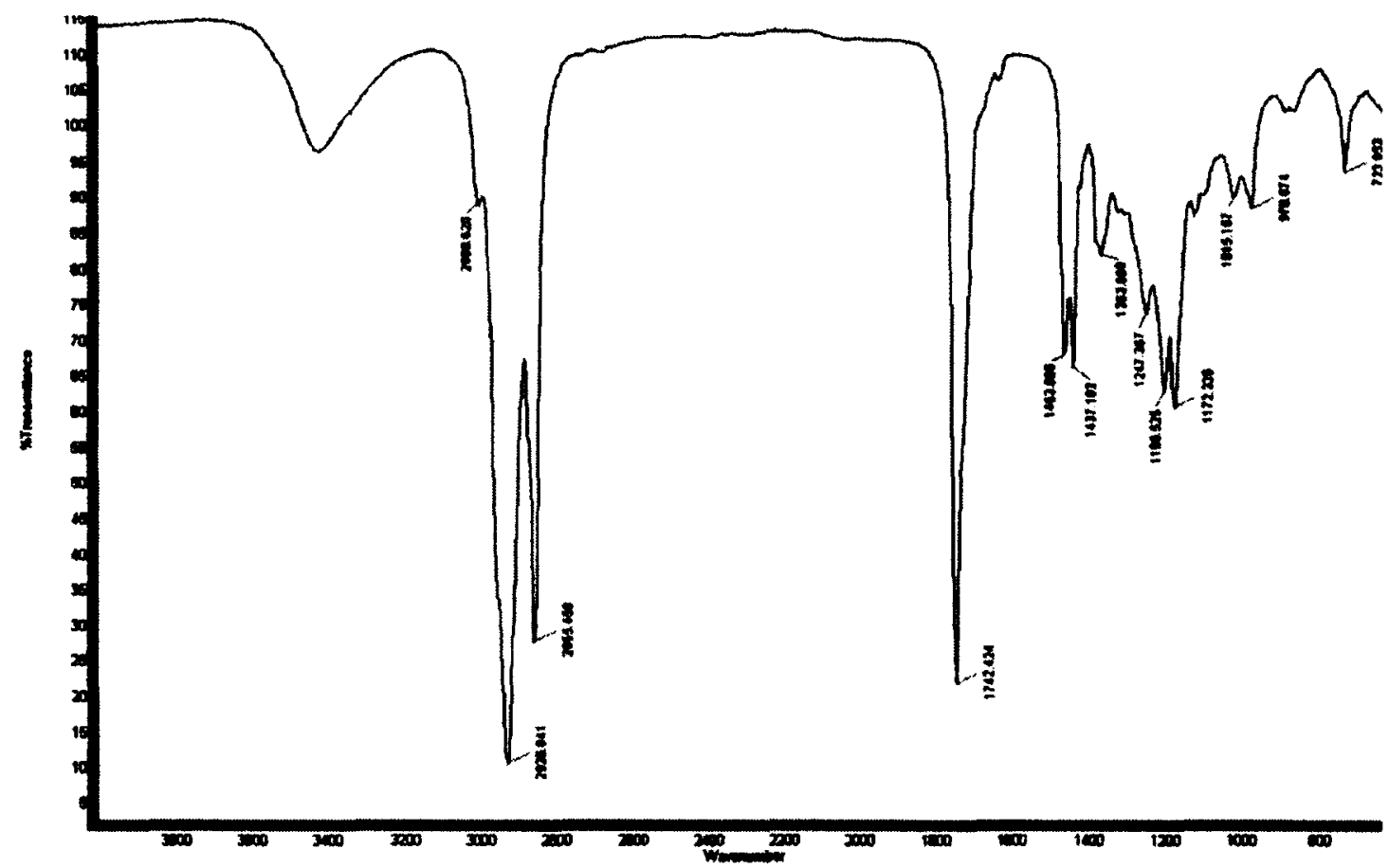

Figure 2.27. IR spectrum of methyl oleate. $\mathrm{OH}$ Stretch at $3400 \mathrm{~cm}^{-1}$ peak due to traces of $\mathrm{H}_{2} \mathrm{O}$. 


\subsubsection{Enzymatic Conversion of Methyl (11S)- and (11R)-[11- $\left.{ }^{2} \mathrm{H}_{1}\right]-$ Octadeca-9Z-} enoate to Corresponding Linoleates, $2 a$ and $2 b$

Saponified samples $(150 \mathrm{mg})$ of methyl $(11 S)$ - and $(11 R)-\left[11-{ }^{2} \mathrm{H}_{1}\right]$ octadeca-9Zenoate dissolved in Tergitol (type NP-40) were incubated at $225 \mathrm{rpm}$ for 6 days at $20^{\circ} \mathrm{C}$ with a $S$. cerevisiae mutant strain pSAS050/DTY10-a2 containing a functional $\Delta^{12}$. desaturase from Caenorhabditis elegans. ${ }^{26}$ In earlier experiments, this particular $\Delta^{12}$. desaturating system has been demonstrated to utilize the exogenous oleic acids as substrate to produce linoleates in relatively good yields. ${ }^{26}$ This yeast strain is also incapable of endogenous fatty acid biosynthesis through a FAS mutation and therefore the chances of dilution of the deuterium labeled linoleic acid product is prevented. The fatty acid mixture containing biosynthetic methyl $\left[11-{ }^{2} \mathrm{H}_{1}\right]$-linoleates were fractionated by reverse-phase HPLC and the title compounds methyl (11S)- and $(11 R)-\left[11-{ }^{2} \mathrm{H}_{1}\right]-$ linoleates were obtained in $\sim 6 \%$ overall yield. GC-MS analysis of this material (Figure 2.28 and 2.29) revealed that a small amount of tetradecanoate was present as impurity. However, the presence of tetradecanoate didn't interfere in our targeted biosynthetic experiment as the $\Delta^{8,10}$-desaturase enzyme doesn't use this particular fatty acid as substrate. The selected ion monitoring chromatograms of the above GC profiles indicated the high deuterium content in the biosynthetic samples and demonstrated that both enantiomers were obtained nearly exclusively in monodeuterio form. 


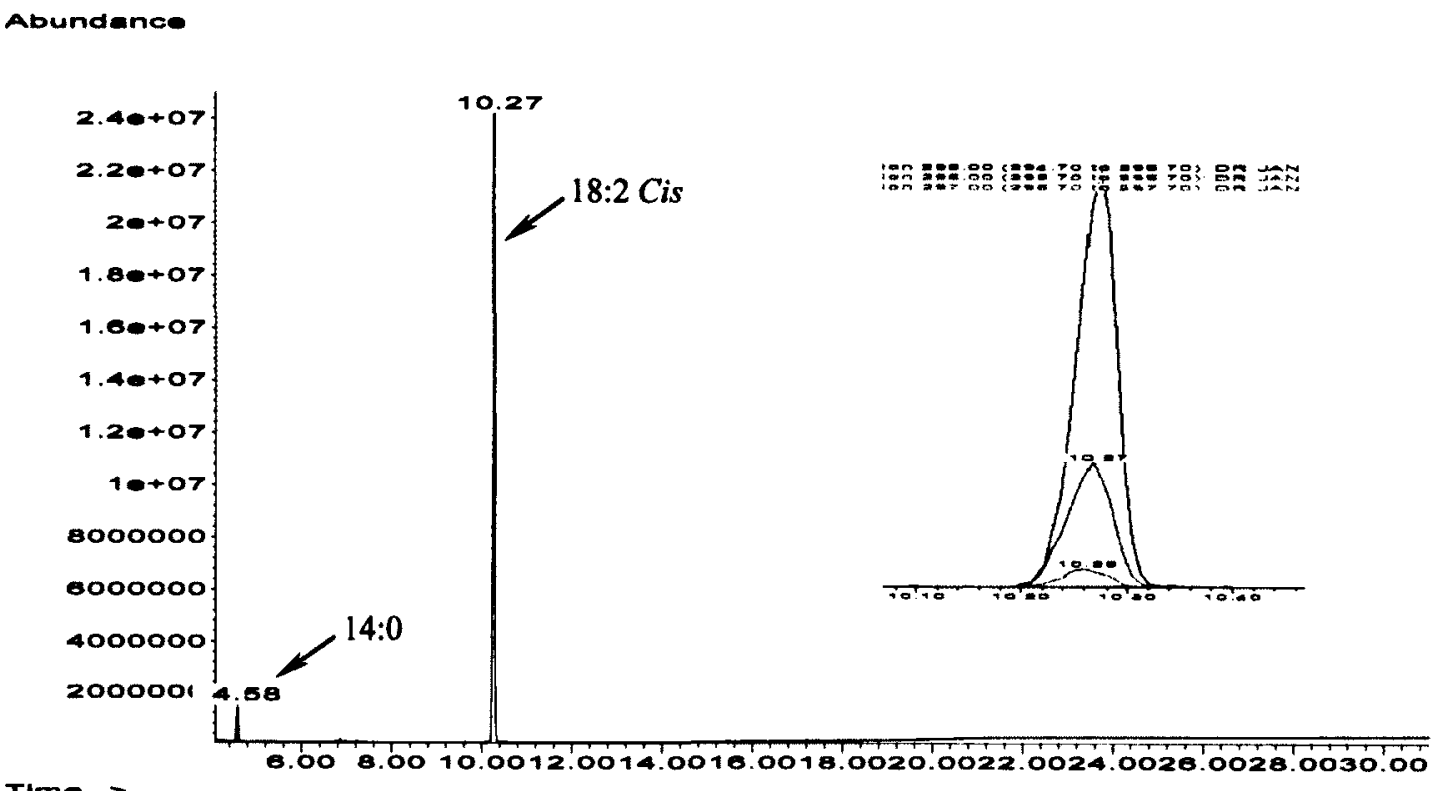

Figure 2.28. GC-MS of biosynthetic methyl $(11 S)-\left[11-{ }^{2} \mathrm{H}_{1}\right]-$-linoleate $2 \mathbf{a}$.

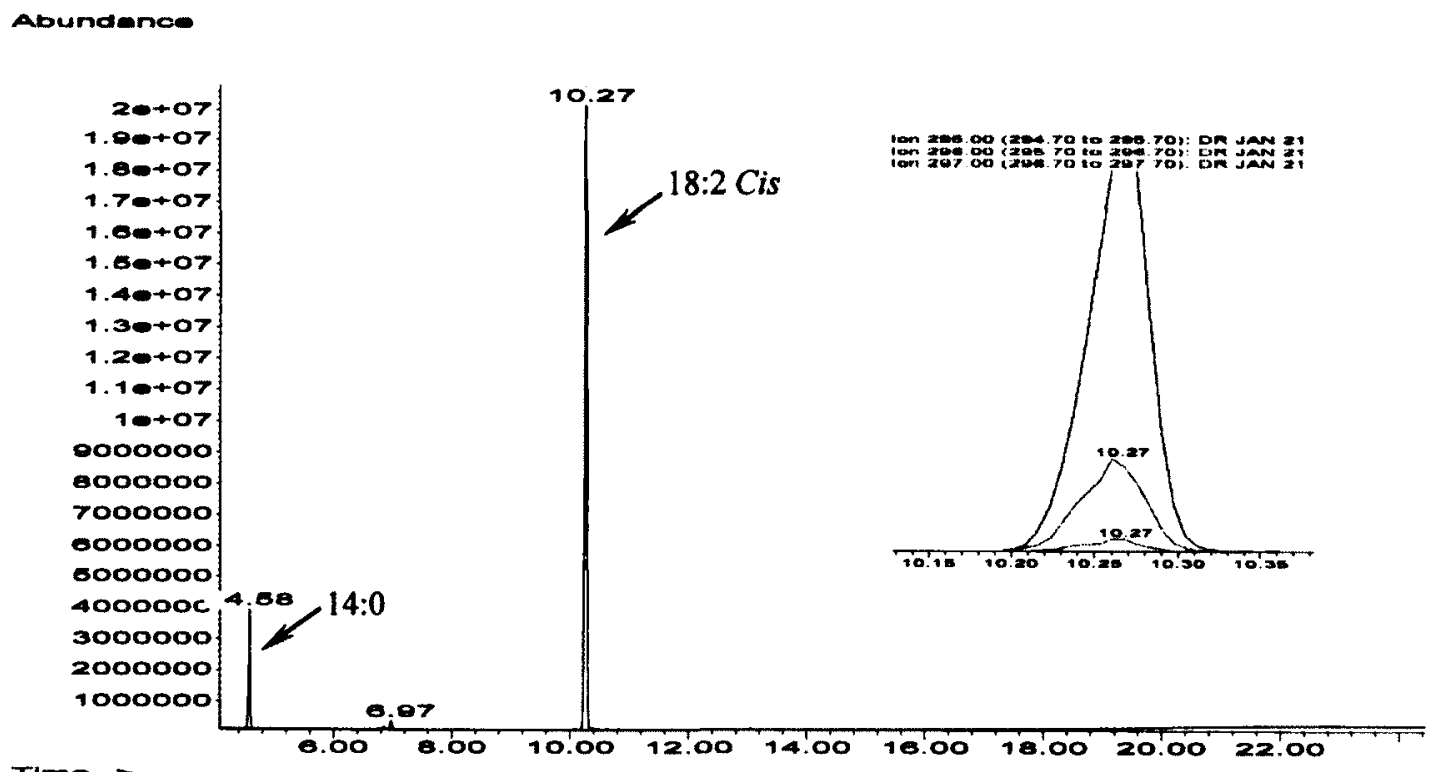

Figure 2.29. GC-MS of biosynthetic methyl $(11 R)-\left[11-{ }^{2} \mathrm{H}_{1}\right]$-linoleate $2 \mathbf{b}$.

The mass spectra of the corresponding GC peak of both enantiomers were found to be essentially identical with each other (Figure 2.30 and 2.31). Appearance of the molecular ion signal at $\mathrm{m} / \mathrm{z} 295\left([\mathrm{M}]^{+}\right)$in both isomers are in accord with the molecular formula 


\section{Abundance}

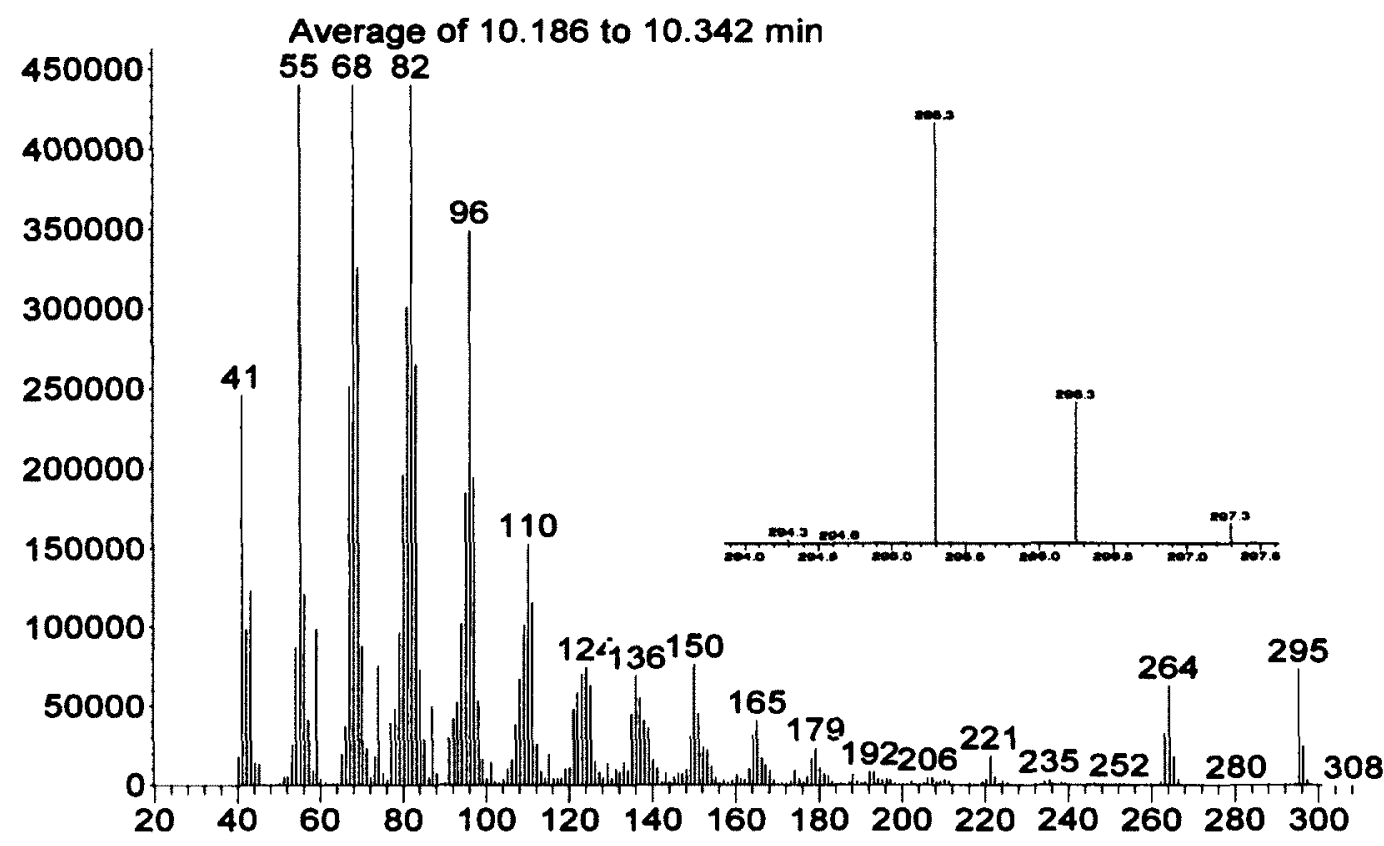

m/z-->

Figure 2.30. Mass spectrum of methyl $(11 S)-\left[11-{ }^{2} \mathrm{H}_{1}\right]$-linoleate 3a.

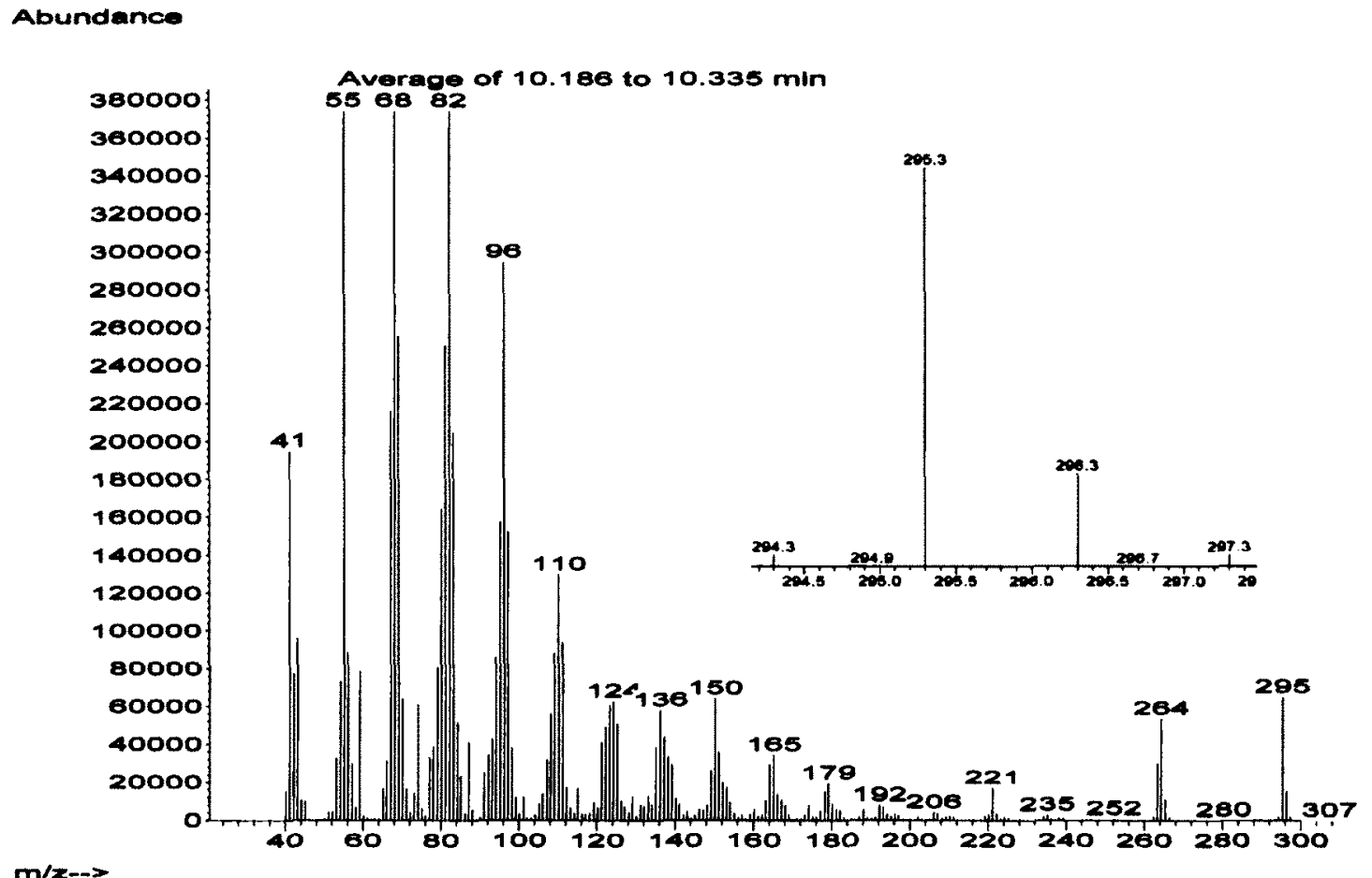

Figure 2.31. Mass spectrum of methyl $(11 R)-\left[11-{ }^{2} \mathrm{H}_{1}\right]$-linoleate $3 \mathrm{~b}$. 
$\mathrm{C}_{19} \mathrm{H}_{33} \mathrm{DO}_{2}$ and clearly demonstrated that the chiral deuterio linoleates consisted mainly as monodeuterated species. Measurement of the deuterium content in the biosynthetic samples $2 \mathrm{a}$ and $\mathbf{2 b}$ revealed the same isotopic purity as observed with the monodeuterio oleates $3 \mathbf{a}$ and $\mathbf{3 b}$.

\subsubsection{Attempted Synthesis of Chiral Methyl $\left[11-{ }^{2} \mathrm{H}_{1}\right]-$ Octadeca-9Z,12Z-Dienoates}

A synthesis of chiral methyl $\left[11-{ }^{2} \mathrm{H}_{1}\right]$-octadeca-9Z,12Z-dienoates $2 \mathrm{a}$ and $\mathbf{2 b}$ has been reported by Crombie and Heavers, ${ }^{173}$ and a modified version was attempted (Figure 2.32):

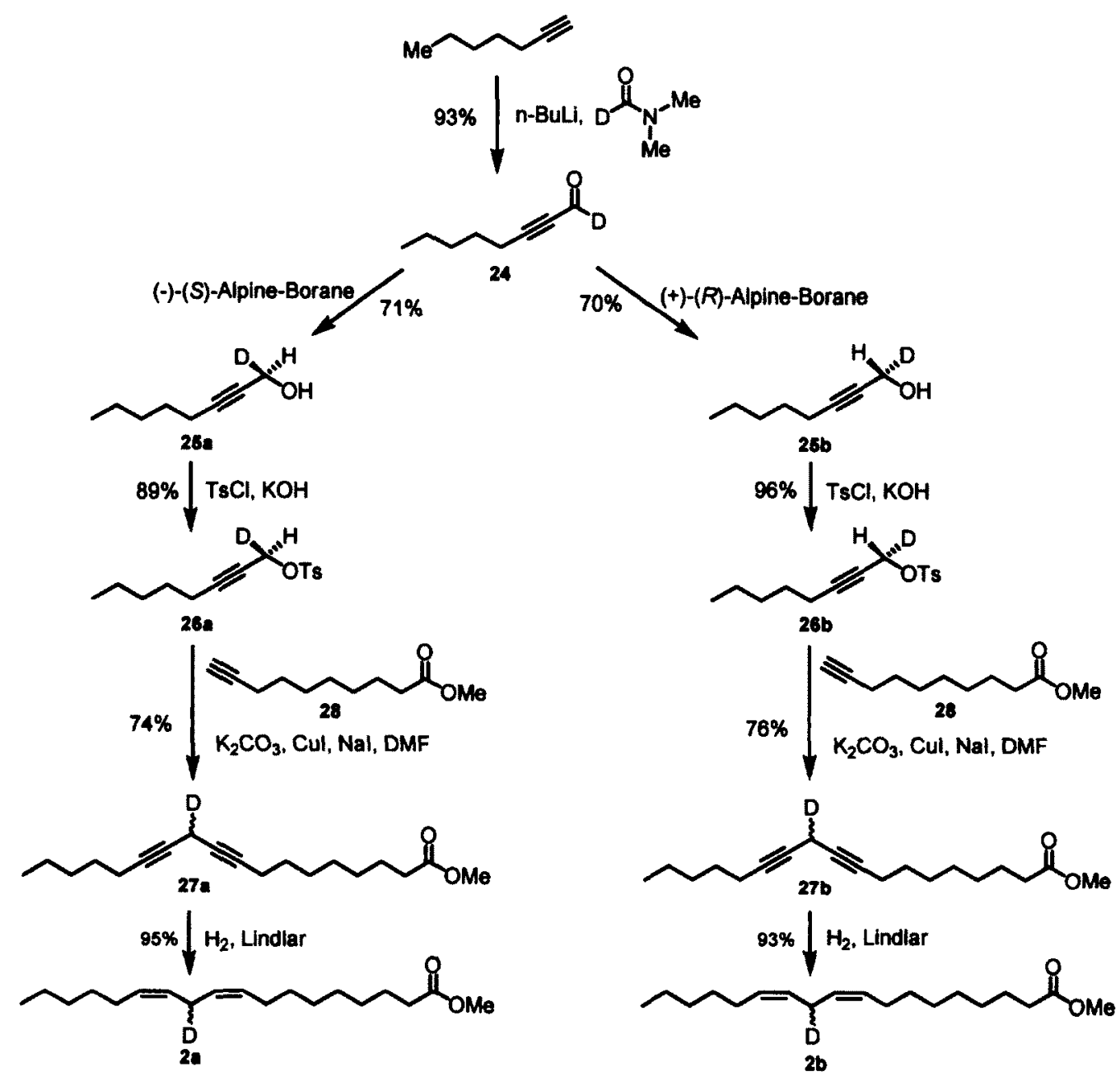

Figure 2.32. Scheme for the attempted synthesis of chiral methyl $\left[11-{ }^{2} \mathrm{H}_{1}\right]$-linoleates. 
The synthesis started with the preparation of one of the key intermediates $\left[1-{ }^{2} \mathrm{H}_{1}\right]-$ 2-octyn-1-al by deprotonation of 1-heptyne with $\mathrm{n}-\mathrm{BuLi}$, followed by addition of the carbanion to a solution of N,N-dimethylformamide- $d_{1}$ to give $24 .{ }^{198}$ This replaced the less efficient Crombie method featuring heptyne acylation with ethyl chloroformate in the presence of Grignard reagent $(\mathrm{EtMgBr}),{ }^{173}$ reduction of the ester with lithium aluminum deuteride and $\mathrm{MnO}_{2}$-mediated oxidation (Figure 2.33).

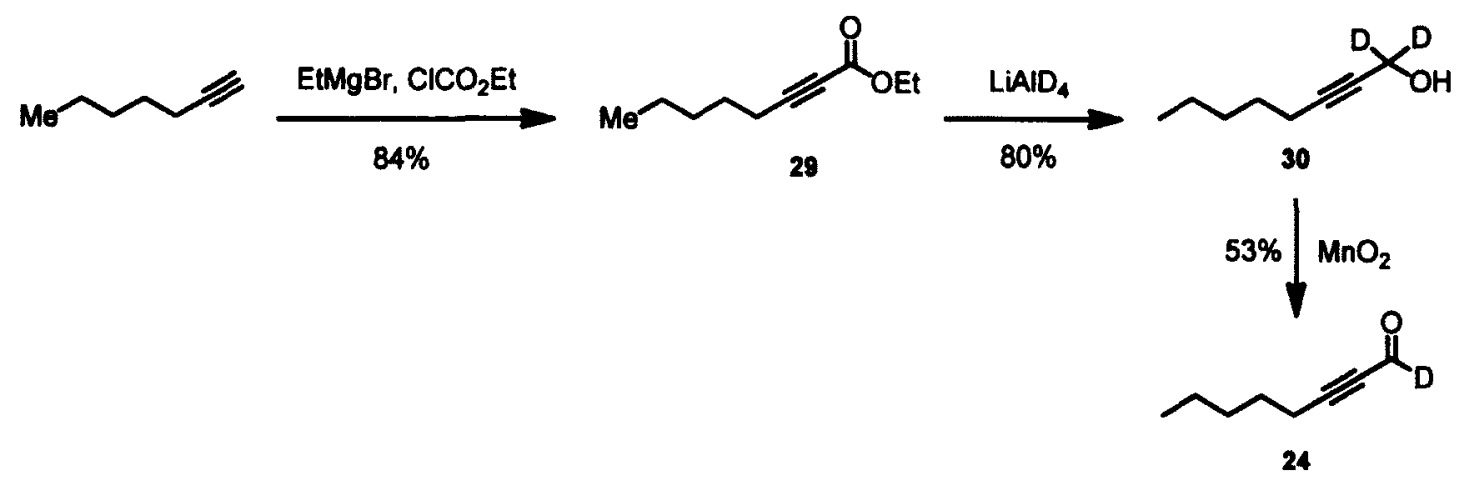

Figure 2.33. Scheme showing the synthesis of $\left[1-^{2} \mathrm{H}_{1}\right]-2$-octyn-1-al by Crombie et al. ${ }^{173}$

The deuterio propargyl aldehyde was then stereoselectively reduced to the corresponding chiral monodeuterated alcohols 25a and 25b using Alpine-Borane ${ }^{\oplus}$ reagents as described above. ${ }^{174,185,188}$ Stereochemical analysis of these products was carried out using Mosher's MTPA esterification method as described previously in Section 2.2.1.3. ${ }^{189}$ As shown in Figure 2.34, the residual $\mathrm{C}-1$ proton resonance in the ${ }^{1} \mathrm{H}$ NMR spectrum of the MTPA ester is broadened due to coupling with geminal deuterium and long range coupled to the methylene group at $\mathrm{C}-4\left({ }^{5} J_{\mathrm{HH}}=\sim 1.9 \mathrm{~Hz}\right)$. It is clear from this analysis that each enantiomer is obtained in high enantiomeric excess and with the expected absolute configuration based on literature precedent. ${ }^{173}$ 


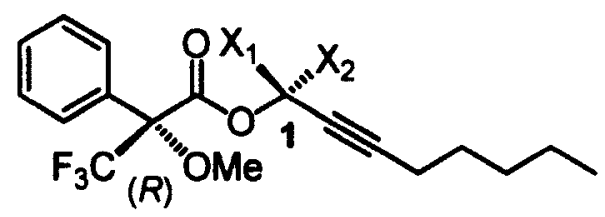

$$
\begin{aligned}
& (1 R)-\left[\mathrm{X}_{1}=\mathrm{H}, \mathrm{X}_{2}=\mathrm{D}\right] ; \delta 4.941(t, J=1.7 \mathrm{~Hz} ., 1 \mathrm{H}) \\
& (1 S)-\left[\mathrm{X}_{1}=\mathrm{D}, \mathrm{X}_{2}=\mathrm{H}\right] ; \delta 4.823(t, J=2.0 \mathrm{~Hz}, 1 \mathrm{H})
\end{aligned}
$$

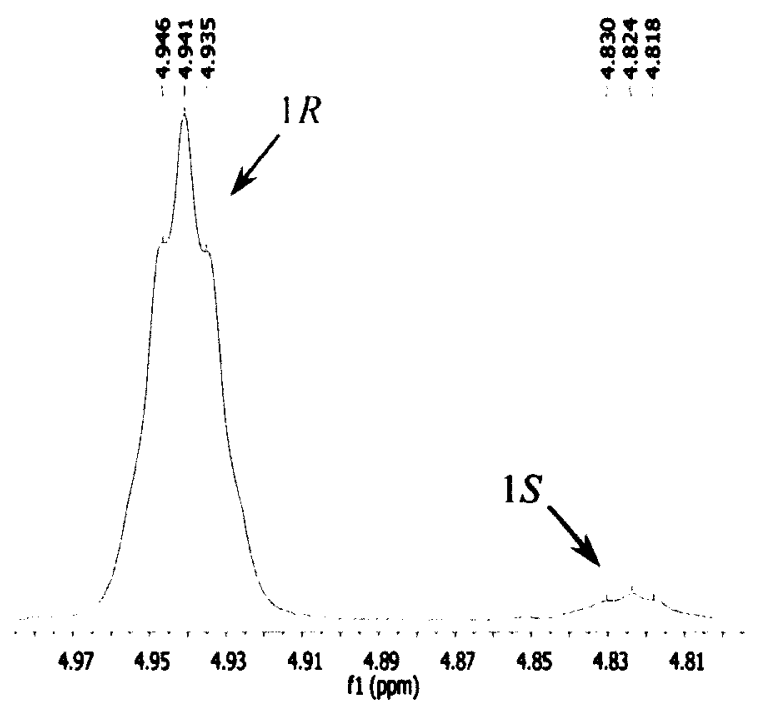

( $R$ )-MTPA-O-CHD- : 95

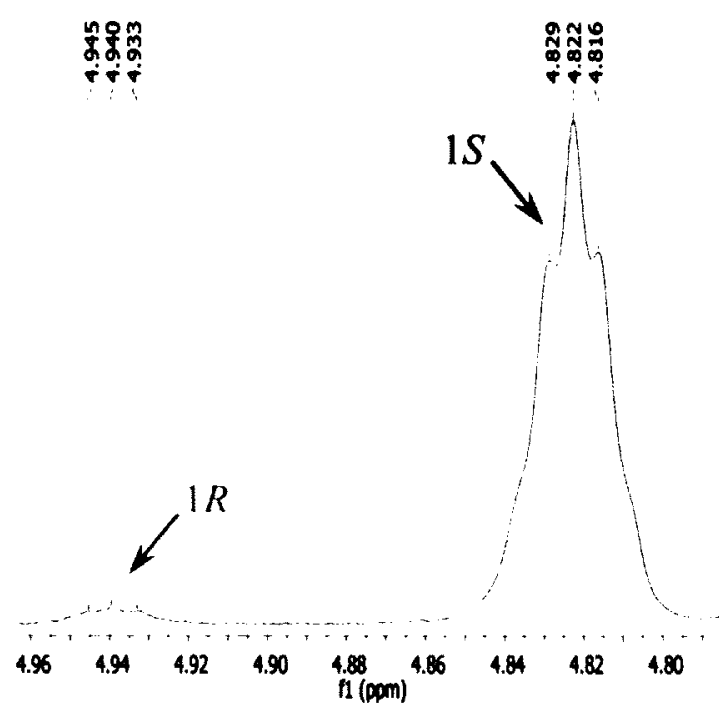

( $(R)$-MTPA- O-CHD- : 3
(1S)-MTPA- O-CHD- : 97

Figure 2.34. Stereochemical analysis of chiral- $d_{1}$-alkyn-1-ols using Mosher's esterification method.

Several attempts to couple the tosylate of the chiral monodeuterated alkynols with the Grignard reagent derived from $\mathrm{H}-\mathrm{C} \equiv \mathrm{C}-\left(\mathrm{CH}_{2}\right)_{7}-\mathrm{CO}_{2} \mathrm{H}$ in the presence of Copper(I) bromide dimethyl sulfide complex, as described by Crombie, were found to be very low yielding processes. ${ }^{173}$ Variations in the amounts of Grignard reagent, tosylate, CuBr-DMS complex or temperature did not lead to significant improvement. Finally, it was decided that the synthesis would be completed ${ }^{175-178,190}$ (Figure 2.32) to generate what was in all likelihood racemic $11-d_{1}$-linoleate due to use of sodium iodide catalysis in the coupling 
step. This material could be used to evaluate the primary deuterium kinetic isotopic effect at $\mathrm{C}-11$ in a manner that was complementary to the methodology employed in a previous work (See Section 1.4.2). ${ }^{158}$ Samples of $(1 R)$ - and $(1 S)-\left[1-{ }^{2} \mathrm{H}_{1}\right]$-2-octyn-1-ol-derived methyl $\left[11-{ }^{2} \mathrm{H}_{1}\right]$-linoleates were obtained as described in Figure 2.32. The proposed structures, chemical and isotopic purity of these compounds were confirmed by comparing the GC-MS (Figure 2.35), ${ }^{1} \mathrm{H}$ and ${ }^{13} \mathrm{C}$ NMR (Figure 2.36 AB), and IR (Figure 2.37 ) spectra with that of non-labeled linoleate ${ }^{192-194}$ as well as with other isotopically substituted linoleates in this series. Representative spectra are given below:

Abundence

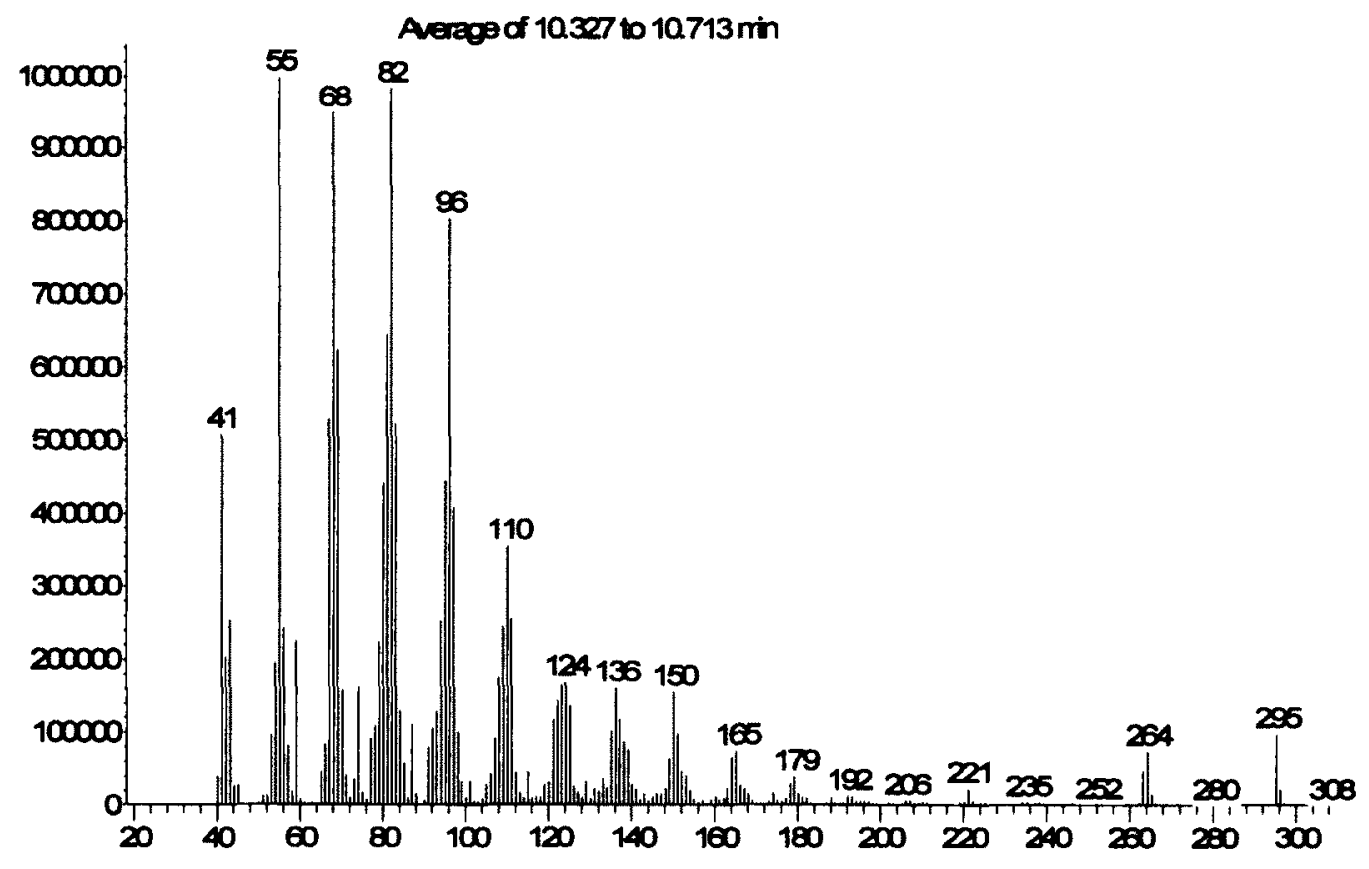

$m z \rightarrow$

Figure 2.35. Mass spectrum of $(1 R)-\left[1-{ }^{2} \mathrm{H}_{1}\right]-2-\mathrm{octyn}-1$-ol-derived methyl $\left[11-{ }^{2} \mathrm{H}_{1}\right]-$ linoleate. 

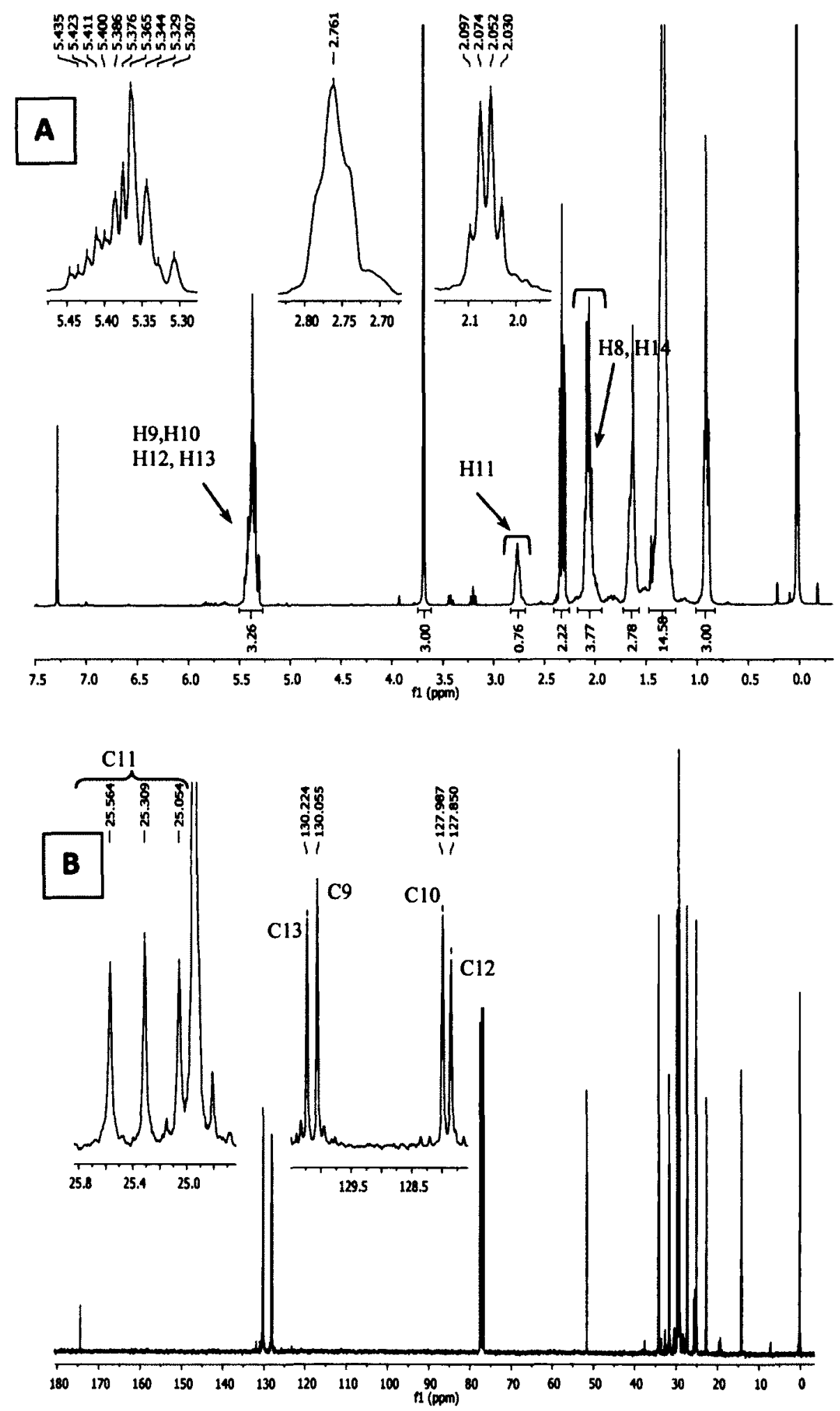

Figure 2.36 A: ${ }^{1} \mathrm{H}$ NMR and B: ${ }^{13} \mathrm{C}$ NMR of methyl $\left[11-{ }^{2} \mathrm{H}_{1}\right]$-linoleate. 


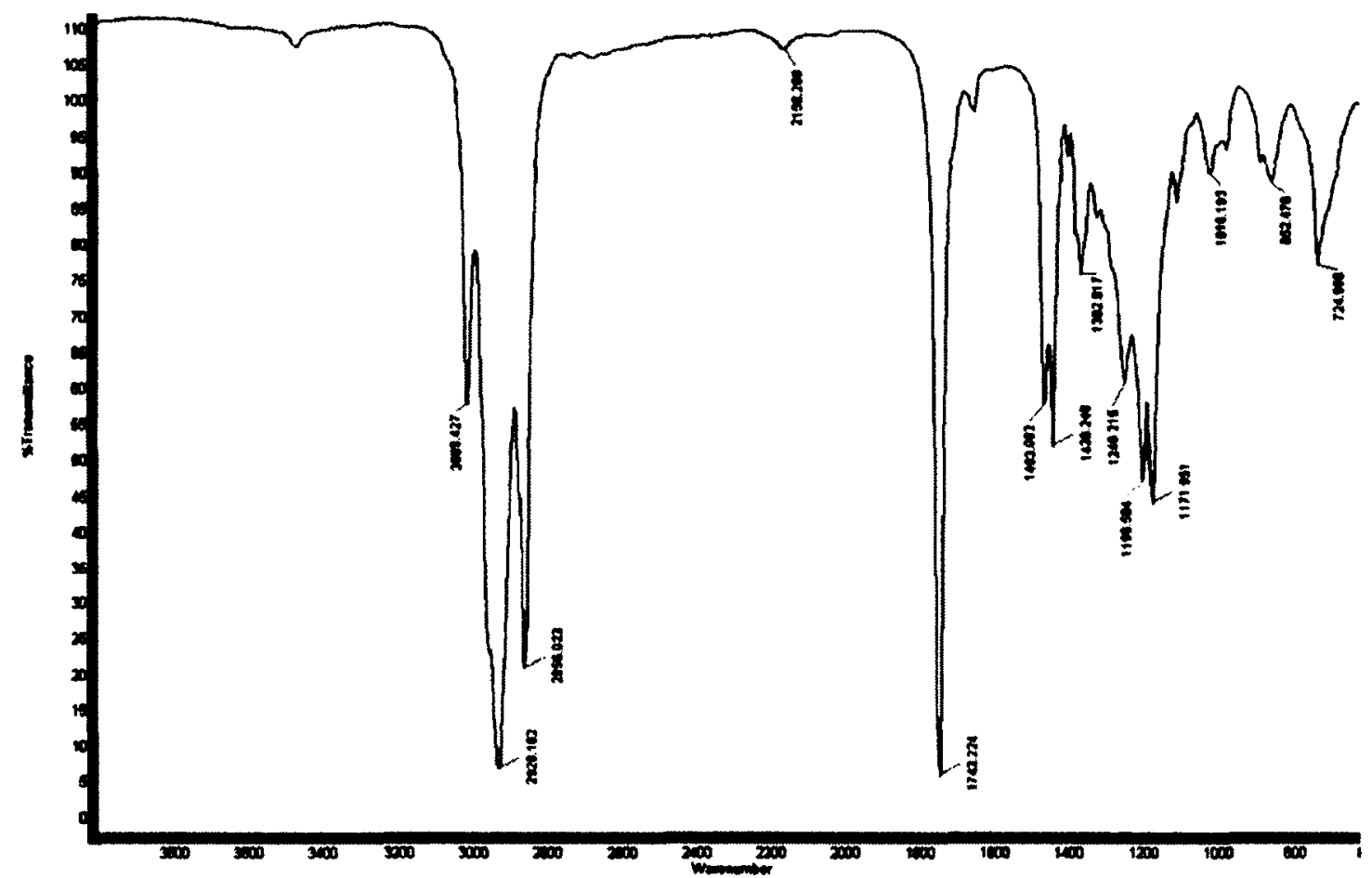

Figure 2.37. IR spectrum of $(1 R)-\left[1-{ }^{2} \mathrm{H}_{1}\right]-2$-octyn-1-ol-derived methyl $\left[11-{ }^{2} \mathrm{H}_{1}\right]$-linoleate. The C-D stretch due to $C_{11} \mathrm{HD}$ at $2158 \mathrm{~cm}^{-1}$ is blue shifted $\left(13 \mathrm{~cm}^{-1}\right)$ relative to that of methyl $(8 R)-\left[8-{ }^{2} \mathrm{H}_{1}\right]$-linoleate.

\subsubsection{Incubation Experiments Using Chiral Methyl $\left[8{ }^{2} \mathrm{H}_{1}\right]-$ and $\left[11-{ }^{2} \mathrm{H}_{1}\right]-$ Linoleates}

\subsubsection{Trial Incubation of $\mathbf{d}_{0}$-Linoleate}

Prior to the incubation of the synthetic chiral monodeuterio linoleic acids, the efficiency of calendate formation from $\mathrm{d}_{0}$-linoleate using cultures of a $S$. cerevisiae mutant expressing the Calendula officinalis $\Delta^{8,10}$-desaturase (Fac2) gene was probed in a preliminary trial experiment. Thus ca. $0.5 \mathrm{~mL}$ of sterilized linoleic acid solution in $10 \%$ Tergitol $(10 \mathrm{mg} / \mathrm{mL})$ was incubated with a $50 \mathrm{~mL}$ growing shake culture $(200 \mathrm{rpm})$ of the $\mathrm{pYJ} / \mathrm{DTY} 10 \mathrm{a} 2$ strain of $S$. cerevisiae at $20^{\circ} \mathrm{C}$ for 3 days and another 3 days at $15^{\circ} \mathrm{C}$. 
These conditions had previously been shown to optimize substrate conversion rates. ${ }^{158} \mathrm{~A}$ corresponding control experiment using $S$. cerevisiae (Fac2- plasmid) was also run. The cellular fatty acid fractions were isolated from the centrifuged yeast cells via a standard hydrolysis $\left(10 \% \mathrm{w} / \mathrm{v} \mathrm{KOH} / \mathrm{MeOH}\right.$ at $80{ }^{\circ} \mathrm{C}$ for $\left.2 \mathrm{~h}\right) /$ methylation $\left(\mathrm{CH}_{2} \mathrm{~N}_{2}\right)$ procedure known not to decompose conjugated fatty ester ${ }^{158}$ and analyzed by GC-MS (Figure 2.38). Comparison of the fatty acid methyl ester (FAME) profile from S. cerevisiae (Fac2plasmid) (data not shown) and the pYJ/DTY10a2 strain of S. cerevisiae (Figure 2.38) revealed that only in the latter culture, was exogenously added linoleic acid converted into the product calendic acid ( $0.6 \%$ of the total cellular fatty acids). This result corresponds well with that observed previously. ${ }^{158}$ The product was identified on the basis of retention times using a reference standard mixture that distinguishes between the triene isomers. ${ }^{158}$ The mass spectrum of the GC peak assigned to methyl calendate (Figure 2.39) revealed the presence of the molecular ion at $\mathrm{m} / \mathrm{z} 292\left([\mathrm{M}]^{+}\right)$and the other fragment ions at $\mathrm{m} / \mathrm{z} 261\left(\left[\mathrm{M}-\mathrm{OCH}_{3}\right]^{+}\right), 163\left(\left[\mathrm{Me}\left(\mathrm{CH}_{2}\right)_{4} \mathrm{CH}=\mathrm{CH}-\mathrm{CH}=\mathrm{CH}-\mathrm{CH}=\mathrm{CH}-\right.\right.$ $\left.\left.\mathrm{CH}_{2}\right]^{+}\right), 149\left(\left[\mathrm{Me}\left(\mathrm{CH}_{2}\right)_{4} \mathrm{CH}=\mathrm{CH}-\mathrm{CH}=\mathrm{CH}-\mathrm{CH}=\mathrm{CH}\right]^{+}\right)$, which are in accordance with the assigned structure and similar to that reported before. ${ }^{63,158}$ Silylation ${ }^{187}$ of a portion of the FAMEs did not reveal hydroxylated product formation as a low-level "error" compound during 1,4-desaturation. A similar examination of the methylated $\mathrm{CH}_{2} \mathrm{Cl}_{2}$ extract of the culture medium did not uncover any hydroxylated fatty acids. 
Abundance

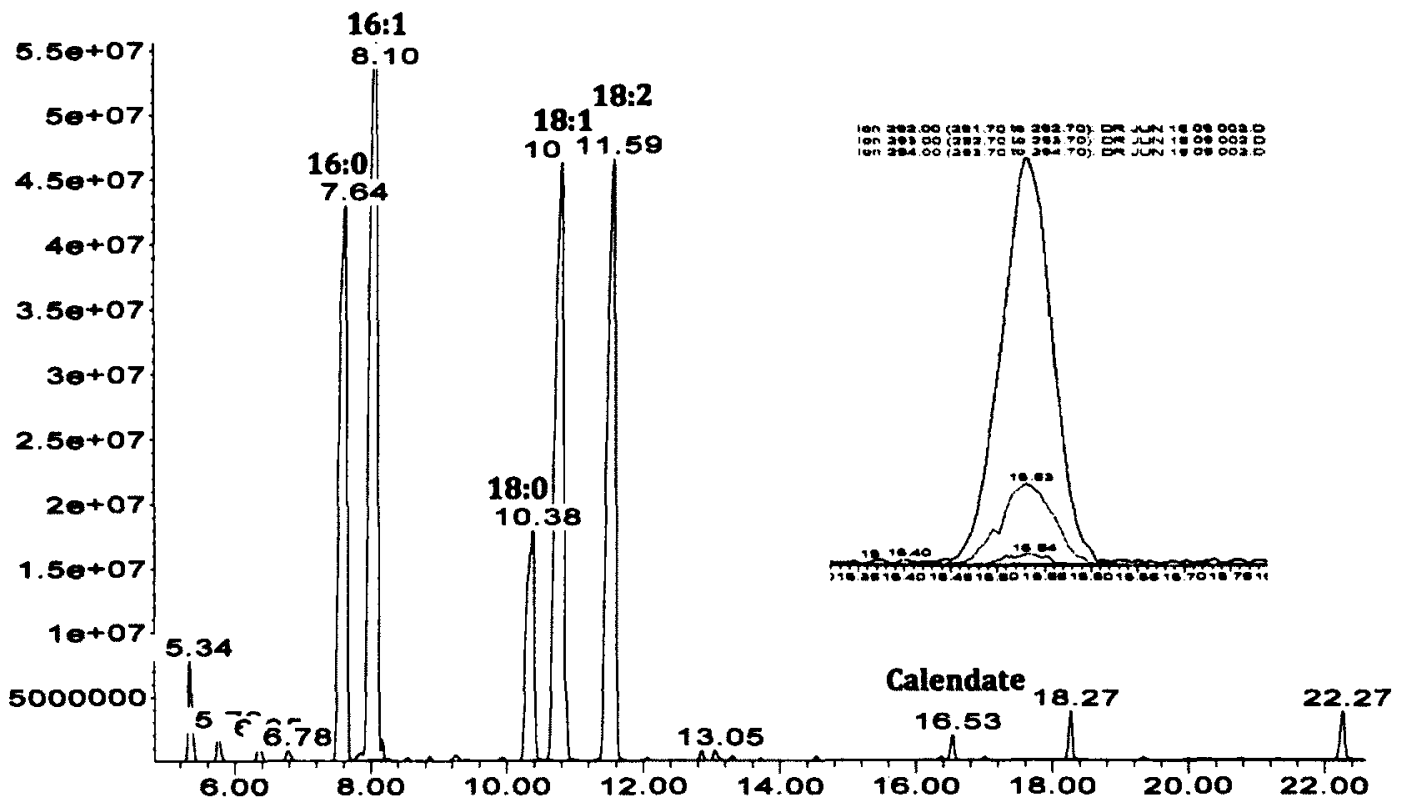

Time-->

Figure 2.38. The cellular fatty acid profile of $S$. cerevisiae grown in a medium supplemented with linoleic acid.

Abundance

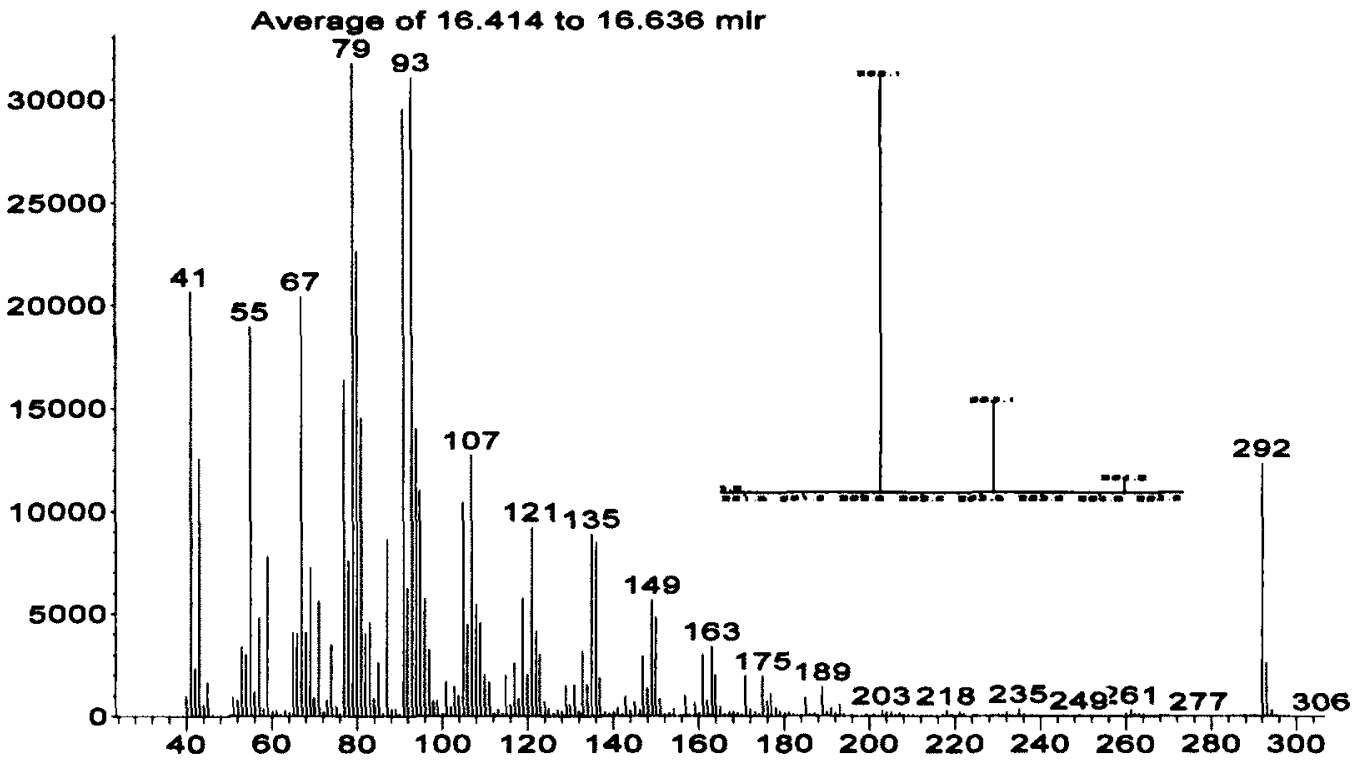

$m / 2->$

Figure 2.39. Mass spectrum of biosynthetic methyl calendate derived from methyl linoleate. 


\subsubsection{Incubation of Chiral $\left[8-{ }^{2} \mathrm{H}_{1}\right]-$ and $\left[11-{ }^{2} \mathrm{H}_{1}\right]-$ Linoleates}

To determine the enantioselectivity of the $\Delta^{8,10}$-desaturase enzyme, the saponified methyl esters of chiral $\left[8-{ }^{2} \mathrm{H}_{l}\right]$-linoleates $\mathbf{1 a}$ and $\mathbf{1 b}$ and chiral $\left[11-{ }^{2} \mathrm{H}_{1}\right]$-linoleates $2 \mathbf{a}$ and 2b (5 mg each) were administered separately, in triplicate, to growing cultures of pYJ/DTY10a2 strain of $S$. cerevisiae $(50 \mathrm{~mL})$ using the conditions identical to that of the trial experiment. The cellular fatty acid fractions were subsequently isolated by hydrolysis/methylation and analyzed by GC-MS to determine the fate of the deuterium label in each case. The $d_{1} / d_{0}$ ratio of the cellular linoleic acid fractions and the $d_{1} / d_{0}$ ratio of the administered compounds were similar (data not shown). This result was not unexpected since $S$. cerevisiae is not able to biosynthesize linoleic acid.

Mass spectral analysis of the calendate product derived from $(8 R)-\left[8-{ }^{2} \mathrm{H}_{1}\right]$-linoleic acid indicated that the most abundant molecular ion appeared at $\mathrm{m} / \mathrm{z} 292$ (Figure 2.40); this represents the overall loss of deuterium during the enzymatic reaction.

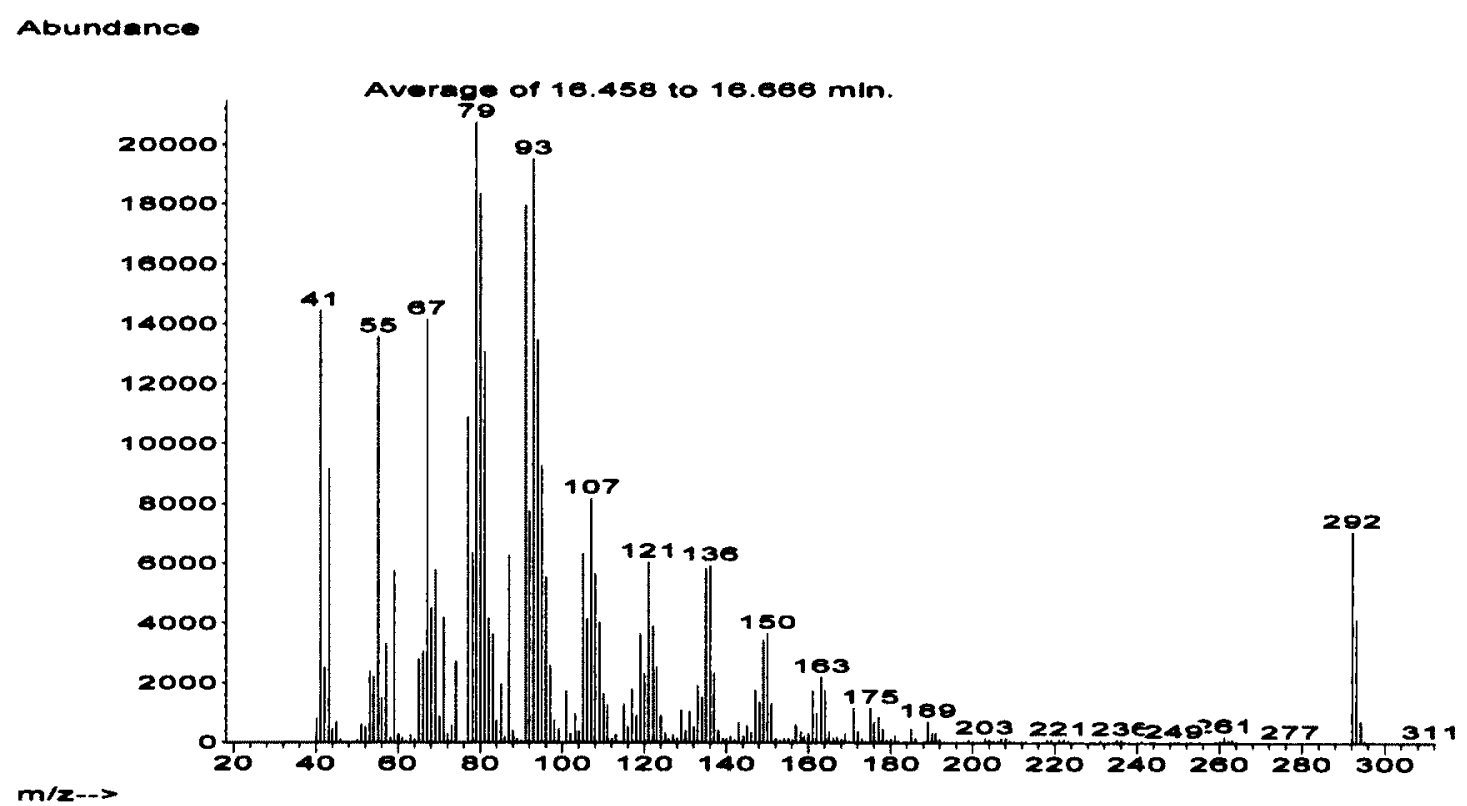

Figure 2.40. Representative mass spectrum of biosynthetic methyl calendate derived from methyl $(8 R)-\left[8-{ }^{2} \mathrm{H}_{1}\right]$-linoleate $1 \mathbf{a}$. 
In contrast, the mass spectrum of the calendate product derived from $(8 S)-\left[8-{ }^{2} \mathrm{H}_{1}\right]$-linoleic acid featured the most abundant molecular ion at $\mathrm{m} / \mathrm{z} 293$ (Figure 2.41); this represents the overall retention of deuterium from the pro $R$ position during the enzymatic reaction.

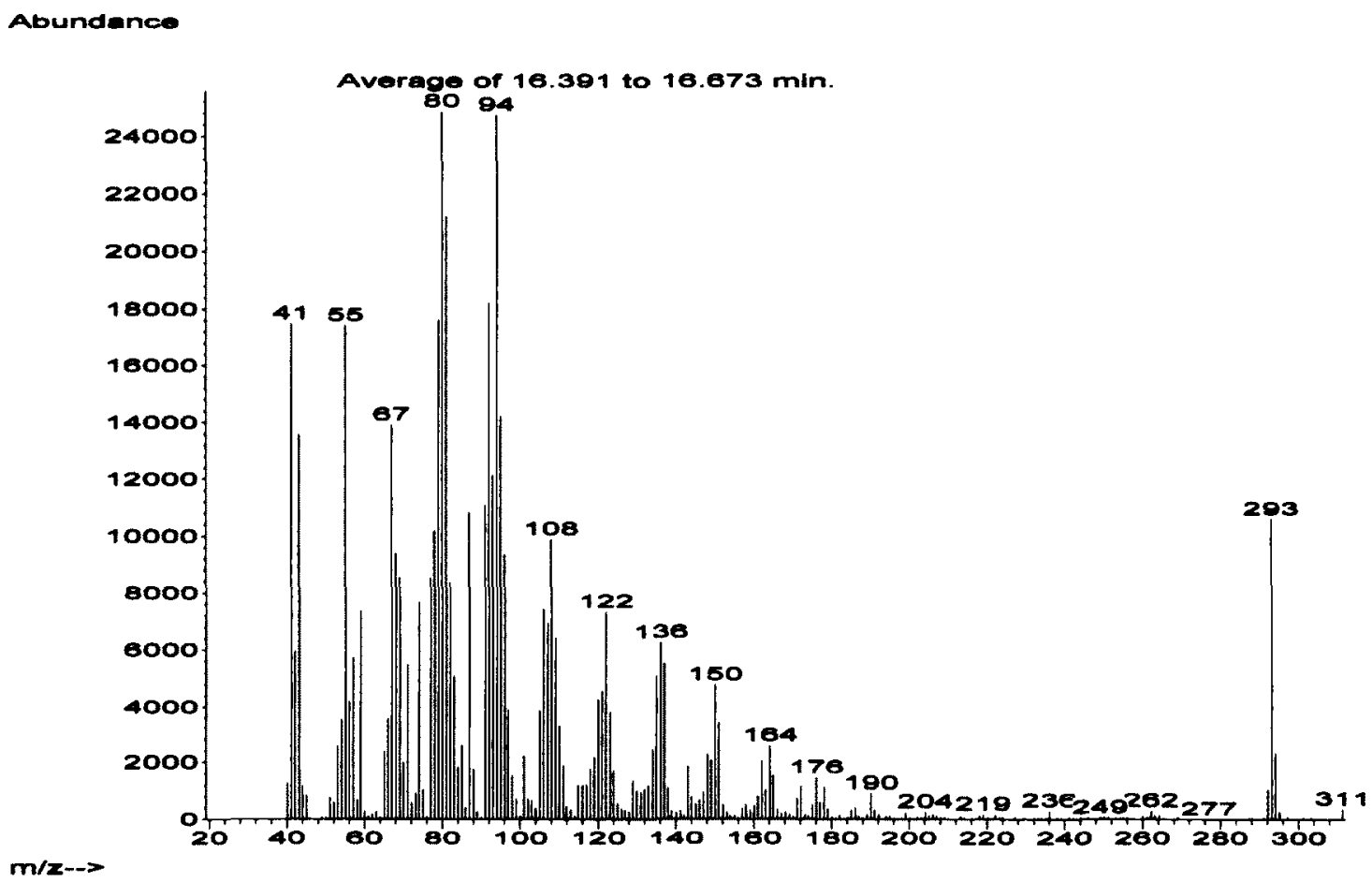

Figure 2.41. Representative mass spectrum of biosynthetic methyl calendate derived from methyl $(8 S)-\left[8-{ }^{2} \mathrm{H}_{1}\right]$-linoleate $\mathbf{1 b}$.

A similar pattern of deuterium incorporation was observed for the calendate derived from $(11 S)-\left[11-{ }^{2} \mathrm{H}_{1}\right]$-linoleic acid $2 \mathrm{a}$ (loss of label) and $(11 R)-\left[11-{ }^{2} \mathrm{H}_{1}\right]$-linoleic acid $2 \mathrm{~b}$ (retention of label) as indicated by the mass spectra shown in Figure 2.42 and Figure 2.43 , respectively. 


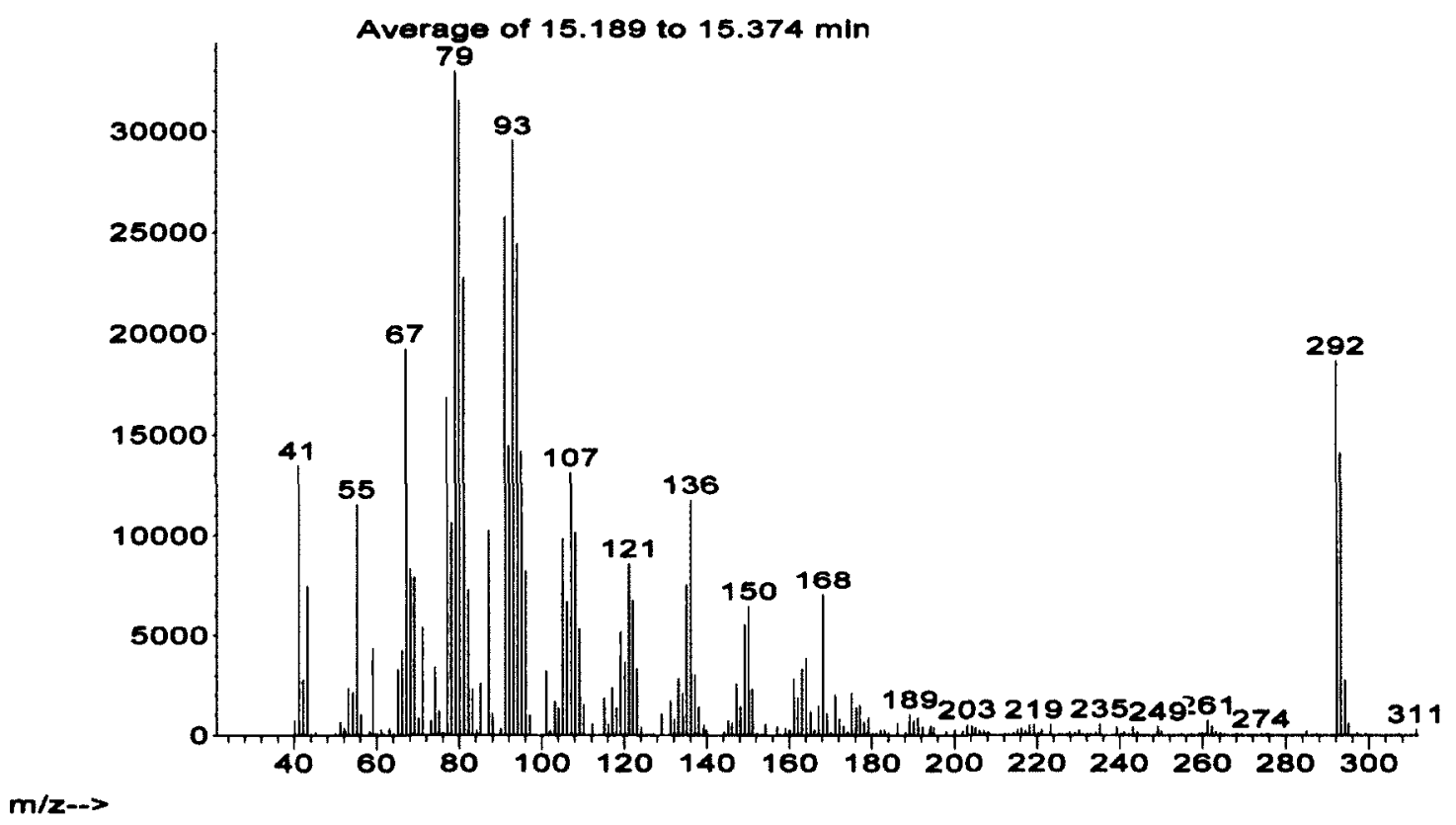

Figure 2.42. Representative mass spectrum of biosynthetic methyl calendate derived from methyl (11S)-[11-2 $\left.\mathrm{H}_{1}\right]$-linoleate $\mathbf{2 a}$.

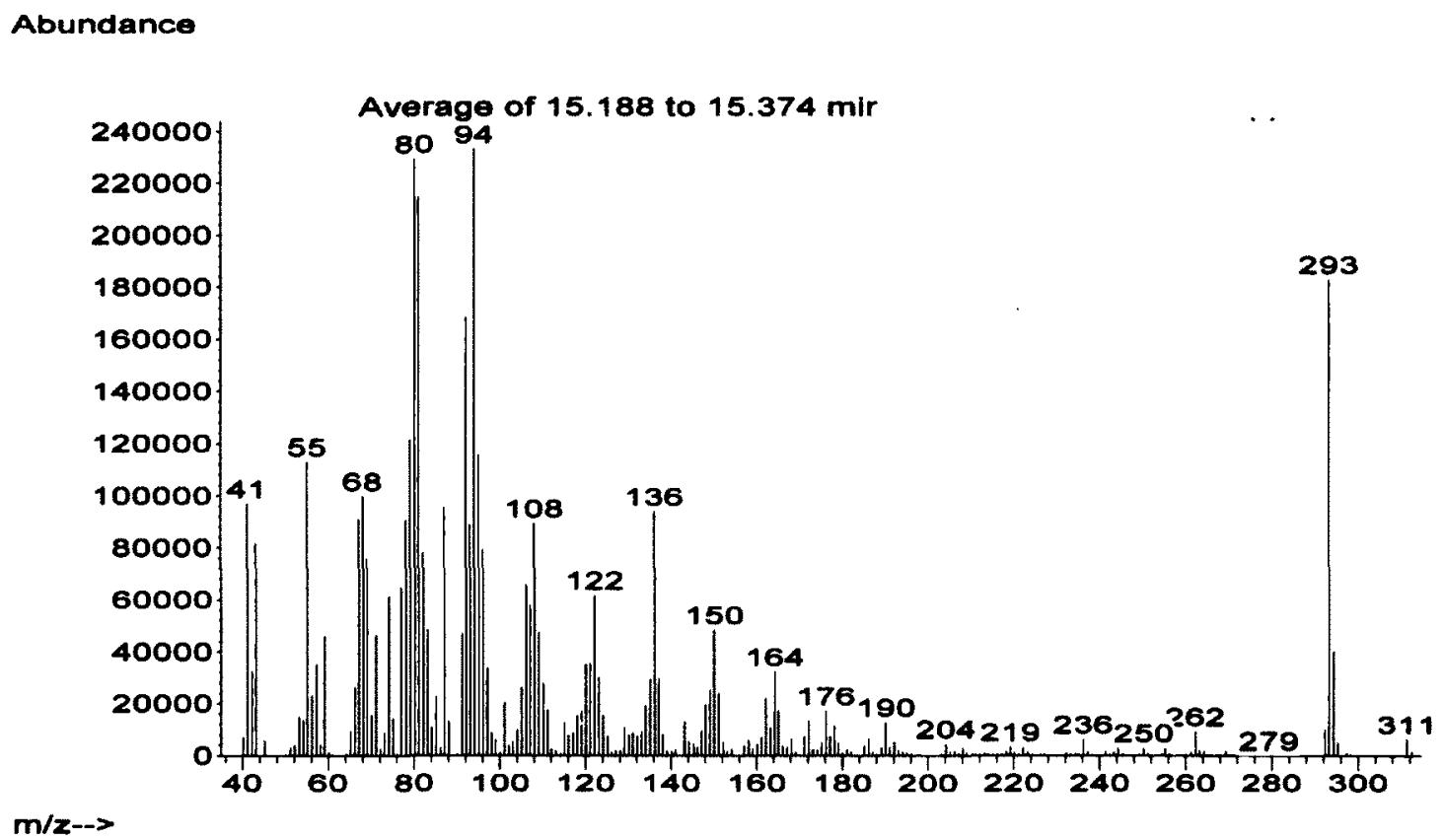

Figure 2.43. Representative mass spectrum of biosynthetic methyl calendate derived from methyl (11R)-[11-2 $\left.\mathrm{H}_{1}\right]$-linoleate $2 \mathbf{b}$. 


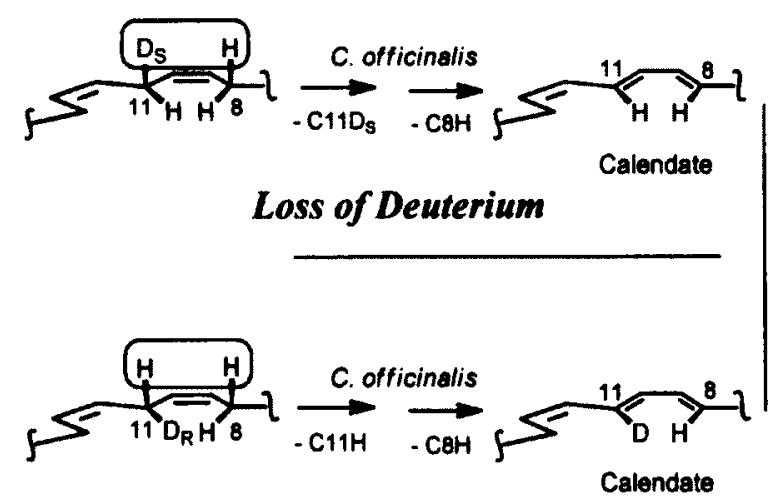

Retention of Deuterium

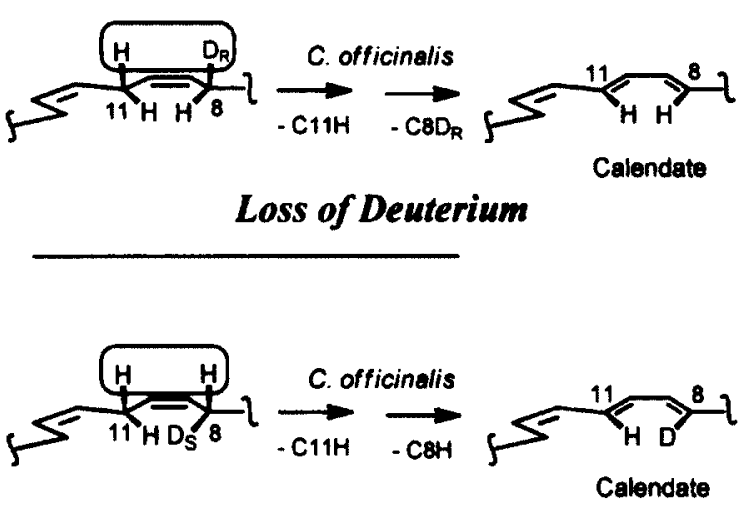

Retention of Deuterium

Figure 2.44 Observed results for calendate formation from stereospecifically deuterated linoleates. Note CIP priority rules dictate that the deuterium atoms that are removed from the $C-8$ and $C-11$ positions are labeled $D_{R}$ and $D_{S}$ respectively.

This result tells us that during calendate biosynthesis, the $\mathrm{C}-8$ and $\mathrm{C}-11$ hydrogens that are removed are topologically equivalent to the hydrogens removed during formation of linoleic acid by the parent $\Delta^{12}$-desaturase $(F A D 2)^{1,112,140}$ as shown by comparing Figure 2.44 with Figure 2.1. This interpretation assumes that the conformations of substrate in each case are similar. Interestingly, this result also correlates well with the observed enantioselectivity for an insect $\Delta^{10,12}$-desaturase that converts a cis-11 ene to the corresponding $(E, E)$-10,12-conjugated diene (Chapter 1, Figure 1.29). ${ }^{40}$ This suggests that the overall contours of the binding site for all these enzymes are similar.

The quantitation of deuterium loss vs. retention was accomplished by analyzing molecular ion clusters of the biosynthetic calendate products (Figures $2.40-2.43$ ) and carrying out the appropriate corrections of ion intensities at $\mathrm{m} / \mathrm{z} 293$ due to $\mathrm{M}+1$ contribution (ca. $21.5 \%$ ) from $d_{0}$ product $(\mathrm{m} / \mathrm{z} 292)$. The $M+1$ correction factors were determined experimentally using $d_{0}$ calendate and compared favourably with the theoretical values. The results of this analysis are given in Table 2.1. It is assumed that 
the enantiomeric purity of the chiral starting materials cannot be better than $90 \%$ ee (see above) and is probably somewhat less due to the lack of perfect stereospecificity in the acetylenation step. This can account for the presence of $5-10 \%$ of $\mathrm{d}_{0}$ product that is obtained in cases where deuterium is retained overall (Experiment \# 2,4).

Table 2.1. Isotopic content of stereospecifically labeled monodeuterated linoleates and $\Delta^{8,10}$-desaturated products (calendate).

\begin{tabular}{|c|c|c|c|c|c|}
\hline \multirow{2}{*}{$\begin{array}{c}\text { Experiment } \\
\#\end{array}$} & \multirow[t]{2}{*}{ Compound } & \multicolumn{2}{|c|}{ Substrates $^{a}$} & \multicolumn{2}{|c|}{ Products $^{a}$} \\
\hline & & $\% \mathrm{~d}_{0}$ & $\% \mathrm{~d}_{1}$ & $\% \mathrm{~d}_{0}$ & $\% d_{1}$ \\
\hline 1 & $(8 R)-\left[8-{ }^{2} \mathrm{H}_{1}\right]-\mathrm{LA}$ & 1.2 & 98.8 & 73.2 & 26.8 \\
\hline 2 & $(8 S)-\left[8-{ }^{2} \mathrm{H}_{1}\right]-\mathrm{LA}$ & 1.1 & 98.9 & 9.6 & 90.4 \\
\hline 3 & $(11 S)-\left[11-{ }^{2} \mathrm{H}_{1}\right]-\mathrm{LA}$ & 0.8 & 99.2 & 61.4 & 38.6 \\
\hline 4 & $(11 R)-\left[11-{ }^{2} \mathrm{H}_{1}\right]-\mathrm{LA}$ & 2.5 & 97.5 & 5.1 & 94.9 \\
\hline
\end{tabular}

\footnotetext{
${ }^{a}$ Each type of chiral incubation was carried out three times and the \% isotopic content of each species is given as an average value of three independent GC-MS runs. The average standard deviation is \pm 0.4 for each species expressed as a percentage.
}

For the cases where deuterium is mainly lost (Exp. \#1,3), the amount of $d_{1}$ product is comparatively much higher $(27-40 \%)$. For the $(11 S)-\mathrm{d}_{1}$-substrate, the apparent reduced stereoselectivity can be accounted for in terms of the known large primary deuterium kinetic isotope effect associated with $\mathrm{C} 11-\mathrm{H}_{S}$ bond cleavage. ${ }^{158}$ Consequently, the $(11 R)-\mathrm{d}_{1}$-contaminant in this sample would react substantially faster than the $(11 S)-\mathrm{d}_{1}$-substrate and lead to enhanced $\% \mathrm{~d}_{1}$ product content at low conversion rates. Since it is known that $\mathrm{C}-8$ bond rupture proceeds without a substantial KIE, the 
appearance of substantial amounts of $\mathrm{d}_{1}$-product in the $(8 R)-\left[8-{ }^{2} \mathrm{H}_{1}\right]$ incubation is puzzling but may be due to an anomalously low substrate $\%$ ee.

\subsubsection{Incubation of Racemic $\left[11-{ }^{2} \mathrm{H}_{1}\right]$-Linoleates}

An independent assessment of the intermolecular primary deuterium kinetic isotope effect for $\mathrm{Cl1}-\mathrm{H}_{\mathrm{S}}$ bond cleavage became possible in serendipitous fashion due to the availability of racemic methyl $\left[11-{ }^{2} \mathrm{H}_{1}\right]$-linoleate (See above). Thus $(1 R)-\left[1-{ }^{2} \mathrm{H}_{1}\right]-2-$ octyn-1-ol-derived- and $(1 S)-\left[1-{ }^{2} \mathrm{H}_{1}\right]-2$-octyn-1-ol-derived methyl $\left[11^{2} \mathrm{H}_{1}\right]$-linoleate samples (5 mg each) were incubated, in duplicate, as a solution in $10 \%$ Tergitol $(10 \mathrm{mg} / \mathrm{mL})$ with a growing culture of pYJ/DTY10a2 strain of $S$. cerevisiae $(50 \mathrm{ml})$ expressing the Fac2 gene under the growth conditions as previously described. The yeast cultures were grown up to the normal extent following which the cells were harvested by centrifugation and hydrolyzed by $\mathrm{KOH}$ in methanol at $80^{\circ} \mathrm{C}$ for $2 \mathrm{~h}$ to recover the cellular fatty acid fraction that was subsequently methylated for the GC-MS analysis. Mass spectral analyses of the GC peak assigned to product calendate derived from all samples of 11-d $d_{1}$ linoleate were essentially the same within experimental error: $78.2 \% \pm 1.0 \mathrm{~d}_{1}$ and $21.8 \%$ $\pm 1.0 \mathrm{~d}_{0}$ (Table 2.2). This indicated that the samples were stereochemically identical i.e. racemic. 
Table 2.2. Intermolecular isotopic discrimination by the Calendula officinalis $\Delta^{8,10}$ desaturase in the 1,4-dehydrogenation of racemic methyl $\left[11-{ }^{2} \mathrm{H}_{1}\right]$-linoleate.

\begin{tabular}{|c|c|c|c|c|c|c|c|}
\hline \multirow[t]{2}{*}{$\begin{array}{l}\text { Position of } \\
\text { Isotope in } d_{1-}- \\
\text { Species }\end{array}$} & \multirow[t]{2}{*}{ Trial } & \multicolumn{3}{|c|}{$\begin{array}{l}\text { Cellular Linoleic acid } \\
\text { Substrate }{ }^{a, b, c}\end{array}$} & \multicolumn{2}{|c|}{$\begin{array}{l}\text { Cellular Calendic } \\
\text { acid Product }{ }^{a, c}\end{array}$} & \multirow[t]{2}{*}{ KIE } \\
\hline & & $\mathrm{d}_{0}$ & $\mathrm{~d}_{1}-(R)$ & $\mathrm{d}_{\mathrm{l}}-(\mathrm{S})$ & $\overline{d_{0}}$ & $d_{1}$ & \\
\hline & 1 & 2.7 & 48.65 & 48.65 & 22.5 & 77.5 & 4.3 \\
\hline \multicolumn{8}{|l|}{$C-11$} \\
\hline & 2 & 2.2 & 48.9 & 48.9 & 21.1 & 78.9 & 4.5 \\
\hline & Average & & & & & & $4.4 \pm 0.1^{d}$ \\
\hline
\end{tabular}

a The isotopic content of both substrate and product were evaluated by considering the $(M+1)$ and $(M-1)$ contributions from $d_{0}$ - and $d_{1}$-species, respectively. ${ }^{b}$ The isotopic ratio of the cellur linoleic acid fractions are similar to that measured prior to the incubation. ${ }^{c}$ The deuterium content (\%) of each species is given as an average value based on two GC-MS runs. ${ }^{d}$ The average KIE (two incubations) \pm standard deviation. The average standard deviation is \pm 0.4 for each species expressed as a percentage.

The product deuterium KIE for $\mathrm{C}-\mathrm{H}$ cleavage at $\mathrm{C}-11$ can be calculated using the following ratio: $:^{199}$

$$
\frac{k_{H}}{k_{D}}=\frac{P_{d_{1}} / P_{H}}{S_{d_{1 R}} / S_{d_{1 S}}}
$$

Where, ${ }^{d_{1}} / P_{H}$ is the ratio of monodeuterated to nondeuterated calendate and $S_{d_{1 R}} / s_{d_{1 S}}$ is the ratio of monodeuterated $(11 R)$ - to $(11 S)$ - $\mathrm{d}_{1}$-linoleate. Since the racemic sample 
contains a small amount of $\mathrm{d}_{0}$-substrate $\left(S_{d_{0}}\right)$ that contributes to $P_{H}$, a modified KIE expression is used:

$$
\frac{k_{H}}{k_{D}}=\frac{\left[\% P_{d_{1}}+\left\{\left(\% P_{d_{1}} \times \% S_{d_{0}}\right) / \% S_{d_{1 R}}\right\}\right] /\left[\% P_{d_{0}}-\left\{\left(\% P_{d_{1}} \times \% S_{d_{0}}\right) / \% S_{d_{1 R}}\right\}\right]}{\left[\left(\% S_{d_{1 R}}+\% S_{d_{0}}\right) / \% S_{d_{1} s}\right]}
$$

The analytical result obtained in this experiment is displayed in Table 2.2, which is showing the involvement of a large primary deuterium KIE $(4.4 \pm 0.1)$ for the $\mathrm{C}-\mathrm{H}$ bond cleavage step at $\mathrm{C}-11$. This value is substantially lower than the previously reported result $(\mathrm{KIE}=5.7 \pm 1.0)$ for the same biotransformation reaction, ${ }^{158}$ where a $\sim 1: 1$ mixture of nondeuterated to regiospecifically dideuterated linoleates was used. This discrepancy can be explained by considering the potential impact of normal $\alpha$-secondary kinetic isotope effects ${ }^{160}$ in the two modes of calculating KIEs (Figure 2.45).
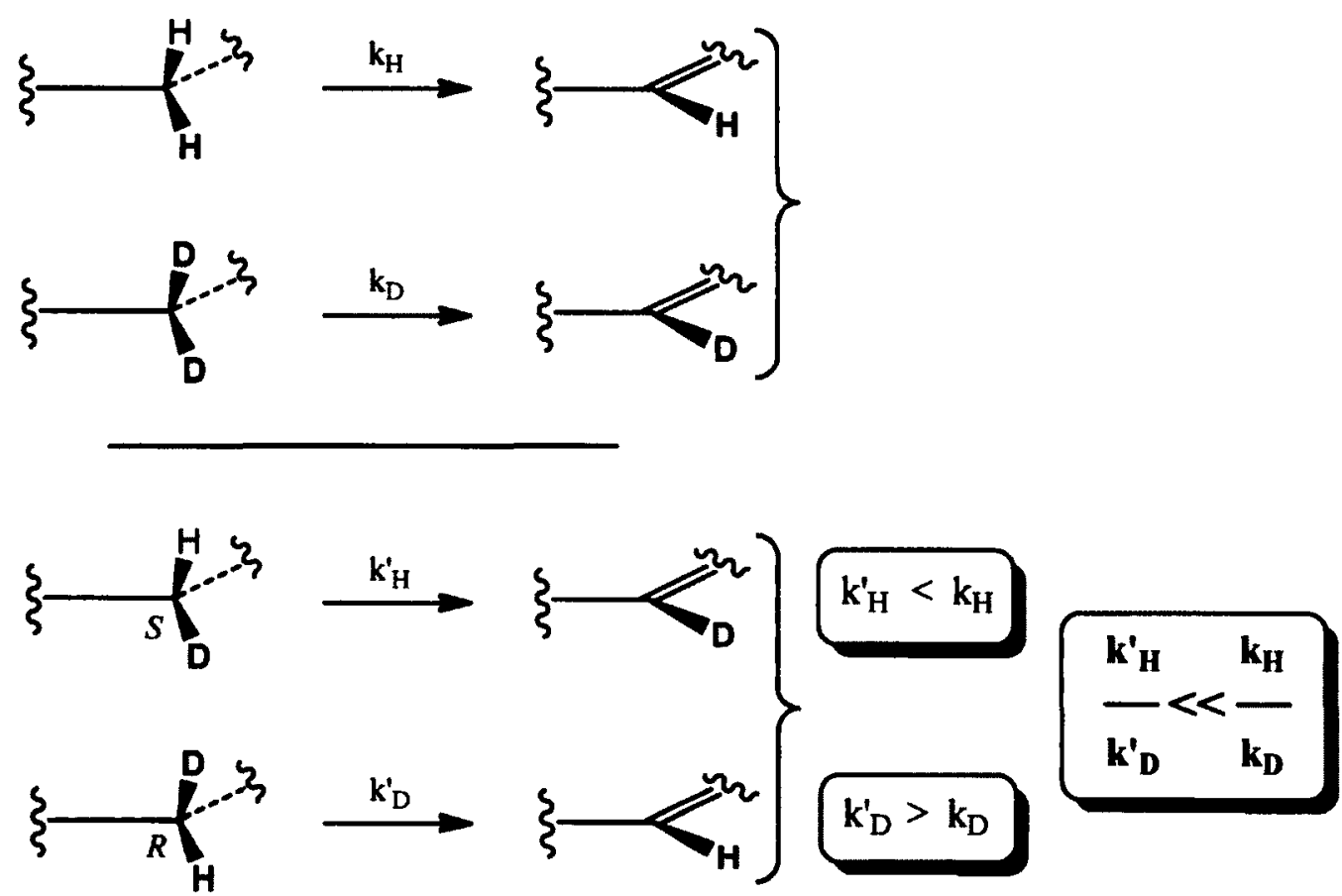

Figure 2.45. Comparison of the relative rates of $\mathrm{C}-\mathrm{H}$ vs. $\mathrm{C}-\mathrm{D}$ bond breaking in the two modes of calculating KIE at C-11. 
The $\alpha$-secondary deuterium isotope effect reduces the rate of $d_{1}$-product formation from $d_{1 S}$ substrate relative to the rate of $d_{0}$-product formation from $d_{0}$-substrate, which in turn reduces the numerator of the ratio $k_{H}^{\prime} / k_{D}^{\prime}$ relative to $k_{H} / k_{D}$. On the other hand, the $\alpha$ secondary deuterium isotope effect reduces the rate of $d_{1}$-product formation from $d_{2}$ substrate relative to $d_{0}$-product formation from $d_{1 R}$ substrate, which in turn increases the denominator of the ratio $\mathrm{k}_{\mathrm{H}}^{\prime} / \mathrm{k}_{\mathrm{D}}^{\prime}$ relative to $\mathrm{k}_{\mathrm{H}} / \mathrm{k}_{\mathrm{D}}$. The net effect is that the KIE calculated using the racemic $11-d_{1}$ samples is $\sim 77 \%$ or $100(1 / 1.15 \times 1 / 1.15)$ of the KIE calculated using $\sim 1: 1$ mixture of nondeuterated to regiospecifically dideuterated linoleates. Values of $\alpha$-secondary isotope effects of 1.15 for iron oxo-mediated hydrogen abstraction reactions are within the expected range. ${ }^{160,199}$

\subsection{Conclusion}

The enantioselectivity of the 1,4-desaturase (FAD2 variant) involved in formation of conjugated fatty acids in plants has been investigated for the first time. This completes a study that was begun by Crombie and coworkers ${ }^{43}$ some 26 years ago. Our investigation established that the formation of calendic acid in Calendula officinalis (pot marigold) involves the initial, isotopically sensitive removal of a pro $S$ hydrogen from C-11 of linoleate and subsequent cleavage of the pro $R \mathrm{C} 8-\mathrm{H}$ bond i.e. two hydrogens are removed in syn fashion from two neighbouring allylic positions of the fatty acid chain to biosynthesize the conjugated trienoic acid (Figure 2.46). 

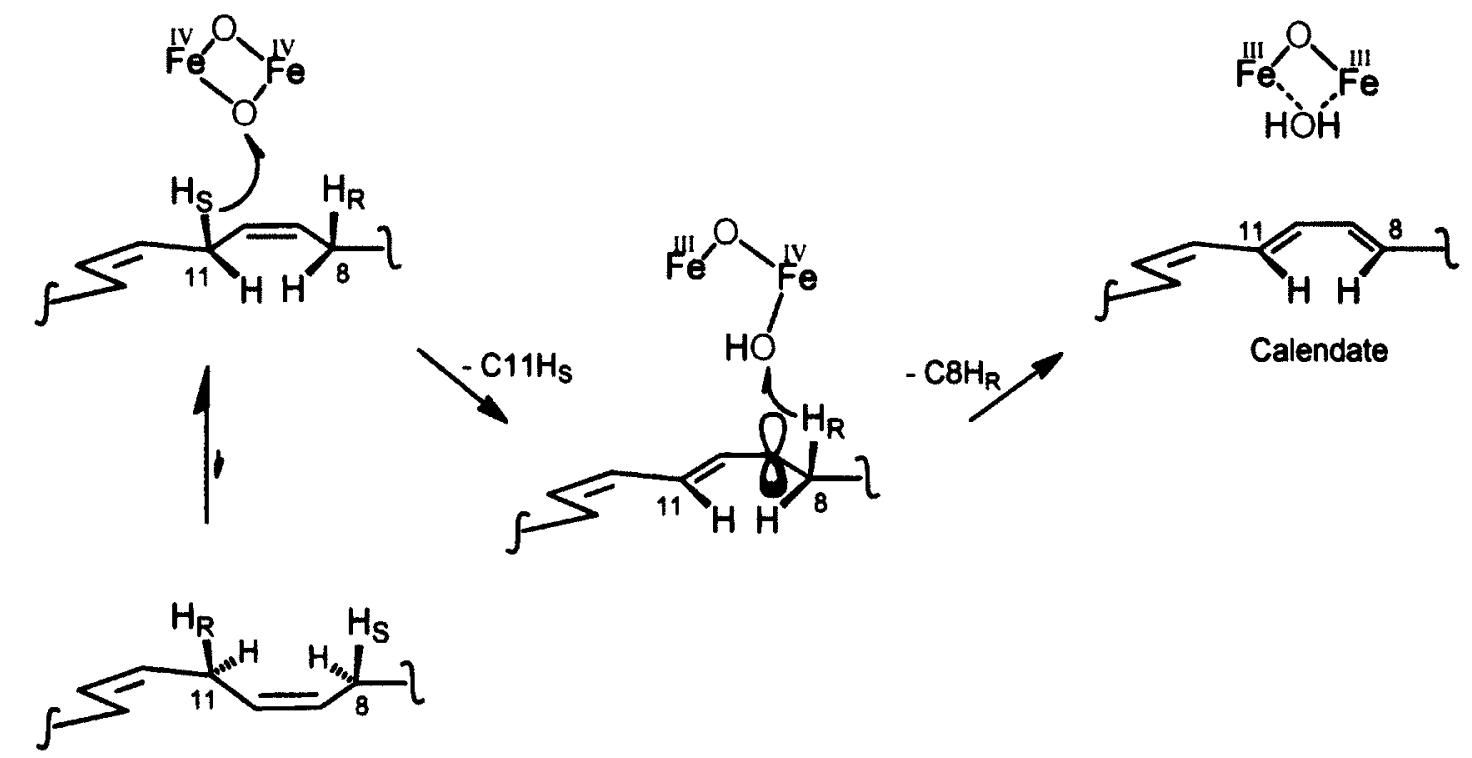

Figure 2.46. Mechanistic model for 1,4-dehydrogenation reaction of linoleate to biosynthesize calendate as it occurs in Calendula officinalis. The double bonds are quasicoplanar.

The observed stereochemistry and cryptoregiochemistry of hydrogen removal in calendate biosynthesis correlates well with that observed for the parent FAD2 enzyme involved in formation of linoleic acid $\left(\mathrm{CH}_{3}-\left(\mathrm{CH}_{2}\right)_{5}-\mathrm{C}_{13}=\mathrm{C}_{12}-\mathrm{CH}_{2}-\mathrm{C}_{10}=\mathrm{C}_{9}-\left(\mathrm{CH}_{2}\right)_{7}-\mathrm{CO}_{2} \mathrm{H}\right)$ from oleate and validates the proposed mechanistic model (Figure 2.1). The results of this investigation strengthens the proposal that there exists a highly conserved, active-site structural motif for the family of membrane-bound FAD2-like enzymes, where the oxidation is initiated by approaching the olefinic plane at or near the C-12 position of substrate from the top $\left(\mathrm{Cl}\right.$ terminus at right hand side of structure $\left(\mathrm{CH}_{3}-\left(\mathrm{CH}_{2}\right)_{5}-\mathrm{C}_{13}=\mathrm{C}_{12^{-}}\right.$ $\left.\mathrm{CH}_{2}-\mathrm{C}_{10}=\mathrm{C}_{9}-\left(\mathrm{CH}_{2}\right)_{7}-\mathrm{CO}_{2} \mathrm{H}\right)$. 


\section{Chapter 3: Mechanistic Studies of $\alpha$-Eleostearate Formation in} Aleurites fordii Hemsl.

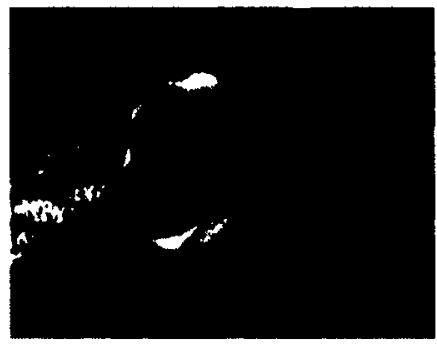

www.southeasternflora.com

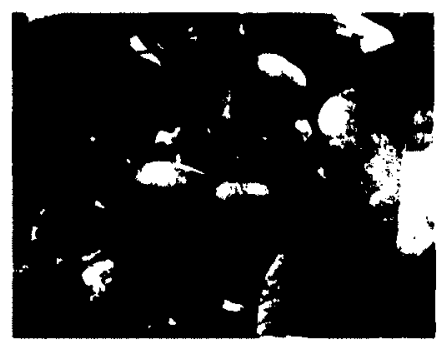

www.botanic.jp

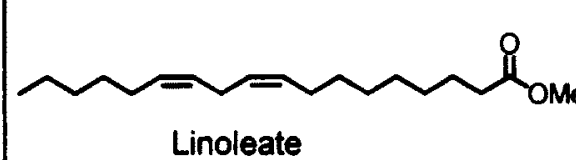

A. fordii Hemsl.

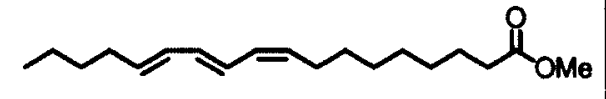

a-Eleostearate

\subsection{Experimental Approach}

To further explore the biosynthesis of conjugated fatty acids involving an apparent 1,4-desaturation reaction, the formation of $\alpha$-eleostearic acid (a calendic acid regioisomer) in the seeds of Aleurites fordii Hemsl. commonly known as Tung tree ${ }^{34}$ was targeted. The mechanism by which this important fatty acid is biosynthesized in the developing seeds of the tung tree has not been explicitly investigated before. Phylogenetic analysis demonstrated that the enzyme tung FADX can be grouped with parent FAD2 and hydroxylases which is consistent with its desaturase activity. ${ }^{34}$ Expression and functional analysis of this FAD2 homologue in yeast $S$. cerevisiae in the presence of the exogenously added linoleic acid showed that the externally supplied linoleic acid is transformed to $\alpha$-eleostearic acid. ${ }^{34}$ One may assume that the formation of $\alpha$-eleostearic acid features the removal of the hydrogens from two non-adjacent 
methylene groups (C-11 and C-14) via an apparent 1,4-dehydrogenation mechanism (hereafter will be called $\Delta^{11,13}$-desaturase, Figure 3.1$)^{34,200}$
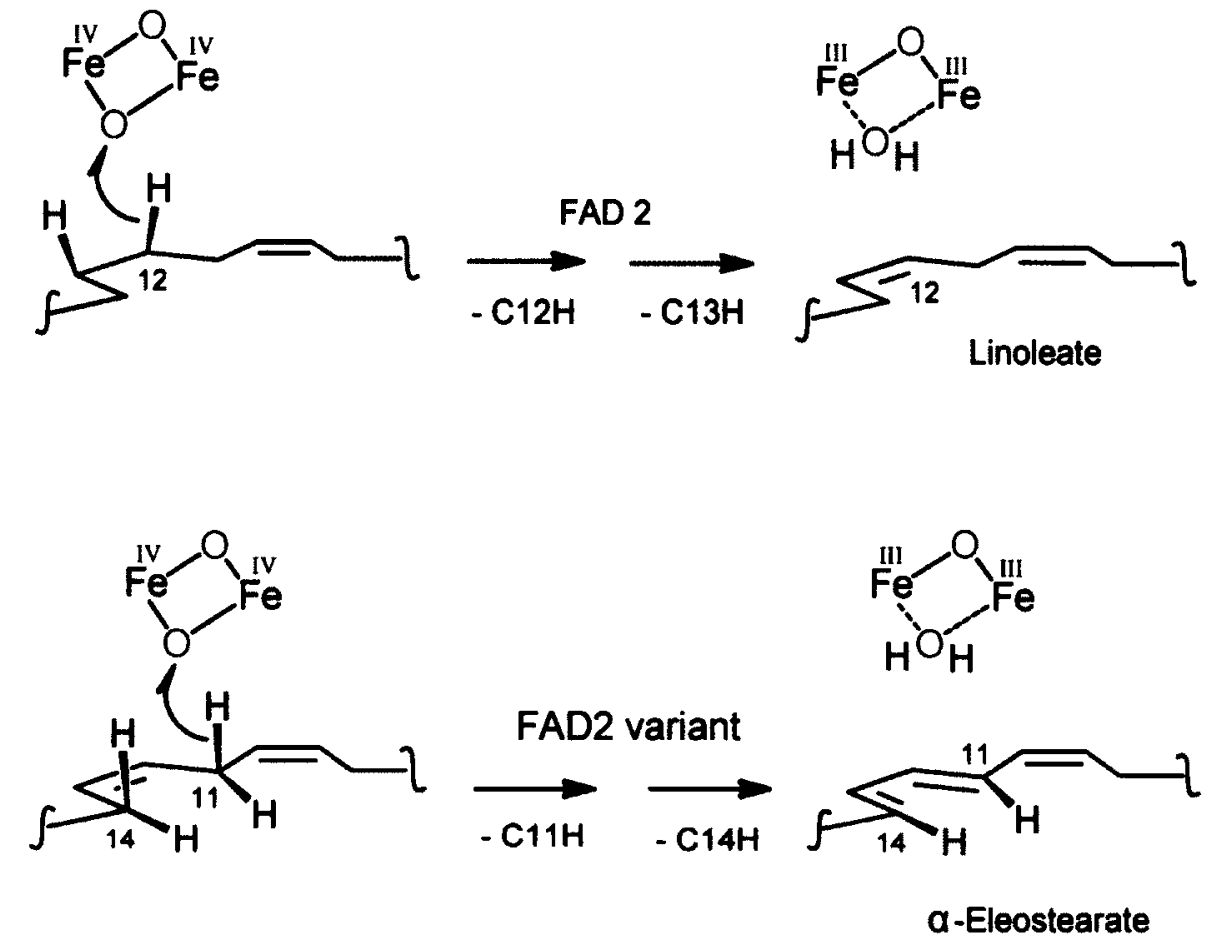

Figure 3.1. A proposed mechanism for the 1,4-desaturase-mediated formation of $\alpha$ eleostearate featuring initial $\mathrm{H}$-abstraction at $\mathrm{C}-11$. The accepted mechanism for parent FAD2 formation is included for the purposes of comparison. The double bonds are quasicoplanar.

We decided to probe the cryptoregiochemistry (site of initial oxidation) of $\Delta^{11,13}$ desaturation by measuring the deuterium kinetic isotope effect (KIE) on the two $\mathrm{C}-\mathrm{H}$ cleavage steps during oxidation - an approach that has already been validated on related systems. As described in Section 1.3.2, the approach is based on the premise that only the cleavage of the first $\mathrm{C}-\mathrm{H}$ bond is likely to be the rate-determining step of the enzyme catalyzed reaction, then substitution of that particular hydrogen with deuterium should in principle generate a primary deuterium KIE (Figure 1.18). On the basis of the similarity between FAD2 and its variant, we propose that $\alpha$-eleostearate formation is initiated at C- 
11, the carbon closest to $\mathrm{C}-12$ (the site of initial oxidation for linoleate formation). The enzymatic isotope effects in our investigation will be determined via intermolecular competitive experiments as described in Section 1.3.2, where a ca. equimolar (1:1) mixture of non-deuterated and regiospecifically dideuterated substrate compete in the same incubation. ${ }^{199} T$ The $d_{1} / d_{0}$ ratio of the olefinic product is then compared with the $d_{2} / d_{0}$ ratio of the substrate. Our approach required the synthesis of regiospecifically dideuterated methyl $\left[11,11-{ }^{2} \mathrm{H}_{2}\right]$ - and $\left[14,14-{ }^{2} \mathrm{H}_{2}\right]$-linoleates (31 and 32) (Figure 3.2) and a $S$. cerevisiae transformant that functionally expresses the $\Delta^{11,13}$-desaturase enzyme from Alurites fordii Hemsl. The latter was constructed through a collaborative effort with the Covello laboratory (NRC- PBI, Saskatoon).

The proposed mechanism for $\alpha$-eleostearate formation as shown in Figure 3.1 predicts that the substrate adopts a conformation that allows the hydrogens at $\mathrm{C}-11$ and C-14 of the fatty acid chain to be removed with the same facial selectivity as observed with the syn removal of hydrogens from $\mathrm{C}-12$ and $\mathrm{C}-13$ positions during linoleic acid formation (FAD2). The correctness of this proposed topology could be tested through the determination of the enantioselectivity of a-eleostearate formation using stereospecifically labeled deuterated fatty acids as mechanistic probes.

Our approach required the use of four stereospecifically monodeuterated linoleates with the chirotopic center in one pair at C-11 and in the other pair at C-14 (Figure 3.2) i.e. methyl $(11 S)-\left[11-^{2} \mathrm{H}_{1}\right]$-octadeca-9Z,12Z-dienoate 2a, methyl (11R)-[11$\left.{ }^{2} \mathrm{H}_{1}\right]$-octadeca-9Z,12Z-dienoate $2 \mathbf{b}$, methyl $(14 S)$-[11- $\left.{ }^{2} \mathrm{H}_{1}\right]$-octadeca-9Z,12Z-dienoate 33a and methyl (14R)-[11- $\left.{ }^{2} \mathrm{H}_{1}\right]$-octadeca-9Z,12Z-dienoate 33b. The former pair of 
enantiomers were available from the calendate experiments (See Chapter 2). The latter compounds were synthesized as described in Section 3.2.3.

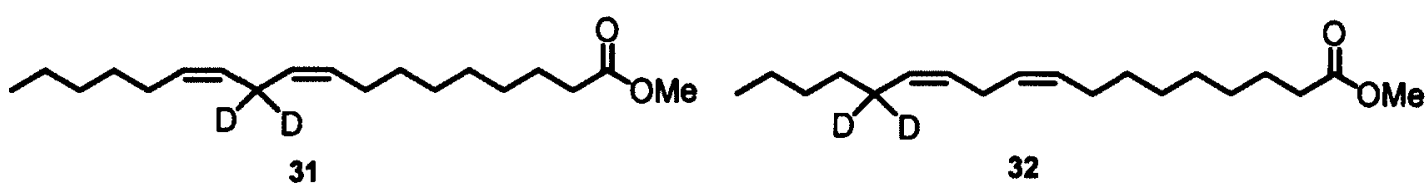<smiles>[2H][C@H](/C=C/CCCCCCCC(=O)OC)[C@H](O)/C=C/CCCCCC</smiles><smiles>[2H]C(/C=C/C/C=C/CCCCCCCC(=O)OC)/C=C/C/C=C/CCCCCCCC(=O)OC</smiles>

Figure 3.2. The dideuterated and chiral monodeuterated linoleates required to investigate the cryptoregiochemistry and stereochemistry $\alpha$-eleostearate formation.

\subsection{Results and Discussion}

\subsubsection{Construction of Yeast Mutant Containing $\Delta^{11,13}$-Desaturase}

\subsubsection{Amplification of the template DNA, Cloning and Expression in $E$. coli}

The plasmid pRTL2 myc-FADX containing the FADX gene that encodes the $\Delta^{11,13}$-desaturase enzyme from Aleurites fordii Hemsl. responsible for the biosynthesis of $\alpha$-eleostearate from linoleate was originally obtained from John M. Dyer (US Department of Agriculture-Agricultural Research Services Southern Regional Research Center, New Orleans, Louisiana). ${ }^{34}$ The plasmid vector was then amplified using the PCR reaction, where a FDX-forward primer (5'-gaagettgtctagaATGGGAGCTGGTGGCCGAATGTCT$\left.3^{\prime}, \mathrm{Tm}=76.1^{\circ} \mathrm{C}\right)$ and FDX-reverse primer $\left(5^{\prime}\right.$-aaggatccACTCCATATCTCGTAACAAGGTCAAACCTC-3', Tm $=69.4{ }^{\circ} \mathrm{C}$ ) were used as described by Dyer $e t$ al. ${ }^{34}$ (the FDXreverse primer contain the BamHI site as indicated in the paper) along with the $2 x$ Phusion high-fidelity PCR master mix (New England BioLabs). During the PCR 
reaction, the cycle was repeated 30 times and the primers were annealed at $72{ }^{\circ} \mathrm{C}$ with the single-stranded DNA template. Following the completion of the cycle, TAQ polymerase was added and the mixture was heated to $72{ }^{\circ} \mathrm{C}$ for 10 minutes to ensure "A overhang" on the PCR products that were then analyzed by agarose gel electrophoresis and visualized by ethidium bromide (Figure 3.3 ).

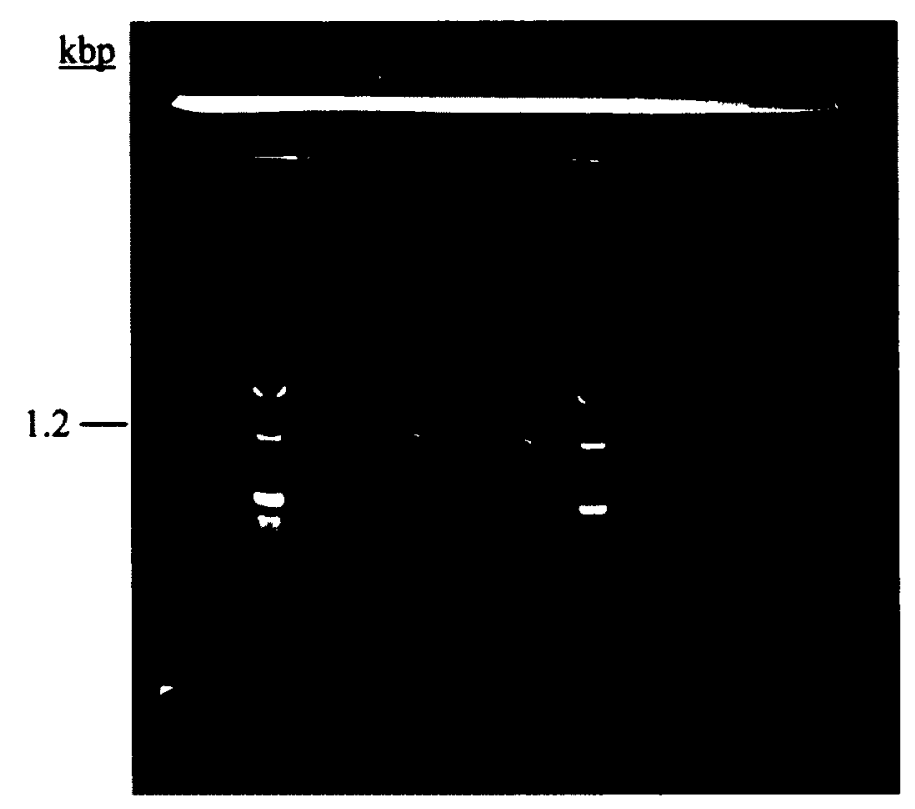

Figure 3.3. Agarose gel electrophoresis of the PCR amplified FADX genes. Expected size of the entire FADX gene was $1.2 \mathrm{~kb}$. The two amplified FADX gene was compared with the reference ladder and showed that the amplification resulted in the production of correct amino acid.

The PCR products were then TA cloned into pYES2.1 vector by using the pYES2.1 TOPO ${ }^{(2)}$ TA Expression Kit from Invitrogen and the recombinant vector was transformed into chemically competent $E$. coli cells. This vector provides for galactoseinducible expression mediated by the GAL1 promoter. The mixture was then plated on LB media plates under ampicillin selection and individual colonies were picked from the well-spaced bacterial colonies to grow in liquid culture media on $\mathrm{LB}_{\text {amp }}(100 \mu \mathrm{g} / \mathrm{mL})$ at $200 \mathrm{rpm}$ in $37^{\circ} \mathrm{C}$. The newly constructed plasmid DNAs were purified by Promega 
Wizard ${ }^{\boxplus}$ Plus Midipreps DNA Purification kit and quantitated by spectrophotometry. The purified plasmid DNA solutions were submitted for sequencing and the sequence data was analyzed by Lasergene SeqMan software and shown to be identical with the reported FADX sequence ${ }^{34}$. The purified plasmid DNA (see Chapter 5) was subsequently inserted into $S$. cerevisiae using the $S$. c. EasyComp transformation kit (Invitrogen).

\subsubsection{Preparation of $S$. cerevisiae mutant expressing $\Delta^{11,13}$-desaturase enzyme from Aleurites fordii Hemsl.}

For transformation, INVSc-1 strain of $S$. cerevisiae was used and selected on SDglucose-uracil selection media plates. Briefly, the freshly prepared cultures of the INVSc-1 strain of $S$. cerevisiae were grown to mid-log phase on YPD medium and the harvested cells were resuspended in highly concentrated solution of lithium acetate (solution II) in order to make the cells competent for transfection using the method described by $S$. c. EasyComp ${ }^{\mathrm{TM}}$ transformation kit (Invitrogen). The competent cells were then cooled to $-80^{\circ} \mathrm{C}$ since better transformation efficiencies have been observed with thawed cells compared to freshly prepared cells. After equilibrating to room temperature, the frozen cells were mixed with the vector DNA and finally diluted with the appropriate transformation solution. The cell/DNA mixture was subsequently mixed thoroughly and incubated at $30{ }^{\circ} \mathrm{C}$ to induce the uptake of DNA. The transformed yeast cells were streaked on SDglucose-uracil selection media plates containing $2 \%$ agar and allowed to grow at $30{ }^{\circ} \mathrm{C}$ for 4 days to yield sufficient discrete colonies. Individual colonies were selected and cultured in selection/induction media, S-Galactose-uracil to prepare the starter culture for incubation experiments with labeled substrates. 


\subsubsection{Cryptoregiochemistry of $\Delta^{11,13}$-Desaturation}

\subsubsection{Synthesis of Methyl $\left[11,11-{ }^{2} \mathrm{H}_{2}\right]$-Linoleate}

Methyl $\left[11,11-{ }^{2} \mathrm{H}_{2}\right]$-linoleate 31 was synthesized in $54 \%$ overall yield starting with 1-heptyne as starting material using the synthetic strategy as depicted in Figure 3.4. A key intermediate $\left[1,1-{ }^{2} \mathrm{H}_{2}\right]-2-o c t y n-1-0 l 30$ was prepared with high deuterium content as previously discussed in Chapter 2 (Figure 2.33).

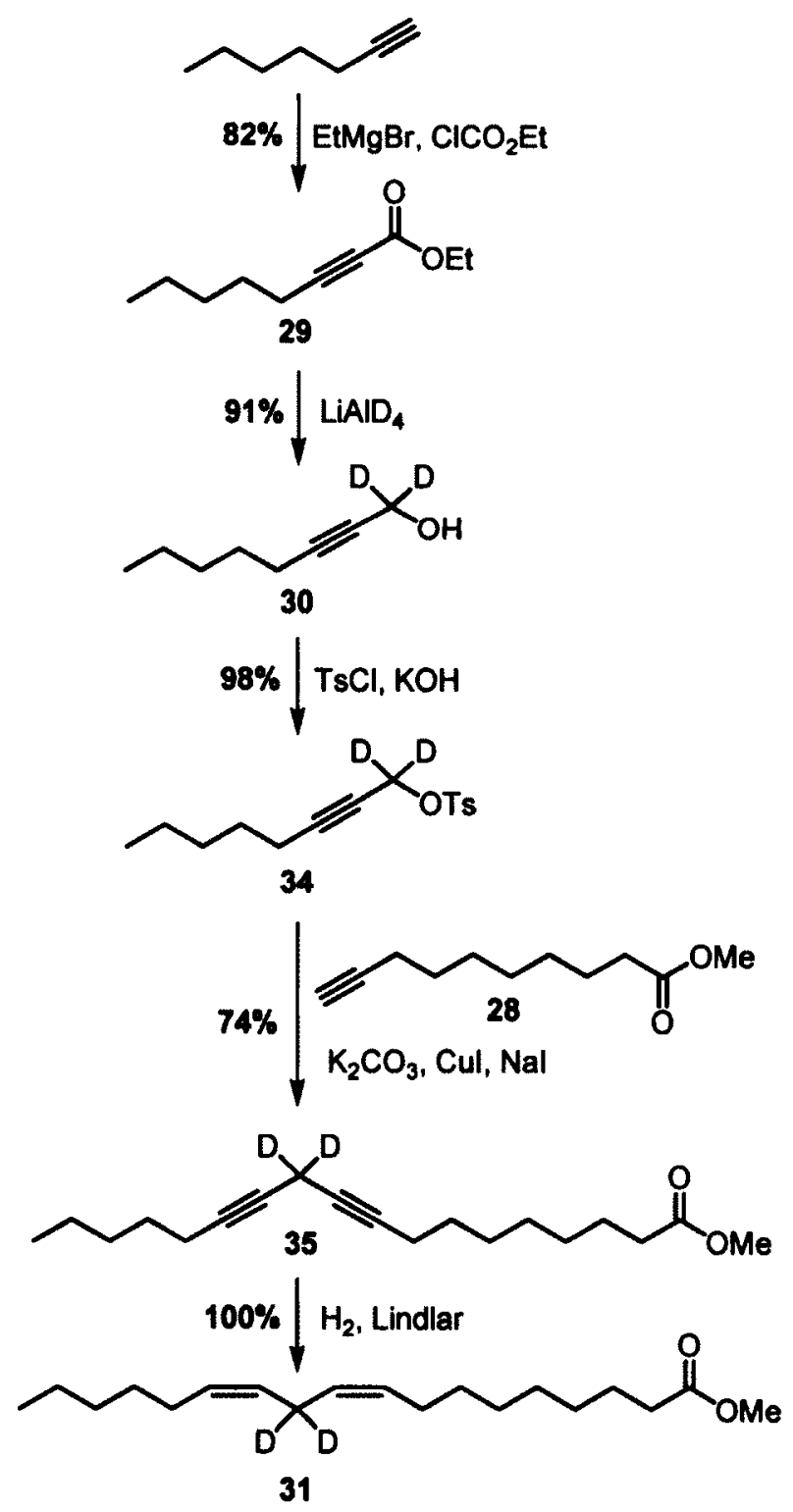

Figure 3.4. Synthesis of methyl $\left[11,11-{ }^{2} \mathrm{H}_{2}\right]$-linoleate. 
This intermediate was elaborated to give the title compound as previously described for the synthesis of racemic $11-\mathrm{d}_{1}$-linoleate (Figure 2.32). Methyl $\left[11,11-{ }^{2} \mathrm{H}_{2}\right]$-linoleate was obtained in high purity following flash column chromatography on silica gel as determined by GC-MS (data not shown). Analysis of the MS revealed that the deuterated substrate consisted mainly of dideuterated species as evidenced by the appearance of the molecular ion peak at $\mathrm{m} / \mathrm{z} 296$ (Figure 3.5) and is in accord with the molecular formula $\mathrm{C}_{19} \mathrm{H}_{32} \mathrm{D}_{2} \mathrm{O}_{2}$.

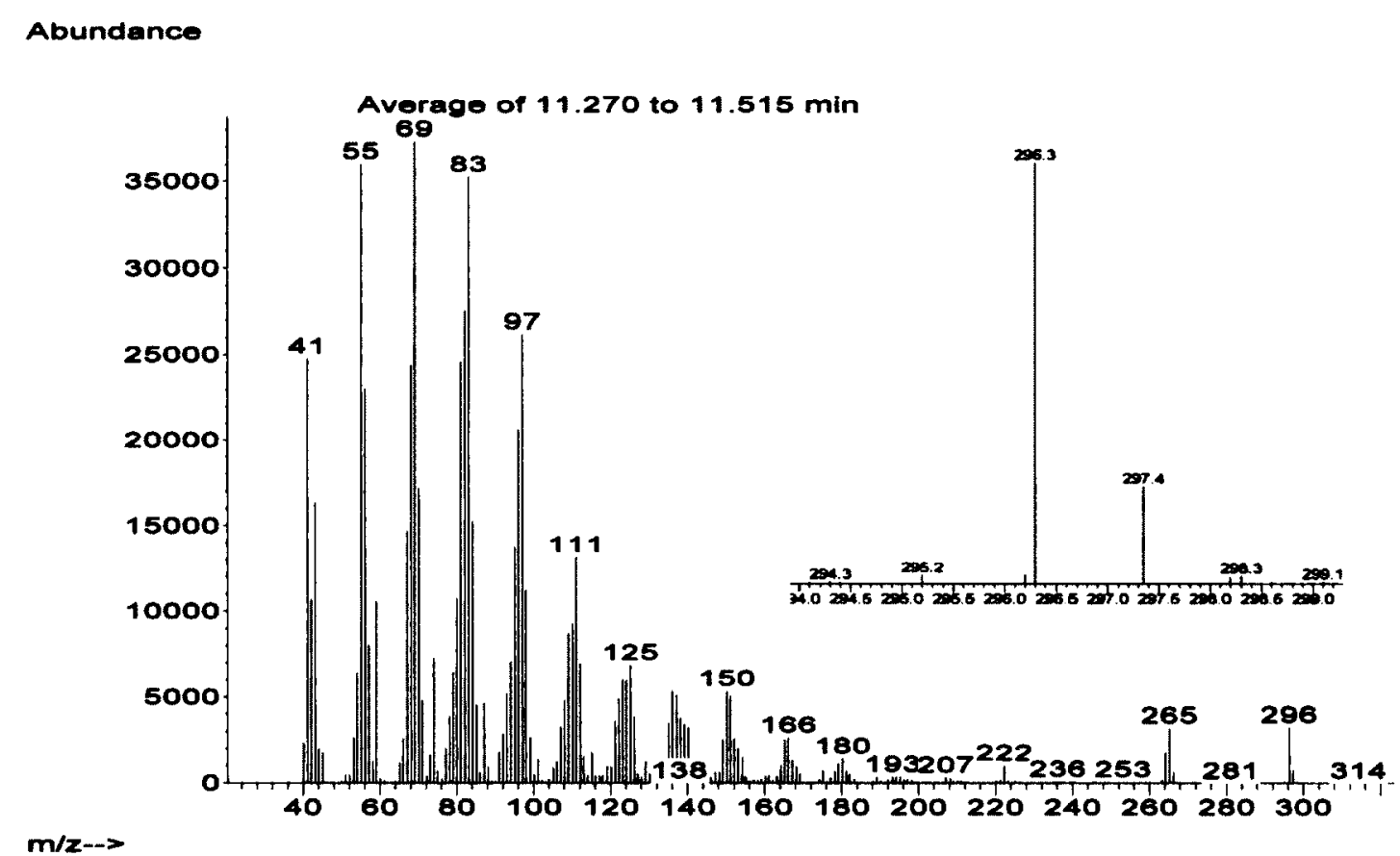

Figure 3.5. Mass spectrum of methyl $\left[11,11-{ }^{2} \mathrm{H}_{2}\right]$-linoleate 31 .

The location of the $\mathrm{CD}_{2}$ group was confirmed by the absence of $\mathrm{C} 11{ }^{1} \mathrm{H}$ resonances (Figure 3.6) that appear at $\delta 2.77 \mathrm{ppm}$ in the ${ }^{1} \mathrm{H}$ spectrum of the non-labeled material (Figure 2.13). The splitting pattern of the multiplet at 5.30-5.45 ppm due to the olefinic protons is also altered. 


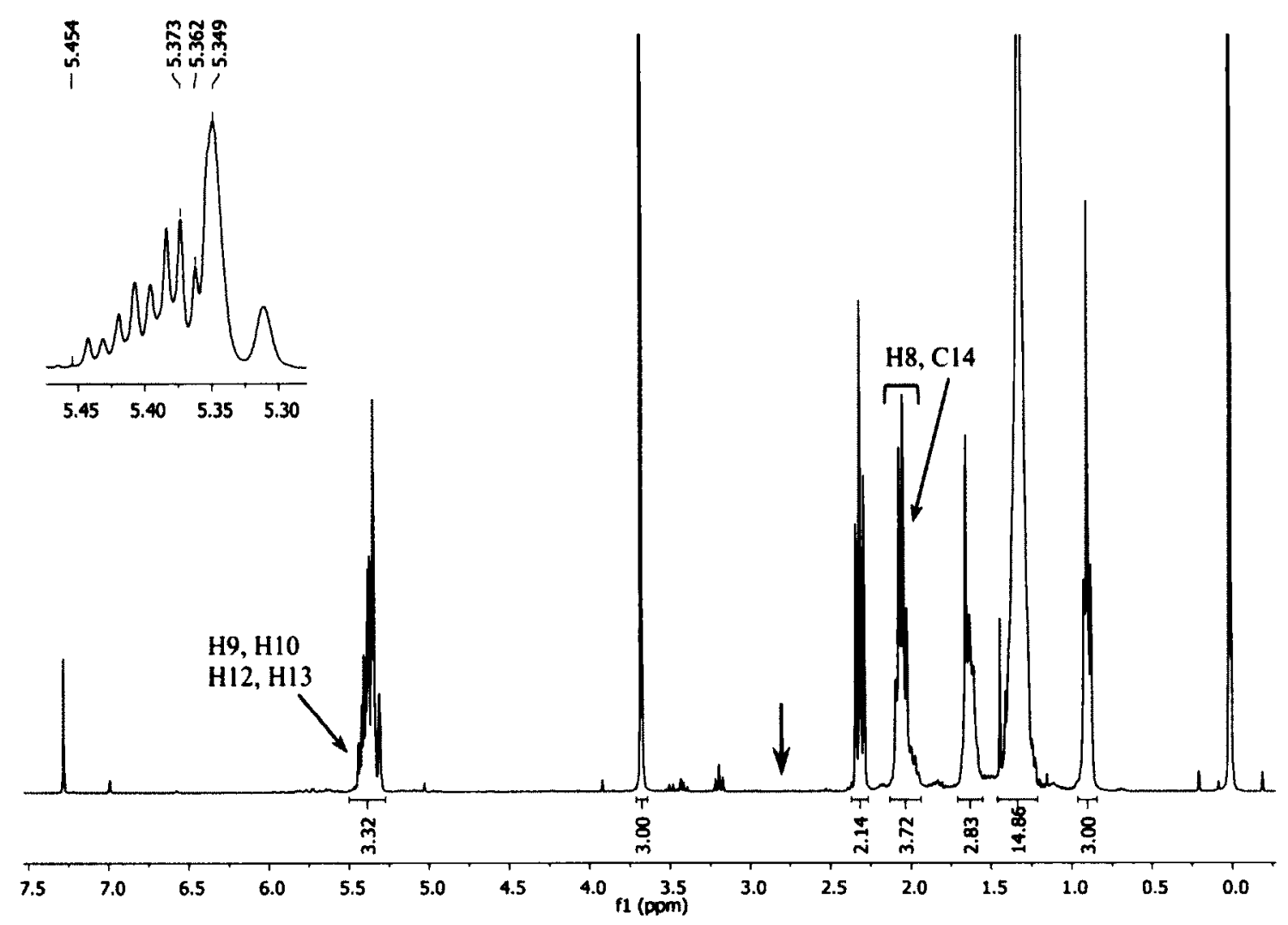

Figure 3.6. ' $\mathrm{H}$ NMR spectrum of methyl $\left[11,11-{ }^{2} \mathrm{H}_{2}\right]$-linoleate. The arrow indicates the positioning of the signals due to the bis-allylic protons at $\mathrm{C}-11$ that are missing here due to dideuteration at this location.

In addition, the dideuterium labeling at $\mathrm{C}-11$ position causes the ${ }^{13} \mathrm{C}$ resonance to split into a pentet due to coupling with the two deuteria $(2 \mathrm{nI}+1$ rule, $\mathrm{I}=1$ and $\mathrm{n}=2)$ and isotope shifted upwards by $0.83 \mathrm{ppm}$ ( $\alpha$-shift). The $\beta$ and $\gamma$-isotope shifts are documented in Appendix A2. 


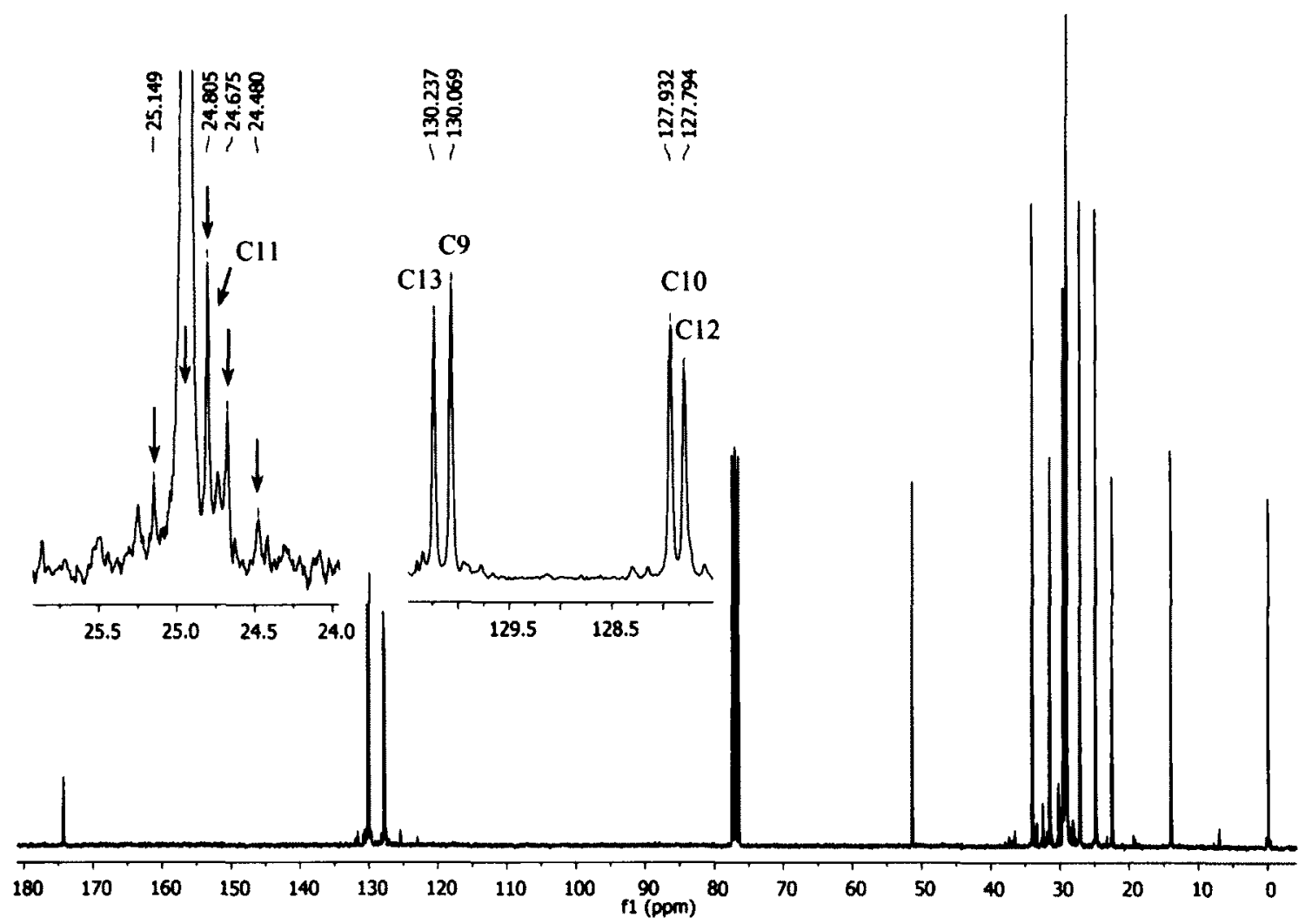

Figure 3.7. ${ }^{13} \mathrm{C}$ NMR spectrum of methyl $\left[11,11-{ }^{2} \mathrm{H}_{2}\right]$-linoleate. The arrows are showing the pentet. The ${ }^{13} \mathrm{C}$ signals due to $\mathrm{C} 9(130.07 \mathrm{ppm}), \mathrm{C} 10$ (127.93 ppm), C12 (127.79 $\mathrm{ppm})$ and $\mathrm{C} 13(130.24 \mathrm{ppm})$ are all reduced in intensity due to the presence of deuterium at $\mathrm{C} 11$. The effect is largest for $\mathrm{C} 10$ and 12.

Additional evidence for the presence of $-\mathrm{CD}_{2}$ unit at the bis-allylic position into the fatty acid chain was obtained via the appearance of two absorption bands at 2200 and 2099 $\mathrm{cm}^{-1}$ in the IR spectrum of the title compound (Figure 3.8). These two absorption bands correspond to the symmetric as well as asymmetric stretching frequencies of the two associated C-D bonds and is absent in the analogous non-deuterated linoleate. 


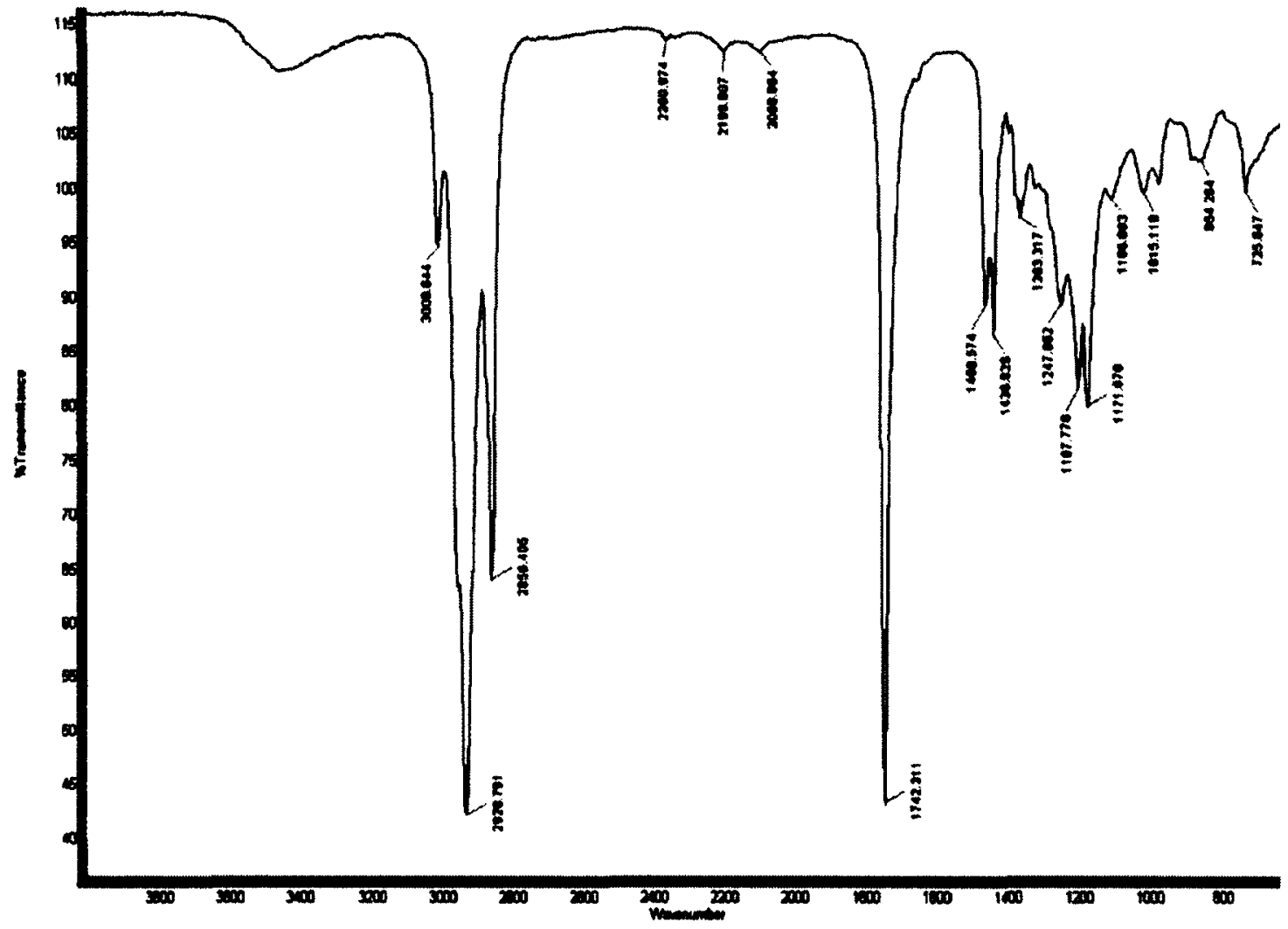

Figure 3.8. IR spectrum of methyl $\left[11,11-{ }^{2} \mathrm{H}_{2}\right]$-linoleate. Asymmetric and symmetric C$D$ stretches due to $C_{14}$ DD appear at 2099 and $2200 \mathrm{~cm}^{-1}$. Peak at $2360 \mathrm{~cm}^{-1}$ is part of a band that may be due to the $\mathrm{CO}_{2}$ stretch.

\subsubsection{Synthesis of Methyl $\left[14,14-{ }^{2} \mathrm{H}_{2}\right]$-linoleate}

Methyl $\left[14,14{ }^{2} \mathrm{H}_{2}\right]$-linoleate 32 was synthesized in $37 \%$ overall yield from the commercially available methyl valerate as starting material (Figure 3.9). The route mimics that outlined for the synthesis of the corresponding chiral 14- $\mathrm{d}_{1}$-linoleates (Figure 3.18) as discussed in the later section. The GC-MS, IR, ${ }^{1} \mathrm{H}$ and ${ }^{13} \mathrm{C}$ NMR data obtained on the purified title compound confirmed the proposed structure, location of deuterium labeling and the high $\mathrm{d}_{2}$-content. The relevant spectra are listed below. 

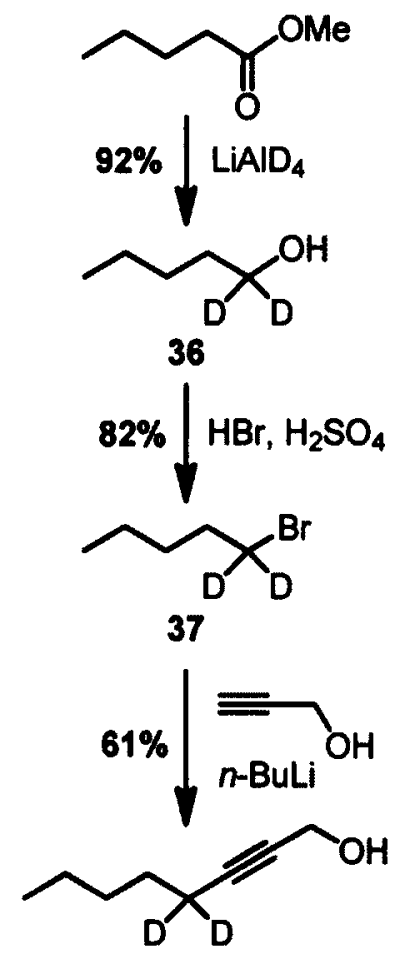

38
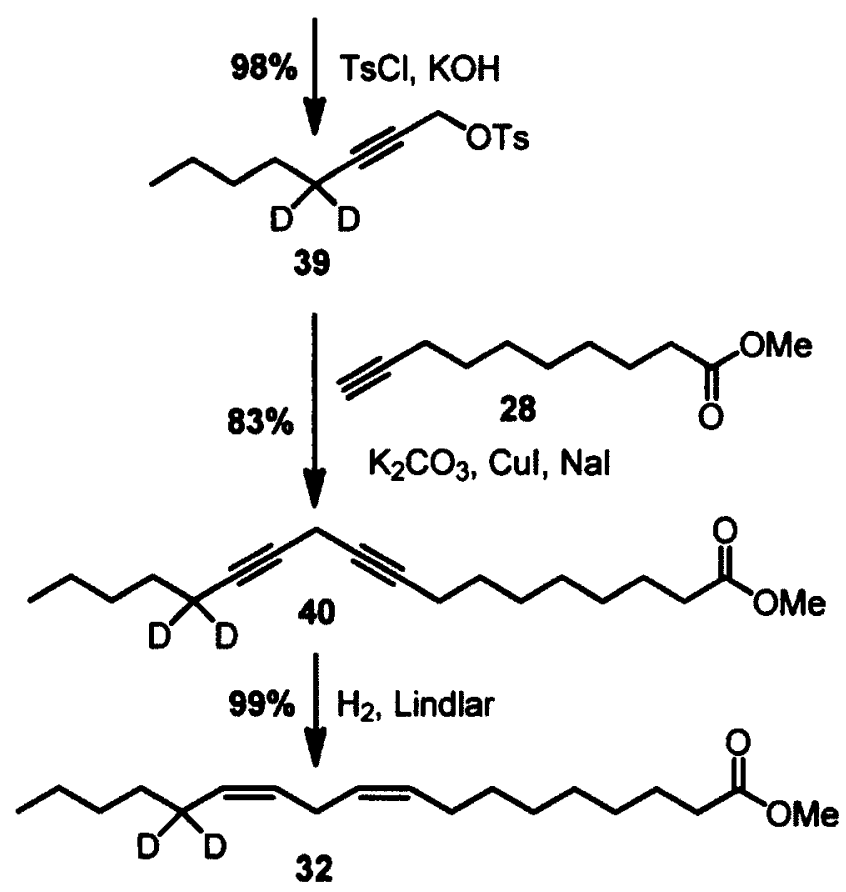

Figure 3.9. Synthesis of methyl $\left[14,14-{ }^{2} \mathrm{H}_{2}\right]$-linoleate. 


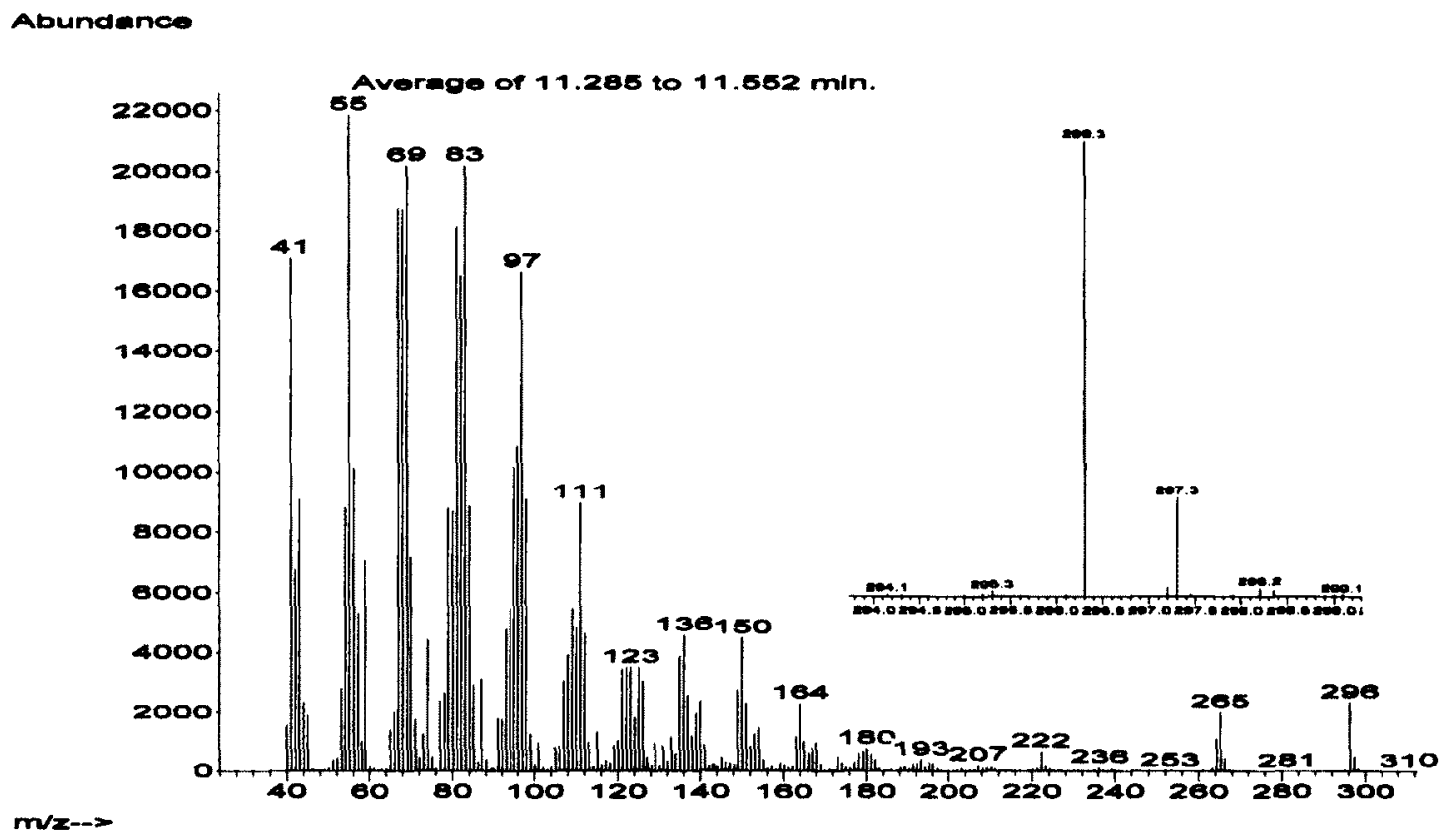

Figure 3.10. Mass spectrum of methyl $\left[14,14-{ }^{2} \mathrm{H}_{2}\right]$-linoleate.

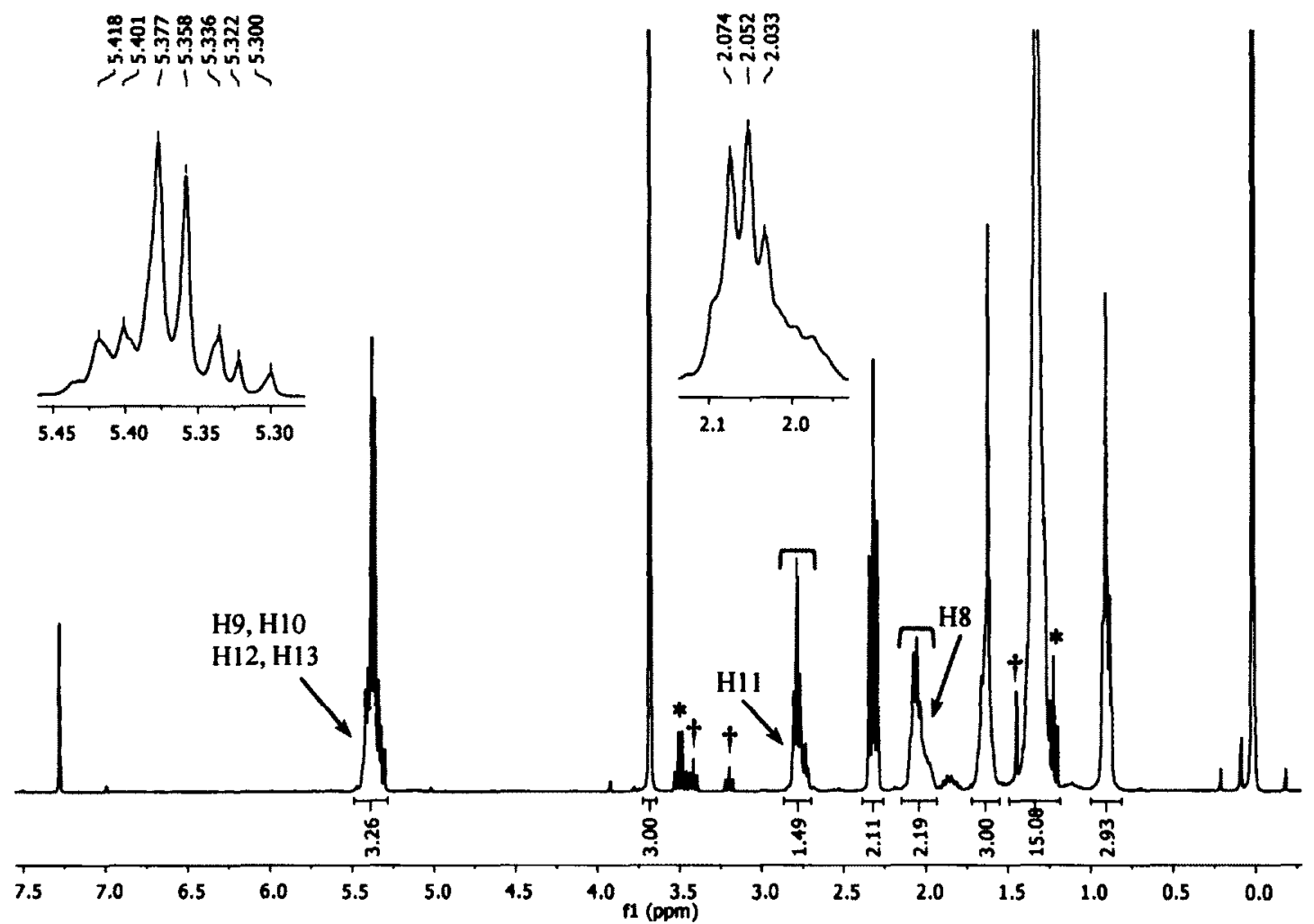

Figure 3.11. ${ }^{1} \mathrm{H}$ NMR spectrum of methyl $\left[14,14-{ }^{2} \mathrm{H}_{2}\right]$-linoleate. Doublet of triplets due to $\mathrm{C}_{14} \mathrm{HH}$ is absent and leaves $\mathrm{C}_{8} \mathrm{HH}$ at 2.05 ppm integrating for $2 \mathrm{H}$. "diethyl ether. ${ }^{\dagger}$ unknown impurities. 

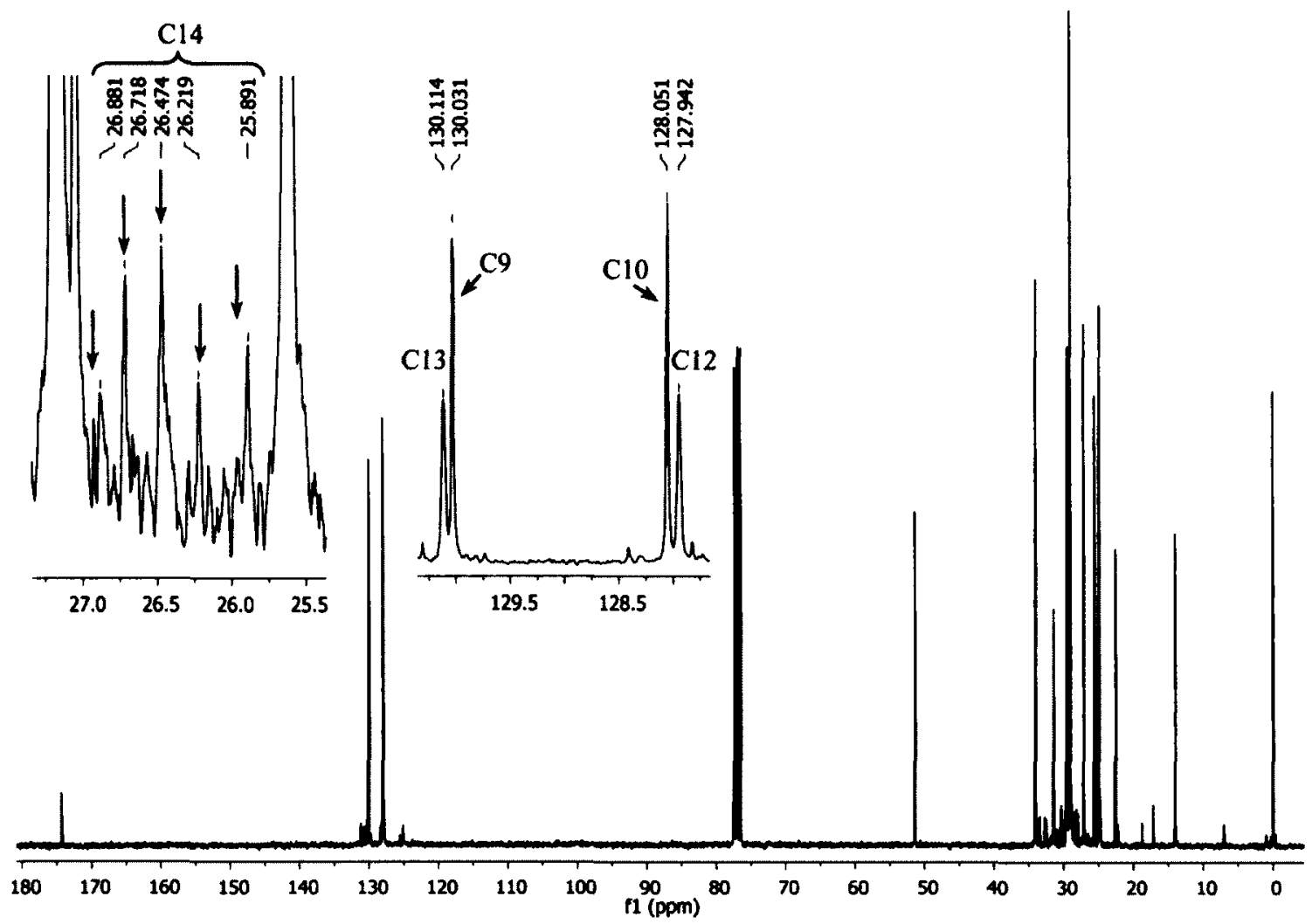

Figure 3.12. ${ }^{13} \mathrm{C}$ NMR spectrum of methyl $\left[14,14-{ }^{2} \mathrm{H}_{2}\right]$-Linoleate. The arrows are showing the pentet which is due to $C 14 D_{2}$ is isotope shifted to $26.47 \mathrm{ppm}$ ( $\alpha$ - shift of $0.719 \mathrm{ppm}$ ) relative to the corresponding signal in non-labelled linoleate. The ${ }^{13} \mathrm{C}$ signals due to $\mathrm{C} 12(127.94 \mathrm{ppm})$ and $\mathrm{C} 13(130.11 \mathrm{ppm})$ are reduced in intensity due to the presence of deuterium at C14. 


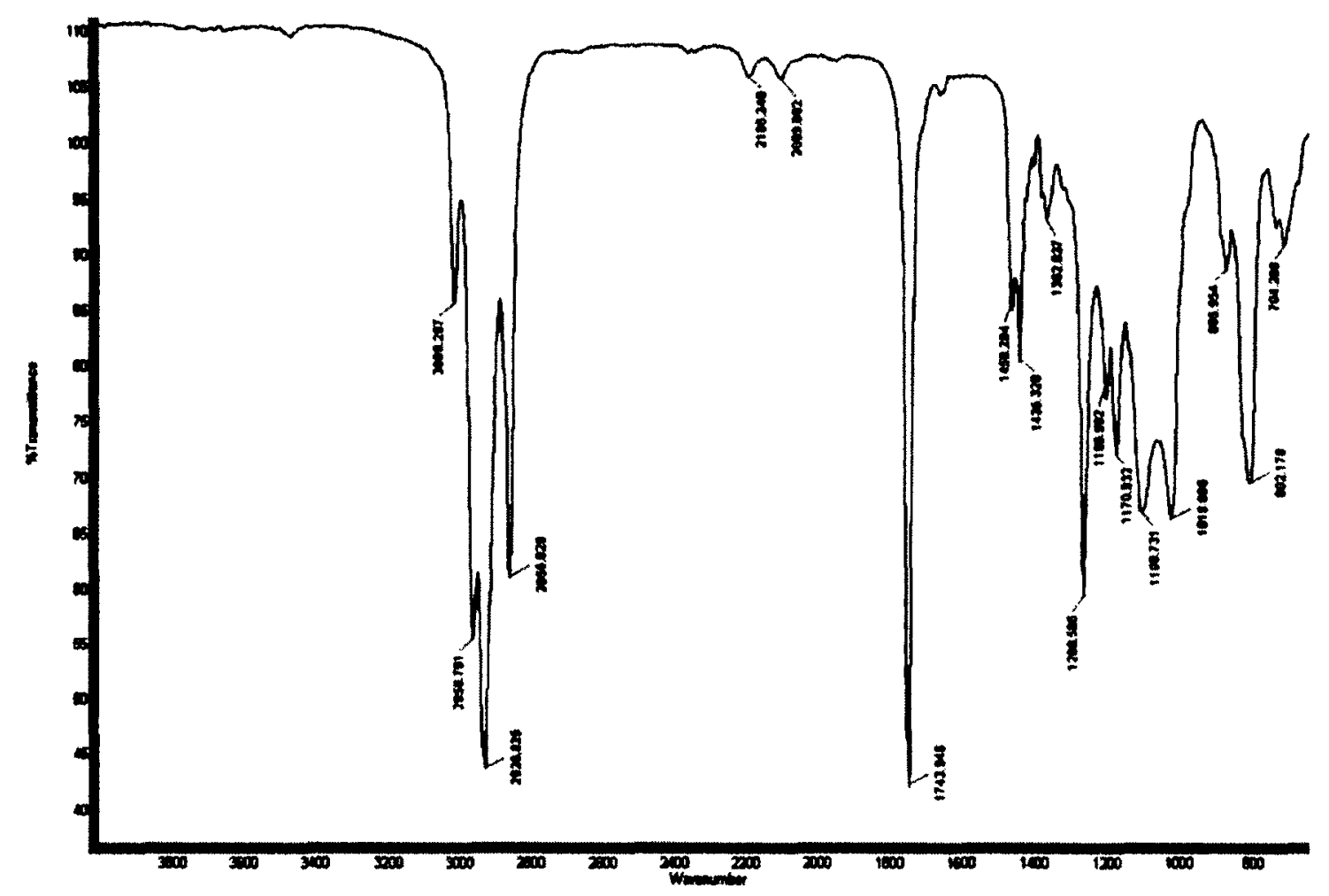

Figure 3.13. IR spectrum of methyl $\left[14,14-{ }^{2} \mathrm{H}_{2}\right]$-linoleate. Asymmetric and symmetric CD stretches due to $C_{14} \mathrm{DD}$ appear at 2099 and $2186 \mathrm{~cm}^{-1}$.

\subsubsection{Incubation of Substrates: Trial Incubation of $d_{0}$-Linoleate}

Before running the intermolecular competition between the $d_{0}$-linoleate and stereospecifically dideuterated linoleates, the efficiency of formation of the $\alpha$-eleostearate product from $d_{0}$-linoleate was examined in a redundant experiment using the newly constructed yeast mutant expressing the $\Delta^{11,13}$-desaturase enzyme (FADX gene) from Aleurites fordii Hemsl. In order to probe this, ca. $0.5 \mathrm{~mL}$ of sterilized linoleic acid solution in $10 \%$ Tergitol $(10 \mathrm{mg} / \mathrm{mL})$ was inoculated with a $50 \mathrm{~mL}$ growing culture of the pRTL2 myc-FADX strain of $S$. cerevisiae and allowed to shake at $20^{\circ} \mathrm{C}$ for 3 days followed by another 3 days at $15^{\circ} \mathrm{C}$ at $200 \mathrm{rpm}$. This incubation condition is similar to that used for the calendate experiments. A corresponding control experiment using only wild type $S$. cerevisiae without having the mutant expression (FADX - plasmid) was also 
run. The cellular fatty acid fractions were subsequently isolated from the centrifuged $S$. cerevisiae cells via a standard hydrolysis $\left(10 \% \mathrm{w} / \mathrm{v} \mathrm{KOH} / \mathrm{MeOH}\right.$ at $80{ }^{\circ} \mathrm{C}$ for $\left.2 \mathrm{~h}\right) /$ methylation $\left(\mathrm{CH}_{2} \mathrm{~N}_{2}\right)$ procedure known not to decompose the conjugated fatty ester ${ }^{158}$ and analyzed by GC-MS (Figure 3.14). Comparison of the fatty acid methyl ester (FAME) profile of the control experiment (FADX - plasmid) (data not shown) with the culture containing the pRTL2 myc-FADX strain of $S$. cerevisiae (Figure 3.14) revealed that only in the latter experiment was the exogenously supplied linoleic acid converted into the product $\alpha$-eleostearic acid to an extent of $2 \%$ of the total cellular fatty acids. The host yeast culture system was also shown to be unable to biosynthesize the substrate linoleic acid endogenously (data not shown here), as is necessary for the type of mechanistic investigations we were using for. The product was identified based on the comparison of the retention times of a reference standard mixture that distinguishes between different triene isomers. ${ }^{158}$ Comparison of the retention time of methyl $\alpha$ eleostearate (at $16.25 \mathrm{~min}$ ) with methyl calendate (at $16.43 \mathrm{~min}$ ) revealed that the former is eluting somewhat faster than the latter under comparable conditions. Analysis of the mass spectrum of the GC peak assigned to methyl $\alpha$-eleostearate (Figure 3.15) revealed that the presence of the molecular ion at $\mathrm{m} / \mathrm{z} 292\left([\mathrm{M}]^{+}\right)$and the other fragment ions at $\mathrm{m} / \mathrm{z} 261\left(\left[\mathrm{M}-\mathrm{OCH}_{3}\right]^{+}\right), 163\left(\left[\mathrm{Me}\left(\mathrm{CH}_{2}\right)_{4} \mathrm{CH}=\mathrm{CH}-\mathrm{CH}=\mathrm{CH}-\mathrm{CH}=\mathrm{CH}-\mathrm{CH}_{2}\right]^{+}\right), \quad 150$ $\left(\left[\mathrm{Me}\left(\mathrm{CH}_{2}\right)_{3} \mathrm{CH}=\mathrm{CH}-\mathrm{CH}=\mathrm{CH}-\mathrm{CH}=\mathrm{CH}-\mathrm{CH}_{2}\right]^{+}\right)$which are in accord with the proposed structure and similar to that reported previously. ${ }^{34}$ No oxygenated intermediate could be detected by silylation of the FAME as low-level "error" compound formation during 1,4desaturation. A similar examination of the methylated $\mathrm{CH}_{2} \mathrm{Cl}_{2}$ extract of the culture medium did not reveal any hydroxylated fatty acids. 


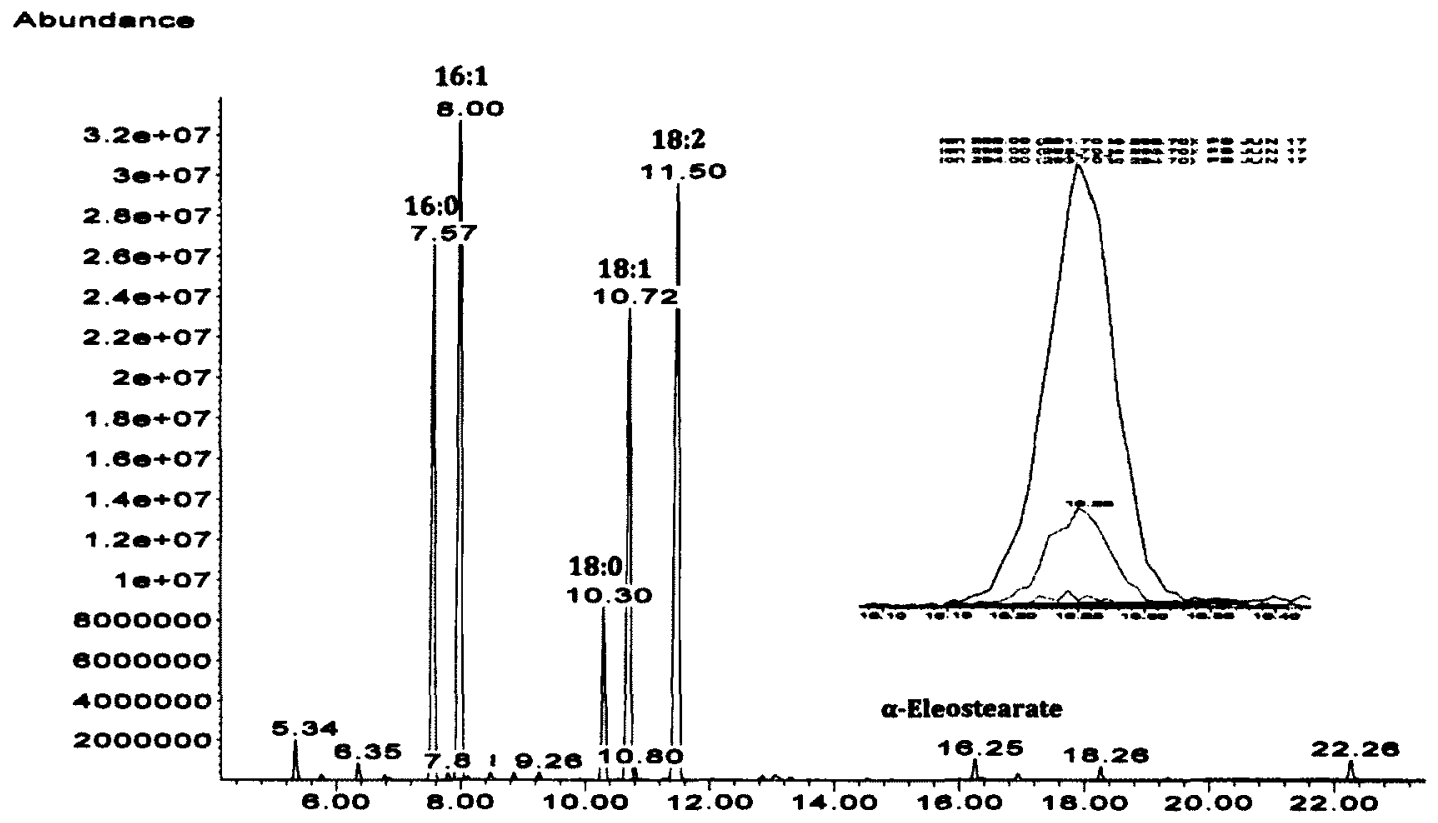

Time-->

Figure 3.14. The cellular fatty acid profile of $S$. cerevisiae grown in a medium supplemented with linoleic acid.

Abundance

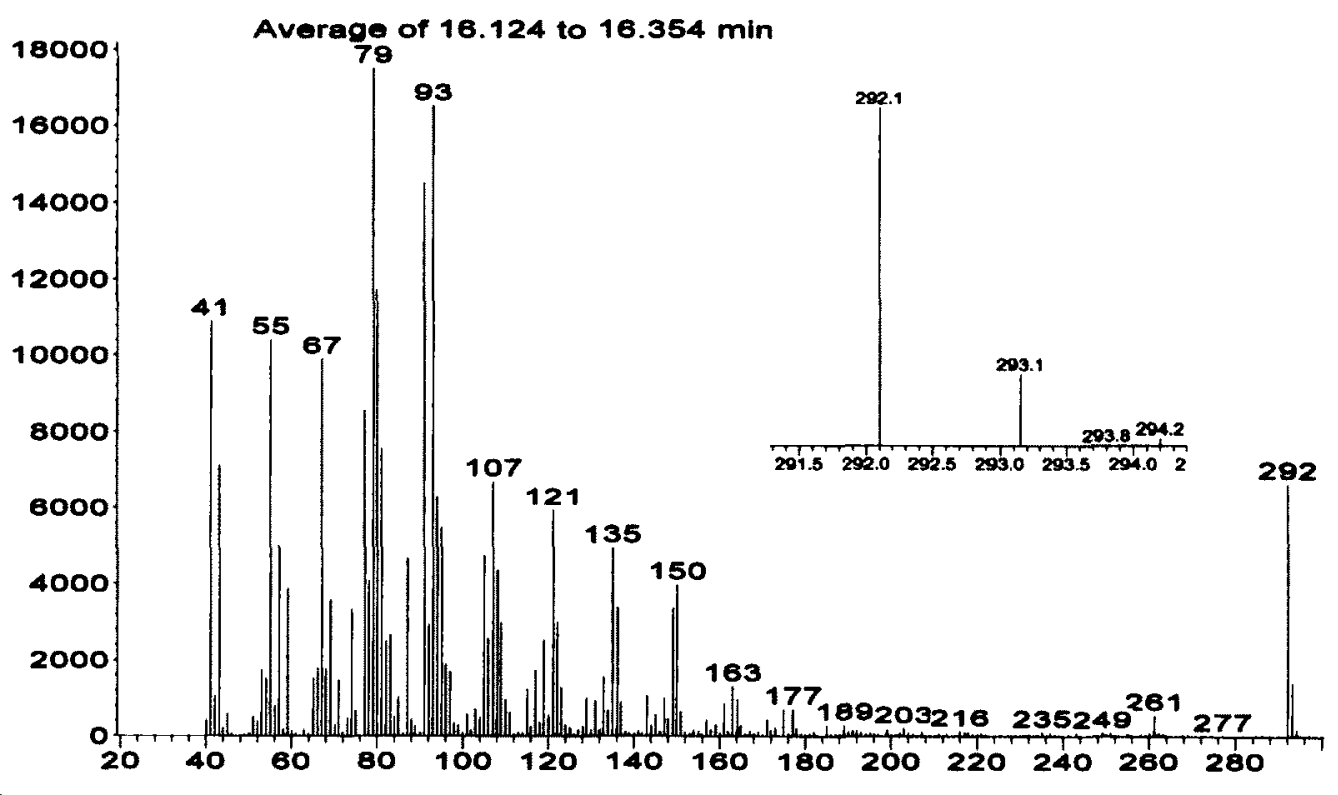

$m / \mathbf{z}->$

Figure 3.15. Mass spectrum of the biosynthetic methyl $\alpha$-eleostearate derived from methyl linoleate. 


\subsubsection{Intermolecular Kinetic Isotope Effect (KIE) Studies}

To determine the intermolecular primary deuterium kinetic isotope effects on each $\mathrm{C}$-H cleavage steps during $\alpha$-eleostearic acid formation by $\Delta^{11,13}$-desaturase enzyme from linoleic acid, a mixture consisting of approximately equivalent amount (1:1) of saponified methyl esters of non-labeled to stereospecifically labeled $\left[11,11-{ }^{2} \mathrm{H}_{2}\right]$ or $\left[14,14-{ }^{2} \mathrm{H}_{2}\right]$ linoleates was incubated as a solution in $10 \%$ Tergitol $(\sim 5 \mathrm{mg}$ each) to the growing cultures of pRTL2 myc-FADX strain of $S$. cerevisiae $(50 \mathrm{~mL})$. A small portion of the fatty acid mixture was withdrawn from the Tergitol solution in order to determine the isotopic deuterium content $\left(d_{0} / d_{2}\right.$ ratio) in the incubated mixtures by GC-MS. For each type of mixture, the incubation was carried out four times and the inoculated cultures were allowed to grow to the normal extent under the conditions identical to that of the trial experiment. The cellular fatty acid fractions were isolated from the harvested cells by the hydrolysis/methylation technique and the deuterium content of the recovered linoleic $\left(d_{0} / d_{2}\right)$ acid as well as the eleostearic acid products $\left(d_{0} / d_{1}\right)$ was assessed by GCMS. Comparison of the $d_{2} / d_{0}$ ratio of the cellular linoleate fraction with that measured in the administered mixture revealed that the ratios were essentially identical in both incubations, indicated that no dilution of the deuterium label occurred during the feeding experiment (data not shown). Maintenance of this ratio before and after the incubation is essential for this type of KIE study and the result was according to the expectation, since the host yeast system has been documented in the trial experiment not able to biosynthesize the $d_{0}$-substrate.

Mass spectral analysis of the GC peak assigned to $\alpha$-eleostearate fraction (Figure 3.16 and 3.17) demonstrated that in both incubations, the product consisted entirely as a 
Abundance

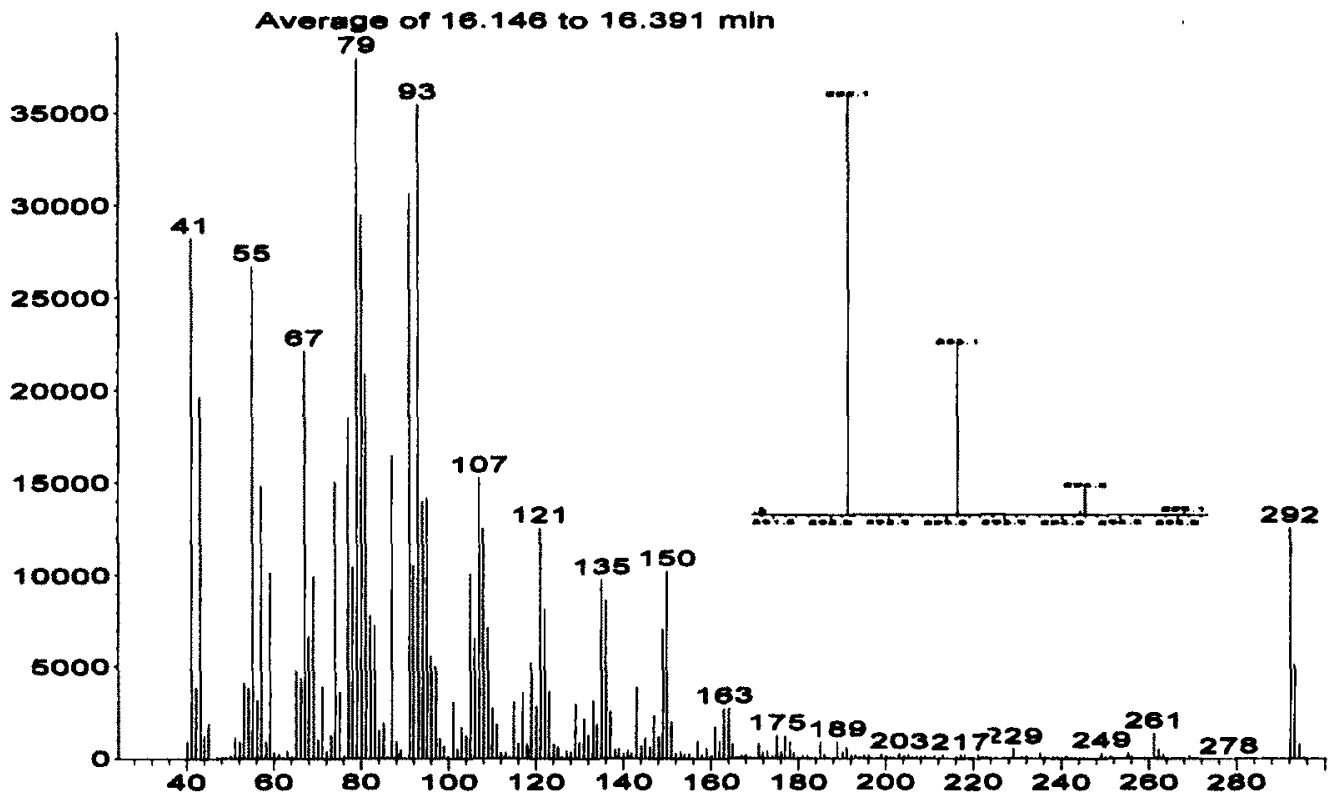

$m / \mathbf{z}->$

Figure 3.16. Representative mass spectrum of biosynthetic $\alpha$-eleostearate derived from 1:1 mixture of $d_{0}$-linoleate and $11-d_{2}$-linoleate.

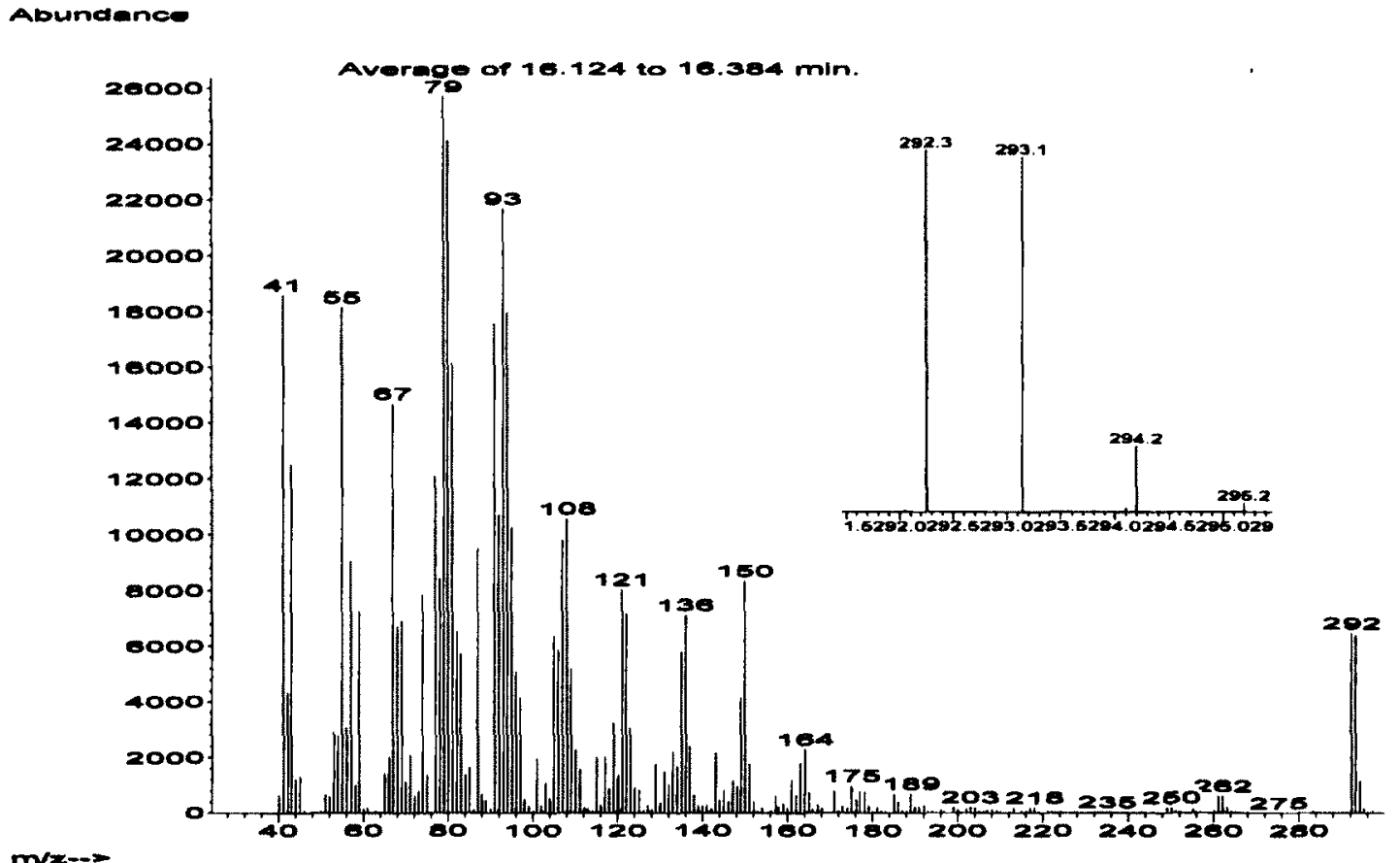

Figure 3.17. Representative mass spectrum of biosynthetic $\alpha$-eleostearate derived from 1:1 mixture of $d_{0}$-linoleate and $14-d_{2}$-linoleate. 
mixture of $d_{0} / d_{1}$ which clearly indicating the loss of one deuterium from $d_{2}$-substrate as expected.

Since, the overall \% conversion was less than $10 \%$ in both incubations as clearly evident from the gas chromatograms of the FAMEs, the product kinetic isotope effects $\left(\mathrm{k}_{\mathrm{H}} / \mathrm{k}_{\mathrm{D}}\right)$ could be calculated. The following equation is used: ${ }^{199}$

$$
\frac{k_{H}}{k_{D}}=\frac{P_{H} / P_{D}}{S_{H}^{0} / S_{D}^{0}}
$$

Where, ${ }_{H} / P_{D}$ is the ratio of the nondeuterated to monodeuterated methyl $\alpha$-eleostearate and $S_{H}^{0} / S_{\mathbb{D}}^{0}$ is the ratio of the nondeuterated to regiospecifically dideuterated $\left[11,11-{ }^{2} \mathrm{H}_{2}\right]-$ or $\left[14,14-{ }^{2} \mathrm{H}_{2}\right]$-linoleate. Thus, the product kinetic isotope effects resolved to:

$$
\frac{k_{H}}{k_{D}}=\frac{\left[\% d_{0} \text { Product }\right] /\left[\% d_{1} \text { Product }\right]}{\left[\% d_{0} \text { Substrate }\right] /\left[\% d_{2} \text { Substrate }\right]}
$$

The analytical data obtained from our competitive experiment is exhibited in Table 3.1. Using the formulation described above, the presence of a large primary deuterium isotope effect $(4.9 \pm 0.4)$ for the carbon-hydrogen bond cleavage at $\mathrm{C}-11$ was calculated, whereas the carbon-hydrogen bond cleavage at C-14 was found to be insensitive to isotopic substitution ( $\mathrm{KIE}=1.12 \pm 0.02$ ). It should be remembered that the magnitude of the 'intrinsic KIE' on C-11 C-H bond cleavage may well be much higher than the determined value, since the estimated value was derived via an intermolecular 
competition that is subjected to partial masking by other enzymatic steps such as, substrate binding or product release. ${ }^{1,201}$ It is also true that the observed isotope effects calculated in this manner typically incorporates a small amount $(\sim 10 \%)$ of a normal $a-$ secondary KIE associated with conversion of an $\mathrm{sp}^{3}$ centre to an $\mathrm{sp}^{2}$ carbon. ${ }^{160}$

Table 3.1. Intermolecular isotopic discrimination by the Aleurites fordii Hemsl. $\Delta^{11,13}$ desaturase mediated 1,4-desaturation of methyl $\left[11,11-{ }^{2} \mathrm{H}_{2}\right]$-linoleate 31 and methyl $\left[14,14^{2} \mathrm{H}_{2}\right]$-linoleate 32 .

\begin{tabular}{|c|c|c|c|c|c|c|}
\hline \multirow[t]{2}{*}{$\begin{array}{l}\text { Position of } \\
\text { Isotope in } \\
d_{2} \text {-Species }\end{array}$} & \multirow[t]{2}{*}{ Trial } & \multicolumn{2}{|c|}{$\begin{array}{c}\text { Cellular Linoleic acid } \\
\text { Substrate } \mathrm{e}^{a, b, c}\end{array}$} & \multicolumn{2}{|c|}{$\begin{array}{c}\text { Cellular Eleostearic acid } \\
\text { Product }^{a . c}\end{array}$} & \multirow[t]{2}{*}{$\mathrm{KIE}$} \\
\hline & & $d_{0}$ & $d_{2}$ & $\mathrm{~d}_{0}$ & $\mathrm{~d}_{1}$ & \\
\hline \multirow{4}{*}{$C-11$} & 1 & 49.7 & 50.3 & 83.8 & 16.2 & 5.2 \\
\hline & 2 & 48.6 & 51.4 & 82.0 & 18.0 & 4.8 \\
\hline & 3 & 49.3 & 50.7 & 83.6 & 16.4 & 5.2 \\
\hline & 4 & 51.1 & 48.9 & 81.8 & 18.2 & 4.3 \\
\hline \multirow{6}{*}{ C-14 } & Average & & & & & $4.9 \pm 0.4^{d}$ \\
\hline & 1 & 54.0 & 46.0 & 56.3 & 43.7 & 1.10 \\
\hline & 2 & 52.8 & 47.2 & 55.6 & 44.4 & 1.12 \\
\hline & 3 & 53.4 & 46.6 & 56.9 & 43.1 & 1.15 \\
\hline & 4 & 52.3 & 47.7 & 54.6 & 45.4 & 1.10 \\
\hline & Average & & & & & $1.12 \pm 0.02^{e}$ \\
\hline
\end{tabular}

${ }^{a}$ The isotopic content of both substrate and product mixtures were evaluated by considering the $(M+1)$, $(M+2)$ as well as (M-1), (M-2) contributions from $d_{0}-, d_{1}$ - and $d_{2}$-species. $d_{0}, d_{1}$ and $d_{2}$ refer to the nondeuterated, monodeuterated and dideuterated species, respectively. ${ }^{b}$ The isotopic composition of the cellur linoleic acid fractions in both incubations were similar to that measured prior to the incubation. ${ }^{c}$ The deuterium content (\%) of each species is given as an average value based on four GC-MS runs. The average standard deviation for these measurements is \pm 0.4 expressed as a percentage. ${ }^{d}$ The average KIE at

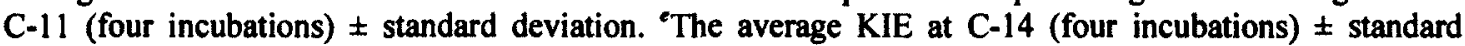
deviation. 
As also described in Chapter 2 (calendate biosynthesis), an independent evaluation of the deuterium isotope effect at C-11 during the biosynthesis of $\alpha$-eleostearate became possible due to the availability of racemic methyl $\left[11-{ }^{2} \mathrm{H}_{1}\right]-$ linoleate. Thus, two independently synthesized samples of methyl $\left[11-{ }^{2} \mathrm{H}_{1}\right]$-linoleates $(\sim 5 \mathrm{mg})$, one derived from $(1 R)-\left[1-{ }^{2} \mathrm{H}_{1}\right]-2-o c t y n-1-0125 a$ and the other from $(1 S)-\left[1-{ }^{2} \mathrm{H}_{1}\right]-2$-octyn-1-ol $25 \mathbf{b}$ were incubated separately with the growing $S$. cerevisiae strain expressing $\Delta^{11,13}$ desaturase enzyme from $A$. fordii Hemsl. After saponification with a growing culture of pRTL2 myc-FADX strain of $S$ cerevisiae $(50 \mathrm{~mL})$ under the optimal incubation conditions as previously described to let the cultures grow to its normal extent. The fatty acid methyl esters (FAMEs) from the harvested yeast cells were isolated as described before and analyzed by GC-MS. Mass spectral analysis of the GC peak assigned to the product $\alpha$-eleostearate derived from both $\left[11-{ }^{2} \mathrm{H}_{1}\right]$-linoleates revealed that the deuterium content was essentially identical (within experimental error): $76.8 \pm 0.4 \mathrm{~d}_{1}-$ and $23.2 \pm$ $0.4 \mathrm{~d}_{0}$-eleostearate. This results confirmed that both of the $\left[11-{ }^{2} \mathrm{H}_{1}\right]$-linoleates were stereochemically alike i.e. racemic. This permitted calculation of the product KIE for CH cleavage at C-11 in the manner discussed in Chapter 2.

$$
\frac{k_{H}}{k_{D}}=\frac{\left[\% P_{d_{1}}+\left\{\left(\% P_{d_{1}} \times \% S_{d_{0}}\right) / \% S_{d_{1 R}}\right\}\right] /\left[\% P_{d_{0}}-\left\{\left(\% P_{d_{1}} \times \% S_{d_{0}}\right) / \% S_{d_{1 R}}\right\}\right]}{\left[\left(\% S_{d_{1 R}}+\% S_{d_{0}}\right) / \% S_{d_{1 S}}\right]}
$$


Table 3.2. Intermolecular isotopic discrimination by the Aleurites fordii Hemsl. $\Delta^{11,13}$ desaturase in the 1,4-dehydrogenation of racemic methyl $\left[11-{ }^{2} \mathrm{H}_{1}\right]$-linoleate.

\begin{tabular}{|c|c|c|c|c|c|c|c|}
\hline \multirow[t]{2}{*}{$\begin{array}{l}\text { Position of } \\
\text { Isotope in } \\
d_{1} \text {-Species }\end{array}$} & \multirow[t]{2}{*}{ Trial $^{a}$} & \multicolumn{3}{|c|}{$\begin{array}{l}\text { Cellular Linoleic acid } \\
\text { Substrate }^{b, c, d}\end{array}$} & \multicolumn{2}{|c|}{$\begin{array}{l}\text { Cellular Eleostearic } \\
\text { acid Product }{ }^{b . d}\end{array}$} & \multirow[t]{2}{*}{$\mathrm{KIE}$} \\
\hline & & $\mathrm{d}_{0}$ & $\mathrm{~d}_{1}-(R)$ & $\mathrm{d}_{1}-(S)$ & $\mathrm{d}_{0}$ & $d_{1}$ & \\
\hline & $1 \mathrm{a}$ & & & & 23.5 & 76.5 & 4.0 \\
\hline \multirow[t]{4}{*}{$C-11$} & $2 a$ & 2.7 & 48.65 & 48.65 & 23.5 & 76.5 & 4.0 \\
\hline & $3 a$ & & & & 23.3 & 76.7 & 4.0 \\
\hline & Average & & & & & & $4.0 \pm 0.1^{e}$ \\
\hline & $1 b$ & & & & 23.4 & 76.6 & 3.8 \\
\hline \multirow[t]{3}{*}{$C-11$} & $2 b$ & 2.2 & 48.9 & 48.9 & 22.4 & 77.6 & 4.1 \\
\hline & $3 b$ & & & & 23.3 & 76.7 & 3.9 \\
\hline & Average & & & & & & $3.9 \pm 0.2^{e}$ \\
\hline
\end{tabular}

${ }^{a}$ Trial la-3a were accomplished by using the $(1 R)-\left[1-{ }^{2} \mathrm{H}_{1}\right]-2$-octyn-1-ol-derived $\left[11-{ }^{2} \mathrm{H}_{1}\right]$-linoleate and trial $1 \mathrm{~b}-3 \mathrm{~b}$ were accomplished by using the $(1 S)-\left[1-{ }^{2} \mathrm{H}_{1}\right]-2$-octyn-1-ol-derived $\left[11-{ }^{2} \mathrm{H}_{1}\right]$-linoleate. ${ }^{b}$ The isotopic content of both substrates and products were evaluated by considering the $(\mathrm{M}+1)$ and $(\mathrm{M}-1)$ contributions from $d_{0}$ - and $d_{1}$-species, respectively. $d_{0}$ and $d_{1}$ refers to non-deuterated and monodeuterated species, respectively. ${ }^{c}$ The isotopic ratio of the cellular linoleate fractions were similar to that measured prior to the incubation. ${ }^{d}$ The deuterium content (\%) of each species is given as an average value based on three GCMS runs. The average standard deviation for these measurements is \pm 0.4 expressed as a percentage. The standard deviation is considered to be similar to the standard error for each individual KIE value. "The average KIE at C-11 (three incubations) \pm standard deviation.

The results clearly point to the existence of a large primary deuterium kinetic isotope effect $(4.0 \pm 0.2)$ for the carbon-hydrogen bond cleavage at $\mathrm{C}-11$ during the formation of $\alpha$-eleostearic acid from linoleic acid. Once again, the calculated value was found to be substantially lower $(\sim 81 \%)$ compared to that obtained using equal amount (1:1) of non-deuterated to $11-\mathrm{d}_{2}$-linoleate $(4.9 \pm 0.4)$ as reported above for the same biotransformation reaction. As described in the calendate Section 2.2.3.3, here also this 
result can be explained by considering the potential impact of $\alpha$-secondary isotope effects on both ways of calculating KIE. ${ }^{160}$ It is interesting to note that the consequence of including the $\alpha$-secondary isotope effects on the primary deuterium KIEs appears to be similar, $81 \%$ vs. 77 \% (calendate KIE) in both cases, as might be expected for reactions following a similar mechanism by the same family of enzymes.

In conclusion, the data presented above (i.e. one large $\mathrm{KIE}$ at $\mathrm{C}-11$ and one small $\mathrm{KIE}$ at C-14) clearly demonstrates the stepwise nature of the 1,4-desaturation reaction to form a-eleostearate. Based on our experimental finding, it can now be described that the $\alpha$-eleostearate formation is initiated by a relatively slow and hence isotopically sensitive hydrogen abstraction at $\mathrm{C}-1 \mathrm{l}$ and completed by a second facile and kinetically unimportant hydrogen removal at the $\mathrm{C}-14$ position. The observed result was similar to that reported for the biosynthesis of calendic acid (Chapter 2), which has also been observed to initiate the oxidation at $\mathrm{C}-11$ of the linoleyl chain. ${ }^{158}$ It is significant that in both cases the cryptic site of initial oxidation is close to $\mathrm{C}-12$, the site of initial oxidation for parent FAD2 ${ }^{1,112}$ enzyme.

The stepwise removal of hydrogens from two non-adjacent carbons during the conjugated double bond formation also matches closely with the pattern observed in insects during the biosynthesis of $(E, E)$-10,12-tetradecadienoic acid from (Z)-11tetradecenoic acid as a component in moth Spodoptera littoralis sex pheromone (Figure 1.29). ${ }^{39}$ The site of initial oxidation for this insect 1,4-desaturase enzyme was revealed to be at $\mathrm{C}-10^{39}$ which is close to $\mathrm{C}-11$, the cryptic site of initial oxidation for its parent $\Delta^{11}$ desaturase $^{139}$ (Figure 1.5). This observation signifies that like tung $\Delta^{11,13}$-desaturase, this 
insect $\Delta^{10,12}$-desaturase enzyme also maintains a close relationship with its parent $\Delta^{11}$ desaturase in terms of cryptoregiochemistry ${ }^{39,139}$ of fatty acid oxidation.

\subsubsection{Stereochemistry of $\Delta^{11,13}$-Desaturation}

\subsubsection{Synthesis of Chiral Methyl $\left[14^{2} \mathrm{H}_{1}\right]-$ Octadeca-9Z,12Z-Dienoates}

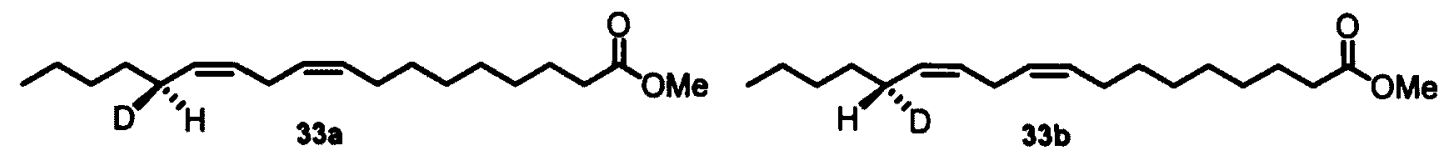

The syntheses of methyl (14S)- and (14R)-[14- $\left.{ }^{2} \mathrm{H}_{1}\right]$-octadeca-9Z,12Z-dienoates

33a and 33b were performed in $\sim 2 \%$ overall yield, starting with commercially available N,N-dimethylformamide- $d_{1}\left(99\right.$ atom \% D) (Figure 3.18). The intermediate $\left[1-{ }^{2} \mathrm{H}_{1}\right]-$ pentanal $41^{198}$ was reduced in asymmetric fashion to the enantiomeric monodeuterio 1pentanols $42 \mathrm{a}$ and $\mathbf{4 2 b}$ using well established Alpine-Borane method described previously in this thesis (Figure 2.5). ${ }^{174,185}$ The absolute configuration as well as the enantiomeric purity of these chirally reduced alcohols was assessed by ${ }^{1} \mathrm{H}$ NMR after derivatization using (S)-MTPA chloride. ${ }^{173,189}$ 


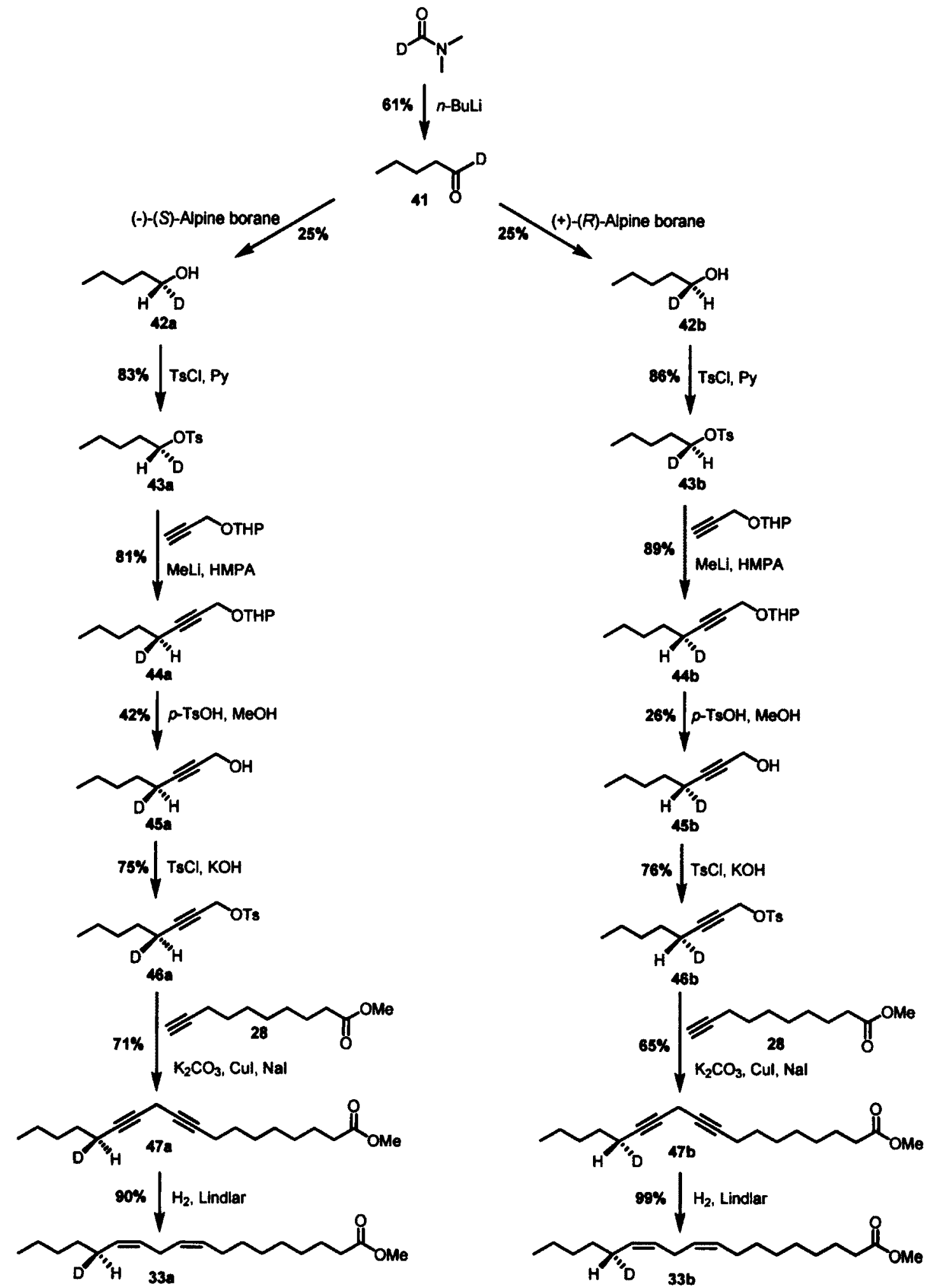

Figure 3.18. Synthesis of chiral methyl $\left[14-{ }^{2} \mathrm{H}_{1}\right]$-linoleates. 
Integration of the diastereotopic ${ }^{1} \mathrm{H}$ resonances assigned to the $\mathrm{C}-1 \mathrm{CHD}$ group of the MTPA ester of $(1 S)-\left[1-{ }^{2} \mathrm{H}_{1}\right]$-pentanol (major) vs. $(1 R)-\left[1-{ }^{2} \mathrm{H}_{1}\right]$-pentanol (minor) shown in the left panel of Figure 3.19 yielded an estimated ee of $90 \%$. The presence of impurities in the $(1 R)-\left[1-{ }^{2} \mathrm{H}_{1}\right]$-pentanol MTPA ester (right panel of Figure 3.19) prevented a similar analysis for this compound. As expected ${ }^{189}$, the C-1 CHD resonance of the $(+)-(R)$-MTPA ester of $(1 S)-\left[1-{ }^{2} \mathrm{H}_{1}\right]$-pentanol appears down field relative to the the corresponding signal of the (1R)-alcohol.

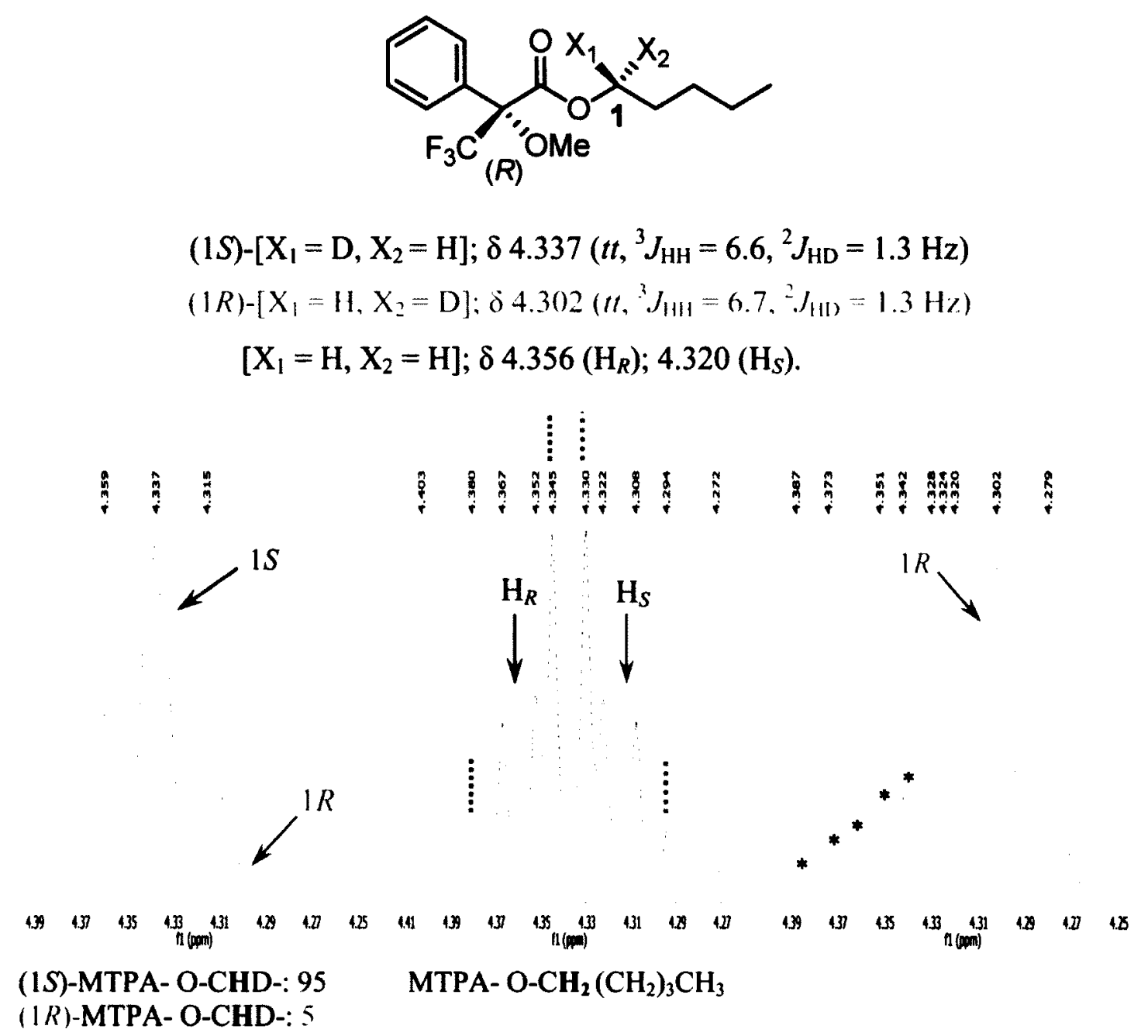

Figure 3.19. ' $\mathrm{H}$ resonances at $\mathrm{C}-1$ of MTPA ester of $(1 S)-,(1 R)-\left[1-{ }^{2} \mathrm{H}_{1}\right]$-pentanol (triplet) and $\mathrm{d}_{0}$-pentanol (AB quartet). ${ }^{*}$ are due to the impurities. $\alpha$-Deuteration causes a small upfield shift $(\sim 0.018,0.019 \mathrm{ppm})$ of the signal due to the geminal proton. 
Analyses of the mass spectra of these chiral deuterio alcohols as TMS derivative $^{187}$ revealed that each enantiomer was obtained mainly as monodeuterated species. The tosylates ${ }^{174,179,184}$ of chiral deuterio 1-pentanols were reacted with the THPderivative of propargyl alcohol in the presence of methyllithium in HMPA to give the THP protected $\left[4-{ }^{2} \mathrm{H}_{1}\right]-2-0 c t y n-1-o l s,{ }^{174} 44 a$ and $44 b$. Displacement of the tosylate by acetylide anions is believed to take place via inversion of configuration and proof for such a claim has been provided as discussed in the next Section 3.2.3.2. Following the deprotection of the THP ether derivatives, the resultant chiral deuterio 2-octyn-1-ols 45a and $45 b^{174,196}$ were tosylated and then coupled ${ }^{175-178,190}$ with methyl 9-decynoate in presence of mild base $\mathrm{K}_{2} \mathrm{CO}_{3}$, copper iodide and sodium iodide in dry DMF. The skipped 1,4-diynoates $47 \mathrm{a}$ and $47 \mathrm{~b}$ generated in this manner were semi-hydrogenated by Lindlar catalyst in hexane to generate the title compounds $\mathbf{3 3 a}$ and $\mathbf{3 3 b}$ as faint-yellowish clear oils after flash chromatography. ${ }^{158,173}$

The proposed structures, chemical and isotopic purity of these compounds were confirmed by comparing the GC-MS (Figure 3.20), ${ }^{1} \mathrm{H}$ NMR (Figure 3.21 ), ${ }^{13} \mathrm{C}$ NMR (Figure 3.22) and IR (Figure 3.23) spectra with that of non-labeled linoleate as well as with other isotopically substituted linoleates in this series. Representative spectra are shown below: 


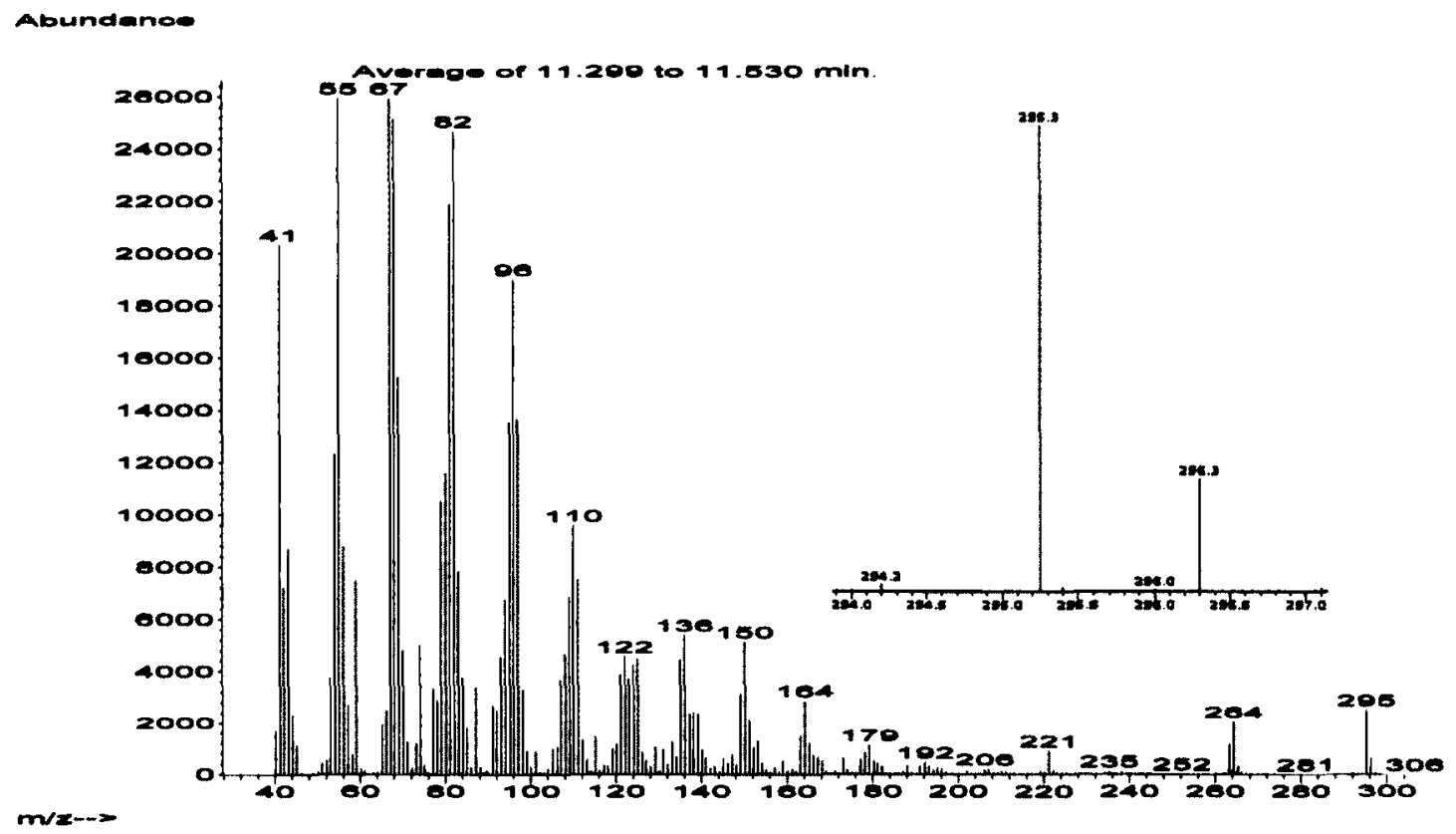

Figure 3.20. Mass spectrum of methyl $(14 S)-\left[14-{ }^{2} \mathrm{H}_{1}\right]$-linoleate 33a. Molecular ion at $\mathrm{m} / \mathrm{z}$ 295.
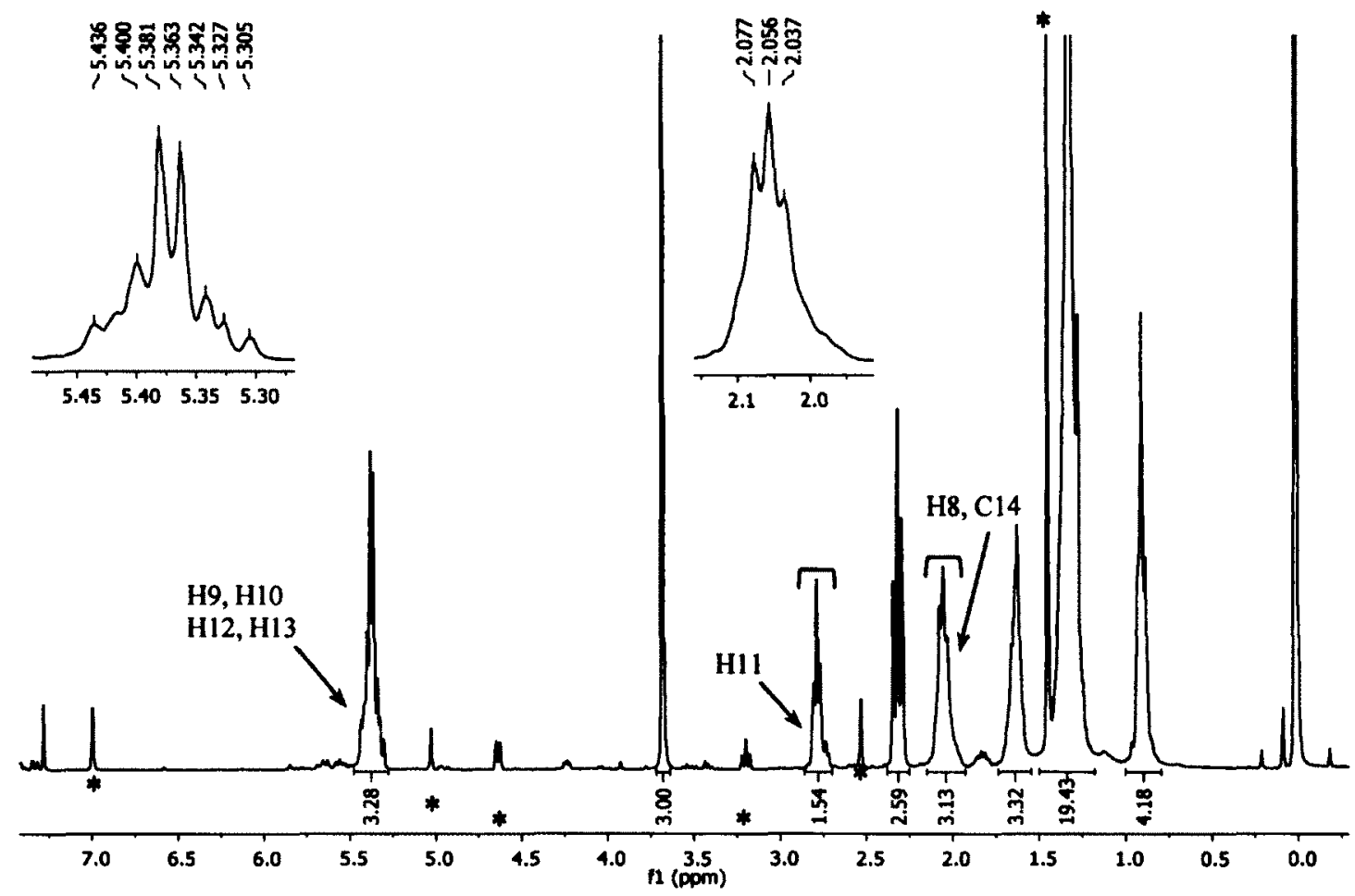

Figure 3.21. ${ }^{1} \mathrm{H}$ NMR spectrum of methyl $(14 S)-\left[14-^{2} \mathrm{H}_{1}\right]$-linoleate 33a. The overlapping resonances due to $\mathrm{C}_{14} \mathrm{HD}$ and $\mathrm{C}_{8} \mathrm{HH}$ at $2.06 \mathrm{ppm}$ is reduced in intensity relative to that of unlabeled linoleate and integrated to $3 \mathrm{H}$ as expected. ${ }^{*}$ are due to the presence of minor amount of impurities. 


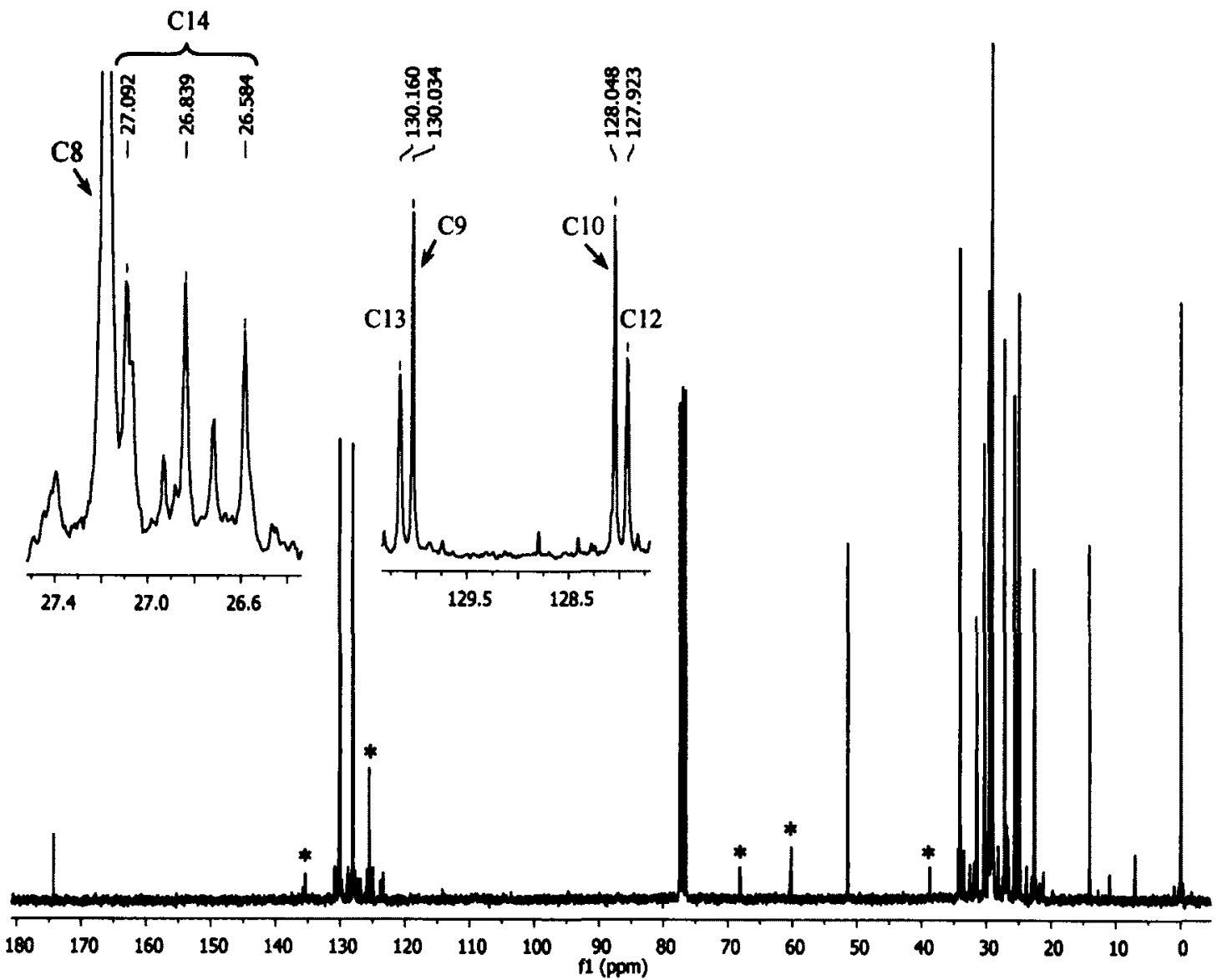

Figure 3.22. ${ }^{13} \mathrm{C}$ NMR spectrum of methyl $(14 S)-\left[14-{ }^{2} \mathrm{H}_{1}\right]$-linoleate 33a. The triplet $\left(J_{\mathrm{CD}}\right.$ $=76.2 \mathrm{~Hz})$ due to $\mathrm{C}_{14} \mathrm{HD}$ at $26.84 \mathrm{ppm}$ is isotope shifted $(-0.359 \mathrm{ppm})$ relative to that of unlabeled linoleate. The ${ }^{13} \mathrm{C}$ signals due to $\mathrm{C} 12$ (127.94 ppm) and $\mathrm{C} 13$ (130.17 ppm) are reduced in intensity due to the presence of deuterium at $\mathrm{C} 14$. "are due to the presence of minor amount of impurities. 


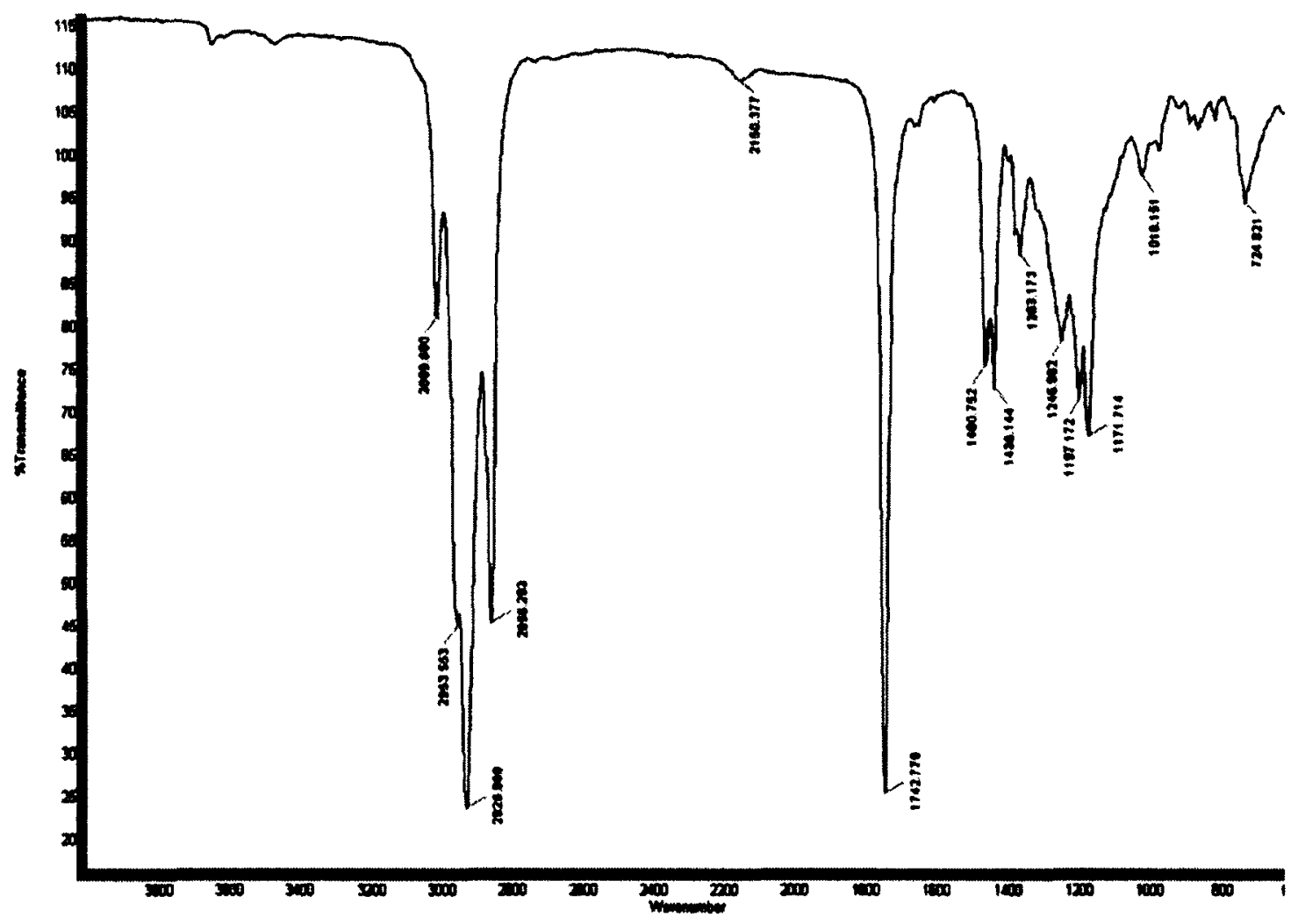

Figure 3.23. IR spectrum of methyl (14S)-[14- $\left.{ }^{2} \mathrm{H}_{1}\right]$-linoleate 33a. The C-D stretch due to $\mathrm{C}_{14} \mathrm{HD}$ appears at $2150 \mathrm{~cm}^{-1}$.

\subsubsection{Stereochemical analysis of Acetylide Anion Attack on Chiral CHD Tosylates}

During the syntheses of chiral monodeuterio fatty acids, it is assumed that the nucleophilic displacement of chiral tosylates by acetylide ion takes place predominantly in $S_{N} 2$ fashion with inversion of configuration. In this section, an attempt to investigate the extent of configurational inversion is described (Figure 3.24). The chiral monodeuterio [4- $\left.{ }^{2} \mathrm{H}_{1}\right]-2-o c t y n-1-o l s ~ 45 a$ and $45 b$ prepared during the synthesis of chiral [14- $\left.{ }^{2} \mathrm{H}_{1}\right]$-linoleates were semi-hydrogenated via Lindlar catalyst to produce the cis-[4${ }^{2} \mathrm{H}_{1}$ ]-2-octen-1-ols 48a and 48b, respectively. ${ }^{202,203}$ The allylic alcohols generated in this fashion were further processed for oxidative cleavage of the olefinic double bond to synthesize the corresponding chiral $\left[2-{ }^{2} \mathrm{H}_{1}\right]$-hexanoic acids $49 \mathrm{a}$ and $49 \mathrm{~b}$ under extremely 
mild conditions via the use of periodic acid $\left(\mathrm{IO}(\mathrm{OH})_{5}\right)$ and the ruthenium(III) chloride hydrate as catalyst. ${ }^{204,205}$ Subsequently, the resultant chiral deuterio hexanoic acids were esterified with the Parker reagent, $(+)-(S)$-methyl mandelate and these esters analyzed by ${ }^{1} \mathrm{H}$ NMR ${ }^{206}$ (Figure 3.25).
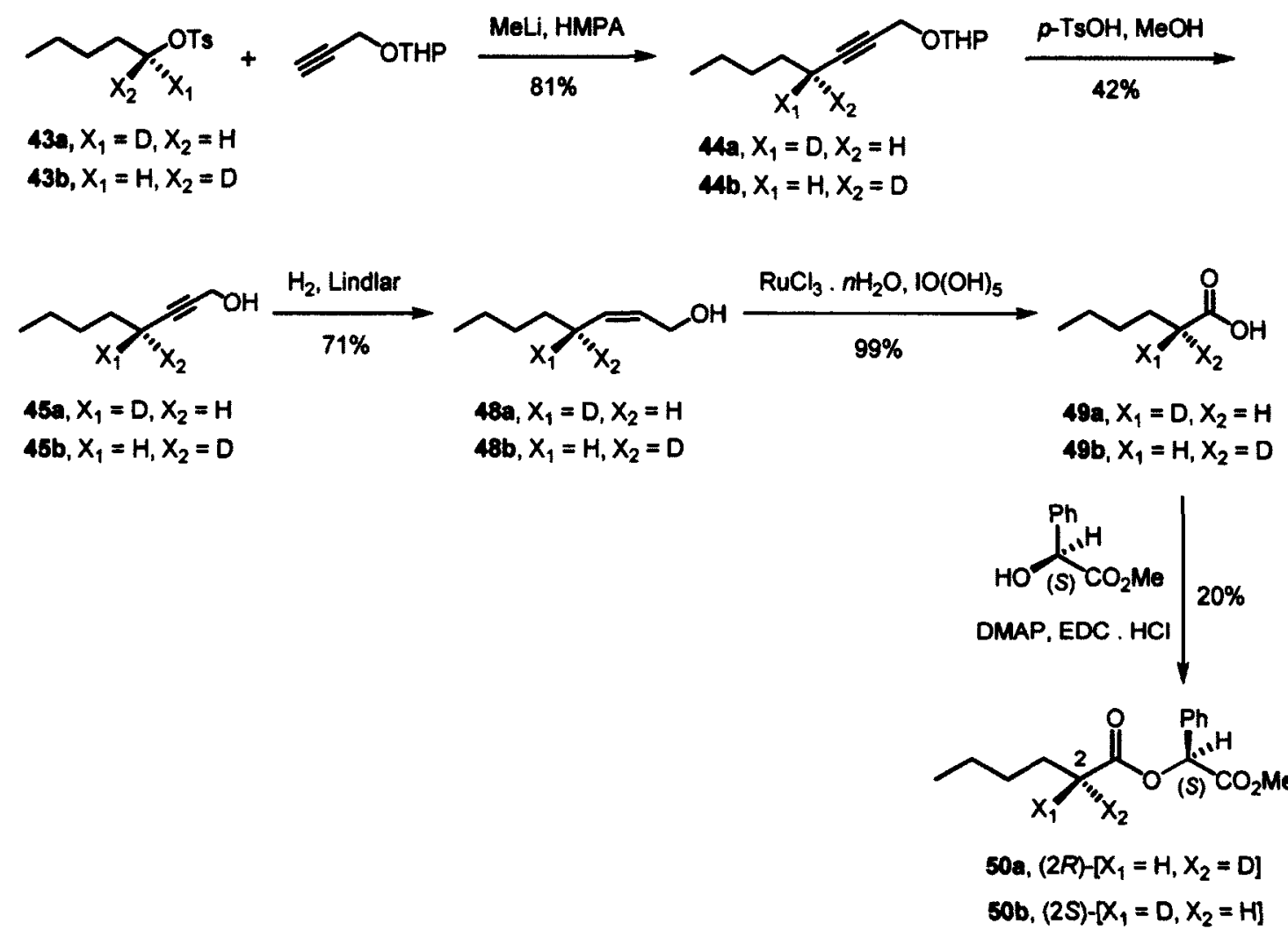

Figure 3.24. Stereochemical analysis of tosylate displacement by alkyne anion.

While a major unknown impurity in the samples of the chiral $d_{1}$-hexanoates prevented accurate assessment of the \% ee of these compounds, it is clear that tosylate displacement did proceed with inversion as the major pathway. As illustrated in Figure 3.25 , it is apparent that in the MMA ester of $(2 R)-\left[2-{ }^{2} \mathrm{H}_{1}\right]$-hexanoic acid, the $\mathrm{H}_{S}$ resonance of $-\mathrm{CHD}$ moiety is shifted upfield due to phenyl shielding relative to the corresponding ${ }^{1} \mathrm{H}$ resonance in the MMA ester of $(2 S)-\left[2-{ }^{2} \mathrm{H}_{1}\right]$-hexanoic acid as expected. 


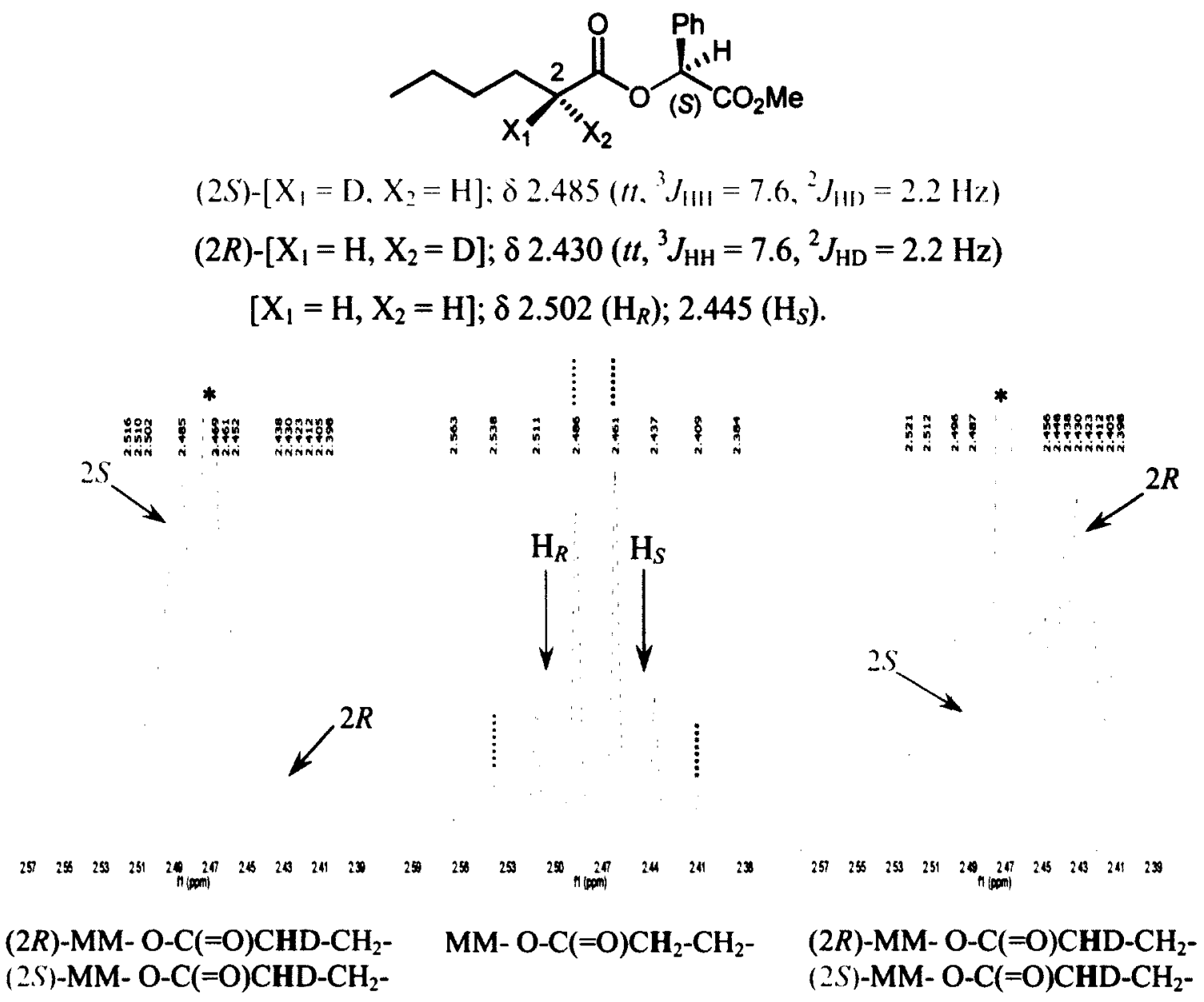

Figure 3.25. ' $\mathrm{H}$ resonances at $\mathrm{C}-2$ of $(S)-\mathrm{O}$ methyl mandelate $(\mathrm{MM})$ ester of $(2 S)-,(2 R)$ $\left[2-{ }^{2} \mathrm{H}_{1}\right]$-hexanoic acid (triplet of triplets) and $\mathrm{d}_{0}$-hexanoic acid (AB quartet). $\alpha$ Deuteration causes a small upfield shift $(\sim 0.017,0.015 \mathrm{ppm})$ of the signal due to the geminal proton. "signals of the major unknown impurity.

\subsubsection{Incubation of Chiral $d_{1}$-Linoleates}

The enantioselectivity of hydrogen removal by $\Delta^{11,13}$-desaturase during $\alpha$ eleostearate formation was investigated in analogous fashion to that described for $\Delta^{8,10}$. desaturation in Chapter 2 (Section 2.2.3.2). Chiral $\left[11-{ }^{2} \mathrm{H}_{1}\right]$-linoleates $2 \mathrm{a}$ and $2 \mathrm{~b}$ and chiral $\left[14^{-2} \mathrm{H}_{1}\right]$-linoleates 33a and 33b $(\sim 5 \mathrm{mg}$ of each saponified substrate) were administered independently, in triplicate and quadruplicate respectively, to growing cultures of pRTL2 myc-FADX strain of $S$. cerevisiae $(50 \mathrm{ml})$. The deuterium content of 
methyl $\alpha$-eleostearate obtained in each experiment was ascertained by GC-MS analysis of the isolated fatty acid methyl ester (FAME) fraction of the collected yeast cells.

Mass spectral analysis of the $\alpha$-eleostearate product derived from $(11 R)-\left[11-{ }^{2} \mathrm{H}_{1}\right]-$ linoleic acid revealed the appearance of the most abundant molecular ion at $\mathrm{m} / \mathrm{z} 292$ (Figure 3.26), this represents the overall loss of deuterium from the fatty acid during enzymatic oxidation.

\section{Abundance}

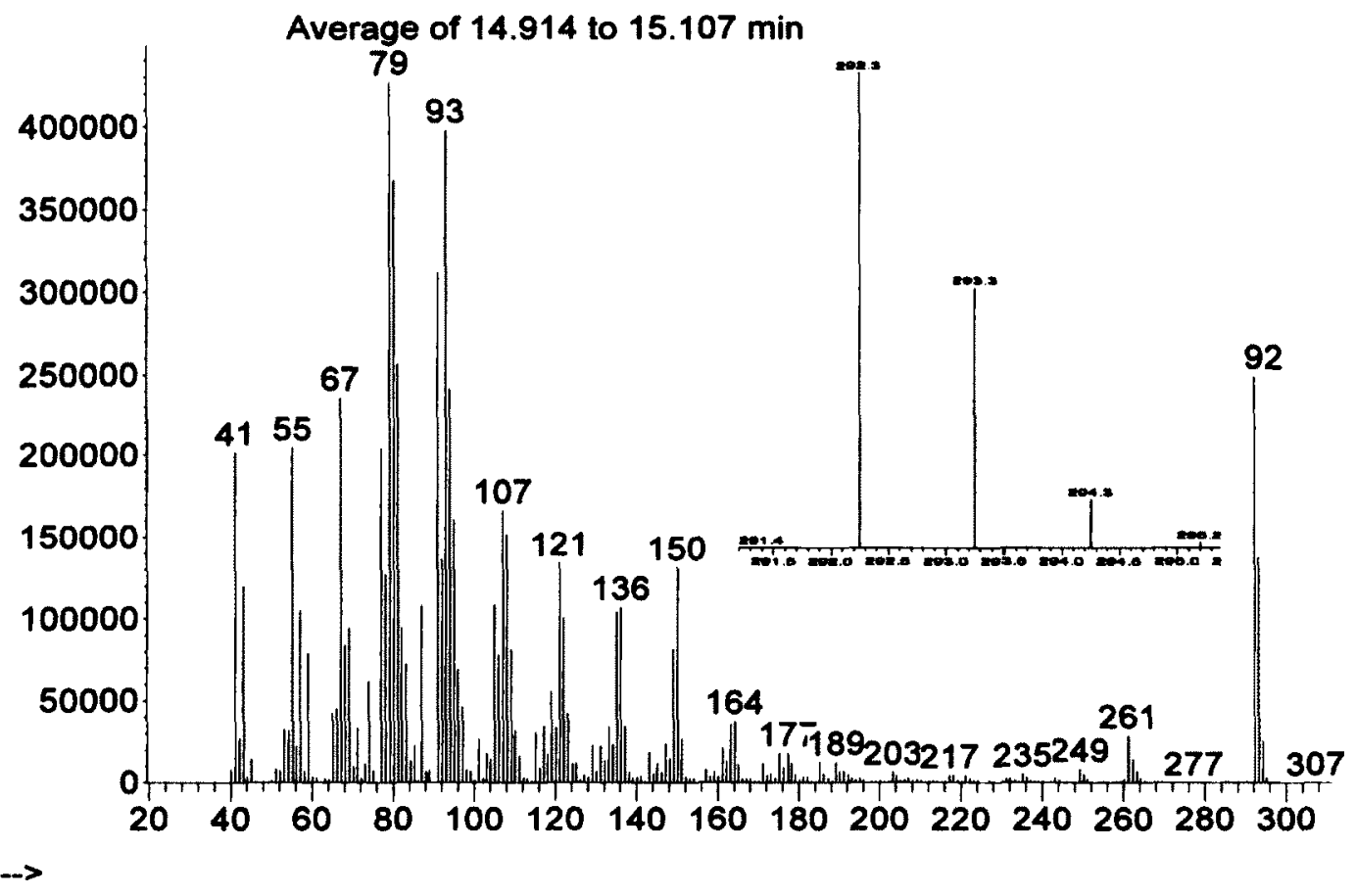

Figure 3.26. Representative mass spectrum of biosynthetic methyl $\alpha$-eleostearate derived from methyl $(11 R)-\left[11-{ }^{2} \mathrm{H}_{1}\right]$-linoleate $2 \mathbf{b}$.

In contrast to the outcome displayed above, the mass spectrum of the $\alpha$ eleostearate derived from $(11 S)-\left[11{ }^{2} \mathrm{H}_{1}\right]$-linoleic acid demonstrated the appearance of the most abundant molecular ion at $\mathrm{m} / \mathrm{z} 293$ (Figure 3.27), this represents the overall 
retention of deuterium in the fatty acid product during enzymatic oxidation when it is positioned at the pro $R$ position of the $\mathrm{d}_{1}$-substrate.

Abundance

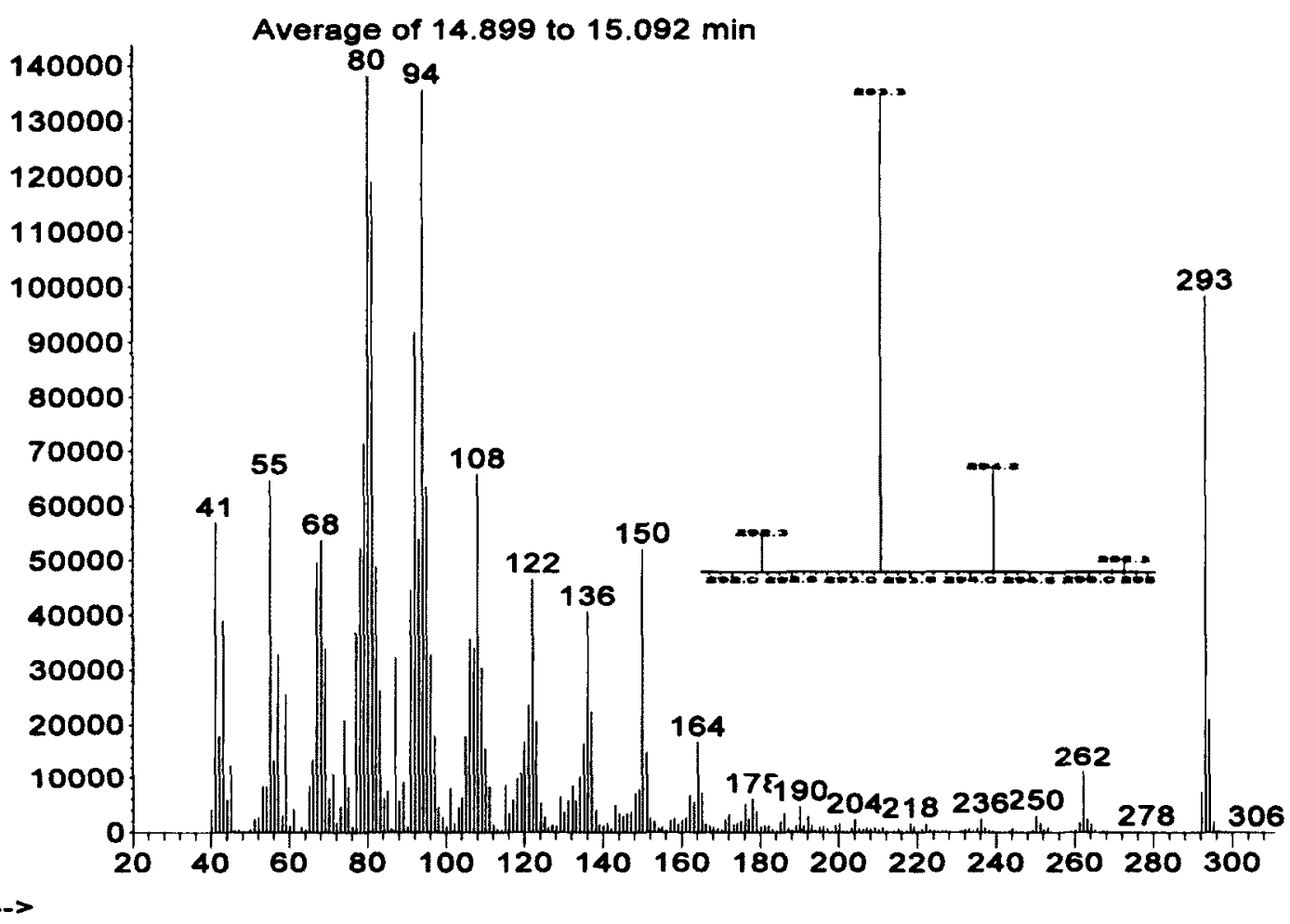

Figure 3.27. Representative mass spectrum of biosynthetic methyl $\alpha$-eleostearate derived from methyl $(11 S)-\left[11^{2} \mathrm{H}_{1}\right]$-linoleate $2 \mathbf{a}$.

A similar pattern of deuterium incorporation was observed for the $\alpha$-eleostearate derived from $(14 R)-\left[14-{ }^{2} \mathrm{H}_{1}\right]$-linoleic acid (loss of label) and $(14 S)-\left[14-{ }^{2} \mathrm{H}_{1}\right]$-linoleic acid (retention of label) as featured by the mass spectra shown in Figure 3.28 and Figure 3.29, respectively. 


\section{Abundance}

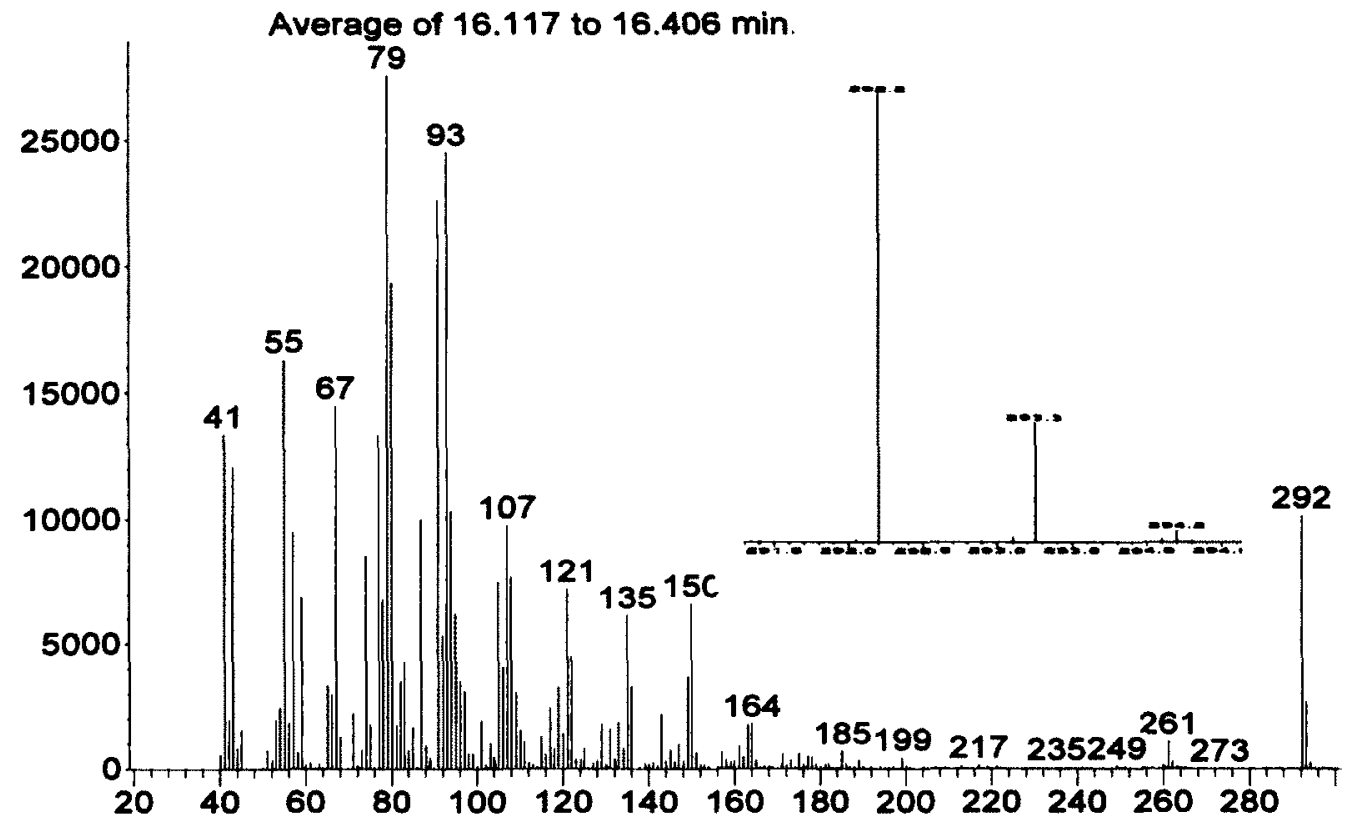

$\mathrm{m} / \mathbf{z}-\mathbf{>}$

Figure 3.28. Representative mass spectrum of biosynthetic methyl $\alpha$-eleostearate derived from methyl $(14 R)-\left[14^{2} \mathrm{H}_{1}\right]$-linoleate $33 \mathrm{~b}$.

Abundance

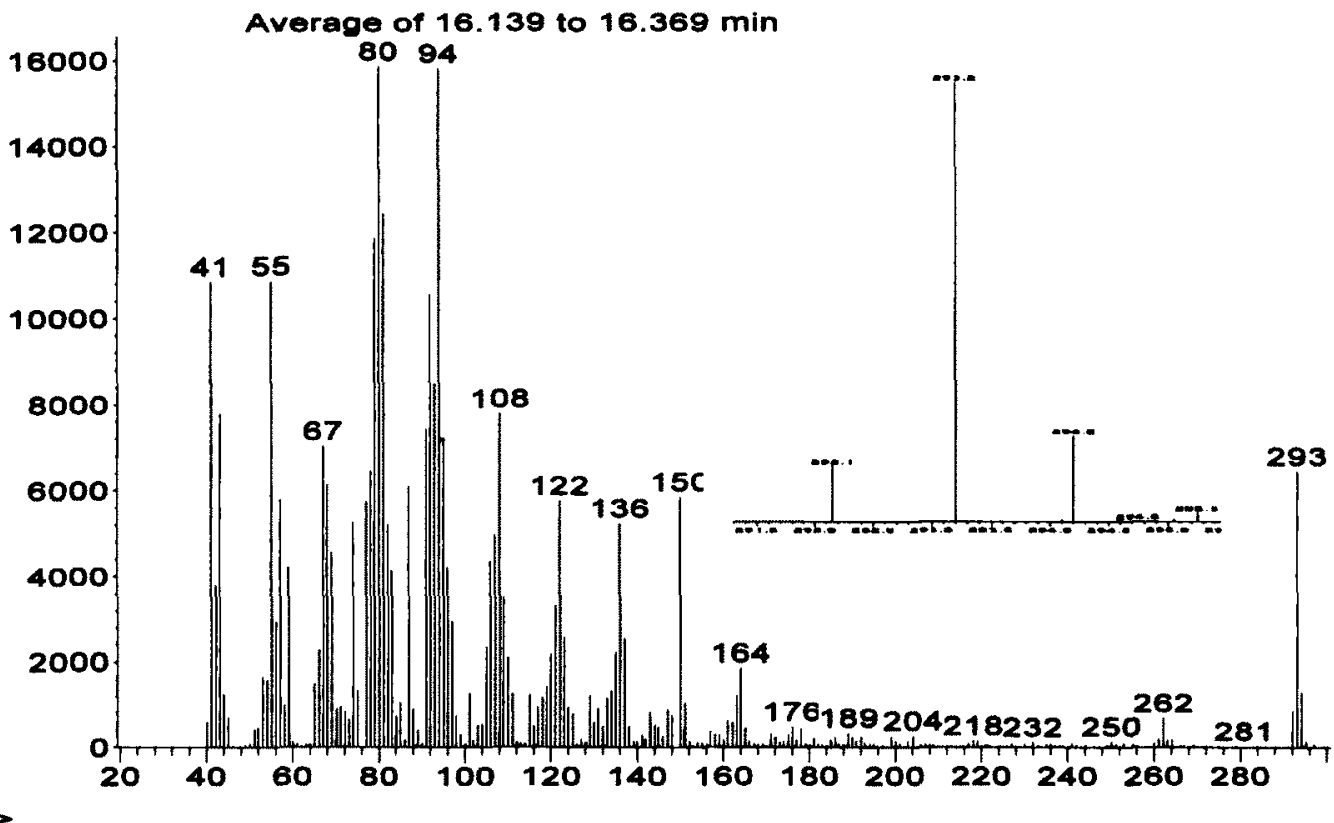

$m / z-->$

Figure 3.29. Representative mass spectrum of biosynthetic methyl $\alpha$-eleostearate derived from methyl $(14 S)-\left[14-{ }^{2} \mathrm{H}_{1}\right]$-linoleate $33 \mathrm{~b}$. 
The quantitative aspects of these results are displayed in Table 3.3. It is interesting to note that the elevated level of $d_{1}$-product derived from $(11 R)-\left[11-{ }^{2} \mathrm{H}_{1}\right]-\mathrm{LA}$ 2b was anticipated due to large primary deuterium KIE (4.0) operating at C-11. This causes the $(11 S)-\mathrm{d}_{1}$ contaminant in this substrate to lose the $11 R$ hydrogen much faster than the corresponding $(R)$-enantiomer loses deuterium from this position.

Table 3.3. Isotopic content of stereospecifically labeled cellular monodeuterated linoleates and $\Delta^{11,13}$-desaturated products ( $\alpha$-eleostearate) .

\begin{tabular}{|c|c|c|c|c|}
\hline \multirow{2}{*}{$\begin{array}{l}\text { Compounds } \\
\text { Name }\end{array}$} & \multicolumn{2}{|c|}{ Substrates } & \multicolumn{2}{|c|}{ Products } \\
\hline & $\% \mathrm{~d}_{0}$ & $\% d_{1}$ & $\% \mathrm{~d}_{0}$ & $\% d_{1}$ \\
\hline$(11 R)-\left[11-{ }^{2} \mathrm{H}_{1}\right]-\mathbf{L} \mathbf{A}^{a}$ & 2.5 & 97.5 & 74.9 & 25.1 \\
\hline$(11 S)-\left[11-{ }^{2} \mathrm{H}_{1}\right]-\mathbf{L A}^{a}$ & 0.8 & 99.2 & 8.2 & 91.8 \\
\hline$(14 R)-\left[14-{ }^{2} \mathrm{H}_{1}\right]-\mathbf{L} \mathbf{A}^{b}$ & 1.2 & 98.8 & 94.3 & 5.7 \\
\hline$(14 S)-\left[14-{ }^{2} \mathrm{H}_{1}\right]-\mathbf{L A}^{b}$ & 1.3 & 98.7 & 10.7 & 89.3 \\
\hline
\end{tabular}

"Incubations of chiral $11-d_{1}$-substrates were carried out three times and the \% isotopic content of each species is given as an average value \pm standard deviation of three independent GC-MS runs. ${ }^{b}$ Incubations of chiral $14-d_{1}-$ substrates were carried out four times and the \% isotopic content of each species is given as an average value \pm standard deviation of four independent GC-MS runs. The average standard deviation for each species is \pm 0.4 expressed as a percentage.

These results show that during the course of $\alpha$-eleostearic acid biosynthesis, linoleic acid loses pro $R$ hydrogens at both $\mathrm{C}-11$ and $\mathrm{C}-14$ positions. Our observation is in accord with the syn dehydrogenation pattern at the two non-adjacent positions, but in 
relation to our predicted model (Figure 3.1 and 3.30) the hydrogens are removed topologically from the opposite side of the olefinic plane compared to that removed during the course of linoleic acid formation by the parent $\Delta^{12}$-desaturase (FAD2) $)^{2.140}$. The observed enantioselectivity also does not correlate with the enantioselectivity identified during calendic acid biosynthesis in Calendula officinalis by $\Delta^{8,10}$-desaturase (Chapter 2, Figure 2.46) and for an insect $\Delta^{10,12}$-desaturase that converts $(Z)$-11-tetradecenoate to the corresponding $(E, E)$-10,12-tetradecadienoate ${ }^{40}$ (Chapter 1.4.1, Figure 1.29).

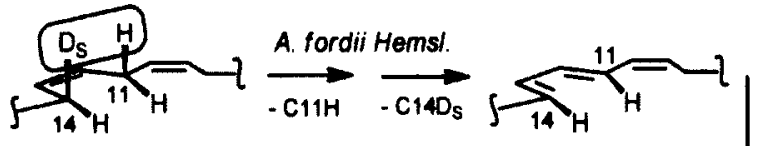

Loss of Deuterium

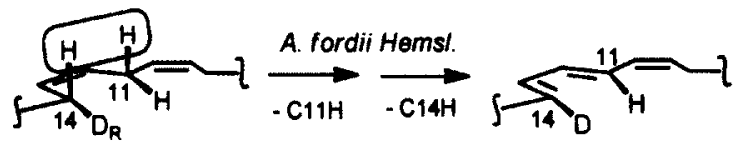

Retention of Deuterium

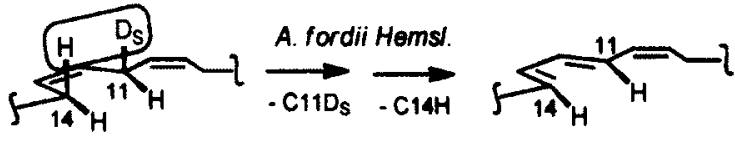

Loss of Deuterium

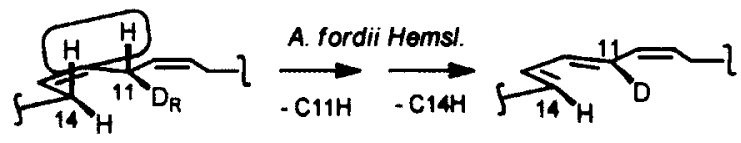

Retention of Deuterium

Figure 3.30. Predicted outcomes for $\alpha$-eleostearate formation from stereospecifically labeled linoleates.

It is difficult to imagine how the diiron peroxo species can abstract hydrogens from below the plane of the olefin, as the associated interactions with its surrounding ligands as well as the amino acid sequence near the active site would have to change dramatically. Looking at the many different fatty acid products that can be generated by the FAD2 family ${ }^{1,2}$ and the enormous enzymatic plasticity of tung FAD2 variant ${ }^{34}$, it is most likely that the enzyme active site cavity can be altered to accommodate different substrate conformations relative to a fixed diiron position. Considering all these factors, we have proposed a revised topological model as shown in Figure 3.31 for the 
explanation of the observed stereochemical outcome in our experiment. In this revised topological model, it is assumed that the substrate can present the pro $R$ hydrogens at $\mathrm{C}$ 11 and $\mathrm{C}-14$ positions of the fatty acid substrate to the diiron peroxo species through rotation at the $\mathrm{C}-10,11$ and $\mathrm{C} 14,15 \mathrm{C}-\mathrm{C}$ bonds. In this conformation, the cryptoregiochemistry of the $\Delta^{11,13}$-desaturase enzyme still remains same at $\mathrm{C}-11 . \quad$ It is understood that the double bonds are quasicoplanar.
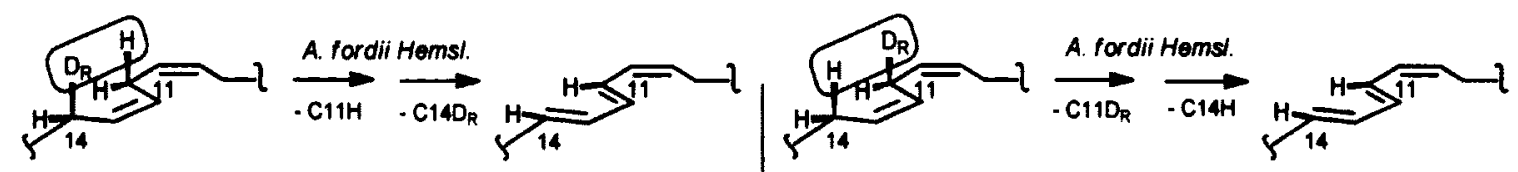

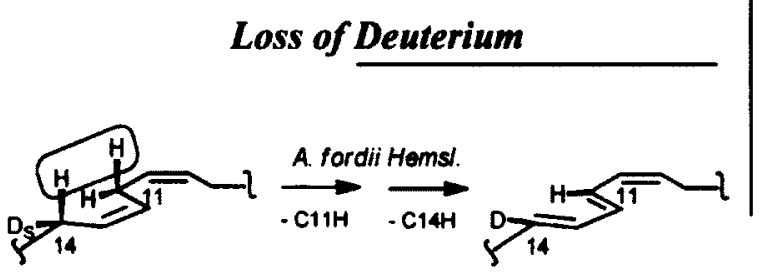

Retention of Deuterium

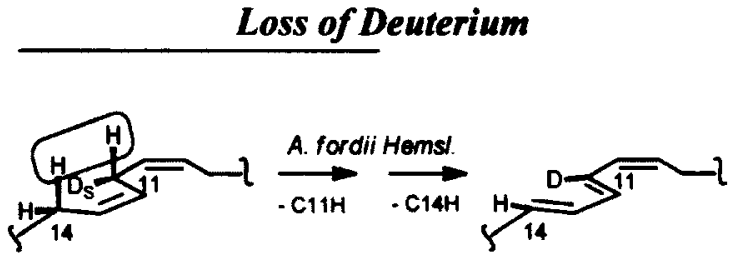

Retention of Deuterium

Figure 3.31. The revised topological model and observed results for eleostearate formation from stereospecifically deuterated linoleates. Note CIP priority rules dictate that the deuterium atoms that are removed from the $\mathrm{C}-11$ and $\mathrm{C}-14$ positions are labeled as both $D_{R}$ and those deuterium atoms retained in the products are labeled as $D_{S}$.

This is not the only case where the enantioselectivity of a desaturase is opposite to expectations. This type of instance was also reported in the literature previously namely during $\Delta^{13}$-desaturation of $(Z)$-11-hexadecenoate and 11-hexadecynoate to biosynthesize $(Z, Z)$-11,13-hexadecadienotate and $(Z)$ - hexadeca-11-yne-13-eneoate respectively in the moth Thaumetopoea pityocampa (Section 1.1.5, Figure 1.6). ${ }^{41,75}$ Using stereospecifically labeled deuterated palmitoyl precursors, it was shown that the $\Delta^{13}$-desaturase removes two pro $S$ hydrogens from $\mathrm{C} 13$ and $\mathrm{C} 14$ positions of $(Z)$-11-hexadecenoate but the pro $R$ 
hydrogens from the 11-alkyne analogue. ${ }^{75} \mathrm{~A}$ topological model for this substratedependent reversal in enantioselectivity was proposed (Figure 3.32).

$\mathbf{A}$

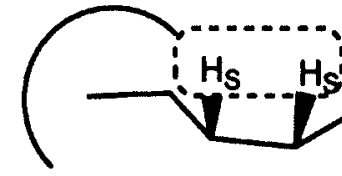

B1
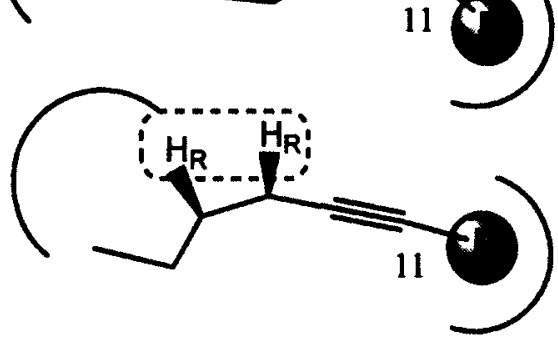

vrs

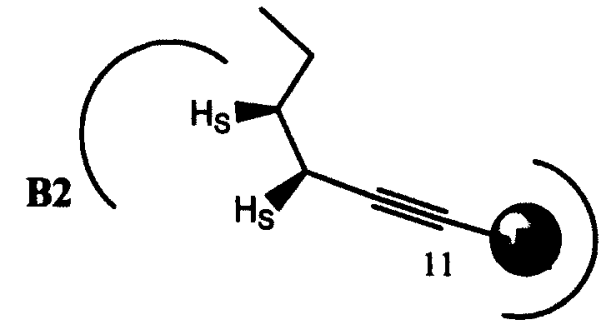

Figure 3.32. Schematic diagram showing the possible conformational arrangement of two different substrates at the binding cavity of $\Delta^{13}$-desaturase in T. pityocampa. A) Good fit for 11-hexenoate substrate; B1) Good fit for 11-hexynoate substrate; B2) Bad fit for 11-hexynoate substrate.

\subsection{Conclusions}

The cryptoregiochemstry and enantioselectivity of another important plant 1,4desaturase enzyme belonging to the family of FAD2 homologue has been determined. The site of initial oxidation (cryptoregiochemistry) for the tung $\Delta^{11,13}$-desaturasemediated formation of $\alpha$-eleostearic acid has been determined to be at $\mathrm{C}-11$, the carbon closest to C-12 which is the site of initial oxidative attack for the parent FAD2 enzyme. Our cryptoregiochemical result correlates well with prediction and to that observed with parent FAD2 and other FAD2 homologues catalyzed formations of various other fatty acids as discussed in Section 1.2.5.

However, the observed enantioselectivity of hydrogen removal doesn't correlate with prediction as well as with the observed enantioselectivity in plant $\Delta^{8,10}$-desaturase and insect $\Delta^{10,12}$-desaturase during the formation of calendic acid and $(E, E)-10,12$ - 
tetradecadienoic acid, respectively. A revised topological model is proposed to explain the reversal in enantioselectivity (Figure 3.33). According to this revised model, the biosynthesis of $\alpha$-eleostearic acid in Aleurites fordii Hemsl. (tung) involves the initial, isotopically sensitive removal of a pro $R$ hydrogen from $\mathrm{C}-11$ of linoleate and subsequent cleavage of the pro $R$ C14-hydrogen bond i.e. two hydrogens are removed in syn fashion from two neighboring allylic positions of the fatty acid chain to generate the conjugated trienoic acid (Figure 3.33).

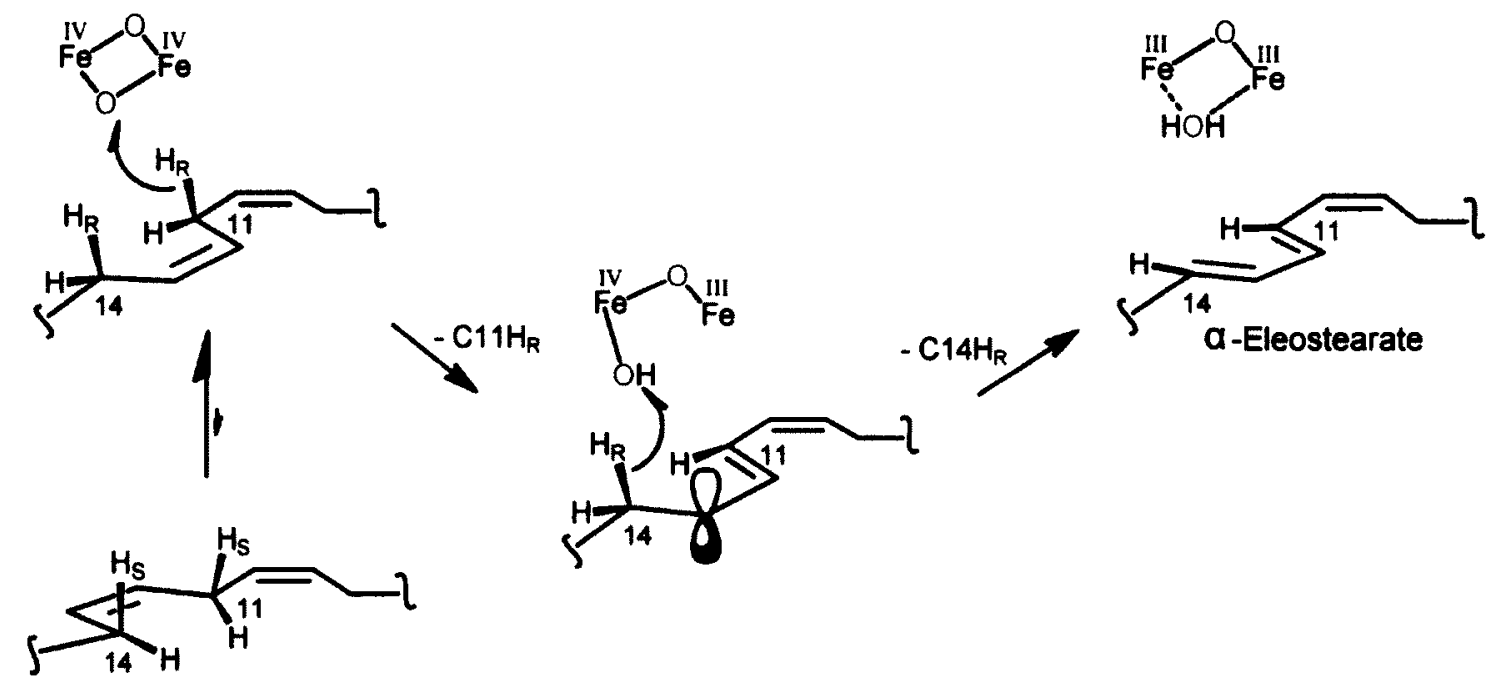

Figure 3.33. Mechanistic model for 1,4-dehydrogenation reaction of linoleate to biosynthesize $\alpha$-eleostearate as it occurs in Aleurites fordii Hemsl. The double bonds are quasi-coplanar. 


\section{Chapter 4: Stereochemistry of Dimorphecolic acid Biosynthesis in Dimorphotheca sinuata and associated KIE Evaluation}

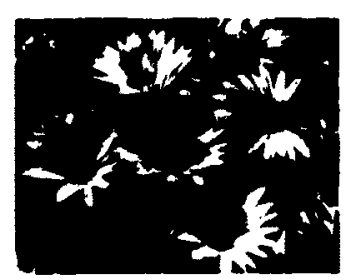

www.pwblog.com

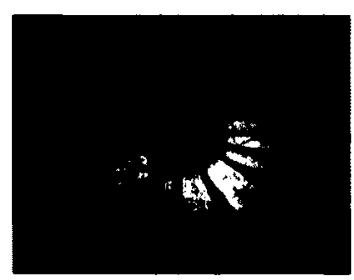

www.panoramio.com

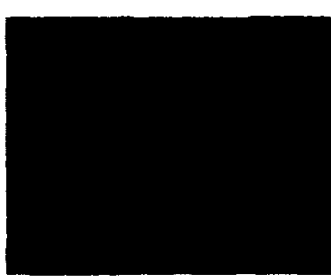

www.kbm.gr

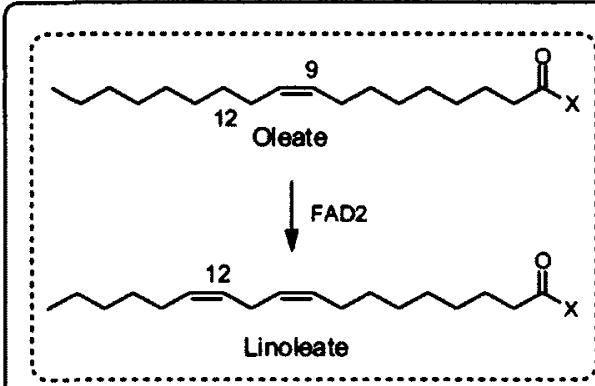

Dimorphotheca sinuata
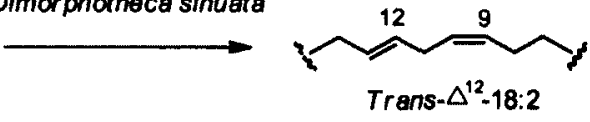

D. sinuata

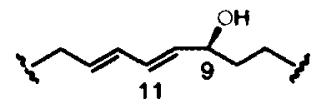

Dimorphecolate

\subsection{Experimental Approach}

In the context of understanding the structure-function relationships of the family of FAD2 homologues that leads to the formation of unusual conjugated fatty acids in plants, the formation of dimorphecolic acid in the seeds of Dimorphotheca sinuata was investigated here. Determination of the mechanism of dimorphecolic acid formation is clearly necessary, as the normally least preferable hydroxyl rebound step dominates over the generally observed second hydrogen removal step. ${ }^{1,2}$ Molecular biological studies revealed that two FAD2-like enzymes (DsFAD2-1 and DsFAD2-2) work in tandem fashion to produce dimorphecolic acid from oleic acid via the 12-trans isomer of linoleate as shown in Figure 4.1. ${ }^{11}$ 

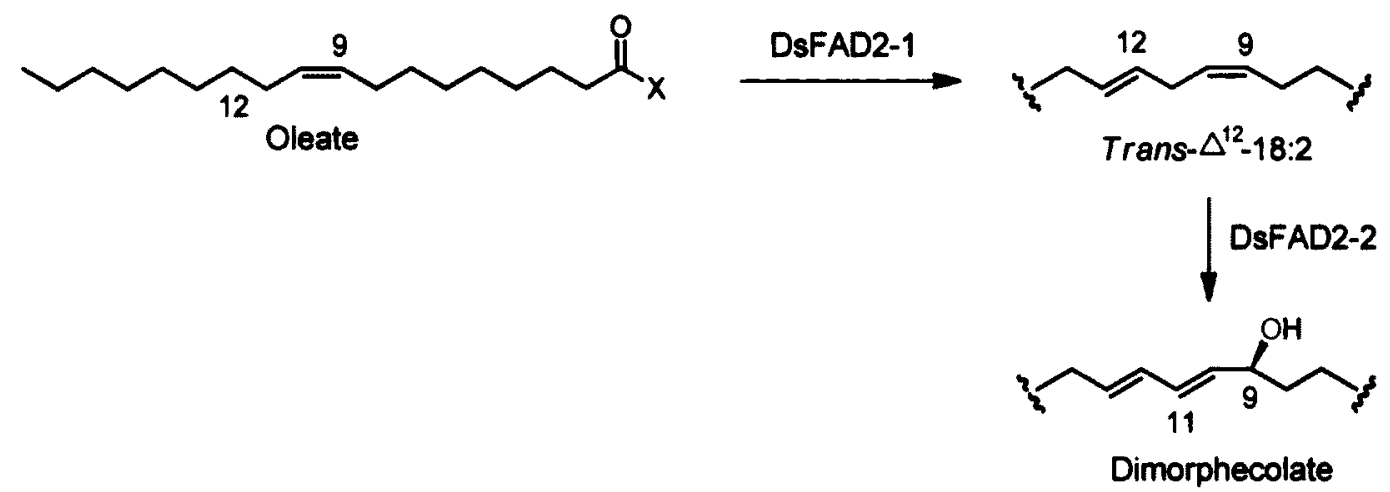

Figure 4.1. The biosynthetic pathway of dimorphecolic acid formation from oleic acid in the seeds of Dimorphotheca sinuata.

Based on the close taxonomical relationship" between Dimorphotheca sinuata and Calendula officinalis and observed high amino acid sequence identity between DsFAD22 and $\Delta^{8,10}$-desaturase enzyme, we propose a mechanistic model for the former enzyme that has been shown in Figure 4.2. The substrate $9 Z, 12 E$-18:2 adopts a conformational arrangement that is similar to that suggested during calendate formation ${ }^{158}$ (see Chapter 2, Figure 2.1) and the reaction starts at $\mathrm{C}-11$ with the removal of a hydrogen atom.

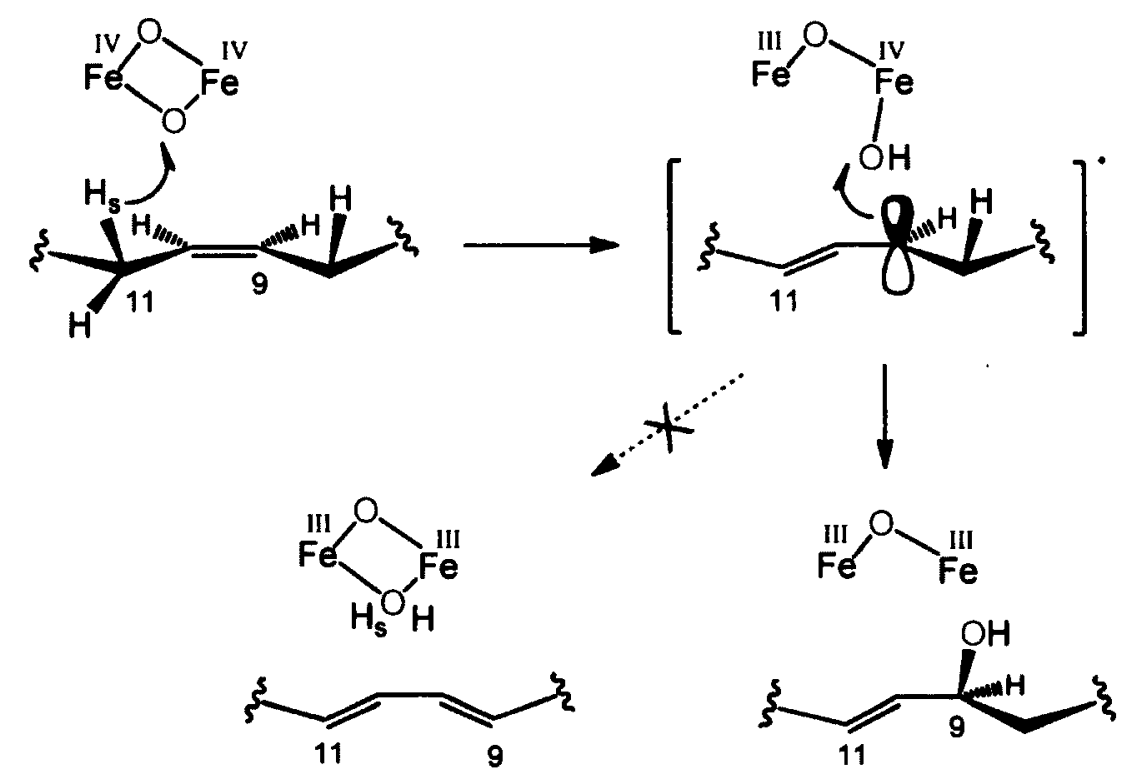

Figure 4.2. Proposed mechanistic model for $\Delta^{9}$-hydroxylation leading to the formation of 9-hydroxy-(E,E)-10,12-octadecadienoic from trans- $\Delta^{12}$-linoleic acid and showing how iron- $\mathrm{OH}$ is not able to reach the $\mathrm{C} 8 \mathrm{H}$ to form calendate. 
The resultant allylic radical is trapped by iron-bound hydroxyl in a regioselective manner and generates the C-9 allylic alcohol that is (S)-configured. ${ }^{11}$

In this spatial arrangement, the removal of hydrogen at $\mathrm{C}-11$ and hydroxyl rebound at C-9 occurs with the same facial selectivity as the syn removal of hydrogens from C-11 and C-8 positions of linoleic acid that was reported during calendate formation (see Chapter 2). The correctness of this proposed topology could be tested through the determination of the enantioselectivity of dimorphecolic acid formation using stereospecifically labeled deuterated fatty acids as mechanistic probes. The availability of a convenient $S$. cerevisiae system for the co-expression of the genes that encoding the enzymes DsFAD2-1 and DsFAD2-2 from Dimorphotheca sinuata allowed the determination to be done in vivo."

Our approach required the use of two stereospecifically monodeuterated oleates, methyl (11S)-[11- $\left.{ }^{2} \mathrm{H}_{1}\right]$-octadec-9Z-enoate $3 \mathbf{a}$ and methyl $(11 R)-\left[11-{ }^{2} \mathrm{H}_{1}\right]$-octadeca-9Zenoate $3 \mathbf{b}$ that were available from the calendate experiment (see Chapter 2 , Section 2.2.2). The availability of methyl $\left[11,11-^{2} \mathrm{H}_{2}\right]$-oleate 51 in Patrick S. Covello's laboratory (NRC - PBI, Saskatoon) prompted us to carry out a measurement of the primary deuterium KIE for $\mathrm{C}-11 \mathrm{H}$ abstraction.

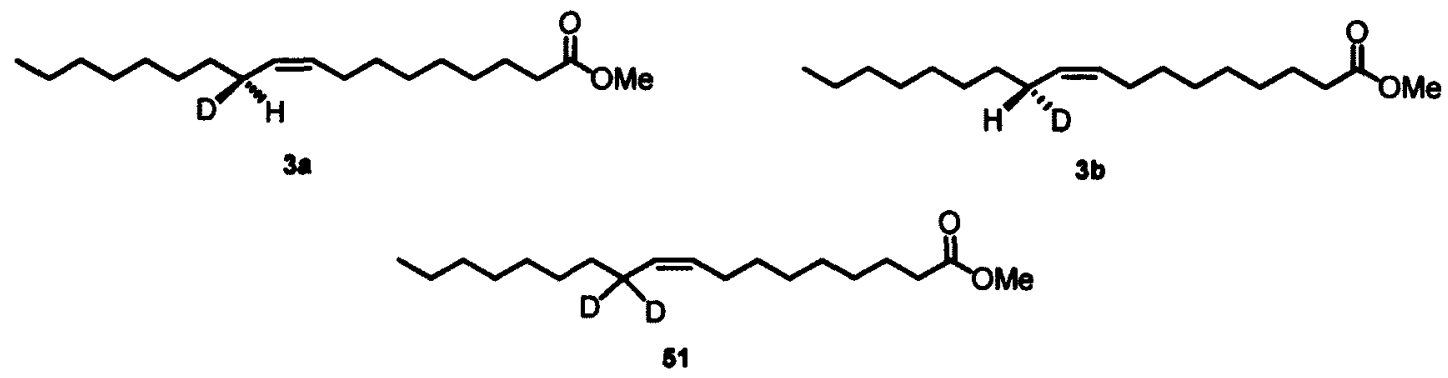

Figure 4.3. The chiral monodeuterated and dideuterated oleate required to investigate the mechanism of dimorphecolate formation. 


\subsection{Incubation Experiments Using Dideuterated Substrates: KIE Studies}

\subsubsection{Trial Incubation of $\mathrm{d}_{0}$-Oleate}

The efficiency of formation of dimorphecolic acid from $d_{0}$-oleate using the cultures of $S$. cerevisiae strain that functionally co-expressing both DsFAD2-1 and DsFAD2-2 genes from Dimorphotheca sinuata was probed in a preliminary trial experiment. Accordingly, ca. $0.5 \mathrm{~mL}$ of sterilized oleic acid solution in $10 \%$ Tergitol (10 $\mathrm{mg} / \mathrm{mL}$ ) was administered to a $50 \mathrm{~mL}$ growing culture of pESC-HIS/YPH499 strain of $S$. cerevisiae and allowed to grow at $28{ }^{\circ} \mathrm{C}$ for 7 days at $310 \mathrm{pm}$. These optimized incubation conditions have been demonstrated to give the best substrate conversion rate and were used throughout the mechanistic studies. A corresponding control experiment under the same incubation conditions, but without the addition of exogenous oleic acid substrate was also run. The cellular fatty acid fractions were isolated from the harvested yeast cells via a standard hydrolysis/methylation protocol as described earlier (see Chapter 2 and 3) and analyzed by GC-MS. For the purpose of analysis the methylated fatty acid extract was silylated using BSTFA/pyridine ${ }^{187}$ to obtain the TMS derivative of methyl dimorphecolate (Figure 4.4). ${ }^{11}$ Silylation of alcohols is known to dramatically enhance their detectability by GC.

The product was identified on the basis of retention time $\left(13.60 \mathrm{~min}\right.$ for $d_{0^{-}}$ molecular ion cluster) of the TMS derivative of methyl dimorphecolate and its comparison with the retention time of a reference standard. ${ }^{11}$ 


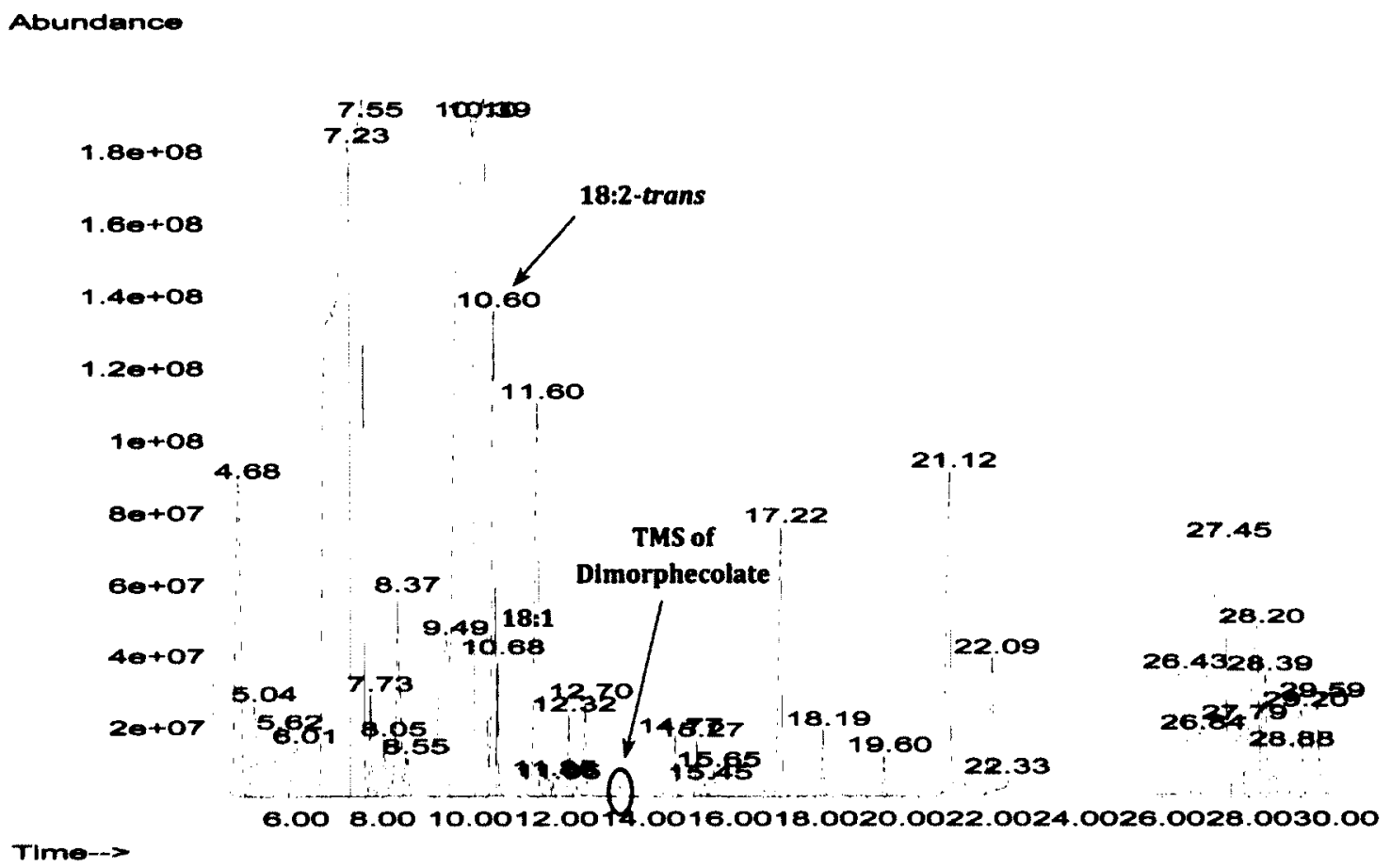

Figure 4.4. The cellular fatty acid profile of $S$. cerevisiae grown in a medium supplemented with oleic acid.

The mass spectrum of the GC peak assigned to silylated derivative of methyl dimorphecolate product (Figure 4.5 ) revealed the presence of the molecular ion at $\mathrm{m} / \mathbf{z}$ $382\left([\mathrm{M}]^{+}\right)$and a diagnostic base peak at $\mathrm{m} / \mathrm{z} 225\left(\left[\mathrm{Me}\left(\mathrm{CH}_{2}\right)_{4} \mathrm{CH}=\mathrm{CH}-\mathrm{CH}=\mathrm{CH}-\mathrm{CH}-\mathrm{O}-\right.\right.$ $\left.\left.\mathrm{Si}\left(\mathrm{CH}_{3}\right)_{3}\right]^{+}\right)$due to the fragmentation between the $\mathrm{C}-8$ and $\mathrm{C}-9$ atoms. The fragmentation pattern is in accord with the assigned structure and similar to that reported before."

The dehydrogenated product $\left(8 E, 10 E, 12 E\right.$-octadeca-trienoic acid) or the $c i s-\Delta^{12}$ isomer of dimorphecolic acid could not be detected as trace "error" compounds in the FAME of the total cellular fatty acids. This observation shows the commitment of DsFAD2-1 and DsFAD2-2 to the production of specific fatty acids. The result matches well with that reported earlier by Cahoon and Kinney. "A similar examination of the methylated $\mathrm{CH}_{2} \mathrm{Cl}_{2}$ extract of the culture medium did not identify any "error" products. 


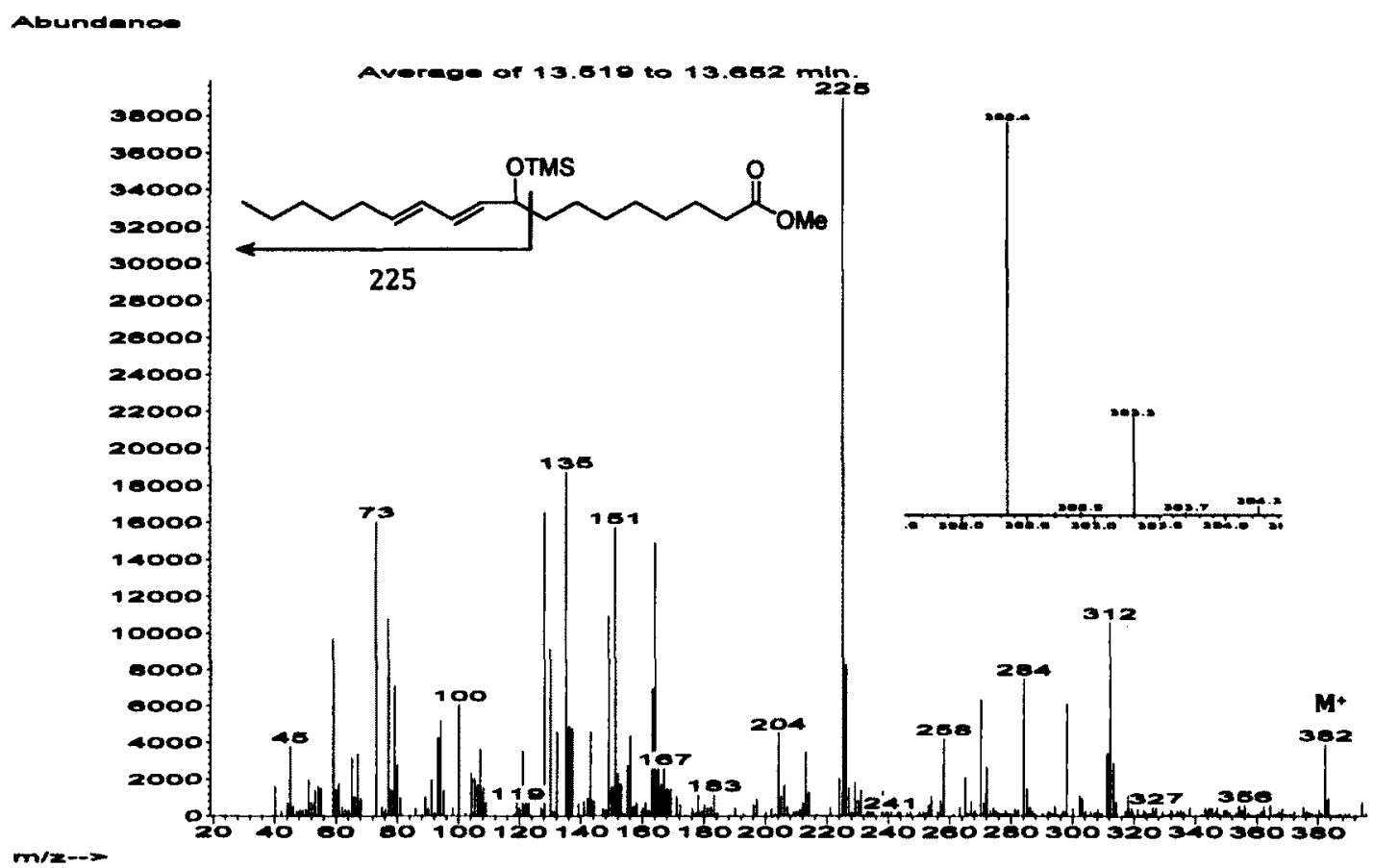

Figure 4.5. Mass spectrum of TMS derivative of the methyl ester of dimorphecolic acid.

Comparison of the fatty acid methyl ester (FAME) profile of the control experiment (no oleate in medium) with the culture expressing the $\mathrm{pESC}-\mathrm{HIS} / \mathrm{YPH} 499$ strain of $S$. cerevisiae (Figure 4.4) revealed that in both incubations the product dimorphecolic acid (as trimethylsilyl derivative) has been produced which is consistent with the endogenous formation of the substrate oleic acid. The amount of dimorphecolic acid formed via the tandem fatty acid oxidation sequence was $\sim 0.5 \%$ of the total cellular fatty acids. The result corresponds well with transformation levels reported previously. ${ }^{11}$

\subsubsection{Intermolecular Kinetic Isotope Effect (KIE) Studies}

The primary deuterium KIE for $\mathrm{C} 11-\mathrm{H}$ cleavage was evaluated in an intermolecular competitive experiment where ca. equimolar (1:1) mixture of $18-d_{3}$-oleate and 11- $d_{2}$-oleate was used. ${ }^{199}$ The $\omega$-tagged 18- $d_{3}$-oleate was used as the "11- $d_{0}$ substrate" since the contribution from endogenous oleate pool could not be controlled. As 
a control, we also performed a set of incubation experiments using a mixture of $18-\mathrm{d}_{3}$ oleate and $\left[9,10-{ }^{2} \mathrm{H}_{2}\right]$-oleate; in this case a KIE of $\sim 1$ was expected since the latter isotopomer does not contain deuterium label at $\mathrm{C}-11$. All labeled compounds were available in the Covello laboratory (NRC - PBI, Saskatoon) and had been synthesized by Pat Tulloch (NRC - Saskatoon) some four decades ago. Sterilized mixtures consisting of saponified methyl esters of $\left[18,18,18-{ }^{2} \mathrm{H}_{3}\right]$-oleate in combination with $\left[11,11-{ }^{2} \mathrm{H}_{2}\right]-$ or $\left[9,10-{ }^{2} \mathrm{H}_{2}\right]$-oleates were administered separately as a solution in $10 \%$ Tergitol $(\sim 5 \mathrm{mg}$ each) to growing cultures of pESC-HIS/YPH499 strain of S. cerevisiae that co-expresses both DsFAD2-1 and DsFAD2-2. For each type of mixture the reaction was run in triplicate. The cellular fatty acid fractions of the harvested cells were isolated by hydrolysis/methylation procedure and treated with BSTFA/pyridine to obtain the TMS derivative of methyl dimorphecolate that was then analyzed by GC-MS. The $d_{3} / d_{2}$ ratio of the recovered oleate fractions was found to be similar to the $d_{3} / d_{2}$ ratio of the administered mixtures. Maintenance of this ratio during the incubation is essential for competitive product KIE measurements as explained earlier (Section 1.3.2). ${ }^{199}$ We can reasonably assume that the rate of trans-olefin at $\mathrm{C} 12$ catalyzed by DsFAD2-1 is not affected by deuterium at $\mathrm{C} 11$ or $\mathrm{C} 9,10$.

Mass spectral analysis of the TMS derivative of the dimorphecolate fraction revealed that when $18-d_{3}-$ oleate and $11-d_{2}$-oleate was used as a substrate, the product (Figure 4.6) consisted of a mixture of $d_{1}$ and $d_{3}$-species, in addition to $d_{0}$ content from endogenous oleate. This indicated the loss of one deuterium from 11- $d_{2}$ substrate as expected. On the other hand, incubation of $18-d_{3}-$ oleate and $9,10-d_{2}$-oleate as a substrate resulted in the formation of the product (Figure 4.7) that contained a mixture of $d_{0}, d_{2}$ and 


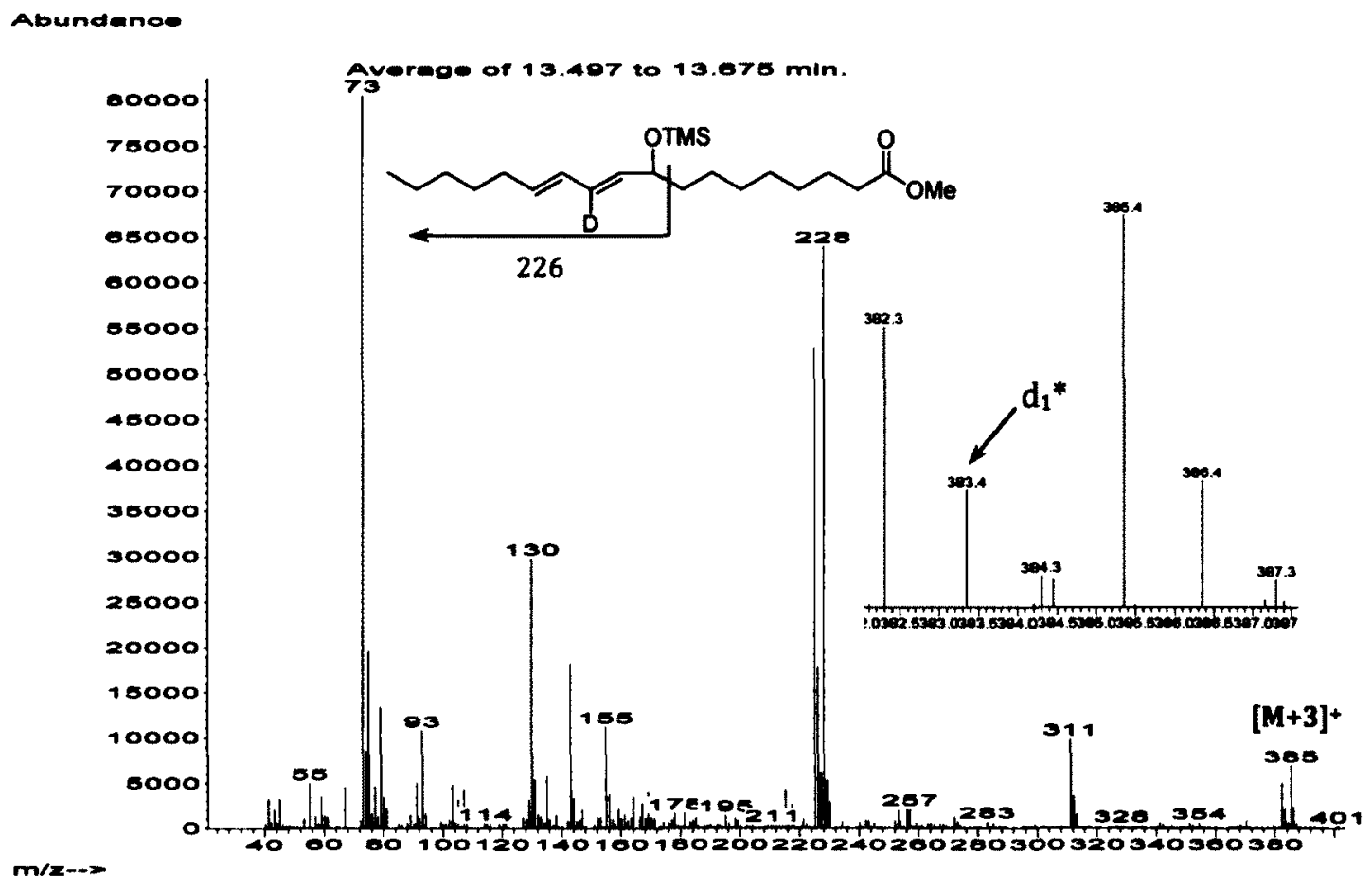

Figure 4.6. Representative mass spectrum of TMS derivative of the methyl ester of dimorphecolic acid derived from 1:1 mixture of 18- $\mathrm{d}_{3}$-oleate and 11- $\mathrm{d}_{2}$-oleate. * The peak at $\mathrm{m} / \mathrm{z} 383$ is enhanced due to presence of $d_{1}$ species in addition to the $d_{0} M+1$ contribution.

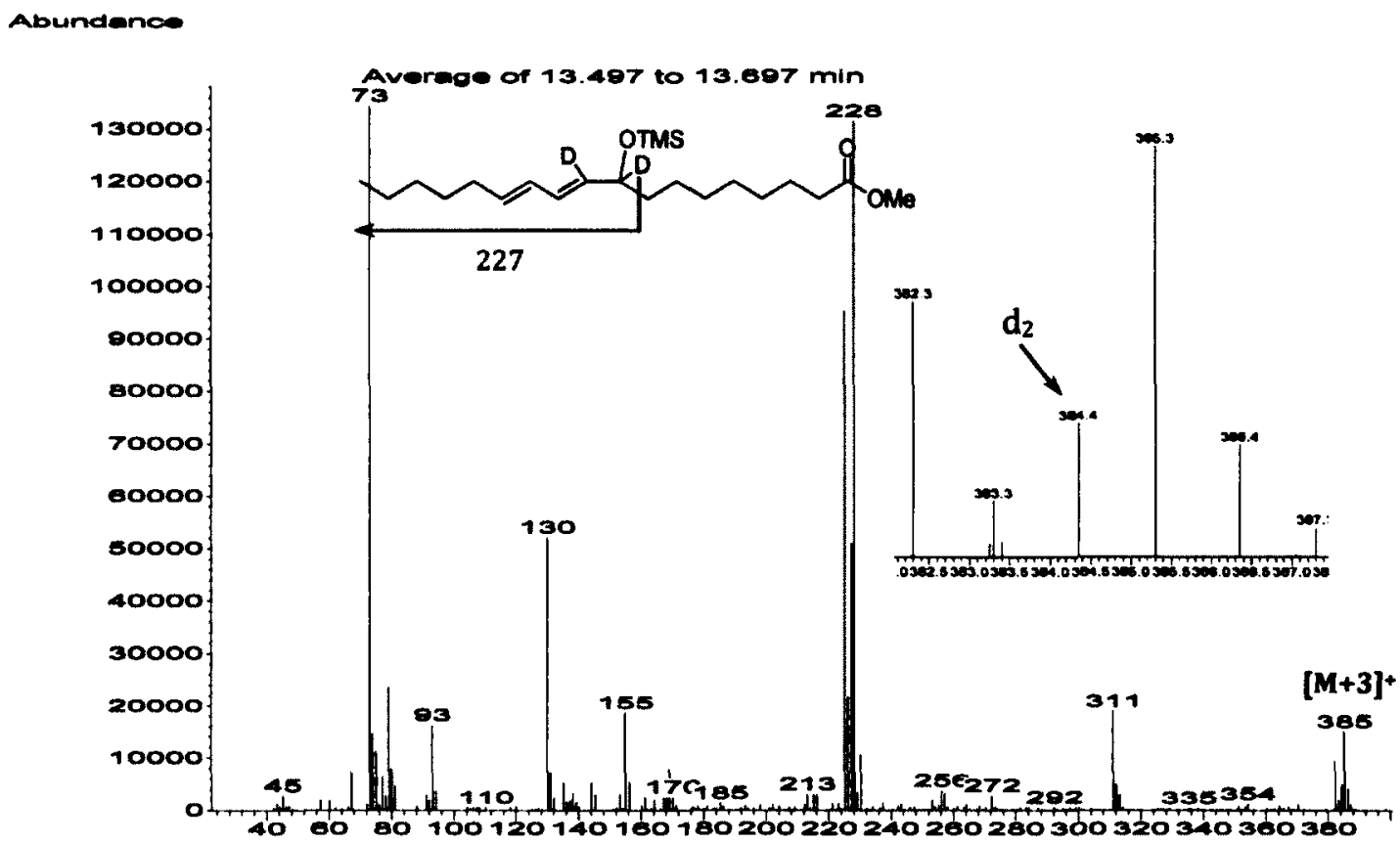

$m / z->$

Figure 4.7. Representative mass spectrum of TMS derivative of the methyl ester of dimorphecolic acid derived from 1:1 mixture of 18- $d_{3}$-oleate and $9,10-d_{2}$-oleate. 
$d_{3}$-species, which indicated the retention of both of the deuterium in the product from $9,10-d_{2}$-substrate as expected.

The primary deuterium product kinetic isotope effect $\left(\mathrm{k}_{\mathrm{H}} / \mathrm{k}_{\mathrm{D}}\right)$ for $\mathrm{C}-\mathrm{H}$ bond cleavage at C-11 was calculated using the following ratio: ${ }^{199}$

$$
\frac{k_{H}}{k_{D}}=\frac{P_{H} / P_{D}}{S_{H}^{0} / S_{D}^{0}}
$$

Where, ${ }^{P_{H}} / P_{D}$ is the ratio of $\left(\left[18,18,18-\mathrm{d}_{3}\right]-\right.$ and $\mathrm{d}_{0}$-dimorphecolate) to $\left[11-\mathrm{d}_{1}\right]-$ dimorphecolate and $S_{H}^{0} / S_{D}^{0}$ is the ratio of $\left(\left[18,18,18-\mathrm{d}_{3}\right]-\right.$ and $\mathrm{d}_{0}$-oleate) to $\left[11,11-\mathrm{d}_{2}\right]-$ oleate. Thus, the above ratio simplifies to:

$$
\frac{k_{H}}{k_{D}}=\frac{\left[\% P_{d_{0}}+\% P_{d_{3}}\right] /\left[\% P_{d_{1}}\right]}{\left[\% S_{d_{0}}+\% S_{d_{3}}\right] /\left[\% S_{d_{2}}\right]}
$$


Table 4.1. Competitive isotopic discrimination in the Dimorphotheca sinuata DsFAD2-1 and DsFAD2-2 desaturase-mediated formation of dimorphecolic acid from $\left[11,11-{ }^{2} \mathrm{H}_{2}\right]-$ oleic acid or $\left[9,10-{ }^{2} \mathrm{H}_{2}\right]$-oleic acid vs. $\left[18,18,18-{ }^{2} \mathrm{H}_{3}\right]$-oleic acid.

\begin{tabular}{|c|c|c|c|c|c|c|c|c|}
\hline \multirow[t]{2}{*}{$\begin{array}{l}\text { Position of } \\
\text { Isotope in } \\
d_{2} \text {-Species }\end{array}$} & \multirow[t]{2}{*}{ Trial } & \multicolumn{3}{|c|}{ Oleate Substrate $e^{a, b, c}$} & \multicolumn{3}{|c|}{$\begin{array}{l}\text { TMS Derivative of } \\
\text { Dimorphecolate Product }{ }^{a c}\end{array}$} & \multirow[t]{2}{*}{ KIE } \\
\hline & & $\mathrm{d}_{0}$ & $d_{2}$ & $d_{3}$ & $\mathrm{~d}_{0}$ & $d_{1}$ & $d_{3}$ & \\
\hline \multirow{4}{*}{$C-11$} & $t_{0}$ & 0.0 & 43.7 & 56.3 & & & & \\
\hline & 1 & 26.2 & 31.2 & 42.6 & 43.0 & 6.0 & 51.0 & 7.1 \\
\hline & 2 & 26.5 & 31.8 & 41.7 & 42.3 & 5.1 & 52.6 & 8.7 \\
\hline & 3 & 25.8 & 31.4 & 42.8 & 42.2 & 5.6 & 52.2 & 7.7 \\
\hline \multirow{2}{*}{\multicolumn{2}{|c|}{ Average }} & & & & & & & $7.8 \pm 0.8^{d}$ \\
\hline & & & & & $d_{0}$ & $d_{2}$ & $d_{3}$ & \\
\hline \multirow{4}{*}{ C- $-9,10$} & $t_{0}$ & 0.0 & 28.3 & 71.7 & & & & \\
\hline & 1 & 30.8 & 17.0 & 52.2 & 35.0 & 18.3 & 46.7 & 0.91 \\
\hline & 2 & 31.7 & 16.3 & 52.0 & 35.0 & 18.8 & 46.2 & 0.84 \\
\hline & 3 & 31.8 & 16.1 & 52.1 & 35.8 & 18.5 & 45.7 & 0.85 \\
\hline \multicolumn{3}{|c|}{ Average } & & & & & & $0.87 \pm 0.04^{e}$ \\
\hline
\end{tabular}

a The isotopic content of both substrate and product mixtures were evaluated by considering the $(M+1)$, $(M+2)$ as well as $(M-1),(M-2)$ contributions from the $d_{0}-, d_{1}-, d_{2}-$ and $d_{3}$-species. $d_{0}, d_{1}, d_{2}$ and $d_{3}$ refer to the non-deuterated, monodeuterated, dideuterated and trideuterated species, respectively. ${ }^{b}$ The isotopic composition of the cellular oleic acid fractions in both incubations were similar to that measured prior to the incubation. ${ }^{c}$ The deuterium content (\%) of each species is given as an average value based on three GC-MS runs. The average standard deviation for these measurements is \pm 0.4 expressed as a percentage. The average KIE at C-11 (three incubations) \pm standard deviation. "The average secondary KIE at C-9,10 (three incubations) \pm standard deviation.

The analytical results obtained from these competitive experiments are displayed in Table 4.1. The existence of a large primary deuterium kinetic isotope effect $(\mathrm{KIE}=7.8$ $\pm 0.8)$ for the $\mathrm{C}-\mathrm{H}$ bond cleavage at $\mathrm{C}-11$ was calculated. In contrast, the secondary kinetic isotope effect due to deuterium at $\mathrm{C}-9$ and $\mathrm{C}-10$ of the fatty acid chain (KIE = 
$0.87 \pm 0.04$ ) was found to be close to unity as expected. The observation of a very large primary deuterium isotope effect on $\mathrm{C}-11$ bond rupture is in accord with what has been observed with other members in the family of FAD2 homologues and indeed all membrane-bound desaturases studied to date (see Section 1.2.5). ${ }^{1,26,112,158}$ In general, the magnitude of the observed kinetic isotope effects are difficult to interpret precisely since partial masking of the intrinsic values by other enzymatic steps such as, substrate binding or product release may be occurring. ${ }^{1,201}$ Primary deuterium KIE that are larger than the theoretical maximum $\left(6.8 \text { at } 28^{\circ} \mathrm{C}\right)^{207}$ such as we have observed here have been observed before in desaturase-mediated reactions $\mathrm{s}^{26,79}$ and may indicate a tunneling phenomenon ${ }^{195}$. In any case, these data can now be used to help interpret the results of the stereochemical investigations as reported in the next section.

\subsection{Incubation of Chiral $d_{1}$-Oleates: Stereochemical Studies}

To determine the enantioselectivity of dimorphecolate formation from oleate in the cascade reaction involving two FAD2 variants, the sterilized solution of saponified methyl esters of chiral $\left[11-{ }^{2} \mathrm{H}_{1}\right]$-oleates $3 \mathrm{a}$ and $3 \mathbf{b}(5 \mathrm{mg}$ each) were administered separately to growing cultures of pESC-HIS/YPH499 strain of S. cerevisiae $(50 \mathrm{~mL})$ under conditions identical to that of the trial experiment. A small portion of the chiral fatty acid solution in Tergitol was also withdrawn prior to the incubation to evaluate the isotopic deuterium content $\left(d_{1} / d_{0}\right)$ of the administered compounds by GC-MS. The cellular fatty acid fraction of the harvested yeast cells were collected by hydrolysis/methylation procedure similar to the trial experiment and treated with 
BSTFA/pyridine ${ }^{187}$ in order to produce the trimethylsilyl derivative of methyl dimorphecolate.

Mass spectral analysis of the TMS derivative of the dimorphecolate product derived from $(11 S)-\left[11-{ }^{2} \mathrm{H}_{1}\right]$-oleic acid $3 \mathrm{a}$ revealed that the most abundant ion in the molecular and base peak cluster appeared at $\mathrm{m} / \mathrm{z} 382$ and 225 , respectively (Figure 4.8); this represents the overall loss of deuterium. In contrast, the mass spectrum of the TMS derivative of the dimorphecolate product derived from $(11 R)-\left[11-{ }^{2} \mathrm{H}_{1}\right]$-oleic acid $3 b$ indicated that the ions at $\mathrm{m} / \mathrm{z} 383$ and 226, respectively (Figure 4.9), were greatly enhanced; this represents the overall retention of deuterium labeling during the enzymatic reaction. The substantial amount of $d_{0}$ product in this mass spectrum can be traced to the substantial amount of endogenous $d_{0}$ oleate that is produced in this yeast mutant. The quantitation of deuterium loss vs. retention at C-11 was accomplished by analyzing the base peak of the TMS derivative of methyl dimorphecolate which is the diagnostic peak for the trimethylsilylated derivative (Figure 4.8 and 4.9) and carrying out the appropriate correction of ion intensity at $\mathrm{m} / \mathrm{z} 226$ due to $M+1$ contribution (ca. $19.3 \%$ ) from its $d_{0-}$ counterpart $(\mathrm{m} / \mathrm{z} 225)$. The $\mathrm{M}+1$ correction factor was determined experimentally using $\mathrm{d}_{0}$-dimorphecolate and compared to the theoretical values. The deuterium content of oleate, the $9 Z, 12 E$-octadecadienoate intermediate and dimorphecolate product is displayed in Table 4.2 . 


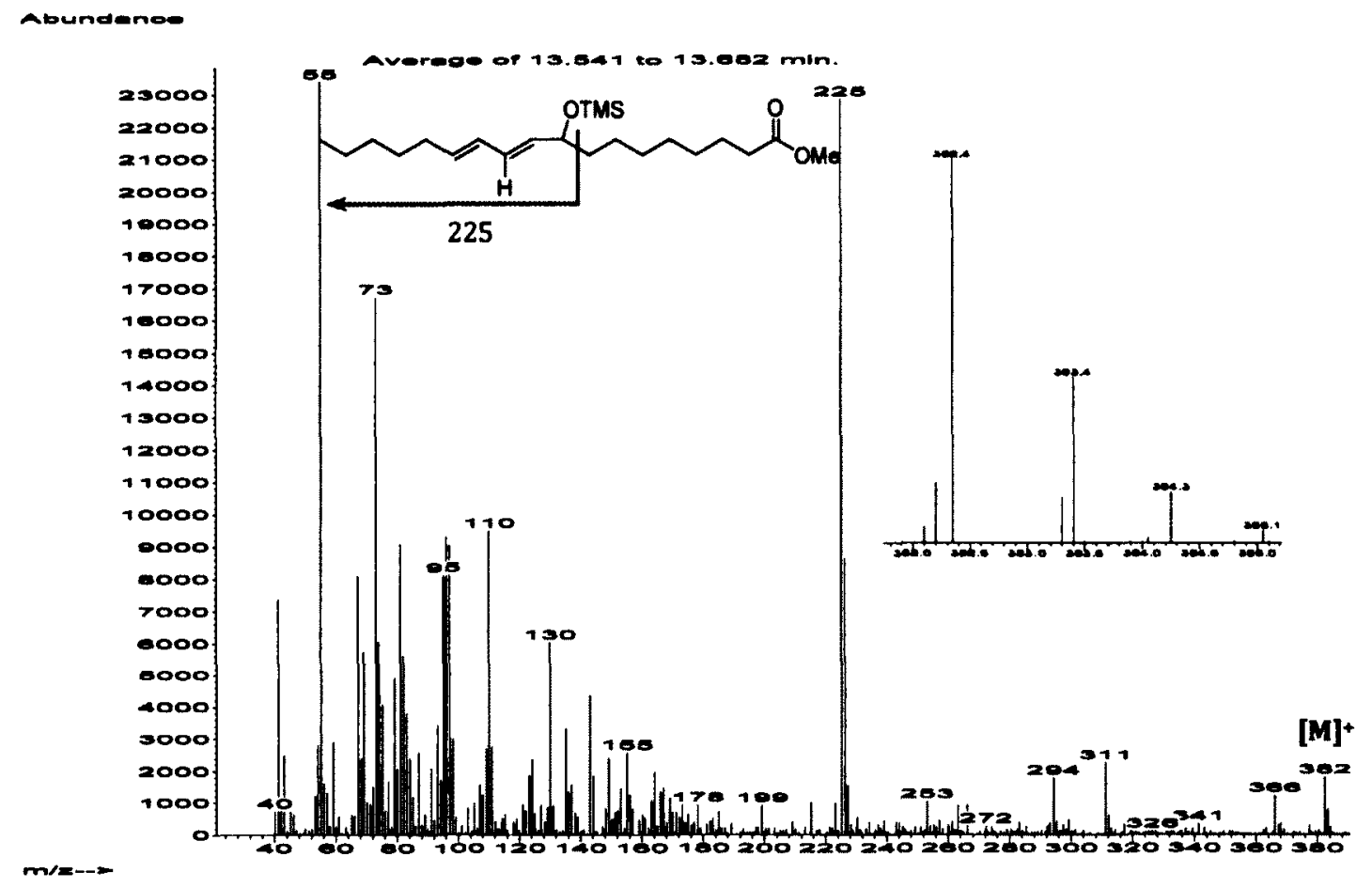

Figure 4.8. Representative mass spectrum of TMS derivative of the methyl ester of dimorphecolic acid derived from methyl (11S)-[11- $\left.{ }^{2} \mathrm{H}_{1}\right]$-oleate $3 a$.

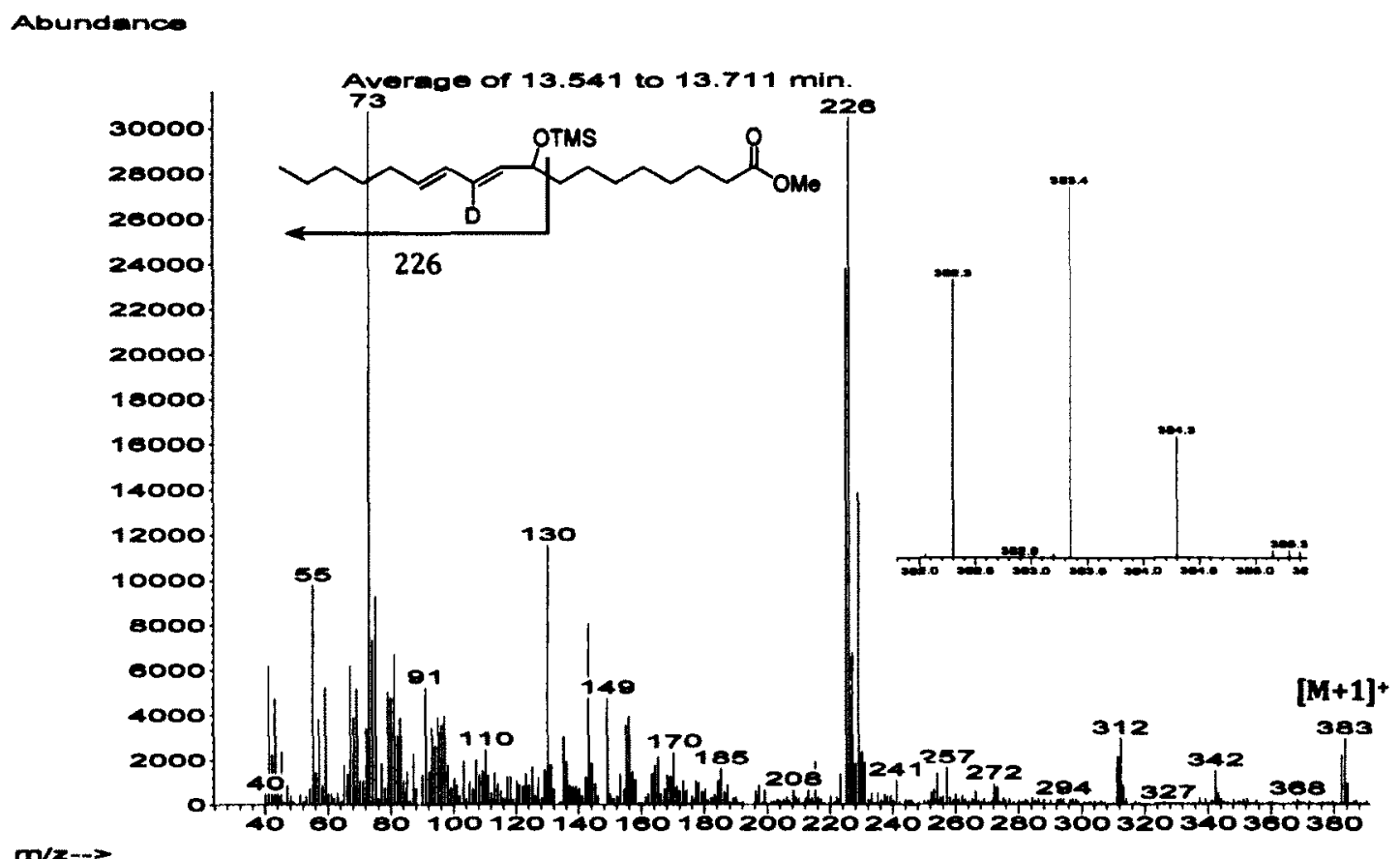

Figure 4.9. Representative mass spectrum of TMS derivative of the methyl ester of dimorphecolic acid derived from methyl $(11 R)-\left[11-{ }^{2} \mathrm{H}_{1}\right]$-oleate $3 b$. 
Table 4.2. Isotopic content of stereospecifically labeled monodeuterated oleates and DsFAD2-1 and DsFAD2-2 involved hydroxylated products (dimorphecolate).

\begin{tabular}{|c|c|c|c|c|}
\hline \multirow[t]{2}{*}{ Compound } & \multicolumn{2}{|c|}{ Cellular Oleate ${ }^{a}$} & \multicolumn{2}{|c|}{$\begin{array}{c}\text { Dimorphecolate } \\
\text { Product }^{a}\end{array}$} \\
\hline & $\% \mathrm{~d}_{0}^{b}$ & $\% \mathrm{~d}_{1}$ & $\% \mathrm{~d}_{0}$ & $\% d_{1}$ \\
\hline$(11 S)-\left[11-{ }^{2} H_{1}\right]-18: 1$ & 39.1 & 60.9 & $\begin{array}{l}84.4 \\
(88)^{c}\end{array}$ & $\begin{array}{l}15.6 \\
(12)^{c}\end{array}$ \\
\hline$(11 R)-\left[11-^{2} \mathrm{H}_{1}\right]-18: 1$ & 39.4 & 60.6 & $\begin{array}{l}48.3 \\
(43)^{c}\end{array}$ & $\begin{array}{l}51.7 \\
(57)^{c}\end{array}$ \\
\hline
\end{tabular}

${ }^{a}$ Incubation of each enantiomer was carried out three times and the \% isotopic content of each species is given as an average value of three independent GC-MS runs. The average standard deviation for these measurements is \pm 0.4 expressed as a percentage. ${ }^{b} \mathrm{~d}_{0}$ Content is due to endogenous oleate production. ${ }^{c}$ Predicted values are based on $80 \%$ ee of the chiral substrates and KIE of 6 at C-11.

The deuterium content of the dimorphecolate product can be rationalized by considering that:

1) $40 \%$ of the oleate substrate is non-deuterated due to endogenous oleate production.

2) The chiral $d_{1}$-substrates may contain as much as $10 \%$ of the minor enantiomer. This is based on $90 \%$ ee of the chiral alcohol intermediate (Figure 3.19) used in the synthesis and a stereospecificity of $~ 90 \%$ in the inversion reaction using acetylide anion (Figure 3.24).

3) C-11-D cleavage is subject to a large KIE isotope effect as demonstrated in the previous section.

The predicted values for $\% d_{0}$ and $\% d_{1}$ of dimorphecolate product derived from the two chiral 11-d $d_{1}$-oleates were calculated as follows: 
For the case when deuterium is lost from (11S) substrate with large KIE

$39.1 \mathrm{~d}_{0}$-Substrate $\stackrel{\mathrm{k}_{\mathrm{H}}}{\longrightarrow} 39.1 \mathrm{~d}_{0}$ - Product

$54.8 \quad(11 S)-\mathrm{d}_{1}-$ Substrate $\stackrel{\mathrm{k}_{\mathrm{D}}}{\longrightarrow} 7.0 \quad \mathrm{~d}_{0}$ - Product $\left(\mathrm{k}_{\mathrm{D}}=\mathrm{k}_{\mathrm{H}} / 7.8\right)$.

$6.1 \quad(11 R)-d_{1}$-Substrate $\stackrel{\mathrm{k}_{H}}{\longrightarrow} 6.1 \quad \mathrm{~d}_{1}$ - Product

$\% \mathrm{~d}_{0}$ Product $=(39.1+7.0) /$ Total $\times 100=46.1 / 52.2 \times 100=88 \%$

$\% d_{1}$ Product $=100-88=12 \%$

For the case when deuterium is retained from $(11 R)$ substrate

$39.4 \mathrm{~d}_{0}$-Substrate $\stackrel{\mathrm{k}_{\mathrm{H}}}{\longrightarrow} 39.4 \mathrm{~d}_{0}$ - Product

$6.1 \quad(11 S)-\mathrm{d}_{1}$-Substrate $\stackrel{\mathrm{k}_{\mathrm{D}}}{\longrightarrow} 0.8 \quad \mathrm{~d}_{0}$ - Product $\left(\mathrm{k}_{\mathrm{D}}=\mathrm{k}_{\mathrm{H}} / 7.8\right)$.

$54.5 \quad(11 R)-\mathrm{d}_{1}$-Substrate $\stackrel{\mathrm{k}_{\mathrm{H}}}{\longrightarrow} 54.5 \mathrm{~d}_{1}$ - Product

$\% \mathrm{~d}_{0}$ Product $=(39.4+0.8) /$ Total $\times 100=40.2 / 94.7 \times 100=43 \%$

$\% \mathrm{~d}_{1}$ Product $=100-43=57 \%$

Inspection of Table 4.2 revealed that the observed results are in reasonable agreement with the predicted deuterium content in both cases, given the uncertainty in the $\%$ ee of the starting materials.

This result signifies that during the course of dimorphecolic acid biosynthesis, the hydroxylase enzyme removes the pro $S$ hydrogen from the C-11 position of trans- $\Delta^{12}$. linoleic acid and delivers the hydroxyl group topologically on the same side of the olefinic plane at C-9 position. Our observed topology of hydrogen removal at C-11 clearly corresponds to the established topology of hydrogen abstraction by the parent FAD2 enzyme during the formation of linoleic acid (Figure 2.1). ${ }^{1,2,140}$ The observed 
stereochemistry also matches the reported enantioselectivity of calendate formation by the same family of FAD2 enzyme, $\Delta^{8,10}$-desaturase which was also found to initiate the bioconjugation by removing the pro $S$ hydrogen at $\mathrm{C}-11$ position of linoleic acid (see Chapter 2). Overall, the result obtained in this experiment further enhance the proposed active site architectural motif for the family of FAD2 homologues that the overall contours of the binding site for all these enzymes are similar.

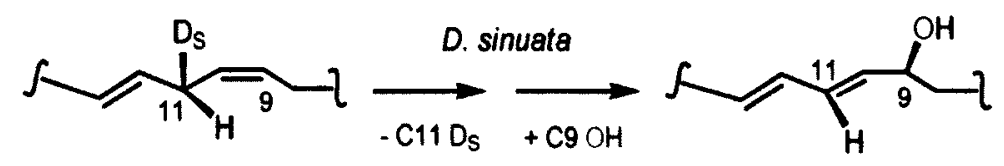

Loss of Deuterium

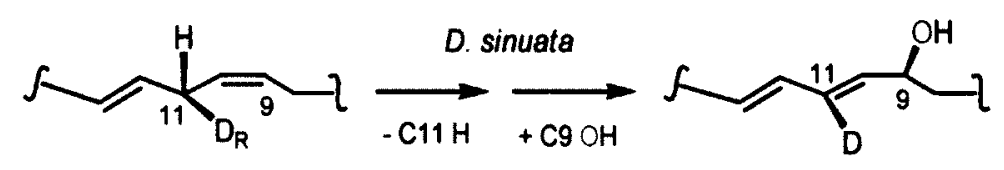

Retention of Deuterium

Figure 4.10. Observed results for dimorphecolate formation from stereospecifically deuterated trans- $\Delta^{12}$-linoleate. Note CIP priority rules dictate that the deuterium atom that is removed from $\mathrm{C}-11$ position is labeled $\mathrm{D}_{\mathrm{s}}$.

Interestingly, this experimental finding also coincides well with the overall topology of another hydroxylated fatty acid formation, namely ricinoleic acid by a different FAD2 variant in the seeds of Ricinus communis $L$., where the desaturase enzyme act as $\Delta^{12}$-hydroxylase and produces the alcohol in a stereoselective manner (Figure 4.11 and 1.26). ${ }^{19,16}$ The regiochemistry of hydroxyl delivery in dimorphecolate formaton may well be dictated by which iron oxygen bond of the diiron dioxo species is cleaved during the initial hydrogen abstraction step. 


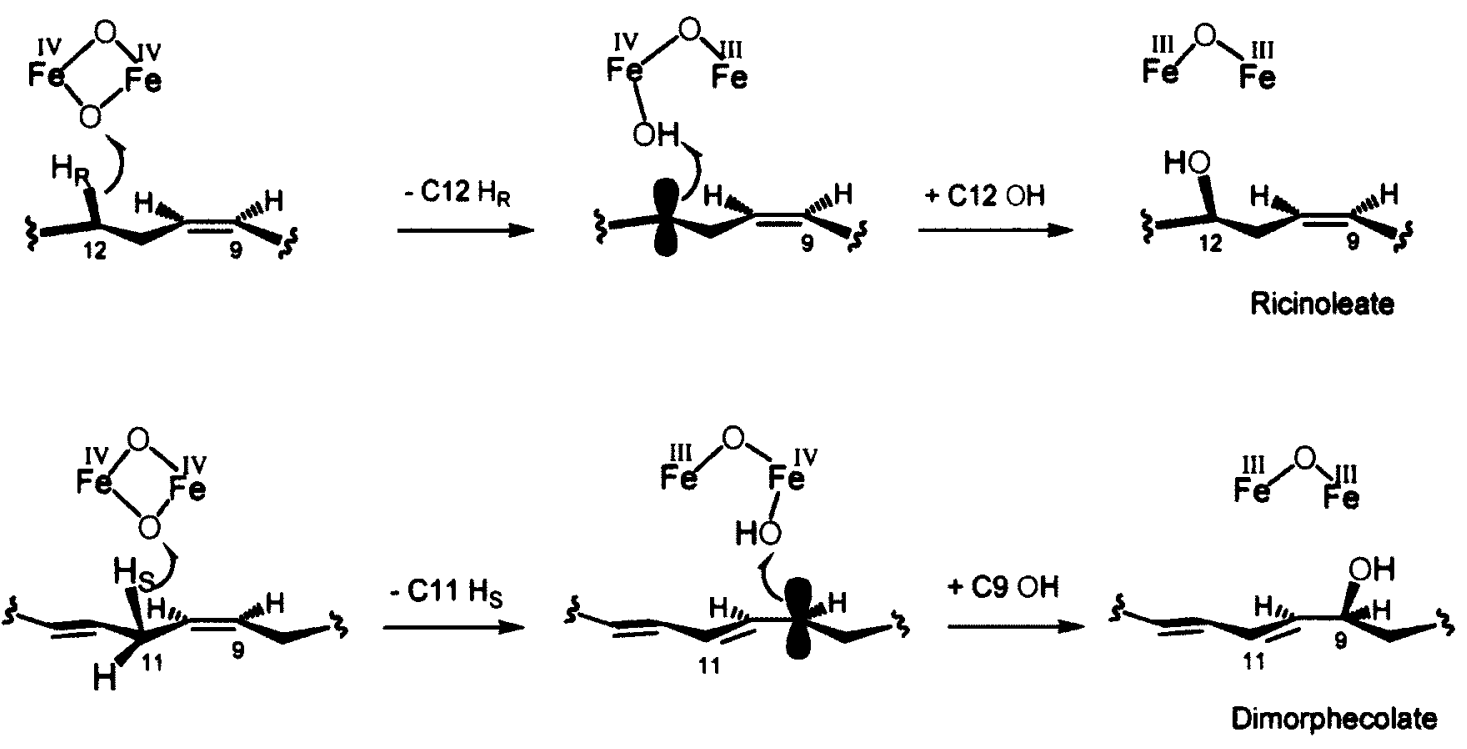

Figure 4.11. Comparison of relative stereochemistry of hydroxylation by FAD2 variants in the biosynthesis of ricinoleate and dimorphecolate.

In the current context of understanding the biosynthesis of unusual hydroxylated fatty acid in plants, it is necessary to mention the serendipitous discovery by Shanklin and coworkers of a new triple mutant T117R/G188L/D280K of soluble plant castor stearoyl-ACP $\Delta^{9}$-desaturase that shows activity analogous to dimorphecolate formation. ${ }^{116}$ It was observed that the mutant desaturase converts oleyl-ACP substrate into a major 9-hydroxy-(10E)-octadecenoic acid.

The mutant desaturase initiates the oxidation at $\mathrm{C}-11$ of oleyl-ACP and generates an allylic intermediate that contains a radical at C-9 which abstracts the hydroxyl group from diiron catalytic center in highly regiospecific fashion (Figure 4.12). $\mathrm{O}^{18}$ labeling experiment demonstrated that the hydroxyl oxygen is well derived from molecular oxygen via the hydroxyl rebound to the radical intermediate at C-9. The mutant desaturase enzyme was also identified to use trans-isomer of oleoyl-ACP as substrate and transform it into two major products, 9-hydroxy-(10Z)-octadecenoic acid and $(9 E, 11 Z)$ - 
CLA isomer. It was proposed that the fate of the reaction outcome from various substrates is primarily governed by the curvature of the substrate binding cavity, which is boomerang shaped with a bend adjacent to the active site (Figure 4.12).
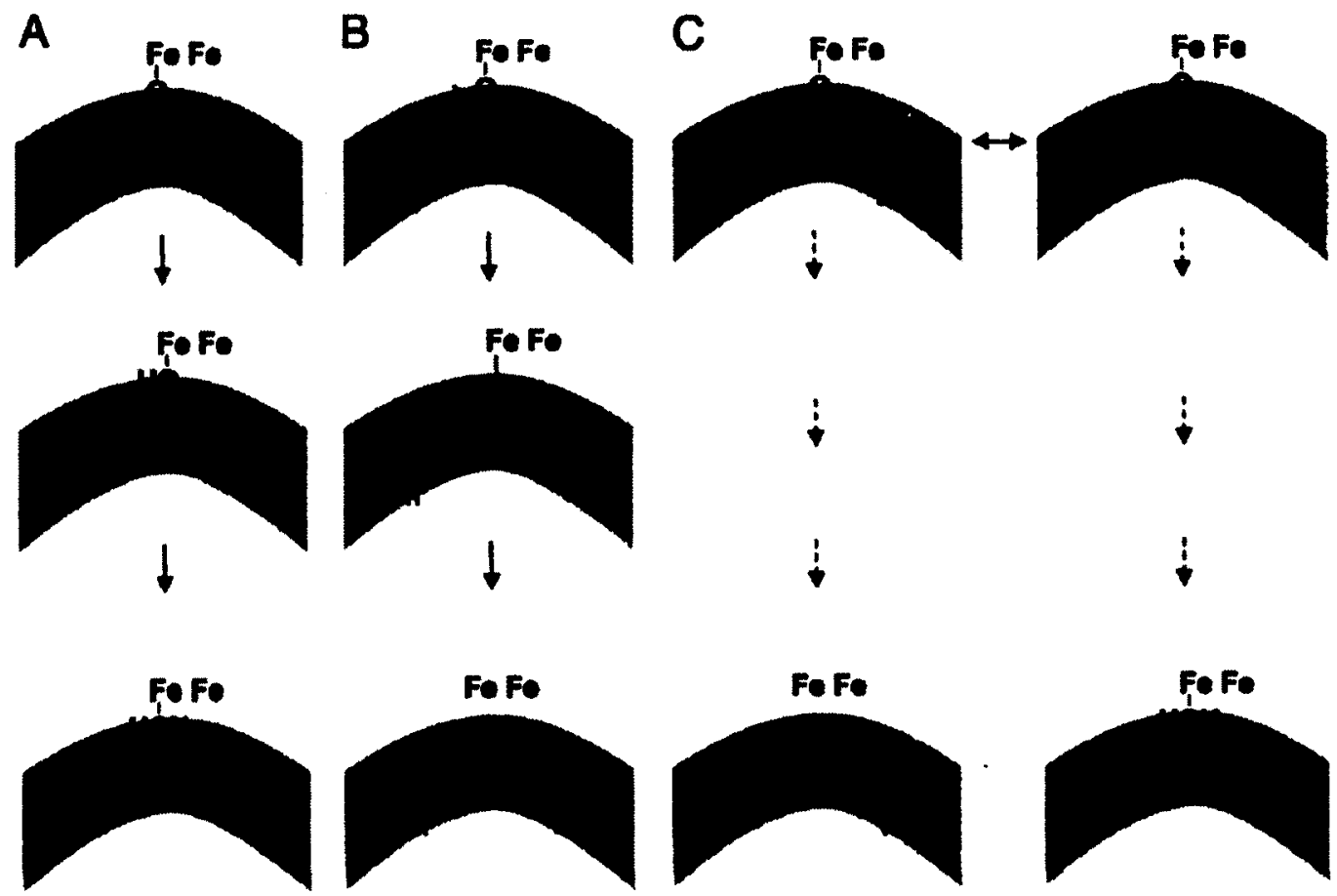

Figure 4.12. Proposed substrate binding modes and reaction outcomes for various substrates with mutant soluble desaturase. The mechanism of (Z)-9-18:A (panel A) and $(E)-10-18: 1-9-\mathrm{OH}$ (panel B) formation are shown in the context of the bend in the desaturase substrate channel (shown in gray) adjacent to the active site oxidant. Postulated substrate binding modes of the nonnatural elaidyl substrate (panel $C$ ) that leads to the formation of $(Z)-10-18: 1-9-\mathrm{OH}$ and $(E)-9-(Z)-11-18: 2$ are shown. The Figure was adapted from Shanklin et al. ${ }^{116}$

A precise positioning of the substrate in this binding curvature is necessary to allow exclusive formation of specific hydroxylated product, as it occurs with oleyl-ACP, which fits nicely in the substrate binding cavity (Figure 4.12B) and allows the hydrogen abstraction to proceed at C-11 followed by stereospecific hydroxylation at C-9 leading to the production of 9-hydroxy-(10E)-octadecenoic acid. Conversely, in case of trans-oleyl isomer because of its inability to completely insert into the substrate binding cavity, the 
corresponding reaction results in the formation of 9-hydroxy-(10Z)-octadecenoic acid as the major and 11-hydroxy-(9E)-isomer as minor product (Figure $4.12 C$ ). It would be of interest to determine more precisely how the DsFAD2-2 hydroxylase favours formation of hydroxylation over dehydrogenation.

\subsection{Conclusions}

The enantioselectivity of another important unusual plant fatty acid biotransformation as it occurs in Dimorphotheca sinuata has been determined in this chapter. Our investigation established that the formation of dimorphecolic acid involved the initial, isotopic sensitive removal of pro $S$ hydrogen from C-11 of trans- $\Delta^{12}$-linleate and generates an allylic radical intermediate that removes the oxygen atom from the catalytic diiron center to hydroxylate in syn fashion at the pro $S$ position (Figure 4.13). A large primary deuterium KIE was observed for the initial hydrogen abstraction.

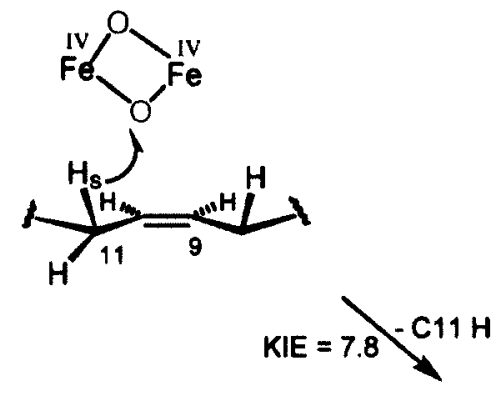

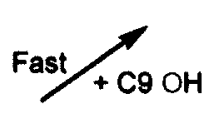
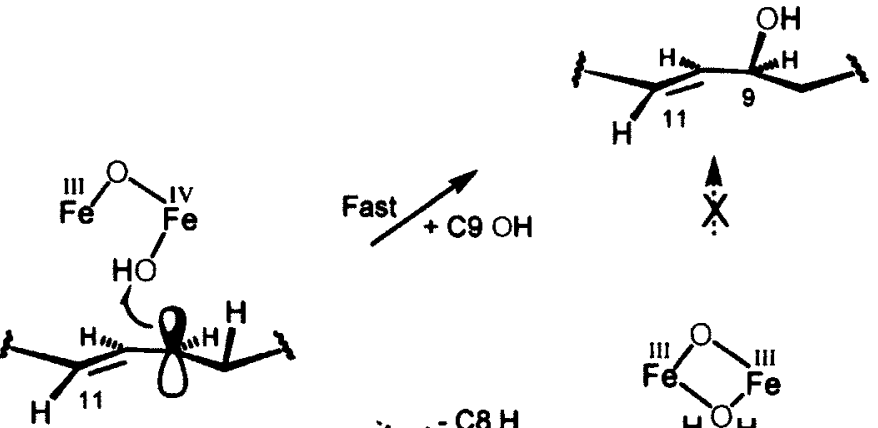
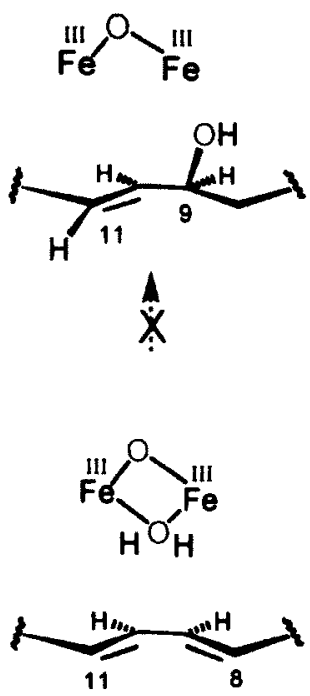

Figure 4.13. Mechanistic model for DsFAD2-2 hydroxylase involved biotransformation of trans- $\Delta^{12}$-linoleate to dimorphecolate as it occurs in the seeds of Dimorphotheca sinuata. 
The observed stereochemistry and site of initial oxidation in dimorphecolate formation correlates well with that observed for the parent FAD2 enzyme involved desaturation of oleate to generate linoleate $\left(\mathrm{CH}_{3}-\left(\mathrm{CH}_{2}\right)_{5}-\mathrm{C}_{13}=\mathrm{C}_{12}-\mathrm{CH}_{2}-\mathrm{C}_{10}=\mathrm{C}_{9}-\left(\mathrm{CH}_{2}\right)_{7}-\right.$ $\mathrm{CO}_{2} \mathrm{H}$ ) and validates the proposed mechanistic model (Figure 4.13). Since no dehydrogenated product could be detected, this militates against the possibility of the presence of a minor pathway involving further dehydrogenation at $\mathrm{C} 9 \mathrm{-C} 8$ position followed by hydration at C-9. The results obtained from this experiment further strengthen the proposal that there exists a highly conserved, active site structural motif for the family of membrane-bound FAD2 homologues. 


\section{Chapter 5: Summary and Future Directions}

\subsection{Summary}

All of the research objectives that were formulated at the outset of this thesis work have been achieved. The results of mechanistic studies of various conjugated fatty acid biosyntheses are as follows:

The enantioselectivity of Calendula officinalis $\Delta^{8,10}$-desaturase (FAD2 variant) - mediated formation of calendic acid from linoleic acid has been determined for the first time using deuterium-labelled mechanistic probes. It was observed that the $\Delta^{8,10}$-desaturase enzyme initiates the oxidation by removing the pro $S$ hydrogen from the $\mathrm{C}-11$ position of linoleic acid and completes the sequence by removing another hydrogen from the non-adjacent pro $R$ position at C-8 in syn fashion. The primary deuterium KIE at C-11 was also assessed using racemic methyl $\left[1-^{2} \mathrm{H}_{1}\right]$-linoleate and the calculated value $(4.4 \pm 0.1)$ was found to be in agreement with that previously reported ${ }^{158}$ when secondary isotope effects are taken into account.

2) We have also investigated the cryptoregiochemistry (site of initial oxidation) and enantioselectivity of Aleurites fordii Hemsl. $\Delta^{11,13}$-desaturase involved in the formation of $\alpha$-eleostearic acid from linoleic acid. The site of initial oxidation for this enzyme has been determined to be at C-11 using KIE methodology; this result is similar to that observed during calendic acid biosynthesis and matches with our prediction. The deuterium kinetic isotope effect at C-11 was also evaluated by using racemic methyl $\left[11^{2} \mathrm{H}_{1}\right]$-linoleate and the anticipated result $(4.0 \pm 0.1)$ is in accord with that estimated using 
approximately $1: 1(\mathrm{w} / \mathrm{w})$ mixture of non-deuterated to regiospecifically dideuterated linoleates. However, using chiral monodeuterated fatty acids as mechanistic probes it was revealed that the enantioselectivity of $\alpha$-eleostearic acid formation doesn't correlate with our prediction. Thus, to explain the stereochemical outcome, a revised substrate conformation was proposed where $\Delta^{11,13}$-desaturase enzyme initiates the oxidation by removing the pro $R$ hydrogen from the $\mathrm{C}-11$ position of linoleic acid and completes the sequence following the removal of non-adjacent pro $R$ hydrogen from the C-14 position in a syn dehydrogenation fashion.

The enantioselectivity of dimorphecolic acid formation from the $12 E$ isomer of linoleic acid in Dimorphotheca sinuata was also investigated. Using chiral $\left[11-{ }^{2} \mathrm{H}_{1]}\right.$-oleates as precursors, it was demonstrated that the oxidation was initiated by isotopically sensitive removal of pro $S$ hydrogen from C-11 position of the trans- $\Delta^{12}$-linoleate substrate to generate an allylic radical that captures ironbound hydroxyl at C-9 in an enantioselective manner from the same side of the olefinic plane.

Overall, the results obtained from these mechanistic studies clearly establish the fact that there exists a highly conserved, active site architectural motif for the family of membranous FAD2 homologues (Figure 4.14), where the oxidation is always initiated by approaching the olefinic plane at or near the $\mathrm{C}-12$ position of the substrate from the top ( $\mathrm{Cl}$ terminus at the right hand side of the structure $\left(\mathrm{CH}_{3}-\left(\mathrm{CH}_{2}\right)_{5}-\mathrm{C}_{13}=\mathrm{C}_{12}-\mathrm{CH}_{2}-\mathrm{C}_{10}=\mathrm{C}_{9}-\right.$ $\left.\left.\left(\mathrm{CH}_{2}\right)_{7}-\mathrm{CO}_{2} \mathrm{H}\right)\right)$ : 


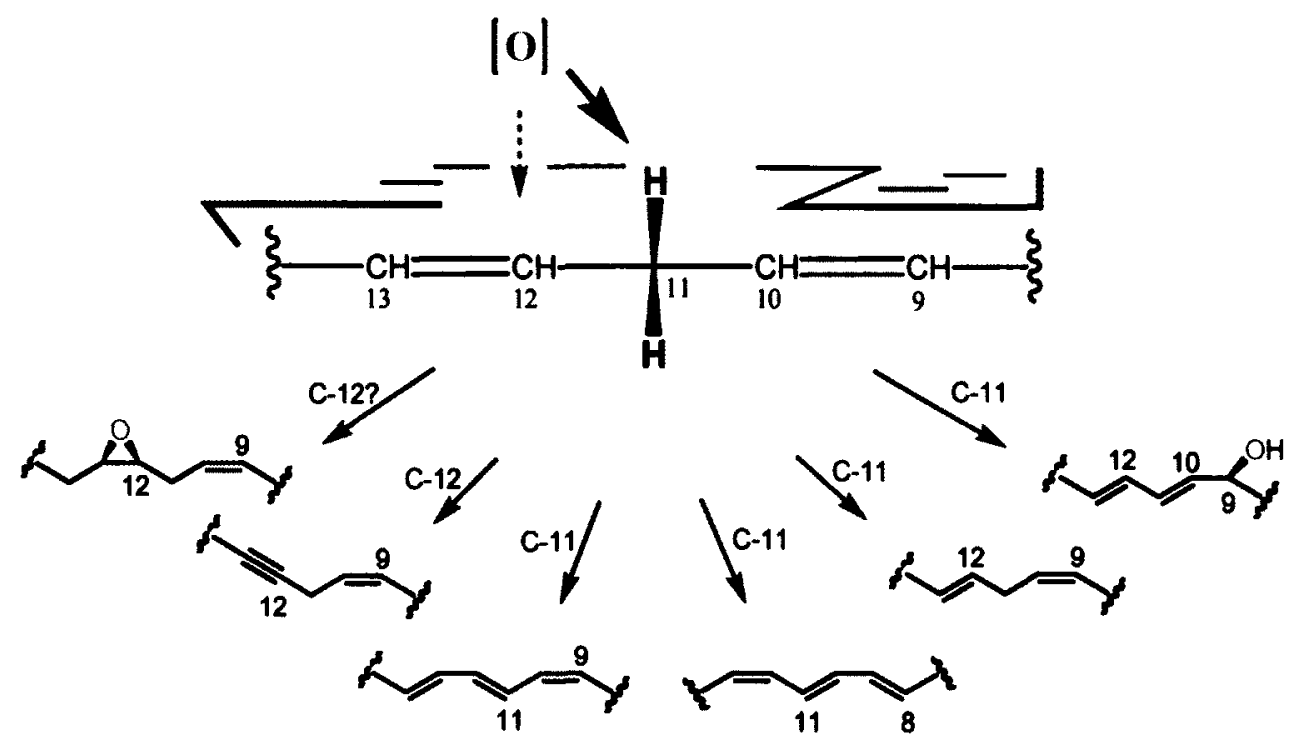

Figure 5.1. Highly conserved active site model of the membrane-bound family of FAD2 homologues showing that the biooxidation is normally initiated via the removal of topologically top hydrogen from the plane of the olefin at the carbon closest to C-12, the site of initial oxidation for parent FAD2 enzyme.

\subsection{Future Directions}

As a part of ongoing research towards the understanding of the structure-function relationships of the family of FAD2-like enzymes, our KIE methodology and chiral deuterium labeled mechanistic probes can be used to further investigate the formation of other unusual fatty acids, such as $\alpha$-parinaric acid (1,4-desaturation) and vernolic acid (epoxidation). In doing so, we hope to correlate the various regio- and enantioselectivities observed for this important set of catalysts with the geometric relationship between oxidant and substrate.

As already discussed in Chapter 3 , since the $\Delta^{11,13}$-desaturase enzyme from Aleurites fordii $\mathrm{Hemsl}^{34}$ exhibits multifunctional enzymatic plasticity by inserting either a trans double bond at $\mathrm{Cl} 2,13$ of an oleate substrate or performing another 1,4-desaturation of $\alpha$-linolenic acid to produce $\alpha$-parinaric acid with the same ease. Therefore, it would be 
interesting to compare the cryptoregiochemistry and stereochemistry of these transformations with that observed for $\alpha$-eleostearic acid biosynthesis. In this manner, the contours of the active site can be further defined.

Experiments could also be performed in the future to determine the origin of oxygen atom in dimorphecolic acid. Also the validity of the proposed active site model for the castor stearoyl-ACP $\Delta^{9}$-desaturase derived triple mutant T117R/G188L/D280K ${ }^{116}$ could be further tested by determining the stereochemistry of hydrogen abstraction and hydroxyl capture.

Finally, the interpretation of the results obtained from these mechanistic studies would be greatly aided by characterizing the membranous FAD2-like enzymes via $\mathrm{X}$-ray crystallography. Analysis of enzyme-substrate complexes will improve our understanding about how the active-site architecture controls the reaction outcome. From a broader point of view, understanding structure-function relationships will assist plant biotechnologists to develop novel transgenic plants and produce lipid-based bio-products with desired properties. 


\section{Chapter 6: Part I Experimental}

\subsection{Materials and Methods}

${ }^{1} \mathrm{H}(300 \mathrm{MHz}),{ }^{13} \mathrm{C}$ NMR $(75 \mathrm{MHz})$, and DEPT NMR spectra were obtained on a Bruker Avance 300 spectrometer. Analytes were dissolved in $\mathrm{CDCl}_{3}$, that was dried by passing the solvent through a short-column packed with neutral alumina (Activated aluminum oxide, Brockmann I standard grade, 150 mesh, 58 A). Typical analyte concentration was $10 \mathrm{mg} / \mathrm{mL}$ for ${ }^{1} \mathrm{H}$ NMR and $20 \mathrm{mg} / \mathrm{mL}$ for ${ }^{13} \mathrm{C}$ NMR and DEPT. Chemical shifts for ${ }^{1} \mathrm{H}$ and ${ }^{13} \mathrm{C}$ NMR spectra are reported in ppm ( $\delta$ ) relative to TMS $(0.00 \mathrm{ppm})$ and standardized to a chemical shift value of $7.2650 \mathrm{ppm}\left({ }^{1} \mathrm{H}\right)$ and 77.0275 ppm $\left({ }^{13} \mathrm{C}\right)$ for residual $\mathrm{CHCl}_{3}\left({ }^{1} \mathrm{H}\right)$ and $\mathrm{CDCl}_{3}\left({ }^{13} \mathrm{C}\right)$. Abbreviations used to assign multiplicity of peaks are as follows: $s=$ singlet, $d=$ doublet, $t=$ triplet, $q=$ quartet, $m=$ multiplet and $b=$ broad. $J$-values are reported in Hertz $(\mathrm{Hz})$. The isotope shifts on the ${ }^{13} \mathrm{C}$ NMR resonances of deuterated compounds are reported in Appendix A and B.

GC-MS data of the synthetic compounds were obtained courtesy of the University of Ottawa's mass spectrometry facility (Dr. Clem Kazakoff) using a Kratos Concept ${ }^{1} \mathrm{H}$ mass spectrometer at $70 \mathrm{eV}$ interfaced with a J \& W $30 \mathrm{~m} \times 0.21 \mathrm{~mm}$, DB-5 capillary column. The temperature program started at $120^{\circ} \mathrm{C}$ and increased at $10{ }^{\circ} \mathrm{C} / \mathrm{min}$ until 320 ${ }^{\circ} \mathrm{C}$ and held for 2 minutes.

GC-MS (EI, $70 \mathrm{eV}$ ) of the biological extracts were obtained at Plant Biotechnology Institute, National Research Council, Saskatoon using an Agilent 5973 Mass Selective Detector coupled to an Agilent 6890 gas chromatograph equipped with J \& W Scientific $30 \mathrm{~m} \times 0.253 \mathrm{~mm}$ DB-23 capillary column (with a film thickness of 0.25 $\mu \mathrm{m})$, where the oven temperature was programmed from $160{ }^{\circ} \mathrm{C}$ to $240{ }^{\circ} \mathrm{C}$ with an 
increment rate of $4{ }^{\circ} \mathrm{C} / \mathrm{min}$ and then isothermal at $240{ }^{\circ} \mathrm{C}$ for 15 minutes with a flow rate of $1.0 \mathrm{~mL} / \mathrm{min}$. Helium flow was set at $30 \mathrm{~m} / \mathrm{s}$. Analyses were carried out under a selected ion monitoring (SIM) mode with a narrow $\mathrm{EI}^{+}$scan range of $280-305 \mathrm{~m} / \mathrm{z}$ used at a rate of 1500 mass units / second that resulted in $\sim 250$ scans/GC peak. The integrated intensities of the individual ions in the pertinent ion cluster were recorded using Agilent MSD Chemstation software and corrected for natural isotopic abundances. The entire GC peak in the integration was carefully included to prevent errors due to fractionation of isotopic species during chromatography.

Purification of the biosynthesized $(11 R)$-and $(11 S)-\left[11-{ }^{2} \mathrm{H}_{1}\right]$-linoleates were carried out on a Hewlett-Packard 1100 series HPLC system (Hewlett-Packard, Pal Alto, CA) equipped with an auto-sampler, solvent degasser, quaternary pump, column heater, and diode array detector (monitored at 205 and $254 \mathrm{~nm}$ ) which were all controlled by HP Chemstation software. The samples were separated on a Phenomenex Gemini-5 $\mu \mathrm{m}$ C18 $110 \mathrm{~A} 250 \mathrm{~mm} \times 10 \mathrm{~mm}$ column, eluting at a rate of $3 \mathrm{~mL} / \mathrm{min}$ isocratically for 5 minutes with acetonitrile followed by $0-30 \%$ acetone gradient in 20 minutes and subsequent flushed with $100 \%$ acetone to rinse and then re-equilibrate. The spectral data obtained for these compounds were in accord with that reported in the literature. The purity of the deuterated fatty acid substrates were estimated using GC-MS by means of an Agilent 5973 Mass Selective Detector coupled to an Agilent 6890 gas chromatograph equipped with J \& W Scientific $30 \mathrm{~m}$ x $0.253 \mathrm{~mm}$ DB-23 capillary column (with a film thickness of $0.25 \mu \mathrm{m}$ ) and controlled by Chemstation software and found that all of them were > 95-99\% chemically pure. 
IR spectra were recorded on a Varian 1000 Scimitar series FT-IR spectrometer and the vibrational frequencies of the chemical bonds were reported in $\mathrm{cm}^{-1}(v)$.

Purification of synthetic compounds by flash chromatography was performed using Merck silica gel (60 A), grade 9385 (230-400 mesh) with an air pressure of ca. 5 psi. Thin layer chromatography was carried out using glass-backed plates coated with Merck silica gel $(60 \mathrm{~A})$. Visualization of TLC spots was achieved by a combination of UV (aromatic compounds), $I_{2}$ vapour (unsaturated compounds) and water spray (universal detection of all compounds) as appropriate.

All reagents obtained commercially were acquired from Sigma-Aldrich and used without purification unless indicated. Freshly purified $p$-toluenesulfonyl chloride was used whenever required: reagent grade $p$-toluenesulfonyl chloride $(10 \mathrm{~g})$ was dissolved in minimum amount of chloroform $(25 \mathrm{~mL})$ and the solution was diluted with five volumes of spectrograde hexane $(125 \mathrm{~mL})$, swirled. The solid precipitate, that formed was removed by gravity filtration. The solvent was evaporated by rotary evaporation and the compound was dried over vacuum. Pyridine was dried over $\mathrm{NaOH}$ pellets. Tetrahydrofuran (THF) and diethyl ether $\left(\mathrm{Et}_{2} \mathrm{O}\right)$ were freshly distilled from $\mathrm{Na}$ benzophenone ketyl. Toluene was freshly distilled from NaH. Dichloromethane was distilled from $\mathrm{CaH}_{2}$. For use in moisture-sensitive reactions, Spectrograde ${ }^{\mathrm{TM}}$ acetone was dried over activated molecular sieves (grade $4 \mathrm{~A}, 1.6 \mathrm{~mm}$ pellets) and under $\mathrm{N}_{2}$ for $1 \mathrm{~h}$. All air and moisture-sensitive reactions were performed under ultra pure $\mathrm{N}_{2}$ grade 5.0. When required, the reaction mixtures were cooled down to 0 to $-4{ }^{\circ} \mathrm{C},-10$ to $-12{ }^{\circ} \mathrm{C},-48$ to $-50{ }^{\circ} \mathrm{C}$ and -68 to $-72{ }^{\circ} \mathrm{C}$ using ice-water, ice- $\mathrm{NaCl}$ salt, acetone-dry ice and isopropanol-dry ice slurry, respectively. Moisture sensitive and reactive reagents were 
added via glass or plastic syringes and 21 gauge needles. Organic extracts were typically worked up by drying over anhydrous $\mathrm{Na}_{2} \mathrm{SO}_{4}$ in a cone filter paper followed by subsequent gravity filtration. The solvents were evaporated in vacuo on a Büchi RE 111 Rotavapor and then dried to constant weight under $\mathrm{N}_{2}$.

All of the non-deuterated compounds prepared in this thesis have been synthesized previously and in each case, the analytical data was compared with the literature values.

Non-deuterated fatty acids ( $>99 \%$ ) were obtained from Nu-Chek Prep, Inc. (Elysian, MN). $\left[11,11-{ }^{2} \mathrm{H}_{2}\right]$-oleic acid, $\left[9,10-{ }^{2} \mathrm{H}_{2}\right]$-oleic acid and $\left[18,18,18-{ }^{2} \mathrm{H}_{3}\right]$-oleic acid were synthesized previously by A. P. Tulloch (NRC-Saskatoon).

\subsection{Synthesis of Deuterium-Labeled Fatty Acids}

\subsubsection{Chiral $\left[8-{ }^{2} \mathrm{H}_{1}\right]$-Linoleates Synthesis}

\subsubsection{Ethyl 8-hydroxyoctanoate}<smiles>CCOC(=O)CCCCCCC(=O)OCC</smiles>

To a stirred solution of diethyl suberate $(8.2 \mathrm{~mL}, 35 \mathrm{mmol})$ in anhydrous THF ( 60 $\mathrm{mL}$ ) was added dropwise a solution of $2.0 \mathrm{M}$ lithium aluminium hydride in THF (9.3 mL, $18.5 \mathrm{mmol})$ at 0 to $-4^{\circ} \mathrm{C}$. After stirring the reaction mixture for $2.5 \mathrm{~h}$ at $0{ }^{\circ} \mathrm{C}$, EtOAc (10 $\mathrm{mL}$ ) was added to quench excess reducing agent and the solvent was removed in vacuo. The residue was suspended in $\mathrm{CH}_{2} \mathrm{Cl}_{2}(70 \mathrm{~mL})$ and poured into an ice-water mixture (75 $\mathrm{mL}$ ). The aqueous layer was acidified with $2.0 \mathrm{M} \mathrm{HCl}$ to $\mathrm{pH} 1$ and the organic layer was separated. The aqueous layer was re-extracted with $\mathrm{CH}_{2} \mathrm{Cl}_{2}(3 \times 50 \mathrm{~mL})$ and the organic 
layers combined. The combined organic phase was worked up in the normal manner to afford a mixture of the desired compound as well as the corresponding diol and starting material. After flash chromatography of the product mixture using EtOAc:hexane (1:4) as eluent, the pure title compound was obtained as a clear colorless liquid $(1.35 \mathrm{~g}, 21 \%)$. bp $85-87{ }^{\circ} \mathrm{C}$ at $0.1 \mathrm{~mm} \mathrm{Hg} ; R_{\mathrm{f}} 0.48$ (EtOAc/hexane, 9:1); IR (film): v $3361(\mathrm{O}-\mathrm{H}), 2933$, $2858(\mathrm{C}-\mathrm{H}), 1735(\mathrm{C}=\mathrm{O}), 1465,1374,1248,1184,1096,1036,860 \mathrm{~cm}^{-1} ;{ }^{1} \mathrm{H}$ NMR $(300$ $\left.\mathrm{MHz}, \mathrm{CDCl}_{3}\right) \delta_{\mathrm{H}} 4.11\left(q, J=7.3 \mathrm{~Hz}, 2 \mathrm{H}, \mathrm{OCH}_{2} \mathrm{Me}\right), 3.63\left(t, J=6.0 \mathrm{~Hz}, 2 \mathrm{H}, \mathrm{CH}_{2} \mathrm{OH}\right)$, $2.28\left(t, J=7.5 \mathrm{~Hz}, 2 \mathrm{H}, \mathrm{CH}_{2} \mathrm{CO}_{2} \mathrm{Et}\right), 1.62\left(t t, J=7.5,6.0 \mathrm{~Hz}, 2 \mathrm{H}, \mathrm{CH}_{2} \mathrm{CH}_{2} \mathrm{CO}_{2} \mathrm{Et}\right), 1.56$ $\left(t t, J=7.0,6.0 \mathrm{~Hz}, 2 \mathrm{H}, \mathrm{CH}_{2} \mathrm{CH}_{2} \mathrm{OH}\right), 1.42(s, 1 \mathrm{H}, \mathrm{OH}), 1.39-1.29(m, 6 \mathrm{H}, \mathrm{C}-4, \mathrm{C}-5, \mathrm{C}-$ 6), $1.25\left(t, J=7.3 \mathrm{~Hz}, 3 \mathrm{H}, \mathrm{CH}_{3}\right) ;{ }^{13} \mathrm{C}$ NMR $\left(100 \mathrm{MHz}, \mathrm{CDCl}_{3}\right) \delta_{\mathrm{C}} 173.89(\mathrm{C}-1), 34.32$ (C-2), 24.86 (C-3), 29.02 (C-4), 29.05 (C-5), 25.53 (C-6), 32.67 (C-7), 62.91 (C-8), 60.19 (-OCH $2 \mathrm{Me}), 14.24\left(\mathrm{CH}_{3}\right)$; EI-MS: $m / z 158\left([\mathrm{M}-32]^{+}\right), 143\left([\mathrm{M}-45]^{+}\right), 124,115,101,96$, 88, 83, 73, 70, 60, 55, 41; TMS Derivative EI-MS: $m / z 259$ ([M - 1] $), 245\left([\mathrm{M}-15]^{+}\right)$, $215\left([\mathrm{M}-45]^{+}\right), 199\left([\mathrm{M}-61]^{+}\right), 173\left([\mathrm{M}-87]^{+}\right), 157,129,115,103,88,75,73,55,43$.

\subsubsection{Ethyl 8-hydroxy- $\left[8,8-{ }^{2} \mathrm{H}_{2}\right]$-octanoate}<smiles>[2H]C([2H])(O)CCCCCCC(=O)OCC</smiles>

The title compound was synthesized from diethyl suberate $(44 \mathrm{~mL}, 188 \mathrm{mmol})$ using lithium aluminium deuteride $(1.0 \mathrm{M}$ in THF, $99.5 \mathrm{~mL}, 99.4 \mathrm{mmol})$ as reducing agent by following the same experimental procedure as used in the synthesis of ethyl 8hydroxyoctanoate. The pure compound was obtained as a clear colorless liquid (13.9 g, $35 \%$ ). bp $85-87^{\circ} \mathrm{C}$ at $0.1 \mathrm{~mm} \mathrm{Hg} ; R_{\mathrm{f}} 0.29$ (EtOAc:hexane, 1:1); and the spectral data of 
the compound was similar to that reported above for the non-deuterated parent compound as well as with that listed in the literature ${ }^{173}$. IR (film): $v 3387(\mathrm{O}-\mathrm{H}), 2981,2930,2858$ (C-H), 2193, 2087 (C-D), 1739 (C=O), 1465, 1373, 1257, 1184, 1108, 1034, 967, 859 $\mathrm{cm}^{-1} ;{ }^{1} \mathrm{H}$ NMR $\left(300 \mathrm{MHz}, \mathrm{CDCl}_{3}\right) \delta_{\mathrm{H}} 4.10\left(q, J=7.1 \mathrm{~Hz}, 2 \mathrm{H}, \mathrm{OCH}_{2} \mathrm{Me}\right), 2.27(t, J=7.5$ $\left.\mathrm{Hz}, 2 \mathrm{H}, \mathrm{CH}_{2} \mathrm{CO}_{2} \mathrm{Me}\right), 1.70(s, 1 \mathrm{H}, \mathrm{OH}), 1.60\left(t t, J=7.5,7.2 \mathrm{~Hz}, 2 \mathrm{H}, \mathrm{CH}_{2} \mathrm{CH}_{2} \mathrm{CO}_{2} \mathrm{Me}\right)$, $1.53\left(t, J=7.3 \mathrm{~Hz}, 2 \mathrm{H}, \mathrm{CH}_{2} \mathrm{CD}_{2} \mathrm{OH}\right), 1.39-1.27(m, 6 \mathrm{H}, \mathrm{C}-4, \mathrm{C}-5, \mathrm{C}-6), 1.23(t, J=7.1$ $\left.\mathrm{Hz}, 3 \mathrm{H}, \mathrm{CH}_{3}\right) ;{ }^{13} \mathrm{C}$ NMR $\left(100 \mathrm{MHz}, \mathrm{CDCl}_{3}\right) \delta_{\mathrm{C}} 173.88(\mathrm{C}-1), 34.31(\mathrm{C}-2), 24.85(\mathrm{C}-3)$, $29.02(\mathrm{C}-4), 29.05$ ( $\delta$-shift - 0.003, C-5), 25.48 ( $\gamma$-shift - 0.051, C-6), 32.45 ( $\beta$-shift $0.217, \mathrm{C}-7), 62.15\left(p, J_{\mathrm{CD}}=21.6 \mathrm{~Hz}, \alpha-\mathrm{shift}-0.755, \mathrm{C}-8\right), 60.18\left(-\mathrm{OCH}_{2} \mathrm{Me}\right), 14.23$ $\left(\mathrm{CH}_{3}\right)$; EI-MS: $m / z 173\left([\mathrm{M}-17]^{+}\right), 158\left([\mathrm{M}-32]^{+}\right), 145\left([\mathrm{M}-45]^{+}\right), 126,99,88,83,70,60$, 5, 40; TMS Derivative EI-MS: $m / z 247\left([\mathrm{M}-15]^{+}\right), 217\left([\mathrm{M}-45]^{+}\right), 201\left([\mathrm{M}-61]^{+}\right), 173$ $\left([\mathrm{M}-89]^{+}\right), 159,131,117,103,83,75,73,55,43$.

\subsubsection{Ethyl 8-oxo- $\left[8-{ }^{2} \mathrm{H}_{1}\right]$ octanoate}
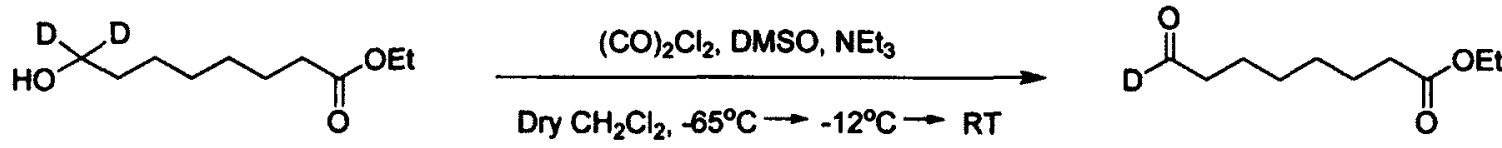

A stirred solution of oxalyl chloride $(6.2 \mathrm{~mL}, 71.0 \mathrm{mmol})$ in $\operatorname{dry} \mathrm{CH}_{2} \mathrm{Cl}_{2}(90 \mathrm{~mL})$ was cooled to $-65^{\circ} \mathrm{C}$ (external temperature) and anhydrous DMSO (10.1 mL, 142.0 mmol) was added dropwise for $20 \mathrm{~min}$. A strongly exothermic reaction took place that turned the reaction mixture viscous after the completion of DMSO addition. The reaction mixture was stirred for an additional 5-10 minutes prior to the addition of ethyl 8hydroxy-[8,8- $\left.{ }^{2} \mathrm{H}_{2}\right]$-octanoate $(6.75 \mathrm{~g}, 35.5 \mathrm{mmol})$ in dry $\mathrm{CH}_{2} \mathrm{Cl}_{2}(30 \mathrm{~mL})$, in portions, at - 
55 to $-60^{\circ} \mathrm{C}$. Immediately after the addition of the alcohol, the reaction mixture turned cloudy white in color and was diluted with dry $\mathrm{CH}_{2} \mathrm{Cl}_{2}(20 \mathrm{~mL})$ to facilitate stirring. Stirring was continued for another $20 \mathrm{~min}$ before warming the reaction mixture to $-12{ }^{\circ} \mathrm{C}$ (external temperature). After stirring for a further $20 \mathrm{~min}$, triethylamine $(29.6 \mathrm{~mL}, 213.1$ mmol) was added and the resultant yellowish reaction mixture was allowed to warm to room temperature and stirred for additional $30 \mathrm{~min}$ before the reaction was terminated. The reaction was worked up by the addition of distilled water $(150 \mathrm{~mL})$ and the organic layer was separated. The aqueous layer was re-extracted with $\mathrm{CH}_{2} \mathrm{Cl}_{2}(3 \times 120 \mathrm{~mL})$ and the combined organic layers were treated in the normal manner to yield a residue that was diluted further with $\mathrm{Et}_{2} \mathrm{O}(180 \mathrm{~mL})$ and washed successively with $2.0 \mathrm{M} \mathrm{HCl}(3 \times 80$ $\mathrm{mL}), \mathrm{H}_{2} \mathrm{O}(2 \times 70 \mathrm{~mL})$ and brine $(3 \times 70 \mathrm{~mL})$. Filtration of the organic phase through anhydrous $\mathrm{Na}_{2} \mathrm{SO}_{4}$ followed by the removal of the solvent in vacuo gave the title compound as a yellowish liquid $(6.16 \mathrm{~g}, 93 \%)$. bp $62-63{ }^{\circ} \mathrm{C}$ at $0.05 \mathrm{~mm} \mathrm{Hg} ; R_{\mathrm{f}} 0.34$ (EtOAc:hexane, 1:3); IR (film): v 2983, 2936, 2860 (C-H), 2070 (C-D), 1735 (C=O, $\left.\mathrm{CO}_{2} \mathrm{Et}\right), 1713(\mathrm{C}=\mathrm{O}, \mathrm{CDO}), 1465,1373,1252,1184,1096,1033,875 \mathrm{~cm}^{-1} ;{ }^{1} \mathrm{H}$ NMR $\left(300 \mathrm{MHz}, \mathrm{CDCl}_{3}\right) \delta_{\mathrm{H}} 4.11\left(q, J=7.3 \mathrm{~Hz}, 2 \mathrm{H}, \mathrm{OCH}_{2} \mathrm{Me}\right), 2.41(t, J=7.4 \mathrm{~Hz}, 2 \mathrm{H}$, $\left.\mathrm{CH}_{2} \mathrm{CDO}\right), 2.28\left(t, J=7.4 \mathrm{~Hz}, 2 \mathrm{H}, \mathrm{CH}_{2} \mathrm{CO}_{2} \mathrm{Et}\right), 1.72-1.54\left(m, 4 \mathrm{H}, \mathrm{CH}_{2} \mathrm{CH}_{2} \mathrm{CDO}\right.$, $\mathrm{CH}_{2} \mathrm{CH}_{2} \mathrm{CO}_{2} \mathrm{Et}$ ), 1.34 (Overlapping $\left.t t, J=7.4,3.0 \mathrm{~Hz}, 4 \mathrm{H}, \mathrm{C}-4, \mathrm{C}-5\right), 1.24(t, J=7.3 \mathrm{~Hz}$, $\left.3 \mathrm{H}, \mathrm{CH}_{3}\right) ;{ }^{13} \mathrm{C}$ NMR $\left(100 \mathrm{MHz}, \mathrm{CDCl}_{3}\right) \delta_{\mathrm{C}} 173.67(\mathrm{C}-1), 34.19(\mathrm{C}-2), 24.68(\mathrm{C}-3), 28.80$ (C-4), $28.75(\mathrm{C}-5), 21.79(\mathrm{C}-6), 43.59\left(t,{ }^{2} J_{\mathrm{CD}}=3.6 \mathrm{~Hz}, \mathrm{C}-7\right), 202.36\left(t, J_{\mathrm{CD}}=26.1 \mathrm{~Hz}\right.$, C-8), $60.19\left(-\mathrm{OCH}_{2} \mathrm{Me}\right), 14.23\left(\mathrm{CH}_{3}\right)$; EI-MS: $m / z 188\left([\mathrm{M}]^{+}\right), 168$ ([M-19] $), 159$ ([M$\left.28]^{+}\right), 143\left([\mathrm{M}-44]^{+}\right), 142\left([\mathrm{M}-45]^{+}\right), 130,123,116,115,102,96,88,74,69,60,55,45$, 41. 


\subsubsection{Ethyl (8S)-8-hydroxy-[8- $\left.{ }^{2} H_{1}\right]$ octanoate}<smiles>[2H]C(=O)CCCCCCC(=O)OCC</smiles>

A 3-neck round-bottom flask equipped with a reflux condenser, stir bar, glass stopper and rubber septa was charged with ethyl 8-oxo-[8- $\left.{ }^{2} \mathrm{H}_{1}\right]$-octanoate $(6.21 \mathrm{~g}, 33.2$ mmol) and anhydrous THF $(90 \mathrm{~mL})$. To the stirred solution was added $(+)-(R)-$ AlpineBorane $(0.5 \mathrm{M}$ in THF, $119.6 \mathrm{~mL}, 59.8 \mathrm{mmol})$ in portions at room temperature. 30 minutes after the addition, the rubber septum was quickly replaced with a glass stopper and the light yellow reaction mixture was refluxed overnight to ensure completion of the reaction. Excess Alpine-Borane ${ }^{\circledR}$ reagent was quenched by the addition of acetaldehyde $(5 \mathrm{~mL})$ and the mixture was stirred for additional $30 \mathrm{~min}$. After replacing the glass stopper was replaced with a one-piece distillation apparatus, THF and the $\alpha$-pinene byproduct was removed under vacuum (at $15 \mathrm{~mm} \mathrm{Hg}$ and $0.02 \mathrm{~mm} \mathrm{Hg}$, respectively). Particularly, to remove $\alpha$-pinene, slight warming $\left(40-45^{\circ} \mathrm{C}\right)$ of the reaction flask was required. The residue was taken up in anhydrous $\mathrm{Et}_{2} \mathrm{O}(150 \mathrm{~mL})$ whilst maintaining an inert atmosphere. The solution was cooled to $0{ }^{\circ} \mathrm{C}$ and ethanolamine $(3.6 \mathrm{~mL}, 59.8 \mathrm{mmol})$ was added dropwise to produce a thick white precipitate that was removed by filtration through celite (grade 521). The precipitate was rinsed with additional portions of ether ( 2 x $50 \mathrm{~mL}$ ) and the combined organic phase was treated in the normal manner to yield the crude compound that was purified by column chromatography on silica gel using a gradient eluent system of EtOAc:hexane (5\%, $10 \%, 15 \%, 20 \%$ and pure EtOAc, respectively). The title compound was obtained as a faint yellowish clear oil $(4.71 \mathrm{~g}, 75$ 
\%). bp $85-87^{\circ} \mathrm{C}$ at $0.1 \mathrm{~mm} \mathrm{Hg} ; R_{\mathrm{f}} 0.26$ (EtOAc:hexane, 1:1); IR (Neat): $v 3416(\mathrm{O}-\mathrm{H})$, 2981, 2932, 2858 (C-H), 2145 (C-D), 1737 (C=O), 1465, 1374, 1254, 1183, 1104, 1034, $941 \mathrm{~cm}^{-1} ;{ }^{1} \mathrm{H}$ NMR $\left(300 \mathrm{MHz}, \mathrm{CDCl}_{3}\right) \delta_{\mathrm{H}} 4.11\left(q, J=7.1 \mathrm{~Hz}, 2 \mathrm{H}, \mathrm{OCH}_{2} \mathrm{Me}\right), 3.59(t t, J$ $=6.5,1.4 \mathrm{~Hz}, 1 \mathrm{H}, \mathrm{CHDOH}), 2.27\left(t, J=7.5 \mathrm{~Hz}, 2 \mathrm{H}, \mathrm{CH}_{2} \mathrm{CO}_{2} \mathrm{Et}\right), 1.55(s, 1 \mathrm{H}, \mathrm{OH})$, $1.61\left(t t, J=7.5,7.3 \mathrm{~Hz}, 2 \mathrm{H}, \mathrm{CH}_{2} \mathrm{CH}_{2} \mathrm{CO}_{2} \mathrm{Et}\right), 1.54(t d, J=6.6,6.5 \mathrm{~Hz}, 2 \mathrm{H}$, $\left.\mathrm{CH}_{2} \mathrm{CHDOH}\right), 1.40-1.28(m, 6 \mathrm{H}, \mathrm{C}-4, \mathrm{C}-5, \mathrm{C}-6), 1.24\left(t, J=7.1 \mathrm{~Hz}, 3 \mathrm{H}, \mathrm{CH}_{3}\right) ;{ }^{13} \mathrm{C}$ NMR (100 MHz, $\left.\mathrm{CDCl}_{3}\right) \delta_{\mathrm{C}} 173.86(\mathrm{C}-1), 34.30(\mathrm{C}-2), 24.84$ (C-3), 29.01 (C-4), 29.04 $(\delta$-shift - 0.008, C-5), $25.50(\gamma$-shift - 0.028, C-6), $32.55(\beta$-shift - 0.118, C-7), $62.50(t$, $J_{\mathrm{CD}}=21.7 \mathrm{~Hz}, \alpha$-shift - 0.403, C-8), $60.17\left(-\mathrm{OCH}_{2} \mathrm{Me}\right), 14.22\left(\mathrm{CH}_{3}\right)$; TMS Derivative EI-MS: $m / z 260\left([\mathrm{M}-1]^{\dagger}\right), 246\left([\mathrm{M}-15]^{+}\right), 216\left([\mathrm{M}-45]^{+}\right), 200\left([\mathrm{M}-61]^{+}\right), 173\left([\mathrm{M}-88]^{+}\right)$, $158,141,130,116,102,88,75,73,55,43$.

\subsubsection{8-Hydroxyoctanoic acid}

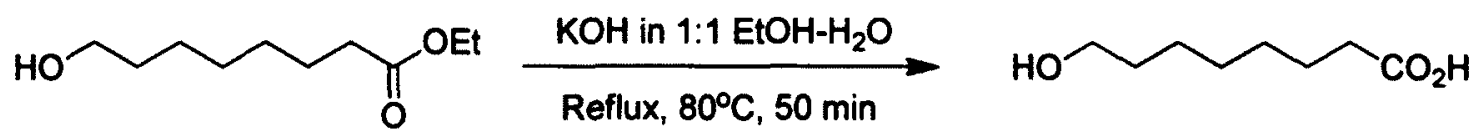

A $2.0 \mathrm{M}$ solution of $\mathrm{KOH}(30 \mathrm{~mL}, 60 \mathrm{mmol})$ in $1: 1 \mathrm{EtOH}-\mathrm{H}_{2} \mathrm{O}$ [prepared by dissolving $5.6 \mathrm{~g}$ of $\mathrm{KOH}$ in $44 \mathrm{~mL}$ of $1: 1 \mathrm{EtOH}-\mathrm{H}_{2} \mathrm{O}$ ] was added to a stirred solution of ethyl 8-hydroxyoctanoate $(1.32 \mathrm{~g}, 7.0 \mathrm{mmol})$ in absolute $\mathrm{EtOH}(20 \mathrm{~mL})$ at room temperature. After stirring at room temperature for $10 \mathrm{~min}$, the reaction mixture was refluxed for 50 minutes at $80{ }^{\circ} \mathrm{C}$ after which the $\mathrm{EtOH}$ was removed in vacuo. The residue was dissolved in $\mathrm{CH}_{2} \mathrm{Cl}_{2} / \mathrm{H}_{2} \mathrm{O}(40: 20 \mathrm{~mL})$ and the aqueous layer was acidified to $\mathrm{pH} 2$ using $2.0 \mathrm{M} \mathrm{HCl}$. The organic layer was separated and the aqueous layer washed with $\mathrm{CH}_{2} \mathrm{Cl}_{2}(3 \times 25 \mathrm{~mL})$. The combined organic layers were worked up in the usual way 
to furnish the desired compound as a white amorphous solid (702 $\mathrm{mg}, 62 \%$ ). $\mathrm{mp} \mathrm{59-61}$ ${ }^{\circ} \mathrm{C} ; R_{\mathrm{f}} 0.32$ (EtOAc:hexane, 19:1); IR (KBr): v $3248(\mathrm{O}-\mathrm{H}), 3500-2600 \mathrm{br}\left(\mathrm{CO}_{2} \mathrm{H}\right), 2937$, $2858(\mathrm{C}-\mathrm{H}), 1709(\mathrm{C}=\mathrm{O}), 1467,1296,1095,1054,907 \mathrm{~cm}^{-1}$; ${ }^{1} \mathrm{H}$ NMR $(300 \mathrm{MHz}$, $\left.\mathrm{CDCl}_{3}\right) \delta_{\mathrm{H}} 6.48-4.38\left(\right.$ br s, $\left.2 \mathrm{H}, \mathrm{OH}, \mathrm{CO}_{2} \mathrm{H}\right), 3.65\left(t, J=6.0 \mathrm{~Hz}, 2 \mathrm{H}, \mathrm{CH}_{2} \mathrm{OH}\right), 2.35(t, J$ $\left.=7.5 \mathrm{~Hz}, 2 \mathrm{H}, \mathrm{CH}_{2} \mathrm{CO}_{2} \mathrm{H}\right), 1.65\left(t t, J=7.5,7.5 \mathrm{~Hz}, 2 \mathrm{H}, \mathrm{CH}_{2} \mathrm{CH}_{2} \mathrm{CO}_{2} \mathrm{H}\right), 1.57(t t, J=7.5$, $\left.6.0 \mathrm{~Hz}, 2 \mathrm{H}, \mathrm{CH}_{2} \mathrm{CH}_{2} \mathrm{OH}\right), 1.36(s-m, 6 \mathrm{H}, \mathrm{C}-4, \mathrm{C}-5, \mathrm{C}-6) ;{ }^{13} \mathrm{C} \mathrm{NMR}\left(75 \mathrm{MHz}, \mathrm{CDCl}_{3}\right) \delta_{\mathrm{C}}$ 179.21 (C-1), 33.92 (C-2), 24.59 (C-3), 28.98 (C-4), 28.98 (C-5), 25.50 (C-6), 32.60 (C7), 62.96 (C-8); bis-TMS Derivative EI-MS: $m / z 303$ ([M-1] $]^{\dagger}$ ), 289 ([M-15]'), 273 ([M$\left.31]^{+}\right), 245,217,199,185,173,157,147,129,117,107,97,83,75,55,45$.

\subsubsection{6 (8S)-8-Hydroxy-[8- $\left.{ }^{2} \mathrm{H}_{1}\right]$-octanoic acid}

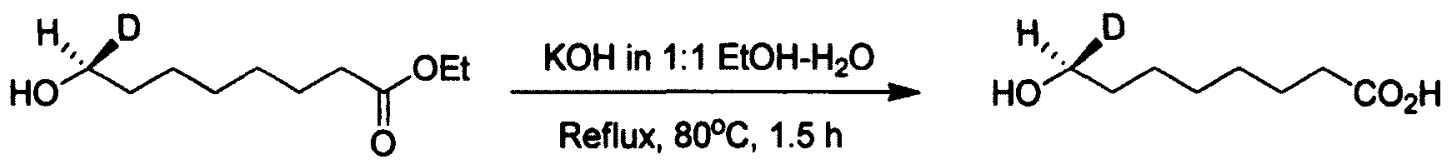

The title compound was prepared from ethyl $(8 S)-8$-hydroxy- $\left[8-{ }^{2} \mathrm{H}_{1}\right]$ octanoate $(4.49 \mathrm{~g}, 23.7 \mathrm{mmol})$ and $\mathrm{KOH}\left(2.0 \mathrm{M}\right.$ in $\left.1: 1 \mathrm{EtOH}-\mathrm{H}_{2} \mathrm{O}, 102.2 \mathrm{~mL}, 0.2 \mathrm{~mol}\right)$ as reagent using the same experimental protocol that used for the synthesis of 8-hydroxyoctanoic acid. The anticipated compound was obtained as a white amorphous solid $(3.27 \mathrm{~g}, 86 \%)$. mp 60-61 ${ }^{\circ} \mathrm{C} ; R_{\mathrm{f}} 0.73\left(\mathrm{MeOH}: \mathrm{CH}_{2} \mathrm{Cl}_{2}, 1: 19\right)$; IR (KBr): v $3393(\mathrm{O}-\mathrm{H}), 3600-2600 \mathrm{br}$ $\left(\mathrm{CO}_{2} \mathrm{H}\right)$, 3019, 2934, 2859 (C-H), 2156 (C-D), 1710 (C=O), 1465, 1412, 1216, 1105, $1064,937 \mathrm{~cm}^{-1} ;{ }^{1} \mathrm{H}$ NMR $\left(300 \mathrm{MHz}, \mathrm{CDCl}_{3}\right) \delta_{\mathrm{H}} 8.87\left(\right.$ br s, $\left.2 \mathrm{H}, \mathrm{OH}, \mathrm{CO}_{2} \mathrm{H}\right), 3.61(t, J=$ $6.4 \mathrm{~Hz}, 1 \mathrm{H}, \mathrm{CHDOH}), 2.37\left(t, J=7.5 \mathrm{~Hz}, 2 \mathrm{H}, \mathrm{CH}_{2} \mathrm{CO}_{2} \mathrm{H}\right), 1.62(t t, J=7.5,7.2 \mathrm{~Hz}, 2 \mathrm{H}$, $\left.\mathrm{CH}_{2} \mathrm{CH}_{2} \mathrm{CO}_{2} \mathrm{H}\right), 1.54\left(t d, J=6.4,5.8 \mathrm{~Hz}, 2 \mathrm{H}, \mathrm{CH}_{2} \mathrm{CHDOH}\right), 1.39-1.29(m, 6 \mathrm{H}, \mathrm{C}-4, \mathrm{C}-$ 5, C-6); ${ }^{13} \mathrm{C}$ NMR (75 MHz, $\mathrm{CDCl}_{3}$ ) $\delta_{\mathrm{C}} 179.14$ (C-1), 33.98 (C-2), 24.59 (C-3), 28.95 (C- 
4), 28.95 ( $\delta$-shift - 0.033, C-5), 25.44 ( $\gamma$-shift - 0.059, C-6), 32.39 ( $\beta$-shift - 0.215, C-7), $62.48\left(t, J_{\mathrm{CD}}=20.3 \mathrm{~Hz}, \alpha\right.$-shift - 0.485, C-8); TMS Derivative EI-MS: $m / z 304\left([\mathrm{M}-1]^{+}\right)$, 290 ([M-15] $), 274$ ([M-31] $\left.]^{+}\right), 246,217,200,186,174,158,147,129,117,108,98,84$, $75,55,45$.

\subsubsection{8-(p-Tolylsulfonyloxy)-octanoic acid}

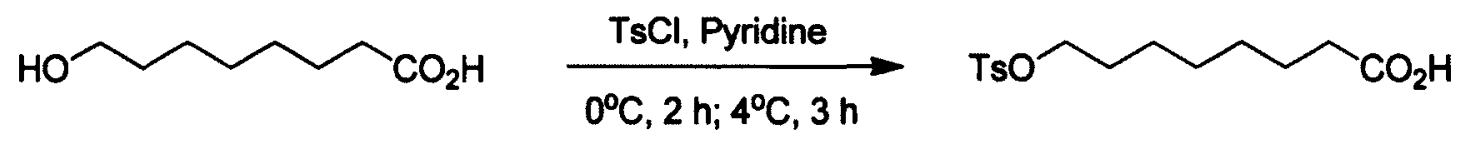

To a stirred solution of 8 -hydroxyoctanoic acid $(408 \mathrm{mg}, 2.6 \mathrm{mmol})$ in anhydrous pyridine $(4 \mathrm{~mL})$ was added dropwise, $p$-toluenesulfonyl chloride $(778 \mathrm{mg}, 4.1 \mathrm{mmol})$ in anhydrous pyridine $(2 \mathrm{~mL})$ at $0{ }^{\circ} \mathrm{C}$. After stirring at $0{ }^{\circ} \mathrm{C}-4{ }^{\circ} \mathrm{C}$ for $5 \mathrm{~h}$, the reaction mixture was poured into $\mathrm{H}_{2} \mathrm{O}(15 \mathrm{~mL})$. The aqueous layer was extracted with $\mathrm{Et}_{2} \mathrm{O}$ (4 x $35 \mathrm{~mL})$ and the combined organic layer was washed successively with $1.0 \mathrm{M} \mathrm{HCl}(3 \times 30$ $\mathrm{mL}$ ), water $(40 \mathrm{~mL})$ and brine $(40 \mathrm{~mL})$. The organic layer was treated in the normal manner to yield the title compound as a colorless oil (655 $\mathrm{mg}, 82 \%) . R_{\mathrm{f}} 0.60$ (EtOAc:hexane, 3:2); IR (film): v 3500-2600br (O-H), 3029, 2936, 2861 (C-H), 1709 $(\mathrm{C}=\mathrm{O}), 1599(\mathrm{C}=\mathrm{C}, \mathrm{Ar}), 1465,1359,1189,1177,1098,914,815 \mathrm{~cm}^{-1}$; ('H NMR $(300$ $\left.\mathrm{MHz}, \mathrm{CDCl}_{3}\right) \delta_{\mathrm{H}} 10.58\left(\right.$ br $\left.s, 1 \mathrm{H}, \mathrm{CO}_{2} \mathrm{H}\right), 4.02\left(t, J=6.0 \mathrm{~Hz}, 2 \mathrm{H}, \mathrm{CH}_{2} \mathrm{OTs}\right), 2.33(t, J=$ $7.5 \mathrm{~Hz}, 2 \mathrm{H}, \mathrm{CH}_{2} \mathrm{CO}_{2} \mathrm{H}$ ), 1.62 (Overlapping $t t, J=7.5,6.0 \mathrm{~Hz}, 4 \mathrm{H}, \mathrm{CH}_{2} \mathrm{CH}_{2} \mathrm{OTs}$, $\left.\mathrm{CH}_{2} \mathrm{CH}_{2} \mathrm{CO}_{2} \mathrm{H}\right), 1.40-1.21(m, 6 \mathrm{H}, \mathrm{C}-4, \mathrm{C}-5, \mathrm{C}-6), 2.46\left(s, 3 \mathrm{H}, \mathrm{CH}_{3} \mathrm{Ar}\right), 7.79(d, J=9.0$ $\mathrm{Hz}, 2 \mathrm{H}, \mathrm{C} \underline{\underline{o}}-\mathrm{Ph}), 7.35(d, J=9.0 \mathrm{~Hz}, 2 \mathrm{H}, \mathrm{C} \underline{m}-\mathrm{Ph}) ;{ }^{13} \mathrm{C} \mathrm{NMR}\left(75 \mathrm{MHz}, \mathrm{CDCl}_{3}\right) \delta_{\mathrm{C}}$ 178.72 (C-1), 33.71 (C-2), 24.48 (C-3), 28.75 (C-4), 28.77 (C-5), 25.16 (C-6), 28.55 (C7), 70.54 (C-8), 144.69 (Cipso - Ph), 127.91 (Co - Ph), 129.84 (C $\underline{m}$ - Ph), 133.20 (Cp - 
$\mathrm{Ph}), 21.66\left(\mathrm{CH}_{3}-\mathrm{Ph}\right)$; EI-MS: $m / z 296\left(-\mathrm{H}_{2} \mathrm{O},[\mathrm{M}-18]^{+}\right), 281,232,207,173,155,125$, $107,91,73,65,41$.

\subsubsection{8 (8S)-8-(p-Tolylsulfonyloxy)-[8- $\left.{ }^{2} \mathrm{H}_{1}\right]-$-octanoic acid}

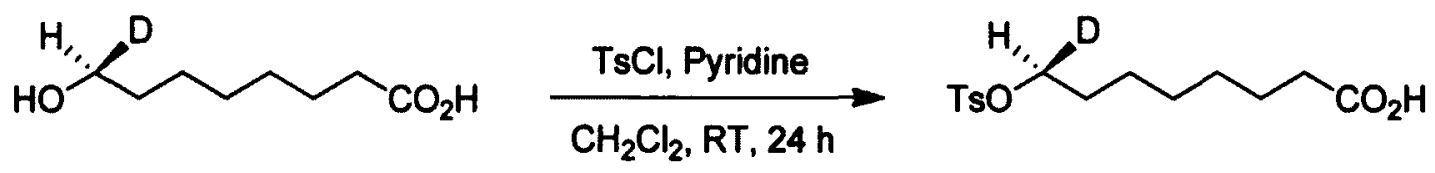

To a mixture of $(8 S)-8$-hydroxy-[8- $\left.{ }^{2} \mathrm{H}_{1}\right]$-octanoic acid $(4.25 \mathrm{~g}, 26.4 \mathrm{mmol})$ and $p$ toluenesulfonyl chloride $(9.06 \mathrm{~g}, 47.5 \mathrm{mmol})$ in dry $\mathrm{CH}_{2} \mathrm{Cl}_{2}(55 \mathrm{~mL})$ was added anhydrous pyridine $(5.4 \mathrm{~mL}, 66 \mathrm{mmol})$ at room temperature and the reaction was stirred for $24 \mathrm{~h}$. The mixture was poured into a saturated solution of $\mathrm{NH}_{4} \mathrm{Cl}(60 \mathrm{~mL})$ and the organic layer was separated after dilution with $\mathrm{CH}_{2} \mathrm{Cl}_{2}(35 \mathrm{~mL})$. The organic layer was then washed with saturated $\mathrm{NH}_{4} \mathrm{Cl}(3 \times 60 \mathrm{~mL})$, saturated $\mathrm{NaCl}(2 \times 60 \mathrm{~mL})$ and dried over anhydrous $\mathrm{Na}_{2} \mathrm{SO}_{4}$. The residue obtained after the removal of the solvent was diluted again with $\mathrm{CH}_{2} \mathrm{Cl}_{2}(50 \mathrm{~mL})$ and washed with $\mathrm{H}_{2} \mathrm{O}(3 \times 50 \mathrm{~mL})$, brine $(50 \mathrm{~mL})$ to decompose and remove remaining excess of $p$-toluenesulfonyl chloride. The title compound was obtained as a pale yellowish oil $\left(6.53 \mathrm{~g}, 78 \%\right.$ ). $R_{\mathrm{f}} 0.11$ (EtOAc:hexane, 3:2); IR (film): v 3500-2700br (O-H), 3028, 2935, 2862 (C-H), 2194 (C-D), 1709 (C=O), 1596 (C=C, Ar), 1455, 1367, 1189, 1176, 1098, 941, $814 \mathrm{~cm}^{-1}$; 'H NMR $(300 \mathrm{MHz}$, $\left.\mathrm{CDCl}_{3}\right) \delta_{\mathrm{H}} 7.79(d, J=7.5 \mathrm{~Hz}, 2 \mathrm{H}, \mathrm{C} \underline{\underline{o}}-\mathrm{Ph}), 7.35(d, J=7.5 \mathrm{~Hz}, 2 \mathrm{H}, \mathrm{C} \underline{m}-\mathrm{Ph}), 4.00(t, J$ $=6.3 \mathrm{~Hz}, 1 \mathrm{H}, \mathrm{CHDOTs}), 2.45\left(s, 3 \mathrm{H}, \mathrm{CH}_{3} \mathrm{Ar}\right), 2.33\left(t, J=7.5 \mathrm{~Hz}, 2 \mathrm{H}, \mathrm{CH}_{2} \mathrm{CO}_{2} \mathrm{H}\right)$, $1.62\left(m, 4 \mathrm{H}, \mathrm{CH}_{2} \mathrm{CHDOTs}, \mathrm{CH}_{2} \mathrm{CH}_{2} \mathrm{CO}_{2} \mathrm{H}\right), 1.39-1.22(m, 6 \mathrm{H}, \mathrm{C}-4, \mathrm{C}-5, \mathrm{C}-6) ;{ }^{13} \mathrm{C}$ NMR (75 MHz, $\left.\mathrm{CDCl}_{3}\right) \delta_{\mathrm{C}} 177.53(\mathrm{C}-1), 34.10(\mathrm{C}-2), 24.71(\mathrm{C}-3), 28.61(\mathrm{C}-4), 28.85(\delta-$ 
shift 0.081, C-5), $25.11\left(\gamma\right.$-shift - 0.045, C-6), $28.58(\beta$-shift $0.029, \mathrm{C}-7), 70.25\left(t, J_{\mathrm{CD}}=\right.$ $22.8 \mathrm{~Hz}, \alpha$-shift - 0.283, C-8), 144.65 (Cipso - Ph), $127.86(\mathrm{C} \underline{o}-\mathrm{Ph}), 129.81(\mathrm{C} \underline{m}-\mathrm{Ph})$, $133.16(\mathrm{C} p-\mathrm{Ph}), 21.62\left(\mathrm{CH}_{3}-\mathrm{Ph}\right)$; EI-MS: $m / z 297\left(-\mathrm{H}_{2} \mathrm{O},[\mathrm{M}-18]^{+}\right), 281,233,207$, $173,155,126,107,91,73,65,41$.

\subsubsection{Dec-9-ynoic acid}

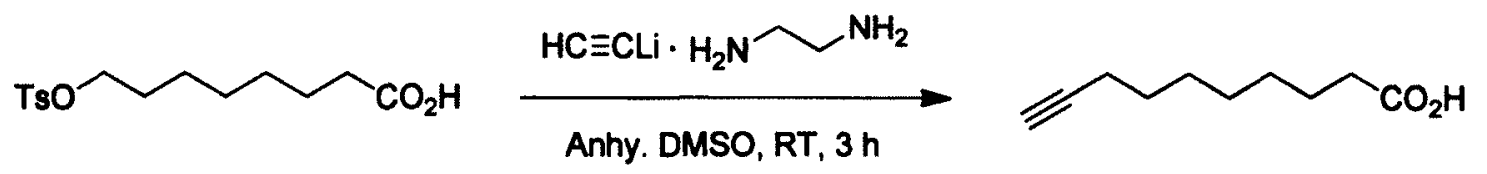

A solution of 8-(p-tolylsulfonyloxy)-octanoic acid (398 $\mathrm{mg}, 1.3 \mathrm{mmol})$ in anhydrous DMSO (4 mL) was added dropwise to a stirred suspension of lithium acetylide-ethylenediamine complex $(466 \mathrm{mg}, 5.1 \mathrm{mmol})$ in DMSO $(8 \mathrm{~mL})$ at room temperature and the reaction mixture was stirred at room temperature for $3 \mathrm{~h}$. Excess reagent was quenched by pouring the mixture on to a chilled ice-1.0 $\mathrm{M} \mathrm{HCl}$ mixture ( 40 $\mathrm{mL}$ ) and the acidity was adjusted to $\mathrm{pH} 2$ using $1.0 \mathrm{M} \mathrm{HCl}$. The aqueous layer was extracted with diethyl ether $(3 \times 30 \mathrm{~mL})$ and the combined organic phase was worked up in the usual way generated the crude compound as an oil (202 $\mathrm{mg}, 95 \%)$ and used further without purification. mp 4-6 ${ }^{\circ} \mathrm{C} ; R_{\mathrm{f}} 0.35\left(\mathrm{MeOH}: \mathrm{CH}_{2} \mathrm{Cl}_{2}, 1: 19\right)$; IR (film): $v 3302$ (terminal $\mathrm{HC} \equiv \mathrm{C}), 3400-2600 \mathrm{br}(\mathrm{O}-\mathrm{H}), 3035,2935,2859(\mathrm{C}-\mathrm{H}), 2118(\mathrm{C} \equiv \mathrm{C}), 1710$ $(\mathrm{C}=\mathrm{O}), 1463,1431,1413,1287,1250,938 \mathrm{~cm}^{-1} ;{ }^{1} \mathrm{H}$ NMR $\left(300 \mathrm{MHz}, \mathrm{CDCl}_{3}\right) \delta_{\mathrm{H}} 11.16$ (br s, $\left.1 \mathrm{H}, \mathrm{CO}_{2} \mathrm{H}\right), 2.35\left(t, J=7.5 \mathrm{~Hz}, 2 \mathrm{H}, \mathrm{CH}_{2} \mathrm{CO}_{2} \mathrm{H}\right), 2.17(t d, J=6.9,2.6 \mathrm{~Hz}, 2 \mathrm{H}$, $\left.\mathrm{CH}_{2} \mathrm{C} \equiv \mathrm{C}\right), 1.93(t, J=2.6 \mathrm{~Hz}, 1 \mathrm{H}, \mathrm{HC} \equiv \mathrm{C}), 1.63(t t, J=7.5,7.2 \mathrm{~Hz}, 2 \mathrm{H}$, $\left.\mathrm{CH}_{2} \mathrm{CH}_{2} \mathrm{CO}_{2} \mathrm{H}\right), 1.52\left(t t, J=7.1,6.9 \mathrm{~Hz}, 2 \mathrm{H}, \mathrm{CH}_{2} \mathrm{CH}_{2} \mathrm{C} \equiv \mathrm{C}\right), 1.46-1.24(m, 6 \mathrm{H}, \mathrm{C}-4$, 
C-5, C-6); ${ }^{13} \mathrm{C}$ NMR (75 MHz, $\mathrm{CDCl}_{3}$ ) $\delta_{\mathrm{C}} 180.44$ (C-1), 34.05 (C-2), 24.57 (C-3), 28.88 (C-4), 28.49 (C-5), 28.36 (C-6), 28.70 (C-7), 18.34 (C-8), 84.60 (C-9), 68.17 (C-10); EIMS: $m / z 149\left([\mathrm{M}-19]^{+}\right), 135\left([\mathrm{M}-33]^{+}\right), 126\left([\mathrm{M}-42]^{+}\right), 121\left([\mathrm{M}-47]^{+}\right), 108\left([\mathrm{M}-60]^{+}\right), 93$ ([M-75] $\left.]^{+}\right), 81,67,60,55,41$.

\subsubsection{0 (8R)-[8- $\left.{ }^{2} \mathrm{H}_{1}\right]-$ Dec-9-ynoic acid}

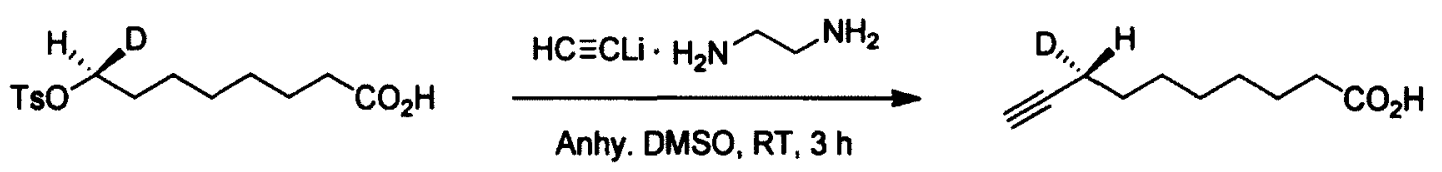

Same experimental procedure has been used for the synthesis of the title compound from (8S)-8-(p-Tolylsulfonyloxy)-[8- $\left.{ }^{2} \mathrm{H}_{1}\right]$-octanoic acid $(4.476 \mathrm{~g}, 14.203$ $\mathrm{mmol})$ and lithium acetylide-ethylenediamine complex $(5.231 \mathrm{~g}, 56.812 \mathrm{mmol})$ as followed to synthesize dec-9-ynoic acid. The desired compound was furnished as a pale yellowish clear liquid that solidifies at $0{ }^{\circ} \mathrm{C}(1.87 \mathrm{~g}, 78 \%) . \mathrm{mp} 4-6{ }^{\circ} \mathrm{C} ; R_{\mathrm{f}} 0.34$ (MeOH: $\mathrm{CH}_{2} \mathrm{Cl}_{2}, 1: 19$ ); IR (film): v 3308 (terminal $\mathrm{HC} \equiv \mathrm{C}$ ), 3500-2700br (O-H), 2933, 2860 (C-H), 2157 (C-D), 2117 (C =C), 1710 (C=O), 1551, 1463, 1412, 1310, 1224, 758 $\mathrm{cm}^{-1}$; ${ }^{1} \mathrm{H}$ NMR $\left(300 \mathrm{MHz}, \mathrm{CDCl}_{3}\right) \delta_{\mathrm{H}} 8.29\left(\right.$ br s, $\left.1 \mathrm{H}, \mathrm{CO}_{2} \mathrm{H}\right), 2.33(t, J=7.5 \mathrm{~Hz}, 2 \mathrm{H}$, $\left.\mathrm{CH}_{2} \mathrm{CO}_{2} \mathrm{H}\right), 2.15(d t, J=7.6,2.6 \mathrm{~Hz}, 1 \mathrm{H}, \mathrm{CHDC} \equiv \mathrm{C}), 1.93(d, J=2.6 \mathrm{~Hz}, 1 \mathrm{H}, \mathrm{HC} \equiv$ C), $1.63\left(t t, J=7.5,7.2 \mathrm{~Hz}, 2 \mathrm{H}, \mathrm{CH}_{2} \mathrm{CH}_{2} \mathrm{CO}_{2} \mathrm{H}\right), 1.51(t d, J=7.6,6.3 \mathrm{~Hz}, 2 \mathrm{H}$, $\mathrm{CH}_{2} \mathrm{CHD}$ ), 1.45-1.24 (m, $\left.6 \mathrm{H}, \mathrm{C}-4, \mathrm{C}-5, \mathrm{C}-6\right) ;{ }^{13} \mathrm{C}$ NMR $\left(75 \mathrm{MHz}, \mathrm{CDCl}_{3}\right) \delta_{\mathrm{C}} 179.39$ (C1), 34.00 (C-2), 24.61 (C-3), 28.88 (C-4), 28.45 ( $\delta$-shift - 0.039, C-5), 28.24 ( $\gamma$-shift $0.115, \mathrm{C}-6), 28.69(\beta$-shift $-0.005, \mathrm{C}-7), 18.04\left(t, J_{\mathrm{CD}}=19.9 \mathrm{~Hz}, \alpha\right.$-shift $\left.-0.305, \mathrm{C}-8\right)$, 84.58 ( $\beta$-shift - 0.012, C-9), 68.17 ( $\gamma$-shift 0.003, C-10); EI-MS: $m / z 169\left([\mathrm{M}]^{+}\right), 150([\mathrm{M}-$ 
$\left.19]^{+}\right), 136\left([\mathrm{M}-33]^{+}\right), 126\left([\mathrm{M}-42]^{+}\right), 122\left([\mathrm{M}-47]^{+}\right), 109\left([\mathrm{M}-60]^{+}\right), 94\left([\mathrm{M}-75]^{+}\right), 82,68$, $60,55,44,41$.

\subsubsection{Methyl dec-9-ynoate}
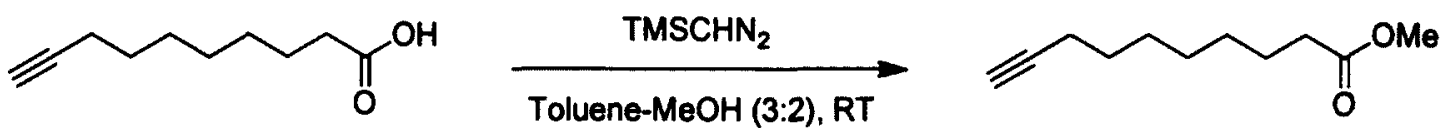

Dec-9-ynoic acid (1.44 g, $5.1 \mathrm{mmol})$ was dissolved in 3:2 toluene/methanol (18 $\mathrm{mL})$ and treated with (trimethylsilyl) diazomethane $(2.0 \mathrm{M}$ in hexanes, $2.6 \mathrm{~mL}, 5.1$ mmol). When the evolution of gas from the reaction mixture was estimated to be complete, an additional few drops of reagent was added until a light yellow color persisted. Excess reagent was destroyed by the addition of a few drops of $\mathrm{AcOH}$. The solvent was removed in vacuo and the crude methyl ester was purified by flash chromatography using $\mathrm{Et}_{2} \mathrm{O}$ :hexane (1:49) as eluent to afford the title compound as a colorless oil (1.33 g, $88 \%$ ). $R_{\mathrm{f}} 0.38$ (EtOAc:hexane, 1:9); IR (film): $v 3298$ (terminal HC $\equiv \mathrm{C}), 2935,2859(\mathrm{C}-\mathrm{H}), 2117(\mathrm{C} \equiv \mathrm{C}), 1741(\mathrm{C}=\mathrm{O}), 1461,1437,1247,1198,1173$, $1098,1016 \mathrm{~cm}^{-1} ;{ }^{1} \mathrm{H}$ NMR $\left(300 \mathrm{MHz}, \mathrm{CDCl}_{3}\right) \delta_{\mathrm{H}} 3.65\left(s, 3 \mathrm{H}, \mathrm{OCH}_{3}\right), 2.29(t, J=7.5$ $\left.\mathrm{Hz}, 2 \mathrm{H}, \mathrm{CH}_{2} \mathrm{CO}_{2} \mathrm{Me}\right), 2.16\left(t d, J=7.0,2.6 \mathrm{~Hz}, 2 \mathrm{H}, \mathrm{CH}_{2} \mathrm{C} \equiv \mathrm{C}\right), 1.92(t, J=2.6 \mathrm{~Hz}, 1$ $\left.\mathrm{H}, \mathrm{HC} \equiv \mathrm{CCH}_{2}\right), 1.61\left(t t, J=7.5,7.3 \mathrm{~Hz}, 2 \mathrm{H}, \mathrm{CH}_{2} \mathrm{CH}_{2} \mathrm{CO}_{2} \mathrm{Me}\right), 1.51(t t, J=7.2,7.0$ $\left.\mathrm{Hz}, 2 \mathrm{H}, \mathrm{CH}_{2} \mathrm{CH}_{2} \mathrm{C} \equiv \mathrm{C}\right), 1.45-1.23(m, 6 \mathrm{H}, \mathrm{C}-4, \mathrm{C}-5, \mathrm{C}-6) ;{ }^{13} \mathrm{C}$ NMR $(75.5 \mathrm{MHz}$, $\left.\mathrm{CDCl}_{3}\right) \delta_{\mathrm{C}} 174.30(\mathrm{C}-1), 34.07(\mathrm{C}-2), 24.88(\mathrm{C}-3), 28.99(\mathrm{C}-4), 28.53$ (C-5), 28.38 (C-6), 28.73 (C-7), 18.37 (C-8), 84.66 (C-9), 68.14 (C-10), 51.48 (-OMe); EI-MS: $m / z 181$ ([M$\left.1]^{+}\right), 167\left([\mathrm{M}-15]^{+}\right), 151\left([\mathrm{M}-31]^{+}\right), 143\left([\mathrm{M}-39]^{+}\right), 135\left([\mathrm{M}-47]^{+}\right), 127,122,108,93,87$, $81,74,67,55,41$. 


\subsubsection{Methyl (8R)-[8- $\left.{ }^{2} \mathrm{H}_{1}\right]-$ dec-9-ynoate}<smiles>[2H][C@@H](C#C)CCCCCCC(=O)O</smiles>
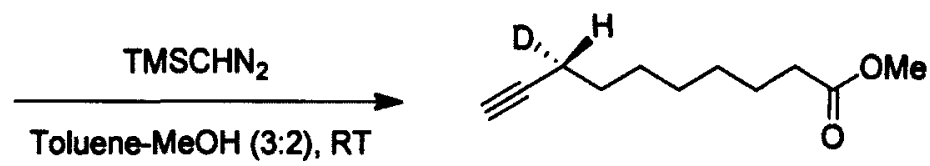

The title compound was synthesized from $(8 R)-\left[8-{ }^{2} \mathrm{H}_{1}\right]-$ dec-9-ynoic acid $(1.77 \mathrm{~g}$, $23.7 \mathrm{mmol}$ ) using the same experimental protocol used for the preparation of methyl dec9-ynoate. The title compound was obtained as a faint yellowish oil (863 mg, $45 \%$ ). $R_{\mathrm{f}}$ 0.28 (Pure $\mathrm{CH}_{2} \mathrm{Cl}_{2}$ ); IR (film): v 3296 (terminal $\mathrm{HC} \equiv \mathrm{C}$ ), 2934, 2859 (C-H), 2145 (C-D), $2117(\mathrm{C} \equiv \mathrm{C}), 1740(\mathrm{C}=\mathrm{O}), 1462,1437,1252,1200,1173,1104,1016,847 \mathrm{~cm}^{-1} ;{ }^{1} \mathrm{H}$ NMR (300 MHz, CDCl $) \delta_{\mathrm{H}} 3.66\left(s, 3 \mathrm{H}, \mathrm{OCH}_{3}\right), 2.30\left(t, J=7.5 \mathrm{~Hz}, 2 \mathrm{H}, \mathrm{CH}_{2} \mathrm{CO}_{2} \mathrm{Me}\right)$, $2.15(m, 1 \mathrm{H}, \mathrm{CHDC} \equiv \mathrm{C}), 1.92(d, J=2.6 \mathrm{~Hz}, 1 \mathrm{H}, \mathrm{HC} \equiv \mathrm{C}), 1.62(t t, J=7.5,7.4 \mathrm{~Hz}, 2$ $\left.\mathrm{H}, \mathrm{CH}_{2} \mathrm{CH}_{2} \mathrm{CO}_{2} \mathrm{Me}\right), 1.51\left(t d, J=7.0,6.8 \mathrm{~Hz}, 2 \mathrm{H}, \mathrm{CH}_{2} \mathrm{CHD}\right), 1.45-1.26(m, 6 \mathrm{H}, \mathrm{C}-4, \mathrm{C}-$ 5, C-6); ${ }^{13} \mathrm{C}$ NMR (75 MHz, $\mathrm{CDCl}_{3}$ ) $\delta_{\mathrm{C}} 174.22$ (C-1), 34.03 (C-2), $24.86(\mathrm{C}-3), 28.97$ (C4), 28.48 ( $\delta$-shift - 0.053, C-5), 28.27 ( $\gamma$-shift - 0.118, C-6), 28.71 ( $\beta$-shift - 0.020, C-7), $18.05\left(t, J_{\mathrm{CD}}=20.0 \mathrm{~Hz}, \alpha\right.$-shift - 0.314, C-8), $84.59(\beta$-shift - 0.062, C-9), 68.14 ( $\gamma$-shift $0.005, \mathrm{C}-10), 51.43$ (-OMe); EI-MS (rel. intensity): $m / z 182\left([\mathrm{M}-1]^{+}\right), 168\left(1,[\mathrm{M}-15]^{+}\right)$, $152\left(4,[\mathrm{M}-31]^{+}\right), 143\left(2,[\mathrm{M}-40]^{\dagger}\right), 136\left(6,[\mathrm{M}-47]^{+}\right), 128(2), 123(12), 109(55), 94(38)$, $87(31), 82(52), 74(100), 68(41), 55(82), 41(73)$. 


\subsubsection{1-(p-Tolylsulfonyloxy)-oct-2-yne ${ }^{173}$}

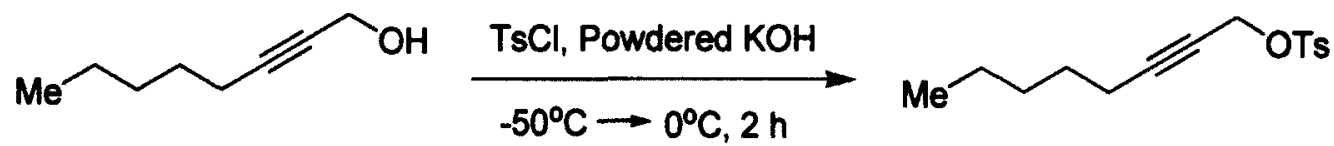

To a stirred solution of 2-octyn-1-ol (500 mg, $4.0 \mathrm{mmol})$ and freshly prepared $p$ toluenesulfonyl chloride (832 $\mathrm{mg}, 4.4 \mathrm{mmol})$ in anhydrous $\mathrm{Et}_{2} \mathrm{O}(12 \mathrm{~mL})$ was added powdered $\mathrm{KOH}(1.92 \mathrm{~g}, 34.1 \mathrm{mmol})$ at $-50^{\circ} \mathrm{C}$. The reaction mixture was stirred at $0{ }^{\circ} \mathrm{C}$ for $2 \mathrm{~h}$ and then poured into $\mathrm{H}_{2} \mathrm{O}(35 \mathrm{~mL})$. The organic layer was separated and the aqueous layer was extracted with $\mathrm{Et}_{2} \mathrm{O}(3 \times 25 \mathrm{~mL})$ and the combined organic phase washed successively with water $(30 \mathrm{~mL})$, brine $(2 \times 30 \mathrm{~mL})$ and filtered through anhydrous $\mathrm{Na}_{2} \mathrm{SO}_{4}$. The solvent was evaporated in vacuo and concentrated to afford the crude compound as a pale yellowish oil (959 mg, $86 \%$ ). $R_{\mathrm{f}} 0.21$ (EtOAc:hexane, 1:4); The spectral data of the compound was similar to that reported in the literature. ${ }^{6}$ IR (film): v 2957, 2933, 2862 (C-H), 2310, 2238 (C三C), 1599 (C=C, Ar), 1456, 1369, $1190,1177,1097,942,838,815 \mathrm{~cm}^{-1} ;{ }^{1} \mathrm{H}$ NMR $\left(300 \mathrm{MHz}, \mathrm{CDCl}_{3}\right) \delta_{\mathrm{H}} 7.81(d, J=8.2$ $\mathrm{Hz}, 2 \mathrm{H}, \mathrm{C} \underline{\underline{o}}-\mathrm{Ph}), 7.34(d, J=8.2 \mathrm{~Hz}, 2 \mathrm{H}, \mathrm{C} \underline{m}-\mathrm{Ph}), 4.70\left(t, J=2.2 \mathrm{~Hz}, 2 \mathrm{H}, \mathrm{CH}_{2} \mathrm{OTs}\right)$, $2.44\left(s, 3 \mathrm{H}, \mathrm{CH}_{3} \mathrm{Ar}\right), 2.06\left(t t, J=7.0,2.2 \mathrm{~Hz}, 2 \mathrm{H}, \mathrm{CH}_{2} \mathrm{C} \equiv \mathrm{C}\right), 1.38(t t, J=7.0,6.9 \mathrm{~Hz}$, $\left.2 \mathrm{H}, \mathrm{CH}_{2} \mathrm{CH}_{2} \mathrm{C} \equiv \mathrm{C}\right), 1.32-1.22\left(m, 4 \mathrm{H}, 2 \times \mathrm{CH}_{2}\right), 0.88\left(t, J=6.8 \mathrm{~Hz}, 3 \mathrm{H}, \mathrm{CH}_{3}\right) ;{ }^{13} \mathrm{C}$ NMR (75 MHz, $\mathrm{CDCl}_{3}$ ) $\delta_{\mathrm{C}} 58.80$ (C-1), 71.76 (C-2), 90.61 (C-3), 18.60 (C-4), 27.73 (C5), 30.89 (C-6), 22.09 (C-7), 13.88 (C-8), 144.80 (Cipso - Ph), 128.09 (Co - Ph), 129.70 $(\mathrm{C} \underline{m}-\mathrm{Ph}), 133.43(\mathrm{C} p-\mathrm{Ph}), 21.61\left(\mathrm{CH}_{3}-\mathrm{Ph}\right) ; \mathrm{EI}-\mathrm{MS}: \mathrm{m} / 2239\left([\mathrm{M}-41]^{+}\right), 225,201,173$ $155\left([\mathrm{M}-125]^{+}\right), 139([\mathrm{M}-141]+), 125,117,107,91,79,65,55,41$. 


\subsubsection{Methyl octadeca-9,12-diynoate}

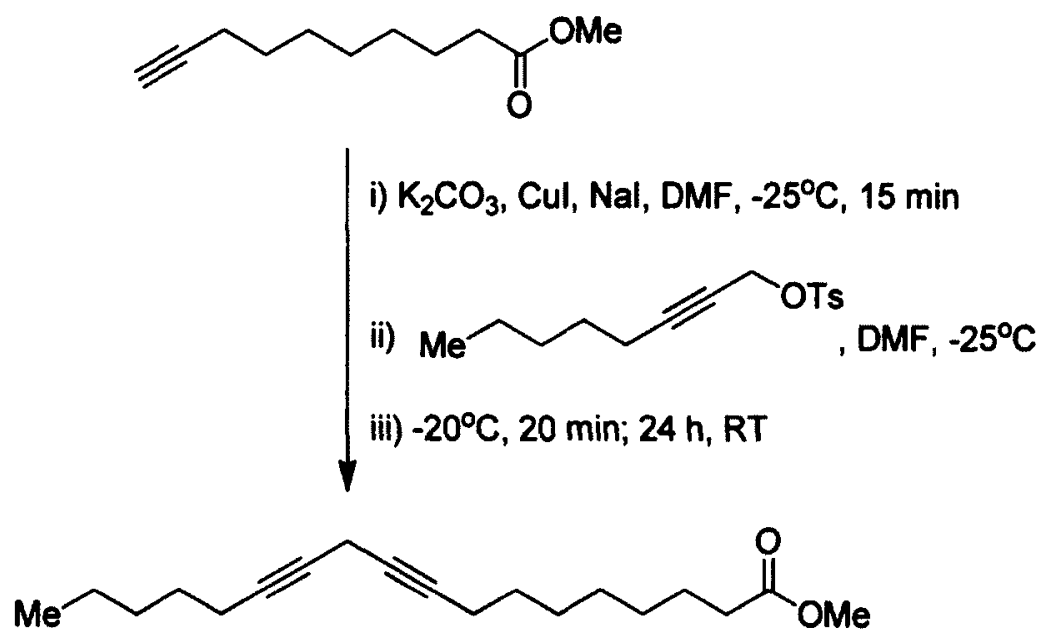

The following components were dissolved in anhydrous DMF $(10 \mathrm{~mL})$ at $-25^{\circ} \mathrm{C}$ : methyl dec-9-ynoate (500 mg, $2.7 \mathrm{mmol}) ; \mathrm{K}_{2} \mathrm{CO}_{3}(378 \mathrm{mg}, 2.7 \mathrm{mmol})$; $\mathrm{Cul}(520 \mathrm{mg}, 2.7$ mmol); and $\mathrm{NaI}(410 \mathrm{mg}, 2.7 \mathrm{mmol})$. After stirring the reaction mixture for 15 minutes at $-25^{\circ} \mathrm{C}$, a solution of 1-(p-tolylsulfonyloxy) oct-2-yne $(766 \mathrm{mg}, 2.7 \mathrm{mmol})$ in anhydrous DMF $(10 \mathrm{~mL})$ was added and stirred was continued for another 20 minutes at $-20^{\circ} \mathrm{C}$ and a further $24 \mathrm{~h}$ at room temperature. The reaction was terminated by pouring the mixture on to a saturated solution of aqueous $\mathrm{NH}_{4} \mathrm{Cl}(50 \mathrm{~mL})$ and the aqueous layer was extracted with $\mathrm{Et}_{2} \mathrm{O}(4 \times 30 \mathrm{~mL})$. The combined organic layers were worked up in the normal manner to afford a residue that was diluted again with hexanes $(30 \mathrm{~mL})$ and washed with $\mathrm{H}_{2} \mathrm{O}(3 \times 25 \mathrm{~mL})$, brine $(3 \times 25 \mathrm{~mL})$ and finally dried over anhydrous $\mathrm{Na}_{2} \mathrm{SO}_{4}$. The solvent was removed in vacuo and the crude compound was purified by flash chromatography using $\mathrm{Et}_{2} \mathrm{O}$ :hexane $(1: 19)$ as eluent to give the title compound as a pale yellowish oil (580 mg, $73 \%$ ). $R_{\mathrm{f}} 0.31$ (EtOAc:hexane, 1:9); IR (film): $v$ 2933, 2859 (C$\mathrm{H}), 2210(\mathrm{C} \equiv \mathrm{C}), 1738(\mathrm{C}=0), 1459,1437,1248,1200,1173,1017,737 \mathrm{~cm}^{-1} ;{ }^{1} \mathrm{H}$ NMR 
$\left(300 \mathrm{MHz}, \mathrm{CDCl}_{3}\right) \delta_{\mathrm{H}} 3.66\left(s, 3 \mathrm{H}, \mathrm{OCH}_{3}\right), 3.11(t t, J=2.3,2.3 \mathrm{~Hz}, 2 \mathrm{H}, \mathrm{C}-11), 2.30(t, J$ $=7.5 \mathrm{~Hz}, 2 \mathrm{H}, \mathrm{CH}_{2} \mathrm{CO}_{2} \mathrm{Me}$ ), 2.15 (Overlapping $t t, J=7.2,2.3 \mathrm{~Hz}, 4 \mathrm{H}, \mathrm{C}-8, \mathrm{C}-14$ ), 1.62 $\left(t t, J=7.5,7.2 \mathrm{~Hz}, 2 \mathrm{H}, \mathrm{CH}_{2} \mathrm{CH}_{2} \mathrm{CO}_{2} \mathrm{Me}\right), 1.55-1.42(m, 4 \mathrm{H}, \mathrm{C}-7, \mathrm{C}-15), 1.43-1.23(m$, $\left.10 \mathrm{H}, 5 \times \mathrm{CH}_{2}\right), 0.89\left(t, J=7.0 \mathrm{~Hz}, 3 \mathrm{H}, \mathrm{CH}_{3}\right) ;{ }^{13} \mathrm{C} \mathrm{NMR}\left(75.5 \mathrm{MHz}, \mathrm{CDCl}_{3}\right) \delta_{\mathrm{C}} 174.28$ (C-1), 34.07 (C-2), 24.90 (C-3), 28.76 (C-4), 28.65 (C-5), 28.65 (C-6), 29.01 (C-7), 18.71 (C-8), 80.37 (C-9), 74.58 (C-10), 9.72 (C-11), 74.44 (C-12), 80.53 (C-13), 18.71 (C-14), 28.47 (C-15), 31.08 (C-16), 22.22 (C-17), 13.99 (C-18), 51.45 (-OMe); EI-MS: (rel. intensity) $\mathrm{m} / \mathrm{z} 259\left(1,[\mathrm{M}-31]^{+}\right), 201$ (2, [M-89] $), 189$ (1, [M-101] $), 173$ (3), 159 (5), 148 (13), 133 (33), 119 (58), 105 (90), 91 (100), 79 (45), 67 (38), 55 (39), 41 (42).

\subsubsection{Methyl (8R)-[8- $\left.{ }^{2} \mathrm{H}_{1}\right]$ octadeca-9,12-diynoate}

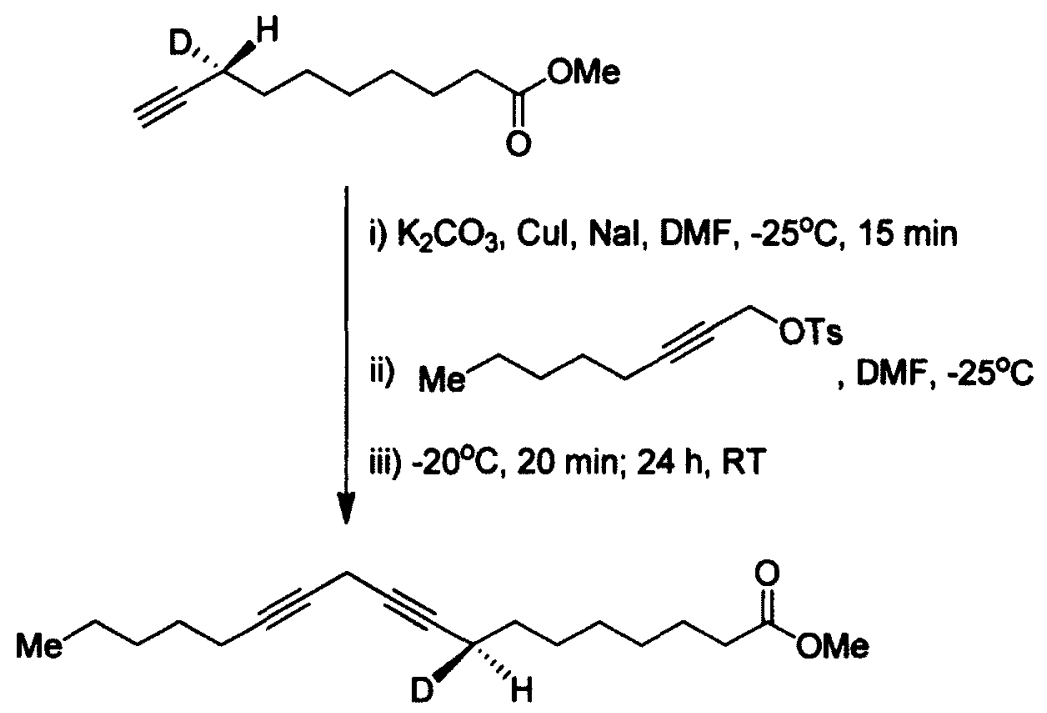

The following components were dissolved in anhydrous DMF $(10 \mathrm{~mL})$ at $-25^{\circ} \mathrm{C}$ : methyl $(8 R)-\left[8-{ }^{2} \mathrm{H}_{1}\right]$ dec-9-ynoate $(500 \mathrm{mg}, 2.7 \mathrm{mmol}) ; \mathrm{K}_{2} \mathrm{CO}_{3}(378 \mathrm{mg}, 2.7 \mathrm{mmol})$; $\mathrm{CuI}$ (520 mg, $2.7 \mathrm{mmol}$ ); and $\mathrm{Nal}(410 \mathrm{mg}, 2.7 \mathrm{mmol})$. After stirring the reaction mixture for 15 minutes at $-25^{\circ} \mathrm{C}$, a solution of 1-(p-tolylsulfonyloxy)-oct-2-yne (766 mg, $\left.2.7 \mathrm{mmol}\right)$ 
in anhydrous DMF (10 mL) was added and stirring was continued for another 20 minutes at $-20{ }^{\circ} \mathrm{C}$ and a further $24 \mathrm{~h}$ at $\mathrm{rt}$. The reaction was worked up as described above for the non-deuterated analogue and the crude compound was purified by flash chromatography using $\mathrm{Et}_{2} \mathrm{O}$ :hexane (1:19) as eluent to give the title compound as a pale yellow oil (580 $\mathrm{mg}, 73 \%$ ). $R_{\mathrm{f}} 0.23$ (EtOAc:hexane, 1:9); IR (film): v 2933, $2859(\mathrm{C}-\mathrm{H}), 2210(\mathrm{C} \equiv \mathrm{C}$ ), $1739(\mathrm{C}=0), 1459,1437,1364,1250,1200,1173,1019 \mathrm{~cm}^{-1} ;{ }^{1} \mathrm{H}$ NMR $(300 \mathrm{MHz}$, $\left.\mathrm{CDCl}_{3}\right) \delta_{\mathrm{H}} 3.65\left(s, 3 \mathrm{H}, \mathrm{OCH}_{3}\right), 3.10(t d, J=3.5,2.4 \mathrm{~Hz}, 2 \mathrm{H}, \mathrm{C}-11), 2.29(t, J=7.5 \mathrm{~Hz}$, $2 \mathrm{H}, \mathrm{CH}_{2} \mathrm{CO}_{2} \mathrm{Me}$ ), 2.14 (Overlapping $\left.t t, J=7.1,2.4 \mathrm{~Hz}, 3 \mathrm{H}, \mathrm{C}-8, \mathrm{C}-14\right), 1.61(t t, J=7.5$, $\left.7.3 \mathrm{~Hz}, 2 \mathrm{H}, \mathrm{CH}_{2} \mathrm{CH}_{2} \mathrm{CO}_{2} \mathrm{Me}\right), 1.55-1.42(m, 4 \mathrm{H}, \mathrm{C}-7, \mathrm{C}-15), 1.41-1.23(m, 10 \mathrm{H}, 5 \mathrm{x}$ $\left.\mathrm{CH}_{2}\right), 0.88\left(t, J=7.1 \mathrm{~Hz}, 3 \mathrm{H}, \mathrm{CH}_{3}\right) ;{ }^{13} \mathrm{C}$ NMR $\left(75.5 \mathrm{MHz}, \mathrm{CDCl}_{3}\right) \delta_{\mathrm{C}} 174.25(\mathrm{C}-1)$, 34.06 (C-2), 24.89 (C-3), 28.76 (C-4), 28.62 ( 8 -shift - 0.036, C-5), 28.55 ( $\gamma$-shift - 0.102, C-6), $29.01\left(\beta\right.$-shift - 0.005, C-7), $18.40\left(t, J_{\mathrm{CD}}=19.9 \mathrm{~Hz}, \alpha\right.$-shift - 0.306, C-8), $80.33(\beta-$ shift - 0.044, C-9), 74.59 ( $\gamma$-shift 0.011, C-10), 9.70 ( $\delta$-shift - 0.02, C-11), 74.44 (C-12), 80.51 (C-13), 18.70 (C-14), 28.46 (C-15), 31.07 (C-16), 22.21 (C-17), 13.97 (C-18), 51.42 (-OMe); EI-MS: $m / z 291\left([\mathrm{M}]^{+}\right), 260\left([\mathrm{M}-31]^{\dagger}\right), 248\left([\mathrm{M}-43]^{+}\right), 235,216,202,188$, $174,160,146,134,120,106,92,79,67,59,55,41$.

\subsubsection{Methyl octadeca-9Z,12Z-dienoate ${ }^{173}$}

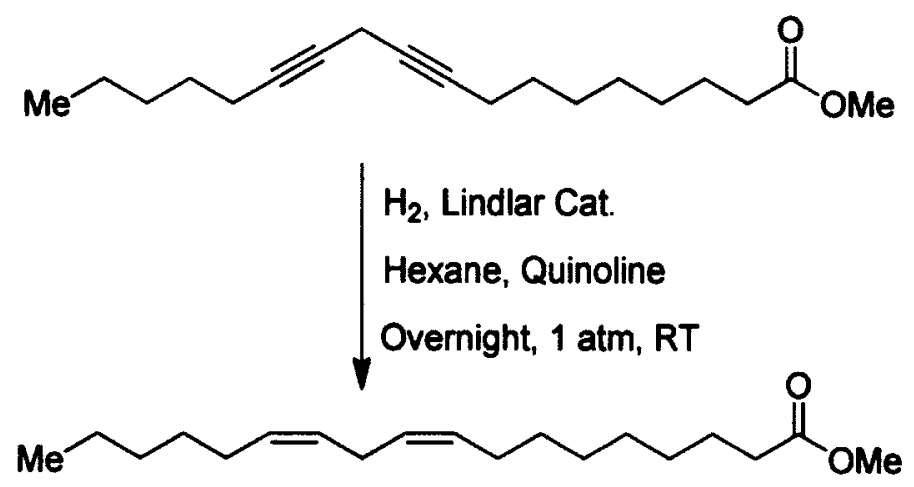


Lindlar catalyst $(16 \mathrm{mg})$ was suspended in a solution of methyl octadeca-9,12diynoate $(50 \mathrm{mg}, 0.2 \mathrm{mmol})$ in hexane $(8 \mathrm{~mL})$ containing $0.00624 \%$ quinoline $(0.4 \mu \mathrm{L})$. The reaction flask was attached to a hydrogenation apparatus and the system was evacuated and flushed with $\mathrm{H}_{2}$ three times. The hydrogenation apparatus was then filled with $\mathrm{H}_{2}$ and the reaction was stirred at 1 atmosphere pressure for $6 \mathrm{~h}$. The catalyst was removed by filtration and washed with hexane $(20 \mathrm{~mL})$. The solvent was removed in vacuo to afford the crude compound that was purified by flash chromatography using $\mathrm{Et}_{2} \mathrm{O}$ :hexane (1:19) as eluent and the final product was resulted as faint yellowish clear oil (49 mg, $96 \%$ ). $R_{\mathrm{f}} 0.44$ (EtOAc:hexane, 1:9); and the spectral data of this compound was similar to that reported in the literature for methyl linoleate. ${ }^{192-194}$ IR (film): $v 2932$, $2859(\mathrm{C}-\mathrm{H}), 2233,2211,1739(\mathrm{C}=\mathrm{O}), 1596(\mathrm{C}=\mathrm{C}), 1459,1437,1248,1198,1173,1018$, $727 \mathrm{~cm}^{-1} ;{ }^{1} \mathrm{H}$ NMR $\left(300 \mathrm{MHz}, \mathrm{CDCl}_{3}\right) \delta_{\mathrm{H}} 5.45-5.27(m, 4 \mathrm{H}, 4 \times \mathrm{HC}=\mathrm{C}), 3.67(s, 3 \mathrm{H}$, $\left.\mathrm{OCH}_{3}\right), 2.77(t, J=5.8 \mathrm{~Hz}, 2 \mathrm{H}, \mathrm{C}-11), 2.30\left(t, J=7.5 \mathrm{~Hz}, 2 \mathrm{H}, \mathrm{CH}_{2} \mathrm{CO}_{2} \mathrm{Me}\right), 2.05(m, 4$ $\left.\mathrm{H}, 2 \times \mathrm{CH}_{2} \mathrm{C}=\mathrm{C}\right), 1.62\left(t t, J=7.5,7.1 \mathrm{~Hz}, 2 \mathrm{H}, \mathrm{CH}_{2} \mathrm{CH}_{2} \mathrm{CO}_{2} \mathrm{Me}\right), 1.41-1.22(m, 14 \mathrm{H}, 7 \mathrm{x}$ $\left.\mathrm{CH}_{2}\right), 0.89\left(t, J=6.8 \mathrm{~Hz}, 3 \mathrm{H}, \mathrm{CH}_{3}\right) ;{ }^{13} \mathrm{C}$ NMR $\left(75.5 \mathrm{MHz}, \mathrm{CDCl}_{3}\right) \delta_{\mathrm{C}} 174.32(\mathrm{C}-1)$, 34.11 (C-2), 24.96 (C-3), 29.11 (C-4), 29.17 (C-5), 29.13 (C-6), 29.60 (C-7), 27.20 (C-8), 130.06 (C-9), 128.06 (C-10), 25.64 (C-11), 127.92 (C-12), 130.23 (C-13), 27.22 (C-14), 29.36 (C-15), 31.54 (C-16), 22.59 (C-17), 14.08 (C-18), 51.45 (-OMe); EI-MS: m/z 294 ([M] $\left.]^{\dagger}\right), 263\left([\mathrm{M}-31]^{+}\right), 220\left([\mathrm{M}-74]^{+}\right), 178,164,150,123,109,95,81,67,55$. 


\subsubsection{Methyl (8R)-[8- $\left.{ }^{2} \mathrm{H}_{1}\right]$-octadeca-9Z,12Z-dienoate}
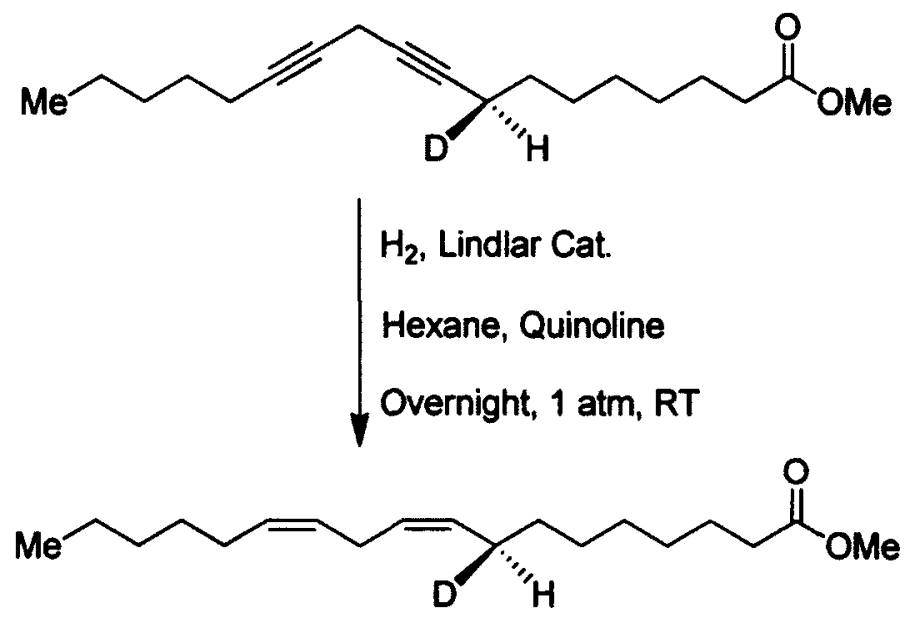

Lindlar catalyst $(50 \mathrm{mg})$ was suspended in a solution of methyl $(8 R)-\left[8-{ }^{2} \mathrm{H}_{1}\right]$ octadeca-9,12-diynoate $(178 \mathrm{mg}, 0.6 \mathrm{mmol})$ in hexane $(16 \mathrm{~mL})$ containing $0.00624 \%$ quinoline $(0.9 \mu \mathrm{L})$. The reaction flask was attached to a hydrogenation apparatus and the system was evacuated and flushed with $\mathrm{H}_{2}$ three times. The hydrogenation apparatus was then filled with $\mathrm{H}_{2}$ and the reaction was stirred at 1 atmosphere pressure for $6 \mathrm{~h}$. The catalyst was removed by filtration and washed with hexane $(30 \mathrm{~mL})$. The solvent was removed in vacuo to afford the crude compound that was purified by flash chromatography using $\mathrm{Et}_{2} \mathrm{O}$ :hexane $(1: 19)$ as eluent and the final product was obtained as a faint yellowish clear oil (180 mg, $99.8 \%$ ). $R_{\mathrm{f}} 0.47$ (EtOAc:hexane, 1:9); IR (film): $v$ 3010, 2955, 2928, 2857 (C-H), 2146 (C-D), 1743 (C=O), 1463, 1436, 1363, 1272, 1171, $1073,728 \mathrm{~cm}^{-1} ;{ }^{1} \mathrm{H}$ NMR $\left(300 \mathrm{MHz}, \mathrm{CDCl}_{3}\right) \delta_{\mathrm{H}} 5.44-5.28(m, 4 \mathrm{H}, 4 \mathrm{x} \mathrm{HC}=\mathrm{C}), 3.67(s, 3$ $\left.\mathrm{H}, \mathrm{OCH}_{3}\right), 2.77(t, J=6.0, \mathrm{~Hz}, 2 \mathrm{H}, \mathrm{C}-11), 2.30\left(t, J=7.5 \mathrm{~Hz}, 2 \mathrm{H}, \mathrm{CH}_{2} \mathrm{CO}_{2} \mathrm{Me}\right), 2.05$ (m, $3 \mathrm{H}, \mathrm{C}-8, \mathrm{C}-14), 1.62\left(m, 2 \mathrm{H}, \mathrm{CH}_{2} \mathrm{CH}_{2} \mathrm{CO}_{2} \mathrm{Me}\right), 1.42-1.23\left(m, 14 \mathrm{H}, 7 \times \mathrm{CH}_{2}\right), 0.89$ $\left(t, J=6.8 \mathrm{~Hz}, 3 \mathrm{H}, \mathrm{CH}_{3}\right) ;{ }^{13} \mathrm{C}$ NMR $\left(75.5 \mathrm{MHz}, \mathrm{CDCl}_{3}\right) \delta_{\mathrm{C}} 174.29(\mathrm{C}-1), 34.11(\mathrm{C}-2)$, 
24.96 (C-3), 29.08 (C-4), 29.17 ( $\delta$-shift 0.006, C-5), 29.13 ( $\gamma$-shift 0.002, C-6), 29.51 ( $\beta$ shift - 0.093, C-7), $26.84\left(t, J_{\mathrm{CD}}=19.2 \mathrm{~Hz}, \alpha\right.$-shift $\left.-0.362, \mathrm{C}-8\right), 130.01(\beta$-shift - 0.051, C-9), 128.08 ( $\gamma$-shift 0.019, C-10), 25.64 ( $\delta$-shift 0.003, C-11), 127.93 (C-12), 130.22 (C13), 27.22 (C-14), 29.36 (C-15), 31.54 (C-16), 22.58 (C-17), 14.07 (C-18), 51.43 (-OMe); EI-MS: $m / z 295\left([\mathrm{M}]^{+}\right), 280\left([\mathrm{M}-15]^{+}\right), 264\left([\mathrm{M}-31]^{+}\right), 235,221$ ([M-74] $\left.]^{+}\right), 207,192$, $179,164,150,136,124,110,96,81,67,55,41$.

\subsubsection{Methyl (8S)-[8- $\left.{ }^{2} \mathrm{H}_{1}\right]$-octadeca-9Z,12Z-dienoate}

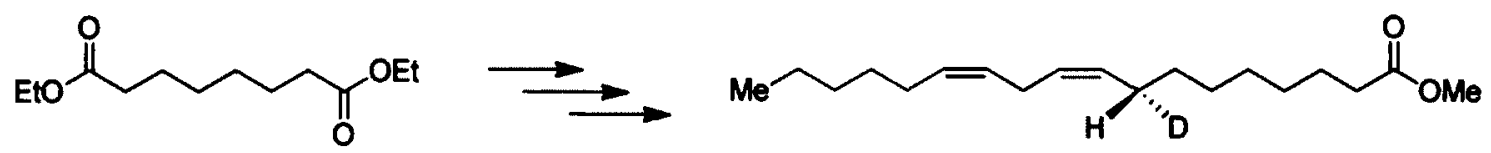

The title compound was synthesized in $3.8 \%$ overall yield from diethyl suberate using the same procedures as described for the synthesis of the corresponding $(R)$ enantiomer. The chiral starting material was prepared by the reduction of ethyl 8-oxo-[8${ }^{2} \mathrm{H}_{1}$ ]-octanoate using a $0.5 \mathrm{M}$ solution of (-)-(S)-Alpine-Borane in THF. $R_{\mathrm{f}} 0.47$ (EtOAc:hexane, 1:9); The analytical data for the title compound was essentially identical to that obtained for the corresponding $(R)$-enantiomer and similar to that reported above with the non-deuterio parent compound (see Section 6.2.1.16). IR (film): $v$ 3010, 2927, 2856 (C-H), 2147 (C-D), 1743 (C=O), 1640 (C=C), 1462, 1436, 1363, 1251, 1199, 1172, $1016,911,727 \mathrm{~cm}^{-1} ;{ }^{1} \mathrm{H}$ NMR (300 MHz, $\left.\mathrm{CDCl}_{3}\right) \delta_{\mathrm{H}}$ 5.44-5.27 (m, $\left.4 \mathrm{H}, 4 \times \mathrm{HC}=\mathrm{C}\right), 3.66$ $\left(s, 3 \mathrm{H}, \mathrm{OCH}_{3}\right), 2.77(t, J=6.0 \mathrm{~Hz}, 2 \mathrm{H}, \mathrm{C}-11), 2.30\left(t, J=7.5 \mathrm{~Hz}, 2 \mathrm{H}, \mathrm{CH}_{2} \mathrm{CO}_{2} \mathrm{Me}\right)$, $2.04(m, 3 \mathrm{H}, \mathrm{C}-8, \mathrm{C}-14), 1.62\left(m, 2 \mathrm{H}, \mathrm{CH}_{2} \mathrm{CH}_{2} \mathrm{CO}_{2} \mathrm{Me}\right), 1.41-1.23\left(m, 14 \mathrm{H}, 7 \times \mathrm{CH}_{2}\right)$, $0.89\left(t, J=6.8 \mathrm{~Hz}, 3 \mathrm{H}, \mathrm{CH}_{3}\right) ;{ }^{13} \mathrm{C}$ NMR $\left(75.5 \mathrm{MHz}, \mathrm{CDCl}_{3}\right) \delta_{\mathrm{C}} 174.28(\mathrm{C}-1), 34.10(\mathrm{C}-$ 2), 24.95 (C-3), 29.08 (C-4), 29.17 ( 8 -shift 0.002, C-5), 29.13 ( $\gamma$-shift - 0.004, C-6), 29.50 
( $\beta$-shift - 0.098, C-7), $26.83\left(t, J_{\mathrm{CD}}=19.2 \mathrm{~Hz}, \alpha\right.$-shift $\left.-0.368, \mathrm{C}-8\right), 130.00(\beta$-shift 0.060, C-9), 128.07 ( $\gamma$-shift 0.012, C-10), 25.63 ( $\delta$-shift 0.004, C-11), 127.92 (C-12), 130.21 (C-13), 27.21 (C-14), 29.36 (C-15), 31.54 (C-16), 22.58 (C-17), 14.07 (C-18), 51.42 (-OMe); EI-MS: $m / z 295$ ([M] $\left.]^{+}\right), 264\left([\mathrm{M}-31]^{+}\right), 235,221$ ([M-74] $\left.]^{+}\right), 207,192,179$, $164,151,136,123,110,96,81,67,55,41$.

\subsubsection{Attempted synthesis of Chiral $\left[11-{ }^{2} \mathrm{H}_{1}\right]-$ Linoleates}

\subsubsection{Ethyl oct-2-ynoate}

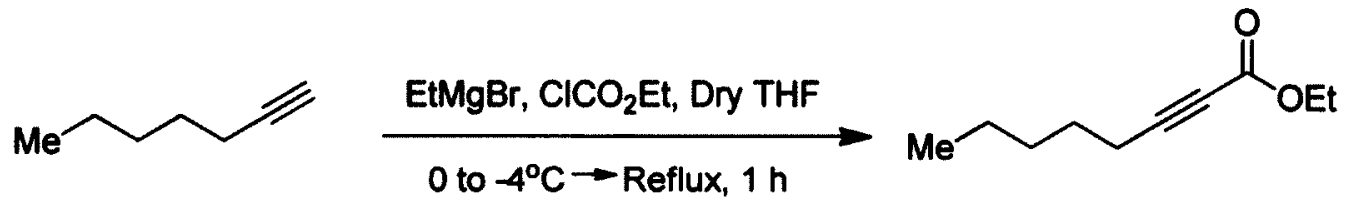

A solution of 1-heptyne $(24.5 \mathrm{~mL}, 187.2 \mathrm{mmol})$ in anhydrous THF $(80 \mathrm{~mL})$ was added dropwise during $1 \mathrm{~h}$ to a stirred solution of ethylmagnesium bromide $(1.0 \mathrm{M}$ in THF, $224.6 \mathrm{~mL}, 224.6 \mathrm{mmol}$ ) in anhydrous THF at 0 to $-5{ }^{\circ} \mathrm{C}$. After the addition, the reaction mixture slowly warmed up to room temperature for $15 \mathrm{~min}$ and subsequently refluxed for $1 \mathrm{~h}$ during which the preformed precipitate dissolved into the yellowish solution. Afterward, the reaction mixture was cooled to room temperature, then to $0{ }^{\circ} \mathrm{C}$ and to that was slowly admitted a solution of ethyl chloroformate $(19.6 \mathrm{~mL}, 205.9 \mathrm{mmol})$ in anhydrous THF $(40 \mathrm{~mL})$ during $1 \mathrm{~h}$. The resultant yellowish reaction mixture was then refluxed for another $1.5 \mathrm{~h}$ during which the solution became deep-brownish in color. The reaction terminated by the addition of a saturated solution of aqueous $\mathrm{NH}_{4} \mathrm{Cl}$ to the icecold reaction mixture. The organic layer was separated in a separatory funnel and the aqueous layer was re-extracted with $\mathrm{Et}_{2} \mathrm{O}(3 \times 150 \mathrm{~mL})$. The collected organic layers 
were combined and worked up in a regular fashion to afford the crude compound that was purified by short-path distillation under vacuum. The purified material was isolated as a colorless oil $(25.8 \mathrm{~g}, 82 \%)$. bp $62-64{ }^{\circ} \mathrm{C}$ at $0.1 \mathrm{~mm} \mathrm{Hg} ; R_{\mathrm{f}} 0.46$ (EtOAc:hexane, 1:4); IR (film): v 2960, 2936, 2873, $2864(\mathrm{C}-\mathrm{H}), 2314,2234(\mathrm{C} \equiv \mathrm{C}), 1713(\mathrm{C}=\mathrm{O}), 1466$, $1367,1252,1076,863,753 \mathrm{~cm}^{-1} ;{ }^{1} \mathrm{H}$ NMR $\left(300 \mathrm{MHz}, \mathrm{CDCl}_{3}\right) \delta_{\mathrm{H}} 4.21(q, J=7.2 \mathrm{~Hz}, 2$ $\left.\mathrm{H}, \mathrm{OCH}_{2} \mathrm{Me}\right), 2.31\left(t, J=7.1 \mathrm{~Hz}, 2 \mathrm{H}, \mathrm{CH}_{2} \mathrm{C} \equiv \mathrm{C}\right), 1.58(t t, J=7.2,7.1 \mathrm{~Hz}, 2 \mathrm{H}$, $\left.\mathrm{CH}_{2} \mathrm{CH}_{2} \mathrm{C} \equiv \mathrm{C}\right), 1.43-1.25\left(m, 4 \mathrm{H}, 2 \times \mathrm{CH}_{2}\right), 1.30\left(t, J=7.2 \mathrm{~Hz}, 2 \mathrm{H}, \mathrm{OCH}_{2} \mathrm{CH}_{3}\right), 0.89$ $\left(t, J=7.0 \mathrm{~Hz}, 3 \mathrm{H}, \mathrm{CH}_{3}\right) ;{ }^{13} \mathrm{C} \mathrm{NMR}\left(75 \mathrm{MHz}, \mathrm{CDCl}_{3}\right) \delta_{\mathrm{C}} 153.86(\mathrm{C}-1), 73.13(\mathrm{C}-2)$, 89.43 (C-3), 18.60 (C-4), 27.21 (C-5), 30.95 (C-6), 22.06 (C-7), 14.01 (C-8), 61.71 ($\left.\mathrm{OCH}_{2} \mathrm{Me}\right), 13.82\left(-\mathrm{OCH}_{2} \mathrm{CH}_{3}\right)$; EI-MS: $m / z 169\left([\mathrm{M}+1]^{+}\right), 153\left([\mathrm{M}-15]^{+}\right), 139\left([\mathrm{M}-29]^{+}\right)$, $123\left(\left[\mathrm{Me}\left(\mathrm{CH}_{2}\right)_{4} \mathrm{C} \equiv \mathrm{CCO}\right]^{+},[\mathrm{M}-45]^{+}\right), 111,95,79,67,55,41$.

\subsubsection{Oct-2-yn-1-ol}

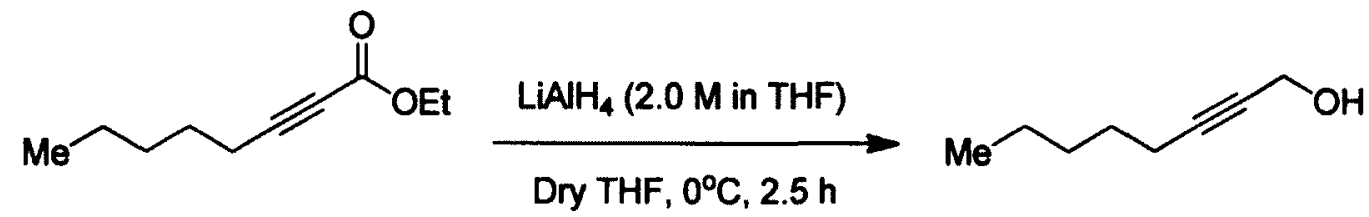

To a stirred solution of ethyl oct-2-ynoate $(6.94 \mathrm{~g}, 41.3 \mathrm{mmol})$ in anhydrous THF $(50 \mathrm{~mL})$ was slowly added a solution of lithium aluminium hydride in anhydrous THF $(2.0 \mathrm{M}, 7.4 \mathrm{~mL}, 14.8 \mathrm{mmol})$ in portions at $0{ }^{\circ} \mathrm{C}$. After stirring the reaction mixture for 2.5 $\mathrm{h}$ at $0{ }^{\circ} \mathrm{C}$ (during which the color of the solution changed to light yellow), excess ethyl acetate $(4 \mathrm{~mL})$ was added to quench any excess of reducing agent. THF was removed in vacuo and the residue was suspended in ether $(50 \mathrm{~mL})$ at $0{ }^{\circ} \mathrm{C}$ to which cold water $(40$ $\mathrm{mL}$ ) was admitted in small portions. The $\mathrm{pH}$ of the solution was adjusted to 1 using 2.0 
$\mathrm{M} \mathrm{HCl}$ and the organic layer was separated using liquid-liquid extraction method. The aqueous layer was re-extracted with ether $(3 \times 40 \mathrm{~mL})$ and the collected organic layers were combined. The combined organic layers were treated in the normal manner to give the crude compound that was purified by flash chromatography using EtOAc:hexane (1:9) as eluent and the title compound was obtained as a colorless liquid (3.28 g, $63 \%)$. bp $48-50^{\circ} \mathrm{C}$ at $0.1 \mathrm{~mm} \mathrm{Hg} ; R_{\mathrm{f}} 0.22$ (EtOAc:hexane, 1:4); IR (film): v $3331(\mathrm{O}-\mathrm{H}), 2958$, 2933, $2861(\mathrm{C}-\mathrm{H}), 2289,2226(\mathrm{C} \equiv \mathrm{C}), 1459,1379,1229,1138,1013,725 \mathrm{~cm}^{-1} ;{ }^{1} \mathrm{H}$ NMR $\left(300 \mathrm{MHz}, \mathrm{CDCl}_{3}\right) \delta_{\mathrm{H}} 4.24\left(t, J=2.2 \mathrm{~Hz}, 2 \mathrm{H}, \mathrm{CH}_{2} \mathrm{OH}\right), 2.20(t t, J=7.2,2.2 \mathrm{~Hz}, 2$ $\left.\mathrm{H}, \mathrm{CH}_{2} \mathrm{C} \equiv \mathrm{C}\right), 1.97-1.81($ br $s, 1 \mathrm{H}, \mathrm{OH}), 1.50\left(t t, J=7.2,7.1 \mathrm{~Hz}, 2 \mathrm{H}, \mathrm{CH}_{2} \mathrm{CH}_{2} \mathrm{C} \equiv \mathrm{C}\right)$, 1.42-1.24 (m, $\left.4 \mathrm{H}, 2 \times \mathrm{CH}_{2}\right), 0.89\left(t, J=7.1 \mathrm{~Hz}, 3 \mathrm{H}, \mathrm{CH}_{3}\right) ;{ }^{13} \mathrm{C}$ NMR $\left(75 \mathrm{MHz}, \mathrm{CDCl}_{3}\right)$ $\delta_{C} 51.29$ (C-1), 78.28 (C-2), 86.50 (C-3), 18.66 (C-4), 28.27 (C-5), 31.01 (C-6), 22.16 (C7), 13.90 (C-8); EI-MS: $m / z 125$ ([M-1] $), 111\left([\mathrm{M}-15]^{+}\right), 95$ ([M-31] $\left.]^{+}\right), 83\left([\mathrm{M}-43]^{+}\right), 79$, $70,55,41$.

\subsubsection{3 $\left[1,1-{ }^{2} \mathbf{H}_{2}\right]-0 \mathrm{ct}-2-\mathrm{yn}-1-\mathrm{ol}$}

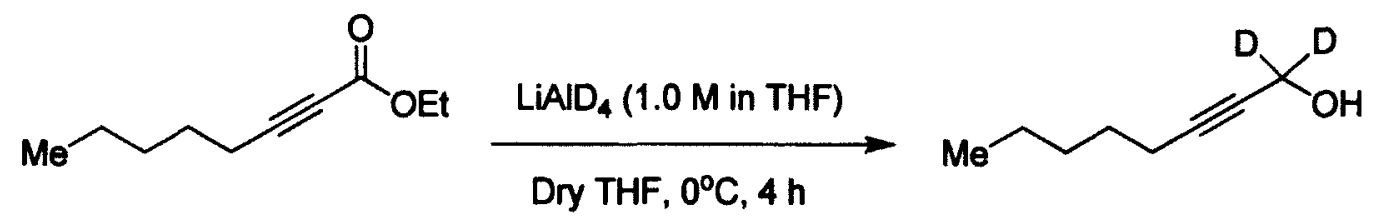

The title compound was prepared using the same experimental protocol as described in 6.2.2.2, from ethyl oct-2-ynoate $(23.34 \mathrm{~g}, 138.9 \mathrm{mmol})$ and lithium aluminium deuteride $(1.0 \mathrm{M}$ in anhydrous $\mathrm{THF}, 50 \mathrm{~mL}, 50 \mathrm{mmol})$ as reagent. The estimated compound was resulted as a colorless liquid $(16.18 \mathrm{~g}, 91 \%)$. bp $48-50{ }^{\circ} \mathrm{C}$ at 0.1 mm Hg; $R_{\mathrm{f}} 0.22$ (EtOAc:hexane, 1:4); IR (film): v 3335 (O-H), 2958, 2933, 2861 (C-H), 
2247,2206 (C三C), 2102 (C-D), 1460, 1379, 1331, 1173, 1080, 1043, 965, $830 \mathrm{~cm}^{-1} ;{ }^{1} \mathrm{H}$ $\operatorname{NMR}\left(300 \mathrm{MHz}, \mathrm{CDCl}_{3}\right) \delta_{\mathrm{H}} 2.20\left(t, J=7.1 \mathrm{~Hz}, 2 \mathrm{H}, \mathrm{CH}_{2} \mathrm{C} \equiv \mathrm{C}\right), 1.72(s, 1 \mathrm{H}, \mathrm{OH}), 1.51$ $\left(t t, J=7.2,7.1 \mathrm{~Hz}, 2 \mathrm{H}, \mathrm{CH}_{2} \mathrm{CH}_{2} \mathrm{C} \equiv \mathrm{C}\right), 1.43-1.24\left(m, 4 \mathrm{H}, 2 \times \mathrm{CH}_{2}\right), 0.89(t, J=7.0$ $\left.\mathrm{Hz}, 3 \mathrm{H}, \mathrm{CH}_{3}\right) ;{ }^{13} \mathrm{C}$ NMR $\left(75 \mathrm{MHz}, \mathrm{CDCl}_{3}\right) \delta_{\mathrm{C}} 50.84\left(p, J_{\mathrm{CD}}=22.5 \mathrm{~Hz}, \alpha\right.$-shift -0.343 , C-1), 78.21 ( $\beta$-shift - 0.059, C-2), 86.59 ( $\gamma$-shift 0.166, C-3), 18.68 ( $\delta$-shift 0.039, C-4), 28.29 (C-5), 31.03 (C-6), 22.19 (C-7), 13.94 (C-8); TMS Derivative EI-MS: m/z 185 ([M$\left.15]^{+}\right), 167\left([\mathrm{M}-33]^{+}\right), 153\left([\mathrm{M}-47]^{+}\right), 143\left([\mathrm{M}-57]^{\dagger}\right), 129,117,109,96,83,75,59,41$.

\subsubsection{2-Octynal}
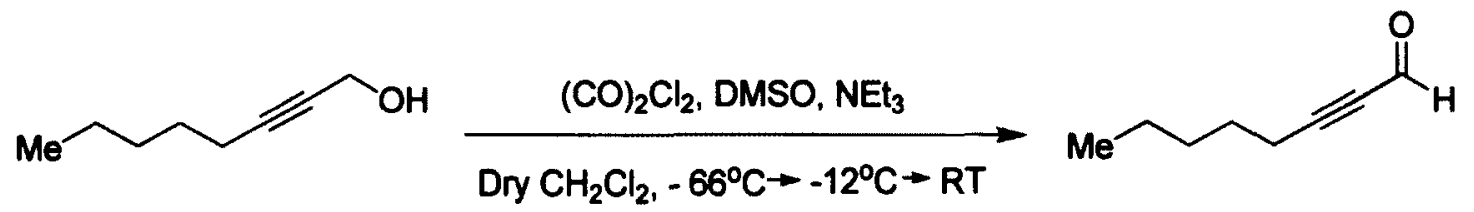

To a three-neck round-botton flask equipped with a magnetic stir bar was charged with oxalyl chloride $(0.7 \mathrm{~mL}, 8 \mathrm{mmol})$ and subsequently diluted with dry $\mathrm{CH}_{2} \mathrm{Cl}_{2}(12$ $\mathrm{mL}$ ). After cooling the solution to $-66^{\circ} \mathrm{C}$ (external temperature), anhydrous DMSO (1.1 $\mathrm{mL}, 15.9 \mathrm{mmol}$ ) was added to it dropwise leading to a strong exothermic reaction. The solution turned more viscous after the addition of DMSO and the reaction mixture was stirred for an additional $5 \mathrm{~min}$ at $-66^{\circ} \mathrm{C}$. To the reaction mixture was added a solution of oct-2-yn-1-ol (500 mg, $4.0 \mathrm{mmol})$ in dry $\mathrm{CH}_{2} \mathrm{Cl}_{2}(10 \mathrm{~mL})$ in portions for $10 \mathrm{~min}$ and the reaction mixture become cloudy, off-white in color. The reaction mixture was then warmed up to $-12{ }^{\circ} \mathrm{C}$ (external) and stirred for additional 30 min before triethylamine (3.3 $\mathrm{mL}, 23.8 \mathrm{mmol})$ was added dropwise. The resultant yellowish reaction mixture was stirred for an additional $10 \mathrm{~min}$ before being warmed to room temperature and stirred for 
another $20 \mathrm{~min}$. The reaction was terminated by pouring the mixture into water $(50 \mathrm{~mL})$ and the organic layer was separated on separatory funnel. The aqueous layer was extracted with dichloromethane $(3 \times 25 \mathrm{~mL})$ and the collected organic layers were treated with $1.0 \mathrm{M} \mathrm{HCl}(40 \mathrm{~mL}), \mathrm{H}_{2} \mathrm{O}(40 \mathrm{~mL})$ and brine $(50 \mathrm{~mL})$. Filtration through anhydrous $\mathrm{Na}_{2} \mathrm{SO}_{4}$ followed by removal of the solvent in rota-evaporator gave the crude compound as a yellowish oil $(24.6 \mathrm{mg}, 5 \%)$. bp $105-110^{\circ} \mathrm{C}$ at $15 \mathrm{~mm} \mathrm{Hg} ; R_{\mathrm{f}} 0.44$ (EtOAc:hexane, 1:9); IR (film): $v$ 2959, 2935, 2863, 2739 (C-H), 2281, 2201 (C $\equiv C), 1671(\mathrm{C}=\mathrm{O}), 1467$, $1388,1138,817 \mathrm{~cm}^{-1} ;{ }^{1} \mathrm{H}$ NMR $\left(300 \mathrm{MHz}, \mathrm{CDCl}_{3}\right) \delta_{\mathrm{H}} 9.18(t, J=0.3 \mathrm{~Hz}, 1 \mathrm{H}, \mathrm{CHO})$, $2.41\left(t d, J=7.2,0.3 \mathrm{~Hz}, 2 \mathrm{H}, \mathrm{CH}_{2} \mathrm{C} \equiv \mathrm{C}\right), 1.61\left(t t, J=7.2,7.1 \mathrm{~Hz}, 2 \mathrm{H}, \mathrm{CH}_{2} \mathrm{CH}_{2} \mathrm{C} \equiv \mathrm{C}\right)$, 1.46-1.23 (m, $\left.4 \mathrm{H}, 2 \times \mathrm{CH}_{2}\right), 0.91\left(t, J=7.1 \mathrm{~Hz}, 3 \mathrm{H}, \mathrm{CH}_{3}\right) ;{ }^{13} \mathrm{C}$ NMR $(75.5 \mathrm{MHz}$, $\left.\mathrm{CDCl}_{3}\right) \delta_{\mathrm{C}} 177.20(\mathrm{C}-1), 81.61(\mathrm{C}-2), 99.33$ (C-3), 19.00 (C-4), 27.15 (C-5), 30.87 (C-6), 22.00 (C-7), 13.76 (C-8); EI-MS (rel. intensity): $m / z 123\left(16,[\mathrm{M}-1]^{+}\right), 109\left(44,[\mathrm{M}-15]^{+}\right)$, $95\left(100,\left[\mathrm{Me}\left(\mathrm{CH}_{2}\right)_{4} \mathrm{C} \equiv \mathrm{C}\right]^{+},[\mathrm{M}-29]^{+}\right), 91(24), 81\left(73,[\mathrm{M}-43]^{+}\right), 79$ (34), 67 (84), 55 (46), $51(22), 41(74), 29(44)$.

\subsubsection{5 $\left[1-{ }^{2} H_{1}\right]-$ Oct-2-ynal}

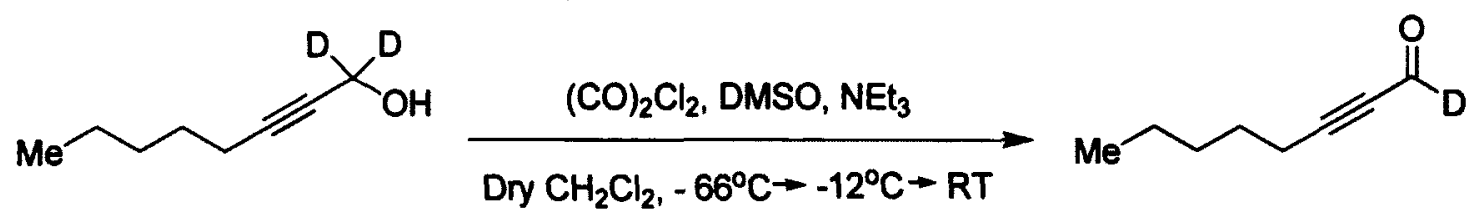

The same experimental procedure was followed to oxidize $\left[1,1 .{ }^{2} \mathrm{H}_{2}\right]-\mathrm{oct}-2-\mathrm{yn}-1-\mathrm{ol}$ (3.05 $\mathrm{mg}, 23.8 \mathrm{mmol}$ ) to the corresponding monodeutrio propargylic aldehyde as described in 6.2.2.4. The reaction resulted in the formation of the expected product as a pale yellowish oil with very poor yield $(149 \mathrm{mg}, 5 \%)$. Therefore, the Swern oxidation 
procedure could not be used to synthesize a large quantity of propargylic aldehyde from the corresponding alcohol. bp $105-108{ }^{\circ} \mathrm{C}$ at $15 \mathrm{~mm} \mathrm{Hg} ; R_{\mathrm{f}} 0.44$ (EtOAc:hexane, 1:9); IR (film): v 2959, 2934, 2863 (C-H), 2322, 2216 (C三C), 2120 (C-D), 1649 (C=O), 1467, $1328,1160,796,728 \mathrm{~cm}^{-1} ;{ }^{1} \mathrm{H}$ NMR $\left(300 \mathrm{MHz}, \mathrm{CDCl}_{3}\right) \delta_{\mathrm{H}} 2.38(t, J=7.2 \mathrm{~Hz}, 2 \mathrm{H}$, $\left.\mathrm{CH}_{2} \mathrm{C} \equiv \mathrm{C}\right), 1.58\left(t t, J=7.2,7.1 \mathrm{~Hz}, 2 \mathrm{H}, \mathrm{CH}_{2} \mathrm{CH}_{2} \mathrm{C} \equiv \mathrm{C}\right), 1.44-1.24\left(m, 4 \mathrm{H}, 2 \times \mathrm{CH}_{2}\right)$, $0.88\left(t, J=7.1 \mathrm{~Hz}, 3 \mathrm{H}, \mathrm{CH}_{3}\right) ;{ }^{13} \mathrm{C} \mathrm{NMR}\left(75.5 \mathrm{MHz}, \mathrm{CDCl}_{3}\right) \delta_{\mathrm{C}} 176.98\left(\mathrm{t}, J_{\mathrm{CD}}=29.3 \mathrm{~Hz}\right.$, $\alpha$-shift - 0.218, C-1), $81.66\left(\mathrm{t},{ }^{2} J_{\mathrm{CD}}=4.9 \mathrm{~Hz}, \beta\right.$-shift $\left.0.049, \mathrm{C}-2\right), 99.30(\gamma$-shift -0.031 , C-3), 19.08 (8-shift 0.082, C-4), 27.22 (C-5), 30.95 (C-6), 22.08 (C-7), 13.90 (C-8); EIMS: $m / 2124\left([\mathrm{M}-1]^{+}\right), 123\left(\left[\mathrm{Me}\left(\mathrm{CH}_{2}\right)_{4} \mathrm{C} \equiv \mathrm{CCO}\right]^{+},[\mathrm{M}-2]^{+}\right), 110\left([\mathrm{M}-15]^{+}\right), 95$ $\left(\left[\mathrm{Me}\left(\mathrm{CH}_{2}\right)_{4} \mathrm{C} \equiv \mathrm{C}\right]^{+},[\mathrm{M}-30]^{+}\right), 93,82\left([\mathrm{M}-43]^{+}\right), 79,68,55,51,41$.

\subsubsection{Oct-2-ynal ${ }^{173}$}

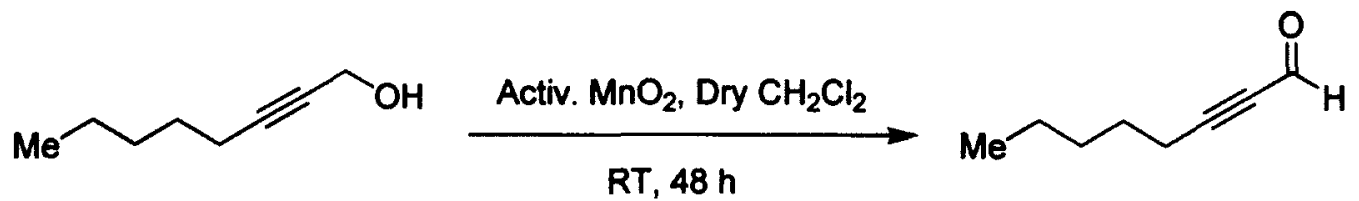

Oct-2-yn-1-ol (785 mg, $6.2 \mathrm{mmol})$ and activated $\mathrm{MnO}_{2}(5.89 \mathrm{~g}, 67.7 \mathrm{mmol})$ were mixed together in dry $\mathrm{CH}_{2} \mathrm{Cl}_{2}(40 \mathrm{~mL})$ and stirred for $48 \mathrm{~h}$ at room temperature. The reaction mixture was filtered through celite on Buchner funnel and the solvent was removed in vacuo to afford the crude compound that was purified by flash chromatography on silica gel using $\mathrm{CH}_{2} \mathrm{Cl}_{2}$ :hexane (1:19) as eluent. The title compound was obtained as a colorless liquid ( $270 \mathrm{mg}, 35 \%)$. bp $105-110^{\circ} \mathrm{C}$ at $15 \mathrm{~mm} \mathrm{Hg} ; R_{\mathrm{f}} 0.35$ (EtOAc:hexane, 1:19); IR (film): v 2959, 2935, 2863, 2739 (C-H), 2281, 2201 (C $\equiv \mathrm{C})$, $1671(\mathrm{C}=0), 1467,1388,1138,817 \mathrm{~cm}^{-1} ;{ }^{1} \mathrm{H}$ NMR $\left(300 \mathrm{MHz}, \mathrm{CDCl}_{3}\right) \delta_{\mathrm{H}} 9.14(t, J=0.3$ 
$\mathrm{Hz}, 1 \mathrm{H}, \mathrm{CHO}), 2.38\left(t d, J=7.1,0.3 \mathrm{~Hz}, 2 \mathrm{H}, \mathrm{CH}_{2} \mathrm{C} \equiv \mathrm{C}\right), 1.57(t t, J=7.2,7.1 \mathrm{~Hz}, 2 \mathrm{H}$, $\left.\mathrm{CH}_{2} \mathrm{CH}_{2} \mathrm{C} \equiv \mathrm{C}\right), 1.43-1.22\left(m, 4 \mathrm{H}, 2 \times \mathrm{CH}_{2}\right), 0.88\left(t, J=7.0 \mathrm{~Hz}, 3 \mathrm{H}, \mathrm{CH}_{3}\right) ;{ }^{13} \mathrm{C}$ NMR $\left(75 \mathrm{MHz}, \mathrm{CDCl}_{3}\right) \delta_{\mathrm{C}} 177.30(\mathrm{C}-1), 81.70$ (C-2), 99.43 (C-3), 19.11 (C-4), 27.24 (C-5), 30.96 (C-6), 22.09 (C-7), 13.87 (C-8).

\subsubsection{7 $\left[1-{ }^{2} \mathrm{H}_{1}\right]-$ Oct-2-ynal}

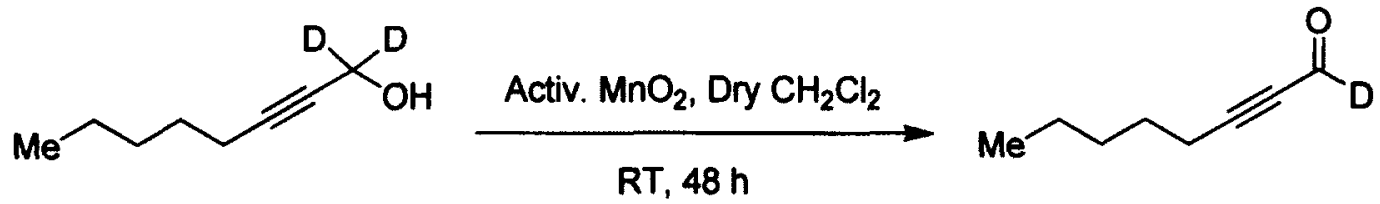

The title compound was obtained in a similar manner to that described in 6.2.2.6, from $\left[1,1-{ }^{2} \mathrm{H}_{2}\right]$-oct-2-yn-1-ol $(9.43 \mathrm{~g}, 73.7 \mathrm{mmol})$ as a colorless oil $(3.22 \mathrm{~g}, 35 \%)$. bp $105-108{ }^{\circ} \mathrm{C}$ at $15 \mathrm{~mm} \mathrm{Hg} ; R_{\mathrm{f}} 0.44$ (EtOAc:hexane, 1:9); IR (film): $v$ 2958, 2933, 2862 (CH), 2321, 2216 (C三C), 2117 (C-D), $1650(\mathrm{C}=0), 1467,1329,1160,1070,796,728 \mathrm{~cm}^{\circ}$ '; ' ${ }^{\mathrm{H}} \mathrm{NMR}\left(300 \mathrm{MHz}, \mathrm{CDCl}_{3}\right) \delta_{\mathrm{H}} 2.41\left(t, J=7.1 \mathrm{~Hz}, 2 \mathrm{H}, \mathrm{CH}_{2} \mathrm{C} \equiv \mathrm{C}\right), 1.61(t t, J=7.2$, 7.1 Hz, $\left.2 \mathrm{H}, \mathrm{CH}_{2} \mathrm{CH}_{2} \mathrm{C} \equiv \mathrm{C}\right), 1.47-1.25\left(m, 4 \mathrm{H}, 2 \times \mathrm{CH}_{2}\right), 0.91\left(t, J=7.1 \mathrm{~Hz}, 3 \mathrm{H}, \mathrm{CH}_{3}\right)$; ${ }^{13} \mathrm{C}$ NMR $\left(75.5 \mathrm{MHz}, \mathrm{CDCl}_{3}\right) \delta_{\mathrm{C}} 177.02\left(t, J_{\mathrm{CD}}=29.3 \mathrm{~Hz}, \alpha\right.$-shift $\left.-0.177, \mathrm{C}-1\right), 81.67(t$, ${ }^{2} J_{\mathrm{CD}}=4.9 \mathrm{~Hz}, \beta$-shift $\left.0.063, \mathrm{C}-2\right), 99.38(\gamma$-shift $0.046, \mathrm{C}-3), 19.10$ ( $\delta$-shift $\left.0.107, \mathrm{C}-4\right)$, 27.24 (C-5), 30.96 (C-6), 22.09 (C-7), 13.86 (C-8); EI-MS: m/z 124 ([M-1] $\left.]^{\dagger}\right), 123$ $\left(\left[\mathrm{Me}\left(\mathrm{CH}_{2}\right)_{4} \mathrm{C} \equiv \mathrm{CCO}\right]^{+},[\mathrm{M}-2]^{+}\right), 110\left([\mathrm{M}-15]^{+}\right), 95\left(\left[\mathrm{Me}\left(\mathrm{CH}_{2}\right)_{4} \mathrm{C} \equiv \mathrm{C}\right]^{+},[\mathrm{M}-30]^{+}\right), 93$, $82\left([\mathrm{M}-43]^{+}\right), 79,68,55,51,41$. 


\subsubsection{2-Nonynal}

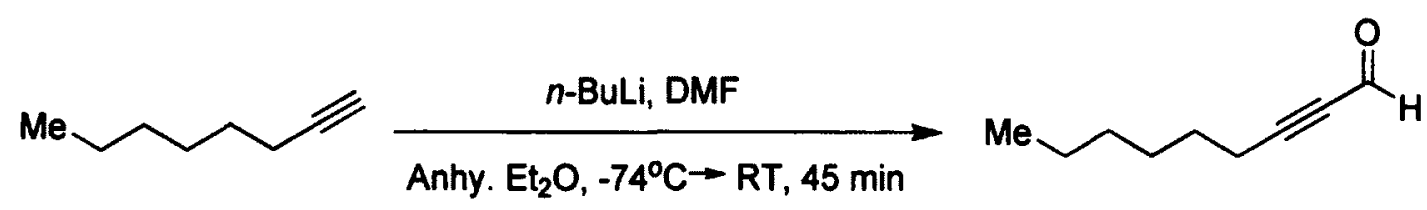

$n$-Butyllithium in hexanes $(2.5 \mathrm{M}$ in hexanes, $4 \mathrm{~mL}, 10 \mathrm{mmol})$ was added dropwise to a stirred solution of 1-octyne $(1.12 \mathrm{~g}, 10 \mathrm{mmol})$ in anhydrous $\mathrm{Et}_{2} \mathrm{O}(10 \mathrm{~mL})$ at $-74^{\circ} \mathrm{C}$ and allowed to stir for 30 minutes. After slowly admitting $\left[1-{ }^{2} \mathrm{H}_{1}\right] \mathrm{N}, \mathrm{N}$ dimethylformamide $(1.2 \mathrm{~mL}, 15 \mathrm{mmol})$ to the reaction mixture, the reaction was warmed up to room temperature and stirred for an additional 30 minutes. Ice-water $(15 \mathrm{ml})$ was added to the reaction mixture and acidified using $3.0 \mathrm{M} \mathrm{HCl}$ to $\mathrm{pH} 3$, followed by neutralization with saturated aqueous $\mathrm{NaHCO}_{3}$ to $\mathrm{pH}$ 7. The organic layer was separated and the aqueous phase was extracted repeatedly with ether $(4 \times 10 \mathrm{ml})$. Combined organic phases were treated with water $(25 \mathrm{~mL})$, brine $(2 \times 25 \mathrm{~mL})$ and finally dried over anhydrous $\mathrm{Na}_{2} \mathrm{SO}_{4}$. The solvent was removed initially by short-path distillation at normal atmospheric pressure and then over to the $\mathrm{N}_{2}$ flash until constant weight was recorded. The anticipated compound was afforded as a yellowish oil ( $1.25 \mathrm{~g}, 90 \%$ yield). $R_{\mathrm{f}} 0.42$ (EtOAc:hexanes, 1:19); IR (film): v 2932, $2860(\mathrm{C}-\mathrm{H}), 2281,2201(\mathrm{C} \equiv \mathrm{C}), 1672(\mathrm{C}=\mathrm{O})$, $1467,1387,1138,824,791,726 \mathrm{~cm}^{-1}$; ${ }^{1} \mathrm{H}$ NMR $\left(300 \mathrm{MHz}, \mathrm{CDCl}_{3}\right) \delta_{\mathrm{H}} 9.17(s, 1 \mathrm{H}$, CHO), $2.40\left(t, J=7.1 \mathrm{~Hz}, 2 \mathrm{H}, \mathrm{CH}_{2} \mathrm{C} \equiv \mathrm{C}\right), 1.59\left(m, 2 \mathrm{H}, \mathrm{CH}_{2} \mathrm{CH}_{2} \mathrm{C} \equiv \mathrm{C}\right), 1.22-1.45(m$, $\left.6 \mathrm{H}, 3 \times \mathrm{CH}_{2}\right), 0.88\left(t, J=6.8 \mathrm{~Hz}, 3 \mathrm{H}, \mathrm{CH}_{3}\right) ;{ }^{13} \mathrm{C} \mathrm{NMR}\left(75.5 \mathrm{MHz}, \mathrm{CDCl}_{3}\right) \delta_{\mathrm{C}}: 177.21(\mathrm{C}-$ 1), 81.63 (C-2), 99.34 (C-3), 19.06 (C-4), 27.45 (C-5), 28.44 (C-6), 31.13 (C-7), 22.40 (C-8), 13.94 (C-9); EI-MS (rel. intensity): m/z 137 (2, [M-1] ${ }^{+}$), 123 (7), 109 (28), 95 (24), 81 (45), 68 (48), 67 (48), 55 (36), 43 (100), 41 (74). 


\subsubsection{9 $\left[1-{ }^{2} H_{1}\right]-$ Oct-2-ynal}
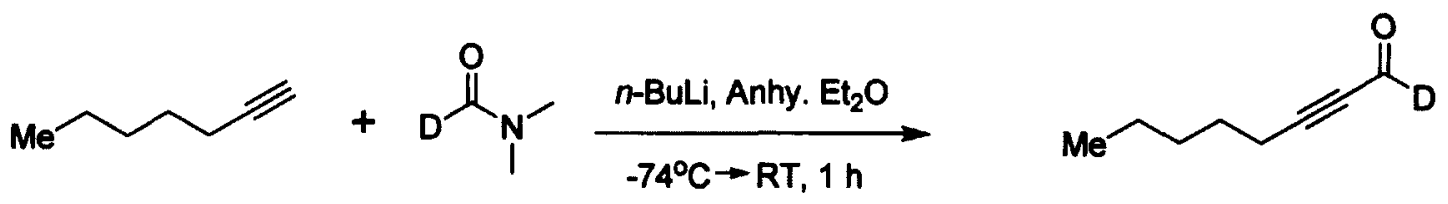

The title compound was synthesized using the same experimental procedure as described in 6.2.2.8, from 1-heptyne $(4.15 \mathrm{~g}, 43.1 \mathrm{mmol})$ and $\left[1-{ }^{2} \mathrm{H}_{1}\right] \mathrm{N}, \mathrm{N}$ dimethylformamide $(5.0 \mathrm{~mL}, 64.6 \mathrm{mmol})$ as a colorless clear oil $(4.87 \mathrm{~g}, 93 \%)$. bp $105-$ $108^{\circ} \mathrm{C}$ at $15 \mathrm{~mm} \mathrm{Hg} ; R_{\mathrm{f}} 0.44$ (EtOAc:hexane, 1:9); IR (film): v 2959, 2934, 2873 (C-H), 2321, $2216(\mathrm{C} \equiv \mathrm{C}), 2119(\mathrm{C}-\mathrm{D}), 1649$ (C=O), 1467, 1328, 1160, 796, $729 \mathrm{~cm}^{-1} ;{ }^{1} \mathrm{H}$ NMR $\left(300 \mathrm{MHz}, \mathrm{CDCl}_{3}\right) \delta_{\mathrm{H}} 2.40\left(t, J=7.1 \mathrm{~Hz}, 2 \mathrm{H}, \mathrm{CH}_{2} \mathrm{C} \equiv \mathrm{C}\right), 1.60(t t, J=7.2,7.1$ $\left.\mathrm{Hz}, 2 \mathrm{H}, \mathrm{CH}_{2} \mathrm{CH}_{2} \mathrm{C} \equiv \mathrm{C}\right), 1.46-1.25\left(m, 4 \mathrm{H}, 2 \times \mathrm{CH}_{2}\right), 0.90\left(t, J=7.1 \mathrm{~Hz}, 3 \mathrm{H}, \mathrm{CH}_{3}\right)$;

${ }^{13} \mathrm{C}$ NMR $\left(75 \mathrm{MHz}, \mathrm{CDCl}_{3}\right) \delta_{\mathrm{C}} 176.96\left(t, J_{\mathrm{CD}}=29.3 \mathrm{~Hz}, \alpha\right.$-shift $\left.-0.233, \mathrm{C}-1\right), 81.63(t$, ${ }^{2} J_{\mathrm{CD}}=4.9 \mathrm{~Hz}, \beta$-shift $\left.-0.024, \mathrm{C}-2\right), 99.30(\gamma$-shift $-0.032, \mathrm{C}-3), 19.06(\delta$-shift $0.062, \mathrm{C}-$ 4), 27.21 (C-5), 30.93 (C-6), 22.06 (C-7), 13.91 (C-8); EI-MS: $m / z 124\left([\mathrm{M}-1]^{+}\right), 123$ $\left(\left[\mathrm{Me}\left(\mathrm{CH}_{2}\right)_{4} \mathrm{C} \equiv \mathrm{CCO}\right]^{+},[\mathrm{M}-2]^{+}\right), 110\left([\mathrm{M}-15]^{+}\right), 95\left(\left[\mathrm{Me}\left(\mathrm{CH}_{2}\right)_{4} \mathrm{C} \equiv \mathrm{C}\right]^{++},[\mathrm{M}-30]^{+}\right), 93$, $82\left([\mathrm{M}-43]^{+}\right), 79,68,55,51,41$.

\subsubsection{Oct-2-yn-1-ol}
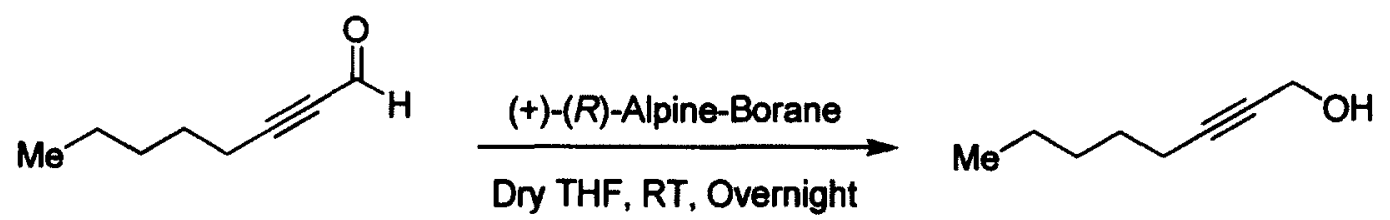

A three-neck round-bottom flask equipped with a reflux condenser, one-piece distillation apparatus, stir bar, and rubber septa was flushed with $\mathrm{N}_{2}$ and charged with 
Oct-2-ynal (504 mg, $4.1 \mathrm{mmol})$. The reactant was diluted with anhydrous THF (17 mL) and to the stirred solution was added a solution of $(+)-(R)$-Alpine-Borane in THF (0.5 $\mathrm{M}, 9 \mathrm{~mL}, 4.5 \mathrm{mmol}$ ) in portions at room temperature. The reaction mixture turned light yellow in color after the completion of addition and was allowed to stir overnight at room temperature. Excess

Borane $^{\circledast}$ reagent was quenched by the addition of excess acetaldehyde $(1 \mathrm{~mL})$ and the mixture was stirred for additional $1 \mathrm{~h}$ to ensure completion of reaction. The rubber septum was quickly replaced with a glass stopper and the solvent (THF) as well as the byproduct $\alpha$-pinene was distilled under vacuum (at $15 \mathrm{~mm}$ and $0.02 \mathrm{~mm} \mathrm{Hg}$, respectively). To remove $\alpha$-pinene, slight warming $\left(40-45^{\circ} \mathrm{C}\right)$ of the reaction flask was required. The residue was diluted with anhydrous $\mathrm{Et}_{2} \mathrm{O}(20 \mathrm{~mL})$ whilst maintaining an inert atmosphere and the solution was subsequently cooled to $0{ }^{\circ} \mathrm{C}$. Ethanolamine $(0.3$ $\mathrm{mL}, 4.5 \mathrm{mmol}$ ) was slowly admitted to the stirred solution leading to the formation of a white precipitate that was removed by filtration through celite on Buchner funnel. The precipitate washed with additional ether $(2 \times 20 \mathrm{~mL})$ and the collected liquids were combined. The combined organic layer was treated in the usual way to give the crude compound that was purified by Kugelrohr distillation under vacuum and the desired alcohol was obtained as a colorless liquid ( $369 \mathrm{mg}, 72 \%$ ). bp $52-55^{\circ} \mathrm{C}$ at $0.1 \mathrm{~mm} \mathrm{Hg} ; R_{\mathrm{f}}$ 0.23 (EtOAc:hexane, 1:3); IR (film): v 3339 (O-H), 2957, 2933, 2861 (C-H), 2289, 2226 $(\mathrm{C} \equiv \mathrm{C}), 1459,1432,1359,1137,1095,1014,725 \mathrm{~cm}^{-1} ;{ }^{1} \mathrm{H} \mathrm{NMR}\left(300 \mathrm{MHz}, \mathrm{CDCl}_{3}\right) \delta_{\mathrm{H}}$ $4.23\left(t, J=2.2 \mathrm{~Hz}, 2 \mathrm{H}, \mathrm{CH}_{2} \mathrm{OH}\right), 2.19\left(t t, J=7.2,2.2 \mathrm{~Hz}, 2 \mathrm{H}, \mathrm{CH}_{2} \mathrm{C} \equiv \mathrm{C}\right), 1.94(s, 1 \mathrm{H}$, OH), $1.50\left(t t, J=7.2,7.1 \mathrm{~Hz}, 2 \mathrm{H}, \mathrm{CH}_{2} \mathrm{CH}_{2} \mathrm{C} \equiv \mathrm{C}\right), 1.42-1.23\left(m, 4 \mathrm{H}, 2 \times \mathrm{CH}_{2}\right), 0.88(t$, $\left.J=6.9 \mathrm{~Hz}, 3 \mathrm{H}, \mathrm{CH}_{3}\right) ;{ }^{13} \mathrm{C}$ NMR $\left(75.5 \mathrm{MHz}, \mathrm{CDCl}_{3}\right) \delta_{\mathrm{C}} 51.19(\mathrm{C}-1), 78.27(\mathrm{C}-2), 86.42$ 
(C-3), 18.64 (C-4), 28.26 (C-5), 30.99 (C-6), 22.14 (C-7), 13.88 (C-8); TMS Derivative EI-MS: $m / z 197\left([\mathrm{M}-1]^{+}\right), 183\left([\mathrm{M}-15]^{+}\right), 165\left([\mathrm{M}-33]^{+}\right), 153\left([\mathrm{M}-45]^{+}\right), 142\left([\mathrm{M}-56]^{+}\right)$, $127,115,109,96,83,75,59,41$.

\subsubsection{1 (1R)-[1- $\left.{ }^{2} \mathrm{H}_{1}\right]-$ Oct-2-yn-1-ol}
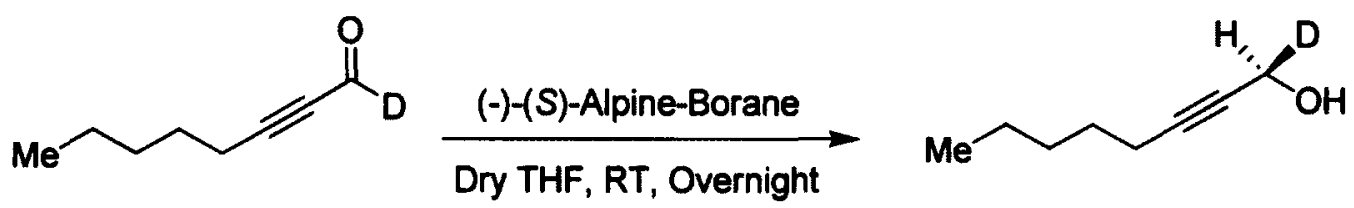

The title compound was prepared in a similar manner to that described in 6.2.2.10, from $\left[1-^{2} \mathrm{H}_{1}\right]-O c t-2-y n a l(4.6 \mathrm{~g}, 36.8 \mathrm{mmol})$ as a colorless liquid $(3.32 \mathrm{~g}, 71 \%)$. bp $51-55^{\circ} \mathrm{C}$ at $0.1 \mathrm{~mm} \mathrm{Hg} ; R_{\mathrm{f}} 0.27$ (EtOAc:hexane, 1:3); IR (film): v $3346(\mathrm{O}-\mathrm{H}), 2957$, 2932, 2872, 2861 (C-H), 2294, 2230 (C 三C), 2180, 2134 (C-D), 1467, 1316, 1142, 1031, 946, $825 \mathrm{~cm}^{-1}$; 'H NMR (300 MHz, $\left.\mathrm{CDCl}_{3}\right) \delta_{\mathrm{H}} 4.23(t t, J=2.3,2.1 \mathrm{~Hz}, 1 \mathrm{H}, \mathrm{CHDOH})$, $2.20\left(t d, J=7.1,2.1 \mathrm{~Hz}, 2 \mathrm{H}, \mathrm{CH}_{2} \mathrm{C} \equiv \mathrm{C}\right), 1.75($ br $s, 1 \mathrm{H}, \mathrm{OH}), 1.51(t t, J=7.2,7.1 \mathrm{~Hz}$, $\left.2 \mathrm{H}, \mathrm{CH}_{2} \mathrm{CH}_{2} \mathrm{C} \equiv \mathrm{C}\right), 1.43-1.25\left(m, 4 \mathrm{H}, 2 \times \mathrm{CH}_{2}\right), 0.89\left(t, J=7.1 \mathrm{~Hz}, 3 \mathrm{H}, \mathrm{CH}_{3}\right) ;{ }^{13} \mathrm{C}$ NMR $\left(75.5 \mathrm{MHz}, \mathrm{CDCl}_{3}\right) \delta_{\mathrm{C}} 51.16\left(t, J_{\mathrm{CD}}=22.6 \mathrm{~Hz}, \alpha\right.$-shift - 0.026, C-1), 78.23 ( $\beta$-shift $-0.032, \mathrm{C}-2), 86.64$ ( $\gamma$-shift 0.219, C-3), 18.70 ( $\delta$-shift 0.063, C-4), 28.30 (C-5), 31.04 (C-6), 22.20 (C-7), 13.96 (C-8); EI-MS: $m / 2126\left([\mathrm{M}-1]^{+}\right), 112$ ([M-15] $\left.]^{+}\right), 98\left([\mathrm{M}-29]^{+}\right)$, 95, 84, 80, 71, 67, 55, 53, 41; TMS Derivative EI-MS: $m / z 198$ ([M-1] $\left.]^{\dagger}\right), 184\left([\mathrm{M}-15]^{+}\right)$, $166\left([\mathrm{M}-33]^{+}\right), 153\left([\mathrm{M}-46]^{+}\right), 143\left([\mathrm{M}-56]^{+}\right), 128,116,109,96,83,75,59,41$. 


\subsubsection{2 (1R)-1-(p-tolylsulfonyloxy)-[1- $\left.{ }^{2} \mathrm{H}_{1}\right]$ oct-2-yne ${ }^{173}$}

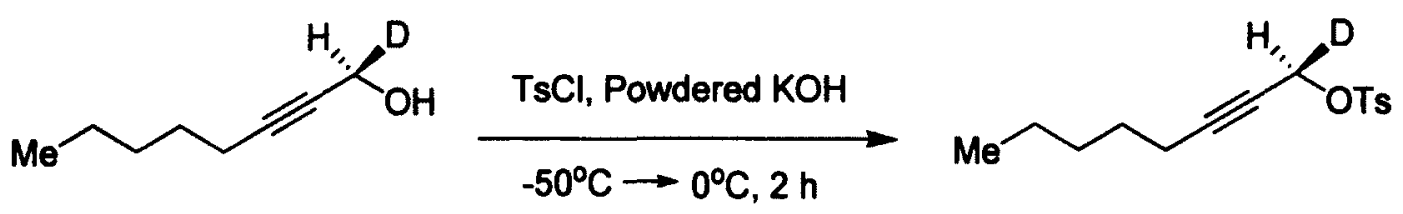

The anticipated compound was obtained in a similar manner to that described in 6.2.1.15, from $(1 R)-\left[1-{ }^{2} \mathrm{H}_{1}\right]$-Oct-2-yn-1-ol $(1.35 \mathrm{~g}, 10.6 \mathrm{mmol})$ as a pale yellowish oil (2.67 g, 89 \%). $R_{\mathrm{f}} 0.41$ (EtOAc:hexane, 1:4); IR (film): v 2957, 2933, $2861(\mathrm{C}-\mathrm{H}), 2312$, $2241(\mathrm{C} \equiv \mathrm{C}), 1598(\mathrm{C}=\mathrm{C}, \mathrm{Ar}), 1458,1369,1190,1178,1097,938,815,738 \mathrm{~cm}^{-1} ;{ }^{1} \mathrm{H}$ NMR $\left(300 \mathrm{MHz}, \mathrm{CDCl}_{3}\right) \delta_{\mathrm{H}} 7.80(d, J=8.2 \mathrm{~Hz}, 2 \mathrm{H}, \mathrm{C} \underline{-}-\mathrm{Ph}), 7.33(d, J=8.2 \mathrm{~Hz}, 2 \mathrm{H}$, $\mathrm{C} \underline{m}-\mathrm{Ph}), 4.68(t t, J=2.2,2.1 \mathrm{~Hz}, 1 \mathrm{H}, \mathrm{CHDOTs}), 2.44\left(s, 3 \mathrm{H}, \mathrm{CH}_{3} \mathrm{Ar}\right), 2.05(t d, J=$ 7.0, 2.2 Hz, $\left.2 \mathrm{H}, \mathrm{CH}_{2} \mathrm{C} \equiv \mathrm{C}\right), 1.37\left(t t, J=7.0,6.9 \mathrm{~Hz}, 2 \mathrm{H}, \mathrm{CH}_{2} \mathrm{CH}_{2} \mathrm{C} \equiv \mathrm{C}\right), 1.31-1.19$ $\left(m, 4 \mathrm{H}, 2 \times \mathrm{CH}_{2}\right), 0.87\left(t, J=6.8 \mathrm{~Hz}, 3 \mathrm{H}, \mathrm{CH}_{3}\right) ;{ }^{13} \mathrm{C} \mathrm{NMR}\left(75.5 \mathrm{MHz}, \mathrm{CDCl}_{3}\right) \delta_{\mathrm{C}} 58.57$ $\left(t, J_{\mathrm{CD}}=23.7 \mathrm{~Hz}, \alpha\right.$-shift - 0.230, C-1), $71.72(\beta$-shift - 0.042, C-2), $90.60(\gamma$-shift - 0.014 , C-3), 18.60 (8-shift 0.002, C-4), 27.74 (C-5), 30.90 (C-6), 22.10 (C-7), 13.90 (C-8), $144.80($ Cipso $-\mathrm{Ph}), 128.09(\mathrm{C} \underline{\underline{o}}-\mathrm{Ph}), 129.70(\mathrm{C} \underline{m}-\mathrm{Ph}), 133.43(\mathrm{C} \underline{p}-\mathrm{Ph}), 21.62\left(\mathrm{CH}_{3}-\right.$ Ph); EI-MS: $m / z 281\left(1,[\mathrm{M}]^{+}\right), 240\left(1,[\mathrm{M}-41]^{+}\right), 226(1), 202(3), 173\left(5,[\mathrm{M}-108]^{+}\right), 155$ $\left(34,[\mathrm{M}-126]^{+}\right), 139\left(30,[\mathrm{M}-142]^{+}\right), 126(5), 118(6), 107(10), 91$ (100), 80 (16), 77 (19), $65(50), 53(22), 41(35)$. 


\subsubsection{Octadeca-9,12-diynoic acid ${ }^{173}$}

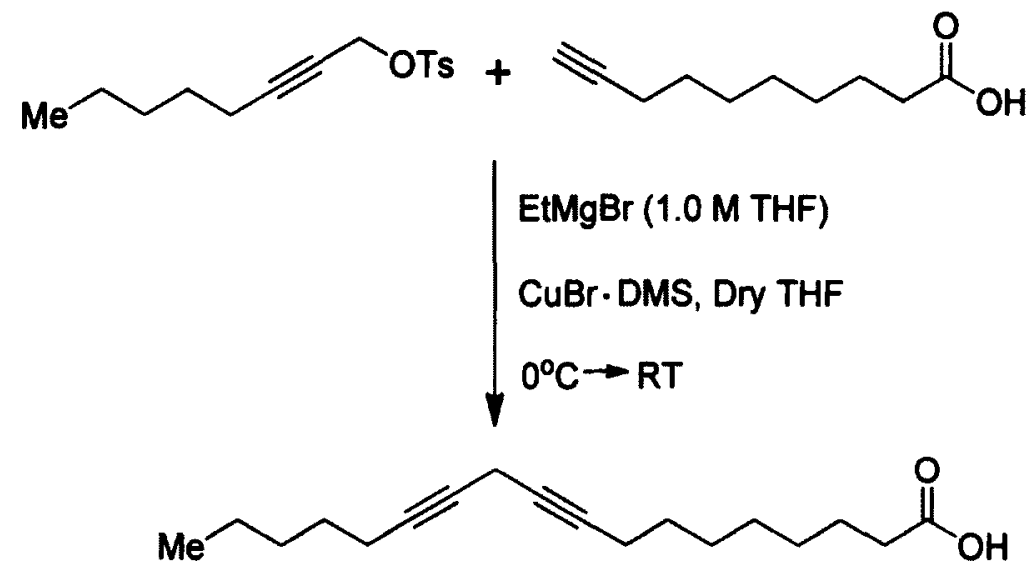

Ethylmagnesium bromide in anhydrous THF (1.0 M, $1.8 \mathrm{~mL}, 1.8 \mathrm{mmol})$ was added dropwise to a stirred solution of dec-9-ynoic acid (142 $\mathrm{mg}, 0.8 \mathrm{mmol})$ in dry THF $(3 \mathrm{~mL})$ at $0{ }^{\circ} \mathrm{C}$. Following the addition, the reaction mixture was heated up to $49^{\circ} \mathrm{C}$ and stirred for 30 minutes. The reaction mixture was then cooled to room temperature and CuBr.DMS (22 mg) complex was added to it in portions during 15 minutes. The reaction mixture was cooled to $0{ }^{\circ} \mathrm{C}$ and to that was added a solution of 1-(p-tolylfonyloxy)-Oct-2yne $(260 \mathrm{mg}, 0.9 \mathrm{mmol})$ in dry THF $(5 \mathrm{~mL})$. After stirring at $0{ }^{\circ} \mathrm{C}$ for $1 \mathrm{~h}$, the reaction was warmed to room temperature and allowed to stir another $3 \mathrm{~h}$. The reaction was quenched by the addition of saturated aqueous solution of $\mathrm{NH}_{4} \mathrm{Cl}(15 \mathrm{~mL})$ and the suspension was diluted with $\mathrm{Et}_{2} \mathrm{O}(10 \mathrm{~mL})$. The organic layer was separated by liquidliquid extraction and the aqueous layer extracted with additional portions of ether $(3 \times 12$ $\mathrm{mL}$ ). The collected organic layers were combined and treated in the normal manner to afford the crude compound as yellowish semi-solid. The crude compound was purified by column chromatography on silica gel using EtOAc:hexane (1:9 in presence of $0.1 \%$ $\mathrm{AcOH})$ to give the purified anticipated compound as a light yellowish oil that crystallized 
at $4{ }^{\circ} \mathrm{C}(79 \mathrm{mg}, 34 \%) . \mathrm{mp}-16^{\circ} \mathrm{C} ; R_{\mathrm{f}} 0.33\left(\mathrm{MeOH}: \mathrm{CH}_{2} \mathrm{Cl}_{2}, 1: 19\right)$; IR (film): $v 3500-2700$ (O-H), 3035, 2934, 2859 (C-H), 2214, 2117 (C $\equiv \mathrm{C}), 1711$ (C=O), 1460, 1413, 1287, $1247,937 \mathrm{~cm}^{-1} ;{ }^{1} \mathrm{H}$ NMR $\left(300 \mathrm{MHz}, \mathrm{CDCl}_{3}\right) \delta_{\mathrm{H}} 3.12(t t, J=2.3,2.3 \mathrm{~Hz}, 2 \mathrm{H}, \mathrm{C}-11), 2.35$ $\left(t, J=7.3 \mathrm{~Hz}, 2 \mathrm{H}, \mathrm{CH}_{2} \mathrm{CO}_{2} \mathrm{H}\right), 2.28-2.10(m, 4 \mathrm{H}, \mathrm{C}-8, \mathrm{C}-14), 1.64(t t, J=7.3,7.0 \mathrm{~Hz}, 2$ $\left.\mathrm{H}, \mathrm{CH}_{2} \mathrm{CH}_{2} \mathrm{CO}_{2} \mathrm{H}\right), 1.58-1.44(m, 4 \mathrm{H}, \mathrm{C}-7, \mathrm{C}-15), 1.42-1.23\left(m, 10 \mathrm{H}, 5 \times \mathrm{CH}_{2}\right), 0.89(t$, $\left.J=7.1 \mathrm{~Hz}, 3 \mathrm{H}, \mathrm{CH}_{3}\right) ;{ }^{13} \mathrm{C} \mathrm{NMR}\left(75.5 \mathrm{MHz}, \mathrm{CDCl}_{3}\right) \delta_{\mathrm{C}} 180.15$ (C-1), 34.02 (C-2), 24.60 (C-3), 28.77 (C-4), 28.65 (C-5), 28.65 (C-6), 28.94 (C-7), 18.72 (C-8), 80.36 (C-9), 74.61 (C-10), 9.73 (C-11), 74.45 (C-12), 80.55 (C-13), 18.72 (C-14), 28.48 (C-15), 31.09 (C$16), 22.23$ (C-17), 14.00 (C-18).

\subsubsection{4 (11S)-[11- $\left.{ }^{2} \mathrm{H}_{1}\right]$-octadeca-9,12-diynoic acid}

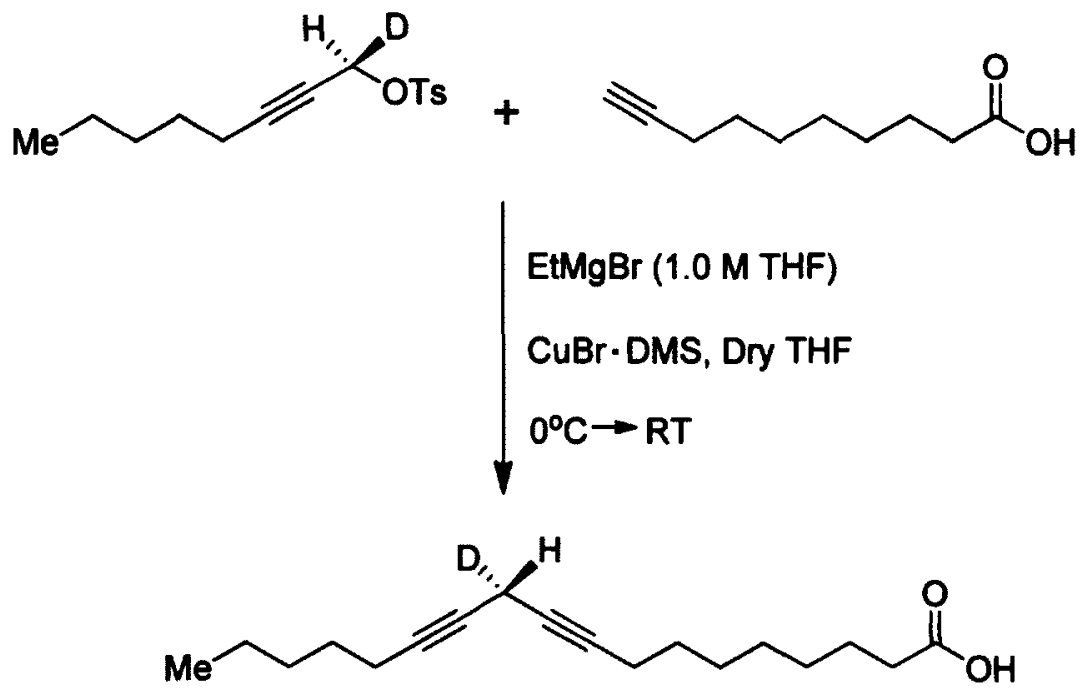

The title compound was obtained in a similar manner to that described in 6.2.2.14, from $(1 R)-1$-( $p$-tolylsulfonyloxy)-[1- $\left.{ }^{2} \mathrm{H}_{1}\right]$-oct-2-yne $(1.11 \mathrm{~g}, 4.0 \mathrm{mmol})$ and dec-9-ynoic acid (600 mg, $3.6 \mathrm{mmol})$ as a faint yellowish oil that solidifies at $4{ }^{\circ} \mathrm{C}(89 \mathrm{mg}, 9 \%) . \mathrm{mp}$ $16{ }^{\circ} \mathrm{C} ; R_{\mathrm{f}} 0.17$ (EtOAc:hexane, $1: 4$ in presence of $0.2 \% \mathrm{AcOH}$ ); IR (film): $v$ 3600-2600 
(O-H), 2933, 2859 (C-H), 2235, $2116(\mathrm{C} \equiv \mathrm{C}), 1710(\mathrm{C}=\mathrm{O}), 1458,1431,1295,1162,929$ $\mathrm{cm}^{-1}$; ${ }^{1} \mathrm{H}$ NMR $\left(300 \mathrm{MHz}, \mathrm{CDCl}_{3}\right) \delta_{\mathrm{H}} 3.10(t t, J=2.1,2.1 \mathrm{~Hz}, 1 \mathrm{H}, \mathrm{C}-11), 2.35(t, J=7.4$ $\mathrm{Hz}, 2 \mathrm{H}, \mathrm{CH}_{2} \mathrm{CO}_{2} \mathrm{H}$ ), 2.26-2.15 (m, $4 \mathrm{H}, \mathrm{C}-8, \mathrm{C}-14$ ), 1.64 (Overlapping $t t, J=7.4,6.5 \mathrm{~Hz}$, $\left.2 \mathrm{H}, \mathrm{CH}_{2} \mathrm{CH}_{2} \mathrm{CO}_{2} \mathrm{H}\right), 1.58-1.46(m, 4 \mathrm{H}, \mathrm{C}-7, \mathrm{C}-15), 1.45-1.24\left(m, 10 \mathrm{H}, 5 \times \mathrm{CH}_{2}\right), 0.88$ $\left(t, J=6.9 \mathrm{~Hz}, 3 \mathrm{H}, \mathrm{CH}_{3}\right) ;{ }^{13} \mathrm{C}$ NMR $\left(75.5 \mathrm{MHz}, \mathrm{CDCl}_{3}\right) \delta_{\mathrm{C}} 179.62$ (C-1), 33.99 (C-2), 24.61 (C-3), 28.72 (C-4), 28.64 (C-5), 28.64 (C-6), 28.91 (C-7), 18.70 ( 8 -shift - 0.018, C8), 80.36 ( $\gamma$-shift 0.003, C-9), 74.57 ( $\beta$-shift - 0.044, C-10), $9.51\left(t, J_{\mathrm{CD}}=20.5 \mathrm{~Hz}, \alpha\right.$-shift $-0.214, \mathrm{C}-11), 74.40$ ( $\beta$-shift - 0.045, C-12), 80.54 ( $\gamma$-shift - 0.003, C-13), 18.70 ( $\delta$-shift $0.018, \mathrm{C}-14), 28.47$ (C-15), 31.08 (C-16), 22.22 (C-17), 13.95 (C-18).

\subsubsection{Methyl octadeca-9,12-diynoate}

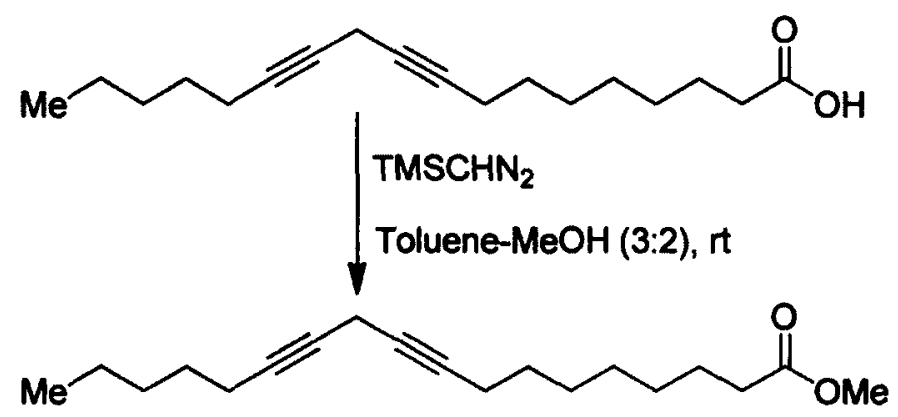

To a stirred solution of octadeca-9,12-diynoic acid $(299 \mathrm{mg}, 1.1 \mathrm{mmol})$ in $3: 2$ toluene-methanol $(10 \mathrm{~mL})$ was added commercially available (trimethylsilyl) diazomethane $(2.0 \mathrm{M}$ in hexanes, $0.6 \mathrm{~mL}, 1.1 \mathrm{mmol})$ dropwise until evolution of the gas from the reaction mixture was estimated to be complete. Few more drops of the reagent were added further to the reaction mixture until a persistent yellow color appeared and the reaction was stirred for another $5 \mathrm{~min}$. Excess diazomethane was quenched by the addition of few drops of acetic acid. The solvent was removed in vacuo and the crude 
compound was purified by flash chromatography using $\mathrm{Et}_{2} \mathrm{O}$ :hexane $(1: 49)$ as eluent to afford the pure compound as a pale yellowish oil ( $243 \mathrm{mg}, 77 \%$ ). $R_{\mathrm{f}} 0.25$ (EtOAc:hexane, 1:32); IR (film): v 2933, 2859 (C-H), 2212 (C $\equiv C), 1740$ (C=0), 1460, 1437, 1247, $1198,1172,1019,728 \mathrm{~cm}^{-1} ;{ }^{1} \mathrm{H}$ NMR $\left(300 \mathrm{MHz}, \mathrm{CDCl}_{3}\right) \delta_{\mathrm{H}} 3.66\left(s, 3 \mathrm{H}, \mathrm{OCH}_{3}\right), 3.11$ (tt, $J=2.4,2.4 \mathrm{~Hz}, 2 \mathrm{H}, \mathrm{C}-11), 2.30\left(t, J=7.5 \mathrm{~Hz}, 2 \mathrm{H}, \mathrm{CH}_{2} \mathrm{CO}_{2} \mathrm{Me}\right.$ ), 2.14 (Overlapping $t t, J=7.1,2.3 \mathrm{~Hz}, 4 \mathrm{H}, \mathrm{C}-8, \mathrm{C}-14), 1.62\left(t t, J=7.5,7.2 \mathrm{~Hz}, 2 \mathrm{H}, \mathrm{CH}_{2} \mathrm{CH}_{2} \mathrm{CO}_{2} \mathrm{Me}\right), 1.486$ $(m, 4 \mathrm{H}, \mathrm{C}-7, \mathrm{C}-15), 1.43-1.22\left(m, 10 \mathrm{H}, 5 \times \mathrm{CH}_{2}\right), 0.89\left(t, J=7.0 \mathrm{~Hz}, 3 \mathrm{H}, \mathrm{CH}_{3}\right) ;{ }^{13} \mathrm{C}$ NMR (75.5 MHz, $\mathrm{CDCl}_{3}$ ) $\delta_{\mathrm{C}} 174.29$ (C-1), 34.07 (C-2), 24.89 (C-3), $28.76(\mathrm{C}-4), 28.65$ (C-5), 28.65 (C-6), 29.01 (C-7), 18.70 (C-8, C-14), 80.37 (C-9), 74.57 (C-10), 9.72 (C11), 74.43 (C-12), 80.53 (C-13), 28.46 (C-15), 31.08 (C-16), 22.22 (C-17), 13.99 (C-18), 51.48 (C-19); EI-MS: (rel. intensity) $m / z 259$ (1, [M-31] $\left.]^{+}\right), 189$ (1, [M-101] $), 175$ (2), 159 (5), 147 (12), 133 (35), 119 (59), 105 (90), 91 (100), 79 (44), 67 (35), 55 (42), 41 (44).

\subsubsection{Methyl (11S)-[11- $\left.{ }^{2} \mathrm{H}_{1}\right]$ octadeca-9,12-diynoate}

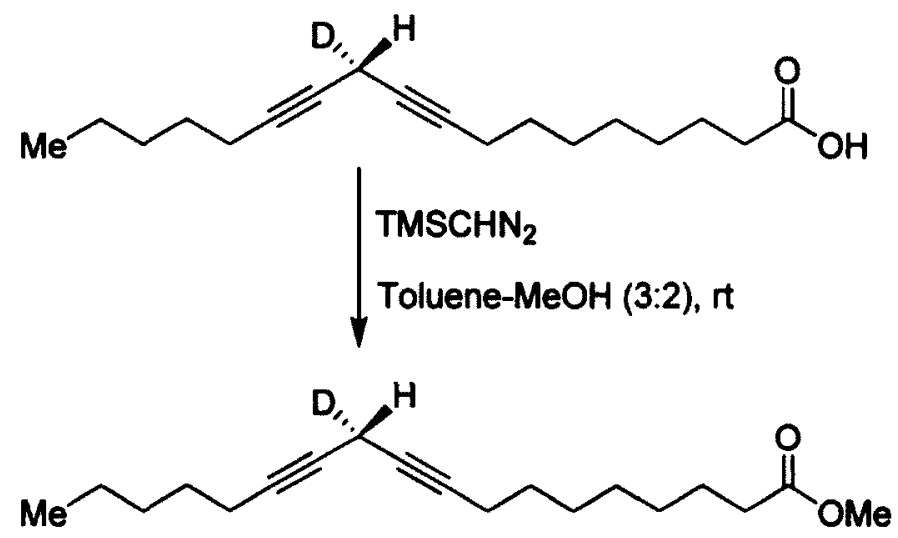

The title compound was synthesized using the same experimental procedure to that described in 6.2.2.16, from $(11 S)-\left[11-{ }^{2} \mathrm{H}_{1}\right]$-octadeca-9,12-diynoic acid $(110 \mathrm{mg}, 0.4$ 
mmol) as a pale-yellowish oil $(92 \mathrm{mg}, 79 \%) . R_{\mathrm{f}} 0.19$ (EtOAc:hexane, 1:19); IR (film): $v$ 2933, 2859 (C-H), 2152 (C-D), 1740 (C=O), 1460, 1436, 1267, 1198, 1173, 1016, 738 $\mathrm{cm}^{-1} ;{ }^{1} \mathrm{H}$ NMR $\left(300 \mathrm{MHz}, \mathrm{CDCl}_{3}\right) \delta_{\mathrm{H}} 3.66\left(s, 3 \mathrm{H}, \mathrm{OCH}_{3}\right), 3.10(t t, J=2.3,2.3 \mathrm{~Hz}, 1 \mathrm{H}$, C-11), $2.30\left(t, J=7.5 \mathrm{~Hz}, 2 \mathrm{H}, \mathrm{CH}_{2} \mathrm{CO}_{2} \mathrm{Me}\right.$ ), 2.14 (Overlapping $t d, J=7.1,2.3 \mathrm{~Hz}, 4 \mathrm{H}$, C-8, C-14), $1.62\left(t t, J=7.5,7.3 \mathrm{~Hz}, 2 \mathrm{H}, \mathrm{CH}_{2} \mathrm{CH}_{2} \mathrm{CO}_{2} \mathrm{Me}\right), 1.56-1.42(m, 4 \mathrm{H}, \mathrm{C}-7, \mathrm{C}-$ 15), 1.42-1.23 (m, $\left.10 \mathrm{H}, 5 \times \mathrm{CH}_{2}\right), 0.89\left(t, J=7.0 \mathrm{~Hz}, 3 \mathrm{H}, \mathrm{CH}_{3}\right) ;{ }^{13} \mathrm{C} \mathrm{NMR}(75.5 \mathrm{MHz}$, $\left.\mathrm{CDCl}_{3}\right) \delta_{\mathrm{C}} 174.25(\mathrm{C}-1), 34.05(\mathrm{C}-2), 24.88(\mathrm{C}-3), 28.75(\mathrm{C}-4), 28.64(\mathrm{C}-5), 28.64(\mathrm{C}-6)$, $29.00(\mathrm{C}-7), 18.69$ ( $\delta$-shift - 0.020, C-8, C-14), $80.35(\gamma$-shift - 0.020, C-9), $74.53(\beta$-shift $-0.042, \mathrm{C}-10), 9.49\left(t, J_{\mathrm{CD}}=20.6 \mathrm{~Hz}, \alpha\right.$-shift $\left.-0.231, \mathrm{C}-11\right), 74.40$ ( $\beta$-shift $-0.041, \mathrm{C}-$ 12), 80.51 ( $\gamma$-shift - 0.020, C-13), 28.46 (C-15), 31.07 (C-16), 22.21 (C-17), 13.98 (C18), 51.43 (-OMe); El-MS: $m / z 260$ ([M-31] $\left.]^{\dagger}\right), 248\left([\mathrm{M}-43]^{+}\right), 235,218,202,188,174$, $160,146,134,120,106,92,79,59,41$.

\subsubsection{Methyl (11S)-[11- $\left.{ }^{2} \mathrm{H}_{1}\right]$ octadeca-9Z,12Z-dienoate ${ }^{173}$}

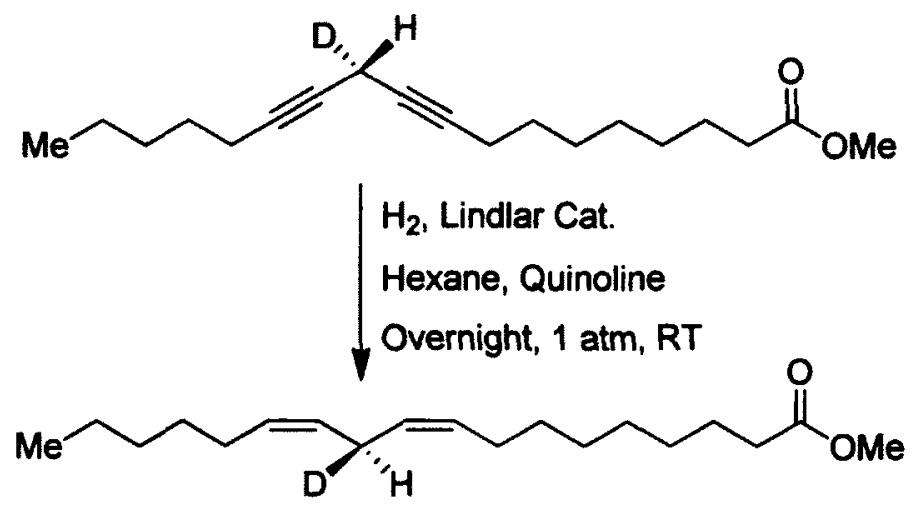

The title compound was obtained in a similar manner to that described in 6.2.1.20, from methyl $(11 S)$-[11- $\left.{ }^{2} \mathrm{H}_{1}\right]$-octadeca-9,12-diynoate $(202 \mathrm{mg}, 0.7 \mathrm{mmol})$ as a paleyellowish clear oil (195 mg, $95 \%$ ). $R_{\mathrm{f}} 0.44$ (EtOAc:hexane, 1:9); The spectral data was 
similar to that reported above wih the non-deuterio parent compound (see Section 6.2.1.16). IR (film): v 3009, 2928, 2856 (C-H), 2163 (C-D), 1743 (C=O), 1463, 1436, $1363,1246,1197,1172,1016,853 \mathrm{~cm}^{-1} ;{ }^{1} \mathrm{H}$ NMR $\left(300 \mathrm{MHz}, \mathrm{CDCl}_{3}\right) \delta_{\mathrm{H}} 5.45-5.28(m, 4$ $\mathrm{H}, 4 \times \mathrm{HC}=\mathrm{C}), 3.67\left(s, 3 \mathrm{H}, \mathrm{OCH}_{3}\right), 2.75(b r t, J=6.8 \mathrm{~Hz}, 1 \mathrm{H}, \mathrm{C}-11), 2.30(t, J=7.5$ $\left.\mathrm{Hz}, 2 \mathrm{H}, \mathrm{CH}_{2} \mathrm{CO}_{2} \mathrm{Me}\right), 2.05(m, 4 \mathrm{H}, \mathrm{C}-8, \mathrm{C}-14), 1.62(t t, J=7.5,7.1 \mathrm{~Hz}, 2 \mathrm{H}$, $\left.\mathrm{CH}_{2} \mathrm{CH}_{2} \mathrm{CO}_{2} \mathrm{Me}\right), 1.42-1.21\left(m, 14 \mathrm{H}, 7 \times \mathrm{CH}_{2}\right), 0.89\left(t, J=6.7 \mathrm{~Hz}, 3 \mathrm{H}, \mathrm{CH}_{3}\right) ;{ }^{13} \mathrm{C} \mathrm{NMR}$ $\left(75 \mathrm{MHz}, \mathrm{CDCl}_{3}\right) \delta_{\mathrm{C}} 174.32(\mathrm{C}-1), 34.11(\mathrm{C}-2), 24.95(\mathrm{C}-3), 29.11(\mathrm{C}-4), 29.17(\mathrm{C}-5)$, 29.13 (C-6), 29.60 (C-7), 27.20 ( $\delta$-shift 0.001, C-8), 130.07 ( $\gamma$-shift 0.010, C-9), 127.998 ( $\beta$-shift - 0.060, C-10), $25.32\left(t, J_{\mathrm{CD}}=19.3 \mathrm{~Hz}, \alpha\right.$-shift - 0.316, C-11), 127.86 ( $\beta$-shift 0.060, C-12), 130.24 ( $\gamma$-shift 0.009, C-13), 27.22 ( $\delta$-shift 0.002, C-14), 29.37 (C-15), 31.54 (C-16), 22.59 (C-17), 14.08 (C-18), 51.45 (-OMe); EI-MS: m/z 295 ([M] $\left.{ }^{+}\right), 280$ $\left([\mathrm{M}-15]^{+}\right), 264\left([\mathrm{M}-31]^{+}\right), 235,221\left([\mathrm{M}-74]^{+}\right), 207,192,179,165,150,136,124,110$, $96,82,68,55,41$.

\subsubsection{Methyl (11R)-[11- $\left.{ }^{2} \mathrm{H}_{1}\right]$ octadeca-9Z,12Z-dienoate}

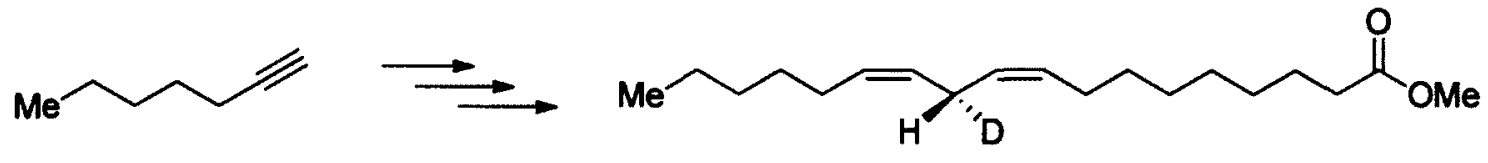

The title compound was synthesized in $4 \%$ overall yield from 1-heptyne using the same procedures as described for the synthesis of the corresponding $(S)$-enantiomer. The chiral starting material was prepared by the reduction of $\left[1-{ }^{2} \mathrm{H}_{1}\right] 2$-octynal using a solution of $(+)-(R)$-Alpine-Borane ${ }^{\circledR} R_{\mathrm{f}} 0.44$ (EtOAc:hexane, 1:9); The analytical data for the title compound was essentially identical to that obtained for the corresponding (S)enantiomer and similar to that reported above for the non-deuterio parent compound (see 
Section 6.2.1.16). IR (film): $\vee$ 3009, 2928, 2856 (C-H), 2158 (C-D), 1743 (C=O), 1463, $1436,1363,1246,1197,1172,1016,852 \mathrm{~cm}^{-1} ;{ }^{1} \mathrm{H}$ NMR $\left(300 \mathrm{MHz}, \mathrm{CDCl}_{3}\right) \delta_{\mathrm{H}} 5.45-5.27$ $(m, 4 \mathrm{H}, 4 \times \mathrm{HC}=\mathrm{C}), 3.66\left(s, 3 \mathrm{H}, \mathrm{OCH}_{3}\right), 2.74(b r t, J=6.6 \mathrm{~Hz}, 1 \mathrm{H}, \mathrm{C}-11), 2.30(t, J=$ $\left.7.5 \mathrm{~Hz}, 2 \mathrm{H}, \mathrm{CH}_{2} \mathrm{CO}_{2} \mathrm{Me}\right), 2.04(m, 4 \mathrm{H}, \mathrm{C}-8, \mathrm{C}-14), 1.70-1.54(t t, J=7.5,6.9 \mathrm{~Hz}, 2 \mathrm{H}$, $\left.\mathrm{CH}_{2} \mathrm{CH}_{2} \mathrm{CO}_{2} \mathrm{Me}\right), 1.41-1.20\left(m, 14 \mathrm{H}, 7 \times \mathrm{CH}_{2}\right), 0.89\left(t, J=6.6 \mathrm{~Hz}, 3 \mathrm{H}, \mathrm{CH}_{3}\right) ;{ }^{13} \mathrm{C} \mathrm{NMR}$ $\left(75 \mathrm{MHz}, \mathrm{CDCl}_{3}\right) \delta_{\mathrm{C}} 174.27$ (C-1), 34.08 (C-2), 24.93 (C-3), 29.09 (C-4), 29.15 (C-5), 29.11 (C-6), 29.58 (C-7), 27.18 ( $\delta$-shift - 0.024, C-8), 130.03 ( $\gamma$-shift - 0.029, C-9), $127.97\left(\beta\right.$-shift - 0.086, C-10), $25.30\left(t, J_{\mathrm{CD}}=19.3 \mathrm{~Hz}, \alpha\right.$-shift - 0.339, C-11), $127.83(\beta-$ shift - 0.086, C-12), 130.20 ( $\gamma$-shift - 0.030, C-13), 27.19 ( $\delta$-shift - 0.023, C-14), 29.34 (C-15), 31.52 (C-16), 22.57 (C-17), 14.05 (C-18), 51.41 (-OMe); EI-MS: m/z 295 ([M] $\left.{ }^{+}\right)$, $280\left([\mathrm{M}-15]^{+}\right), 264$ ([M-31] $\left.]^{+}\right), 235,221\left([\mathrm{M}-74]^{+}\right), 207,192,179,165,150,136,124$, $110,96,82,68,55,41$.

\subsubsection{Chiral $\left[11-{ }^{2} H_{1}\right]$-Linoleates (via $\Delta^{12}$-Desaturation of Chiral [11- ${ }^{2} \mathbf{H}_{1}$ ]-Oleates}

\subsubsection{2-(8-Bromooctyl-1-oxy)tetrahydropyran ${ }^{196}$}

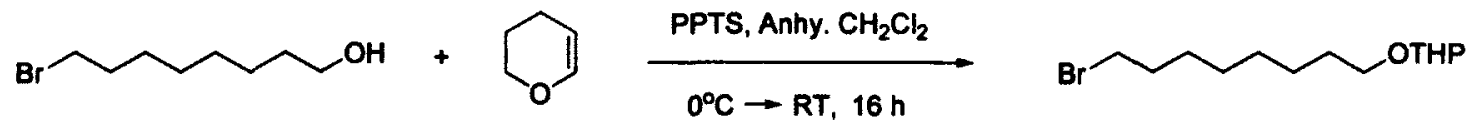

3,4-Dihydro-2H-pyran $(2.7 \mathrm{~mL}, 29.9 \mathrm{mmol})$ and pyridinium $p$-toluenesulfonate

$(0.9 \mathrm{~g}, 15 \mathrm{~mol} \%)$ was added to a stirred solution of 8-bromooctan-1-ol $(5.0 \mathrm{~g}, 23.9$ mmol) in anhydrous $\mathrm{CH}_{2} \mathrm{Cl}_{2}(116 \mathrm{~mL})$ at $0{ }^{\circ} \mathrm{C}$. After stirring the reaction mixture for another 30 minutes at $0{ }^{\circ} \mathrm{C}$, it was slowly warmed up to room temperature and allowed to stir for another $16 \mathrm{~h}$. During stirring, the reaction mixture turned into light brownish in 
color. The reaction mixture was poured into a solution of saturated $\mathrm{NaHCO}_{3}(120 \mathrm{~mL})$ and extracted with $\mathrm{CH}_{2} \mathrm{Cl}_{2}(40 \mathrm{~mL})$. The aqueous layer was re-extracted using $\mathrm{CH}_{2} \mathrm{Cl}_{2}$ (3 $x 60 \mathrm{~mL}$ ) and the combined organic layers were worked up in the normal manner to give a crude THP ether that was purified by flash chromatography using EtOAc:hexane (1:13) as eluent. A pale yellow oil was obtained (7.0 g, $99.8 \%$ ). $R_{\mathrm{f}} 0.31$ (EtOAc:hexane, 1:9); IR (film): v 2935, 2856 (C-H), 1465, 1454, 1440, 1352, 1200, 1135, 1120, 1078, 1034, $987,869 \mathrm{~cm}^{-1} ;{ }^{1} \mathrm{H}$ NMR $\left(300 \mathrm{MHz}, \mathrm{CDCl}_{3}\right) \delta_{\mathrm{H}} 4.57(t, J=3.6 \mathrm{~Hz}, 1 \mathrm{H}$, OCHO), 3.92$3.81(m, 1 \mathrm{H}, \mathrm{CHO}), 3.78-3.67(m, 1 \mathrm{H}, \mathrm{CHO}), 3.56-3.44(m, 1 \mathrm{H}, \mathrm{CHO}), 3.44-3.32(m, 1$ $\mathrm{H}, \mathrm{CHO}$ ), $3.40\left(t, J=6.9 \mathrm{~Hz}, 2 \mathrm{H}, \mathrm{CH}_{2} \mathrm{Br}\right.$ ), 1.85 (overlapping $t d$ and $t t J=7.1,7.1 \mathrm{~Hz}, 4$ H, C-7, C-2'), 1.65-1.46 (m, $\left.6 \mathrm{H}, 3 \times \mathrm{CH}_{2}\right), 1.46-1.26\left(m, 8 \mathrm{H}, 4 \times \mathrm{CH}_{2}\right) ;{ }^{13} \mathrm{C}$ NMR (75 $\left.\mathrm{MHz}, \mathrm{CDCl}_{3}\right) \delta_{\mathrm{C}} 62.32(\mathrm{C}-1), 29.67(\mathrm{C}-2), 26.11(\mathrm{C}-3), 28.67$ (C-4), $28.08(\mathrm{C}-5), 29.23$ (C-6), 32.77 (C-7), 33.92 (C-8), 98.83 (C-1'), 30.76 (C-2'), 19.68 (C-3'), 25.49 (C-4'), $67.56\left(\mathrm{C}-5^{\prime}\right)$.

\subsubsection{2-(8-Iodooctyl-1-oxy)tetrahydropyran ${ }^{196}$}

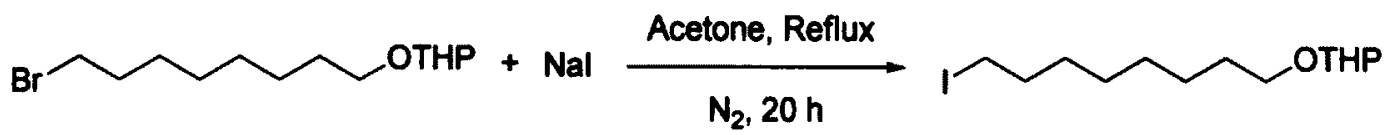

To a stirred solution of 2-(8-bromooctyl-1-oxy)tetrahydropyran (7.06 g, 24.1 $\mathrm{mmol})$ in dry acetone $(250 \mathrm{~mL})$ was added $\mathrm{Nal}(7.21 \mathrm{~g}, 48.1 \mathrm{mmol})$ and the reaction mixture was refluxed for $20 \mathrm{~h}$. A white precipitate, that formed was removed by filtration and the solvent was evaporated. The residue was diluted with $\mathrm{CH}_{2} \mathrm{Cl}_{2}(140 \mathrm{~mL})$, transferred to a separatory funnel and extracted with water $(160 \mathrm{~mL})$. The aqueous layer was re-extracted with $\mathrm{CH}_{2} \mathrm{Cl}_{2}(2 \times 140 \mathrm{~mL})$ and the organic layers were combined. The 
combined organic layers were worked up as usual to afford a crude sample, that was purified by flash chromatography using ethyl acetate-hexane (1:13) as eluent to furnish pure 2-(8-iodooctyl-1-oxy)tetrahydropyran as a pale yellow non-viscous liquid $(7.26 \mathrm{~g}$, 89 \%). $R_{\mathrm{f}} 0.33$ (EtOAc:hexane, 1:9); IR (film): $v$ 2932, 2855 (C-H), 1464, 1453, 1440, $1352,1200,1135,1120,1078,1034,984,869 \mathrm{~cm}^{-1} ;{ }^{1} \mathrm{H}$ NMR $\left(300 \mathrm{MHz}, \mathrm{CDCl}_{3}\right) \delta_{\mathrm{H}} 4.57$ $(t, J=3.5 \mathrm{~Hz}, 1 \mathrm{H}, \mathrm{OCHO}), 3.92-3.81(m, 1 \mathrm{H}, \mathrm{CHO}), 3.78-3.67(m, 1 \mathrm{H}, \mathrm{CHO}), 3.56-$ $3.44(m, 1 \mathrm{H}, \mathrm{CHO}), 3.44-3.32(m, 1 \mathrm{H}, \mathrm{CHO}), 3.18\left(t, J=7.0 \mathrm{~Hz}, 2 \mathrm{H}, \mathrm{CH}_{2} \mathrm{I}\right), 1.81$ (Overlapping $t d$ and $t t=7.1,7.1 \mathrm{~Hz}, 4 \mathrm{H}, 2 \times \mathrm{CH}_{2}, \mathrm{C}-7, \mathrm{C}-2$ '), $1.66-1.46(m, 6 \mathrm{H}, 3 \times$ $\left.\mathrm{CH}_{2}\right), 1.45-1.25\left(m, 8 \mathrm{H}, 4 \times \mathrm{CH}_{2}\right):{ }^{13} \mathrm{C} \mathrm{NMR}\left(75 \mathrm{MHz}, \mathrm{CDCl}_{3}\right) \delta_{\mathrm{C}} 62.35(\mathrm{C}-1), 30.43(\mathrm{C}-$ 2), 26.14 (C-3), 28.46 (C-4), 29.69 (C-5), 29.23 (C-6), 33.52 (C-7), 7.21 (C-8), 98.85 (C1'), 30.79 (C-2'), 19.71 (C-3'), 25.51 (C-4'), 67.59 (C-5'); EI-MS: m/z 339 ([M-1] $\left.]^{+}\right), 267$ ([M-73] $\left.]^{+}\right), 239$ (- OTHP, [M-101] $\left.]^{+}\right), 212,197,183,169,155,127,101,85$ ([OTHP] $\left.]^{+}\right)$, $69,55,41$.

\subsubsection{1-[1,1- $\left.{ }^{2} \mathbf{H}_{2}\right]-$ Octanol ${ }^{179}$}

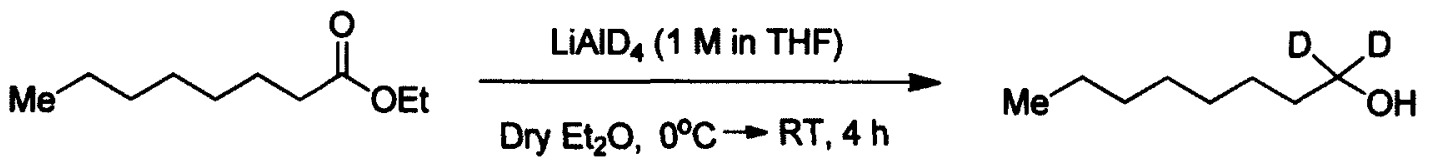

A $1.0 \mathrm{M}$ solution of lithium aluminium deuteride in anhydrous THF $(50 \mathrm{ml}, 50$ mmol) was added dropwise to a stirred solution of ethyl octanoate $(7.83 \mathrm{~g}, 45.5 \mathrm{mmol})$ in anhydrous diethyl ether $(50 \mathrm{~mL})$ at $0^{\circ} \mathrm{C}$. The reaction mixture was stirred at $0{ }^{\circ} \mathrm{C}$ for $2 \mathrm{~h}$ and then at room temperature for another $3 \mathrm{~h}$. Ethyl acetate $(4 \mathrm{~mL})$ was added to quench any excess of reducing agent, THF was removed by rotary evaporation. The solids were suspended in ether $(200 \mathrm{~mL})$ at $0{ }^{\circ} \mathrm{C}$ to which cold water $(120 \mathrm{~mL})$ was added in small 
portions. The $\mathrm{pH}$ of the solution was adjusted to 1 using $2 \mathrm{M} \mathrm{HCl}$ and the organic layer was separated. The aqueous layer was extracted with ether $(2 \times 90 \mathrm{~mL})$ and the combined organic layers were treated in the usual manner to obtain the title compound as a colorless oil ( $5.95 \mathrm{~g}, 99 \%$ ). bp $194-197{ }^{\circ} \mathrm{C} ; R_{\mathrm{f}} 0.23$ (EtOAc:hexane, 1:3); The spectral data of this material was similar to that reported in the literature for the non-deuterated parent compound ${ }^{2}$. IR (film): v 3331 (O-H), 2957, 2926, 2856 (C-H), 2197, 2093 (C-D), $1466,1379,1297,1134,1093,968,723 \mathrm{~cm}^{-1} ;{ }^{1} \mathrm{H}$ NMR $\left(300 \mathrm{MHz}, \mathrm{CDCl}_{3}\right) \delta_{\mathrm{H}} 1.54$ (br t, $\left.J=6.9 \mathrm{~Hz}, 2 \mathrm{H}, \mathrm{CH}_{2} \mathrm{CD}_{2} \mathrm{OH}\right), 1.45(s, 1 \mathrm{H}, \mathrm{OH}), 1.38-1.21\left(m, 10 \mathrm{H}, 5 \mathrm{x} \mathrm{CH}_{2}\right), 0.87(t, J$ $\left.=6.8 \mathrm{~Hz}, 3 \mathrm{H}, \mathrm{CH}_{3}\right) ;{ }^{13} \mathrm{C} \mathrm{NMR}\left(75.5 \mathrm{MHz}, \mathrm{CDCl}_{3}\right) \delta_{\mathrm{C}} 62.26\left(p, J_{\mathrm{CD}}=21.5 \mathrm{~Hz}, \alpha-\right.$ shift $0.370, \mathrm{C}-1), 32.58$ ( $\beta$-shift - 0.063, C-2), $25.70(\gamma$-shift - 0.028, C-3), $29.40(\delta$-shift 0.027, C-4), 29.27 (C-5), 31.81 (C-6), 22.64 (C-7), 14.06 (C-8); TMS Derivative EI-MS: m/z $204\left([\mathrm{M}]^{+}\right), 189\left(\left[\mathrm{Me}\left(\mathrm{CH}_{2}\right)_{6} \mathrm{CD}_{2} \mathrm{OSiMe}_{2}\right]^{+},[\mathrm{M}-15]^{+}\right), 131\left(\left[\mathrm{Me}\left(\mathrm{CH}_{2}\right)_{6} \mathrm{CD}_{2} \mathrm{O}\right]^{+},[\mathrm{M}-73]^{+}\right)$, $117,105,103,91,75,70,55,43,41$.

\subsubsection{4 $1-\left[1-{ }^{2} \mathbf{H}_{1}\right]$ Octanal ${ }^{183}$}

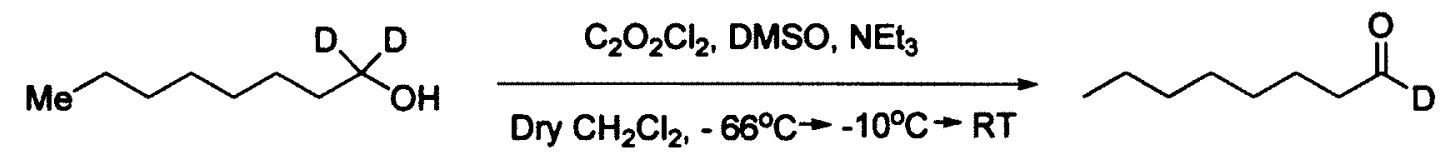

A three-neck $500 \mathrm{ml}$ round-botton flask, equipped with a magnetic stir bar was purged with $\mathrm{N}_{2}$, prior to the addition of oxalyl chloride $(11.1 \mathrm{~mL}, 127.2 \mathrm{mmol})$. After diluting the reagent with dry $\mathrm{CH}_{2} \mathrm{Cl}_{2}\left(90 \mathrm{~mL}\right.$ ), the solution was cooled to $-72{ }^{\circ} \mathrm{C}$ (external temperature) and anhydrous DMSO (18.1 $\mathrm{mL}, 254.3 \mathrm{mmol})$ was added in portions. The reaction was strongly exothermic and turned viscous after the DMSO addition was complete. The reaction mixture was stirred for additional 5-10 minutes after which was 
added a solution of 1-[1,1- $\left.{ }^{2} \mathrm{H}_{2}\right]$-octanol $(8.41 \mathrm{~g}, 63.6 \mathrm{mmol})$ in dry $\mathrm{CH}_{2} \mathrm{Cl}_{2}(40 \mathrm{~mL})$ dropwise for 30 minutes at -65 to $-70^{\circ} \mathrm{C}$. Soon after the addition of the alcohol the reaction mixture become cloudy and was stirred for additional 15 minutes before being warmed to $-12{ }^{\circ} \mathrm{C}$ (external temperature) and stirred for another $3 \mathrm{~h} . \mathrm{NEt}_{3}(53.1 \mathrm{~mL}$, $381.5 \mathrm{mmol}$ ) was added and the resultant yellowish reaction mixture was stirred for an additional 40 minutes before being allowed to warm to room temperature and stirred for another $1.5 \mathrm{~h}$. The reaction was worked up by the addition of distilled water $(300 \mathrm{~mL})$ and the mixture was transferred to a separatory funnel. The organic layer was diluted with another $40 \mathrm{~mL}$ of $\mathrm{CH}_{2} \mathrm{Cl}_{2}$ before being collected. The aqueous layer was extracted several times with dichloromethane $(3 \times 120 \mathrm{~mL})$ and the combined organic layers treated in the normal manner to give the crude compound $(7.89 \mathrm{~g}, 96 \%)$. This material was purified by column chromatography on silica gel using ethyl acetate-hexane $(1: 19)$ as eluting solvent to provide the title compound as a pale yellow oil (4.52 g, $55 \%$ ). bp 170$172{ }^{\circ} \mathrm{C} ; R_{\mathrm{f}} 0.44$ (EtOAc:hexane, 1:19); The spectral data of the product was similar to that reported in the literature for the non-deuterated parent compound. ${ }^{3}$ IR (film): $v 2956$, 2926, 2856 (C-H), 2184, 2090 (C-D), 1731 (C=O), 1466, 1378, 1211, 1159, 1071, 967, $723 \mathrm{~cm}^{-1} ;{ }^{1} \mathrm{H}$ NMR $\left(300 \mathrm{MHz}, \mathrm{CDCl}_{3}\right) \delta_{\mathrm{H}} 2.41\left(t, J=7.4 \mathrm{~Hz}, 2 \mathrm{H}, \mathrm{CH}_{2} \mathrm{CDO}\right), 1.62(t t, J$ $\left.=7.4,7.3 \mathrm{~Hz}, 2 \mathrm{H}, \mathrm{CH}_{2} \mathrm{CH}_{2} \mathrm{CDO}\right), 1.39-1.20\left(m, 8 \mathrm{H}, 4 \times \mathrm{CH}_{2}\right), 0.87(t, J=6.8 \mathrm{~Hz}, 3 \mathrm{H}$, $\left.\mathrm{CH}_{3}\right) ;{ }^{13} \mathrm{C}$ NMR $\left(75.5 \mathrm{MHz}, \mathrm{CDCl}_{3}\right) \delta_{\mathrm{C}} 202.53\left(t, J_{\mathrm{CD}}=26.1 \mathrm{~Hz}\right.$, $\alpha$-shift - 0.357, C-1), $43.72\left(t,{ }^{2} J_{\mathrm{CD}}=3.6 \mathrm{~Hz}, \beta\right.$-shift - 0.102, C-2), $22.05(\gamma$-shift 0.038, C-3), 29.11 ( $\delta$-shift 0.053, C-4), 29.00 (C-5), 31.61 (C-6), 22.56 (C-7), 14.01 (C-8); EI-MS: $m / z 111$ $\left(\left[\mathrm{Me}\left(\mathrm{CH}_{2}\right)_{6} \mathrm{C}\right]^{+},[\mathrm{M}-18]^{+}\right), 101\left([\mathrm{M}-28]^{+}\right), 96\left([\mathrm{M}-33]^{+}\right), 84,82,69,58,55,45,41,39,30$. 


\subsubsection{5 (1R)-[1- $\left.{ }^{2} \mathrm{H}_{1}\right]-$ Octanol ${ }^{185,208}$}
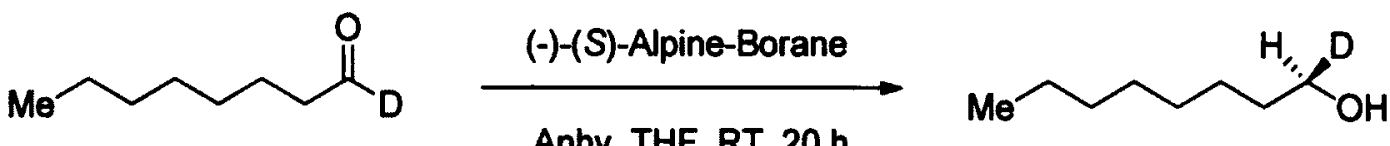

Anhy. THF, RT, $20 \mathrm{~h}$

A 3-neck round-bottom flask equipped with a reflux condenser, a one-piece distillation apparatus, stir bar, and rubber septa was flushed with $\mathrm{N}_{2}$. The flask was then charged with monodeuterio-aldehyde $(6.53 \mathrm{~g}, 50.6 \mathrm{mmol})$ that was then diluted with anhydrous THF (120 mL). A $0.5 \mathrm{M}$ solution of (-)-(S)-Alpine-Borane in THF (152 mL, $75.8 \mathrm{mmol}$ ) was added slowly to the stirred solution at room temperature and the reaction mixture became light yellow in color. The reaction mixture was stirred at room temperature for another $40 \mathrm{~h}$. Excess Alpine-Borane reagent was quenched by the addition of excess acetaldehyde $(5 \mathrm{~mL})$ and the mixture was stirred for additional $1 \mathrm{~h}$ to ensure completion of the reaction. The rubber septum was quickly replaced with a glass stopper and the solvent (THF) as well as the byproduct $\alpha$-pinene was distilled under vacuum (at $15 \mathrm{~mm}$ and $0.02 \mathrm{~mm} \mathrm{Hg}$, respectively). To remove $\alpha$-pinene, slight warming $\left(40-45^{\circ} \mathrm{C}\right)$ of the reaction flask was required. The residue was taken up in anhydrous $\mathrm{Et}_{2} \mathrm{O}(150 \mathrm{~mL})$ whilst maintaining an inert atmosphere. The solution was cooled to $0{ }^{\circ} \mathrm{C}$ and ethanolamine $(4.6 \mathrm{~mL}, 75.8 \mathrm{mmol})$ was added dropwise to produce a white precipitate that was removed by filtration through celite 521 . The precipitate rinsed with additional portions of ether $(2 \times 25 \mathrm{~mL})$. The combined organic phase was treated in the normal manner to yield the crude compound that was then purified by column chromatography on silica gel using a gradient eluent system of EtOAc:hexane (4\%, $6 \%$, $8 \%$ and $10 \%)$. The title compound was obtained as a clear colorless liquid $(6.37 \mathrm{~g}, 96$ 
\%). bp 194-197 ${ }^{\circ} \mathrm{C} ; R_{\mathrm{f}} 0.28$ (EtOAc:hexane, 1:3); and the spectral data of the compound was similar to that reported above for the dideuterated compound (see Section 6.2.3.3) as well as with that of the non-deuterated parent compound reported in the literature ${ }^{3}$. IR (film): v 3345 (O-H), 2926, 2858 (C-H), 2169 (C-D), 1468, 1452, 1411, 1326, 1300, $1166,1040,1003 \mathrm{~cm}^{-1}$; ${ }^{1} \mathrm{H}$ NMR $\left(300 \mathrm{MHz}, \mathrm{CDCl}_{3}\right) \delta_{\mathrm{H}} 3.62(t t, J=6.6,1.4 \mathrm{~Hz}, 1 \mathrm{H}$, $\mathrm{CHDOH}), 1.55$ (Overlapping $\left.t d, J=6.8,6.6 \mathrm{~Hz}, 2 \mathrm{H}, \mathrm{CH}_{2} \mathrm{CHDOH}\right), 1.43(s, 1 \mathrm{H}, \mathrm{OH}$ ), 1.40-1.23 (m, $\left.10 \mathrm{H}, 5 \times \mathrm{CH}_{2}\right), 0.88\left(t, J=6.7 \mathrm{~Hz}, 3 \mathrm{H}, \mathrm{CH}_{3}\right) ;{ }^{13} \mathrm{C}$ NMR $(75.5 \mathrm{MHz}$, $\left.\mathrm{CDCl}_{3}\right) \delta_{\mathrm{C}} 62.68\left(t, J_{\mathrm{CD}}=21.7 \mathrm{~Hz}, \alpha\right.$-shift 0.046, C-1), 32.71 ( $\beta$-shift $\left.0.063, \mathrm{C}-2\right), 25.73$ $(\gamma$-shift 0.004, C-3), 29.41 ( $\delta$-shift 0.030, C-4), 29.28 (C-5), 31.82 (C-6), 22.66 (C-7), 14.09 (C-8); TMS Derivative EI-MS: $m / z 203\left([\mathrm{M}]^{+}\right), 188\left(\left[\mathrm{Me}\left(\mathrm{CH}_{2}\right)_{6} \mathrm{CHDOSiMe}_{2}\right]^{+}\right.$, $\left.[\mathrm{M}-15]^{+}\right), 130\left(\left[\mathrm{Me}\left(\mathrm{CH}_{2}\right)_{6} \mathrm{CHDO}\right]^{+},[\mathrm{M}-73]^{+}\right), 116,104,102,90,75,70,55,43,41$.

\subsubsection{6 (1R)-1-(p-Tolylsulfonyloxy)-[1- $\left.{ }^{2} \mathbf{H}_{1}\right]-\operatorname{Octane}^{179}$}

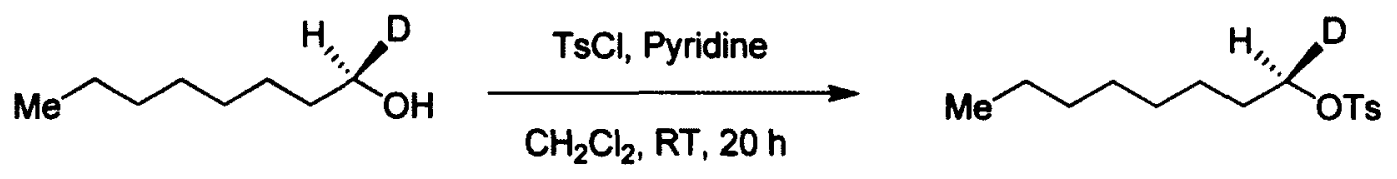

To a solution of $(1 R)-\left[1-{ }^{2} \mathrm{H}_{1}\right]$-octanol $(3.57 \mathrm{~g}, 27.2 \mathrm{mmol})$ and $p$-toluenesulfonyl chloride $(5.71 \mathrm{~g}, 30.0 \mathrm{mmol})$ in dry $\mathrm{CH}_{2} \mathrm{Cl}_{2}(55 \mathrm{~mL})$ was added dropwise anhydrous pyridine ( $3.1 \mathrm{~mL}, 38.1 \mathrm{mmol}$ ) at room temperature. The reaction was stirred for $24 \mathrm{~h}$ and then poured into a saturated solution of $\mathrm{NH}_{4} \mathrm{Cl}(50 \mathrm{~mL})$. The organic layer was diluted with $\mathrm{CH}_{2} \mathrm{Cl}_{2}(25 \mathrm{~mL})$ that was separated and washed successively with saturated $\mathrm{NH}_{4} \mathrm{Cl}$ $(3 \times 50 \mathrm{~mL})$ and saturated $\mathrm{NaCl}(2 \times 50 \mathrm{~mL})$. Filtration through anhydrous $\mathrm{Na}_{2} \mathrm{SO}_{4}$ followed by removal of solvent in vacuo afforded the crude compound that was purified by flash chromatography using ethyl acetate-hexane (1:9) as eluent to yield the pure 
tosylate $(6.26 \mathrm{~g}, 81 \%) . R_{\mathrm{f}} 0.44$ (EtOAc:hexane, 1:4); and the spectral data of the compound was similar to that reported in the literature for the non-deuterated parent compound. IR (film): $v$ 2928, $2858(\mathrm{C}-\mathrm{H}), 1596(\mathrm{C}=\mathrm{C}, \mathrm{Ar}), 1455,1366,1190,1176$, $1098,943,814 \mathrm{~cm}^{-1} ;{ }^{1} \mathrm{H}$ NMR $\left(300 \mathrm{MHz}, \mathrm{CDCl}_{3}\right) \delta_{\mathrm{H}} 7.79(d, J=8.2 \mathrm{~Hz}, 2 \mathrm{H}, \mathrm{C} \underline{\underline{o}}-\mathrm{Ph})$, $7.34(d, J=8.2 \mathrm{~Hz}, 2 \mathrm{H}, \mathrm{C} \underline{m}-\mathrm{Ph}), 4.00(t, J=6.5 \mathrm{~Hz}, 1 \mathrm{H}$, CHDOTs$), 2.45(s, 3 \mathrm{H}$, $\left.\mathrm{CH}_{3} \mathrm{Ar}\right), 1.62\left(t d, J=6.7,6.5 \mathrm{~Hz}, 2 \mathrm{H}, \mathrm{CH}_{2} \mathrm{CHDOTs}\right), 1.35-1.14\left(m, 10 \mathrm{H}, 5 \times \mathrm{CH}_{2}\right)$, $0.87\left(t, J=6.9 \mathrm{~Hz}, 3 \mathrm{H}, \mathrm{CH}_{3}\right) ;{ }^{13} \mathrm{C} \mathrm{NMR}\left(75 \mathrm{MHz}, \mathrm{CDCl}_{3}\right) \delta_{\mathrm{C}} 70.39\left(t, J_{\mathrm{CD}}=22.6 \mathrm{~Hz}, \mathrm{C}-\right.$ 1), 28.68 (C-2), 25.28 (C-3), 29.03 (C-4), 28.86 (C-5), 31.68 (C-6), 22.58 (C-7), 14.06 (C-8), 144.61 (C $\underline{i p s o}$ - Ph), 129.79 (C $\underline{\mathrm{C}}-\mathrm{Ph}), 127.87(\mathrm{C} \underline{m}-\mathrm{Ph}), 133.24(\mathrm{C} \underline{\underline{p}}-\mathrm{Ph}), 21.62$ $\left(\mathrm{CH}_{3}-\mathrm{Ph}\right)$; EI-MS: $m / z$ 173, 155, 139, 113, 107, 91, 84, 70, 65, 56, 41.

\subsubsection{7 (3S)-[3- $\left.{ }^{2} \mathrm{H}_{1}\right]-$ Dec-1-yne ${ }^{173}$}

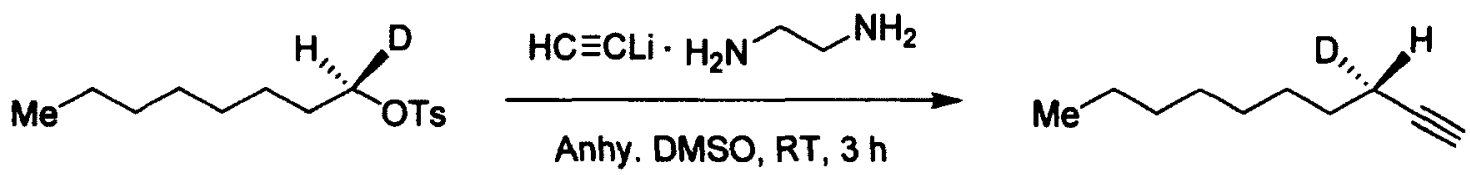

A solution of $(1 R)-1-(p$-tolylsulfonyloxy $)-\left[1-{ }^{2} \mathrm{H}_{1}\right]$-octane $(6.17 \mathrm{~g}, 21.6 \mathrm{mmol})$ in anhydrous DMSO $(20 \mathrm{~mL})$ was added dropwise to a stirred suspension of lithium acetylide-ethylenediamine complex $(2.59 \mathrm{~g}, 28.1 \mathrm{mmol})$ in DMSO $(20 \mathrm{~mL})$ at room temperature. The reaction mixture was stirred for $3 \mathrm{~h}$. The reaction was worked up by pouring into an ice-water mixture $(100 \mathrm{~mL})$ and $\mathrm{pH}$ of the solution was adjusted to 1 using $1.0 \mathrm{M} \mathrm{HCl}$. The aqueous layer was extracted with diethyl ether $(3 \times 40 \mathrm{~mL})$ and the combined organic phase was worked up in the usual manner to yield the crude compound. This material was purified by flash chromatography using $\mathrm{Et}_{2} \mathrm{O}$-pentane (1:32) as eluent to obtain the pure title compound as a light yellowish oil $(1.17 \mathrm{~g}, 39 \%)$. 
bp $174-178{ }^{\circ} \mathrm{C} ; R_{\mathrm{f}} 0.83$ (Pure $\mathrm{Et}_{2} \mathrm{O}$ ); and the spectral data of the title compound was similar to that obtained using the authentic non-deuterated parent compound. IR (film): $v$ 3314 (HC $\equiv \mathrm{C}), 2956,2925,2855$ (C-H), 2164 (C-D), 2120 (C $\equiv \mathrm{C}), 1465,1378,1299$, $1060,967,724 \mathrm{~cm}^{-1} ;{ }^{1} \mathrm{H}$ NMR $\left(300 \mathrm{MHz}, \mathrm{CDCl}_{3}\right) \delta_{\mathrm{H}} 2.17(m, 1 \mathrm{H}, \mathrm{CHDC} \equiv \mathrm{C}), 1.93(d$, $J=2.6 \mathrm{~Hz}, 1 \mathrm{H}, \mathrm{HC} \equiv \mathrm{C}), 1.51\left(t d, J=7.0,7.0 \mathrm{~Hz}, 2 \mathrm{H}, \mathrm{CH}_{2} \mathrm{CHD}\right), 1.46-1.35(m, 2 \mathrm{H}$, $\left.\mathrm{CH}_{2}, \mathrm{C}-9\right), 1.28\left(m, 8 \mathrm{H}, 4 \times \mathrm{CH}_{2}, \mathrm{C}-5, \mathrm{C}-6, \mathrm{C}-7, \mathrm{C}-8\right), 0.89\left(t, J=6.7 \mathrm{~Hz}, 3 \mathrm{H}, \mathrm{CH}_{3}\right) ;{ }^{13} \mathrm{C}$ NMR (75.5 MHz, $\left.\mathrm{CDCl}_{3}\right) \delta_{\mathrm{C}} 68.03$ ( $\gamma$-shift 0.005, C-1), 84.77 ( $\beta$-shift 0.003, C-2), 18.13 $\left(t, J_{\mathrm{CD}}=20.0 \mathrm{~Hz}, \alpha\right.$-shift $\left.-0.283, \mathrm{C}-3\right), 28.43(\beta$-shift $-0.091, \mathrm{C}-4), 28.76(\gamma$-shift - 0.031, C-5), 29.10 ( $\delta$-shift 0.001, C-6), 29.19 (C-7), 31.85 (C-8), 22.67 (C-9), 14.09 (C-10); EIMS: $m / z 124\left(\left[\mathrm{CH}_{2}\left(\mathrm{CH}_{2}\right)_{5} \mathrm{CHDC} \equiv \mathrm{CH}\right]^{+},[\mathrm{M}-15]^{+}\right), 110\left(\left[\mathrm{CH}_{2}\left(\mathrm{CH}_{2}\right)_{4} \mathrm{CHDC} \equiv \mathrm{CH}\right]^{+}\right.$, $\left.[\mathrm{M}-29]^{+}\right), 96\left(\left[\mathrm{CH}_{2}\left(\mathrm{CH}_{2}\right)_{3} \mathrm{CHDC} \equiv \mathrm{CH}\right]^{+},[\mathrm{M}-43]^{+}\right), 82\left(\left[\mathrm{CH}_{2}\left(\mathrm{CH}_{2}\right)_{2} \mathrm{CHDC} \equiv \mathrm{CH}\right]^{+}\right.$, $\left.[\mathrm{M}-57]^{+}\right), 68\left(\left[\mathrm{CH}_{2} \mathrm{CH}_{2} \mathrm{CHDC} \equiv \mathrm{CH}\right]^{+},[\mathrm{M}-71]^{+}\right), 55,41$.

\subsubsection{2-((11S)-[11- $\left.{ }^{2} \mathrm{H}_{1}\right]-$ Octadeca-9-ynyl-1-oxy)tetrahydropyran ${ }^{196}$}

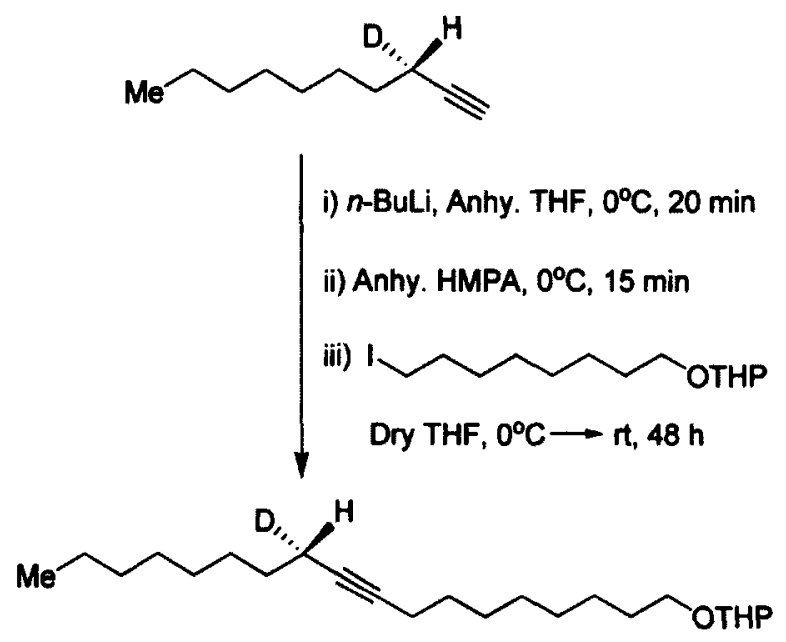

$n$-BuLi (2.5 M in hexanes; $4.2 \mathrm{~mL}, 10.4 \mathrm{mmol}$ ) was added dropwise to a stirred solution of $(3 S)-\left[3-{ }^{2} \mathrm{H}_{1}\right]$-decyne $(1.29 \mathrm{~g}, 9.3 \mathrm{mmol})$ in dry THF $(17 \mathrm{~mL})$ at $0{ }^{\circ} \mathrm{C}$. After 20 
min, HMPA $(17 \mathrm{~mL})$ was added to the reaction mixture and the solution changed from orange to wine red in color. After stirring the reaction mixture for another $15 \mathrm{~min}$, a solution of 2-(8-iodooctyl-1-oxy)tetrahydropyran ( $3.78 \mathrm{~g}, 11.1 \mathrm{mmol})$ in anhydrous THF $(17 \mathrm{~mL})$ was added, and the solution turned yellow-orange in color. The reaction mixture was kept at $0{ }^{\circ} \mathrm{C}$ for $1.5 \mathrm{~h}$ (during which the solution become much darker in color) and then allowed to stir at $\mathrm{rt}$ for another $48 \mathrm{~h}$. THF was removed in vacuo, the residue was diluted with $\mathrm{Et}_{2} \mathrm{O}(40 \mathrm{~mL})$ and the solution was poured into an ice-water mixture (140 $\mathrm{mL}$ ). The organic layer was separated and the aqueous layer was extracted with diethyl ether $(3 \times 90 \mathrm{~mL})$. The combined organic layers were worked up in the normal manner to afford the crude compound that was purified by column chromatography on silica gel using a gradient eluent system (pentane, $4 \% \mathrm{Et}_{2} \mathrm{O}$-pentane, $4 \%$ EtOAc-hexane, $8 \%$ EtOAc-hexane, $12 \%$ EtOAc-hexane and $16 \%$ EtOAc-hexane). The title compound was obtained as a pale yellowish liquid $\left(1.88 \mathrm{~g}, 58 \%\right.$ ). $R_{\mathrm{f}} 0.30$ (EtOAc: hexane, $1: 11$ ); and the spectral data of the compound was similar to that reported in the literature for the nondeuterated parent compound. ${ }^{1}$ IR (film): v 2930, 2856, 2804 (C-H), 2145 (C-D), 1465, $1456,1352,1201,1136,1121,1078,1034,990,906,870 \mathrm{~cm}^{-1} ;{ }^{1} \mathrm{H}$ NMR $(300 \mathrm{MHz}$, $\left.\mathrm{CDCl}_{3}\right) \delta_{\mathrm{H}} 4.57(t, J=3.6 \mathrm{~Hz}, 1 \mathrm{H}, \mathrm{OCHO}), 3.92-3.81(m, 1 \mathrm{H}, \mathrm{CHO}), 3.78-3.68(m, 1 \mathrm{H}$, CHO), 3.56-3.45 ( $m, 1 \mathrm{H}, \mathrm{CHO}$ ), 3.44-3.32 ( $m, 1 \mathrm{H}, \mathrm{CHO}$ ), 2.13 (Overlapping $t, J=6.3$ $\mathrm{Hz}, 3 \mathrm{H}, \mathrm{C}-8, \mathrm{C}-11), 1.93-1.19\left(m, 30 \mathrm{H}, 15 \times \mathrm{CH}_{2}\right), 0.88\left(t, J=6.7 \mathrm{~Hz}, 3 \mathrm{H}, \mathrm{CH}_{3}\right) ;{ }^{13} \mathrm{C}$ NMR (75.5 MHz, CDCl 3 ) $\delta_{C} 67.67$ (C-1), 30.80 (C-2), 29.15 (C-3), 29.40 (C-4), 29.08 (C-5), 28.85 (C-6), 29.15 (C-7), 18.76 (C-8), 80.19 (C-9), 80.28 (C-10), $18.47\left(t, J_{\mathrm{CD}}=\right.$ $19.6 \mathrm{~Hz}, \mathrm{C}-11), 29.15$ (C-12), 28.82 (C-13), 29.12 (C-14), 29.24 (C-15), 31.86 (C-16), 22.67 (C-17), 14.11 (C-18), 98.84 (C-1'), 29.75 (C-2'), 19.72 (C-3'), 25.52 (C-4'), 62.33 
(C-5); EI-MS: $\left.m / z 351\left(\left[\mathrm{M}^{+}\right), 308\left([\mathrm{M}-43]^{+}\right), 278\left([\mathrm{M}-73]^{+}\right), 266 \text { (-OTHP, [M-85] }\right]^{+}\right)$, $252\left(\left[\mathrm{CHDC} \equiv \mathrm{C}\left(\mathrm{CH}_{2}\right)_{8} \mathrm{OTHP}\right]^{+}\right), 237\left(\left[\mathrm{C} \equiv \mathrm{C}\left(\mathrm{CH}_{2}\right)_{8} \mathrm{OTHP}\right]^{++}\right), 224,195,153,136$, $124,110,101,96,85,67,55,41$.

\subsubsection{9 (11S)-[11- $\left.{ }^{2} \mathbf{H}_{1}\right]-$ Octadec-9-yn-1-ol ${ }^{196}$}

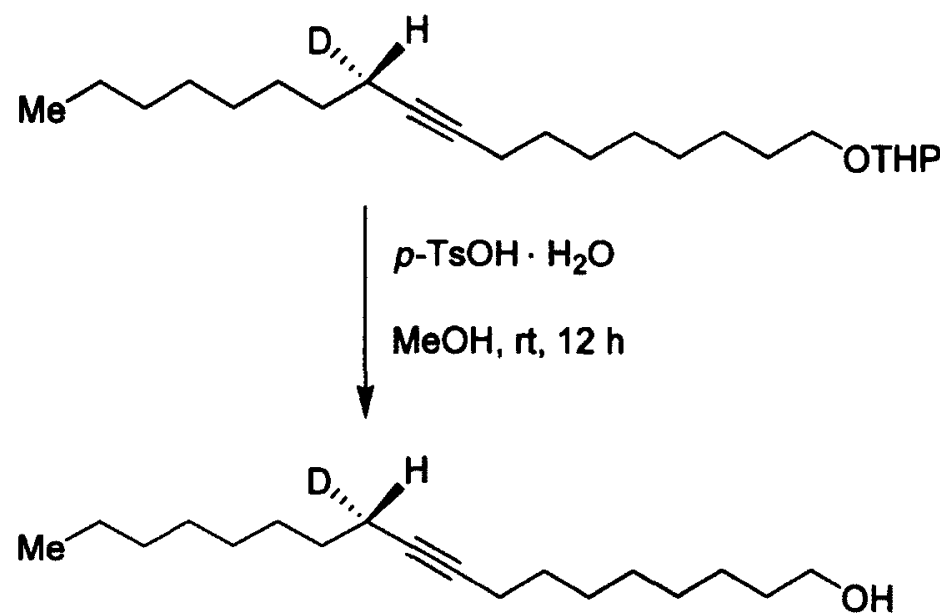

To a stirred solution of 2-((11S)-[11- $\left.{ }^{2} \mathrm{H}_{1}\right]$-octadeca-9-ynyl-1-oxy)tetrahydropyran $(1.78 \mathrm{~g}, 5.1 \mathrm{mmol})$ in methanol $(40 \mathrm{~mL})$ was added $p$-toluenesulfonic acid, monohydrate ( $97 \mathrm{mg}, 10 \mathrm{~mol} \%, 0.5 \mathrm{mmol}$ ) and allowed to stir for overnight at room temperature. Reaction mixture was poured into a saturated solution of $\mathrm{NaHCO}_{3}(15 \mathrm{~mL})$ and methanol was removed in vacuo. The residue was diluted with $\mathrm{Et}_{2} \mathrm{O}(30 \mathrm{~mL})$ and the organic layer was separated. The aqueous phase was re-extracted with $\mathrm{Et}_{2} \mathrm{O}(3 \times 20 \mathrm{~mL})$ and the collected organic layers were washed with brine $(3 \times 25 \mathrm{~mL})$ until the washings were found neutral with litmus paper. The solvent was removed in vacuo after drying over anhydrous $\mathrm{Na}_{2} \mathrm{SO}_{4}$ and concentrated to furnish the crude compound as a crystalline white solid (1.34 g, estimated yield $99 \%$ ). The crude compound was used for further reaction without purification. $R_{\mathrm{f}} 0.25$ (EtOAc:hexane, 1:3); and the spectral data of the compound 
was similar to that reported in the literature for the non-deuterated parent compound. ${ }^{1}$ IR (film): v $3342(\mathrm{O}-\mathrm{H}), 2928,2856(\mathrm{C}-\mathrm{H}), 2145$ (C-D), 1465, 1378, 1057, $723 \mathrm{~cm}^{-1} ;{ }^{1} \mathrm{H}$ NMR $\left(300 \mathrm{MHz}, \mathrm{CDCl}_{3}\right) \delta_{\mathrm{H}} 3.64\left(t, J=6.5 \mathrm{~Hz}, 2 \mathrm{H}, \mathrm{CH}_{2} \mathrm{OH}\right), 2.13$ (Overlapping $t, J=$ 6.3 Hz, 3 H, C-8, C-11), $1.56\left(t t, J=6.8,6.5 \mathrm{~Hz}, 2 \mathrm{H}, \mathrm{CH}_{2} \mathrm{CH}_{2} \mathrm{OH}\right.$ ), 1.45 (Overlapping $t t$, $J=6.6,6.3 \mathrm{~Hz}, 4 \mathrm{H}, \mathrm{C}-7, \mathrm{C}-12), 1.41-1.22\left(m, 18 \mathrm{H}, 9 \times \mathrm{CH}_{2}\right), 0.88(t, J=6.7 \mathrm{~Hz}, 3 \mathrm{H}$, $\left.\mathrm{CH}_{3}\right) ;{ }^{13} \mathrm{C} \mathrm{NMR}\left(75.5 \mathrm{MHz}, \mathrm{CDCl}_{3}\right) \delta_{\mathrm{C}} 63.05(\mathrm{C}-1), 32.78(\mathrm{C}-2), 25.70(\mathrm{C}-3), 29.33$ (C4), 29.07 (C-5), 28.85 (C-6), 29.14 (C-7), 18.744 (C-8), 80.17 (C-9), 80.28 (C-10), 18.46 $\left(t, J_{\mathrm{CD}}=19.8 \mathrm{~Hz}, \mathrm{C}-11\right), 29.14(\mathrm{C}-12), 28.78(\mathrm{C}-13), 29.14(\mathrm{C}-14), 29.23(\mathrm{C}-15), 31.85$ (C-16), 22.67 (C-17), 14.11 (C-18); EI-MS: m/z 339 ([M-1]+), 324 ([M-16]+), 296 ([M$44]+), 268,248,225,183,169,153,135,121,103,96,82,75,68,55,41$.

\subsubsection{0 (11S)-[11- $\left.{ }^{2} \mathbf{H}_{1}\right]-$ Octadec-9-ynoic acid ${ }^{196}$}

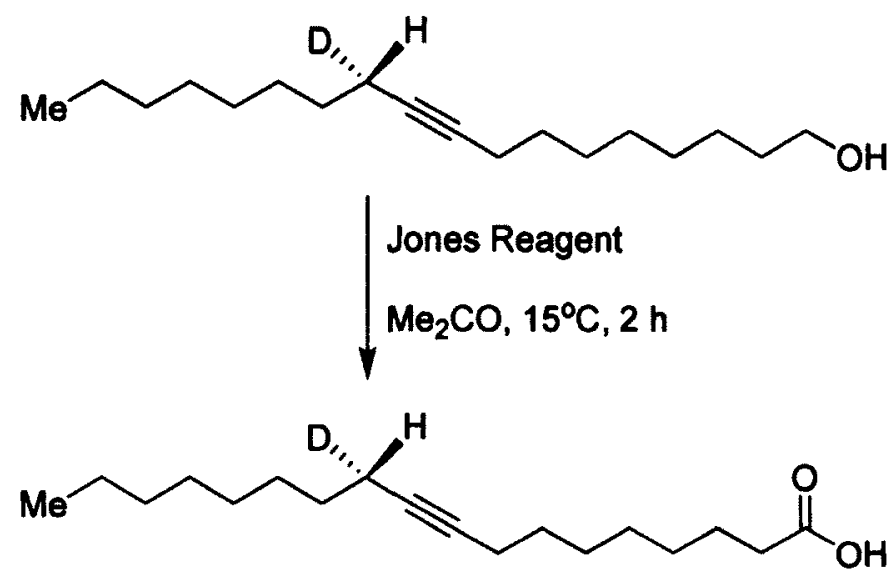

Freshly prepared Jones reagent $(4.1 \mathrm{~mL})$ [prepared by mixing $\mathrm{CrO}_{3}(16 \mathrm{~g}), \mathrm{H}_{2} \mathrm{O}$ $(10 \mathrm{~mL})$ and $\left.3.0 \mathrm{M} \mathrm{H}_{2} \mathrm{SO}_{4}(60 \mathrm{~mL})\right]$ was slowly admitted to a stirred solution of (11S)[11- $\left.{ }^{2} \mathrm{H}_{1}\right]$-octadeca-9-yn-1-ol $(1.37 \mathrm{~g}, 5.1 \mathrm{mmol})$ in acetone $(110 \mathrm{~mL})$. During addition, temperature of the reaction mixture was maintained below $25{ }^{\circ} \mathrm{C}$ (using an ice-water bath) and the reaction was stirred for $2 \mathrm{~h}$ at $\mathrm{rt}$. Excess oxidant was destroyed by passing 
$\mathrm{SO}_{2}$ through the reaction mixture to yield an emerald green color and the precipitate was dissolved in $\mathrm{H}_{2} \mathrm{O}(50 \mathrm{~mL})$. The aqueous layer was extracted with $\mathrm{Et}_{2} \mathrm{O}(3 \times 40 \mathrm{~mL})$ and the combined organic layers were washed with water $(50 \mathrm{~mL})$ and saturated $\mathrm{NaCl}$ (made slightly acidic to $\mathrm{pH} 5$ by adding few drops of $2.0 \mathrm{M} \mathrm{HCl}$ ) until the aqueous layer was colorless. The solvent was removed in vacuo and the residue was further diluted with hexane and worked up in the usual manner to afford the title compound as an amorphous white solid (1.426 g, $99 \%) . R_{\mathrm{f}} 0.28\left(\mathrm{MeOH}: \mathrm{CH}_{2} \mathrm{Cl}_{2}, 1: 19\right)$; and the spectral data of the compound was similar to that reported in the literature for the non-deuterated parent compound. ${ }^{1}$ IR (KBr): v 3500-2800br (O-H), 3020, 2930, $2858(\mathrm{C}-\mathrm{H}), 1711(\mathrm{C}=\mathrm{O})$, $1463,1413,1286,1216,1125,1074,914,758 \mathrm{~cm}^{-1} ;{ }^{1} \mathrm{H}$ NMR $\left(300 \mathrm{MHz}, \mathrm{CDCl}_{3}\right) \delta_{\mathrm{H}}$ $11.18\left(b r s, 1 \mathrm{H}, \mathrm{CO}_{2} \mathrm{H}\right.$ ), $2.36\left(t d, J=7.5,1.9 \mathrm{~Hz}, 2 \mathrm{H}, \mathrm{CH}_{2} \mathrm{CO}_{2} \mathrm{H}\right.$ ), 2.14 (Overlapping $t$, $J=6.3 \mathrm{~Hz}, 3 \mathrm{H}, \mathrm{C}-8, \mathrm{C}-11), 1.65\left(t t, J=7.5,6.3 \mathrm{~Hz}, 2 \mathrm{H}, \mathrm{CH}_{2} \mathrm{CH}_{2} \mathrm{CO}_{2} \mathrm{H}\right), 1.53-1.21(m$, $\left.20 \mathrm{H}, 10 \times \mathrm{CH}_{2}\right), 0.88\left(t, J=7.0 \mathrm{~Hz}, 3 \mathrm{H}, \mathrm{CH}_{3}\right) ;{ }^{13} \mathrm{C} \mathrm{NMR}\left(75.5 \mathrm{MHz}, \mathrm{CDCl}_{3}\right) \delta_{\mathrm{C}} 179.79$ (C-1), 33.98 (C-2), 24.64 (C-3), 29.07 (C-4), 28.97 (C-5), 28.87 (C-6), 29.07 (C-7), 18.73 (C-8), 80.08 (C-9), $80.36(\mathrm{C}-10), 18.47\left(t, J_{\mathrm{CD}}=19.9 \mathrm{~Hz}, \mathrm{C}-11\right), 28.62(\mathrm{C}-12), 28.78(\mathrm{C}-$ 13), 29.15 (C-14), 29.24 (C-15), 31.86 (C-16), 22.68 (C-17), 14.12 (C-18); EI-MS: $m / z$ $284\left(+2 \mathrm{H},[\mathrm{M}+3]^{+}\right), 267\left(-\mathrm{OH} ;[\mathrm{M}-14]^{+}\right), 253\left([\mathrm{M}-28]^{+}\right), 241,224,210,197,183,174$, $167\left(\left[\mathrm{C} \equiv \mathrm{C}\left(\mathrm{CH}_{2}\right)_{7} \mathrm{CO}_{2} \mathrm{H}\right]^{+}\right), 153,147,137,123,110,96,82,68,60,55,41$. 


\subsubsection{Methyl (11S)-[11- $\left.{ }^{2} \mathrm{H}_{1}\right]-$ Octadec-9-ynoate}

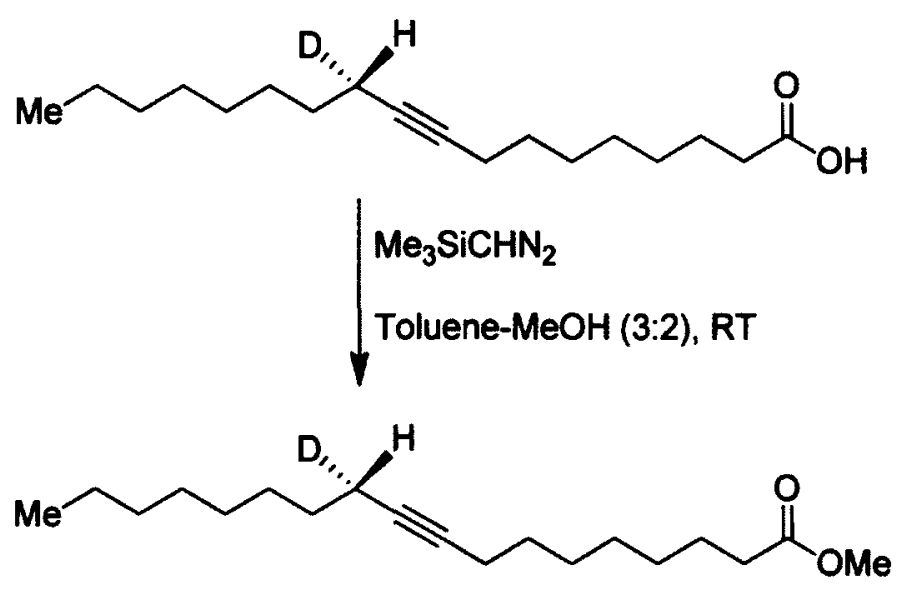

The deuterated fatty acid $(1.44 \mathrm{~g}, 5.1 \mathrm{mmol})$ was dissolved in $3: 2$ toluene/methanol $(18 \mathrm{~mL})$ and treated with (trimethylsilyl) diazomethane $(2.0 \mathrm{M}$ in hexanes, $2.6 \mathrm{~mL}, 5.1 \mathrm{mmol}$ ) as described above for the methylation of decynoic acid. The crude product was purified by flash chromatography using EtOAc:hexane $(1: 49)$ as eluent and the title compound was obtained as colorless oil (1.33 g, $88 \%) . R_{\mathrm{f}} 0.37$ $\left(\mathrm{Et}_{2} \mathrm{O}\right.$ :hexane, 1:3); and the spectral data of the compound was similar to that reported in the literature for the non-deuterated parent compound. ${ }^{197}$ IR (film): v 2929, 2856 (C-H), 2145 (C-D), 1743 (C=O), 1463, 1436, 1362, 1247, 1197, 1171, 1098, 1017, $725 \mathrm{~cm}^{-1} ;{ }^{1} \mathrm{H}$ $\operatorname{NMR}\left(300 \mathrm{MHz}, \mathrm{CDCl}_{3}\right) \delta_{\mathrm{H}} 3.66\left(s, 3 \mathrm{H}, \mathrm{OCH}_{3}\right), 2.30\left(t, J=7.5 \mathrm{~Hz}, 2 \mathrm{H}, \mathrm{CH}_{2} \mathrm{CO}_{2} \mathrm{Me}\right)$, 2.13 (Overlapping $t, J=6.2 \mathrm{~Hz}, 3 \mathrm{H}, \mathrm{C}-8, \mathrm{C}-11$ ), $1.62(t t, J=7.5,7.3 \mathrm{~Hz}, 2 \mathrm{H}$, $\left.\mathrm{CH}_{2} \mathrm{CH}_{2} \mathrm{CO}_{2} \mathrm{Me}\right), 1.46(m, 4 \mathrm{H}, \mathrm{C}-7, \mathrm{C}-12), 1.41-1.19\left(m, 16 \mathrm{H}, 8 \times \mathrm{CH}_{2}\right), 0.88(t, J=6.7$ $\mathrm{Hz}, 3 \mathrm{H}, \mathrm{CH}_{3}$ ); ${ }^{13} \mathrm{C}$ NMR (75.5 MHz, $\mathrm{CDCl}_{3}$ ) $\delta_{\mathrm{C}} 174.28$ (C-1), 34.08 (C-2), $24.92(\mathrm{C}-3)$, 29.08 (C-4), 29.06 (C-5), 28.86 (C-6), 29.08(C-7), 18.73 (C-8), 80.09 (C-9), 80.31 (C$10), 18.47\left(t, J_{\mathrm{CD}}=19.7 \mathrm{~Hz}, \mathrm{C}-11\right), 28.65(\mathrm{C}-12), 28.80(\mathrm{C}-13), 29.15(\mathrm{C}-14), 29.24(\mathrm{C}-$ 15), 31.86 (C-16), 22.68 (C-17), 14.11 (C-18), 51.44 (-OMe); EI-MS: m/z 295 ([M]'), 
$264\left([\mathrm{M}-31]^{+}\right), 246\left([\mathrm{M}-49]^{+}\right), 221\left([\mathrm{M}-74]^{+}\right), 211,197,179,165,153,137,123,110,96$, $82,69,55,41$.

\subsubsection{Methyl (11S)-[11- $\left.{ }^{2} \mathrm{H}_{1}\right]-$ Octadeca-9Z-enoate}

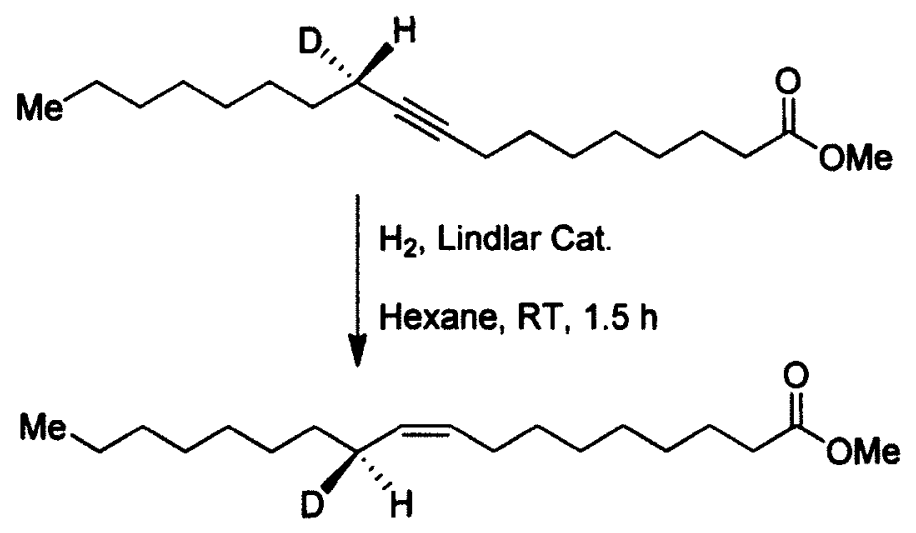

Methyl (11S)-[11- $\left.{ }^{2} \mathrm{H}_{1}\right]$-octadeca-9-ynoate $(100 \mathrm{mg}, 0.3 \mathrm{mmol})$ in hexane $(10 \mathrm{~mL})$ was hydrogenated essentially as described above using Lindlar catalyst $(17 \mathrm{mg})$ and $1.5 \mathrm{~h}$ reaction time. The final product was obtained as clear colorless oil (100 mg, $99 \%) . R_{\mathrm{f}}$ 0.53 (EtOAc:hexane, 1:9); and the spectral data of the monodeuterated compound was similar to the non-deuterated parent compound reported in the literature. ${ }^{192-194,197}$ IR (film): v 3004, 2926, 2855 (C-H), 2149 (C-D), 1744 (C=O), 1464, 1436, 1362, 1246, $1196,1171,1016,724 \mathrm{~cm}^{-1} ;{ }^{1} \mathrm{H}$ NMR $\left(300 \mathrm{MHz}, \mathrm{CDCl}_{3}\right) \delta_{\mathrm{H}} 5.42-5.28(m, 2 \mathrm{H}, 2 \mathrm{x}$ $\mathrm{HC}=\mathrm{C}), 3.67(s, 3 \mathrm{H}, \mathrm{OCH}), 2.30\left(t, J=7.5 \mathrm{~Hz}, 2 \mathrm{H}, \mathrm{CH}_{2} \mathrm{CO}_{2} \mathrm{Me}\right.$ ), 2.01 (Overlapping $t t$ and $t d, J=6.0,6.0 \mathrm{~Hz}, 3 \mathrm{H}, \mathrm{C}-8, \mathrm{C}-11), 1.62\left(t t, J=7.5,7.3 \mathrm{~Hz}, 2 \mathrm{H}, \mathrm{CH}_{2} \mathrm{CH}_{2} \mathrm{CO}_{2} \mathrm{Me}\right)$, 1.39-1.21 $\left(m, 20 \mathrm{H}, 10 \times \mathrm{CH}_{2}\right), 0.88\left(t, J=6.7 \mathrm{~Hz}, 3 \mathrm{H}, \mathrm{CH}_{3}\right) ;{ }^{13} \mathrm{C}$ NMR $(75.5 \mathrm{MHz}$, $\left.\mathrm{CDCl}_{3}\right) \delta_{\mathrm{C}} 174.32(\mathrm{C}-1), 34.12(\mathrm{C}-2), 24.97(\mathrm{C}-3), 29.11(\mathrm{C}-4), 29.17$ (C-5), 29.15 (C-6), 29.70 (C-7), 27.18 ( $\delta$-shift 0.011, C-8), 129.78 ( $\gamma$-shift 0.027, C-9), 129.97 ( $\beta$-shift $0.037, \mathrm{C}-10), 26.87\left(t, J_{\mathrm{CD}}=19.3 \mathrm{~Hz}, \alpha\right.$-shift - 0.352, C-11), $29.70(\beta$-shift - 0.078, C-12), 
$29.32(\gamma$-shift - 0.022, C-13), 29.55 ( $\delta$-shift 0.007, C-14), 29.34 (C-15), 31.93 (C-16), 22.70 (C-17), 14.13 (C-18), 51.44 (-OMe); EI-MS: $m / z 297$ ([M] $\left.{ }^{+}\right), 266$ ([M-31] $\left.]^{+}\right), 265$ ($\left.\mathrm{MeOH}^{\circ},[\mathrm{M}-32]^{+}\right), 236\left([\mathrm{M}-61]^{+}\right), 223\left([\mathrm{M}-74]^{+}\right), 208,181\left([\mathrm{M}-116]^{+}\right), 167,152,139$, $123,110,98,83,74,69,55,41$.

\subsubsection{Methyl (11R)-[11- $\left.{ }^{2} \mathrm{H}_{1}\right]-$ Octadeca-9Z-enoate}

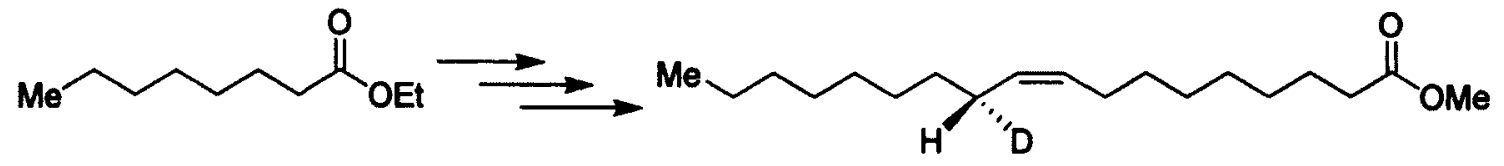

The title compound was synthesized in $7.3 \%$ overall yield from ethyl octanoate using the same procedures as described for the synthesis of the corresponding (S)enantiomer. The chiral starting material was prepared by the reduction of $1-\left[1-{ }^{2} \mathrm{H}_{1}\right]-$ octanal using $(+)-(R)$-Alpine-Borane. $R_{\mathrm{f}} 0.53$ (EtOAc:hexane, 1:9); The analytical data for the title compound was essentially identical to that obtained for the corresponding (S)enantiomer and similar to that reported in the literature for the non-deuterio compound. ${ }^{192-194,197}$ IR (film): v 3004, 2926, 2855 (C-H), 2147 (C-D), 1744 (C=O), 1465, $1436,1248,1197,1171,1018,725 \mathrm{~cm}^{-1} ;{ }^{1} \mathrm{H}$ NMR $\left(300 \mathrm{MHz}, \mathrm{CDCl}_{3}\right) \delta_{\mathrm{H}} 5.41-5.27(m, 2$ $\mathrm{H}, 2 \times \mathrm{HC}=\mathrm{C}), 3.67\left(s, 3 \mathrm{H}, \mathrm{OCH}_{3}\right), 2.31\left(t, J=7.6 \mathrm{~Hz}, 2 \mathrm{H}, \mathrm{CH}_{2} \mathrm{CO}_{2} \mathrm{Me}\right), 2.01$ (Overlapping $t t$ and $t d, J=6.0,6.0 \mathrm{~Hz}, 3 \mathrm{H}, \mathrm{C}-8, \mathrm{C}-11), 1.62(t t, J=7.6,7.3 \mathrm{~Hz}, 2 \mathrm{H}$,

$\left.\mathrm{CH}_{2} \mathrm{CH}_{2} \mathrm{CO}_{2} \mathrm{Me}\right), 1.39-1.21\left(m, 20 \mathrm{H}, 10 \times \mathrm{CH}_{2}\right), 0.88\left(t, J=6.7 \mathrm{~Hz}, 3 \mathrm{H}, \mathrm{CH}_{3}\right) ;{ }^{13} \mathrm{C}$ NMR (75.5 MHz, CDCl $) \delta_{\mathrm{C}} 174.34$ (C-1), 34.12 (C-2), 24.97 (C-3), 29.11 (C-4), 29.17 (C-5), 29.14 (C-6), 29.70 (C-7), 27.18 ( $\delta$-shift 0.007, C-8), 129.78 ( $\gamma$-shift 0.025, C-9), $129.97\left(\beta\right.$-shift - 0.039, C-10), $26.87\left(t, J_{\mathrm{CD}}=19.3 \mathrm{~Hz}, \alpha\right.$-shift - 0.356, C-11), $29.70(\beta-$ shift - 0.081, C-12), 29.31 ( $\gamma$-shift - 0.024, C-13), 29.55 ( $\delta$-shift 0.006, C-14), 29.34 (C- 
15), 31.92 (C-16), 22.70 (C-17), 14.13 (C-18), 51.46 (-OMe); EI-MS: m/z 297 ([M] ${ }^{\dagger}$ ), $266\left([\mathrm{M}-31]^{+}\right), 265\left(-\mathrm{MeOH} ;[\mathrm{M}-32]^{+}\right), 236\left([\mathrm{M}-61]^{+}\right), 223\left([\mathrm{M}-74]^{+}\right), 208,181([\mathrm{M}-$ $\left.116]^{+}\right), 167,152,139,123,110,98,84,74,69,55,41$.

\subsubsection{Chiral $\left[14-^{2} \mathrm{H}_{1}\right]$-Linoleate Synthesis}

\subsubsection{1-Pentanal}<smiles>CN(C)C=O</smiles>

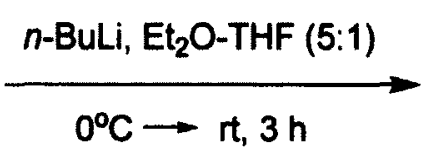<smiles>CCCCC=O</smiles>

A 2.5 M solution of $n$-butyllithium in hexane $(54.5 \mathrm{~mL}, 135.6 \mathrm{mmol})$ was added dropwise over a period of 50-55 minutes to a stirred solution of N,N-dimethylformamide $(10 \mathrm{~mL}, 129.2 \mathrm{mmol})$ in a $5: 1$ mixture of anhydrous $\mathrm{Et}_{2} \mathrm{O}-\mathrm{THF}(120 \mathrm{~mL})$ at $0{ }^{\circ} \mathrm{C}$. The reaction was exothermic and the reaction mixture turned viscous and become off-white in color after the addition was complete. The reaction mixture was warmed up to $\mathrm{rt}$ and allowed to stir for $3 \mathrm{~h}$. The solvent was removed in vacuo and the residue was diluted with ether $(120 \mathrm{~mL})$ that was poured into chilled $\mathrm{H}_{2} \mathrm{O}(110 \mathrm{~mL})$. After acidifying the aqueous layer with $3.0 \mathrm{M} \mathrm{HCl}$, the organic layer was separated and the aqueous layer was extracted with $\mathrm{Et}_{2} \mathrm{O}(3 \times 80 \mathrm{~mL})$. The combined organic layers were washed successively with water $(120 \mathrm{~mL})$, saturated $\mathrm{NaHCO}_{3}(120 \mathrm{~mL})$, brine $(2 \times 120 \mathrm{~mL})$ and finally dried over anhydrous $\mathrm{Na}_{2} \mathrm{SO}_{4}$. The solvent was initially removed by short-path fractional distillation at atmospheric pressure and when the solvent was judged to evaporated; the title compound was distilled using diethyl suberate as a high boiling chase. The title compounds was obtained as a pure colorless liquid $(6.90 \mathrm{~g}, 62 \%)$. bp $100-102{ }^{\circ} \mathrm{C} ; R_{\mathrm{f}} 0.48$ 
(EtOAc:hexane, 1:3); IR (film): v 2960, 2935, 2875, 2821 (C-H), 2720 (C-H, CHO), 1727 (C-O), 1467, 1412, 1391, 1147, 1120, 1001, $888 \mathrm{~cm}^{-1} ;{ }^{1} \mathrm{H}$ NMR (300 MHz, $\mathrm{CDCl}_{3}$ ) $\delta_{\mathrm{H}} 9.75(t, J=1.9 \mathrm{~Hz}, 1 \mathrm{H}, \mathrm{CHO}), 2.41\left(t d, J=7.4,1.9 \mathrm{~Hz}, 2 \mathrm{H}, \mathrm{CH}_{2} \mathrm{CHO}\right), 1.60(t t, J=$ 7.4, 7.4 Hz, $\left.2 \mathrm{H}, \mathrm{CH}_{2} \mathrm{CH}_{2} \mathrm{CHO}\right), 1.35\left(q t, J=7.4,7.3 \mathrm{~Hz}, 2 \mathrm{H}, \mathrm{CH}_{2} \mathrm{CH}_{3}\right), 0.91(t, J=7.3$ $\left.\mathrm{Hz}, 3 \mathrm{H}, \mathrm{CH}_{3}\right) ;{ }^{13} \mathrm{C}$ NMR (75.5 MHz, $\mathrm{CDCl}_{3}$ ) $\delta_{\mathrm{C}} 202.88$ (C-1), 43.59 (C-2), 24.13 (C-3), $22.25(\mathrm{C}-4), 13.74(\mathrm{C}-5)$.

\subsubsection{1-[1- $\left.{ }^{2} \mathrm{H}_{1}\right]-$ Pentanal ${ }^{209}$}

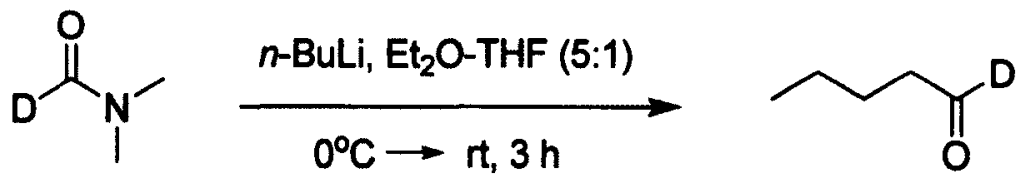

The title compound was synthesized from $\left[1-{ }^{2} \mathrm{H}_{1}\right] \mathrm{N}, \mathrm{N}$-dimethylformamide $(10.6$ $\mathrm{mL}, 135.0 \mathrm{mmol}$ ) using the same experimental procedure to that described above during the preparation of 1-pentanal. The title compound was obtained as a pure colorless liquid (7.19 g, 61\%). bp $99-104{ }^{\circ} \mathrm{C} ; R_{\mathrm{f}} 0.48$ (EtOAc:hexane, 1:3); and the spectral data of the monodeuterated compound was similar to that of the non-deuterated parent compound reported above. IR (film): v 2964, 2875 (C-H), 2191, 2071 (C-D), 1715 (C=O), 1467, $1406,1380,1094,954 \mathrm{~cm}^{-1} ;{ }^{1} \mathrm{H}$ NMR $\left(300 \mathrm{MHz}, \mathrm{CDCl}_{3}\right) \delta_{\mathrm{H}} 2.41(t, J=7.3 \mathrm{~Hz}, 2 \mathrm{H}$, $\left.\mathrm{CH}_{2} \mathrm{CDO}\right), 1.60\left(t t, J=7.5,7.3 \mathrm{~Hz}, 2 \mathrm{H}, \mathrm{CH}_{2} \mathrm{CH}_{2} \mathrm{CDO}\right), 1.34(q t, J=7.5,7.3 \mathrm{~Hz}, 2 \mathrm{H}$, $\left.\mathrm{CH}_{2} \mathrm{Me}\right), 0.91\left(t, J=7.3 \mathrm{~Hz}, \mathrm{CH}_{3}\right) ;{ }^{13} \mathrm{C} \mathrm{NMR}\left(75.5 \mathrm{MHz}, \mathrm{CDCl}_{3}\right) \delta_{\mathrm{C}} 202.63\left(t, J_{\mathrm{CD}}=25.9\right.$ $\mathrm{Hz}, \alpha$-shift $-0.251, \mathrm{C}-1), 43.43\left(t,{ }^{2} J_{\mathrm{CD}}=3.6 \mathrm{~Hz}, \beta\right.$-shift - 0.156, C-2), $24.10(\gamma$-shift 0.023, C-3), 22.27 ( $\delta$-shift 0.016, C-4), 13.77 (C-5); EI-MS: $m / z 85\left([\mathrm{M}-2]^{+}\right), 70$ ([M$\left.17]^{+}\right), 57\left([\mathrm{M}-30]^{+}\right), 41,28$. 


\subsubsection{1-Pentanol}

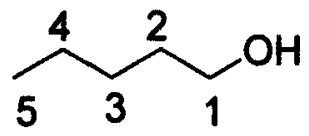

The spectral data and physical characteristics of the compound was recorded using the authentic sample to establish the formation of the synthetic deuterated materials as well as to calculate the isotopic shifts. bp $138-140{ }^{\circ} \mathrm{C} ; R_{\mathrm{f}} 0.23$ (EtOAc:hexane, $1: 3$ ); IR (film): v $3338(\mathrm{O}-\mathrm{H}), 2958,2932,2873(\mathrm{C}-\mathrm{H}), 1467,1379,1116,1076,1056,1006,889$, $729 \mathrm{~cm}^{-1} ;{ }^{1} \mathrm{H}$ NMR $\left(300 \mathrm{MHz}, \mathrm{CDCl}_{3}\right) \delta_{\mathrm{H}} 3.59\left(t, J=6.5 \mathrm{~Hz}, 2 \mathrm{H}, \mathrm{CH}_{2} \mathrm{OH}\right), 2.19-2.06$ (br $s, 1 \mathrm{H}, \mathrm{OH}), 1.54\left(t t, J=6.8,6.5 \mathrm{~Hz}, 2 \mathrm{H}, \mathrm{CH}_{2} \mathrm{CH}_{2} \mathrm{OH}\right), 1.39-1.25\left(m, 4 \mathrm{H}, 2 \times \mathrm{CH}_{2}\right)$, $0.88\left(t, J=6.8 \mathrm{~Hz}, 3 \mathrm{H}, \mathrm{CH}_{3}\right) ;{ }^{13} \mathrm{C} \mathrm{NMR}\left(75.5 \mathrm{MHz}, \mathrm{CDCl}_{3}\right) \delta_{\mathrm{C}} 62.81(\mathrm{C}-1), 32.38(\mathrm{C}-2)$, 27.88 (C-3), 22.44 (C-4), 13.97 (C-5); EI-MS: m/z 87 ([M-1] $\left.]^{+}\right), 70$ ([M-18] $\left.]^{+}\right), 55$ ([M$\left.33]^{+}\right), 42,31,29,27$.

\subsubsection{4 (1S)-[1- $\left.{ }^{2} \mathrm{H}_{1}\right]-$ pentanol}

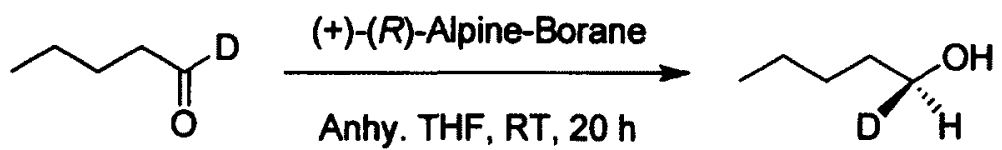

The title compound was synthesized from monodeuterated $\left[1-{ }^{2} \mathrm{H}_{1}\right]$-pentanal $(5.36$ $\mathrm{g}, 61.5 \mathrm{mmol})$ and $(+)-(R)$-Alpine-Borane ${ }^{\circledast}(185 \mathrm{~mL}, 92.3 \mathrm{mmol})$ using the same experimental protocol as that described above for the synthesis of $(1 R)-\left[1-{ }^{2} \mathrm{H}_{1}\right]$-octanol. The crude compound was purified by flash chromatography using $\mathrm{Et}_{2} \mathrm{O}$ :pentane (1:9) as eluent and the pure monodeuterated alcohol was isolated as colorless oil $(1.35 \mathrm{~g}, 25 \%)$. bp 138-140 ${ }^{\circ} \mathrm{C} ; R_{\mathrm{f}} 0.18$ (EtOAc:hexane, 1:3); and the spectral data of the monodeuterated 
compound was similar to that reported above for the non-deuterated parent compound. IR (film): v 3339 (O-H), 2959, 2931, 2874 (C-H), 2153 (C-D), 1467, 1379, 1132, 1067, $1015,940,736 \mathrm{~cm}^{-1} ;{ }^{1} \mathrm{H}$ NMR $\left(300 \mathrm{MHz}, \mathrm{CDCl}_{3}\right) \delta_{\mathrm{H}} 3.59(t t, J=6.8,1.5 \mathrm{~Hz}, 1 \mathrm{H}$, $\mathrm{CHDOH}), 2.47(s, 1 \mathrm{H}, \mathrm{OH}), 1.53\left(t t, J=6.9,6.8 \mathrm{~Hz}, 2 \mathrm{H}, \mathrm{CH}_{2} \mathrm{CHD}\right), 1.38-1.24(m, 4 \mathrm{H}$, $\left.2 \times \mathrm{CH}_{2}\right), 0.87\left(t, J=6.9 \mathrm{~Hz}, 3 \mathrm{H}, \mathrm{CH}_{3}\right) ;{ }^{13} \mathrm{C} \mathrm{NMR}\left(75.5 \mathrm{MHz}, \mathrm{CDCl}_{3}\right) \delta_{\mathrm{C}} 62.56\left(t, J_{\mathrm{CD}}=\right.$ $21.6 \mathrm{~Hz}, \alpha$-shift - 0.252, C-1), 32.32 ( $\beta$-shift - 0.069, C-2), 27.87 ( $\gamma$-shift - 0.017, C-3), 22.46 ( $\delta$-shift $0.018, \mathrm{C}-4), 14.00(\mathrm{C}-5)$; EI-MS: $m / z 88\left([\mathrm{M}-1]^{+}\right), 71\left([\mathrm{M}-18]^{+}\right), 56([\mathrm{M}-$ $\left.33]^{+}\right), 42,32,29,27$; TMS Derivative EI-MS: $m / z 161\left([\mathrm{M}]^{+}\right), 146\left([\mathrm{M}-15]^{+}\right), 133$ ([M$\left.28]^{+}\right), 116\left([\mathrm{M}-45]^{+}\right), 104,90,75,59,45$.

\subsubsection{1-(p-Tolylsulfonyloxy)-pentane $e^{173}$}

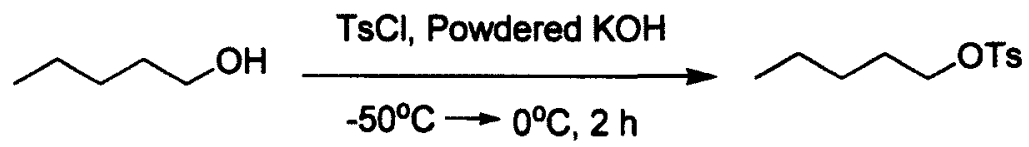

To a stirred solution of 1-pentanol $(1.5 \mathrm{~g}, 17.0 \mathrm{mmol})$ and $p$-toluenesulfonyl chloride $(4.05 \mathrm{~g}, 21.3 \mathrm{mmol})$ in anhydrous $\mathrm{Et}_{2} \mathrm{O}(50 \mathrm{~mL})$ was added powdered $\mathrm{KOH}(8.2$ $\mathrm{g}, 146.2 \mathrm{mmol}$ ) at $-50{ }^{\circ} \mathrm{C}$. The reaction mixture was stirred at $0{ }^{\circ} \mathrm{C}$ for $2 \mathrm{~h}$, poured into $\mathrm{H}_{2} \mathrm{O}(50 \mathrm{~mL})$ and worked up as described above for the preparation of 1-(ptolylsulfonyloxy)-oct-2-yne to afford a colorless clear liquid (3.62 $\mathrm{g}, 88 \%), R_{\mathrm{f}} 0.41$ (EtOAc:hexane, 1:4); IR (film): v 2959, 2933, 2873 (C-H), 1599 (C=C, Ar), 1467, 1360, $1177,1098,961,913,815,762 \mathrm{~cm}^{-1} ;{ }^{1} \mathrm{H}$ NMR $\left(300 \mathrm{MHz}, \mathrm{CDCl}_{3}\right) \delta_{\mathrm{H}} 7.78(d, J=8.2 \mathrm{~Hz}$, $2 \mathrm{H}, \mathrm{C} \underline{o}-\mathrm{Ph}), 7.34(d, J=8.2 \mathrm{~Hz}, 2 \mathrm{H}, \mathrm{C} \underline{m}-\mathrm{Ph}), 4.01\left(t, J=6.5 \mathrm{~Hz}, 2 \mathrm{H}, \mathrm{CH}_{2} \mathrm{OTs}\right), 2.44$ $\left(s, 3 \mathrm{H}, \mathrm{CH}_{3} \mathrm{Ar}\right), 1.63\left(t t, J=6.9,6.5 \mathrm{~Hz}, 2 \mathrm{H}, \mathrm{CH}_{2} \mathrm{CH}_{2} \mathrm{OTs}\right), 1.36-1.18(m, 4 \mathrm{H}, 2 \mathrm{x}$ $\left.\mathrm{CH}_{2}\right), 0.84\left(t, J=7.0 \mathrm{~Hz}, 3 \mathrm{H}, \mathrm{CH}_{3}\right) ;{ }^{13} \mathrm{C} \mathrm{NMR}\left(75.5 \mathrm{MHz}, \mathrm{CDCl}_{3}\right) \delta_{\mathrm{C}} 70.68(\mathrm{C}-1), 28.47$ 
(C-2), 27.40 (C-3), 21.98 (C-4), 13.78 (C-5), 144.61 (Cipso - Ph), 129.77 (Co - Ph), $127.83(\mathrm{C} \underline{m}-\mathrm{Ph}), 133.19(\mathrm{C} p-\mathrm{Ph}), 21.58\left(\mathrm{CH}_{3}-\mathrm{Ph}\right) ;$ EI-MS: $\mathrm{m} / z 242\left([\mathrm{M}]^{+}\right), 185,173$, $155,139,107,91,77,65,55,41$.

\subsubsection{6 (1S)-1-(p-Tolylsulfonyloxy)-[1- $\left.{ }^{2} \mathrm{H}_{1}\right]-$ pentane $^{173}$}

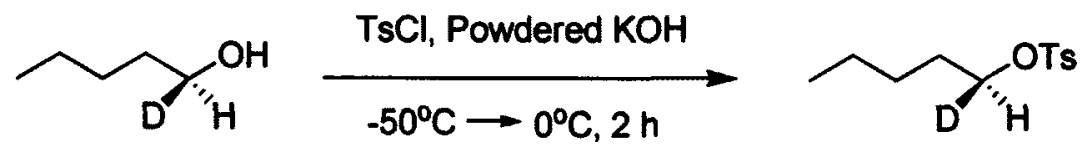

The anticipated compound was synthesized in a similar manner to that described in 6.2.4.5, from $(1 S)$-[1 $\left[{ }^{2} \mathrm{H}_{1}\right]$-pentanol $(1.35 \mathrm{~g}, 10.6 \mathrm{mmol})$ as a colorless clear oil $(2.67 \mathrm{~g}$, 89\%). $R_{\mathrm{f}} 0.41$ (EtOAc:hexane, 1:4); and the spectral data of the compound was similar to the non-deuterated parent compound reported above. IR (film): v 2959, 2933, 2873 (CH), 2193 (C-D), 1599 (C=C, Ar), 1458, 1364, 1189, 1178, 1098, 943, 816, $755 \mathrm{~cm}^{-1} ;{ }^{1} \mathrm{H}$ NMR $\left(300 \mathrm{MHz}, \mathrm{CDCl}_{3}\right) \delta_{\mathrm{H}} 7.78(d, J=8.2 \mathrm{~Hz}, 2 \mathrm{H}, \mathrm{C} \underline{\underline{a}}-\mathrm{Ph}), 7.34(d, J=8.2 \mathrm{~Hz}, 2 \mathrm{H}$, C $\underline{m}-\mathrm{Ph}), 3.99(t t, J=6.5,1.2 \mathrm{~Hz}, 1 \mathrm{H}, \mathrm{CHDOTs}), 2.44\left(s, 3 \mathrm{H}, \mathrm{CH}_{3} \mathrm{Ar}\right), 1.62(t d, J=$ 6.9, $\left.6.5 \mathrm{~Hz}, 2 \mathrm{H}, \mathrm{CH}_{2} \mathrm{CHDOTs}\right), 1.36-1.18\left(m, 4 \mathrm{H}, 2 \times \mathrm{CH}_{2}\right), 0.84(t, J=7.0 \mathrm{~Hz}, 3 \mathrm{H}$, $\left.\mathrm{CH}_{3}\right) ;{ }^{13} \mathrm{C}$ NMR $\left(75.5 \mathrm{MHz}, \mathrm{CDCl}_{3}\right) \delta_{\mathrm{C}} 70.37\left(t, J_{\mathrm{CD}}=22.8 \mathrm{~Hz}, \alpha\right.$-shift $\left.-0.309, \mathrm{C}-1\right)$, 28.37 ( $\beta$-shift - 0.10, C-2), 27.38 ( $\gamma$-shift - 0.023, C-3), 21.99 ( $\delta$-shift 0.005, C-4), 13.77

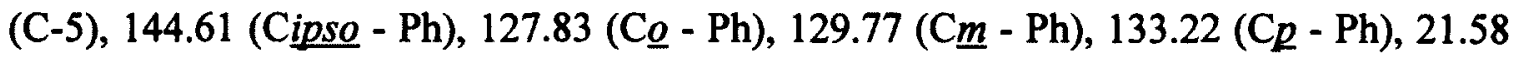
$\left(\mathrm{CH}_{3}-\mathrm{Ph}\right)$; EI-MS: $m / z 243\left([\mathrm{M}]^{+}\right), 186,173,155,139,107,91,77,71,65,41$. 


\subsubsection{2-((4R)-[4- $\left.{ }^{2} \mathrm{H}_{1}\right]-$-octa-2-ynyl-1-oxy)tetrahydropyran ${ }^{174}$}

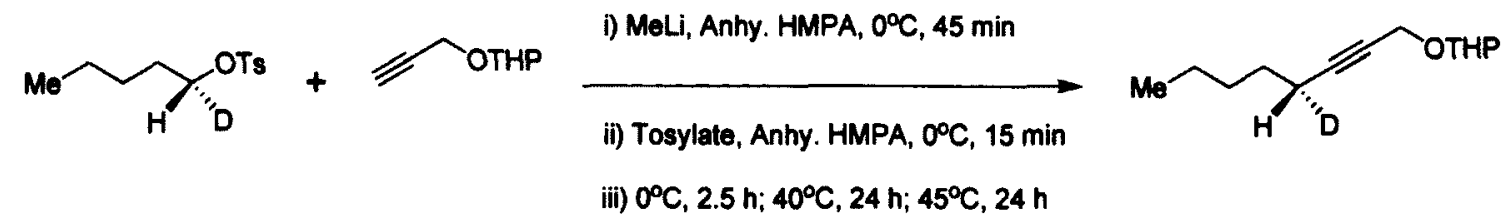

Methyl lithium (1.6 $\mathrm{M}$ in $\left.\mathrm{Et}_{2} \mathrm{O}, 7.3 \mathrm{~mL}, 11.7 \mathrm{mmol}\right)$ was added dropwise to a stirred solution of the tetrahydro-2-(2-propynyloxy)-2H-pyran (1.64 $\mathrm{g}, 11.7 \mathrm{mmol})$ in dry HMPA $(10 \mathrm{~mL})$ at $0{ }^{\circ} \mathrm{C}$. After stirring the reaction mixture for 45 minutes at $0{ }^{\circ} \mathrm{C}$ during which the reaction mixture turned from yellow to deep purple, a solution of $(1 S)-1-(p-$ tolylsulfonyloxy)-[1 $\left.{ }^{2} \mathrm{H}_{1}\right]$-pentane $(2.35 \mathrm{~g}, 9.6 \mathrm{mmol})$ in dry HMPA $(5 \mathrm{~mL})$ was added dropwise over a period of 15 minutes. During stirring, the solution color changed from green to brown. The reaction mixture was stirred at $0{ }^{\circ} \mathrm{C}$ for $2.5 \mathrm{~h}$, at $40{ }^{\circ} \mathrm{C}$ for $24 \mathrm{~h}$ and at $45^{\circ} \mathrm{C}$ for another $24 \mathrm{~h}$. The reaction was quenched by pouring the mixture into an icewater mixture $(30 \mathrm{~mL})$, stirred for 15 minutes and additional $\mathrm{H}_{2} \mathrm{O}(100 \mathrm{~mL})$ was added to it. The suspension was transferred to a separatory funnel and the organic layer was diluted with 1:3 mixture of $\mathrm{Et}_{2} \mathrm{O}$-pentane $(40 \mathrm{~mL})$. The organic layer was separated and the aqueous phase was extracted with additional 1:3 mixture of $\mathrm{Et}_{2} \mathrm{O}$-pentane $(3 \times 40$ $\mathrm{mL}$ ). The collected organic layers were combined and worked up in the usual manner to result the crude compound that was purified by flash chromatography using $\mathrm{Et}_{2} \mathrm{O}$ :hexane (1:9) as eluent. The pure compound was obtained as a light yellowish oil (1.83 $\mathrm{g}, 89 \%)$. $R_{\mathrm{f}} 0.21$ (EtOAc:hexane, 1:19); and the spectral data of the compound was similar to that reported in the literature for the non-deuterated parent compound. ${ }^{10}$ IR (film): $v 2943$, $2872(\mathrm{C}-\mathrm{H}), 2237$ (C $\equiv \mathrm{C}), 2118$ (C-D), 1455, 1443, 1357, 1202, 1121, 1030, 903, 871, 
$816 \mathrm{~cm}^{-1} ;{ }^{1} \mathrm{H}$ NMR $\left(300 \mathrm{MHz}, \mathrm{CDCl}_{3}\right) \delta_{\mathrm{H}} 4.81(t d, J=4.5,3.2 \mathrm{~Hz}, 1 \mathrm{H}, \mathrm{OCHO}), 4.34-$ 4.16 ( $m$, overlapping $\left.d d d, 2 \mathrm{H}, \mathrm{CH}_{2} \mathrm{OTHP}\right), 3.84\left(m, 1 \mathrm{H}, \mathrm{CHO}\left(5^{\prime}\right)\right)$, 3.67-3.47 (m, $1 \mathrm{H}$, CHO (5')), $2.19(t t, J=7.0,2.3 \mathrm{~Hz}, 1 \mathrm{H}, \mathrm{CHDC} \equiv \mathrm{C}), 1.94-1.45\left(m, 8 \mathrm{H}, 4 \times \mathrm{CH}_{2}, \mathrm{C}-5\right.$, C-2', C-3', C-4'), 1.42-1.24 (m, $\left.4 \mathrm{H}, 2 \times \mathrm{CH}_{2}, \mathrm{C}-6, \mathrm{C}-7\right), 0.89\left(t, J=6.7 \mathrm{~Hz}, 3 \mathrm{H}, \mathrm{CH}_{3}\right)$; ${ }^{13} \mathrm{C}$ NMR $\left(75.5 \mathrm{MHz}, \mathrm{CDCl}_{3}\right) \delta_{\mathrm{C}} 54.64(\delta$-shift - 0.003, C-1), $79.76(\gamma$-shift - 0.002, C-2), 88.97 ( $\beta$-shift 2.212, C-3), $18.50\left(t, J_{\mathrm{CD}}=20.0 \mathrm{~Hz}, \alpha\right.$-shift - 0.295, C-4), $28.20(\beta$-shift $0.101, \mathrm{C}-5), 31.02(\gamma$-shift - 0.032, C-6), 22.18 ( 8 -shift - 0.004, C-7), 13.96 (C-8), 97.55 (C-1'), 30.30 (C-2'), 19.14 (C-3'), 25.39 (C-4'), 61.99 (C-5'); EI-MS: m/z 210 ([M-1] $\left.]^{+}\right)$, $182\left([\mathrm{M}-29]^{+}\right), 168\left([\mathrm{M}-43]^{+}\right), 154\left([\mathrm{M}-57]^{+}\right), 140\left([\mathrm{M}-71]^{+}\right), 127,111,101,94,85,80$, $68,55,41$.

\subsubsection{8 (4R)-[4-- $\left.\left.{ }^{2} \mathrm{H}_{1}\right]-2-\mathrm{Octyn}-1-\mathrm{ol}\right]^{174}$}

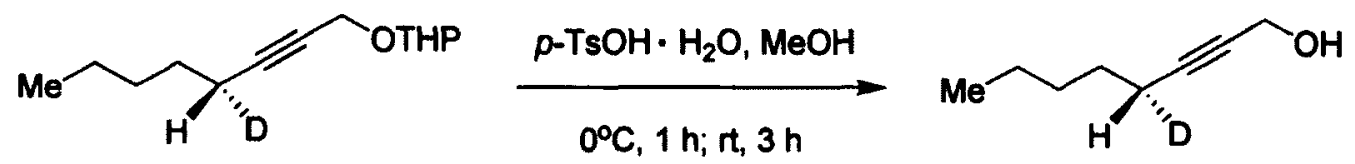

To a stirred solution of 2-((4R)-[4- $\left.\left.{ }^{2} \mathrm{H}_{1}\right]-\mathrm{octa}-2-\mathrm{ynyl}-1-\mathrm{oxy}\right) \operatorname{tetrahydropyran}(1.71$ $\mathrm{g}, 8.1 \mathrm{mmol})$ in methanol $(40 \mathrm{~mL})$ was added $p$-toluenesulfonic acid, monohydrate (28 $\mathrm{mg}$ ). The resulting reaction mixture turned cloudy, but became clear after 20 minutes of stirring. After stirring the reaction for $1 \mathrm{~h}$ at $0{ }^{\circ} \mathrm{C}$, it was warmed up to room temperature and allowed to stir another $3 \mathrm{~h}$. Saturated aqueous solution of $\mathrm{NaHCO}_{3}(9 \mathrm{~mL})$ was added to the reaction mixture and methanol was removed in vacuo. The resulting residue was extracted with $\mathrm{Et}_{2} \mathrm{O}(3 \times 25 \mathrm{~mL})$ and the collected organic layers were combined. Combined organic layers were worked up with brine $(3 \times 25 \mathrm{~mL})$ until the washings were found neutral to $\mathrm{pH}$ paper. After filtering the ether layer through anhydrous $\mathrm{Na}_{2} \mathrm{SO}_{4}$, the 
solvent was removed in vacuo and concentrated to afford the crude compound (646 mg, $63 \%)$. The crude compound was purified by flash chromatography using EtOAc:hexane (1:19) as eluent and pure compound was obtained as a light yellowish clear liquid (267 $\mathrm{mg}, 26 \%$ ). bp $50-54{ }^{\circ} \mathrm{C}$ at $0.1 \mathrm{~mm} \mathrm{Hg} ; R_{\mathrm{f}} 0.23$ (EtOAc:hexane, 1:3); the spectral data of the compound was similar to that reported above for the non-deuterated parent compound (see Section 6.2.2.2) and with the closely related compound in the literature ${ }^{10}$. IR (film): $v$ 3340 (O-H), 2958, 2932, 2862 (C-H) 2275, 2237 (C 三C), 2147 (C-D), 1469, 1348, $1161,1114,1010,722 \mathrm{~cm}^{-1} ;{ }^{1} \mathrm{H}$ NMR $\left(300 \mathrm{MHz}, \mathrm{CDCl}_{3}\right) \delta_{\mathrm{H}} 4.25(d d, J=5.8,2.2 \mathrm{~Hz}, 2$ $\left.\mathrm{H}, \mathrm{CH}_{2} \mathrm{OH}\right), 2.19(t t, J=7.0,2.2 \mathrm{~Hz}, 1 \mathrm{H}, \mathrm{CHDC} \equiv \mathrm{C}), 1.64(b r t, J=5.8 \mathrm{~Hz}, 1 \mathrm{H}, \mathrm{OH})$, $1.50\left(t d, J=7.0,6.9 \mathrm{~Hz}, 2 \mathrm{H}, \mathrm{CH}_{2} \mathrm{CHD}\right), 1.43-1.24\left(m, 4 \mathrm{H}, 2 \mathrm{x} \mathrm{CH}_{2}\right), 0.90(t, \mathrm{~J}=7.0 \mathrm{~Hz}$, $\left.3 \mathrm{H}, \mathrm{CH}_{3}\right) ;{ }^{13} \mathrm{C}$ NMR $\left(75.5 \mathrm{MHz}, \mathrm{CDCl}_{3}\right) \delta_{\mathrm{C}} 51.42(\delta$-shift 0.229, C-1), $78.28(\gamma$-shift $0.013, \mathrm{C}-2), 86.63$ ( $\beta$-shift $0.209, \mathrm{C}-3), 18.41\left(t, J_{\mathrm{CD}}=20.0 \mathrm{~Hz}, \alpha\right.$-shift $\left.-0.230, \mathrm{C}-4\right)$, 28.20 ( $\beta$-shift - 0.056, C-5), 31.01 ( $\gamma$-shift 0.021, C-6), 22.20 ( $\delta$-shift $0.055, C-7), 13.95$ (C-8); TMS Derivative EI-MS (rel. intensity): $m / z 198\left(1,[\mathrm{M}-1]^{+}\right), 184\left(26,[\mathrm{M}-15]^{+}\right)$, $166\left(2,[\mathrm{M}-33]^{+}\right), 154\left(100,[\mathrm{M}-45]^{+}\right), 143\left(6,[\mathrm{M}-56]^{+}\right), 128(14), 115(6), 110(6), 97$ (16), 83 (5), 75 (52), $59(9), 41$ (13).

\subsubsection{9 (4R)-1-(p-Tolylsulfonyloxy)-[4-- $\left.{ }^{2} H_{1}\right]-0 c t-2-$ yne $^{173}$}

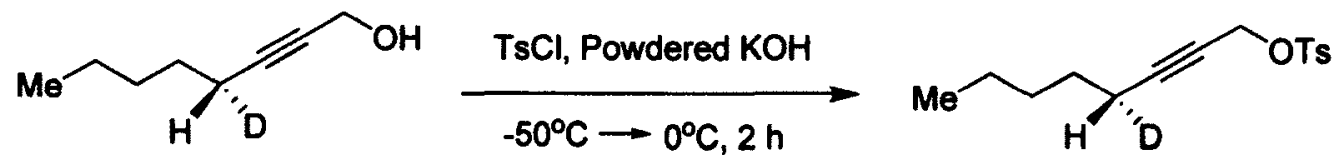

The title compound was synthesized from (4R)-[4- $\left.{ }^{2} \mathrm{H}_{1}\right]-2$-octyn-1-ol (213 mg, 1.7 mmol) and p-toluenesulfonyl chloride ( $400 \mathrm{mg}, 2.1 \mathrm{mmol}$ ) using the same experimental procedure that used for the preparation of 1-(p-tolylsulfonyloxy)-oct-2-yne. The desired 
compound was obtained as a pale yellowish oil ( $356 \mathrm{mg}, 76 \%$ ). $R_{\mathrm{f}} 0.41$ (EtOAc:hexane, 1:4); and the spectral data of the monodeuterated compound was similar to that reported with the non-deuterated parent compound (see Section 6.2.1.13). ${ }^{6}$ IR (film): $v$ 2957, 2932, 2863 (C-H), 2244 (C 三C), 2153 (C-D), 1599 (C=C, Ar), 1457, 1370, 1190, 1177, $1097,940,815 \mathrm{~cm}^{-1} ;{ }^{1} \mathrm{H}$ NMR $\left(300 \mathrm{MHz}, \mathrm{CDCl}_{3}\right) \delta_{\mathrm{H}} 7.82(d, J=8.2 \mathrm{~Hz}, 2 \mathrm{H}, \mathrm{Co}-\mathrm{Ph})$, $7.34(d, J=8.2 \mathrm{~Hz}, 2 \mathrm{H}, \mathrm{C} \underline{m}-\mathrm{Ph}), 4.70\left(d, J=2.3 \mathrm{~Hz}, 2 \mathrm{H}, \mathrm{CH}_{2} \mathrm{OTs}\right), 2.45(s, 3 \mathrm{H}$, $\left.\mathrm{CH}_{3} \mathrm{Ar}\right), 2.05(t t, J=7.0,2.3 \mathrm{~Hz}, 1 \mathrm{H}, \mathrm{CHDC} \equiv \mathrm{C}), 1.38(t d, J=7.0,6.6 \mathrm{~Hz}, 2 \mathrm{H}$, $\left.\mathrm{CH}_{2} \mathrm{CHD}\right), 1.33-1.21\left(m, 4 \mathrm{H}, 2 \times \mathrm{CH}_{2}\right), 0.88\left(t, J=6.7 \mathrm{~Hz}, 3 \mathrm{H}, \mathrm{CH}_{3}\right) ;{ }^{13} \mathrm{C}$ NMR $(75.5$ $\left.\mathrm{MHz}, \mathrm{CDCl}_{3}\right) \delta_{\mathrm{C}} 58.82(\delta$-shift 0.019, C-1), $71.80(\gamma$-shift 0.041, C-2), 90.63 ( $\beta$-shift $0.019, \mathrm{C}-3), 18.34\left(t, J_{\mathrm{CD}}=20.0 \mathrm{~Hz}, \alpha\right.$-shift $\left.-0.253, \mathrm{C}-4\right), 27.67(\beta$-shift $-0.066, \mathrm{C}-5)$, 30.90 ( $\gamma$-shift 0.003, C-6), 22.12 ( 8 -shift 0.030, C-7), 13.91 (C-8), 144.82 (Cipso - Ph), $128.12(\mathrm{C} \underline{\underline{o}}-\mathrm{Ph}), 129.72(\mathrm{C} \underline{m}-\mathrm{Ph}), 133.48(\mathrm{C} \underline{p}-\mathrm{Ph}), 21.64\left(\mathrm{CH}_{3}-\mathrm{Ph}\right)$; EI-MS (rel. intensity): $m / z 281\left(1,[\mathrm{M}]^{+}\right), 239\left(1,[\mathrm{M}-42]^{+}\right), 226(1), 202(1), 172\left(3,[\mathrm{M}-109]^{+}\right), 155$ (30, [M-126] $), 139\left(27,[\mathrm{M}-142]^{+}\right), 126(4), 117$ (5), 107 (10), 91 (100), 80 (17), 77 (19), $65(54), 53(27), 41(40)$.

\subsubsection{Methyl (14R)-[14- $\left.{ }^{2} \mathrm{H}_{1}\right]$-octadeca-9,12-diynoate ${ }^{176}$}

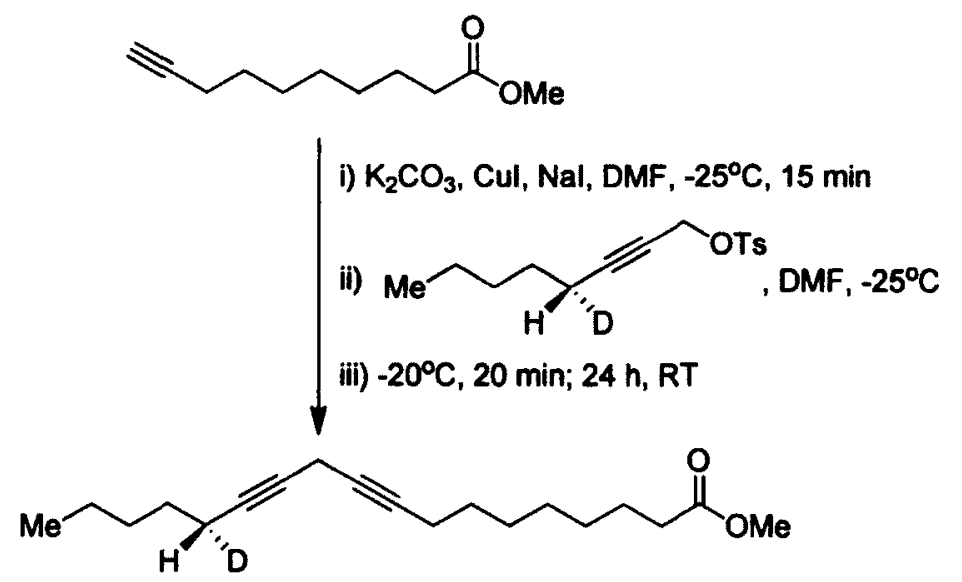


The following components were mixed in anhydrous DMF $(6 \mathrm{~mL})$ at $-25^{\circ} \mathrm{C}$ in the following order: methyl 9-decynoate $(113 \mathrm{mg}, 0.6 \mathrm{mmol}) ; \mathrm{K}_{2} \mathrm{CO}_{3}(86 \mathrm{mg}, 0.6 \mathrm{mmol})$; CuI (118 mg, $0.6 \mathrm{mmol})$; and $\mathrm{NaI}(93 \mathrm{mg}, 0.6 \mathrm{mmol})$. After stirring the reaction mixture for 15 minutes at $-25{ }^{\circ} \mathrm{C}$, a solution of $(4 R)-1-\left(p\right.$-tolylsulfonyloxy)-[4- $\left.{ }^{2} \mathrm{H}_{1}\right]$-oct-2-yne (168 $\mathrm{mg}, 0.6 \mathrm{mmol})$ in anhydrous DMF $(6 \mathrm{~mL})$ was added and stirred for another 20 minutes at $-20^{\circ} \mathrm{C}$. The reaction mixture was slowly warmed up to room temperature and kept stirring for next $24 \mathrm{~h}$. The reaction was quenched by pouring the mixture into a saturated solution of aqueous $\mathrm{NH}_{4} \mathrm{Cl}(40 \mathrm{~mL})$ and the aqueous layer was extracted with $\mathrm{Et}_{2} \mathrm{O}(4 \times 25 \mathrm{~mL})$. The collected ether layers were combined and worked up in the normal manner to afford a residue that was diluted with hexane $(30 \mathrm{~mL})$ and washed further with $\mathrm{H}_{2} \mathrm{O}(3 \times 20 \mathrm{~mL})$, brine $(3 \times 25 \mathrm{~mL})$. After drying the organic phase over anhydrous $\mathrm{Na}_{2} \mathrm{SO}_{4}$, the solvent was removed in vacuo to give the crude compound that was purified by flash chromatography using $\mathrm{Et}_{2} \mathrm{O}$ :hexane $(1: 49)$ as eluent. The title compound was isolated as a faint yellowish clear oil $(117 \mathrm{mg}, 65 \%) . R_{\mathrm{f}} 0.43\left(\mathrm{Et}_{2} \mathrm{O}\right.$ :hexane, $\left.1: 3\right)$; and the spectral data of the monodeuterated compound was similar to that reported above in Section 6.2.1.14 for the non-deuterated parent compound. IR (film): v 2933, 2859 (C-H), $2211(\mathrm{C} \equiv \mathrm{C}), 1740(\mathrm{C}=\mathrm{O}), 1459,1437,1248,1198,1172,1021,882,727 \mathrm{~cm}^{-1} ;{ }^{1} \mathrm{H}$ $\operatorname{NMR}\left(300 \mathrm{MHz}, \mathrm{CDCl}_{3}\right) \delta_{\mathrm{H}} 3.66\left(s, 3 \mathrm{H}, \mathrm{OCH}_{3}\right), 3.11(t d, J=3.5,2.3 \mathrm{~Hz}, 2 \mathrm{H}, \mathrm{C}-11)$, $2.29\left(t, J=7.5 \mathrm{~Hz}, 2 \mathrm{H}, \mathrm{CH}_{2} \mathrm{CO}_{2} \mathrm{Me}\right.$ ), 2.14 (Overlapping $t t, J=7.1,2.4 \mathrm{~Hz}, 3 \mathrm{H}, \mathrm{C}-8, \mathrm{C}-$ 14), $1.61\left(t t, J=7.5,6.9 \mathrm{~Hz}, 2 \mathrm{H}, \mathrm{CH}_{2} \mathrm{CH}_{2} \mathrm{CO}_{2} \mathrm{Me}\right), 1.55-1.42(m, 4 \mathrm{H}, \mathrm{C}-7, \mathrm{C}-15), 1.42-$ $1.21\left(m, 10 \mathrm{H}, 5 \times \mathrm{CH}_{2}\right), 0.88\left(t, J=7.1 \mathrm{~Hz}, 3 \mathrm{H}, \mathrm{CH}_{3}\right) ;{ }^{13} \mathrm{C} \mathrm{NMR}\left(75.5 \mathrm{MHz}, \mathrm{CDCl}_{3}\right) \delta_{\mathrm{C}}$ 174.23 (C-1), 34.03 (C-2), 24.86 (C-3), 28.73 (C-4), 28.62 (C-5), 28.62 (C-6), 28.98 (C7), 18.67 (C-8), 80.32 (C-9), 74.55 (C-10), 9.68 ( 8 -shift -0.045, C-11), 74.53 ( $\gamma$-shift - 
$0.015, \mathrm{C}-12), 80.46$ ( $\beta$-shift $-0.073, \mathrm{C}-13), 18.38\left(t, J_{\mathrm{CD}}=19.9 \mathrm{~Hz}, \alpha\right.$-shift $\left.-0.326, \mathrm{C}-14\right)$, 28.34 ( $\beta$-shift $-0.125, \mathrm{C}-15), 31.02$ ( $\gamma$-shift $-0.061, \mathrm{C}-16), 22.19(\delta$-shift $-0.031, \mathrm{C}-17)$, 13.95 (C-18), 51.41 (-OMe); EI-MS: $m / z 291$ ([M] $\left.]^{+}\right), 260$ ([M-31] $\left.]^{+}\right), 248$ ([M-43] $\left.]^{+}\right), 235$, $216,202,188,174,160,146,134,120,106,92,79,67,59,55,41$.

\subsubsection{Methyl (14R)-[14- $\left.{ }^{2} \mathrm{H}_{1}\right]-$ octadeca-9Z,12Z-dienoate ${ }^{173}$}

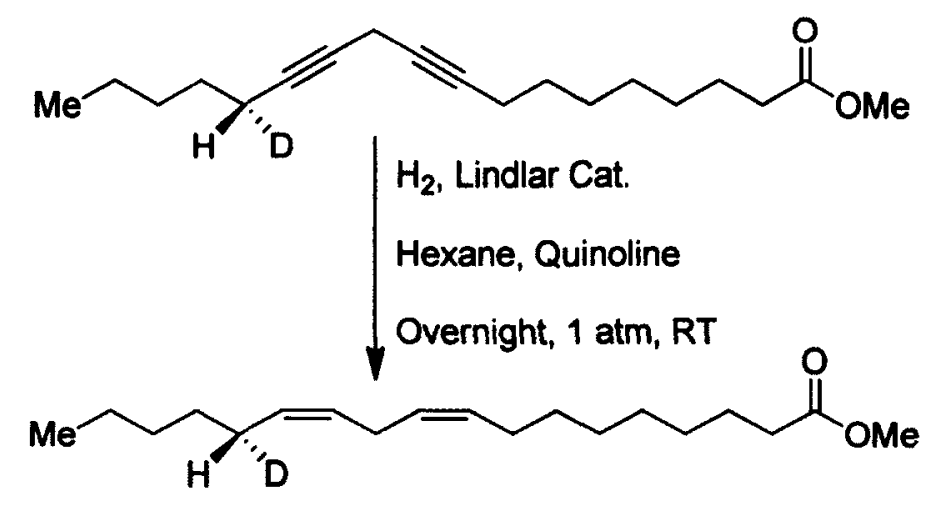

The experimental procedure used for the preparation of the title compound from methyl $(14 R)-\left[14-{ }^{2} \mathrm{H}_{1}\right]$-octadeca-9,12-diynoate $(83 \mathrm{mg}, 0.3 \mathrm{mmol})$ was identical to that described above for the preparation of $(8 R)-\left[8{ }^{2} \mathrm{H}_{1}\right]$ isotopomer (see Section 6.2.1.17). The title compound was isolated as a faint yellowish clear liquid ( $84 \mathrm{mg}, 99.8 \%$ ). $R_{\mathrm{f}} 0.44$ (EtOAc:hexane, 1:9); and the spectral data of the monodeuterated compound was similar to that obtained with the non-deuterated parent compound (see Section 6.2.1.16). IR (film): v 3010, 2954, 2927, 2856 (C-H), 2150 (C-D), 1743 (C=O), 1461, 1436, 1247, $1197,1172,1018,725 \mathrm{~cm}^{-1} ;{ }^{1} \mathrm{H}$ NMR $\left(300 \mathrm{MHz}, \mathrm{CDCl}_{3}\right) \delta_{\mathrm{H}} 5.45-5.27(m, 4 \mathrm{H}, 4 \mathrm{x}$ $\mathrm{HC}=\mathrm{C}), 3.67\left(s, 3 \mathrm{H}, \mathrm{OCH}_{3}\right), 2.77(t, J=6.00 \mathrm{~Hz}, 2 \mathrm{H}, \mathrm{C}-11), 2.30(t, J=7.5 \mathrm{~Hz}, 2 \mathrm{H}$, $\left.\mathrm{CH}_{2} \mathrm{CO}_{2} \mathrm{Me}\right), 2.04(m, 3 \mathrm{H}, \mathrm{C}-8, \mathrm{C}-14), 1.63\left(m, 2 \mathrm{H}, \mathrm{CH}_{2} \mathrm{CH}_{2} \mathrm{CO}_{2} \mathrm{Me}\right), 1.42-1.21(m, 14$ $\left.\mathrm{H}, 7 \times \mathrm{CH}_{2}\right), 0.89\left(t, J=6.8 \mathrm{~Hz}, 3 \mathrm{H}, \mathrm{CH}_{3}\right) ;{ }^{13} \mathrm{C} \mathrm{NMR}\left(75.5 \mathrm{MHz}, \mathrm{CDCl}_{3}\right) \delta_{\mathrm{C}} 174.30(\mathrm{C}-$ 
1), 34.11 (C-2), 24.95 (C-3), 29.11 (C-4), 29.16 (C-5), 29.13 (C-6), 29.60 (C-7), 27.19 (C-8), 130.05 (C-9), 128.06 (C-10), 25.64 ( $\delta$-shift - 0.001, C-11), 127.94 ( $\gamma$-shift 0.016, C-12), 130.17 ( $\beta$-shift $-0.053, \mathrm{C}-13), 26.85\left(t, J_{\mathrm{CD}}=19.2 \mathrm{~Hz}, \alpha\right.$-shift $\left.-0.363, \mathrm{C}-14\right)$, 29.27 ( $\beta$-shift - 0.096, C-15), 31.51 ( $\gamma$-shift - 0.031, C-16), 22.59 ( $\delta$-shift 0.001, C-17), 14.07 (C-18), 51.43 (-OMe); EI-MS: $m / z 295$ ([M] $\left.]^{+}\right), 264$ ([M-31] $\left.]^{+}\right), 235,221$ ([M-74] $\left.]^{+}\right)$, $207,192,179,164,150,136,121,110,96,81,67,55,41$.

\subsubsection{Methyl (14S)-[14- $\left.{ }^{2} \mathrm{H}_{1}\right]$ octadeca-9Z,12Z-dienoate ${ }^{173}$}

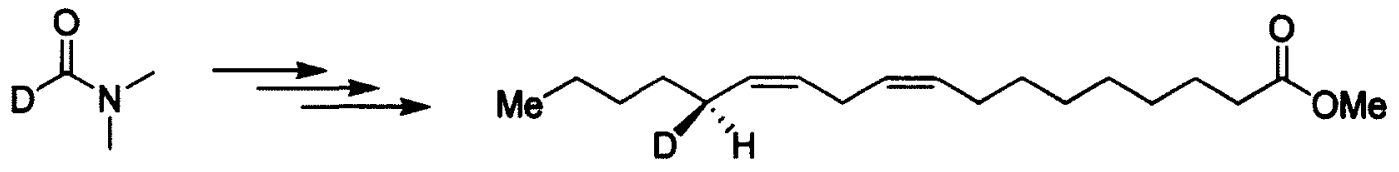

The title compound was synthesized in $1.5 \%$ overall yield from $\left[1-{ }^{2} \mathrm{H}_{1}\right] \mathrm{N}, \mathrm{N}$ dimethylformamide using the same procedures as described for the synthesis of the corresponding $(R)$-enantiomer. The chiral starting material was prepared by the reduction of $\left[1-{ }^{2} \mathrm{H}_{1}\right]$ pentanal using $(+)-(S)$-Alpine-Borane ${ }^{2}, R_{\mathrm{f}} 0.44$ (EtOAc:hexane, 1:9); The analytical data for the title compound was essentially identical to that obtained for the corresponding $(R)$-enantiomer and similar to that reported above with the non-deuterated parent compound (see Section 6.2.1.16). IR (film): v 3010, 2927, 2856 (C-H), 2155 (CD), 1743 (C=O), 1460, 1436, 1247, 1197, 1172, 1019, $726 \mathrm{~cm}^{-1}$; 'H NMR (300 MHz, $\left.\mathrm{CDCl}_{3}\right) \delta_{\mathrm{H}} 5.44-5.26(m, 4 \mathrm{H}, 4 \times \mathrm{HC}=\mathrm{C}), 3.67\left(s, 3 \mathrm{H}, \mathrm{OCH}_{3}\right), 2.77(t, J=5.9 \mathrm{~Hz}, 2 \mathrm{H}$, C-11), $2.31\left(t, J=7.6 \mathrm{~Hz}, 2 \mathrm{H}, \mathrm{CH}_{2} \mathrm{CO}_{2} \mathrm{Me}\right), 2.04(m, 3 \mathrm{H}, \mathrm{C}-8, \mathrm{C}-14), 1.61(m, 2 \mathrm{H}$, $\left.\mathrm{CH}_{2} \mathrm{CH}_{2} \mathrm{CO}_{2} \mathrm{Me}\right), 1.41-1.19\left(m, 14 \mathrm{H}, 7 \times \mathrm{CH}_{2}\right), 0.89\left(t, J=6.7 \mathrm{~Hz}, 3 \mathrm{H}, \mathrm{CH}_{3}\right) ;{ }^{13} \mathrm{C} \mathrm{NMR}$ (75.5 MHz, $\left.\mathrm{CDCl}_{3}\right) \delta_{\mathrm{C}} 174.31$ (C-1), $34.11(\mathrm{C}-2), 24.96(\mathrm{C}-3), 29.11(\mathrm{C}-4), 29.17$ (C-5), 29.13 (C-6), 29.60 (C-7), 27.20 (C-8), 130.05 (C-9), 128.06 (C-10), 25.64 ( $\delta$-shift 0.001, 
C-11), $127.94(\gamma$-shift $0.018, \mathrm{C}-12), 130.17(\beta$-shift $-0.051, \mathrm{C}-13), 26.86\left(t, J_{\mathrm{CD}}=19.2\right.$ $\mathrm{Hz}, \alpha$-shift $-0.359, \mathrm{C}-14), 29.27$ ( $\beta$-shift $-0.092, \mathrm{C}-15), 31.51(\gamma$-shift $-0.028, \mathrm{C}-16)$, 22.59 ( $\delta$-shift 0.005, C-17), 14.07 (C-18), 51.43 (-OMe); EI-MS: $m / z 295\left([\mathrm{M}]^{+}\right), 264$ $\left([\mathrm{M}-31]^{+}\right), 235,221\left([\mathrm{M}-74]^{+}\right), 207,192,179,164,150,136,121,110,96,81,67,55,41$.

\subsubsection{Assessment of Enantiomeric Purity of the Chiral Synthetic Intermediates}

\subsubsection{1 (4R)-[4- $\left.{ }^{2} \mathrm{H}_{1}\right]-2-$ octen-1-ol}

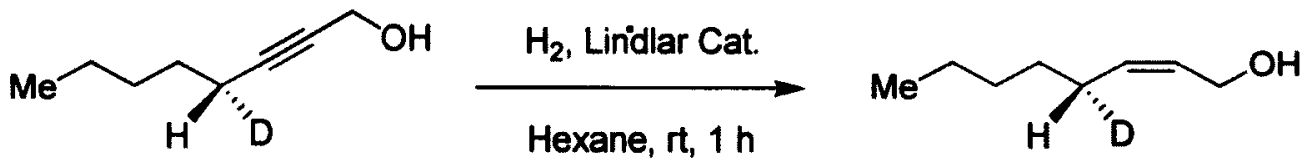

Lindlar catalyst $(18 \mathrm{mg})$ was added to a solution of $(4 R)-\left[4-{ }^{2} \mathrm{H}_{1}\right]-2-\mathrm{octyn}-1-\mathrm{ol}(50$ $\mathrm{mg}, 0.4 \mathrm{mmol})$ in Spectrograde hexane $(10 \mathrm{~mL})$ containing quinoline $(180 \mu \mathrm{L}, 2 \mathrm{~mol} \%$ with respect to solvent). Hydrogenation was allowed to proceed as described above for 1 $\mathrm{h}$ at atmospheric pressure and the reaction mixture worked up in the normal manner to yield the crude alcohol product as a brownish liquid (37 $\mathrm{mg}, 71 \%$ ). $R_{\mathrm{f}} 0.25$ (EtOAc:hexane, 1:3); and the spectral data of the compound was similar to that reported in the literature for the non-deuterated parent compound. ${ }^{1} \mathrm{H}$ NMR $\left(300 \mathrm{MHz}, \mathrm{CDCl}_{3}\right) \delta_{\mathrm{H}}$ 5.69-5.49 $(m, 2 \mathrm{H}, 2 \times \mathrm{HC}=\mathrm{C}), 4.19\left(d, J=5.8 \mathrm{~Hz}, 2 \mathrm{H}, \mathrm{CH}_{2} \mathrm{OH}\right), 2.05(t d t, \mathrm{~J}=7.0,1.9$ $\mathrm{Hz}, 1 \mathrm{H}, \mathrm{CHDC}=\mathrm{C}), 1.42-1.19\left(m, 6 \mathrm{H}, 3 \times \mathrm{CH}_{2}\right), 0.89\left(t, J=6.8 \mathrm{~Hz}, 3 \mathrm{H}, \mathrm{CH}_{3}\right) ;{ }^{13} \mathrm{C}$ NMR (75.5 MHz, $\left.\mathrm{CDCl}_{3}\right) \delta_{\mathrm{C}} 58.77$ ( $\delta$-shift 0.275, C-1), 128.34 ( $\gamma$-shift 0.027, C-2), 133.17 ( $\beta$-shift 0.072, C-3), $27.03\left(t, J_{\mathrm{CD}}=19.2 \mathrm{~Hz}, \alpha\right.$-shift - 0.327, C-4), $29.18(\beta$-shift - 
0.074, C-5), 31.37 ( $\gamma$-shift - 0.013, C-6), 22.50 ( $\delta$-shift 0.019, C-7), 14.01 (C-8); EI-MS: $m / z 201\left([\mathrm{M}-1]^{+}\right), 186\left([\mathrm{M}-16]^{+}\right), 172\left([\mathrm{M}-30]^{+}\right), 157\left([\mathrm{M}-45]^{+}\right), 144,129,114,101,82$, $75,73,68,59,55,41$.

\subsubsection{2 (2R)-[2- $\left.{ }^{2} \mathrm{H}_{1}\right]-$ Hexanoic acid ${ }^{204,210}$}

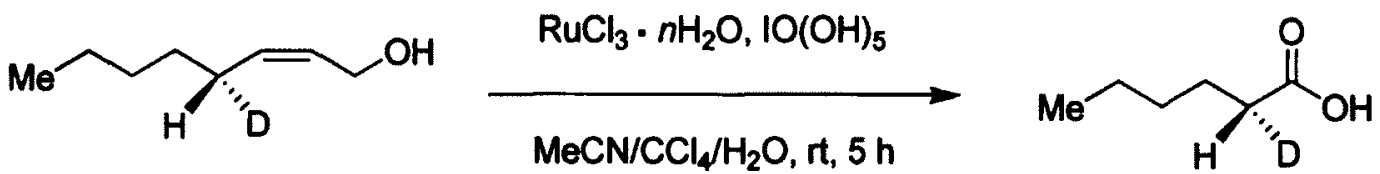

To a stirred solution of $(4 R)-\left[4-{ }^{2} \mathrm{H}_{1}\right]-2$-octen-1-ol $(29 \mathrm{mg}, 0.2 \mathrm{mmol})$ in $2: 2: 3$ $\mathrm{CH}_{3} \mathrm{CN}(1.2 \mathrm{~mL}), \mathrm{CCl}_{4}(1.2 \mathrm{~mL})$ and $\mathrm{H}_{2} \mathrm{O}(1.8 \mathrm{~mL})$ was added periodic acid $(266 \mathrm{mg}, 1.2$ $\mathrm{mmol}$ ) and the reaction was allowed to proceed at room temperature for $3 \mathrm{~min} . \mathrm{RuCl}_{3}$. $n \mathrm{H}_{2} \mathrm{O}(3 \mathrm{mg}, 0.01 \mathrm{mmol})$ was added to the reaction mixture and stirring at room temperature was continued for another $5 \mathrm{~h}$ during which the solution turned from brown to blackish in color. The product was isolated by extraction with ether $(3 \times 10 \mathrm{~mL})$ and the combined organic extracts were dried over $\mathrm{Na}_{2} \mathrm{SO}_{4}$. The solvent was evaporated in vacuo to give the crude compound ( $26 \mathrm{mg}$, estimated $99 \%$ ) that was used in the next step without purification. $R_{\mathrm{f}} 0.80$ (MeOH: $\left.\mathrm{CH}_{2} \mathrm{Cl}_{2}, 1: 19\right)$; The spectral data of the compound was similar to that of the non-deuterated parent compound reported in the literature. ${ }^{1} \mathrm{H}$ NMR $\left(300 \mathrm{MHz}, \mathrm{CDCl}_{3}\right) \delta_{\mathrm{H}} 9.03\left(\right.$ br s, $\left.1 \mathrm{H}, \mathrm{CO}_{2} \mathrm{H}\right), 2.33(t t, J=7.6,2.2 \mathrm{~Hz}, 1 \mathrm{H}$, $\left.\mathrm{CHDCO}_{2} \mathrm{H}\right), 1.64\left(t d, J=7.2,6.8 \mathrm{~Hz}, 2 \mathrm{H}, \mathrm{CH}_{2} \mathrm{CHD}\right), 1.40-1.20\left(m, 4 \mathrm{H}, 2 \times \mathrm{CH}_{2}\right), 0.90$ $\left(t, J=6.9 \mathrm{~Hz}, 3 \mathrm{H}, \mathrm{CH}_{3}\right) ;{ }^{13} \mathrm{C} \mathrm{NMR}\left(75.5 \mathrm{MHz}, \mathrm{CDCl}_{3}\right) \delta_{\mathrm{C}} 180.14$ ( $\beta$-shift $\left.-0.576, \mathrm{C}-1\right)$, $33.74\left(t, J_{\mathrm{CD}}=19.6 \mathrm{~Hz}, \alpha\right.$-shift $\left.-0.371, \mathrm{C}-2\right), 24.31(\beta$-shift $-0.053, \mathrm{C}-3), 31.18(\gamma$-shift - 
0.029, C-4), 22.29 ( $\delta$-shift -0.006, C-5), 13.87 (C-6); TMS Derivative EI-MS: $m / z 188$ $\left([\mathrm{M}-1]^{+}\right), 176\left([\mathrm{M}-13]^{+}\right), 147\left([\mathrm{M}-42]^{+}\right), 100\left([\mathrm{M}-89]^{+}\right), 73\left([\mathrm{M}-116]^{+}\right), 59,45,28$.

\subsubsection{3 (S)-2-Methoxy-2-oxo-1-phenylethyl $(2 R)-\left[2-{ }^{2} \mathbf{H}_{1}\right]-$ hexanoate $^{206}$}

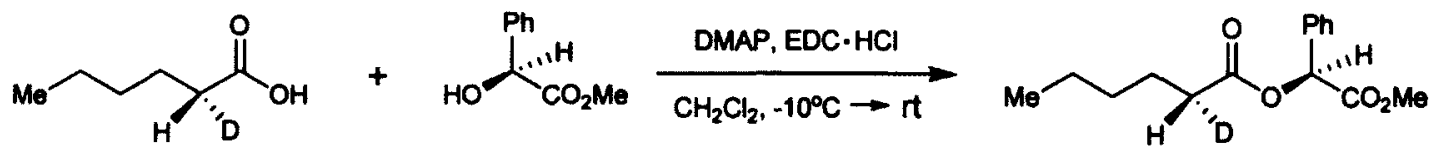

$(+)-(S)$-Methyl 2-hydroxy-2-phenylethanoate $(31 \mathrm{mg}, 0.2 \mathrm{mmol})$ and $\mathrm{N}-(3-$ dimethylaminopropyl)- $\mathrm{N}^{\prime}$-ethylcarbodiimide hydrochloride $(36 \mathrm{mg}, 0.2 \mathrm{mmol})$ were added to a stirred solution of $(2 R)-\left[2-{ }^{2} \mathrm{H}_{1}\right]$-hexanoic acid $(22 \mathrm{mg}, 0.2 \mathrm{mmol})$ and 4 (dimethylamino)pyridine $(5 \mathrm{mg})$ in $\mathrm{CH}_{2} \mathrm{Cl}_{2}(5 \mathrm{~mL})$ at $-10{ }^{\circ} \mathrm{C}$. The resultant mixture was stirred at $-10^{\circ} \mathrm{C}$ for $1 \mathrm{~h}$ and at room temperature for $3 \mathrm{~h}$. The suspension was diluted with $\mathrm{CH}_{2} \mathrm{Cl}_{2}$ and washed with water $(3 \times 15 \mathrm{~mL})$, brine $(2 \times 15 \mathrm{~mL})$ and filtered through anhydrous $\mathrm{Na}_{2} \mathrm{SO}_{4}$. The solvent was removed under reduced pressure and the crude compound was purified by flash chromatography using ethyl acetate:hexane (1:9) as eluent to furnish the pure compound as a light yellowish oil $(16 \mathrm{mg}$, overall yield from $(4 R)$-[4- $\left.{ }^{2} \mathrm{H}_{1}\right]$-2-octyn-1-ol: $20 \%$ ). $R_{\mathrm{f}} 0.40$ (EtOAc:hexane, 1:4); The spectral data of the compound was similar to that reported before with the analogous material. ${ }^{9} \mathrm{H}$ NMR $\left(300 \mathrm{MHz}, \mathrm{CDCl}_{3}\right) \delta_{\mathrm{H}} 7.48(d d, J=6.6,3.3 \mathrm{~Hz}, 1 \mathrm{H}, \mathrm{C} \underline{-}-\mathrm{Ph}), 7.43-7.35(m, 4 \mathrm{H}, \mathrm{C} \underline{m}-$ $\mathrm{Ph}, \mathrm{C} \underline{\mathrm{a}}-\mathrm{Ph}), 5.940(s, 1 \mathrm{H}, \mathrm{CHPhCO} 2 \mathrm{Me}), 3.73\left(s, 3 \mathrm{H}, \mathrm{OCH}_{3}\right), 2.472(2 S, t t, J=7.6,2.2$ $\left.\mathrm{Hz}, 1 \mathrm{H}, \mathrm{CHDCO}_{2} \mathrm{R}^{\prime}, 26.002 \%\right), 2.415\left(2 R, t t, J=7.6,2.2 \mathrm{~Hz}, 1 \mathrm{H}, \mathrm{CHDCO}_{2} \mathrm{R}^{\prime}, 73.998\right.$ \%), $1.691\left(t d, J=7.6,7.1 \mathrm{~Hz}, 2 \mathrm{H}, \mathrm{CH}_{2} \mathrm{CHD}\right), 1.42-1.19\left(m, 4 \mathrm{H}, 2 \times \mathrm{CH}_{2}\right), 0.89(t, J=$ $\left.7.0 \mathrm{~Hz}, 3 \mathrm{H}, \mathrm{CH}_{3}\right)$. 


\subsubsection{4 (S)-2-Methoxy-2-oxo-1-phenylethyl (2S)-[2- $\left.{ }^{2} \mathrm{H}_{1}\right]$ hexanoate ${ }^{206}$}<smiles>[2H]C([2H])[C@H]([2H])C#CCO</smiles>

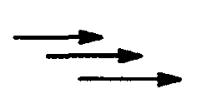<smiles>[2H]C([2H])(OC(=O)[C@@H](O)CCCC)c1ccccc1</smiles>

The title compound was synthesized in $14 \%$ overall yield from $(4 R)-\left[4-{ }^{2} \mathrm{H}_{1}\right]-2-$ octyn-1-ol using the same procedures as described for the synthesis of the corresponding $(S, R)$-diastereomer. The analytical data for the title compound is given here: $R_{\mathrm{f}} \mathbf{0 . 4 0}$ (EtOAc:hexane, 1:4); The spectral data of the compound was similar to that reported before with the analogous material. ${ }^{206}{ }^{1} \mathrm{H}$ NMR $\left(300 \mathrm{MHz}, \mathrm{CDCl}_{3}\right) \delta_{\mathrm{H}} 7.48(d d, J=6.6$, 3.3 Hz, $1 \mathrm{H}, \mathrm{C} \underline{p}-\mathrm{Ph}), 7.43-7.35(m, 4 \mathrm{H}, \mathrm{C} \underline{\underline{o}}-\mathrm{Ph}, \mathrm{C} \underline{m}-\mathrm{Ph}), 5.941(s, 1 \mathrm{H}$, $\left.\mathrm{CHPhCO}_{2} \mathrm{Me}\right), 3.73\left(s, 3 \mathrm{H}, \mathrm{OCH}_{3}\right), 2.470\left(2 S, t t, J=7.4,2.2 \mathrm{~Hz}, 1 \mathrm{H}, \mathrm{CHDCO}_{2} \mathrm{R}^{\prime}\right.$, $86.401 \%), 2.415\left(2 R, t t, J=7.4,2.2 \mathrm{~Hz}, 1 \mathrm{H}, \mathrm{CHDCO}_{2} \mathrm{R}^{\prime}, 13.599 \%\right), 1.691(t d, J=7.4$, $\left.7.2 \mathrm{~Hz}, 2 \mathrm{H}, \mathrm{CH}_{2} \mathrm{CHD}\right), 1.42-1.21\left(m, 4 \mathrm{H}, 2 \times \mathrm{CH}_{2}\right), 0.90\left(\mathrm{t}, J=7.0 \mathrm{~Hz}, 3 \mathrm{H}, \mathrm{CH}_{3}\right) ;{ }^{13} \mathrm{C}$ $\operatorname{NMR}\left(75.5 \mathrm{MHz}, \mathrm{CDCl}_{3}\right) \delta_{\mathrm{C}} 174.06(\mathrm{C}-1), 33.60\left(t, J_{\mathrm{CD}}=19.7 \mathrm{~Hz}, \mathrm{C}-2\right), 24.36(\mathrm{C}-3)$, 31.11 (C-4), 22.24 (C-5), 13.85 (C-6), $74.21\left(\mathrm{CHCO}_{2} \mathrm{Me}\right), 169.38\left(\mathrm{CO}_{2} \mathrm{Me}\right), 52.53$ $\left(\mathrm{OCH}_{3}\right), 138.35($ Cipso $-\mathrm{Ph}), 129.16(\mathrm{C} \underline{\underline{a}}-\mathrm{Ph}), 128.75(\mathrm{C} \underline{m}-\mathrm{Ph}), 127.57(\mathrm{C} \underline{p}-\mathrm{Ph})$.

\subsubsection{5 (R)-[1(S)- $\left.{ }^{2} \mathrm{H}_{1}\right]-$ pentyl 3,3,3-trifluoro-2-methoxy-2-phenylpropanoate ${ }^{189}$}

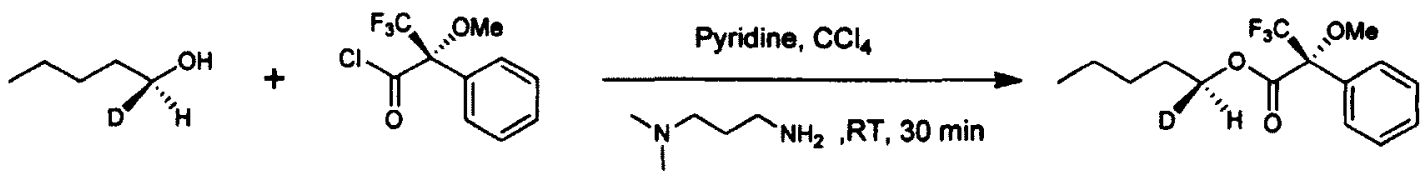

To an oven-dried $5 \mathrm{~mL}$ reaction vial were added dry pyridine $(440 \mu \mathrm{L}),(+)-(S)-\alpha-$ methoxy- $\alpha$-trifluoromethylphenylacetyl chloride $(50 \mathrm{mg}, 0.2 \mathrm{mmol}$ ), carbon tetrachloride $(430 \mu \mathrm{L})$ and the substrate $1-(1 S)-\left[1-{ }^{2} \mathrm{H}_{1}\right]-$ pentanol $(13 \mathrm{mg}, 0.1 \mathrm{mmol})$, accordingly. After 
stirring the reaction mixture for $30 \mathrm{~min}$ at $\mathrm{rt}$ the reaction was judged to be complete when crystalline pyridine hydrochloride ceased to precipitate. At this point, excess 3dimethylamino-1-propylamine $(50 \mu \mathrm{L})$ was added and stirring was continued for additional $10 \mathrm{~min}$. The reaction mixture was diluted with ether $(10 \mathrm{~mL})$ and washed successively with chilled $2.0 \mathrm{M} \mathrm{HCl}(8 \mathrm{~mL})$, chilled saturated $\mathrm{Na}_{2} \mathrm{CO}_{3}(8 \mathrm{~mL})$ and brine $(8 \mathrm{~mL})$. The solvent was removed in vacuo after filtration through anhydrous $\mathrm{Na}_{2} \mathrm{SO}_{4}$ and the filtrate was diluted with $\mathrm{CCl}_{4}$ and evaporated three times to remove last traces of ether. The product was obtained as a light yellowish clear oil ( $40 \mathrm{mg}, 92 \%) . R_{\mathrm{f}} 0.57$ (EtOAc:hexane, 1:4); ${ }^{1} \mathrm{H}$ NMR $\left(300 \mathrm{MHz}, \mathrm{CDCl}_{3}\right) \delta_{\mathrm{H}} 7.54(d d, J=6.5,2.6 \mathrm{~Hz}, 2 \mathrm{H}, \mathrm{Co}$ $\mathrm{Ph}), 7.46-7.38(m, 3 \mathrm{H}, 2 \times \mathrm{Cm}-\mathrm{Ph}, \mathrm{C} p-\mathrm{Ph}), 4.322(t t, J=6.6,1.4 \mathrm{~Hz}, 1 \mathrm{H}$, CHDOMTPA, $96.622 \%), 4.287(t t, J=6.7,1.3 \mathrm{~Hz}, 1 \mathrm{H}$, CHDOMTPA, $3.378 \%), 3.57$ $\left(q, J=1.2 \mathrm{~Hz}, 3 \mathrm{H}, \mathrm{OCH}_{3}\right), 1.697\left(t d, J=7.0,6.6 \mathrm{~Hz}, 2 \mathrm{H}, \mathrm{CH}_{2} \mathrm{CHDOMTPA}\right), 1.39-1.24$ $\left(m, 4 \mathrm{H}, 2 \times \mathrm{CH}_{2}, \mathrm{C}-3, \mathrm{C}-4\right), 0.89\left(t, J=6.8 \mathrm{~Hz}, 3 \mathrm{H}, \mathrm{CH}_{3}\right)$.

\subsubsection{6 $(R)-\left[1(R)-{ }^{2} \mathbf{H}_{1}\right]-$ pentyl 3,3,3-trifluoro-2-methoxy-2-phenylpropanoate ${ }^{189}$}

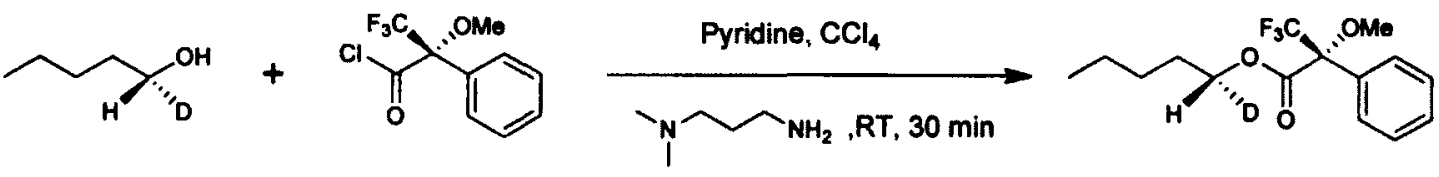

The title compound was synthesized in $89 \%$ overall yield from $1-(1 S)-\left[1{ }^{2} \mathrm{H}_{1}\right]-$ pentanol using the same procedure as described for the synthesis of the corresponding $(R, S)$-diastereomer. The analytical data for the title compound is given here: $R_{\mathrm{f}} 0.57$ (EtOAc:hexane, 1:4); ${ }^{1} \mathrm{H}$ NMR $\left(300 \mathrm{MHz}, \mathrm{CDCl}_{3}\right) \delta_{\mathrm{H}} 7.54(d d, J=6.6,2.5 \mathrm{~Hz}, 2 \mathrm{H}, \mathrm{C} \underline{\mathrm{o}}-$ $\mathrm{Ph}), 7.46-7.38(m, 3 \mathrm{H}, 2 \times \mathrm{C} \underline{m}-\mathrm{Ph}, \mathrm{C} p-\mathrm{Ph}), 4.322(t t, J=6.7,1.4 \mathrm{~Hz}, 1 \mathrm{H}$, CHDOMTPA, $3.378 \%$ \%), $4.287(t t, J=6.7,1.3 \mathrm{~Hz}, 1 \mathrm{H}$, CHDOMTPA, $96.622 \%), 3.57$ 
$\left(q, J=1.1 \mathrm{~Hz}, 3 \mathrm{H}, \mathrm{OCH}_{3}\right), 1.697\left(t d, J=7.0,6.7 \mathrm{~Hz}, 2 \mathrm{H}, \mathrm{CH}_{2} \mathrm{CHDOMTPA}\right), 1.39-1.24$ $\left(m, 4 \mathrm{H}, 2 \mathrm{X} \mathrm{CH}_{2}, \mathrm{C}-3, \mathrm{C}-4\right), 0.89\left(t, J=6.8 \mathrm{~Hz}, 3 \mathrm{H}, \mathrm{CH}_{3}\right)$.

\subsubsection{7 (R)-[1(R)- $\left.{ }^{2} \mathrm{H}_{1}\right]-$-Oct-2-yne 3,3,3-trifluoro-2-methoxy-2-phenylpropanoate ${ }^{189}$}

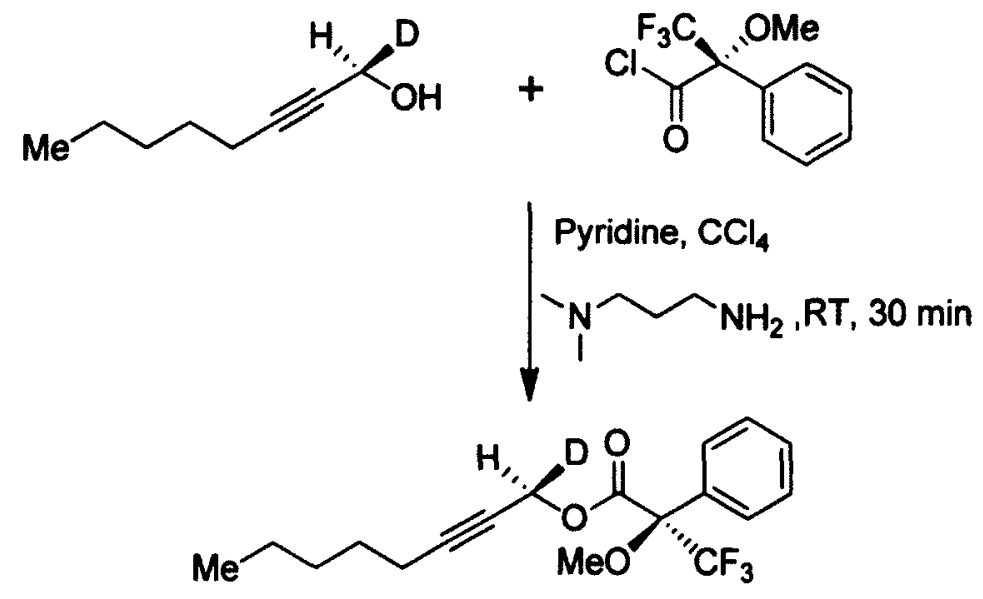

To a small round-bottom flask the following reagents were added according to the order: anhydrous pyridine $(0.4 \mathrm{~mL}, 5.3 \mathrm{mmol}), \quad(+)-(S)-\alpha$-methoxy- $\alpha-$ trifluoromethylphenylacetyl chloride $(50 \mathrm{mg}, 0.2 \mathrm{mmol})$, carbon tetrachloride $(0.4 \mathrm{~mL}$, $4.4 \mathrm{mmol})$ and the substrate alcohol, $(1 R)-\left[1-{ }^{2} \mathrm{H}_{1}\right]$ oct-2-yn-1-ol $(18 \mathrm{mg}, 0.1 \mathrm{mmol})$. The reaction was then allowed to stir for 30 minutes at room temperature (completion of the reaction was evidenced by no more formation of crystalline pyridine hydrochloride) subsequently was added excess 1,4-diaminobutane $(0.04 \mu \mathrm{L}, 0.4 \mathrm{mmol})$ and stirred for additional 5 minutes. The reaction mixture was diluted with diethyl ether $(10 \mathrm{~mL})$ before being washed with chilled $2.0 \mathrm{M} \mathrm{HCl}(8 \mathrm{~mL})$, chilled saturated aqueous $\mathrm{Na}_{2} \mathrm{CO}_{3}(8 \mathrm{~mL})$, saturated $\mathrm{NaCl}(8 \mathrm{~mL})$ and finally dried over anhydrous $\mathrm{Na}_{2} \mathrm{SO}_{4}$. The solvent was removed in vacuo and the content was diluted with $\mathrm{CCl}_{4}$ and evaporated three times to remove the last traces of ether. The product was obtained as a colorless liquid $(47 \mathrm{mg}, 96$ 
\%). The ${ }^{1} \mathrm{H}$ NMR spectrum was recorded with the crude sample and compared with reference standard to calculate the enantiomeric excess. ${ }^{1} \mathrm{H}$ NMR $\left(300 \mathrm{MHz}, \mathrm{CDCl}_{3}\right) \delta_{\mathrm{H}}$ 7.59-7.47 (m, $2 \mathrm{H}, \mathrm{C} \underline{o}-\mathrm{Ph}), 7.44-7.35$ (m, $3 \mathrm{H}, 2 \times \mathrm{Cm}-\mathrm{Ph}, \mathrm{C} \underline{p}-\mathrm{Ph}), 4.927$ (1R, $t, J=$ $1.7 \mathrm{~Hz}, 1 \mathrm{H}$, CHDOMTPA, $95.548 \%), 4.810(1 S, t, J=1.8 \mathrm{~Hz}, 1 \mathrm{H}$, CHDOMTPA, $4.452 \%), 3.59\left(q, J=1.2 \mathrm{~Hz}, 3 \mathrm{H}, \mathrm{OCH}_{3}\right), 2.204\left(t t, J=7.1,2.13 \mathrm{~Hz}, 2 \mathrm{H}, \mathrm{CH}_{2} \mathrm{C} \equiv \mathrm{C}\right)$, 1.58-1.49 (m, $\left.2 \mathrm{H}, \mathrm{CH}_{2} \mathrm{CH}_{2} \mathrm{C} \equiv \mathrm{C}\right), 1.38-1.28\left(m, 4 \mathrm{H}, 2 \times \mathrm{CH}_{2}\right), 0.89(t, J=7.2 \mathrm{~Hz}, 3 \mathrm{H}$, $\left.\mathrm{CH}_{3}\right)$.

\subsubsection{8 (R)-[1(S)- $\left.{ }^{2} \mathrm{H}_{1}\right]-$ Oct-2-yne 3,3,3-trifluoro-2-methoxy-2-phenylpropanoate ${ }^{189}$}

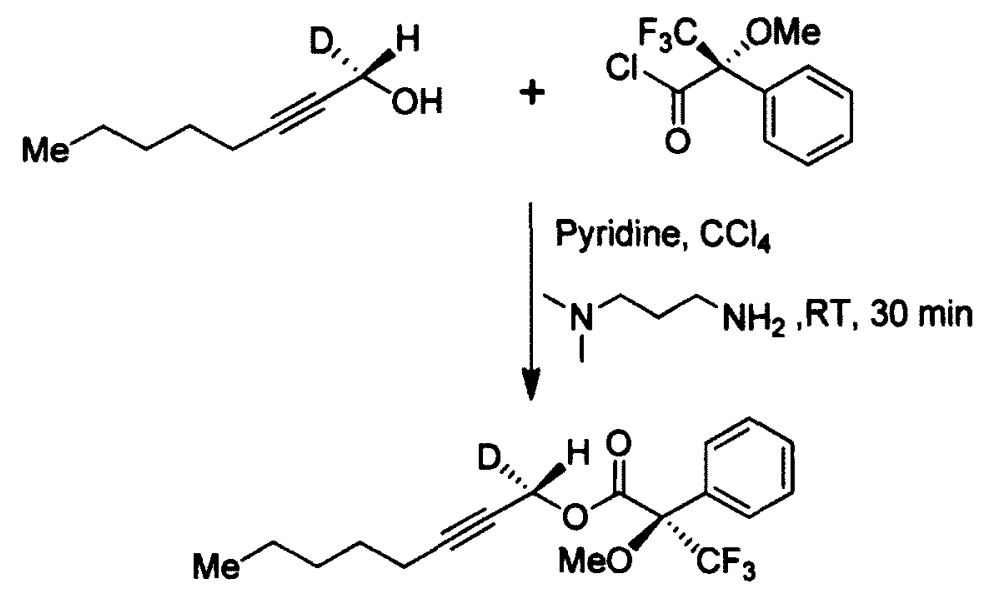

The title compound was synthesized in $95 \%$ overall yield from $(1 S)-\left[1-{ }^{2} \mathrm{H}_{1}\right]-2-$ octyn-1-ol using the same procedure as described for the synthesis of the corresponding $(R, R)$-diastereomer. The analytical data for the title compound is given here: ${ }^{1} \mathrm{H}$ NMR $\left(300 \mathrm{MHz}, \mathrm{CDCl}_{3}\right) \delta_{\mathrm{H}}$ 7.57-7.47 (m, $\left.2 \mathrm{H}, \mathrm{C} \underline{\underline{o}}-\mathrm{Ph}\right)$, 7.44-7.36 (m, $3 \mathrm{H}, 2$ x C$\underline{m}-\mathrm{Ph}, \mathrm{C} \underline{p}-$ $\mathrm{Ph}), 4.925(1 R, t, J=1.8 \mathrm{~Hz}, 1 \mathrm{H}$, CHDOMTPA, $3.108 \%), 4.808(1 S, t, J=1.9 \mathrm{~Hz}, 1 \mathrm{H}$, CHDOMTPA, $96.892 \%), 3.59\left(q, J=1.2 \mathrm{~Hz}, 3 \mathrm{H}, \mathrm{OCH}_{3}\right), 2.205(t t, J=7.1,1.9 \mathrm{~Hz}, 2$ 
$\left.\mathrm{H}, \mathrm{CH}_{2} \mathrm{C} \equiv \mathrm{C}\right), 1.60-1.52\left(m, 2 \mathrm{H}, \mathrm{CH}_{2} \mathrm{CH}_{2} \mathrm{C} \equiv \mathrm{C}\right), 1.40-1.27\left(m, 4 \mathrm{H}, 2 \times \mathrm{CH}_{2}\right), 0.89(t$, $\left.J=6.0 \mathrm{~Hz}, 3 \mathrm{H}, \mathrm{CH}_{3}\right)$.

\subsubsection{Methyl $\left[11,11-{ }^{2} \mathrm{H}_{2}\right]$-Linoleate Synthesis}

\subsubsection{1 $\left[1,1-^{2} \mathrm{H}_{2}\right]-1-\left(p-\right.$ Tolylsulfonyloxy)-oct-2-yne ${ }^{173}$}

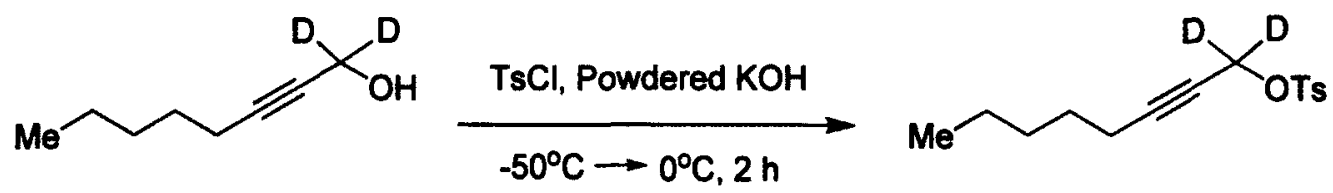

The estimated compound was synthesized from $\left[1,1-{ }^{2} \mathrm{H}_{2}\right]-2-o c t y n-1-\mathrm{ol}(2.5 \mathrm{~g}$, $19.5 \mathrm{mmol}$ ) using the similar procedure as described in 6.2.1.13, as a clear colorless liquid (5.4 g, $98 \%$ ). $R_{\mathrm{f}} 0.46$ (EtOAc:hexane, 1:4); and the spectral data of this material was similar to that reported above for the non-deuterated material (see Section 6.2.1.13). IR (film): v 2957, 2933, 2862 (C-H), 2255 (C 三C), 2154 (C-D), 1598 (C=C, Ar), 1458, $1367,1190,1177,1098,954,846 \mathrm{~cm}^{-1} ;{ }^{1} \mathrm{H}$ NMR $\left(300 \mathrm{MHz}, \mathrm{CDCl}_{3}\right) \delta_{\mathrm{H}} 7.81(d, J=8.2$ $\mathrm{Hz}, 2 \mathrm{H}, \mathrm{C} \underline{o}-\mathrm{Ph}), 7.34(d, J=8.2 \mathrm{~Hz}, 2 \mathrm{H}, \mathrm{C} \underline{m}-\mathrm{Ph}), 2.45\left(s, 3 \mathrm{H}, \mathrm{CH}_{3} \mathrm{Ar}\right), 2.06(t, J=$ $\left.7.0 \mathrm{~Hz}, 2 \mathrm{H}, \mathrm{CH}_{2} \mathrm{C} \equiv \mathrm{C}\right), 1.38\left(t t, J=7.0,6.9 \mathrm{~Hz}, 2 \mathrm{H}, \mathrm{CH}_{2} \mathrm{CH}_{2} \mathrm{C} \equiv \mathrm{C}\right), 1.33-1.18(m, 4$ $\left.\mathrm{H}, 2 \times \mathrm{CH}_{2}\right), 0.88\left(t, J=6.7 \mathrm{~Hz}, 3 \mathrm{H}, \mathrm{CH}_{3}\right) ;{ }^{13} \mathrm{C} \mathrm{NMR}\left(75.5 \mathrm{MHz}, \mathrm{CDCl}_{3}\right) \delta_{\mathrm{C}} 58.33(p$, $J_{\mathrm{CD}}=21.7 \mathrm{~Hz}, \alpha$-shift $\left.-0.475, \mathrm{C}-1\right), 71.68(\beta$-shift $-0.085, \mathrm{C}-2), 90.58(\gamma$-shift $-0.029, \mathrm{C}-$ 3), 18.59 ( $\delta$-shift - 0.003, C-4), 27.74 (C-5), 30.90 (C-6), 22.09 (C-7), 13.90 (C-8), 144.80 (Cipso - Ph), $128.09(\mathrm{C} \underline{\underline{a}}-\mathrm{Ph}), 129.70(\mathrm{C} \underline{m}-\mathrm{Ph}), 133.44(\mathrm{C} \underline{p}-\mathrm{Ph}), 21.63\left(\mathrm{CH}_{3}-\right.$ Ph); EI-MS: $m / z 241$ ([M-41]+), 227 ([M-55] $\left.]^{+}\right), 203,173$ ([M-109] $\left.]^{+}\right), 155$ ([M-127] $)$, $139\left([\mathrm{M}-143]^{+}\right), 119,107,91,77,65,54,41$. 


\subsubsection{Methyl $\left[11,11-{ }^{2} \mathrm{H}_{2}\right]$-octadeca-9,12-diynoate}

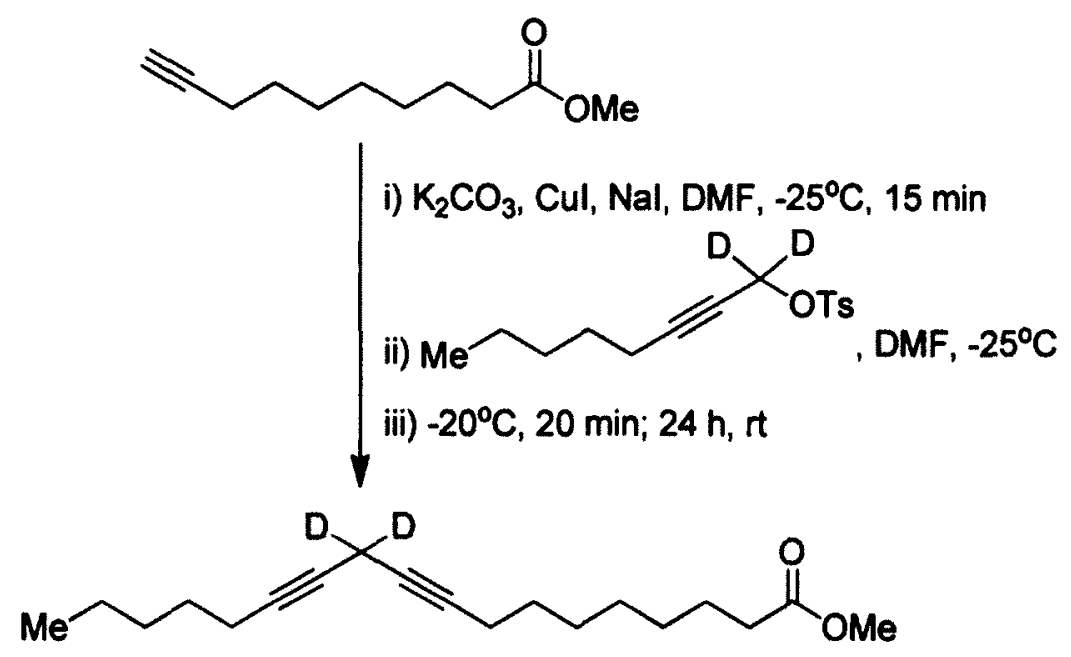

The title compound was obtained in a similar manner to that described in 6.2.1.14, from $\left[1,1-{ }^{2} \mathrm{H}_{2}\right]-1-(p$-tolylsulfonyloxy)-oct-2-yne $(2.64 \mathrm{~g}, 9.3 \mathrm{mmol})$ and methyl 9decynoate $(1.7 \mathrm{~g}, 9.3 \mathrm{mmol})$ as a colorless clear oil $(2.04 \mathrm{~g}, 74 \%) . \quad R_{\mathrm{f}} 0.21$ (EtOAc:hexane, 1:19); and the spectral data of the compound was similar to that reported above for the non-deuterated parent compound (see Section 6.2.1.14). IR (film): $v 2933$, $2859(\mathrm{C}-\mathrm{H}), 1741(\mathrm{C}=\mathrm{O}), 1461,1436,1247,1198,1172,1098,1016,726 \mathrm{~cm}^{-1} ;{ }^{1} \mathrm{H}$ NMR $\left(300 \mathrm{MHz}, \mathrm{CDCl}_{3}\right) \delta_{\mathrm{H}} 3.66\left(s, 3 \mathrm{H}, \mathrm{OCH}_{3}\right), 2.29\left(t, J=7.5 \mathrm{~Hz}, 2 \mathrm{H}, \mathrm{CH}_{2} \mathrm{CO}_{2} \mathrm{Me}\right), 2.14$ (Overlapping $t, J=7.0 \mathrm{~Hz}, 4 \mathrm{H}, \mathrm{C}-8, \mathrm{C}-14$ ), $1.61(t t, J=7.5,7.2 \mathrm{~Hz}, 2 \mathrm{H}$, $\left.\mathrm{CH}_{2} \mathrm{CH}_{2} \mathrm{CO}_{2} \mathrm{Me}\right), 1.48(m, 4 \mathrm{H}, \mathrm{C}-7, \mathrm{C}-15), 1.42-1.23\left(m, 10 \mathrm{H}, 5 \times \mathrm{CH}_{2}\right), 0.88(t, J=7.0$ $\left.\mathrm{Hz}, 3 \mathrm{H}, \mathrm{CH}_{3}\right) ;{ }^{13} \mathrm{C}$ NMR $\left(75.5 \mathrm{MHz}, \mathrm{CDCl}_{3}\right) \delta_{\mathrm{C}} 174.24(\mathrm{C}-1), 34.04(\mathrm{C}-2), 24.88(\mathrm{C}-3)$, 28.75 (C-4), 28.63 (C-5), 28.63 (C-6), 29.00 (C-7), 18.68 ( $\delta$-shift - 0.029, C-8), $80.35(\gamma-$ shift - 0.023, C-9), 74.51 ( $\beta$-shift - 0.069, C-10), $9.28\left(p, J_{\mathrm{CD}}=20.0 \mathrm{~Hz}, \alpha\right.$-shift -0.445 , C-11), 74.37 ( $\beta$-shift - 0.067, C-12), 80.51 ( $\gamma$-shift - 0.023, C-13), 18.68 ( $\delta$-shift - 0.029, C-14), 28.45 (C-15), 31.06 (C-16), 22.20 (C-17), 13.97 (C-18), 51.42 (-OMe); EI-MS: 
$m / z 292\left([\mathrm{M}]^{+}\right), 277\left([\mathrm{M}-15]^{+}\right), 261\left([\mathrm{M}-31]^{+}\right), 205,189,177,161,149,135,121,107$, $93,79,74,67,59,43$.

\subsubsection{Methyl $\left[11,11-{ }^{2} \mathrm{H}_{2}\right]$-octadeca-9Z,12Z-dienoate ${ }^{173}$}

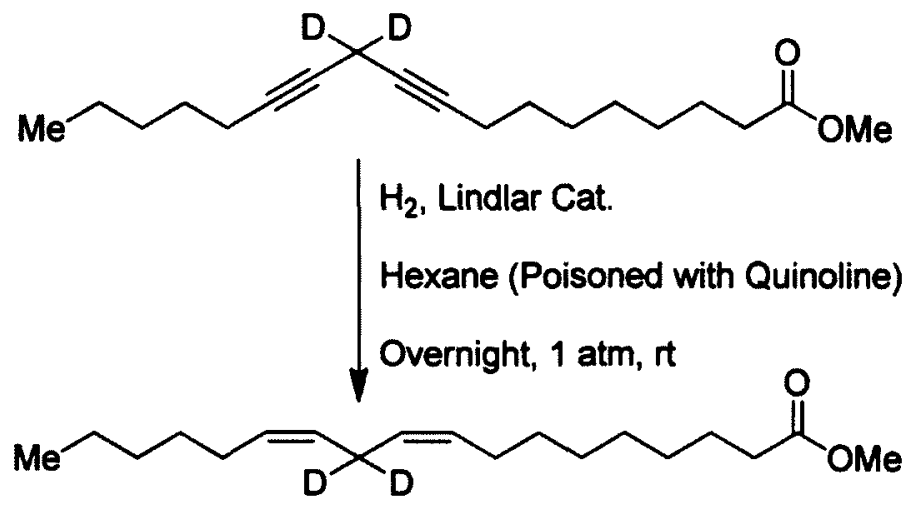

The title compound was synthesized using the similar procedure that described in 6.2.1.16, from methyl $\left[11,11-{ }^{2} \mathrm{H}_{2}\right]$-octadeca-9,12-diynoate $(250 \mathrm{mg}, 0.9 \mathrm{mmol})$ as a colorless oil (253 mg, $100 \%$ ). $R_{\mathrm{f}} 0.45$ (EtOAc:hexane, 1:9); and the spectral data of the dideuterated material was similar to that reported above for the non-deuterated parent compound (see Section 6.2.1.16). IR (film): v 3009, 2929, 2856 (C-H), 2200, 2099 (CD), $1742(\mathrm{C}=\mathrm{O}), 1461,1437,1248,1198,1172,1015,726 \mathrm{~cm}^{-1}$; ${ }^{1} \mathrm{H}$ NMR $(300 \mathrm{MHz}$, $\left.\mathrm{CDCl}_{3}\right) \delta_{\mathrm{H}} 5.46-5.28(m, 4 \mathrm{H}, 4 \times \mathrm{xC}=\mathrm{C}), 3.66\left(s, 3 \mathrm{H}, \mathrm{OCH}_{3}\right), 2.30(t, J=7.5 \mathrm{~Hz}, 2 \mathrm{H}$, $\mathrm{CH}_{2} \mathrm{CO}_{2} \mathrm{Me}$ ), 2.05 (Overlapping $\left.t d, J=7.0,6.8 \mathrm{~Hz}, 4 \mathrm{H}, \mathrm{C}-8, \mathrm{C}-14\right), 1.62(t t, J=7.5,7.1$ $\left.\mathrm{Hz}, 2 \mathrm{H}, \mathrm{CH}_{2} \mathrm{CH}_{2} \mathrm{CO}_{2} \mathrm{Me}\right), 1.42-1.20\left(m, 14 \mathrm{H}, 7 \times \mathrm{CH}_{2}\right), 0.89\left(t, J=6.8 \mathrm{~Hz}, 3 \mathrm{H}, \mathrm{CH}_{3}\right)$; ${ }^{13} \mathrm{C}$ NMR $\left(75 \mathrm{MHz}, \mathrm{CDCl}_{3}\right.$ ) $\delta_{\mathrm{C}} 174.33$ (C-1), 34.10 (C-2), 24.94 (C-3), 29.10 (C-4), 29.16 (C-5), 29.12 (C-6), 29.60 (C-7), 27.20 ( $\delta$-shift - 0.005, C-8), 130.07 ( $\gamma$-shift 0.014, C-9), 127.93 ( $\beta$-shift - 0.124, C-10); $24.81\left(p, J_{\mathrm{CD}}=9.8 \mathrm{~Hz}, \alpha\right.$-shift $\left.-0.829, \mathrm{C}-11\right), 127.80(\beta-$ shift - 0.124, C-12), 130.24 ( $\gamma$-shift 0.013, C-13), 27.21 ( $\delta$-shift - 0.009, C-14), 29.36 (C- 
15), 31.53 (C-16), 22.58 (C-17), 14.07 (C-18), 51.45 (-OMe); EI-MS: $m / z 296\left([\mathrm{M}]^{+}\right)$, $281\left([\mathrm{M}-15]^{+}\right), 265\left([\mathrm{M}-31]^{+}\right), 236,222\left([\mathrm{M}-74]^{+}\right), 207,193,180,165,150,137,125$, $111,97,83,69,55,41$.

\subsubsection{Methyl $\left[14,14-{ }^{2} \mathbf{H}_{2}\right]$-Linoleate Synthesis}

\subsubsection{1 $\left[1,1-{ }^{2} \mathrm{H}_{2}\right]-$ pentanol}

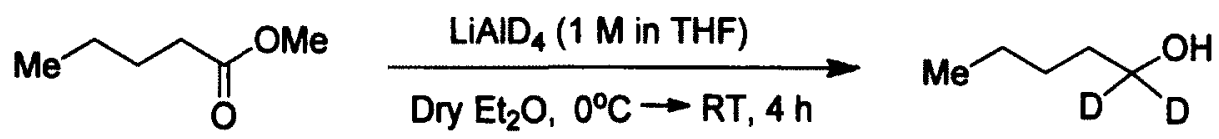

The title compound was synthesized by following the similar procedure that described in 6.2.3.3, from methyl valerate $(6.0 \mathrm{~g}, 51.7 \mathrm{mmol})$ using lithium aluminium deuteride $(1.0 \mathrm{M}$ in anhydrous THF, $57 \mathrm{ml}, 56.8 \mathrm{mmol})$ as reagent and the estimated compound was obtained as a clear colorless liquid $(4.25 \mathrm{~g}, 92 \%)$. bp $138-140{ }^{\circ} \mathrm{C} ; R_{\mathrm{f}} 0.18$ (EtOAc:hexane, 1:3); and the spectral data of the compound was similar to that reported above for the non-deuterated compound (see Section 6.2.4.3). IR (film): v $3336(\mathrm{O}-\mathrm{H})$, 2959, 2930, 2862 (C-H), 2194, 2093 (C-D), 1467, 1379, 1161, 1130, 1083, $970 \mathrm{~cm}^{-1} ;{ }^{1} \mathrm{H}$ NMR (300 MHz, $\left.\mathrm{CDCl}_{3}\right) \delta_{\mathrm{H}} 1.96(s, 1 \mathrm{H}, \mathrm{OH}), 1.53\left(b r t, J=6.4 \mathrm{~Hz}, 2 \mathrm{H}, \mathrm{CH}_{2} \mathrm{CD}_{2}\right)$, 1.39-1.26 (m, $\left.4 \mathrm{H}, 2 \times \mathrm{CH}_{2}\right), 0.89\left(t, J=6.8 \mathrm{~Hz}, 3 \mathrm{H}, \mathrm{CH}_{3}\right) ;{ }^{13} \mathrm{C}$ NMR $(75.5 \mathrm{MHz}$, $\left.\mathrm{CDCl}_{3}\right) \delta_{\mathrm{C}} 62.14\left(p, J_{\mathrm{CD}}=21.5 \mathrm{~Hz}, \alpha\right.$-shift $\left.-0.669, \mathrm{C}-1\right), 32.20(\beta$-shift $-0.187, \mathrm{C}-2)$, 27.85 ( $\gamma$-shift - 0.035, C-3), 22.46 ( $\delta$-shift 0.013, C-4), 13.99 (C-5); TMS Derivative EIMS: $m / z 162\left([\mathrm{M}]^{+}\right), 147\left([\mathrm{M}-15]^{+}\right), 131\left([\mathrm{M}-31]^{+}\right), 117\left([\mathrm{M}-45]^{+}\right), 105,91,75,59,45$. 


\subsubsection{1-Bromopentane ${ }^{211}$}

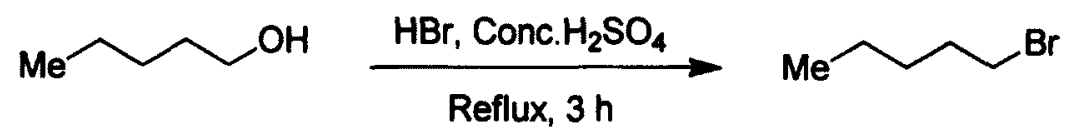

To a stirred solution of $\mathrm{HBr}\left(48 \%\right.$ in $\left.\mathrm{H}_{2} \mathrm{O}, 7.5 \mathrm{~g}, 44.5 \mathrm{mmol}\right)$ and concentrated $\mathrm{H}_{2} \mathrm{SO}_{4}(2.2 \mathrm{~mL})$ was added 1-pentanol $(3.08 \mathrm{~g}, 35 \mathrm{mmol})$ followed by conc. $\mathrm{H}_{2} \mathrm{SO}_{4}(1.0$ $\mathrm{mL}$ ) in several portions and allowed the reaction mixture to reflux for $3 \mathrm{~h}$. Direct shortpath distillation of the contents in vacuo $(15 \mathrm{~mm} \mathrm{Hg})$ afforded the title compound as a colorless clear liquid ( $4.56 \mathrm{~g}, 86 \%$ ). bp $28-30^{\circ} \mathrm{C}$ at $15 \mathrm{~mm} \mathrm{Hg} ; R_{\mathrm{f}} 0.59$ (EtOAc:hexane, 1:19); IR (film): v 2960, 2933, 2873, 2861 (C-H), 1466, 1296, 1250, 1208, 1036, 920, $729 \mathrm{~cm}^{-1}$; ' $\mathrm{H}$ NMR $\left(300 \mathrm{MHz}, \mathrm{CDCl}_{3}\right) \delta_{\mathrm{H}} 3.41\left(t, J=6.9 \mathrm{~Hz}, 2 \mathrm{H}, \mathrm{CH}_{2} \mathrm{Br}\right), 1.87(t t, J=$ 7.1, $\left.6.9 \mathrm{~Hz}, 2 \mathrm{H}, \mathrm{CH}_{2} \mathrm{CH}_{2} \mathrm{Br}\right), 1.48-1.28\left(m, 4 \mathrm{H}, 2 \times \mathrm{CH}_{2}\right), 0.92(t, J=7.1 \mathrm{~Hz}, 3 \mathrm{H}$, $\mathrm{CH}_{3}$ ); ${ }^{13} \mathrm{C}$ NMR (75.5 MHz, $\mathrm{CDCl}_{3}$ ) $\delta_{\mathrm{C}} 34.01$ (C-1), 32.56 (C-2), 30.34 (C-3), 21.89 (C4), 13.91 (C-5); EI-MS: $m / z 151\left(\mathrm{Br}^{81},[\mathrm{M}-1]^{+}\right), 149\left(\mathrm{Br}^{79},[\mathrm{M}-1]^{+}\right), 121\left([\mathrm{M}-30]^{+}\right), 119$ $\left([\mathrm{M}-30]^{+}\right), 109\left([\mathrm{M}-42]^{+}\right), 107\left([\mathrm{M}-42]^{+}\right), 81,79,69,55,53,41$.

\subsubsection{1-[1,1- $\left.{ }^{2} \mathrm{H}_{2}\right]-$ Bromopentane}

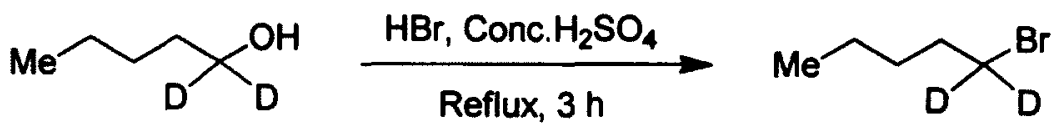

The title compound was synthesized in a similar manner to that described in 6.2.7.2, from $\left[1,1-{ }^{2} \mathrm{H}_{2}\right]$-pentanol $(4.23 \mathrm{~g}, 47.0 \mathrm{mmol})$ as colorless liquid $(5.88 \mathrm{~g}, 82 \%)$. bp $40-44{ }^{\circ} \mathrm{C}$ at $15 \mathrm{~mm} \mathrm{Hg} ; R_{\mathrm{f}} 0.59$ (EtOAc:hexane, 1:19); and the spectral data of this material was similar to that reported above for the non-deuterated parent compound and 
the analogous compound reported in the literature. ${ }^{14}$ IR (film): $v$ 2959, 2933, 2873, 2861 (C-H), 2158 (C-D), 1466, 1380, 1217, 1121, 992, 932, $731 \mathrm{~cm}^{-1}$; ${ }^{1} \mathrm{H}$ NMR $(300 \mathrm{MHz}$, $\left.\mathrm{CDCl}_{3}\right) \delta_{\mathrm{H}} 1.85\left(b r t, J=7.1 \mathrm{~Hz}, 2 \mathrm{H}, \mathrm{CH}_{2} \mathrm{CD}_{2} \mathrm{Br}\right), 1.48-1.28\left(m, 4 \mathrm{H}, 2 \times \mathrm{CH}_{2}\right), 0.91(t, J$ $\left.=7.1 \mathrm{~Hz}, 3 \mathrm{H}, \mathrm{CH}_{3}\right) ;{ }^{13} \mathrm{C}$ NMR $\left(75.5 \mathrm{MHz}, \mathrm{CDCl}_{3}\right) \delta_{\mathrm{C}} 33.46\left(p, J_{\mathrm{CD}}=23.0 \mathrm{~Hz}, \alpha\right.$-shift $0.543, \mathrm{C}-1), 32.32$ ( $\beta$-shift - 0.243, C-2), 30.27 ( $\gamma$-shift - 0.068, C-3), $21.89(\delta$-shift $0.008, \mathrm{C}-4), 13.89(\mathrm{C}-5)$; EI-MS: $m / z 153\left(\mathrm{Br}^{81},[\mathrm{M}-1]^{+}\right), 151\left(\mathrm{Br}^{79},[\mathrm{M}-1]^{+}\right), 121$ ([M$\left.32]^{+}\right), 119\left([\mathrm{M}-32]^{+}\right), 109\left([\mathrm{M}-44]^{+}\right), 107\left([\mathrm{M}-44]^{+}\right), 81,79,71,55,51,42$.

\subsubsection{2-Octyn-1-ol ${ }^{212}$}

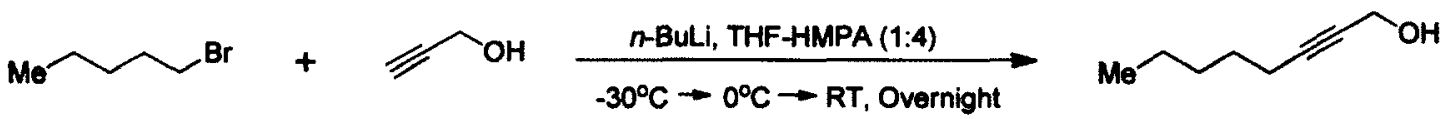

$n$-Butyllithium $(2.5 \mathrm{M}$ in hexane, $21 \mathrm{~mL}, 52.3 \mathrm{mmol})$ was added dropwise to a stirred solution of propargyl alcohol $(1.4 \mathrm{~mL}, 23.8 \mathrm{mmol})$ in anhydrous THF-HMPA $(24: 19.5 \mathrm{~mL})$ at -25 to $30^{\circ} \mathrm{C}$. After stirring the reaction mixture for $30 \mathrm{~min}$ at $0{ }^{\circ} \mathrm{C}$, a solution of 1-bromopentane $(4.0 \mathrm{~g}, 26.2 \mathrm{mmol})$ in anhydrous HMPA $(20 \mathrm{~mL})$ was added to it in portions over $25-30 \mathrm{~min}$, keeping the temperature below $0{ }^{\circ} \mathrm{C}$. The reaction mixture was warmed up to room temperature and stirred overnight. The reaction was terminated by pouring the mixture to chilled water $(120 \mathrm{~mL})$ and the aqueous layer was extracted several times with ether $(4 \times 60 \mathrm{~mL})$. The combined organic layers were washed with $\mathrm{H}_{2} \mathrm{O}(80 \mathrm{~mL})$, saturated $\mathrm{NaCl}(2 \times 80 \mathrm{~mL})$ and dried over anhydrous $\mathrm{Na}_{2} \mathrm{SO}_{4}$. The solvent was removed in vacuo to afford the crude compound as a yellowish oil (2.68 g, $61 \%$ ). bp $48-50{ }^{\circ} \mathrm{C}$ at $0.1 \mathrm{~mm} \mathrm{Hg} ; R_{\mathrm{f}} 0.25$ (EtOAc:hexane, 1:3); IR (film): $v$ 3351 (O-H), 2957, 2932, 2860 (C-H), 2285, 2224 (C三C), 1460, 1299, 1198, 1136, 
1029, $987 \mathrm{~cm}^{-1} ;{ }^{1} \mathrm{H}$ NMR $\left(300 \mathrm{MHz}, \mathrm{CDCl}_{3}\right) \delta_{\mathrm{H}} 4.21\left(t, J=2.2 \mathrm{~Hz}, 2 \mathrm{H}, \mathrm{CH}_{2} \mathrm{OH}\right), 2.36$ $(s, 1 \mathrm{H}, \mathrm{OH}), 2.17\left(t t, J=7.2,2.2 \mathrm{~Hz}, 2 \mathrm{H}, \mathrm{CH}_{2} \mathrm{C} \equiv \mathrm{C}\right), 1.48(t t, J=7.2,7.1 \mathrm{~Hz}, 2 \mathrm{H}$, $\left.\mathrm{CH}_{2} \mathrm{CH}_{2} \mathrm{C} \equiv \mathrm{C}\right), 1.40-1.22\left(m, 4 \mathrm{H}, 2 \times \mathrm{CH}_{2}\right), 0.87\left(t, J=7.1 \mathrm{~Hz}, 3 \mathrm{H}, \mathrm{CH}_{3}\right) ;{ }^{13} \mathrm{C}$ NMR $\left(75 \mathrm{MHz}, \mathrm{CDCl}_{3}\right) \delta_{\mathrm{C}} 51.13(\mathrm{C}-1), 78.30(\mathrm{C}-2), 86.34$ (C-3), 18.63 (C-4), 28.26 (C-5), 30.98 (C-6), 22.13 (C-7), 13.85 (C-8); TMS Derivative EI-MS: $m / z 197$ ([M-1] $\left.]^{+}\right), 183$ $\left([\mathrm{M}-15]^{+}\right), 165\left([\mathrm{M}-33]^{+}\right), 153\left([\mathrm{M}-45]^{\dagger}\right), 142\left([\mathrm{M}-56]^{+}\right), 127,115,109,96,83,75,59$, 41.

\subsubsection{5 $\left.\left[4,4-{ }^{2} \mathrm{H}_{2}\right]-0 \mathrm{ct}-2-\mathrm{yn}-1-\mathrm{o}\right]^{212}$}

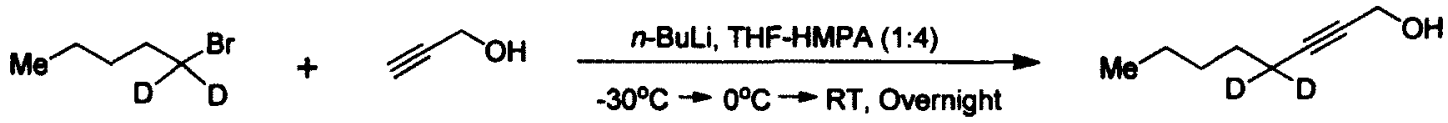

The anticipated compound was obtained in a similar manner as described in 6.2.7.4, from 1-[1,1- $\left.{ }^{2} \mathrm{H}_{2}\right]$-bromopentane $(4.0 \mathrm{~g}, 26.2 \mathrm{mmol})$ and propargyl alcohol $(1.33$ $\mathrm{g}, 23.8 \mathrm{mmol}$ ). The crude compound was purified by flash chromatography using EtOAchexane (1:9) as eluent to give the pure compound as a clear colorless oil $(1.86 \mathrm{~g}, 61 \%)$. bp $50-53{ }^{\circ} \mathrm{C}$ at $0.1 \mathrm{~mm} \mathrm{Hg} ; R_{\mathrm{f}} 0.33$ (EtOAc:hexane, 1:3); and the spectral data of the title compound was found similar to that reported above for the non-deuterated counterpart. IR (film): v 3336 (O-H), 2931, 2862 (C-H), 2245, 2201 (C $\equiv C), 2100$ (C-D), 1459, $1379,1234,1184,1040,999 \mathrm{~cm}^{-1} ;{ }^{1} \mathrm{H}$ NMR $\left(300 \mathrm{MHz}, \mathrm{CDCl}_{3}\right) \delta_{\mathrm{H}} 4.24(d, J=5.8 \mathrm{~Hz}, 2$ $\left.\mathrm{H}, \mathrm{CH}_{2} \mathrm{OH}\right), 1.78(t, J=5.8 \mathrm{~Hz}, 1 \mathrm{H}, \mathrm{OH}), 1.49\left(t, J=6.7 \mathrm{~Hz}, 2 \mathrm{H}, \mathrm{CH}_{2} \mathrm{CD}_{2}\right), 1.42-1.22$ $\left(m, 4 \mathrm{H}, 2 \times \mathrm{CH}_{2}\right), 0.89\left(t, J=7.1 \mathrm{~Hz}, 3 \mathrm{H}, \mathrm{CH}_{3}\right) ;{ }^{13} \mathrm{C} \mathrm{NMR}\left(75.5 \mathrm{MHz}, \mathrm{CDCl}_{3}\right) \delta_{\mathrm{C}} 51.36$ ( $\delta$-shift 0.175, C-1), 78.31 ( $\gamma$-shift 0.040, C-2), $86.57\left(\beta\right.$-shift 0.147, C-3), $18.11\left(p, J_{\mathrm{CD}}=\right.$ $19.9 \mathrm{~Hz}, \alpha$-shift - 0.528, C-4), 28.10 ( $\beta$-shift - 0.159, C-5), 30.98 ( $\gamma$-shift - 0.013, C-6), 
22.19 ( $\delta$-shift 0.046, C-7), 13.93 (C-8); EI-MS: $m / z 127$ ([M-1] $\left.]^{+}\right), 113$ ([M-15] ), 97 ([M$\left.31]^{+}\right), 85\left([\mathrm{M}-43]^{+}\right), 80,72,67,57,41$; TMS Derivative EI-MS: $m / z 199\left([\mathrm{M}-1]^{+}\right), 185$ $\left([\mathrm{M}-15]^{+}\right), 167\left([\mathrm{M}-33]^{+}\right), 155\left([\mathrm{M}-45]^{+}\right), 144\left([\mathrm{M}-56]^{+}\right), 129,115,109,98,83,75,59$, 41.

\subsubsection{6 $\left[4,4-{ }^{2} \mathrm{H}_{2}\right]-1-\left(p-\right.$ Tolylsulfonyloxy)-oct-2-yne $\mathrm{e}^{173}$}

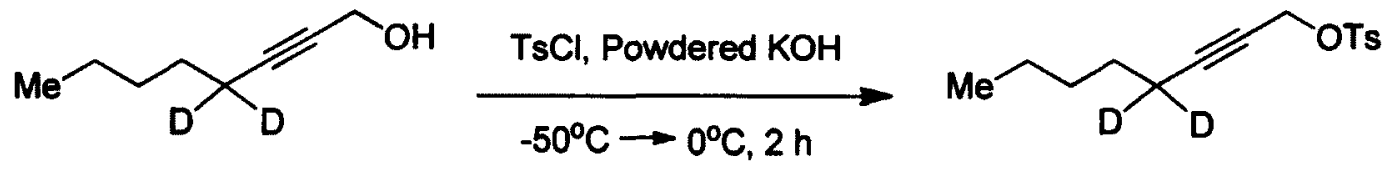

The title compound was synthesized from $\left[4,4-{ }^{2} \mathrm{H}_{2}\right]-2$-octyn-1-ol $(1.5 \mathrm{~g}, 11.7$ mmol) using the same experimental procedure that used in 6.2.1.13, and the purified compound was isolated as a pale yellowish oil $\left(3.23 \mathrm{~g}, 98 \%\right.$ ). $R_{\mathrm{f}} 0.41$ (EtOAc:hexane, 1:4); and the spectral data of the di-deuterio material was similar to that reported above for the non-deuterated compound. IR (film): $v$ 2958, 2932, $2863(\mathrm{C}-\mathrm{H}), 2251$ (C $\equiv \mathrm{C})$, 2107 (C-D), 1599 (C=C, Ar), 1456, 1370, 1190, 1177, 1097, 941, 838, $815 \mathrm{~cm}^{-1} ;{ }^{1} \mathrm{H}$ $\operatorname{NMR}\left(300 \mathrm{MHz}, \mathrm{CDCl}_{3}\right) \delta_{\mathrm{H}} 7.82(d, J=8.2 \mathrm{~Hz}, 2 \mathrm{H}, \mathrm{C} \underline{\underline{a}}-\mathrm{Ph}), 7.34(d, J=8.2 \mathrm{~Hz}, 2 \mathrm{H}$, $\mathrm{C} \underline{m}-\mathrm{Ph}), 4.70\left(s, 2 \mathrm{H}, \mathrm{CH}_{2} \mathrm{OTs}\right), 2.45\left(s, 3 \mathrm{H}, \mathrm{CH}_{3} \mathrm{Ar}\right), 1.37(b r t, J=6.2 \mathrm{~Hz}, 2 \mathrm{H}$, $\left.\mathrm{CH}_{2} \mathrm{CD}_{2}\right), 1.33-1.19\left(m, 4 \mathrm{H}, 2 \times \mathrm{CH}_{2}\right), 0.88\left(t, J=6.8 \mathrm{~Hz}, 3 \mathrm{H}, \mathrm{CH}_{3}\right) ;{ }^{13} \mathrm{C}$ NMR $(75.5$ $\left.\mathrm{MHz}, \mathrm{CDCl}_{3}\right) \delta_{\mathrm{C}} 58.81(\delta$-shift $0.005, \mathrm{C}-1), 71.81(\gamma$-shift 0.052, C-2), 90.60 ( $\beta$-shift $0.013, \mathrm{C}-3), 18.05\left(p, J_{\mathrm{CD}}=20.0 \mathrm{~Hz}, \alpha\right.$-shift $\left.-0.548, \mathrm{C}-4\right), 27.56(\beta$-shift $-0.171, \mathrm{C}-5)$, $30.86\left(\gamma\right.$-shift - 0.033, C-6), 22.11 ( $\delta$-shift 0.02, C-7), $13.90(\mathrm{C}-8), 144.81\left(\mathrm{C}_{\text {ipse }}-\mathrm{Ph}\right)$, 128.12 $\left(\mathrm{C}_{\varrho}-\mathrm{Ph}\right), 129.71\left(\mathrm{C}_{m}-\mathrm{Ph}\right), 133.4775\left(\mathrm{C}_{\mathrm{p}}-\mathrm{Ph}\right), 21.63\left(\mathrm{CH}_{3}-\mathrm{Ph}\right)$; EI-MS (rel. intensity): $m / z 282\left(1,[\mathrm{M}]^{+}\right), 239\left(1,[\mathrm{M}-43]^{+}\right), 227$ (1), 203 (1), $173\left(4,[\mathrm{M}-109]^{+}\right), 155$ 
(38, [M-127] $), 139\left(56,[\mathrm{M}-143]^{+}\right), 127(6), 117(8), 107(10), 91(100), 81(11), 77(11)$, $65(40), 55(19), 41(24)$.

\subsubsection{Methyl $\left[14,14-{ }^{2} \mathrm{H}_{2}\right]$-octadeca-9,12-diynoate}

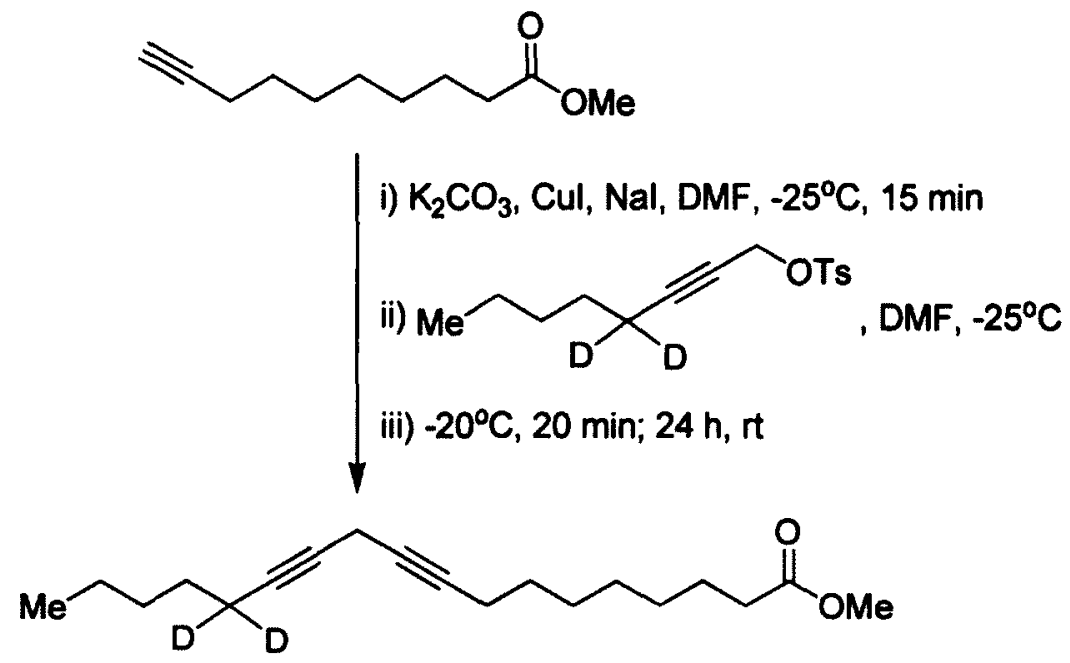

The title compound was synthesized by following the similar route that described in 6.2.1.14, from $\left[4,4-^{2} \mathrm{H}_{2}\right]-1$-(p-tolylsulfonyloxy)-oct-2-yne $(3.0 \mathrm{~g}, 10.6 \mathrm{mmol})$ and methyl 9-decynoate $(1.94 \mathrm{~g}, 10.6 \mathrm{mmol})$ as a light yellowish clear oil $(2.58 \mathrm{~g}, 83 \%) . R_{\mathrm{f}}$ $0.21\left(\mathrm{Et}_{2} \mathrm{O}\right.$ :hexane, 1:19); and the spectral data of the title compound was similar to that reported above for the non-deuterated compound (see Section 6.2.1.14). IR (film): $v$ 2932, 2859 (C-H), 2204 (C $\equiv C)$, 2185, 2098 (C-D), 1740 (C=O), 1462, 1436, 1363, $1315,1248,1198,1172,1018 \mathrm{~cm}^{-1} ;{ }^{1} \mathrm{H}$ NMR $\left(300 \mathrm{MHz}, \mathrm{CDCl}_{3}\right) \delta_{\mathrm{H}} 3.66\left(s, 3 \mathrm{H}, \mathrm{OCH}_{3}\right)$, $3.11(t, J=2.2 \mathrm{~Hz}, 2 \mathrm{H}, \mathrm{C}-11), 2.29\left(t, J=7.5 \mathrm{~Hz}, 2 \mathrm{H}, \mathrm{CH}_{2} \mathrm{CO}_{2} \mathrm{Me}\right), 2.14(t t, J=7.0,2.2$ $\mathrm{Hz}, 2 \mathrm{H}, \mathrm{C}-8), 1.63\left(t t, J=7.5,7.3 \mathrm{~Hz}, 2 \mathrm{H}, \mathrm{CH}_{2} \mathrm{CH}_{2} \mathrm{CO}_{2} \mathrm{Me}\right), 1.472(m, 4 \mathrm{H}, \mathrm{C}-7, \mathrm{C}-15)$, $1.42-1.21\left(m, 10 \mathrm{H}, 5 \times \mathrm{CH}_{2}\right), 0.88\left(t, J=7.0 \mathrm{~Hz}, 3 \mathrm{H}, \mathrm{CH}_{3}\right) ;{ }^{13} \mathrm{C}$ NMR $(75.5 \mathrm{MHz}$, $\left.\mathrm{CDCl}_{3}\right) \delta_{\mathrm{C}} 174.27(\mathrm{C}-1), 34.06(\mathrm{C}-2), 24.90(\mathrm{C}-3), 28.76(\mathrm{C}-4), 28.65(\mathrm{C}-5), 28.65$ (C-6), 29.01 (C-7), 18.70 (C-8), 80.35 (C-9), 74.59 (C-10), 9.71 (ס-shift - 0.019, C-11), 74.47 
$(\gamma$-shift $0.030, \mathrm{C}-12), 80.47$ ( $\beta$-shift - 0.062, C-13), $18.12\left(p, J_{\mathrm{CD}}=20.0 \mathrm{~Hz}, \alpha\right.$-shift 0.588, C-14), 28.27 ( $\beta$-shift - 0.195, C-15), 31.03 ( $\gamma$-shift - 0.056, C-16), 22.22 ( $\delta$-shift 0.002, C-17), 13.97 (C-18), 51.43 (-OMe); EI-MS: $m / z 291$ ([M-1] $\left.{ }^{\dagger}\right), 281$ ([M-11] $\left.]^{+}\right), 262$ ([M-30] $\left.]^{+}\right), 248$ ([M-44] $\left.]^{+}\right), 241,221,205,191,175,161,147,135,121,107,93,79,67$, 59,41 .

\subsubsection{Methyl $\left[14,14-{ }^{2} \mathrm{H}_{2}\right]$-octadeca-9Z,12Z-dienoate ${ }^{173}$}

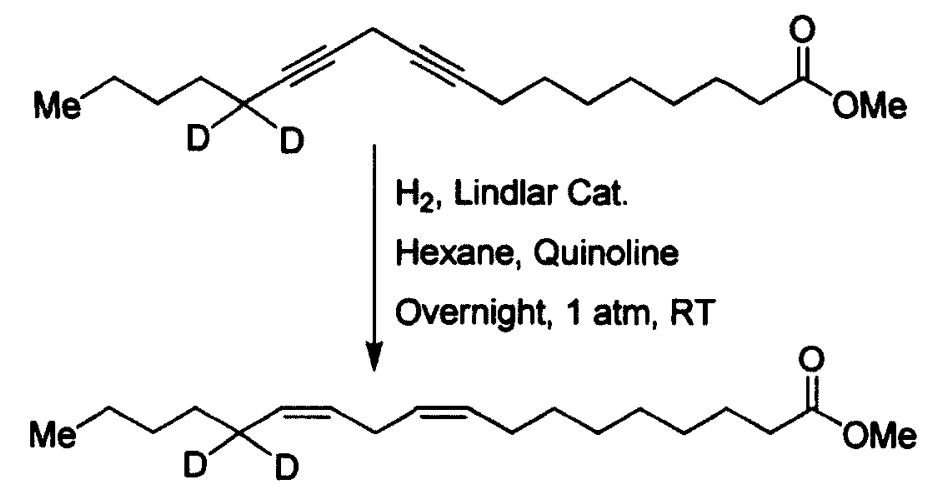

The title compound was obtained in a similar manner to that described in 3.2.1.16, from methyl $\left[14,14-{ }^{2} \mathrm{H}_{2}\right]$-octadeca-9,12-diynoate $(57 \mathrm{mg}, 0.2 \mathrm{mmol})$ as a pale yellowish clear oil (59 $\mathrm{mg}, 99 \%) . R_{\mathrm{f}} 0.45$ (EtOAc/hexane, 1:9); and the spectral data of the dideuterated compound was similar to that reported above for the monodeuterated (see Section 6.2.4.11 \& 6.2.4.12) as well as non-deuterated parent compound (see Section 6.2.1.16). IR (film): v 3009, 2959, 2927, 2856 (C-H), 2186, 2099 (C-D), 1744 (C=O), $1459,1436,1261,1171,1101,1019,802 \mathrm{~cm}^{-1}$; ${ }^{1} \mathrm{H}$ NMR $\left(300 \mathrm{MHz}, \mathrm{CDCl}_{3}\right) \delta_{\mathrm{H}} 5.44-5.27$ $(m, 4 \mathrm{H}, 4 \times \mathrm{HC}=\mathrm{C}), 3.66\left(s, 3 \mathrm{H}, \mathrm{OCH}_{3}\right), 2.77(t, J=5.6 \mathrm{~Hz}, 2 \mathrm{H}, \mathrm{C}-11), 2.30(t, J=7.5$ $\left.\mathrm{Hz}, 2 \mathrm{H}, \mathrm{CH}_{2} \mathrm{CO}_{2} \mathrm{Me}\right), 2.05(t d, J=6.4,6.4 \mathrm{~Hz}, 2 \mathrm{H}, \mathrm{C}-8), 1.62(t t, J=7.5,7.0 \mathrm{~Hz}, 2 \mathrm{H}$, $\left.\mathrm{CH}_{2} \mathrm{CH}_{2} \mathrm{CO}_{2} \mathrm{Me}\right), 1.40-1.22\left(m, 14 \mathrm{H}, 7 \times \mathrm{CH}_{2}\right), 0.89\left(t, J=6.8 \mathrm{~Hz}, 3 \mathrm{H}, \mathrm{CH}_{3}\right) ;{ }^{13} \mathrm{C}$ NMR 
(75.5 MHz, $\mathrm{CDCl}_{3}$ ) $\delta_{\mathrm{C}} 174.30$ (C-1), 34.12 (C-2), 24.96 (C-3), 29.11 (C-4), 29.17 (C-5),

29.13 (C-6), 29.60 (C-7), 27.20 (C-8), 130.05 (C-9), 128.08 (C-10), 25.65 (ס-shift 0.014, C-11), $127.97\left(\gamma\right.$-shift 0.051, C-12), 130.14 ( $\beta$-shift - 0.090, C-13), $26.50\left(p, J_{\mathrm{CD}}=18.6\right.$ Hz, $\alpha$-shift - 0.719, C-14), 29.17 ( $\beta$-shift - 0.195, C-15), 31.49 ( $\gamma$-shift - 0.053, C-16), 22.59 ( $\delta$-shift 0.006, C-17), 14.06 (C-18), 51.42 (-OMe); EI-MS: $m / z 296\left([\mathrm{M}]^{+}\right), 281$ $\left([\mathrm{M}-15]^{+}\right), 265\left([\mathrm{M}-31]^{+}\right), 236,222\left([\mathrm{M}-74]^{+}\right), 207,193,180,164,150,136,121,111$, $97,81,74,67,55,41$.

\section{Chapter 6: Part II Biological Experiments}

\subsection{General}

All components of the biological media were purchased from Difco with the exception of D-glucose, D-galactose, Tergitol (type NP-40), diazald, BSTFA, pyridine, potassium hydroxide and the spectrograde organic solvents which were obtained from Sigma-Aldrich Canada LTD. (Oakville, Ontario, Canada). The CSM-URA or "dropout powder" was obtained from MP Biomedicals, LLC. All buffers and salts were purchased from Merck (Kirkland, Quebec, Canada). Distilled deionized water $\left(\mathrm{ddH}_{2} \mathrm{O}\right)$ was used in the preparation of all media and obtained using a Millipore Milli $Q$ water system (CD0F01205) equipped with a Super C Carbon cartridge, two ion exchange cartridges, an Organex Q cartridge and a Milli Pak Filter. All glassware was sterilized in an Amsco Eagle series (model 2021) gravity autoclave at $120^{\circ} \mathrm{C}$ for 30 minutes. All biological transfers were performed in sterile laminar-flow fume hood under a Bunsen burner flame. All cultures were grown in either a New Brunswick Scientific G25 incubator shaker, or a 
LabLine Instruments Environ-Shaker model \#3597. Centrifugations were carried out either on a Sorvall RC 5C Plus floor centrifuge, Beckmann GS 6 tabletop centrifuge or an Eppendorf 5414D bench top centrifuge at ca. $4{ }^{\circ} \mathrm{C}$ (PBI, NRC, Saskatoon).

The following $S$. cerevisiae strains, used in this thesis were available in the laboratory of Dr. Patrick S. Covello (PBI, NRC-CNRC, Saskatoon): S. cerevisiae pSAS050/DTY10-a2 (MATa, fas2A::LEU2, can1-100, ura3-1, ade2-1, his3-11, his3-15, pSAS050) for enzymatic conversion of chiral 11- $\mathrm{d}_{1}$-oleic acid to chiral 11- $\mathrm{d}_{1}$-linoleic acid; S. cerevisiae pYJ/DTY10-a2 (MATa, fas2A::LEU2, can1-100, ura3-1, ade2-1, his311, his3-15) containing the plasmid pYJ comprised of the vector pYES2 and a Fac2 gene (expressing $\Delta^{8,10}$-fatty acid desaturase) from Calendula officinalis for the conversion of linoleate to calendate; yeast strain pESC-HIS/YPH499 derived from the strain YPH499 using the vector $\mathrm{pESC}$-HIS (Stratagene) i.e. co-expressing the two cDNAs of DsFAD2-1 and DsFAD2-2 fatty acid desaturases for the conversion of oleate to dimorphecolate via the formation of trans $-\Delta^{12}$-linoleate.

For the construction of the tung 1,4-desaturase (FADX)-containing yeast strain, 2x Phusion high-fidelity PCR master mix for PCR reaction was ordered from New England BioLabs, Inc. pYES2.1 TOPO ${ }^{\top}$ TA Expression Kit from Invitrogen was used to TA clone the PCR product into pYES2.1 vector and transformed into chemically competent E. coli cells. The plasmid DNAs were purified by Promega Wizard ${ }^{\circledR}$ Plus Midipreps DNA Purification system. The yeast strain INVSc-1 was obtained from Invitrogen, Carlsbad, California. The purified plasmid DNA was transformed into $S$. cerevisiae using a $S$. cerevisiae EasyComp transformation kit from Invitrogen. 


\subsection{General Procedures}

\subsubsection{Saponification of Fatty Acid Substrates}

Fatty acid methyl ester was weighed in Teflon sealed, $12 \mathrm{~mL}$ culture tube and 3 $\mathrm{mL}$ of $10 \% \mathrm{KOH}$ in $\mathrm{MeOH}$ and $10 \% \mathrm{ddH}_{2} \mathrm{O}$ was added. The fatty ester was dispersed in the solution by vortexing the tube and flushed under $\mathrm{N}_{2}$ for about 4 minutes to remove any trace of $\mathrm{O}_{2}$. The tube was sealed tightly and allowed to heat at $80^{\circ} \mathrm{C}$ for $2 \mathrm{hrs}$. It was removed from the heating block and cooled on ice for about 2 minutes. The alkaline fatty acid solution was then diluted with $2 \mathrm{~mL}$ of deionized water and acidified with acetic acid $(400 \mu \mathrm{L})$. Two $\mathrm{mL}$ of hexane was added and the crude fatty acid was extracted by vortexing the culture tube vigorously followed by centrifugation at $2000 \mathrm{rpm}$ for 1 minute. The aqueous layer was re-extracted with hexanes $(2 \times 2 \mathrm{~mL})$ and the collected organic layers were combined and subsequently dried over anhydrous $\mathrm{Na}_{2} \mathrm{SO}_{4}$. After removing the solvent under a stream of $\mathrm{N}_{2}$ at $25{ }^{\circ} \mathrm{C}$, the neat free fatty acid sample was weighed. Yields were typically $>95 \%$.

\subsubsection{Incubation Experiments}

An equal volume of ethanol was added to the neat free fatty acid sample in a 12 $\mathrm{mL}$ glass culture tube that was sealed and heated at $80^{\circ} \mathrm{C}$ for 10 minutes to sterilize. A sterile solution of $10 \%$ Tergitol in $\mathrm{ddH}_{2} \mathrm{O}$ (filter sterilized) was added to the tube to make a final concentration of $5-10 \mathrm{mg} / \mathrm{mL}$ of the fatty acid. The sample was vortexed, the tube was sealed and heated again at $80^{\circ} \mathrm{C}$ for 5 minutes at which time the solution was clear.

A typical incubation experiment was carried out as follows: $0.5 \mathrm{~mL}$ of the above fatty acid-Tergitol solution containing the desired fatty acid was transferred to a sterile $250 \mathrm{~mL}$ Erlenmeyer flask containing $50 \mathrm{~mL}$ of the growth media (SDgalactose - uracil) 
and $1 \mathrm{~mL}$ of the starter yeast culture. The Erlenmeyer flask was plugged with cotton wool, covered with tinfoil and placed in a shaking incubator. The incubation period, temperature and shake speed varied with each organism and is specified in the appropriate Results and Discussion sections. Cultures typically became turbid at the end of the incubation period. We have observed that the addition of exogenous fatty acid did not affect the growth of the yeast strain when compared with the control culture.

\subsubsection{Extraction of the Cellular Fatty Acids}

Fatty acids were isolated from the harvested yeast cells by using standard hydrolysis-methylation protocol. The yeast cultures $(50 \mathrm{~mL})$ of each incubation experiments were removed from the shaker and transferred to Falcon ${ }^{\mathrm{TM}}$ tubes $(50 \mathrm{~mL})$. The yeast cells were pelleted by centrifugation at $3000 \mathrm{x} \mathrm{g}$ for 5 minutes and the supernatants were carefully decanted from each centrifuge tubes. The yeast pellets were first washed with $10 \mathrm{~mL}$ of $10 \%$ Tergitol solution and the mixtures were transferred to $12 \mathrm{~mL}$ culture tubes. The solutions were centrifuged at $3000 \times \mathrm{g}$ for 5 minutes, the supernatants were carefully discarded and the pellets were washed another two times with deionized water $(10 \mathrm{~mL})$. The harvested cells were first saponified, non-saponifiable lipids were removed and then the partially purified free fatty acids were methylated. To accomplish this, the yeast pellets $(\sim 1 \mathrm{~g})$ were diluted with $2 \mathrm{~mL}$ of $10 \% \mathrm{KOH}$ in $\mathrm{MeOH}$ in each tube and flushed with $\mathrm{N}_{2}$ to remove any trace of $\mathrm{O}_{2}$. The culture tubes were then quickly sealed and vortexed to disperse the material. The mixtures were saponified by heating the solution at $80{ }^{\circ} \mathrm{C}$ for $2 \mathrm{hr}$ which leads to the lyses of the cells and saponification of any esters. The culture tubes were cooled down to room temperature and $2 \mathrm{~mL}$ of $\mathrm{dH}_{2} \mathrm{O}$ was added to each tube. The aqueous layer was extracted with 
hexanes $(2 \times 2 \mathrm{~mL})$ by vortexing the mixtures to remove any nonsaponifiable lipids. In this particular step, centrifugation at $3000 \mathrm{x}$ g for 2 minutes helped to get a clear phase separation. The aqueous layer was neutralized with $50 \%$ acetic acid, diluted with $2 \mathrm{~mL}$ of deionized water and extracted with hexanes $(3 \times 2 \mathrm{~mL})$. The organic layers were combined and the solvent was removed under a stream of $\mathrm{N}_{2}$ to yield the fatty acid residue as free long-chain carboxylic acid for GC-MS analysis.

\subsubsection{Derivatization of Fatty Acids for GC-MS Analysis}

Fatty acids were derivatized to fatty acid methyl ester (FAME) for GC-MS analysis. An ice-cold solution of diazomethane was used for that purpose and was prepared by the following procedure: to a diazomethane generator were added $2.5 \mathrm{~g}$ of $\mathrm{KOH}$ in $4 \mathrm{~mL}$ of water and $5 \mathrm{~mL}$ of $\mathrm{EtOH}$ and the mixture was heated up to $65^{\circ} \mathrm{C}$ using a water bath. A solution of $2.5 \mathrm{~g}$ of Diazald ${ }^{\mathrm{TM}}$ in $30 \mathrm{~mL} \mathrm{Et}_{2} \mathrm{O}$ was slowly admitted to the mixture using a dropping funnel and the freshly prepared diazomethane solution was collected by condensation from a dry-ice cold finger to an ice-cold RBF as a bright yellow liquid. Once the Diazald ${ }^{\mathrm{TM}}$ addition was completed and the distillation had ceased, $1 \mathrm{~mL}$ ether was added until a clear distillate appeared.

Freshly prepared ice-cold diazomethane solution was then slowly added to the crude fatty acid solutions until the yellow color persisted. The solvent and excess reagent was removed under a stream of $\mathrm{N}_{2}$ and the residual fatty acid methyl esters (FAME) were finally taken in $100 \mu \mathrm{L}$ of hexane for GC-MS analysis. Use of diazomethane to methylate the fatty acid samples containing sensitive functionalities was found to be most suitable over other methylation techniques such as Fischer esterification, as it esterifies under extremely mild condition. When analyzing fatty acid methyl ester samples containing 
dimorphecolate, the FAMEs were silylated using BSTFA and pyridine. ${ }^{187}$ For silylation, 0.2-5.0 mg of the FAMEs, $0.2-0.4 \mathrm{~mL}$ of $\mathrm{N}, \mathrm{O}$-bis(trimethylsilyl)trifluoroacetamide and 0.1-0.2 mL of pyridine were combined in a $1.0 \mathrm{~mL}$ reacti-vial $^{\mathrm{TM}}$; the mixture was shaken at room temperature for 30 seconds and subsequently heated at $60^{\circ} \mathrm{C}$ for 10 minutes to ensure completion of the reaction.

\subsection{Microbial synthesis of methyl (11R)- and (11S)-[11- $\left.{ }^{2} \mathbf{H}_{1}\right]$-octadeca- 9Z,12Z-dienoate by $\Delta^{12}$-desaturation of corresponding methyl octadeca-9Z-enoates}

The yeast strain pSAS050/DTY10-a2 (MATa, fas2 $\triangle:: L E U 2$, canl-100, ura3-1, ade2-1, his3-11, his3-15, pSAS050) used for this experiment is defective in FAS (Fatty Acid Synthase) to prevent endogenous oleic from diluting the deuterium labeled 18:2 product and functionally expresses a $\Delta^{12}$-desaturase gene. Methyl $(11 R)-\left[11-{ }^{2} \mathrm{H}_{1}\right]$ oleate (163 mg) was saponified with $2 \mathrm{~mL}$ of $10 \% \mathrm{KOH}$ in methanol and $10 \%$ deionized water in a sealed $12 \mathrm{~mL}$ glass culture tube at $80^{\circ} \mathrm{C}$ for 2 hours. The mixture was cooled to room temperature, acidifed with $2 \mathrm{~mL}$ of $6 \mathrm{M} \mathrm{HCl}$ and extracted with $3 \times 2 \mathrm{~mL}$ of hexane. The combined hexane layer was dried over anhydrous $\mathrm{Na}_{2} \mathrm{SO}_{4}$ and the solvent was removed under a stream of $\mathrm{N}_{2}$ to recover $150 \mathrm{mg}$ of free fatty acid. $100 \mu \mathrm{L}$ of ethanol was added and the tube was heated to $80^{\circ} \mathrm{C}$ for 20 minutes to sterilize, then $10 \mathrm{~mL}$ of sterile $10 \%$ Tergitol was added to make a $15 \mathrm{mg} / \mathrm{mL}$ solution. The solution was warmed until dissolved/emulsified to give a clear solution. 10 flasks each containing $150 \mathrm{~mL}$ of SDgalactose-uracil media was inoculated with $1 \mathrm{~mL}$ of stationary phase starter culture of the pSAS050/DTY10-a2 yeast strain. $1 \mathrm{~mL}$ of oleic acid/Tergitol mixture was then 
added. The cultures were capped with a foam plug, covered with tinfoil and shaken at $225 \mathrm{rpm}$ for 6 days at $20^{\circ} \mathrm{C}$. The cultures were spun down by centrifugation; the pellets were washed with $10 \mathrm{~mL}$ of water and pelleted into a glass culture tube. The pellets were suspended in $2 \mathrm{~mL}$ of $10 \%$ methanolic- $\mathrm{KOH}$, sealed to vortex and heated at $80^{\circ} \mathrm{C}$ for 2 hours to saponify the harvested cells. The mixture was cooled to room temperature, $2 \mathrm{~mL}$ of deionized water added and acidified with $2 \mathrm{~mL}$ of $6 \mathrm{M} \mathrm{HCl}$ before extraction with $3 \times 2$ $\mathrm{mL}$ of hexane. The combined hexane extracts were dried under nitrogen and esterified with $2 \mathrm{~mL}$ of $1 \% \mathrm{H}_{2} \mathrm{SO}_{4}$ in dry methanol for $1 \mathrm{~h}$ at $60{ }^{\circ} \mathrm{C}$. The mixture was cooled; $2 \mathrm{~mL}$ of water was added and the fatty acid methyl esters were extracted with $3 \times 2 \mathrm{~mL}$ of hexane. The organic layers from all 10 cultures were combined, the solvent was evaporated under nitrogen and the crude FAME (147 mg in total) was dissolved in 300 $\mu \mathrm{L}$ of $50 \%$ acetone:acetonitrile mixture. The purification was carried out on a HewlettPackard 1100 series HPLC system equipped with an auto-sampler, solvent degasser, quaternary pump, column heater, and diode array detector (monitored at 205 and $254 \mathrm{~nm}$ ) controlled by HP Chemstation software. The crude sample was divided $6 \times 50 \mu \mathrm{L}$ aliquots and each aliquot injected on a Gemini-5 $\mu \mathrm{m}$ C18 $110 \mathrm{~A} 250 \mathrm{~mm} \times 10 \mathrm{~mm}$ column, eluting at a rate of $3 \mathrm{~mL} / \mathrm{min}$ isocratically for 5 minutes with acetonitrile, followed by a $0-30 \%$ acetone gradient for 20 minutes and finally with $100 \%$ acetone to rinse and re-equilibrate. Methyl $(11 R)-\left[11-{ }^{2} \mathrm{H}_{1}\right]$-linoleate was collected between 12 and 13 minutes and the unreacted labeled oleate was recovered between 14 and 16 minutes. After removing the solvent (acetone:acetonitrile), pure methyl $(11 R)-\left[11-{ }^{2} \mathrm{H}_{1}\right]$-linoleate (10 mg, $7 \%$ of the total crude FAME) was obtained. The exact same procedure was followed to convert methyl $(11 S)-\left[11-{ }^{2} \mathrm{H}_{1}\right]$-oleate into methyl $(11 S)-\left[11-{ }^{2} \mathrm{H}_{1}\right]-$ linoleate. In 
this case, $8 \mathrm{mg}$ of pure methyl $(11 S)-\left[11-{ }^{2} \mathrm{H}_{1}\right]$-linoleate was recovered after purification $7 \%$ of the total crude FAME.

\subsection{Construction of the Tung 1,4-Desaturase (FADX)-containing Yeast Mutant}

\subsubsection{Amplification of the Template Plasmid DNA using PCR Reaction}

The DNA plasmid pRTL2 myc-FADX, containing the tung (Aleurites fordii Hemsl.) seed FADX gene, that encodes the $\Delta^{11,13}$-fatty acid conjugase/desaturase enzyme was a generous gift from J. M. Dyer (US Department of Agriculture-Agricultural Research Services Southern Regional Research Center, New Orleans, Louisiana) and amplified using the PCR reaction using a FDX-forward primer (5'gaagettgtctagaATGGGAGCTGGTGGCCGAATGTCT-3') and a FDX-reverse primer (5'-aaggatccACTCCATATCTCGTAACAAGGTCAAACCTC-3').

$50 \mu \mathrm{L}$ of PCR mixture was set up on ice using the following components, which were added in the following order:

\begin{tabular}{|l|l|}
\hline Component & $50 \mu \mathrm{L}$ reaction \\
\hline Sterile water & $22 \mu \mathrm{L}$ (to make the mixture up to $50 \mu \mathrm{l})$ \\
\hline $2 \times$ Phusion $^{\Phi}$ high-fidelity master mix & $25 \mu \mathrm{L}$ \\
\hline FADX forward primer, $\mathrm{Tm}=76.1^{\circ} \mathrm{C}$ & $1 \mu \mathrm{L}(50$ pmoles $)$ \\
\hline FADX reverse primer, $\mathrm{Tm}=69.4^{\circ} \mathrm{C}$ & $1 \mu \mathrm{L}(50$ pmoles $)$ \\
\hline $\begin{array}{l}\text { pRTL2-myc-FADX plasmid as } \\
\text { template DNA }\end{array}$ & $1 \mu \mathrm{L}(0.5 \mathrm{ng})$ \\
\hline
\end{tabular}


$2 \mathrm{x}$ Phusion ${ }^{\circledR}$ high-fidelity PCR master mix is a convenient $2 \mathrm{x}$ mix containing Phusion high-fidelity DNA polymerase $(0.04 \mathrm{U} / \mu \mathrm{L})$, nucleotides $(400 \mu \mathrm{L}$ of each $\mathrm{dNTP})$ and optimized 2x Phusion HF reaction buffer including $\mathrm{MgCl}_{2}$. The PCR reaction was then started on a thermal cycler after mixing the contents of the tube and amplified by using the following cycling parameters:

\begin{tabular}{|l|c|c|c|}
\hline \multirow{2}{*}{ Cycle step } & \multicolumn{2}{|c|}{ 2-Step protocol } & \multirow{2}{*}{ Cycles } \\
\cline { 2 - 3 } & Temperature & Time & \\
\hline Step 1. Initial denaturation & $98^{\circ} \mathrm{C}$ & $30 \mathrm{sec}$ & 1 \\
\hline Step 2. Denaturation & $98{ }^{\circ} \mathrm{C}$ & $10 \mathrm{sec}$ & 30 \\
Step 3. Annealing & $72{ }^{\circ} \mathrm{C}$ & $30 \mathrm{sec}$ & \\
Step 4. Extension & $72{ }^{\circ} \mathrm{C}$ & $30 \mathrm{sec}$ & \\
\hline Step 5. Final extension & $72^{\circ} \mathrm{C}$ & $10 \mathrm{~min}$ & 1 \\
& $4{ }^{\circ} \mathrm{C}$ & hold & \\
\hline
\end{tabular}

$1 \mu \mathrm{L}$ of Taq polymerase (thermostable DNA polymerase) was added to the mixture at step 5 and heated up to $72{ }^{\circ} \mathrm{C}$ for 10 minutes to ensure A-overhang on PCR products. The Phusion DNA polymerase has the ability to stabilize primer-template hybridization. The annealing temperature used is the lowest primer $\mathrm{Tm}+3{ }^{\circ} \mathrm{C}=72{ }^{\circ} \mathrm{C}$ for 30 seconds. The final extension of 10 minutes at $72{ }^{\circ} \mathrm{C}$ after the last cycle was included to ensure that all PCR products are full length and 3' adenylated. After the completion of amplification, 10 $\mu \mathrm{L}$ of PCR product was removed from the reaction and analyzed by agarose gel electrophoresis. DNA was visualized by ethidium bromide staining. A single, discrete band was obtained. 


\subsubsection{TOPO ${ }^{\circledR}$ Cloning of PCR Products and Expression in E. coli}

$\mathrm{TOPO}$ cloning technology provides an opportunity to construct the required PCR product, recombined into the TOPO vector, and then transform the recombinant vector into E. coli easily. pYES2.1 TOPO TA Expression Kit was used to TA clone the PCR product into pYES2.1 vector and transform TOP10 chemically competent one shot E. coli cells.

\subsubsection{1a LB Medium}

$10 \mathrm{~g}$ Tryptone (1\%), $5 \mathrm{~g}$ yeast extract $(0.5 \%)$ and $10 \mathrm{~g} \mathrm{NaCl}(1 \%)$ were dissolved into $950 \mathrm{~mL}$ of deionized water in a $2 \mathrm{~L}$ sterile bottle and the $\mathrm{pH}$ of the solution was adjusted to 7.0 using $\mathrm{NaOH}$. After adjusting the final volume to $1 \mathrm{~L}$, the solution was autoclaved on liquid cycle for 20 minutes at $120^{\circ} \mathrm{C}$ and 15 psi. The solution was cooled and ampicillin was added as antibiotic $(100 \mu \mathrm{g} / \mathrm{mL})$ and stored at room temperature $\left(\mathrm{LB}_{\text {amp }}\right)$.

\subsubsection{1b LB agar Plates}

$10 \mathrm{~g}$ Tryptone (1\%), $5 \mathrm{~g}$ yeast extract $(0.5 \%), 10 \mathrm{~g} \mathrm{NaCl}(1 \%)$ and $15 \mathrm{~g}$ Bacto agar $(1.5 \%)$ were dissolved into $935 \mathrm{~mL}$ of deionized water in a $2 \mathrm{~L}$ sterile bottle and the $\mathrm{pH}$ of the solution was adjusted to 7.0 using $\mathrm{NaOH}$. After adjusting the final volume to 1 $\mathrm{L}$, the solution was autoclaved on liquid cycle for 20 minutes at $120^{\circ} \mathrm{C}$ and $15 \mathrm{psi}$. The solution was cool down to $55^{\circ} \mathrm{C}$ before $100 \mathrm{mg}$ of ampicillin was added as antibiotic $(100 \mu \mathrm{g} / \mathrm{mL})$, stirred well and poured into $10 \mathrm{~cm}$ petri plates $(\sim 30 \mathrm{ml} /$ plate). Plates were allowed to harden, inverted and stored at $4{ }^{\circ} \mathrm{C}$ in the dark for future use. 


\subsubsection{1c SOC Medium}

$2 \%$ Tryptone, $0.5 \%$ yeast extract, $10 \mathrm{mM} \mathrm{NaCl}, 2.5 \mathrm{mM} \mathrm{KCl}, 10 \mathrm{mM} \mathrm{MgCl}_{2}, 10$ $\mathrm{mM} \mathrm{MgSO}_{4}$ and $20 \mathrm{mM}$ glucose added together to make a final volume of $6 \mathrm{~mL}$.

\subsubsection{Bacterial Transformation}

Before starting the cloning reaction, SOC media was warmed to room temperature, the ( $\left(\mathrm{LB}_{\text {amp }}\right)$ agar plate was placed in the incubator to warm up to $37^{\circ} \mathrm{C}$ for 30 minutes, a water bath was equilibrated to $42{ }^{\circ} \mathrm{C}$ for the chemical transformation, the vial of TOP 10 chemically competent one shot ${ }^{\circledR} E$. coli cells ( $50 \mu \mathrm{L}$ each) was thawed on ice.

\subsection{Preparation of $\mathrm{TOPO}^{\oplus}$ Cloning Reaction}

For each transformation, $2 \mu \mathrm{L}$ of sterile $\mathrm{ddH}_{2} \mathrm{O}$ was added to a mixture of $2 \mu \mathrm{L}$ of fresh PCR product and $1 \mu \mathrm{L}$ of salt solution to make a final volume of $5 \mu \mathrm{L}$ and the TOPO Cloning reaction was started by gently mixing $1 \mu \mathrm{L}$ of TOPO ${ }^{\infty}$ vector pYES2.1 with the combination. The reaction was then incubated for 5 minutes at room temperature then placed on ice and proceeds immediately for One Shot ${ }^{\circledR}$ chemical transformation.

\subsection{Chemical Transformation}

$2 \mu \mathrm{L}$ of the TOPO Cloning reaction was pipetted directly into the vial containing the TOP 10 One Shot ${ }^{\Phi}$ chemically competent $E$. coli cells $(50 \mu \mathrm{L})$ and mixed by gently tapping the vial. The reaction mixtures were incubated on ice for 30 minutes, removed from the ice bath and given a heat-shock treatment for 30 seconds at $42{ }^{\circ} \mathrm{C}$ without shaking the vial and then placed immediately back on ice. Room temperature SOC media ( $250 \mathrm{ul}$ ) was added to the tube. After sealing the vial, the reaction was shaken at $200 \mathrm{rpm}$ for 1 hour at $37^{\circ} \mathrm{C}$, removed from the incubator and $5 \mu \mathrm{L}$ was spread equally on two pre- 
warmed $\left(\mathrm{LB}_{\mathrm{amp}}\right)$ plates. The plates were inverted and incubated for overnight at $37^{\circ} \mathrm{C}$. From one of the two plates, 10 well-spaced bacterial colonies were picked and incubated overnight in $15 \mathrm{~mL}$ of liquid culture $\left(\mathrm{LB}_{\text {amp }}\right)(100 \mu \mathrm{g} / \mathrm{mL})$ in $50 \mathrm{~mL}$ sterile Falcon ${ }^{\mathrm{TM}}$ tubes at $200 \mathrm{rpm}$ at $37^{\circ} \mathrm{C}$. The plasmids were purified and submitted to DNA Services at PBI for sequencing.

\subsection{Long-term storage}

A single colony was picked up from the plate(s) that contains the correct clone and was inoculated into $2 \mathrm{~mL}$ of LB media under ampicillin selection $(100 \mu \mathrm{g} / \mathrm{mL})$. After growing the culture(s) to saturation $\left(\mathrm{OD}_{600}=1-2\right), 0.85 \mathrm{~mL}$ of the culture was pipetted to a cryovial containing $0.15 \mathrm{~mL}$ of sterile glycerol and stored at $-80^{\circ} \mathrm{C}$.

\subsubsection{Plasmid DNA Purification}

Small scale purification of the plasmid DNA known as "minipreps" is commonly used in molecular biology procedures. The plasmid DNA was purified by using Promega Wizard ${ }^{\oplus}$ Plus Midipreps DNA Purification kit. The following procedures were used in the purification:

\subsubsection{Preparation of Bacterial Lysate}

The overnight grown cultures of $E$. Coli $(15 \mathrm{~mL})$ in $50 \mathrm{~mL}$ conical tubes were pelleted by centrifugation at $10,000 \times \mathrm{g}$ for 10 minutes at $4{ }^{\circ} \mathrm{C}$. The supernatants were carefully decanted and discarded and the centrifuge tubes were blotted upside down with a paper towel to remove excess liquid. The pellets were suspended in Cell Resuspension Solution ( $3 \mathrm{~mL}$ each) and the cell lumps broken up using an applicator stick. Cell Lysis Solution ( $3 \mathrm{~mL}$ ) was added to each Falcon ${ }^{\mathrm{TM}}$ tube and left $3-5$ minutes for efficient lysis of the bacterial cells. The tubes were capped and inverted 4 times for proper mixing 
which made the cell suspension completely clear. Subsequently, Neutralization Solution ( $3 \mathrm{~mL}$ ) was added to the tubes and the solutions were mixed again by inverting the capped tubes 4 times. The lysed cells were centrifuged at $14,000 \times \mathrm{g}$ for 15 minutes at 4 ${ }^{\circ} \mathrm{C}$ to produce a tight pellet and the supernatant liquid containing the plasmid DNAs were carefully decanted off from the top of the white precipitates to the new $50 \mathrm{~mL}^{\text {Falcon }}{ }^{\mathrm{TM}}$ tubes.

\subsubsection{Plasmid DNA Purification}

Wizard ${ }^{\$}$ Midipreps DNA Purification resin was thoroughly shaken and $10 \mathrm{~mL}$ resin solution was added to the plasmid DNA solutions. The resin/DNA mixtures were swirled before being transferred to the Midicolumns that were attached to the vacuum manifold ports. The vacuum was applied and when all liquids from the reservoir have been judged to pass through the column the vacuum was released. A total of $30 \mathrm{~mL}$ of column wash solution was passed through the column and the resin was allowed to dry under vacuum suction for 30 seconds. The Midicolumns were removed from the vacuum manifold, separated from the reservoirs and placed in $1.5 \mathrm{~mL}$ microcentrifuge tubes that were then centrifuged at $10,000 \times \mathrm{g}$ for 2 minutes (to remove any residual Column Wash Solution). The Midicolumns were transferred to new microcentrifuge tubes $(1.5 \mathrm{~mL})$ and to each was added $100 \mu \mathrm{L}$ of preheated $\left(80^{\circ} \mathrm{C}\right)$ nuclease-free water. After $1 \mathrm{~min}$, the plasmid DNA was isolated by centrifugation at $10,000 \times \mathrm{g}$ for 20 seconds. The Midicolumns were removed from the microcentrifuge tubes and discarded. The supernatant was centrifuged for additional 5 minutes at $10,000 \times g$ to separate the resin fines and the supernatant containing the plasmid DNA was carefully pipetted to new microcentrifuge tubes. The solutions containing the purified plasmid DNAs in water were 
finally stored at $-20{ }^{\circ} \mathrm{C}$. Purified plasmid was quantitated by spectrophotometer and submitted for sequencing to DNA services at PBI. The sequence data was analyzed using Lasergene SeqMan software to verify that the sequence was correctly translated.

tttggctagaATGGGAGCTGGTGGCCGAATGTCTGTTGCCCCCAACAACAGCAAGTGCGAGAAAAAGGAAAGCAGAAG CGTGAAACGAGTCCCACACACAAAGCCTCCATTCACTCTTGGCCAATTAAAGCAAGCCATACCGTCCCATTGCTTTAA ACGCTCCCTTCTTCGCTCCTTCTCTTATGTTGTTTATGACCTTTCTTTAAGTTTATTTTCTACTCCATTGCCACCACCTA CTTCCATCTCCTCCCTTCTCCGATAACCTACATCGCTTGGCCTGTCTACTGGGCTTTCCAAGGCTGCATTCTCACTAGT GITIGGGTGCTCGGCCATGAATGTGGTCACCATGCTTITAGCGAATATAATTGGCTTGACGACACTATTGGCCTAATT CTCCACTCTTCACTTCTAGTTCCATACTITTCATTTAAAATTAGCCATCGTCGCCATCACTCTAACATTGCATCACTTGA ACGCGACGAGGTGTTTGTCCCTAGGCTCAAGTCTGCAATTCCATGGTATTCCAAGTACCTCAACAACCCACCAGGCC GAGCTTTAACCCTTGTAGCTACACTCTTCATTGGCTGGCCTTTATATTTAGCCTTCAATGTTICGGGCCGATACTATGA TCGCTITGCCTGCCATTATGATCCTTATAGTCCTATATATTCTGATAGAGAAAGGCTTCAGATTTACATTTCTGATGCT ATGATTTCGTTGCAGCTTATGTGTTGTATAAGATTGCCATGGCAAAAGGGCTAGCATGGCTGGTATGTATCTATGG GGTACCATTGCTTATTGTTAATGCTCTTGTTGTGACTATCACATCCTTGCAGCACACCCACGTTGCATTGCCACATTAT GACTCCTCAGAATGGGATTGGCTTCGGGGAGGTTAGCAACAGTAGATAGAGATTATGGAGTGTTCAATAAGATTT TCCATAATGCTACAGATACTCATGTAATACACCATCTATTCTCTTCAATGCCTCATTATCATGGAGTGGAAGCTACTAG AGCAATCAAGCCGATATTGGGTGACTATTATCTGTTTGATGATACTCCTATTCATGTGGCACTGTGGAGGGAGGCAA AGGAGTGTCTGTTIGTTGAGCCAGATGAGGGAGATAACAACAATGGTGTTITCTGGTATAGTAATAAGITTGAggat CC

Figure 6.1. Sequence of the FADX from tung (Aleurites fordii Hemsl.) inserted in the pYES2.1 plasmid. The capital letters indicates open reading frame (ORF) and the lower case is untranslated.

MGAGGRMSVAPNNSKCEKKESRSVKRVPHTKPPFTLGQLKQAIPSHCFKRSLLRSFSWVYDLSLSFIFYSIATT YFHLLPSPITYIAWPVYWAFQGCILTSVWVLGHECGHHAFSEYNWLDDTIGLILHSSLLVPYFSFKISHRRHHSN IASLERDEVFVPRLKSAIPWYSKYLNNPPGRALTLVATLFIGWPLYLAFNVSGRYYDRFACHYDPYSPIYSDRERL QIYISDAMIFVAAYVLYKIAMAKGLAWLVCIYGVPLLIVNALWVTITSLQHTHVALPHYDSSEWDWLRGGLATV DRDYGVFNKIFHNATDTHVIHHLFSSMPHYHGVEATRAIKPILGDYYLDDTPIHVALWREAKECLFVEPDEG DNNNGVFWYSNKF

Note: amino acid 122 D (Asp) translated from "gat" (FADX) and from "gac" (FADX19).

Figure 6.2. Translated gene sequence of tung FADX from Aleurites fordii Hemsl.

\subsubsection{Transformation of S. cerevisiae}

The FADX plasmid DNA was then transformed into the INVSc-1 strain of $S$. cerevisiae using the method described in the manual for $S$. cerevisiae EasyComp 
transformation kit (Invitrogen) and selected on SDglucose-uracil selection media plates. The following protocol was used in the preparation of competent $S$. cerevisiae cells that were transformed with the purified plasmid DNA.

\subsubsection{1a Stock Solution}

$200 \mathrm{~g}$ of dextrose (D-glucose) was dissolved in $800 \mathrm{~mL}$ of $\mathrm{ddH}_{2} \mathrm{O}$ to make a final volume of $1 \mathrm{~L}$. The solution was filtered into a sterilized $2 \mathrm{~L}$ bottle and stored at $4{ }^{\circ} \mathrm{C}$.

\subsubsection{1b YPD Solution}

$10 \mathrm{~g}$ of yeast extract $(1 \%), 20 \mathrm{~g}$ of bacto-peptone $(2 \%)$ and $20 \mathrm{~g}$ of dextrose (2 \%) [D-glucose] were dissolved in $950 \mathrm{~mL}$ of $\mathrm{ddH}_{2} \mathrm{O}$ to make a final volume of $1 \mathrm{~L}$. The solution was filtered into a sterile $2 \mathrm{~L}$ bottle. The YPD medium was stored at room temperature for future use.

\subsubsection{1c YPD Agar Medium}

$10 \mathrm{~g}$ of yeast extract (1\%), $20 \mathrm{~g}$ of bacto-peptone ( $2 \%$ ) were dissolved in $900 \mathrm{~mL}$ of $\mathrm{ddH}_{2} \mathrm{O}$ and $20 \mathrm{~g}$ of bacto-agar ( $2 \%$ ) was added to it. The solution was filtered into a sterile $2 \mathrm{~L}$ bottle. $100 \mathrm{~mL}$ of stock solution ( $2 \% \mathrm{D}$-glucose) was added to the bottle, swirled well. The solution was then poured into sterile petri plates $(\sim 30 \mathrm{ml} /$ plate $)$ to make the YPD plates and stored in the dark at $4{ }^{\circ} \mathrm{C}$ for future use.

\subsubsection{Preparation of Competent Yeast Cells}

The INVSc-1 strain of $S$. cerevisiae was streaked on a YPD (Yeast Extract Peptone Dextrose) plate and incubated at $28-30^{\circ} \mathrm{C}$ for 2 days. Solution I (wash solution) and II (Lithium cation solution for making cells competent) were removed from the $S$. cerevisiae EasyComp Transformation Kit and allowed to equilibrate to room temperature. $10 \mathrm{~mL}$ of YPD solution was transferred into a $50 \mathrm{~mL}$ sterile Falcon ${ }^{\mathrm{TM}}$ tube 
and to that was added the INVSc-1 strain of $S$. cerevisiae using a platinum loop that was flamed before use. The yeast culture was allowed to grow overnight at $28{ }^{\circ} \mathrm{C}$ in a shaking incubator at $250 \mathrm{rpm}$ to an $\mathrm{OD}_{600}$ of between 3.0 and 5.0. The cells from the overnight grown culture were diluted to an $\mathrm{OD}_{600}$ of 0.2 to 0.4 in a total volume of $10 \mathrm{~mL}$ of YPD. The diluted YPD culture was again allowed to grow in a shaking incubator at $28-30^{\circ} \mathrm{C}$ for $4-5$ hrs at $250 \mathrm{rpm}$ until the $\mathrm{OD}_{600}$ reached a value of 0.6 to 1.0 . The cells were then pelleted by centrifugation at $500 \mathrm{x} \mathrm{g}$ for 5 minutes at room temperature and the supernatant was discarded. The cell pellet was resuspended in $10 \mathrm{~mL}$ of Solution I (wash solution) and after 2 minutes, pelleted by centrifugation at $500 \mathrm{xg}$ for 5 minutes at room temperature. The supernatant was discarded and the cell pellet was resuspended for the final time in $1 \mathrm{~mL}$ of Solution II (Lithium cation solution for making cells competent). The competent cells were aliquoted into several $1.5 \mathrm{~mL}$ sterile screw-capped microcentrifuge tubes, each containing $50 \mu \mathrm{L}$ of solution and placed in a Styrofoam box (to avoid damage to the cell wall, it is important that the cells freeze slowly) and stored at $-80{ }^{\circ} \mathrm{C}$ for future use (higher transformation efficiencies have been observed with the frozen cells compared to freshly prepared cells).

\subsubsection{Transformation of the Competent Cells}

The Solution III (transformation solution) from the $S$. cerevisiae EasyComp Transformation Kit was equilibrated to room temperature. Five selective SD-ura agar plates were equilibrated to room temperature. One $1.5 \mathrm{~mL}$ microcentrifuge tube containing $50 \mu \mathrm{L}$ of competent cells was defrosted at room temperature and to that was added $4 \mu \mathrm{L}$ of purified FADX plasmid DNA solution (vector DNA) followed by $500 \mu \mathrm{L}$ of room temperature solution III. The cell/DNA mixture was mixed thoroughly by 
vigorously vortexing the tube and subsequently incubated in a water bath for $1 \mathrm{hr}$ at 30 ${ }^{\circ} \mathrm{C}$. During heating in the water bath, the transformation reaction was mixed by vortexing the tube vigorously every 15 minutes. The microcentrifuge tube was removed from the water bath and by using a sterile spreader the transformed yeast was streaked on 2 SDuracil selection media plates each containing 10 and $100 \mu \mathrm{L}$ of cell/DNA mixture. The plates were then incubated at $30^{\circ} \mathrm{C}$ for 4 days during which each transformation yielded sufficient number of discrete colonies (each transformation yielded at least 100 colonies).

\subsubsection{Starter Culture}

A starter culture was prepared from a single colony of INVSc-1 strain of $S$. cerevisiae that was grown on a plate of selection media (SD-uracil $+2 \%$ agar) ${ }^{213}$ and functionally expressing the fatty acid $\Delta^{11,13}$-desaturase/conjugase enzyme of Aleurites fordii Hemsl. A platinum loop was flamed and the colony was transferred from the plate to $2 \mathrm{~mL}$ liquid selection/induction media (SDgalactose-ura) and grown to stationary phase for 2 days at $28^{\circ} \mathrm{C}$ in a rotary shaking incubator set at $150 \mathrm{rpm}$. The starter culture was kept at $5{ }^{\circ} \mathrm{C}$ for up to 3 weeks. The selection media (solid media) for the petri plates was prepared from $20 \mathrm{~g}$ of glucose $(20 \%), 6.8 \mathrm{~g}$ of Difco bacto-Peptone $(0.7 \%)$ [Yeast nitrogen base without amino acids], $1.5 \mathrm{~g}$ of CSM-URA or "dropout powder" (1.5 g, 0.2 \%) [Complete supplemental mixture without uracil, the essential base and amino acid mixture for the yeast strain to grow] and $20 \mathrm{~g}$ of Difco bacto-agar (2\%) were dissolved in $951 \mathrm{~mL}$ of $\mathrm{ddH}_{2} \mathrm{O}$ to make a $1 \mathrm{~L}$ solution, filtered in a $2 \mathrm{~L}$ Erlenmeyer flask that was plugged with cotton wool, covered with tinfoil and autoclaved on liquid cycle for 20 minutes to sterilize. The solution was cool down to $55^{\circ} \mathrm{C}$ and poured into several $10 \mathrm{~cm}$ petri plates which were stored at $4^{\circ} \mathrm{C}$ in the dark for future use. The selection/induction 
media was prepared as follows: $20 \mathrm{~g}$ of galactose (2\%) [99 \% (D)-(+)-galactose], Difco bacto-Peptone ( $6.8 \mathrm{~g}, 0.7 \%)$ [Yeast nitrogen base without amino acids] and CSM-URA or "dropout powder" $(1.5 \mathrm{~g}, 0.2 \%)$ [Complete supplemental mixture without uracil, the essential base and amino acid mixture for the yeast strain to grow] were dissolved in 971 $\mathrm{mL}$ of $\mathrm{ddH}_{2} \mathrm{O}$ to make a $1 \mathrm{~L}$ solution, filtered in a $2 \mathrm{~L}$ Erlenmeyer flask that was capped with cotton wool, covered with tinfoil and autoclaved to sterilize. 


\section{Appendix A1.}

\section{${ }^{13} \mathrm{C}$ NMR data for methyl $\left[8-{ }^{2} \mathrm{H}_{1}\right]$-octadec-9Z,12Z-dienoate and corresponding synthetic intermediates: Deuterium Isotope Shifts (ppm)}

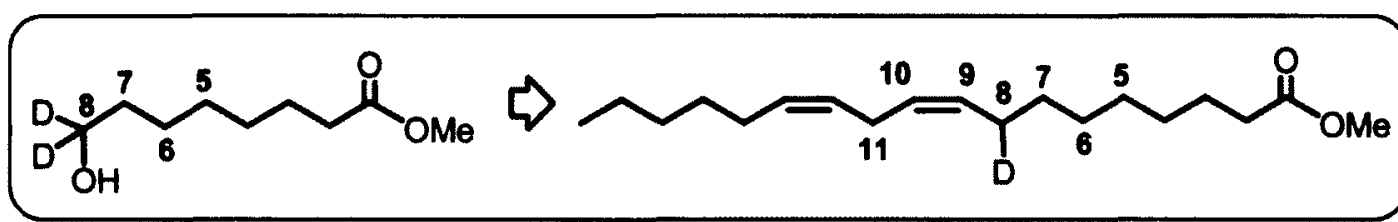

\begin{tabular}{|c|c|c|c|c|c|c|c|}
\hline Compound & $\mathrm{C} 11$ & $\mathrm{ClO}$ & C9 & C8 & $\mathrm{C} 7$ & $\mathrm{C} 6$ & $\mathrm{C} 5$ \\
\hline Et 8-OH-octanoate & & & & 62.91 & 32.67 & 25.53 & 29.05 \\
\hline Et 8-d $d_{1}-80 \mathrm{H}-$ octanoate & & & & $\begin{array}{l}62.50 \\
(\alpha:-0.40)\end{array}$ & $\begin{array}{l}32.55 \\
(\beta:-0.12)\end{array}$ & $\begin{array}{l}25.50 \\
(\gamma:-0.03)\end{array}$ & 29.04 \\
\hline Et $8-\mathrm{d}_{2}-8 \mathrm{OH}-\mathrm{octanoate}$ & & & & $\begin{array}{l}62.15 \\
(\alpha:-0.76)\end{array}$ & $\begin{array}{l}32+5 \\
(\beta:-0.22)\end{array}$ & $\begin{array}{l}25.45 \\
(\gamma:-0.05)\end{array}$ & 29.05 \\
\hline Me dec-9-ynoate & & 68.14 & 84.66 & 18.37 & 28.73 & 28.38 & 28.53 \\
\hline Me 8-d $d_{1}$-dec-9-ynoate & & $\begin{array}{l}68.18 \\
(\gamma:+0.04)\end{array}$ & $\begin{array}{l}84.63 \\
(\beta:-0.03)\end{array}$ & $\begin{array}{l}18.09 \\
(\alpha:-0.28)\end{array}$ & $\begin{array}{l}28.75 \\
(\beta:+0.02)\end{array}$ & $\begin{array}{l}28.31 \\
(\gamma:-0.07)\end{array}$ & 28.52 \\
\hline $\begin{array}{l}\text { Me octadec-9,12- } \\
\text { diynoate } \\
\text { Me 8-d,-octadec-9,12- }\end{array}$ & 9.72 & 74.58 & 80.37 & 18.71 & 29.01 & 28.65 & 28.65 \\
\hline diynoate & 9.71 & $\begin{array}{l}74.60) \\
(\gamma:+0.02)\end{array}$ & $\begin{array}{l}80.34 \\
(\beta:-0.03)\end{array}$ & $\begin{array}{l}18.41) \\
(\alpha:-0.31)\end{array}$ & $\begin{array}{l}28.02 \\
(\beta:+\mathbf{0 . 0 1})\end{array}$ & $\begin{array}{l}28.56 \\
(\gamma:-0.09)\end{array}$ & 28.63 \\
\hline $\begin{array}{l}\text { Me octadec-9Z,12Z- } \\
\text { dienoate } \\
\text { Me 8-d,-octadec-9Z }\end{array}$ & 25.64 & 128.06 & 130.06 & 27.20 & 29.60 & 29.13 & 29.17 \\
\hline 12Z-dienoate & 28.64 & $\begin{array}{l}128.08 \\
(\gamma:+0.02)\end{array}$ & $\begin{array}{l}1.30 .01 \\
(\beta:-0.05)\end{array}$ & $\begin{array}{l}26.84 \\
(\alpha:-0.36)\end{array}$ & $\begin{array}{l}24.5 / \\
(\beta:-0.09)\end{array}$ & $\begin{array}{l}29.08 \\
(\gamma:-\mathbf{0 . 0 3})\end{array}$ & 29.17 \\
\hline
\end{tabular}


Appendix A2.

\section{${ }^{13} \mathrm{C}$ NMR data for methyl $\left[11-{ }^{2} \mathrm{H}_{1}\right]-$ and $\left[11-{ }^{2} \mathrm{H}_{2}\right]-0 c t a d e c-9 Z, 12 Z$-dienoate and corresponding synthetic intermediates: Deuterium Isotope Shifts (ppm)}

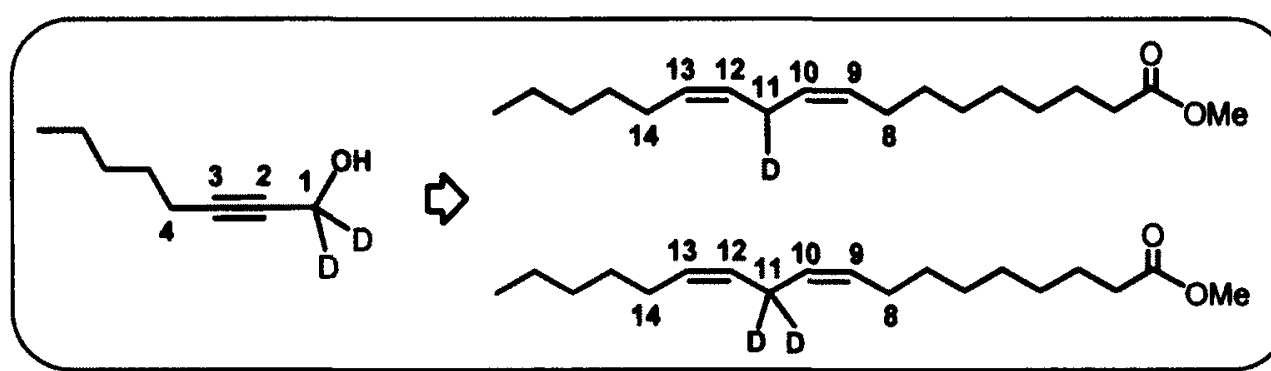

\begin{tabular}{|c|c|c|c|c|c|c|c|}
\hline Compound & $\begin{array}{l}\text { C14 } \\
\text { (C4) }\end{array}$ & $\begin{array}{l}\mathrm{C} 13 \\
\text { (C3) }\end{array}$ & $\begin{array}{l}\mathrm{C} 12 \\
\text { (C2) }\end{array}$ & $\begin{array}{l}\text { C11 } \\
\text { (C1) }\end{array}$ & $\mathrm{ClO}$ & C9 & $\mathrm{C} 8$ \\
\hline 2-octyn-1-al & 19.00 & 99.33 & 81.61 & 177.20 & & & \\
\hline$\left|-d_{1}-2-o c t y n-1-a\right|$ & 19.08 & $\begin{array}{l}99.30 \\
(\gamma:-0.03)\end{array}$ & $\begin{array}{l}81.6 n \\
(\beta:+0.05)\end{array}$ & $\begin{array}{l}176.98 \\
(\alpha:-0.22)\end{array}$ & & & \\
\hline 2-octyn-1-ol & 18.66 & 86.50 & 78.28 & 51.29 & & & \\
\hline $1-d_{1}-2-o c t y n-1-o l$ & 18.65 & $\begin{array}{l}86.49 \\
(\gamma:-0.01)\end{array}$ & $\begin{array}{l}7 \times .24 \\
(\beta:-0.04)\end{array}$ & $\begin{array}{l}51.01 \\
(\alpha:-0.28)\end{array}$ & & & \\
\hline $1,1-d_{2}-2-o c t y n-1-o l$ & 18.68 & $\begin{array}{l}86.59 \\
(\gamma:+0.09)\end{array}$ & $\begin{array}{l}78.21 \\
(\beta:-0.07)\end{array}$ & $\begin{array}{l}50.84 \\
(\alpha:-0.35)\end{array}$ & & & \\
\hline $\begin{array}{l}\text { Me octadec-9,12- } \\
\text { diynoate } \\
\text { Me } 11-d_{1} \text {-octadec-9,12- }\end{array}$ & 18.71 & 80.53 & 74.44 & 9.72 & 74.58 & 80.37 & 18.71 \\
\hline diynoate & 18.69 & $\begin{array}{l}8(1.51 \\
(\gamma:-0.02)\end{array}$ & $\begin{array}{l}74.411 \\
(\beta:-0.04)\end{array}$ & $\begin{array}{l}9.49 \\
(\alpha:-0.23)\end{array}$ & $\begin{array}{l}74.5 \\
(\beta:-0.05)\end{array}$ & $\begin{array}{l}x(0.35 \\
(\gamma:-0.02)\end{array}$ & 18.69 \\
\hline $\begin{array}{l}\text { Me II-d } d_{2} \text {-octadec-9,12- } \\
\text { diynoate }\end{array}$ & 18.68 & $\begin{array}{l}80.51 \\
(\gamma:-0.02)\end{array}$ & $\begin{array}{l}74.37 \\
(\beta:-0.07)\end{array}$ & $\begin{array}{l}9.28 \\
(\alpha:-0.44)\end{array}$ & $\begin{array}{l}74.51 \\
(\beta:-0.07)\end{array}$ & $\begin{array}{l}x(1) .35 \\
(\gamma:-0.02)\end{array}$ & 18.68 \\
\hline $\begin{array}{l}\text { Me octadec-9Z,12Z- } \\
\text { dienoate } \\
\text { Me 11-d -octadec- }\end{array}$ & 27.22 & 130.23 & 127.92 & 25.64 & 128.06 & 130.06 & 27.20 \\
\hline $9 Z, 12 Z$-dienoate & 27.22 & $\begin{array}{l}130.24 \\
(\gamma:+0.01)\end{array}$ & $\begin{array}{l}127.86 \\
(\beta:-0.06)\end{array}$ & $\begin{array}{l}25.32 \\
(\alpha:-0.32)\end{array}$ & $\begin{array}{l}128.00) \\
(\beta:-0.06)\end{array}$ & $\begin{array}{l}130.017 \\
(\gamma:+0.01)\end{array}$ & 27.20 \\
\hline $\begin{array}{l}\text { Me 11-d } \text { d }_{2} \text {-octadec- } \\
\text { 9Z,12Z-dienoate }\end{array}$ & 27.21 & $\begin{array}{l}13(1.24 \\
(\gamma:+0.01)\end{array}$ & $\begin{array}{l}127.51 \\
(\beta:-0.12)\end{array}$ & $\begin{array}{l}24.81 \\
(\alpha:-0.83)\end{array}$ & $\begin{array}{l}127.93 \\
(8:-0.13)\end{array}$ & $\begin{array}{l}l 30.07 \\
(\gamma:+0.01)\end{array}$ & 27.20 \\
\hline
\end{tabular}




\section{Appendix A3.}

${ }^{13} \mathrm{C}$ NMR data for methyl $\left[11-{ }^{2} \mathrm{H}_{1}\right]-$ octadec-9Z-enoate and corresponding synthetic intermediates: Deuterium Isotope Shifts (ppm)
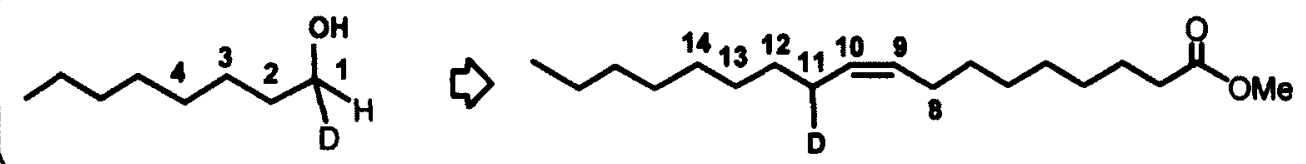

\begin{tabular}{|c|c|c|c|c|c|c|c|}
\hline Compound & $\begin{array}{l}\mathrm{Cl4} \\
\text { (C4) }\end{array}$ & $\begin{array}{l}\mathrm{C} 13 \\
\text { (C3) }\end{array}$ & $\begin{array}{l}\mathrm{C} 12 \\
\text { (C2) }\end{array}$ & $\begin{array}{l}\text { C11 } \\
\text { (C1) }\end{array}$ & $\mathrm{C} 10$ & C9 & C8 \\
\hline octan-1-ol & 29.33 & 25.70 & 32.78 & 63.07 & & & \\
\hline 1-d $\mathrm{d}_{1}$-octan-1-ol & 29.34 & 25.66 & 32.04 & 62.61 & & & \\
\hline & & $(\gamma:-0.04)$ & $(\beta:-0.14)$ & $(\alpha:-0.46)$ & & & \\
\hline $1,1-d_{2}-o c t a n-1-o l$ & 29.35 & 25.65 & 32.53 & 62.26 & & & \\
\hline & & $(\gamma:-0.05)$ & $(\beta:-0.25)$ & $(\alpha:-0.81)$ & & & \\
\hline 1-decyne & 29.10 & 28.79 & 28.52 & 18.41 & 84.76 & 68.02 & \\
\hline 3-d - -1-decyne & 29.10 & $\begin{array}{l}2 x .76 \\
(\gamma:-0.03)\end{array}$ & $\begin{array}{l}2 \times .43 \\
(\beta:-0.09)\end{array}$ & $\begin{array}{l}18.13 \\
(\alpha:-0.28)\end{array}$ & $\begin{array}{l}54.77 \\
(\beta:+0.01)\end{array}$ & $\begin{array}{l}68: 013 \\
(y:+0.01)\end{array}$ & \\
\hline \multirow{2}{*}{$\begin{array}{l}\text { Me octadec-9Z-enoate } \\
\text { Me } 11 \text { - } d_{1} \text {-octadec-9Z- } \\
\text { enoate }\end{array}$} & 29.54 & 29.34 & 29.78 & 27.22 & 130.01 & 129.76 & 27.17 \\
\hline & 29.55 & $\begin{array}{l}24.31 \\
(\gamma:-0.03)\end{array}$ & $\begin{array}{l}28,70 \\
(\beta:-0.08)\end{array}$ & $\begin{array}{l}26.8^{7} \\
(\alpha:-0.35)\end{array}$ & $\begin{array}{l}129.47 \\
(\beta:-0.04)\end{array}$ & $\begin{array}{l}1.29 .7 \gamma \\
(\gamma:+0.02)\end{array}$ & 27.18 \\
\hline
\end{tabular}


Appendix A4.

\section{${ }^{13} \mathrm{C}$ NMR data for methyl $\left[14-{ }^{2} \mathrm{H}_{1}\right]-$ and $\left[14-{ }^{2} \mathrm{H}_{2}\right]-0 \mathrm{ctadec}-9 Z, 12 Z$-dienoate and corresponding synthetic intermediates: Deuterium Isotope Shifts (ppm)}

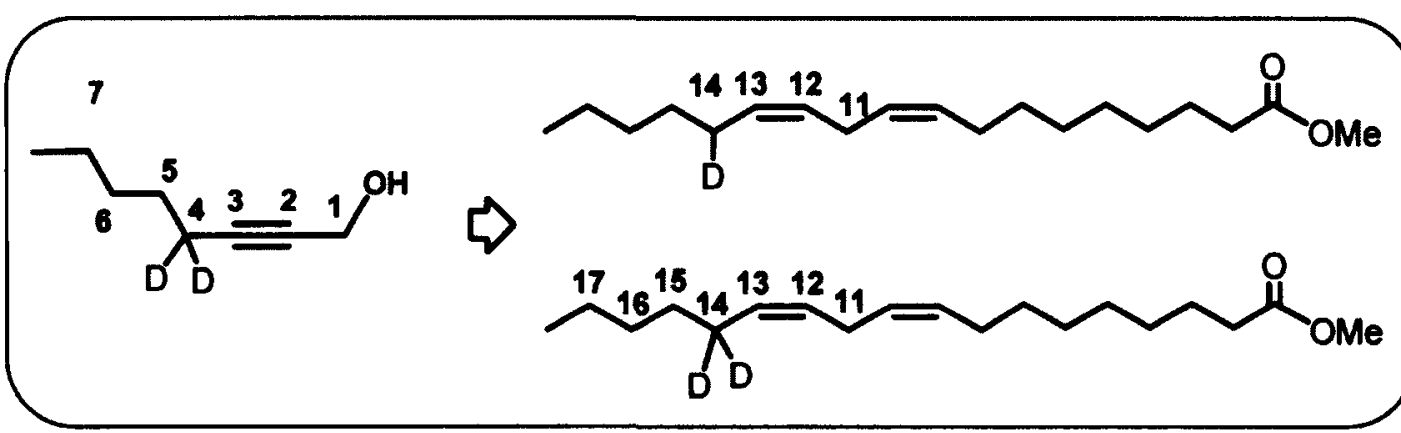

\begin{tabular}{|c|c|c|c|c|c|c|c|}
\hline Compound & $\begin{array}{l}\text { C17 } \\
\text { (C7) }\end{array}$ & $\begin{array}{l}\text { C16 } \\
\text { (C6) }\end{array}$ & $\begin{array}{l}\text { C15 } \\
\text { (C5) }\end{array}$ & $\begin{array}{l}\text { C14 } \\
\text { (C4) }\end{array}$ & $\begin{array}{l}\text { C13 } \\
\text { (C3) }\end{array}$ & $\begin{array}{l}\mathrm{C12} \\
\text { (C2) }\end{array}$ & $\begin{array}{l}\text { C11 } \\
\text { (C1) }\end{array}$ \\
\hline 2-octyn-1-ol & 22.14 & 30.99 & 28.26 & 18.64 & 86.42 & 78.27 & 51.19 \\
\hline 4-d $\mathrm{d}_{1}-2$-octyn-1-ol & 22.14 & $\begin{array}{l}30.95 \\
(\gamma:-0.04)\end{array}$ & $\begin{array}{l}28.15 \\
(\beta:-0.11)\end{array}$ & $\begin{array}{l}18.35 \\
(\alpha:-0.29)\end{array}$ & $\begin{array}{l}86.55 \\
(\beta:+0.13)\end{array}$ & $\begin{array}{l}78.23 \\
(\gamma:-0.04)\end{array}$ & 51.33 \\
\hline 4,4-d d $_{2}-2-$ octyn-1-ol & 22.14 & $\begin{array}{l}30.9 .3 \\
(\gamma:-0.06)\end{array}$ & $\begin{array}{l}28.115 \\
(\beta:-0.21)\end{array}$ & $\begin{array}{l}18.11 \\
(\alpha:-0.53)\end{array}$ & $\begin{array}{l}86.52 \\
(\beta:+0.10)\end{array}$ & $\begin{array}{l}7 k .26 \\
(\gamma:-0.01)\end{array}$ & 51.31 \\
\hline $\begin{array}{l}\text { Me octadec-9,12- } \\
\text { diynoate } \\
\text { Me } 14-d_{1} \text {-octadec-9,12- }\end{array}$ & 22.22 & 31.08 & 28.47 & 18.71 & 80.53 & 74.44 & 9.72 \\
\hline Me 14 14d & 22.23 & $\begin{array}{l}31.06 \\
(\gamma:-0.02)\end{array}$ & $\begin{array}{l}28.38 \\
(\beta ;-0.09)\end{array}$ & $\begin{array}{l}18.42 \\
(\alpha:-0.29)\end{array}$ & $\begin{array}{l}(6) .50) \\
(\beta:-0.03)\end{array}$ & $\begin{array}{l}74.47 \\
(\gamma:+0.03)\end{array}$ & 9.72 \\
\hline $\begin{array}{l}\text { Me } 14,14-d_{2} \text {-octadec- } \\
9,12 \text {-diynoate }\end{array}$ & 22.22 & $\begin{array}{l}31.113 \\
(\gamma:-0.05)\end{array}$ & $\begin{array}{l}2 \times .27 \\
(\beta:-0.20)\end{array}$ & $\begin{array}{l}18.12 \\
(\alpha:-0.59)\end{array}$ & $\begin{array}{l}80.47 \\
(\boldsymbol{\beta}:-\mathbf{0 . 0 6})\end{array}$ & $\begin{array}{l}74.47 \\
(\gamma:+0.03)\end{array}$ & 9.71 \\
\hline $\begin{array}{l}\text { Me octadec-9Z,12Z- } \\
\text { dienoate } \\
\text { Me 14-d } \\
\text {-octadec- }\end{array}$ & 22.59 & 31.54 & 29.36 & 27.22 & 130.23 & 127.92 & 25.64 \\
\hline $\begin{array}{l}\text { 9Z,12Z-dienoate } \\
\text { Me 14,14-d -octadec- }\end{array}$ & 22.59 & $\begin{array}{l}3 / .51 \\
(\gamma:-0.03)\end{array}$ & $\begin{array}{l}24.27 \\
(\beta:-0.09)\end{array}$ & $\begin{array}{l}26.86 \\
(\alpha:-0.36)\end{array}$ & $\begin{array}{l}1.301 .17 \\
(\beta:-0.06)\end{array}$ & $\begin{array}{l}127.94 \\
(\gamma:+0.02)\end{array}$ & 25.64 \\
\hline $9 Z, 12 Z$-dienoate & 22.59 & $\begin{array}{l}31.49 \\
(\gamma:-0.05)\end{array}$ & $\begin{array}{l}29.17 \\
(\beta:-0.19)\end{array}$ & $\begin{array}{l}26.50 \\
(\alpha:-0.72)\end{array}$ & $\begin{array}{l}\mid 3(1) .3 \\
(\beta:-0.10)\end{array}$ & $\begin{array}{l}127.96 \\
(\gamma:+0.04)\end{array}$ & 25.65 \\
\hline
\end{tabular}




\section{References:}

1. Buist, P. H. Nat. Prod. Rep. 2004, 21, 249.

2. Buist, P. H. In Comprehensive Natural Products II Chemistry and Biology; Mander, L.; Lui, H.-W., Eds.; Elsevier: Oxford, 2010, 1, pp. 5-33.

3. Buist, P. H. Nat. Prod. Rep. 2007, 24, 1110.

4. Buist, P. H. Wiley Encyclopedia of Chemical Biology; Begley, T. P., Ed.; John Wiley \& Sons: Hoboken, 2009, 2, pp. 1-7.

5. Gunstone, F. D.; Harwood, J. L.; Dijkstra, A. J. Lipid Handbook, $3^{\text {rd }}$ Ed.; Taylor CRC Press: Boca Raton, 2007, pp. 37-141.

6. Weete, J. D. Lipid Biochemistry of Fungi and Other Organisms, New York: Plenum Press, 1980.

7. Gurr, M. I.; Harwood, J. L. Lipid Biochemistry: An Introduction, $4^{\text {th }}$ ed., Chapman and Hall: London, 1991.

8. Millar, A. A.; Smith, M. A.; Kunst, L. Trends Plants Sci. 2000, 5, 95.

9. Jaworski, J.; Cahoon, E. B. Curr. Opin. Plant Biol. 2003, 6, 178.

10. Lee, M.; Lenman, M.; Banas, A.; Bafor, M.; Singh, S. et al Science, 1998, 280, 915.

11. Cahoon, E. B.; Kinney, A. J. J. Biol. Chem. 2004, 279, 12495.

12. Van de Loo, F. J.; Broun, P.; Turner, S.; Summerville, C. Proc. Natl. Acad. Sci. USA, 1995, 92, 6743 .

13. Van de Loo, F. J.; Fox, B. G.; Somerville, C. Lipid Metabolism in Plants; Moore, T. S. Jr., Ed., CRC Press: Boca Raton, 1993, pp. 91-126.

14. Hoffmann-Roder, A.; Krause, N. Angew. Chem. Int. Ed. 2004, 43, 1196.

15. Bagby, M. O.; Jr. Smith, C. R.; Wolff, I. A. J. Org. Chem. 1965, 30, 4227.

16. Broadwater, J. A.; Whittle, E.; Shanklin, J. J. Biol. Chem. 2002, 277, 15613.

17. Lu, C.; Wallis, J. G.; Browse, J. BMC Plant Biol. 2007, 7, 42.

18. Nunn, J. R. J. Chem. Soc. 1952, 313. 
19. Yano, I.; Morris, L. J.; Nichols, B. W.; James, A. T. Lipids, 1972, 7, 35.

20. Gomez, F. E.; Bauman, D. E.; Ntambi, J. M.; Fox, B. G. Biochim. Biophys. Res. Commun. 2003, 300, 316.

21. Jeffcoat, R.; Pollard, M. R. Lipids, 1977, 12, 480.

22. Johnson, A. R.; Pearson, J. A.; Shenstone, F. S.; Fogert, A. C. Nature, 1967, 214, 1244.

23. Fogert, A.C.; Johnson, A. R.; Pearson. J. A. Lipids, 1972, 7, 335.

24. Billault, I.; Duan, J. R.; Guiet, S.; Robins, R. J. J. Biol. Chem. 2005, 280, 17645.

25. Cahoon, E. B.; Ripp, K. G.; Hall, S. E.; McGonigle, B. Plant Physiol. 2002, 128, 615.

26. Reed, D. W.; Polichuk, D. R.; Buist, P. H.; Ambrose, S. J. et al J. Am. Chem. Soc. 2003, 125, 10635.

27. Minto, R. E.; Blacklock, B. J. Prog. Lipid Res. 2008, 47, 233.

28. Cahoon, E. B.; Schnurr, J. A.; Huffman, E. A.; Minto, R. E. Plant J. 2003, 34, 671.

29. Sperling, P.; Lee, M.; Thomas, G.; Zahringer, U.; Stymne, S.; Heinz, E. Eur. J. Biochem. 2000, 267, 3801 .

30. Bohlmann, F.; Burkhardt, T.; Zdero, C. Naturally Occurring Acetylenes; Academic Press: London, 1973.

31. Christensen, L. P.; Lam, J. Phytochemistry, 1991, 30, 2453.

32. Shi Shun, A. L. K.; Tykwinski, R. R. Angew. Chem. Int. Ed. 2006, 45, 1034.

33. Damste, J. S. S.; Strous, M.; Rijpstra, W. I.; Hopmans, E. C.; Geenevasen, J. A.; Van Duin, A. C.; Van Niftrik, L. A.; Jetten, M. S. Nature, 2002, 419, 708.

34. Dyer, J. M.; Chapital, D. C.; Kuan, J. et al Plant Physiol. 2002, 130, 2027.

35. Corl, B. A.; Baumgard, L. H.; Dwyer, D. A.; Griinari, J. M.; Phillips, B. S.; Bauman, D. E. J. Nutr. Biochem. 2001, 12, 622.

36. Zheng, W.; Wise, M.; Wyrick, A. Metz, J.; Yuan, L.; Gerwick, W. Arch. Biochem. Biophys. 2002, 401, 11 . 
37. Wise, M. L.; Hamberg, M.; Gerwick, W. H. Biochemistry, 1994, 33, 15223.

38. Griinari, J. M.; Bauman, D. E. Advances in Conjugated Linoleic Acid Research; AOCS Press, Champaign, IL, pp. 180-200.

39. Rodriguez, S.; Camps, F.; Fabrias, G. J. Org. Chem. 2001, 66, 8052.

40. Rodriguez, S.; Clapes, P.; Camps, F.; Fabrias, G. J. Org. Chem. 2002, 67, 2228.

41. Serra, M.; Pina, B.; Abad, J. L.; Camps, F.; Fabrias, G. Proc. Natl. Acad. Sci. USA, 2007, 104, 16444.

42. Liu, L.; Hammond, E. G.; Nikolau, B. J. Plant Physiol. 1997, 113, 1343.

43. Crombie, L.; Holloway, S. L. J. Chem. Soc. Perkin Trans. 1 1985, 2425.

44. Booth, R. G.; Kon, S. K.; Dann, W. J.; Moore, T. Biochem. J. 1935, 29, 133.

45. Parodi, P. W. J. Dairy Sci. 1977, 60, 1550.

46. Yuan, G.; Sun, H.; Sinclair, A. J.; Li, D. Eur. J. Lipid Sci. Technol. 2009, 111, 537.

47. Park, Y.; Pariza, M. W. Food Res. Int. 2007, 40, 311.

48. Chardingy, J. M.; Hasselwander, O.; Genty, M.; Kraemer, M.; Ptock, A.; Sebedio, J. L. Lipids, 2003, 38(9), 895.

49. Liavonchanka, A.; Hornung, E.; Feussner, I.; Rudolph, M. G. Proc. Natl. Acad. Sci. USA, 2006, 103(8), 2576-2581.

50. Park, Y. J. Food Comp. Anal. 2009, 225, S4.

51. Corl, B. A.; Barbano, D. M.; Bauman, D. E.; Ip, C. J. Nutr. Biochem. 2003, 133, 2893.

52. Belury, M. A.; Nutr. Rev. 1995, 53, 83.

53. Park, Y.; Albright, K. J.; Storkson, J. M.; Liu, W.; Pariza, M. W. J. Food Sci. 2007, 72, S612.

54. Kelly, N. S.; Hubbard, N. E.; Erickson, K. L. J. Nutr. 2007, 137, 2599.

55. Bauman, D. E.; Baumgard, L. H.; Corl, B. A.; Griinari, J. M. Proc. Am. Soc. Animal Sci. 1999, 1. 
56. Kemp, P.; Lander, D. J. J. Gen. Microbiol. 1984, 130, 527; Kepler, C. R.; Hirons, K. P. McNeill, J. J.; Tove, S. B. J. Biol. Chem. 1966, 241, 1350.

57. Ha, Y. L.; Grimm, N. K.; Pariza, M. W. Carcinogenesis, 1987, 8, 1881.

58. Pariza, M. W.; Hargraves, W. A. Carcinogenesis, 1985, 6, 591.

59. Tsuzuki, T.; Tokuyama, Y.; Igarashi, M. et al J. Nutr. 2004, 134, 2634.

60. Igarashi, M.; Miyazawa, T. Cancer Lett. 2000, 148, 173.

61. Tsuzuki, T.; Tokuyama, Y.; Igarashi, M.; Miyazawa, T. Carcinogenesis, 2004, $25,1417$.

62. Cahoon, E. B.; Dietrich, C. R.; Meyer, K. Damude, H. G.; Dyer, J. M.; Kinney, A. J. Phytochemistry, 2006, 67, 1166.

63. Cahoon, E. B.; Ripp, K. G.; Hall, S. E.; Kinney, A. J. J. Biol. Chem. 2001, 276, 2637.

64. Hennessy, A. A.; Ross, R. P.; Devery, R. Lipids, 2011, 46, 105.

65. Koba, K.; Akahoshi, A.; Yamasaki, M. Tanaka, K. et al Lipids, 2002, 37, 343.

66. Yamasaki, M.; Kitagawa, T.; Koyanagi, N. et al Nutrition, 2006, 22, 54.

67. Al-Madaney, M. M.; Kramer, J. K.; Deng, Z.; Vanderhoek, J. Y. Biochim. Biophys. Acta, 2003, 1635, 75.

68. Li, G.; Barnes, D.; Butz, D.; Bjorling, D.; Cook, M. E. J. Lipid Res. 2005, 46, 2134.

69. Lai, C.; Yin, J.; Li, D.; Zhao, L.; Chen, X. Archives Animal Nutr. 2005, 59, 41.

70. Ritzenthaler, K. L.; McGuire, M. K. et al J. Nutr. 2001, 131, 1548.

71. Serra, M.; Pina, B.; Bujons, J.; Camps, F.; Fabrias, G. Insect Biochem. Mol. Biol. 2006, 36, 634 .

72. Oldham, N. J.; Boland, W. Naturwissrnschaften, 1996, 83, 248.

73. Howard, R. W.; Blomquist, G. J. Ann. Rev. Entomol. 2005, 50, 371.

74. Tillman, J. A.; Seybold, S. J.; Jurenka, R. A.; Blomquist, G. J. Insect Biochem. Mol. Biol. 1999, 29, 481. 
75. Abad, J. L.; Camps, F.; Fabrias, G. J. Am. Chem. Soc. 2007, 129, 15007.

76. Rodriguez, S.; Hao, G.; Liu, W.; Pina, B.; Rooney, A. P. Camps, F.; Roelofs, W. L.; Fabrias, G. Insect Biochem. Mol. Biol. 2004, 34, 1315.

77. Serra, M.; Gauthier, L. T.; Fabrias, G.; Buist, P. H. Insect Biochem. Mol. Biol. 2006, 36, 822 .

78. Villorbina, G.; Roura, L.; Camps, F.; Joglar, J.; Fabrias, G. J. Org. Chem. 2003, $68,2820$.

79. Abad, J. L.; Camps, F.; Fabrias, G. Angew. Chem. Int. Ed. 2000, 39, 3279.

80. Moto, K.; Suzuki, M. G.; Hull, J. J.; Kurata, R. et al Proc. Natl. Acad. Sci. USA, 2004, 101, 8631.

81. Abad, J. L.; Villorbina, G.; Fabrias, G.; Camps, F. J. Org. Chem. 2004, 69, 7108.

82. Smith, C. R. Jr. In Progress in the Chemistry of Fats and Other Lipids; Holman, R. T. Ed.; Pergammon: London, 1970, 11, 137.

83. Napier, J. A. Annu. Rev. Plant Biol. 2007, 58, 295.

84. Cahoon, E. B.; Shockey, J. M.; Dietrich, C. R.; Gidda, S. K.; Mullen, R. T.; Dyer, J. M. Curr. Opin. Plant Biol. 2007, 10, 236.

85. Broun, P.; Gettner, S.; Somerville, C. Annu. Rev. Nutr. 1999, 19, 197.

86. Cloez, Compt. Rend., 1875, 81, 469; 1876, 82, 501; 1876, 83, 943.

87. Boeseken and Ravenswaay, Rec. Trav. Chim., 1925, 44, 241.

88. Crombie, L.; Jacklin, A. G. J. Chem. Soc. 1957, 1632.

89. Yang, P.; Li, X.; Shipp, M.; Shockey, J. M.; Cahoon, E. B. BMC Plant Biol. 2010, $10,250$.

90. Hopkins, C. Y.; Chisholm, M. J. J. Am. Oil. Chem. Soc. 1968, 45, 176.

91. Chisholm, M. J.; Hopkins, C. Y. Can. J. Biochem. 1967, 45, 1081.

92. Cahoon, E. B.; Carlson, T. J.; Ripp, K. G. et al, Proc. Natl. Acad. Sci. USA, 1999, 96, 12935.

93. Qiu, X.; Reed, D. W.; Hong, H.; Mackenzie, S. L.; Covello, P. S. Plant Physiol. 2001, $125,847$. 
94. Gunstone, F. D.; Subbarao, R. Chem. Phys. Lipids, 1967, 1, 349.

95. Bagby, M. O.; Smith, C. R. Jr.; Wolff, I. A. Lipids, 1966, 1, 263.

96. Formo, M. W. In Bailey's Industrial Oil and Fat Products; Swern, D. Ed.; John Wiley \& sons: New York, 1979, pp. 708-709.

97. Sonntag, N. O. V. In Bailey's Industrial Oil and Fat Products; Swern, D. Ed.; John Wiley \& sons: New York, 1979, pp. 289-477.

98. Yasui, Y.; Hosokawa, M.; Kohno, H.; Tanaka, T.; Miyashita, K. Anticancer Res. 2006, 26, 1855.

99. Brown, D. J.; Dattner, A. M. Archives of dermatology, 1998, 134, 1401.

100. Ley, S. V.; Meek, G. J. Chem. Soc. Perkin Trans. 1, 1997, 1125.

101. Palmer, C. N. A.; Wolf, C. R. FEBS Letters, 1998, 431, 476.

102. Steenbergen, R. H. G.; Drummen, G. P. C.; Kamp, J. A. F.; Post, J. A. Biochim. Biophys. Acta, 1997, 1330, 127.

103. Zaheer, A.; Sahu, S. K.; Ryken, T. C.; Traynelis, V. C. Neurochem. Res. 2007, 32, 115-124.

104. Shanklin, J.; Cahoon, E. B. Annu. Rev. Plant Physiol. Plant Mol. Biol. 1998, 49, 611.

105. Brenner, R. R. Prostaglandins, Leukotrienes Essent. Fatty Acids, 2003, 68, 71.

106. Breslow, R. Chem. Soc. Rev. 1972, 1, 553.

107. Bloomfield, D. K.; Bloch, K. Biochem. Biophys. Acta, 1958, 30, 220.

108. Bloomfield, D. K.; Bloch, K. J. Biol. Chem. 1960, 235, 337.

109. Schroepfer, G. J.; Bloch, K. J. Biol. Chem. 1965, 240, 54.

110. Behrouzian, B.; Buist, P. H. Curr. Opin. Chem. Biol. 2002, 6, 577.

111. Behrouzian, B.; Fauconnot, L.; Daligault, F.; Nugier-Chauvin, C.; Patin, H.; Buist, P. H. Eur. J. Biochem. 2001, 268, 3545.

112. Buist, P. H.; Behrouzian, B. J. Am. Chem. Soc. 1998, 120, 871. 
113. Fox, B. G.; Shanklin, J.; Ai, J.; Loehr, T. M.; Sanders-Loehr, J. Biochemistry, 1994, 33, 12776.

114. Shanklin, J.; Whittle, E.; Fox, B. G. Biochemistry, 1994, 33, 12787.

115. Shanklin, J.; Achim, C.; Schmidt, H.; Fox, B. G.; Munck, E. Proc. Natl. Acad. Sci. USA, 1997, 94, 2981.

116. Whittle, E. J.; Tremblay, A. E.; Buist, P. H.; Shanklin, J. Proc. Natl. Acad. Sci. USA, 2008, 105, 14738.

117. Guy, J. E.; Whittle, E. J.; Kumaran, D.; Lindqvist, Y.; Shanklin, J. J. Biol. Chem. 2007, 282, 19863.

118. Lindqvist, Y.; Huang, W.; Schneider, G.; Shanklin, J. EMBO J. 1996, 15, 4081.

119. Moche, M.; Shanklin, J.; Ghoshal, A.; Lindqvist, Y. J. Biol. Chem. 2003, 278, 25072.

120. Dyer, D. H.; Lyle, K. S.; Rayment, I.; Fox, B. G. Protein Sci. 2005, 14, 1508.

121. Cahoon, E. B.; Shanklin, J.; Ohlrogge, J. B. Proc. Natl. Acad. Sci. USA, 1992, 89, 11184.

122. Cahoon, E. B.; Becker, C. K.; Shanklin, J.; Ohlrogge, J. B. Plant Physiol. 1994, $106,807$.

123. Cahoon, E. B.; Cranmar, A. M.; Shanklin, J.; Ohlrogge, J. B. J. Biol. Chem. 1994, $269,27519$.

124. Shanklin, J.; Somerville, C. Proc. Natl. Acad. Sci. USA, 1991, 88, 2510.

125. Thompson, G. A.; Scherer, D. E.; Aken, S. F.; Kenny, J. W. Proc. Natl. Acad. Sci. USA, 1991, 88, 2578.

126. Baik, M. H.; Newcomb, M.; Friesner, R. A.; Lippard, S. J. Chem. Rev. 2003, 103, 2385.

127. Behrouzian, B.; Savile, C. K.; Dawson, B.; Buist, P. H.; Shanklin, J. J. Am. Chem. Soc. 2002, 124, 3277.

128. Strittmatter, P.; Spatz, L.; Corcoran, D.; Rogers, M. J.; Setlow, B.; Redline, R. Proc. Natl. Acad. Sci. USA, 1974, 71, 4565.

129. Enoch, H. G.; Catala, A.; Strittmatter, P. J. Biol. Chem. 1976, 251, 5095. 
130. Mason, H. S. Adv. Enzymol. 1957, 19, 79.

131. Shanklin, J.; Achim, C.; Schmidt, H.; Fox, B.; Munck, E. Proc. Natl. Acad. Sci. USA, 1997, 94, 2981.

132. Sperling, P.; Heinz, E. Eur. J. Lipid Sci. Technol. 2001, 103, 158.

133. Thiede, M.; Ozols, J.; Strittmatter, P. J. Biol. Chem. 1986, 261, 13230.

134. Stukey, J. E.; McDonough, V. M.; Martin, C. E. J. Biol. Chem. 1990, 265, 20144.

135. Mayer, K. M.; McCorkle, S. R.; Shanklin, J. BMC Bioinformatics, 2005, 2, 284.

136. Heinz, E. Biosynthesis of Ployunsaturated Fatty Acids. In Lipid Metabolism in Plants; T. S. Morre, Jr., Ed.; CRC Press: Boca Raton, 1993; pp 33-89.

137. Schwartzbeck, J. L.; Jung, S.; Abbott, A. G. et al Phytochemistry, 2001, 57, 643.

138. Covello, P. S.; Reed, D. W. Plant Physiol. 1996, 111, 223.

139. Pinilla, A.; Camps, F.; Fabrias, G. Biochemistry, 1999, 38, 15272.

140. Morris, L. J.; Harris, R. V.; Kelly, W.; James, A. T. Biochem. J. 1968, 19, 673.

141. Shu, L.; Nesheim, J. C.;Kauffmann, K.; Munck, E.; Lipscomb, J. D.; Que, L. Jr., Science 1997, 275, 515.

142. Cook, G. K.; Mayer, J. M. J. Am. Chem. Soc. 1994, 116, 1855.

143. Light, R. J.; Lennarz, W. J.; Bloch, K. J. Biol. Chem. 1962, 237, 1793.

144. Brett, D.; Howling, D.; Morris, L. J.; James, A. T. Arch Biochem Biophys. 1971, 143, 535.

145. Stoffel, W.; Assmann, G.; Bister, K. Hoppe-Seyler's Z. Physiol. Chem. 1971, 352, 1531.

146. Navarro, I. F.; Fabrias, G.; Camps, F. J. Am. Chem. Soc. 1997, 119, 11335.

147. Rawlings, L.; Reese, P. B.; Ramer, S. E.; Vederas, J. C. J. Am. Chem. Soc. 1989, 111,3382 .

148. McInnes, A. G.; Walter, J. A.; Wright, J. L. C. Tetrahedron, 1983, 39, 3515.

149. Buist, P. H.; Marecak, D. M. J. Am. Chem. Soc., 1991, 113, 5877. 
150. Buist, P. H.; Marecak, D. M. Can. J. Chem. 1994, 72, 176.

151. Buist, P. H.; Marecak, D. M. J. Am. Chem. Soc. 1992, 114, 5073.

152. Pirkle, W. H.; Beare, S. D.; Muntz, R. L. Tett. Lett. 1974, 26, 2295.

153. Tremblay, A. E.; Tan, N.; Whittle. E.; Hodgson, D. J.; Dawson, B.; Buist, P. H.; Shanklin, J. Org. Biomol. Chem. 2010, 8, 1322.

154. Seco, J. M.; Latypov, S.; Quinoa, E.; Riguera, R. Tett. Lett. 1994, 35, 2921.

155. Lao, K. Y. Y.; Hodgson, D. J.; Dawson, B.; Buist, P. H. Bioorg. Med. Chem. Lett. 2005, 15, 2799.

156. Tremblay, A. E.; Lao, K. Y. Y.; Hodgson, D. J.; Dawson, B.; Buist, P. H. Bioorg. Med. Chem. Lett. 2009, 19, 5146.

157. Buist, P. H.; Behrouzian, B. J. Am. Chem. Soc. 1996, 118, 6295.

158. Reed, D. W.; Savile, C. K.; Qiu, X; Buist, P. H.; Covello, P. S. Eur. J. Biochem. 2002, 269, 5024.

159. Beckmann, C.; Rattke, J.; Oldham, N. J.; Sperling, P.; Heinz, E.; Boland, W. Angew. Chem. Int. Ed. 2002, 41, 2298.

160. Jones, J. P.; Trager, W. F. J. Am. Chem. Soc. 1987, 109, 2171.

161. Behrouzian, B.; Buist, P. H.; Shanklin, J. Chem. Commun. 2001, 401.

162. Lyle, K. S.; Haas, J. A.; Fox, B. Biochemistry, 2003, 42, 5857.

163. Fauconnot, L.; Buist, P. H. J. Org. Chem. 2001, 66, 1210.

164. Buist, P. H.; Marecak, D. M.; Dawson, B.; Black, B. Can. J. Chem. 1996, 74, 453.

165. Hodgson, D. J.; Lao, K. Y. Y.; Dawson, B.; Buist, P. H. Helv. Chim. Acta, 2003, 86,3688 .

166. Behrouzian, B.; Hodgson, D.; Savile, C. K.; Dawson, B.; Buist, P. H.; Shanklin, J. Mag. Res. Chem. 2002, 40, 524.

167. Gakh, Y. G.; Gakh, A. A.; Gronenborn, A. M. Mag. Res. Chem., 2000, 38, 551.

168. Carvalho, F.; Gauthier, L. T.; Hodgson, D. J.; Dawson, B.; Buist, P. H. Org. Biomol. Chem. 2005, 3, 3979. 
169. Hodgson, D. J.; Buist, P. H. Tetrahedron Asymmetry, 2003, 14, 641.

170. Zhou, J.; Kelly, E. L.; Bachmann, B. O.; Gunsior, M.; Townsend, C. A.; Solomon, E. I. J. Am. Chem. Soc. 2001, 123, 7388.

171. Rettie, E.; Rettenmeier, A. W.; Howald, W. N.; Baillie, T. A. Science, 1987, 235, 890.

172. Broun, P.; Shanklin, J.; Whittle, E.; Somerville, C. Science, 1998, 282, 1315.

173. Crombie, L.; Heavers, A. D. J. Chem. Soc. Perkin Trans. 1, 1992, 1929.

174. Schwab, J. M.; Klassen, J. B. J. Am. Chem. Soc. 1984, 106, 7217.

175. Durand, S.; Parrain, J.; Santelli, M. J. Chem. Soc. Perkin Trans. 1, 2000, 253.

176. Jeffery, T.; Gueugnot, S.; Linstrumelle, G. Tett. Lett. 1992, 33, 5757.

177. Caruso, T.; Spinella, A. Tetrahedron, 2003, 59, 7787.

178. Tedeschi, C.; Saccavini, C.; Maurette, L.; Soleilhavoup, M.; Chauvin, R. J. Organomet. Chem. 2003, 670, 151.

179. Fujita, K.; Yorimitsu, H.; Shinokubo, H.; Oshima, K. J. Am. Chem. Soc. 2004, 126,6776 .

180. Fu, Y.; Weng, Y.; Hong, W.; Zhang, Q. Synlett. 2011, (6), 809.

181. Jacquot, C.; McGinley, C. M.; Plata, E.; Holman, T. R.; van der Donk, W. A. Org. Biomol. Chem. 2008, 6, 4242.

182. Savile, C. K.; Reed, D. W.; Meesapyodsuk, D.; Covello, P. S.; Buist, P. H. J. Chem. Soc., Perkin Trans. 1, 2001, 1116.

183. Mancuso, A. J.; Huang, S.; Swern, D. J. Org. Chem. 1978, 43, 2480.

184. Rawlings, B. J.; Reese, P. B.; Ramer, S. E.; Vederas, J. C. J. Am. Chem. Soc. $1989,111,3382$.

185. Midland, M. M.; Greer, S.; Tramontano, A.; Zderic, S. A. J. Am. Chem. Soc. 1979, 101, 2352; Midland, M. M.; Tramontano, A.; Zderic, S. A. J. Am. Chem. Soc. 1977, 99, 5211.

186. Lorca, M.; Kuhn, D.; Kurosu, M. Tett. Lett. 2001, 42, 6243. 
187. Chambez, E. M.; Homing, E. C. Anal. Letters, 1968, 1, 201; Klebe, J. F. J. Am. Chem. Soc. 1966, 88, 3390.

188. Midland, M. M.; McDowell, D. C.; Hatch, R. L.; Tramontano, A. J. Am. Chem. Soc. 1980, 102, 867.

189. Dale, J. A.; Dull, D. L.; Mosher, H. S. J. Org. Chem. 1969, 34, 2543-2549; Dale, J. A.; Mosher, H. S. J. Am. Chem. Soc. 1973, 95, 512.

190. Spinella, A.; Caruso, T.; Martino, M.; Sessa, C. Synlett. 2001, (12), 1971.

191. Presser, A.; Hüfner, A. Monatshefte für Chemie, 2004, 135, 1015.

192. Gunstone, F. D.; Pollard, M. R.; Scrimgeour, C. M.; Vedanayagam, H. S. Chem. Phys. Lipids, 1977, 18, 115.

193. Hallgren, B.; Ryhage, R.; Stenhagen, E. Acta. Chem. Scand. 1959, 13, 845.

194. Gunstone, F. D.; Lie Ken Jie, M.; Wall, R. T. Chem. Phys. Lipids, 1969, 3, 297.

195. Nagel, Z. D.; Klinman, J. P. Chem. Rev. 2006, 106, 3095.

196. Fletcher, S.; Ahmad, A.; Perouzel, E.; Heron, A. et al J. Med. Chem. 2006, 49, 349.

197. Frost, D. J.; Gunstone, F. D. Chem. Phys. Lipids, 1975, 15, 53.

198. Kuroda, H.; Hanaki, E.; Izawa, H.; Kano, M.; Itahashi, H. Tetrahedron, 2004, 60, 1913.

199. Melander, L.; Saunders, W. H. Jr. Reaction Rates of Isotopic Molecules, $2^{\text {nd }}$ Ed.; Wiley and Sons: Hoboken, 1979, pp 91-129.

200. Fritsche, K.; Hornung, E.; Peitzsch, N.; Renz, A.; Feussner, I. FEBS Letters, 1999, 462, 249.

201. Northrop, D. B. Annu. Rev. Biochem. 1981, 50, 103.

202. Schomaker, J. M.; Pulgam, V. R.; Borhan, B. J. Am. Chem. Soc. 2004, 126, 13600 .

203. Iwasaki, G.; Sano, M.; Sodeoka, M.; Yoshida, K.; Shibasaki, M. J. Org. Chem. 1988, 53, 4864.

204. Griffith, W. P.; Kwong, E. Synth. Commun. 2003, 33, 2945. 
205. Ashby, E. C.; Goel, A. B. J. Org. Chem. 1981, 46, 3936.

206. Parker, D. J. Chem. Soc. Perkin Trans. II, 1983, 83.

207. Maskill, H. The Physical Basis of Organic Chemistry, Oxford University Press, USA, 1985, pp 367-404.

208. Dussault, P. H.; Eary, C. Y.; Woller, K. R. J. Org. Chem. 1999, 64, 1789.

209. Olah, G. A.; Prakash, G. K. S.; Arvanaghi, M. Synthesis, 1984, (3), 228.

210. Ashby, E. C.; Goel, A. B. J. Org. Chem. 1981, 46, 3936.

211. Savile, C. K.; Reed, D. W.; Buist, P. H. et al J. Chem. Soc. Perkin Trans. I, 2001, 1116.

212. Sakai, T.; Hamamoto, H.; Mori, K. Agri. Biol. Chem. 1986, 50, 1621.

213. Dauenpen, M.; Reed, D. W.; Savile, C. K.; Buist, P. H. et al Biochemistry, 2000, 39, 11948. 